\title{
IntechOpen
}

\section{Current Directions in ADHD and Its Treatment}

\author{
Edited by Jill M. Norvilitis
}





\section{CURRENT DIRECTIONS IN ADHD AND ITS TREATMENT}

Edited by Jill M. Norvilitis 


\section{Current Directions in ADHD and Its Treatment}

http://dx.doi.org/10.5772/1213

Edited by Jill M. Norvilitis

\section{Contributors}

Somnath Banerjee, Nada Pop-Jordanova, Mohammad Ali Nazari, Jose Armando Vidarte Claros, Consuelo Vélez Alvarez, Lise Aagaard, Ebba Holme Hansen, Sarina Grosswald, Fred Travis, Goran B. W. Soderlund, Sverker Sikstrom, Claire Advokat, Christine Vinci, Nitin Patel, Harsha Patel, Mita Patel, Antigone Papavasiliou, Irene Nikaina, Georgia Kleidaria, Aspa Paspali, Julio Cesar Flores Lazaro, María Alejandra Salgado Soruco, Paul Glaser, Greg Gerhardt, Barbara Felt, Elizabeth Hastings, Gideon David Charach, Tilla Olivier, Ana Gomes

\section{(c) The Editor(s) and the Author(s) 2012}

The moral rights of the and the author(s) have been asserted.

All rights to the book as a whole are reserved by INTECH. The book as a whole (compilation) cannot be reproduced, distributed or used for commercial or non-commercial purposes without INTECH's written permission.

Enquiries concerning the use of the book should be directed to INTECH rights and permissions department (permissions@intechopen.com).

Violations are liable to prosecution under the governing Copyright Law.

\section{(c) BY}

Individual chapters of this publication are distributed under the terms of the Creative Commons Attribution 3.0 Unported License which permits commercial use, distribution and reproduction of the individual chapters, provided the original author(s) and source publication are appropriately acknowledged. If so indicated, certain images may not be included under the Creative Commons license. In such cases users will need to obtain permission from the license holder to reproduce the material. More details and guidelines concerning content reuse and adaptation can be foundat http://www.intechopen.com/copyright-policy.html.

\section{Notice}

Statements and opinions expressed in the chapters are these of the individual contributors and not necessarily those of the editors or publisher. No responsibility is accepted for the accuracy of information contained in the published chapters. The publisher assumes no responsibility for any damage or injury to persons or property arising out of the use of any materials, instructions, methods or ideas contained in the book.

First published in Croatia, 2012 by INTECH d.o.o.

eBook (PDF) Published by IN TECH d.o.o.

Place and year of publication of eBook (PDF): Rijeka, 2019.

IntechOpen is the global imprint of IN TECH d.o.o.

Printed in Croatia

Legal deposit, Croatia: National and University Library in Zagreb

Additional hard and PDF copies can be obtained from orders@intechopen.com

Current Directions in ADHD and Its Treatment

Edited by Jill M. Norvilitis

p. cm.

ISBN 978-953-307-868-7

eBook (PDF) ISBN 978-953-51-6736-5 


\section{We are IntechOpen, \\ the world's leading publisher of Open Access books}

Built by scientists, for scientists

\section{$4,100+$}

Open access books available

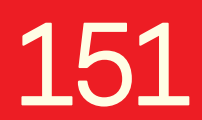

Countries delivered to
$116,000+$

International authors and editors
$120 \mathrm{M}+$

Downloads

Our authors are among the

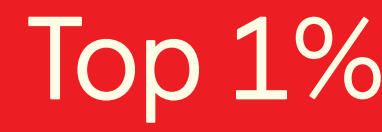

most cited scientists

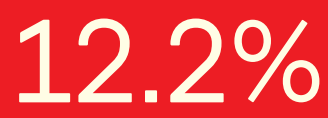

Contributors from top 500 universities

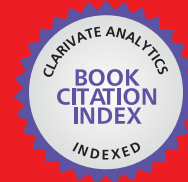

WEB OF SCIENCE ${ }^{\mathrm{TM}}$

Selection of our books indexed in the Book Citation Index in Web of Science ${ }^{\mathrm{TM}}$ Core Collection (BKCI)

Interested in publishing with us?

Contact book.department@intechopen.com

Numbers displayed above are based on latest data collected.

For more information visit www.intechopen.com

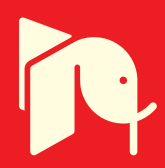





\section{Meet the editor}

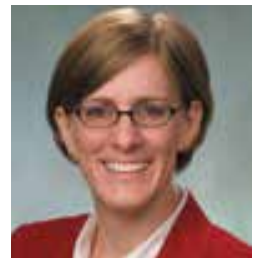

Dr Jill M. Norvilitis PhD is a Professor of Psychology at Buffalo State College in Buffalo, NY. She earned her undergraduate degree in psychology from Hope College in Holland, Michigan and her MA and PhD in Clinical Psychology from Wayne State University in Detroit, Michigan. In 2010, she was recognized with the SUNY Chancellor's Award for Excellence in Teaching. Dr.

Norvilitis is a Clinical Psychologist who specializes in behavior disorders of childhood. Her research focuses on predictors of social and academic adjustment among college students with Attention Deficit Hyperactivity Disorder (ADHD) and perceptions of ADHD cross-culturally. She is the author of over 30 journal articles, book chapters, and edited volumes and she has given over 80 conference and invited presentations. 



\section{Contents}

Preface XI

Part 1 Treatment Considerations 1

Chapter 1 ADHD in Children and Adolescents:

A Good Practice Guidance 3

Somnath Banerjee

Chapter 2 ADHD and Comorbid Conditions 25

Nitin Patel, Mita Patel and Harsha Patel

Chapter 3 Comorbidity in ADHD:

A Neuropsychological Perspective 47

Julio César Flores Lázaro and María Alejandra Salgado Soruco

Chapter 4 ADHD and Sleep Problems in Children 61

Elizabeth Hastings and Barbara T. Felt

Part 2 Psychopharmacology: Mechanisms and Effects 8

Chapter 5 The Neuropsychopharmacology

of Stimulants: Dopamine and ADHD 91

Paul E.A. Glaser and Greg A. Gerhardt

Chapter 6 Trends in the Prescribing and Adverse

Drug Reactions Patterns of Psychostimulants

Among Danish Children and Adolescents 111

Lise Aagaard and Ebba Holme Hansen

Chapter 7 Do Stimulant Medications for Attention-Deficit /Hyperactivity Disorder (ADHD) Enhance Cognition? 125

Claire Advokat and Christine Vinci

Chapter 8 Motor Skills in Children with ADHD:

Comparative Study from the Farmacological Treatment 157

Jose Armando Vidarte Claros and Consuelo Velez Alvarez 
Chapter 9 Methylphenidate and Dyslipidemia 185

Gideon Charach, Nechemia Kaysar, Alexander Rabinovich, Ori Argov and Moshe Weintraub

Part 3 Non-Medication Interventions 193

Chapter 10 ADHD and Stress: The Role of Meditation to Reduce Stress, and Improve Brain Function and Behavior Regulation 195 Sarina J. Grosswald and Fred Travis

Chapter 11 The Value of Coached Behaviour Modification in the Effective Management of Attention Deficit Hyperactivity Disorder (ADHD) 211 Tilla Olivier and Ana Gomes

Chapter 12 Distractor or Noise?

The Influence of Different Sounds on Cognitive Performance in Inattentive and Attentive Children 233

Göran Söderlund and Sverker Sikström

Part 4 EEG Biofeedback 247

Chapter 13 QEEG Characteristics and Biofeedback Modalities in Children with ADHD 249 Nada Pop-Jordanova

Chapter 14 EEG Findings in ADHD and the Application of EEG Biofeedback in Treatment of ADHD 269 Mohammad Ali Nazari

Chapter 15 The Effect of Psycho-Educational Therapy on Electroencephalographic Biofeedback Scores in Attention Deficit Hyperactivity Disorder 287 Irene Nikaina, Aspa Paspali, Georgia Kleidaria and Antigone Papavasiliou 


\section{Preface}

Attention Deficit Hyperactivity Disorder is one of the most widely diagnosed behavior disorders in childhood, with a worldwide pooled prevalence of $5.29 \%$ (Polanczyk et al., 2007). It is also one of the most controversial diagnoses with concerns raised about whether it is a cultural construct or a neurobehavioral disorder (Barkley et al., 2004, Timimi et al., 2004) and whether it is over diagnosed or under diagnosed. The treatment of ADHD is also a matter of ongoing research and debate, with considerable data supporting both psychopharmacological and behavioral approaches. Researchers continue to search for new interventions to be used in conjunction with or in place of the more traditional approaches. These interventions run the gamut from social skills training, to cognitive behavioral interventions ,to meditation to neuropsychologicallybased techniques.

This goal of this volume is to explore the state-of-the-art in considerations of the treatment of ADHD around the world. The 16 chapters in this volume represent the work of 27 researchers in countries spanning the globe from Iran to Columbia, Denmark to South Africa, and the United States. Some of the chapters in this book provide a new way of looking at well-established treatments while others examine treatments that are seeking the confirmation of the research community. This broad survey covers issues related to comorbidity that affect the treatment choices that are made, the effects of psychopharmacology, and non-medication treatments, with a special section devoted to the controversial new treatment, neurofeedback.

The first section of the book provides an overview of treatment of ADHD and comorbid conditions that may be of concern. Chapter 1, "ADHD in Children and Adolescents: A Good Practice Guidance" provides a summary of the assessment and diagnosis of ADHD. It also concisely describes the management of the disorder, covering such topics as choices and safety in medication, non-medicinal treatments, and a consideration of how comorbid conditions may affect treatment. Chapter 2, "ADHD and Comorbid Conditions," examines in more depth the identification and treatment of psychological problems that are associated with ADHD, such as depression, anxiety, bipolar disorder, and oppositional defiant disorder. Chapter 3, "Comorbidity in ADHD: A Neuropsychological Perspective," takes a different approach to the issue from the first two chapters by examining the subtypes of ADHD as related to different neuropsychological profiles. These profiles are in turn related to 
different comorbid cognitive and behavioral conditions. The final chapter in the first section, "ADHD and Sleep Problems in Children," focuses on the need for the consideration of sleep problems in children with ADHD because of the overlap between the two categories and the implications for treatment of ADHD.

The second section of the volume examines the psychopharmacological treatment of ADHD. Chapter 5, "The Neuropsychology of Stimulants: Dopamine and ADHD," examines how dopamine's role in ADHD is related to the action of psychostimulant medications, as well as the role of other neurotransmitters and other medications. Chapter 6, "Trends in the Prescribing and Adverse Drug Reaction Patterns of Psychostimulants among Danish Children and Adolescents," takes advantage of the availability of data regarding the individual use of medication in Denmark to explore both changes in prescription rates over a 10 year period and the prevalence of adverse reactions to the various medications. Chapter 7, "Do Stimulant Medications for Attention Deficit Hyperactivity Disorder (ADHD) Enhance Cognition?," reviews the research on this topic and presents the authors' own data that indicate that, though stimulants improve attention and concentration, changes in other areas of cognitive and academic functioning are inconsistent such that stimulants do not appear to improve learning. Chapter 8, "Motor Skills in Children with ADHD: Comparative Study from the Pharmacological Treatment," summarizes the literature indicating that $50 \%$ of children with ADHD have motor skill problems and describes the authors' own research. Although such problems are common, the effect of medication and exercise on ADHD symptoms and motor disturbance remains unclear. Chapter 9, "Methylphenidate and Dyslipidemia," examines the relationship between the drug and lipid profiles, concluding that methylphenidate is related to some positive changes in total cholesterol and lipoproteins.

The third section of the book moves from psychostimulants to an examination of nonmedication treatments. Chapter 10, "Non-Medication Treatments of ADHD," provides an overview of a variety of interventions, summarizing the techniques and research regarding efficacy. Chapter 11, "ADHD and Stress: The Role of Meditation to Reduce Stress, and Improve Brain Function and Behavior Regulation," reports the results of two studies examining Transcendental Meditation's effects on ADHD symptomatology that indicate that children with ADHD are able to learn the technique and that meditation may reduce stress and improve executive functions. Chapter 12, "The Value of Coached Behavior Modification in the Effective Management of Attention Deficit Hyperactivity Disorder (ADHD)," describes research supporting the use of coaches in the treatment of ADHD. Such coaches are not replacements for therapists, but come alongside those with ADHD to help them learn to manage the practical challenges that arise in the pursuit of goals. Chapter 13, "Distractor or Noise? The Influence of Different Sounds on Cognitive Performance in Inattentive and Attentive Children," reports the results of a study examining the differential response of children with and without inattention symptoms to background auditory noise. The authors report that such noise improves cognitive performance in children with inattentive symptoms and provide a theoretical framework for understanding this phenomenon. 
The final section of the book explores the fairly new field of neurofeedback. Neurofeedback, which uses quantitative EEG in biofeedback training, is a controversial technique. Critics point to the lack of controlled research on the topic, but it remains popular with practitioners and clients. Grants investigating the technique are currently funded by the National Institutes of Health. Clearly, more research on this technique is needed and, in that spirit, three chapters here address neurofeedback. Chapter 14, "QEEG Characteristics and Biofeedback Modalities in Children with ADHD," describes the use of QEEG in the diagnosis of ADHD, with results from a study highlighting the differences among subgroups of the disorder. The chapter also provides a brief overview of neurofeedback for ADHD. Chapter 15, "EEG Findings in ADHD and the Application of EEG Biofeedback in Treatment of ADHD," provides a more extensive description of EEG biofeedback, including summaries of the treatment protocols. Chapter 16, "The Effect of Psycho-Educational Therapy on Electroencephalographic Biofeedback Scores in Attention Deficit Hyperactivity Disorder," reports the results of a study examining the effect of a combination of neurofeedback and a cognitive-behavioral academic intervention. They found that a combination of treatments accelerated the improvement in brain activity.

Overall, the chapters presented here tap intomuch of the breadth of this field. I believe that there is something in this volume for everyone interested in the treatment of ADHD, from students examining the topic for the first time to researchers and practitioners looking for inspiration for new research questions or potential interventions. I hope that the chapters spark new thoughts and, perhaps, debates.

This book is the result of the work of many individuals. I am particularly grateful for the assistance of Ms. Adriana Pecar and Mr. Igor Babic for their assistance in coordinating this book. I also thank all of the authors who contributed to this volume.

Jill M. Norvilitis

Department of Psychology,

Buffalo State College,

Buffalo, NY 14222,

USA

\section{References}

Barkley, R. A., Duncan, M., Loney, J., Milich, R., Werry, J., Cunningham, C....Hay, D. (2004). Critique or misrepresentation? A reply to Timimi et al. Clinical Child and Family Psychology Review, 7, 65-69.

Polanczyk, G., Silva de Lima, M., Lessa Horta, B., Biederman, J., \& Rohde, L. A. (2007). The worldwide prevalence of ADHD: A systematic review and metaregression analysis. American Journal of Psychiatry, 164, 6, 942-948.

Timimi, S., Moncrieff, J., Jureidini, J., Leo, J., Cohen, D., Whitfield, C...White, R. (2004). A critique of the International Consensus Statement on ADHD. Clinical Child and Family Psychology Review, 7, 59-63. 



\section{Part 1}

\section{Treatment Considerations}





\title{
ADHD in Children and Adolescents: A Good Practice Guidance
}

\author{
Somnath Banerjee \\ Community Paediatrician, East Kent Hospitals University NHS Foundation Trust, \\ Convenor, UK George Still Forum (National Paediatric ADHD Interest Group) \\ United Kingdom
}

\section{Introduction}

The syndrome of restless, inattentive, and impulsive behaviour known as Attention Deficit Hyperactivity Disorder (ADHD) or Hyperkinetic Disorder (HKD) is a common neurodevelopmental problem in children and adolescents. Children affected with this disorder are at risk of academic failure, substance abuse, and criminality in adolescence and adulthood. ADHD is a reflection of an underlying deficit that may have several ultimate causes such as genetic predisposition, psycho-physiological factors and psychological dysfunction, but a common pathway at the behavioural level (Hill \& Cameron, 1999). By labelling children with difficult behaviour as suffering from a disorder, we make it easier to treat and this carries with it the danger of over-diagnosis (Orford, 1998). On the other hand, under-diagnosis is also an issue (Dopheide, 2001).

\section{Incidence}

Prevalence of ADHD estimates that 3 to $7 \%$ children would meet the criteria of Diagnostic and Statistical Manuel-IV (DSM-IV) of American Psychiatric Association's diagnostic criteria (APA, 1994). The ratio of boys to girls is between 3:1 and 9:1 but this may decrease with age (Swanson et al., 1998). Part of the difference between sexes may be referral bias (Beiderman et al., 1996). Follow-up studies of children with ADHD find that $15 \%$ still have the full diagnosis at 25 years, and another $50 \%$ are in partial remission, with some symptoms persisting (Faraone et al., 2006).

\section{Aetiology}

No genetic marker has been identified in children with ADHD. Neuroimaging studies confirm abnormalities in those regions of the brain that are implicated in ADHD (Swanson et al., 1998; Castellanos et al., 2002). These studies report significantly smaller asymmetrical prefrontal and basal ganglia structures, in children with ADHD. Correlations of magnetic resonance imaging-based anatomical measures and specific-task performance in children suggest that the right prefrontal cortex is involved in inhibiting attentional and behavioural responses, whereas the basal ganglia seem to be involved in the execution of these responses (Konrad \& Eickhoff, 2010). One third of affected individuals have at least one parent who 
suffers from similar symptoms. ADHD is also associated with low birth weight $(<1500 \mathrm{~g})$; tobacco and alcohol use during pregnancy (SIGN, 2001). Although ADHD is highly heritable, no specific susceptibility gene has been identified (Franke et al., 2009).

\section{Symptoms}

The symptoms of ADHD are excessive and impairing levels of activity, inattention, and impulsiveness. Children have great difficulty remaining seated when required in structured situations such as in the classroom or at the dinner table. They fail to pay attention to instructions in academic and social situations. The difference between ADHD and normal behaviour is the degree of impairment. The alarming signals may be a child who is academically under-achieving because of his/her behaviour, despite having a normal intellect or a child exhibiting behaviour problems both at home and in school, which are considerably worse than would be expected for the standard of parenting and home environment. The symptoms of ADHD change through out the lifecycle. Hyperactivity and impulsivity may decrease as patients get older but the demands on their attention may increase.

\section{Assessment}

Concern about the future quality of life is heightened when parents observe their children struggling behaviourally at home, academically in school and socially on the playground. When told that a child has ADHD, parents are relieved by finding a reason for their child's difficulties. The diagnosis confirms that it is not their fault. Not all children and young people presenting with difficult behaviour will warrant a specialist referral. This depends on the severity of child's difficulties. ADHD can be provisionally diagnosed in preschool children but it should be confirmed after the child has started school. Some argue that problems with inattention, hyperactivity and impulsivity are the results of cultural phenomena (Block, 1977). Yet this point of view appears to be changing with the acceptance of ADHD as a cross-cultural disorder (Sandberg, 1996). Some children are inherently more inclined to be inattentive, impulsive and hyperactive than others. Such traits may run in families e.g. parents, siblings, and extended family members and there seems to be a genetic predisposition to them. Mental traits can be inherited just as height and weight. What we are dealing with are normal human variations that are only understood as being disorders when they are in conflict with cultural expectations and norms. The rating scales are an essential tool to obtain information but are not a substitute for a diagnosis. Reports from parents and teachers may not agree on the types of behaviours. This does not mean they are necessarily inaccurate; it may be attributed to the fact that the parents and the teachers are seeing the children in different settings. There are many rating scales available, most of which have been developed in the USA. The assessment should include information gathering. Apart from obtaining the completed rating scales, information should be obtained from the school about peer relationship and the child's academic progress. Information is gathered about child's current difficulties; family and social history; child's developmental and medical history and the educational progress. While interviewing parents, one needs to obtain a comprehensive knowledge of each parent's medical and psychiatric history. Family situations, such as a single working parent, separated or divorced parents, or reconstituted families where one or both parents have remarried, all affect the child. 


\subsection{Medical assessment}

Since symptoms of ADHD can occur due to a wide variety of underlying conditions, a comprehensive approach to the evaluation of a child with ADHD is recommended (Reiff $e t$ al., 1993, AACAP, 1997)). Medical assessment should include a perinatal history, behavioural/developmental history, family history (SIGN, 2001) and a physical examination for any contraindications for possible medication use, such as some cardiac dysrhythmias. Perinatal history should include about pregnancy and birth, maternal exposure to drugs, alcohol and smoking cigarettes, cannabis etc and high-risk pregnancy (e. g. prematurity, LBW). Behavioural/developmental history should include developmental milestones, difficulty engaging in quiet play and problems with obeying commands. Family History should include history of ADHD, drug or alcohol abuse, psychiatric illnesses, problems with the law, specific precipitant where symptoms can be dated, events precipitating ADHD symptoms such as neglect, physical or sexual abuse, parenting issues and cardiac arrhythmias or sudden death especially in 35 years or younger age, home Environment (key caregivers, frequent moves or frequent change in foster care, frequent changes of school, home environment and family dynamics e. g. single family, same gender partners), interpersonal relationship/s in the family, Poor or crowded housing. History should be obtained about time spent on TV-watching, computer and video games. History should also be obtained about peer relationships, academic under-achievement, truancy, does the child enjoy school, ask the school age child if she/he thinks she/he has trouble concentrating, review current school report as well as those from earlier years and psychometric evaluation by education psychologists for cognitive abilities and academic achievement levels. History of sleep pattern needs to be obtained. Eating History should include appetite and eating habits and joining the other members for dinner. Physical examination is done to document a baseline growth parameters (height and weight) which should be plotted on a centile chart and at each follow-up visit if the child is prescribed medication, blood pressure and pulse rate are recorded and plotted on the centile chart, cardiac examination including auscultation for murmurs and femoral pulses, dysmorphic features suggestive of Fetal Alcohol Syndrome (FAS) or other genetic conditions, cutaneous stigmata, such as café au lait spots, bruising or other evidence of injury, tonsillar hypertrophy suggestive of mouth breathing, neurologic exam and age-appropriate mental status exam, tics, play skills (particularly problem solving), observing the child/parent interaction, co-ordination tasks and handwriting, menstrual/pubertal status in adolescents and if deemed necessary, psychological assessment. The assessment process should take account of the other conditions, which may better account for the challenging behaviour (AACAP, 1997). These might include medical disorders such as sleep apnoea, seizure disorders, developmental disorders (e. g. Intellectual disability (Learning disability, LD ), Specific Learning Difficulty (SpLD) formerly dyslexia and Developmental Coordination Disorder (DCP) formerly dyspraxia), brain injury, use of other medications (e. g. anti-epileptic drug) or sensory impairments; mental health disorders such as Oppositional Defiant Disorder (ODD)/Conduct disorder (CD), anxiety/depression, adjustment disorder, attachment disorder or substance abuse. Other conditions include Autism Spectrum Disorder (ASD) and the normal active preschool child. In addition to the history mentioned above, evaluation of an adolescent should also include eliciting history regarding use of alcohol and drugs, cigarette smoking, number of accidents and speeding tickets, sexual activity and spending history. The above assessments are not indicative of ADHD but may help to rule 
out the possibility of other under-lying medical or developmental conditions mimicking ADHD symptoms.

\section{Guidelines}

The guidelines for assessment and treatment have been issued by American Academy of Child and Adolescent Psychiatry (AACAP, 2007), the American Academy of Pediatrics (AAP, 2001), the European Guidelines (Taylor et al., 2004, Banaschewski et al., 2006), National Institute for Health and Clinical Excellence (NICE, 2008) and the Scottish Intercollegiate Guideline Network (SIGN, 2004). While there is a degree of consensus among these publications, there seems to be some international difference such as NICE recommends drug treatment in severe ADHD, whereas the American guidelines advice to start medication and later other management strategy may be considered (CADDRA, 2010).

\section{Diagnosis}

ADHD is a clinical diagnosis for which there are no tests. It is important to gather the information from parents/carers and school. The diagnosis is made by using the diagnosis criteria either of DSM -IV (APA, 2000) or ICD -10 (WHO, 1992). Both major systems of classification identify identical 18 symptoms (table 1 ).

\begin{tabular}{|c|c|c|}
\hline Inattention (IN) & Hyperactivity $(\mathrm{H})$ & Impulsivity (IMP) \\
\hline Fails to attend to details & $\begin{array}{l}\text { Fidgets with hands or } \\
\text { feet }\end{array}$ & \\
\hline Difficulty sustaining attention & $\begin{array}{l}\text { Leaves seat in } \\
\text { classroom }\end{array}$ & \\
\hline Does not seem to listen & Runs about or climbs & \\
\hline Fails to finish & $\begin{array}{l}\text { Difficulty playing } \\
\text { quietly }\end{array}$ & \\
\hline Difficulty organising tasks & $\begin{array}{l}\text { Motor access (on the } \\
\text { go, in DSM-IV) }\end{array}$ & \\
\hline Avoids sustained effort & $\begin{array}{l}\text { Talks excessively } \\
\text { (DSM-IV) }\end{array}$ & Talks excessively (ICD-10) \\
\hline Loses things & & $\begin{array}{l}\text { Blurts out answers to } \\
\text { questions }\end{array}$ \\
\hline $\begin{array}{l}\text { Distracted by extraneous } \\
\text { stimuli }\end{array}$ & & Difficulty waiting turn \\
\hline Forgetful & & $\begin{array}{l}\text { Interrupts or intrudes on } \\
\text { others }\end{array}$ \\
\hline
\end{tabular}

Table 1. Symptom domains for ADHD in DSM-IV and ICD-10

\subsection{Differences between the two major diagnostic manuals (table 2)}

7.1.1 In the symptom domains of Inattention, Hyperactivity, and Impulsivity; an ICD-10 diagnosis of HKD needs some symptoms from all three groups whereas DSM-IV ADHD does not, but instead specifies subtypes if symptoms are from only one domain. HKD is broadly similar to severe type of ADHD. 
7.1.2 Because of the high rate of conduct disorder, ICD-10 uses the presence or absence of conduct disorder as the basis for the main subdivision of HKD. DSM-IV does allow the diagnosis of conduct disorder as a comorbid condition.

7.1.3 Another difference between the two classifications is the use of other conditions as exclusion criteria. ICD-10 aims at a single diagnosis. DSM-IV aims to recognize, as many diagnoses as there are symptoms.

\begin{tabular}{|l|l|}
\hline \multicolumn{1}{|c|}{ HKD (ICD-10) } & $\begin{array}{l}\text { Six or Six or more from IN domain, three or more from } \\
\text { H domain and one or more from IMP domain. }\end{array}$ \\
\hline ADHD \& subtypes (DSM-IV) & \\
\hline Combined type & $\begin{array}{l}\text { Six or Six or more from IN domain and six or more } \\
\text { from the H / IMP domain. }\end{array}$ \\
\hline Inattentive type & $\begin{array}{l}\text { Six or Six or more from IN domain and less than six } \\
\text { from H / IMP domain+ / H / IMP less than 6 }\end{array}$ \\
\hline Hyperactive/Impulsive & $\begin{array}{l}\text { Six or Six or more from H / IMP domain and less than } \\
\text { six from IN domain. }\end{array}$ \\
\hline
\end{tabular}

IN: Inattention; H: Hyperactivity; IMP: Impulsivity

Table 2. HKD diagnosis and ADHD diagnosis subtypes

\subsection{Limitations of diagnosis criteria}

Although the DSM-IV and ICD-10 are widely used as diagnostic tools, there are a number of limitations (AAP, 2000).

7.2.1 The numbers of symptoms required to make the diagnosis of ADHD / HKD does not vary with the severity of the symptoms or with the age. Therefore an older child with a few severe symptoms may not meet the diagnostic criteria, whereas a younger child with many less severe symptoms may meet the criteria, even though the former may experience more functional impairment. For a child with few severe symptoms, the diagnosis of ADHD not otherwise specified that is included in DSM-IV may be used, but the manual provides no criteria for when to make this diagnosis.

7.2.2 The requirements that symptoms occur in at least two settings can be problematic at times. It may hinder the diagnosis for children who may have significant difficulties with attention in school but do not have problems at home.

7.2.3 Although the criteria include symptoms be present before 7 years is useful in highlighting that ADHD rarely is the correct diagnosis when inattention, hyperactivity, or impulsivity is occurring for the first time in adolescence or in adulthood; however, it may be problematic in excluding children whose limited attention spans becomes more noticeable when they start secondary school because of increased school and home work.

7.2.4 The terms "significant" and "some impairment" are subjective and unclear.

7.2.5 The diagnosis is also environmentally dependent, which explains why clinicians will see differences in the child's behaviour between school and home settings. 
7.2.6 It is a challenge to make a diagnosis when there are no explicit criteria for defining what is typical for a particular age?

7.2.7 Currently the major diagnosis criteria are only applicable to adolescents up to 18 years of age. There are no diagnosis criteria for adults.

\section{Management}

Following diagnosis of ADHD, written information and available option of various management strategies, website addresses, and contact details of the local support groups should be given to the parents and the child's school regarding the condition and its management. Parents/carers should be informed that ADHD is a neuro-behavioural condition with a possible genetic aetiology which is the result of low or imbalanced levels of specific neurotransmitters in certain areas of the brain. Abnormally low levels of these neurotransmitters are associated with the impairments that are the hallmarks of ADHD. The need to rule out other possible diagnoses should be explained. Subsequently various treatment options need to be explored. Ensure that the patient and family have had an adequate opportunity to be educated about ADHD (CADDRA, 2010). Do ask the family to find out more about ADHD through reputable websites and recommended reading. They need to be informed of the symptoms that indicate a diagnosis and the aims and rationale for treatment (with an understanding that no medication eliminates all the symptoms of ADHD and that other strategies are also indicated as part of management). There needs to be a discussion of the risks and benefits of the advised therapy and the alternatives. There should also be discussion regarding the potential risks of no therapy. It is important to describe to the parents the findings obtained from the assessment, including a clear statement about the diagnosis and the basis on which the diagnosis is made. The family should be told that a copy of the clinic letter, with their consent, will be sent to the school.

The child's surroundings should support routines and decrease distractions. Consistent ageappropriate limit setting is important. Retaining a positive, enjoyable relationship with their child improves the child's self-esteem. Thus, doing things that the child enjoys is important. Parents/carers need to help the child to develop appropriate social behaviours with peers and adults. Whenever possible, an attempt should be made to work with both parents so that they are consistent with each other in their approaches. Family support should aim to improve relationships within the family, promote parental empowerment and develop strategies to manage behaviour, e.g. through a parenting group. In addition, families should be advised of ADHD parent support groups existing in their area. In many cases careful management of behaviour and advice to parents and teachers and working with the child to control impulsiveness and maintain concentration may be sufficient to manage the problems (AAP, 2001). Where these are not deemed to be sufficient, medication may be tried. Liaison with the school should help inform behaviour management strategies within the classroom. Further support from behaviour specialist teachers may be sought where necessary. The child's school and the school nurse should be notified at the start of medication. Any change of the dosage must be notified to school and school nurse by the prescribing doctor, rather than simply being relayed to them by the parents or the child. Individual counselling or group work may be offered to the child to address issues of low self-esteem as well as to promote social skills and the peer relationships and offer skills in the area of anger management. Time should be spent with the child/young person to help them understand 
what ADHD is and if medication is to be used, how it works and its side effects. In the more severe cases, and usually following behavioural interventions, a trial of medication may be considered. ADHD is a chronic condition requiring access to long-term treatment and support, sometimes over many years. However, the level and type of support needed can vary. The principal aims of treatment are to promote the child's development and to reduce secondary difficulties.

\subsection{Non psychopharmacolical interventions}

This treatment is a non-drug intervention and is recommended in mild and moderate degree of severity of ADHD (NICE, 2008). The therapist consults with parents and teachers to train them to change the learning environment for the child. It means to physically calming the child, to enable them to stay still, even in a group. The goal is to teach parents and teachers to use rewards and punishment consistently and effectively. Together with the therapist, the parents and teacher find rewards that will motivate the child to work. Group based parenting programmes can help parents/carers to develop their skills and boost confidence in managing challenging and difficult behaviour of their children. Some parenting programmes include Incredible Years, which use Webster Stratton programme for parents of children aged $2-8$ years (Incredible years), Triple $\mathrm{P}$ for parents and the programme run by barnardo's (Barnardo's). Using effective communication skills to develop a loving, guiding, limit setting, consequence providing that rewards the desired behaviour and eliminates the undesired behaviour is the long term aim of non psychopharmacological intervention.

\subsection{Medications}

Advice and support about the child's self esteem, peer group progress, academic progress and family relationships will also be required even if medication is used. A positive response to medication is not diagnostic and a good response to drugs does not validate the diagnosis. The presently available medications are symptomatic therapies; they are not able to cure the condition. Medication aims to reduce the core symptoms and to improve the effectiveness of other interventions. Medication treatment should focus on behavioural improvement and not on getting better grades in school; grades may be the net result of a many factors, including learning disabilities, motivation and family attitudes.

Drugs used in the treatment of ADHD are grouped into two major categories: stimulants and non stimulants. Methylphenidate (MPH) and Atomoxetine (ATX) are not licensed for use in children less than six years of age or in adults. ATX is licensed after 6 years of age and may be continued in adulthood where they have been started in adolescence. Dexamfetamine (DEX) may be prescribed after 3 years of age. Stimulants (MPH and DEX) are not licensed for children with marked anxiety, agitation or tension, symptoms or family history of tics or Tourette's syndrome, hyperthyroidism, angina or cardiac arrhythmia, glaucoma or thyrotoxicosis. Stimulants are controlled by the Misuse of Drugs Act 1971 and are subject to the regulations for Controlled Drugs. For details the practitioners are advised to consult the European treatment guidelines (Taylor et al. 2004, Banaschewski et al, 2006). Parents should have been explained the advantages and disadvantages of various available drugs. Concerns and questions parents may have regarding both effects and side effects need to be addressed. An explanation of what medication can and cannot do, and what 
other interventions are available should be given. Parents/carers should be told that not every medication is suitable for every patient. The guiding principle of drug intervention is to achieve the optimum response with the lowest dose possible that does not give side effects. Therefore, start with a low dose and gradually increased until the maximum recommended dose level is reached or target symptoms have improved or side effects appear. There are recommendations for dosing for different medications in accordance with their licenses. Where there are comorbidities, additional or multiple medications may be used. The summary of product characteristics (SPC) contains specific advice about a drug.

The most important development in ADHD occurred in 1937 with the discovery of the beneficial effect of DEX on attention and behaviour among children (Bradley, 1937). The main breakthrough came in 1957 with the introduction of MPH and subsequently numerous trials confirmed safety and efficacy of stimulants (Laufer, 1971; Eisenberg, 1972). The mechanism of action of both the stimulants; DEX and MPH is similar; they act principally as inhibitors of the dopamine-uptake mechanism in the brain. DEX, in addition, promotes the release of this neurotransmitter, thus stimulating dopaminergic mechanisms. The stimulants also act on norepinephrine-containing neurones to promote an increased release of this monoamine. The most extensively used and researched stimulant is $\mathrm{MPH}$, a derivative of DEX which has a rapid onset of action, short half-life. Dosage and frequency requirements vary with each individual child and are independent of the weight or the age. Stimulants are rapidly absorbed and easily cross the blood- brain barrier. If there is a lack of improvement or substantial side effects, another ADHD drug may be considered another (e.g., MPH vs. $\mathrm{DEX}$ ). If a patient is responding well to one medication, it is advised that another medication should not be tried to see if there is a better response. Table 3 describes the properties of the ADHD drugs.

\begin{tabular}{|c|c|c|c|c|c|}
\hline \multicolumn{1}{|c|}{ Agent } & Onset & Max effect & Duration & Half-life & Dosage/day \\
\hline Short-acting MPH & & & & & \\
\hline MPH-IR $^{2}$ & $20-30 \mathrm{~min}$ & $1-2 \mathrm{hrs}$ & $3-5 \mathrm{hrs}$ & $2-3 \mathrm{hrs}$ & $2.5-60 \mathrm{mg}$ \\
\hline DEX $^{3}$ & $20-60 \mathrm{~min}$ & $1-2 \mathrm{hrs}$ & $6 \mathrm{hrs}$ & $4-6 \mathrm{hrs}$ & $1.25-40 \mathrm{mg}$ \\
\hline Long-acting MPH & & & & \\
\hline Concerta XL $^{4}$ & $20-30 \mathrm{~min}$ & $2 \mathrm{hrs}$ & $12 \mathrm{hrs}$ & $3.5 \mathrm{hrs}$ & $18-54 \mathrm{mg}$ \\
\hline Equasym XL $^{\text {Medikinet XL }}$ & $20-30 \mathrm{~min}$ & $1-2 \mathrm{hrs}$ & $8 \mathrm{hrs}$ & $2 \mathrm{hrs}$ & $10-60 \mathrm{mg}$ \\
\hline MPH-SR $^{5}$ & $1-30 \mathrm{~min}$ & $1-2 \mathrm{hrs}$ & $8 \mathrm{hrs}$ & $2 \mathrm{hrs}$ & $10-60 \mathrm{mg}$ \\
\hline Non stimulant & & $2 \mathrm{hrs}$ & $5-6 \mathrm{hrs}$ & $2-6 \mathrm{hrs}$ & $20-60 \mathrm{mg}$ \\
\hline ATX & $1 \mathrm{wk}$ & $1-2 \mathrm{hrs}$ & $24 \mathrm{hrs}$ & $\begin{array}{l}3.6 \mathrm{hrs} \text { in } \\
\text { rapid } \\
\text { metabolis } \\
\text { ers }\end{array}$ & $\begin{array}{l}0.5 \mathrm{mg} / \mathrm{kg} \times 7 \\
\text { days; then } \\
1.2 \mathrm{mg} / \mathrm{kg} ; \\
\text { max } \\
100 \mathrm{mg} / \mathrm{day}\end{array}$ \\
\hline
\end{tabular}

$\mathrm{MPH}^{1}=$ methylphenidate, $\mathrm{MPH}-\mathrm{IR}^{2}=$ methylphenidate-immediate release, $\mathrm{DEX}^{3}=$ dexamfetamine, Concerta $\mathrm{XL}^{4}=$ licensed up to $54 \mathrm{mg}$ per day, $\mathrm{MPH}^{-\mathrm{SR}^{5}}=$ methylphenidate slow release, $\mathrm{ATX}^{6}=$ atomoxetine

Table 3. Properties of ADHD drugs. 


\subsubsection{Medications}

The license status of ADHD medications varies in the different European countries. On average the drugs licensed and available in different European countries are: Methylphenidate immediate release (IR) available as 5, 10 and $20 \mathrm{mg}$ tablets (5 and $20 \mathrm{mg}$ only available as Medikinet and methylphenidate tablets).

Medikinet ${ }^{\circledR}$ XL (methylphenidate SR) available as 5, 10, 20, 30, and 40mg capsules.

Equasym $^{\circledR}$ XL (methylphenidate SR) available as 10, 20 and $30 \mathrm{mg}$ capsules.

Concerta ${ }^{\circledR} \mathrm{XL}$ (methylphenidate SR) available as 18,27 and $36 \mathrm{mg}$ tablets.

Dexedrine ${ }^{\circledR}$ (Dexamfetamine) available as: $5 \mathrm{mg}$ tablets.

Strattera ${ }^{\circledR}$ (atomoxetine) available as $10,18,25,40,60$ and $80 \mathrm{mg}$ capsules.

\subsubsection{Methylphenidate Immediate Release (MPH IR)}

$\mathrm{MPH}$ is a central nervous system stimulant. The maximum recommended dosage is $0.7 \mathrm{mg}$ per $\mathrm{Kg}$ per dose or $2.1 \mathrm{mg}$ per $\mathrm{Kg}$ daily in divided doses. It is licensed from six years to eighteen years of age. MPH IR is active for about four hours after the last dose taken.

\subsubsection{Methylphenidate sustained release (MPH SR)}

Recently, there is an influx of sustained release stimulants. The first one that came in the market is OROS MPH, or Concerta XL (Tri-layer capsule-shaped tablets). This tablet has an osmotic membrane and overcoat of $\mathrm{MPH}$, has two compartments for $\mathrm{MPH}$, and a push compartment. It also has a laser-drilled hole that allows the release of medication at a prespecified pace. This medication was developed to replace 3 doses of immediate-release MPH given 4 hours apart; therefore, this drug is active for about 12 hours. The tablet shell may appear in the faeces $20 \% \mathrm{MPH}$ is excreted due to an osmotic pump action and therefore 18 mg tablet gives $15 \mathrm{mg}$ IR The second MPH reformulation that came to market is marketed as Equasym XL which has a 30\% short-acting bead, 70\% long-acting bead. It is a Biphasic Release Bead-Delivery System drug. This medication was designed to replace 2 doses of $\mathrm{MPH}$. It is advised to swallow whole or empty capsule contents onto one spoonful of apple sauce or similar soft food such as yoghurt The third MPH compound that came to UK market is called Medikinet XL. It has Bimodal Release for Once-Daily Dosing and has a 50\% short-acting bead, $50 \%$ long-acting bead, so this medication is designed also to work for 6 to 8 hours to cover the school day. This capsule is either swallowed whole or capsule content can be emptied onto one spoonful of apple sauce or similar soft food Table 4 describes the immediate and extended release proportions of MPH. Ritalin slow Release (Ritalin SR) is not licensed in UK, but sometimes used. It is available as $20 \mathrm{mg}$ tablets.

\begin{tabular}{|l|l|l|l|l|l|l|l|l|}
\hline \multicolumn{4}{|l|}{ Concerta XL } & \multicolumn{4}{l|}{ Equasym XL } & \multicolumn{3}{l|}{ Medikinet XL } \\
\hline Tablet & IR 22\% & ER 78\% & Capsule & IR 30\% & ER 70\% & Capsule & IR 50\% & ER 50\% \\
\hline- & - & - & $10 \mathrm{mg}$ & $3 \mathrm{mg}$ & $7 \mathrm{mg}$ & $10 \mathrm{mg}$ & $5 \mathrm{mg}$ & $5 \mathrm{mg}$ \\
\hline $18 \mathrm{mg}$ & $4 \mathrm{mg}$ & $14 \mathrm{mg}$ & $20 \mathrm{mg}$ & $6 \mathrm{mg}$ & $14 \mathrm{mg}$ & $20 \mathrm{mg}$ & $10 \mathrm{mg}$ & $10 \mathrm{mg}$ \\
\hline $27 \mathrm{mg}$ & $6 \mathrm{mg}$ & $21 \mathrm{mg}$ & $30 \mathrm{mg}$ & $9 \mathrm{mg}$ & $21 \mathrm{mg}$ & $30 \mathrm{mg}$ & $15 \mathrm{mg}$ & $15 \mathrm{mg}$ \\
\hline $36 \mathrm{mg}$ & $8 \mathrm{mg}$ & $28 \mathrm{mg}$ & $40 \mathrm{mg}$ & $812 \mathrm{mg}$ & $28 \mathrm{mg}$ & $40 \mathrm{mg}$ & $20 \mathrm{mg}$ & $20 \mathrm{mg}$ \\
\hline $45 \mathrm{mg}$ & $10 \mathrm{mg}$ & $35 \mathrm{mg}$ & $50 \mathrm{mg}$ & $15 \mathrm{mg}$ & $35 \mathrm{mg}$ & $50 \mathrm{mg}$ & $25 \mathrm{mg}$ & $25 \mathrm{mg}$ \\
\hline $54 \mathrm{mg}$ & $12 \mathrm{mg}$ & $42 \mathrm{mg}$ & $60 \mathrm{mg}$ & $18 \mathrm{mg}$ & $42 \mathrm{mg}$ & $60 \mathrm{mg}$ & $30 \mathrm{mg}$ & $30 \mathrm{mg}$ \\
\hline
\end{tabular}

Table 4. Relative proportions of Immediate-Release (IR) and Extended-Release (ER) methylphenidate 


\subsubsection{Dexamfetamine (DEX)}

DEX is also a central nervous system stimulant. Its effect and adverse event profile are similar to MPH. The initial dose may be $2.5 \mathrm{mg}$ once or twice daily and increased if necessary by weekly increments of $5-10 \mathrm{mg}$ in the daily dose. The maximum licensed dose is $40 \mathrm{mg}$ daily in divided dosage.

\subsubsection{Safety of stimulant medications}

Although some parents express concern that stimulants may cause drug abuse or dependence, a review of long-term studies of stimulants and substance abuse showed that drug abuse was less likely in children with ADHD who were treated with stimulants compared with those who were not (Wilens et al., 2003). Patients or parents/carers, who are at risk for substance abuse/drug-diversion, should not be prescribed short-acting stimulants until precautionary measures have been put. One of the most common reasons for noncompliance is related to a lack of awareness or understanding of the side effects and lack of liaison between the clinician and parents. The common side-effects reported with MPH are insomnia, decreased appetite, pain in abdomen and headache. They are often mild and transient, and may be alleviated by reducing or adjusting the dosage. Many parents complain that their children are 'picky eaters'. In addition, both stimulant and non-stimulant ADHD medication can further suppresses appetite. Moreover children with ADHD may not sit still long enough to finish their meals. Common sense dictates that while balanced diet is not likely to cure ADHD, nutritious food would improve overall health, and thus indirectly benefit behaviour and attention. Unwanted effects of appetite suppression can be avoided by advising the drug to be taken after breakfast and lunch. Problems with sleep are a common complaint among ADHD patients. A decrease in sleep quality and/or quantity may lead to worsening of behaviour. It is therefore important to screen for sleep difficulties. The acronym BEARS (Owens \& Dalzell, 2008) is useful for this purpose: Bedtime resistance and delayed sleep onset, Excessive day time sleepiness, Awakenings during night, Regularity, pattern and duration of sleep and Snoring and other symptoms of sleepdisordered breathing. The causes of sleep problems include anxiety, ODD, sleep disorders (Obstructive Sleep Apnoea, Delayed Sleep Phase Syndrome (DSPS) and Restless Leg Syndrome). Stimulants may increase the difficulty of falling asleep. In DSPS, teenager falls asleep later than the expected time, has a normal sleep at night but wakes up late the next day. Sleep is optimized by maintaining a quiet and comfortable sleep environment. Exposures to TV, computer games or internet chat lines disrupt the initiation of sleep. The bed is not for watching TV, eating, or doing homework. No vigorous exercise within two hours of bedtime is recommended. Avoid drinks containing caffeine such as chocolate, coffee, tea and cola in the late afternoon and evening and advice a bath before bed to help relax. Melatonin is a natural hormone produced by the pineal gland in the brain. It is a sleep inducer and helps to fall asleep at night. Certain foods are rich in melatonin such as oats, rice, sweet corn, barley and tomatoes. Melatonin 2-10 mg may be administered 30-60 minutes before the bedtime for children with significant difficulty getting to sleep. There is evidence that melatonin is safe with short term use, but additional studies are needed to determine its long term safety (Buscemi et al. 2006). A very rare but important adverse reaction is bone marrow suppression. A routine full blood count is not warranted unless there is a clinical indication. The most commonly reported adverse effects with ATX are appetite decrease, headache, somnolence, nausea, vomiting and abdominal pain. Hostility 
(predominantly aggression, oppositional behaviour and anger) is an uncommon adverse effect with ATX. Aggression is a common side-effect of stimulants. Stimulants are advised to avoid in those with suicidal tendency and suicidal ideation, although suicidal attempt (including completed suicide) is very rare. Patients with emergent suicidal ideation or behaviour during treatment should be evaluated immediately by their physician. Patients on ATX should be closely monitored for the appearance or worsening of aggressive behaviour, hostility or emotional lability. A full list of potential adverse effects is listed in the summary of product characteristic (SPC) of individual drugs. They are also available online on the Electronic Medicines Compendium website (http://www.medicines.org.uk/emc/). Side effects which may warrant dose omission until discussed with an ADHD specialist include raised blood pressure, increase in seizure frequency in patients with epilepsy, heart rhythm changes, blurred eyesight or evidence of rare blood disorders. Anxiety is a commonly occurring adverse event with MPH. Clinical evaluation for anxiety or agitation should precede use of $\mathrm{MPH}$ and patients should be regularly monitored for the emergence or worsening of these symptoms regularly. A caution is needed to use ADHD drugs in women of childbearing age as effects of ADHD medications on the foetus and on breast-feeding are unknown. As per the SPC, ATX or MHP should not be used during pregnancy unless the benefit outweighs the risk and DEX is contra-indicated in pregnancy and during lactation. Full list of precautions / contra-indications are included in the SPC. ATX should be discontinued in patients with jaundice or laboratory evidence of liver injury. Very rarely, liver toxicity with elevated hepatic enzymes and bilirubin has been reported. Uses of all ADHD medications with MAOIs are contraindicated. It is good practice to explain to a teenager about the condition and the option of various drugs to gain her/his confidence. The use of medications may protect them from poor social skills. Combining medications for ADHD with illicit drugs or alcohol could be dangerous as the effects may be exaggerated.

\subsubsection{Is there any tolerance to stimulants?}

Acute tolerance, or tachyphylaxis means in order to maintain the response in the neuron, we need to increase the concentration of medication to maintain the response. In fact, two of the available stimulants, Concerta $\mathrm{XL}$ and Equasym $\mathrm{XL}$, use the principle of what's called an ascending curve, meaning that the concentration of $\mathrm{MPH}$ goes up across the day to counteract acute tolerance. So the concentration rises across the day. Thus the issue of acute tachyphylaxis has been considered in the development of some of the compounds available today.

\subsubsection{Non-stimulant}

Atomoxetine hydrochloride (ATX) is a non stimulant drug with no abuse potential and is effective for 24 hours. It is a selective inhibitor of noradrenaline reuptake licensed for the treatment of ADHD in children aged 6 years and older, adolescents and adults. It may be useful in children who do not respond or develop severe side effects to stimulants. Certain situations such as comorbidity with tics, Tourette's syndrome or substance abuse would support ATX as a first line option. It is available as Strattera ${ }^{\circledR} 10,18,25,40,60 \& 80 \mathrm{mg}$ capsules. The recommended starting dose in six years or older and adolescents with body weight up to $70 \mathrm{~kg}$ is $0.5 \mathrm{mg} / \mathrm{kg} /$ day (the dosing is weight based). The initial dose should be maintained for a minimum of 7 days prior to upward dose titration according to clinical response and tolerability. The dose is usually administered as a single daily dose but can 
also be given in two dived doses. The maintenance dose is $1.2 \mathrm{mg} / \mathrm{kg} /$ day (depending on the available dosage strengths). No additional benefit has been demonstrated for doses higher than $1.2 \mathrm{mg} / \mathrm{kg} /$ day. The plasma half-life is 3.6 hours in extensive metabolizers and 21 hours in poor metabolizers. It is not a schedule II drug. Strattera has been studied in trials involving over 4000 children and adolescents with ADHD. Peak efficacy occurs between 2 and 6 weeks after initiation, in contrast to the stimulants, which provide a response within hours. It is safe, well tolerated, and effective in 6 published trials in children and adolescents (Corman et al., 2004). Labelling for ATX includes "Black Box" warning for severe liver injury since February 2005, and for suicidality since November 2005, both of which reflect a pooled analysis of short-term placebo-controlled clinical trials found an average risk of suicidal ideation of $0.4 \%$ of children and adolescents (Wooltorton, 2005). Although uncommon, suicidal ideation is reported to be significantly more frequent in paediatric ADHD patients treated with ATX compared to those treated with placebo (Bangs et al., 2008).

\subsubsection{Choosing between stimulant and nonstimulant drug}

Tasks that require mental effort change over the years. In childhood there may only be a need to treat during daytime while in adolescents, the need to cover the evenings may be necessary. This may be critical for tasks such as driving. When selecting stimulants Vs nonstimulants, it is helpful to assess and compare the different side effect profiles. Another consideration when choosing a drug category is the onset of action. When patients require rapid response, stimulants are the treatments of choice. Non stimulant may require two to six weeks to show a treatment response. MPH/ DEX/ATX dependence is not a problem in the drug therapy of ADHD.

\subsubsection{Monitoring}

Heart rate, blood pressure, height and weight should be checked regularly and recorded on a growth chart. Drug related side effects should be checked at each clinic visit. Blood tests should not be viewed as routine. Patients and their families/carers need to be educated about ADHD and prescribed drug. Biases against the use of ADHD medications are often due to misinformation regarding side effects. Alternatively, parents/carers may have excessive expectation from the drug therapy which may lead to disappointment. It is important to inform parents/carers that medication is a part of the holistic approach to the management of ADHD. Obtain the completed rating scales to monitor treatment response. Advice the parents to stop the medication during weekends and during school holidays if growth appears to be adversely affected. A telephone call may be beneficial to follow up the prescribed ADHD medication. Once a stable optimal dose has been determined, the ideal medication follow-up is six months. Non-compliance to treatment may be related to lack of frequency of follow-up. A monitoring form such as in appendix 1 may be used in the clinics.

\subsubsection{Polypharmacy}

When a clinician feels that a second medication is needed, it is advised to begin with an ADHD medication that is known to combine safely with the second medication. For example, in the selection of an ADHD medication for a patient with severe conduct disorder and aggressive behaviour, a psychostimulant could be combined with an atypical antipsychotic (Turgay, 2005). MPH SPC has a caution around this combination. Some of the 
side effects related to drug interaction occur because of competition for liver enzymes that metabolise the drug. MPH increases plasma concentrations of phenytoin and delays intestinal absorption of phenytoin, phenobarbitone and ethosuximide. MPH inhibits metabolism of tricyclic antidepressants and warfarin. ATX can be combined with stimulants to augment the effect in the case that the clinician feels the patient has not achieved an adequate response (Wilens et al., 2009). Such a combination should be initiated by a clinician with specialization in ADHD. The combinations of ADHD drugs are used sometimes in clinical practice although it is not recommended. Results from the handful of studies suggest that combining stimulant therapy with nonstimulant alternatives may result in more significant symptom reductions in patients for whom monotherapy is less than optimal. There are no studies to suggest that combing stimulant with a non-stimulant increases the risk of cardiac side-effects. Combination usage of stimulant and non-stimulant is not included in the SPCs and hence appropriate safety and efficacy have not been determined.

\subsubsection{Length of treatment}

Many children will need to be on medication for years, hence the need to be clear about the diagnosis and review the diagnosis if required.

\subsubsection{Compliance}

Between a third and a half of medicines that are prescribed for long-term conditions are not used as recommended (NICE, 2009). Psychoeducation is the most useful means of ensuring compliance. The aim should be to get the adolescent to take responsibility for his/her own medications. Parents involvement may be necessary to ensure that medication is taken as scheduled. Once-daily dosing improves compliance.

\subsection{Non psychopharmacological interventions}

ADHD patients may take longer time to integrate socially acceptable habits into their lives. They are at significant risk of being involved with bullying as a bully, as a victim or both. The key factor is to create a positive environment that motivates the individual. When families are reluctant to use medications or there are side-effects behavioural treatments alone can be a viable alternative, provided that both parents and teachers are willing to undertake the effort required. National Institute for Health and Clinical excellence (NICE) recommends group parent training for ADHD with moderate levels of impairment (NICE, 2008). Parents/carers should be informed that children with ADHD may have additional social, academic, and emotional problems. Interventions such as additional help in academic work, social skills training, individual psychotherapy, parent training, family therapy or explaining to the child about ADHD, removing guilt, low self-esteem may be needed for the child and family.

\subsection{Practice point}

Make sure to review the child's strengths, not just his/her areas of weaknesses. This establishes a rapport with the child and family that makes future visits easier and can aid intervention planning. If there are any signs or symptoms of a physical illness that may be a factor in explaining the clinical symptoms, this takes precedence in the evaluation. Begin the 
interview by talking about the child's strengths. Ask the child to draw a picture of themselves and then their family on the same page. This helps to determine the child's perspective of the family. Note any unusual perceptual differences like drawing themselves bigger than the parents. Make appropriate referral if one or both of the parents need an assessment for ADHD or other psychiatric disturbance if it appears evident.

\section{Treatment consideration in common comorbidities}

It is estimated that at least $65 \%$ of children with ADHD have one or more comorbid conditions (Biederman et al., 1991). When there is a comorbid psychiatric disorder, it is generally advised that the ADHD should be treated first. However, if comorbidity puts the patient at risk for harm to others or to himself/herself, then this comorbidity takes precedence for treatment.

\subsection{Oppositional Defiant Disorder (ODD)}

In childhood, the most common comorbid disorder is ODD in as many as $40 \%$ of ADHD children (Taylor et al., 2004). ODD is characterized by the child's inability to accept parental authority and the strong need to be in control. Distinguishing between normal adolescent self-assertion and ODD may not always be easy. Treatment of the ADHD may not resolve all ODD symptomatology. Strategies leading to positive reinforcement and targeting positive goals are often useful. Use of time-out and appropriate strategies that are applied with consistency also help to deal with the oppositional defiance. Behavioural interventions are effective, but they need to be consistent and ongoing.

\subsection{Conduct Disorder (CD)}

The risk for the development of CD in children with both ADHD and ODD is two to three times greater (Barkley, 2004). Behavioural interventions are necessary for this disorder. Comorbid CD also puts children at risk for gravitating towards other children with similar problems. Strategies that promote positive peer relationships and effective empathy development are indicated. A medication trial may be advised in conjunction with comprehensive psychosocial treatment.

\subsection{Intellectual Disability (Learning Disability, LD)}

Children with ADHD frequently fall below control groups on standardised achievement tests (Barkley et al., 1990). Children with ADHD often have weaknesses in the cognitive areas of executive functioning, working memory and processing speed. If LD is documented, it may need more one to one support for the age-appropriate educational progress. Children and adolescents with an IQ less than 50 should not ordinarily be prescribed stimulants as they are usually sensitive to the side effects.

\subsection{Aggression}

Verbal and physical aggression is not uncommon in ADHD. The most common reason why children with ADHD would act aggressively is a combination of ADHD with either ODD or $\mathrm{CD}$. Treating the ADHD is usually the first step. However, aggression might be part of 
another diagnosis. Behavioural interventions and all ADHD medications may decrease aggressive behaviour. If needed, new generation antipsychotic medications can be tried. A study has shown that risperidone is effective in controlling ADHD, ODD and CD (Aman et al., 2004). Monitoring of metabolic changes, weight gain, and extra-pyramidal side effects are necessary if an antipsychotic is used.

\subsection{Bipolar Disorder (BD)}

This is an uncommon disorder in childhood. BD should be considered as the primary diagnosis if there are prominent, episodic, cycling mood symptoms. BD may be suggested by a strong family history of $\mathrm{BD}$ or depression and paradoxical response to stimulants (worsening of mood or rage symptoms).If BD is suspected, referral to a Child and Adolescent Psychiatrist is recommended (CADDRA, 2010).

\subsection{Pervasive Developmental Disorder (PDD)}

PDD presents with difficulties in social communication, social interaction and stereotyped, repetitive behaviour. Clinical symptoms of PDD supersede that of ADHD and should be the primary diagnosis and can co-exist. The FDA recently approved the use of risperidone in controlling aggressive and self-injurious behaviour and irritability (Taylor et al., 2004).

\subsection{Depression}

Many patients with depression may present with transitional inattention, short-term memory problems, irritability and impulsivity (Voeller, 2004). When the depression is associated with problem in the social environment, treatment strategies include individual and family therapy. Stimulants may produce a mild antidepressant effect in some patients, while they may worsen mood in others. All of the drugs used to treat ADHD have the potential to unmask a mood disorder or to cause mood symptoms.

\subsection{Anxiety}

Anxiety in ADHD can manifest as Generalized Anxiety Disorder, Social Phobia, Separation Anxiety Disorder, Post Traumatic Stress Disorder (PTSD) or Obsessive-Compulsive Disorder (OCD). PTSD may be a misdiagnosed as ADHD as there are similar symptom complex. PTSD is likely if there is no clear family history of ADHD or pre-morbid symptoms of ADHD prior to the traumatic situation.

\subsection{Tic disorders}

Stimulant medications can be used to treat ADHD with tic disorders, but caution should be exercised as tics may be exacerbated in some children. If tics appear with stimulants, consider lowering amount/discontinue or change to a non stimulant. The MPH SPC has warnings for tics and anxiety. There are currently no warnings relating to the use of atomoxetine in tics or anxiety. If OCD exists then the combination with ADHD may be part of a Tic Disorder (e.g., Tourette's syndrome) so it is important to look for motor and phonic tics. 


\subsection{Epilepsy}

Seizure control is first priority as numbers of seizures are directly related to processing and attention difficulties. Structural abnormality in brain is probably a risk factor for epilepsy with comorbid ADHD. Moreover uncontrolled seizures cause disturbed sleep, which in turn may result in attention difficulties during the day. Side effects of some anti epileptic drugs such as topiramate, vigabatrin, gabapentine are known to increase aggression in LD and many children with epilepsy are likely to have LD. Stimulants seem to be safe in children with well controlled seizures. ADHD children are more prone for unprovoked seizures then the normal population (Hersdorffern et al., 2004). The SPC for MPH state it may lower the convulsive threshold in patients with prior history of seizures and in patients with prior EEG abnormalities.

\subsection{Substance Use/Abuse Disorder (SUD)}

ADHD patients are at increased risk of using illicit substances. It is essential that a history for substance abuse is explored with the individual alone. Ask whether their friends use drugs or alcohol. A positive response suggests they are likely to be at high risk for substance use. By treating ADHD, there is better outcome in comorbid SUD.

\section{Cardiac risks of drugs}

Sudden death with ADHD medications is very similar to those with sudden death in the general population. Structural heart diseases, history of syncope, family history of sudden death/exercise induced sudden death are clues, which can help to suspect a higher risk. The usefulness of ECG screening in patients being treated with drugs is unknown. The small but unproven potential contribution of ADHD drugs to the rare incidence of sudden death in children must be weighed against the clinical benefit of the medication. In a child or adolescent with ADHD, who has no cardiac symptoms, the risk of cardiac adverse events from ADHD medications is very low. The American Heart Association Recommends (Vetter et al., 2008) that before therapy with psychotherapeutic agents is initiated, a careful history should be obtained with special attention to fainting or dizziness particularly with exercise, complaint of chest pain or shortness of breath with exercise and about seizures. The family history should focus on the long QT syndrome, sudden cardiac death or heart attack in members below 35 years of age and history of Marfan syndrome. Presence of these symptoms/risk factors warrants a cardiovascular evaluation by a cardiologist before initiation of drug. Patients should be asked the occurrence of any of the cardiac symptoms during the follow up visits. The physical examination should include checking heart rate and blood pressure.

\section{Complimentary and Alternative Medicine (CAM) in ADHD}

Over the years, a great deal of media attention has focused on diets for treatment of ADHD. Some suggested a "few foods" approach elimination diet if psychological interventions are not effective (Carter et al., 1993; Hill \& Taylor, 2001). Most of these dietary manipulations involve eliminating additives (Feingold diet) and foods incriminated to increase hyperactivity, such as sugar, chocolate and caffeine or common food allergens such as wheat, milk and eggs. Several double-blind placebo-controlled studies have failed to 
support beneficial effect of dietary manipulation on the behaviour, except possibly in a very small percentage of children (Egger et al., 1992; Wolraich et al., 1995). Few studies have reported behavioural improvement with hypoallergenic diets (Kaplan et al., 1989; Egger et al., 1992; Boris et al., 1994). The results of these studies require further replication before dietary intervention can be considered efficacious. A working group of the American Academy of Child and Adolescent Psychiatry has stated "Given the minimal evidence of efficacy and extreme difficulty of inducing children and adolescents to comply with restricted diet, they should not be recommended (AACAP, 1997). Current evidence suggest that diets are arduous to implement and some may be nutritionally deficient (DTB, 1995) and a restriction or elimination of diet in children with ADHD is not recommended (SIGN, 2001). The available best evidence practice is that a response to food may show change in mood state (irritability) rather than ADHD symptoms per se. An additive-free diet, low in sugar, and avoiding foods that are suspected of exacerbating symptoms is often tried by families to help improve ADHD symptoms. As long as needs for essential nutrients are met, these diets are safe, although their effectiveness in individual children is difficult to predict.

\section{Long term outcomes}

It was thought that hyperactivity simply goes away by adolescence. Although hyperactivity lessens with time but it is often replaced by problems of antisocial behaviour. There appears to be three different patterns of outcomes- resolution of symptoms in young adulthood in about $30 \%$, persistence of some symptoms in about $40 \%$ and severe dysfunction associated with persistent symptoms, substance abuse and antisocial behaviour in 30\% (Cantwell, 1996). A prospective study in London community survey found that hyperactive behaviour was a strong risk factor for later psychiatric diagnosis, antisocial behaviour, and social and peer problems, even after allowing for a coexistent CD (Taylor et al. 1996).

\section{Summary}

ADHD is a persistent and impairing disorder. Although its origin is uncertain, biological, psychological, and social factors are implicated. The first step in the management is accurate diagnosis. Behavioural modification and educational approaches are strategies of first choice. Medication can provide respite from the symptoms during which time other essential aspects of therapy can be implemented. Advice and support about behaviour management and attention to the child's self esteem, peer group interaction, academic progress and family relationships are required even if medication is used. Children with ADHD are cared for at home. Parents are both part of the team and carer for the child and the family needs care themselves. As their child's primary carers, they must be included in the decision of any treatment plans. Some parents collect endless opinions. Sometimes the initial counselling or diagnosis was inadequate and questions that could have been answered were ignored or sidestepped. There are many parents who want specific advice on what more they themselves can do to help the child. For parents of an ADHD child, the number of possible interventions can be extremely confusing. They are likely to hear about a host of treatment options that lack scientific support. 


\section{Key points}

- $\quad$ ADHD is a common behavioural disorder with clear diagnosis criteria.

- ADHD co-exists with other conditions in a high proportion.

- Treatment options for ADHD include behaviour management, medications and educational modification.

- Early recognition and treatment of ADHD may result in less antisocial behaviour, criminality and substance abuse in later life.

\section{Appendix 1.}

\section{Side-effects Questionnaire (parents/carers)}

Child's Name: $D O B$ :

Date:

All medication has side-effects; some are more troublesome than others. We want to make sure that children who are taking medication do not suffer.

For each item, please tick on each line how much that statement applies to your child over the last seven days according to your own observations

0 is not at all

1 is a few occasions only

2 is about half the time

3 is most of the time

4 is all the time

\begin{tabular}{|l|l|l|l|l|l|}
\hline \multicolumn{1}{|c|}{ Symptom } & 0 & 1 & 2 & 3 & 4 \\
\hline Talks less than usual & & & & & \\
\hline Poor appetite & & & & & \\
\hline Irritable & & & & & \\
\hline Complains of stomach ache & & & & & \\
\hline Complains of headache & & & & & \\
\hline Drowsy & & & & & \\
\hline Looks sad, miserable & & & & & \\
\hline Looks anxious & & & & & \\
\hline Seems unsteady & & & & & \\
\hline Excited & & & & & \\
\hline Angry & & & & & \\
\hline Has nightmares & & & & & \\
\hline Displays twitches (tics) & & & & & \\
\hline
\end{tabular}

Is there anything else you would like to add?

Thank you very much 


\section{References}

Aman, M.G, Binder C, Turgay A (2004). Risperidone effects in the presence/absence of psychostimulant medication in children with ADHD, other disruptive behavior disorders, and subaverage IQ. J Child Adolesc Psychopharmacol, 14(2): 243-54.

American Academy of Child and Adolescent Psychiatry (1997). Practice Parameters for the Assessment and Treatment of Children, adolescents, and Adults with AttentionAmerican American Academy of Child and Adolescent Psychiatry (2007). Practice parameter for the use of stimulant medications in the treatment of children, adolescents and adults. J Am Acad. Child Adolesc Psychiatry; 36 (10): 85S- 121S.

American Psychiatric Association (2000). Diagnostic and Statistical Manual of Mental Disorders, fourth edition, Text Revision. Washington.

American Academy of Pediatrics (2000). Diagnosis and Evaluation of the Child With Attention Deficit/Hyperactivity Disorder. Pediatrics; 105: 1158- 70.

American Academy of Pediatrics (2001). Clinical Practice Guideline: Treatment of the School-Aged Child With Attention-Deficit / Hyperactivity Disorder. Pediatrics; 108: 1033-44.

Banaschewski, T, Coghill D, Santosh P, Zuddas A, Asherson P, Buitelaar J, et al. (2006). Long-acting medications for the hyperkinetic disorders: A systematic review and European treatment guideline. Eur Child Adolesc Psychiatry; 15: 476-95.

Bangs M E, Tauscher-Wisniewski S, Polzer J (2008). Meta-analysis of suicide-related behaviour-events in patients treated with atomoxetine, J Am Acad Child Adolesc Psychiatry, 47 (2): 209- 218.

Barcley R A, DuPaul G J, McMurray M B (1990). Comprehensive evaluation of attention deficit disorder with and without hyperactivity as defined by research criteria, J Consult Clin Psychol; 58: 775- 89.

Barkley, R.A (2005). Attention Deficit Hyperactivity Disorder: A Clinical Handbook (third Edition). Guildford Press, New York.

Barnardo's. Accessed on accessed 4.06.11 available at http://www.palmersvilletraining.co.uk/_explore/search.html

Block J H. (1977). Hyperactivity: A cultural perspective. Journal of Learning Disabilities; 10: 236-240.

Biederman J, Newcom J, Sprich S (1991). Comorbidity of attention deficit hyperactivity disorder with conduct, depressive, anxiety and other disorders. Am J Psychiatry; 148: 564- 77.

Biederman J, Faraone S V, milberger S, et al.(1996). Predictors of persistence and remissions of ADHD into adolescence: results from a four-year prospective follow-up study. J Am Acad Child Adolesc Psychiatry; 35: 343-51.

Boris M, Mandel F S (1994). Foods and additives are common causes of the attention deficit hyperactive disorder in children. Annals of Allergy; 74: 462-8.

Bradley, C (1997). The behavior of children receiving benzedrine. American Journal of Psychiatry; 94: 577-85

Buscemi N, Vandermeer B, Hooton N, Pandya R, Tjosvold L, Hartling L, et al. (2006). Efficacy and safety of exogenous melatonin for secondary sleep disorders and sleep disorders accompanying sleep restriction: meta-analysis. BMJ, 332: 385- 88.

Canadian Attention Deficit Hyperactivity Disorder Resource Alliance (CADDRA) (2010) Assessed on accessed on 5/06/2011, available at www.caddra.ca 
Cantwell D P (1996). Attention deficit disorder: A review of the past 10 years. J Am Acad Child Adoles Psychiatry; 35: 978-87.

Carter C M, Urbanowicz M, Hemsley R, Mantilla L, Strobel S, Graham P J, Taylor E (1993). Effects of a few food diet in attention deficit disorder. Arch Dis Child; 69: 564- 68.

Castellanos F X, Lee P P, Sharp W, Jeffries N O, Greenstein D K, Clasen L S, et al, (2002). Developmental Tragectories of Brain Volume Abnormalities in Children and Adolescents With Attention-Deficit/Hyperactivity Disorder. JAMA; 288: 1740- 48.

Coghill D R (2005). Growth in European children with ADHD: results from ADORE. Program and abstracts of the 52nd Annual Meeting of the American Academy of Child and Adolescent Psychiatry; October 18-23, Toronto, Ontario, Canada. Symposium 42E.

Corman SL, Fedutes BA, Culley CM (2004). Atomoxetine: the fist nonstimulant for the management of attention-deficit/hyperactivity disorder. Am J Health Syst Pharm, 2391-2399.

Dopheide J A (2001). ADHD Part I: Current Status, Diagnosis, Etiology/Pathophysiology. AphA - American Pharmaceutical Association 148th Annual Meeting, http://www.medscape.com/Medscape/CNO/2001/AphA/Apha-01.html (Accessed on 8/11/2001).

Drug and Therapeutics Bulletin (1995). The management of hyperactive children. DTB; 33: 57-60.

Egger J, Stolla A, McEwen L M (1992). Controlled trial of hypo sensitisation in children with food-induced hyperkinetic syndrome. Lancet; 339:1150-3.

Eisenberg J. The clinical use of stimulant drugs in children. Paediatrics 1972; 49: 709-15.

Faraone SV, Biederman J, Mick E (2006). The age-dependent decline of attention deficit hyperactivity disorder: a meta-analysis of follow-up studies. Psychol Med;36:15965.

Franke B, Neale B M, Faraone S V (2009). Genome-wide association studies in ADHD. Hum Genet; 126: 13- 15.

Hersdorffern CD, Ludvigsson P, Olafsson E, Gudmundsson G, Kjartansson O, Hauser A (2004). ADHD as a Risk Factor for Incident Unprovoked Seizures and Epilepsy in Children. Arch Gen Psychiatry, 61: 731- 36.

Hill P. \& Cameron M (1999). Recognising hyperactivity: a guide for the cautious clinician. Child Psychol and Psychiatric Review; 4: 50-60.

Hill P, Taylor E (2001). An auditable protocol for treating ADHD. Arch Dis Child; 84:404-9.

Kaplan B J, McNicol J, Conte R A (1989). dietary replacement in preschool-aged hyperactive boys. Pediatrics; 83:7-17.

Konrad K, Eickhoff S B (2010) Is the ADHD brain wired differently? A review on structural and functional connectivity in attention deficit hyperactivity disorder. Hum Brain Mapp; 31: 904- 16

Laufer M W. Long term management and some follow up findings on the use of drugs with minimal cerebral syndromes. Journal of Learning Disabilities 1971; 4: 519-22.

National Institute for Health and Clinical Excellence. Attention deficit hyperactivity disorder (2008). Diagnosis and management of ADHD in children, young people and adults. Clinical Guideline 72.www.nice.org.uk. 
National Institute for Health and Clinical Excellence (2009). Medicines adherence: Involving patients in decisions about prescribed medicines and supporting adherence. Clinical guideline 76, 2009. www.nice.org.uk/CG76 (accessed on 18 April 2010).

Orford E (1998). Commentary: Diagnosis needs tightening. BMJ; 316: 1595-6.

Owens, JA and Dalzell V (2005). Use of the 'BEARS' sleep screening tool in a pediatric residents' continuity clinic: a pilot study. Sleep Med, 6(1): 63-9.

Reiff M I, Banez G A, Culbert T P (1993). Children who have attentional disorders: diagnosis and evaluation. Pediatr Rev; 12: 455- 65.

Sandberg S (1996). Hyperkinetic or attention deficit disorder. British Journal of Psychiatry; 169: 10-17.

Scottish Intercollegiate Guidelines Network (2001). Attention Deficit and Hyperkinetic Disorders in Children and Young People. A national clinical guideline 52. www.sign.ac.uk

Shea, S, Turgay A, Carroll A, Achulz M, Orlik M, Smith I, et al, (2004). Risperidone in the treatment of disruptive behavioral symptoms in children with autistic and other pervasive developmental disorders. Pediatrics, 114: 634-41.

Swanson J M, Sergeant J A, Taylor E, Sonuga-Barke E J S, Jensen P S, Cantwell D P (1998). Attention-deficit hyperactivity disorder and hyperkinetic disorder. The Lancet, 351: 429-33.

Swanson JM, Elliott GR, Greenhill LL, Wigal T, Arnold LE, Vitiello B, et al. (2007). Effects of Stimulant Medication on Growth Rates Across 3 Years in the MTA Follow-up. J Am Acad Child Adolesc Psychiatry, 46 (8):1-12.

Taylor E, Chadwick O, Heptinstall E, danckaerts M (1996). Hyperactivity and conduct problems as risk factors for adolescents development. J Am Acad Child Adolesc Psychiatry; 35: 1213-26.

Taylor E, Sergeant J, Doepfner M, Gunning B, Overmeyer S, Mobius H J, et al. (2004). European clinical guidelines for hyperkinetic disorder - first update. Eur Adolesc Psychiatry 13:1/7-1/30.

Turgay, A (2005). Treatment of comorbidity in conduct disorder with Attention-Deficit /Hyperactivity Disorder (ADHD) (Special Report). Essential Psychopharmacology, 6(5): 277-290.

Vetter VL, Elia J, Erickson C, Berger S, Blum N, Uzark K, et al. (2008). Cardiovascular Monitoring of Children and Adolescents With Heart Disease Receiving Stimulant Drugs: A Scientific Statement From the American Heart Association Council on Cardiovascular Disease in the Young Congenital Cardiac Defects Committee and the Council on Cardiovascular Nursing. Circulation, 117: 2407- 23.

Voeller K K J (2004). Attention-Deficit Hyperactivity Disorder (ADHD. J Child Neurol; 19 (10): 798-814.

Webster Stratton programme

http:/ / www.incredibleyears.com/ (accessed 4.06.11).

Wilens TE, Faraone SV, Biederman J, Gunawardene S (2003). Does stimulant therapy of attention-deficit/hyperactivity disorder beget later substance abuse? a metaanalytic review of the literature. Pediatrics, 111:179-185.

Wilens TE, Hammerness P, Utzinger L, Schillinger M, Geogiopoulous A, Doyle R et al. (2009). An Open Study of Adjunct OROS-Methylphenidate in Children and 
Adolescents Who Are Atomoxetine Partial Responders: I Effectiveness. J Child Adolesc Pschycopharmacology, 19; 485-92.

Wolraich M L, Wilson D B, White J W (1995). the effect of sugar on behavior or cognition in children. JAMA; 274:1617-21.

World Health Organization (1992). The ICD-10 classification of mental and behavioural disorders: clinical descriptions and diagnostic guidelines, Geneva.

Wooltorton E (2005). Suicidal ideation among children taking atomoxetine (Strattera). CMAJ; 173: 1447. 


\title{
ADHD and Comorbid Conditions
}

\author{
Nitin Patel, Mita Patel and Harsha Patel \\ University of Missouri Health Care, \\ USA
}

\section{Introduction}

ADHD stands for Attention Deficit Hyperactivity Disorder. Comorbidities is the medical term for two or more disorders that occur at the same time with the primary disease or disorder. In this article we will talk about ADHD comorbidity.

ADHD (Attention Deficit Hyperactivity) is the most underdiagnosed condition in children. It is also a misdiagnosed disorder. The main reason for this is comorbidity since the comorbid conditions have many similar symptoms to ADHD. The million-dollar question though is: Where does ADHD stop and where do comorbid conditions take over?

ADHD affects 3-6\% of school children (Mirsky, AF 2001). The risk of comorbidity with psychiatric disorders is high, and the presence of comorbid conditions warrants a special consideration in treatment of patient with these disorders. A child specifically with ADHD will likely have other difficulties.

Two-thirds of US children with ADHD will have a comorbid learning disorder, another mental health disorder, or a neuro-developmental disorder. Unraveling psychiatric comorbidity can often be a chicken or egg conundrum. Which came first: the ADHD or the supposed comorbidity? The presence of comorbidity conditions makes the diagnosis of ADHD more difficult.

The survey published by National Survey of Children Health, which involved over 60,000 children ages 6-17 years including over 5,000 with ADHD, showed that psychiatric and physical comorbidities were very common in children with ADHD. (Larson 2011)

Overall, $67 \%$ of ADHD children had at least one other mental health or neurodevelopmental disorder compared to $11 \%$ of children without ADHD. $33 \%$ had one comorbidity disorder, $16 \%$ had two, and $18 \%$ had three or more. ADHD was associated with elevated prevalence of the following (Phend):

1. Learning disorders ( $46 \%$ vs $5 \%)$

2. Conduct disorder ( $27 \%$ vs $2 \%)$

3. Anxiety $(18 \%$ vs $2 \%)$

4. Depression ( $14 \%$ vs $1 \%)$

5. Speech problems $12 \%$ vs $3 \%)$

6. Autism spectrum Disorder (6\% vs $0.6 \%)$

7. Epilepsy/Seizures (2.6\% vs $0.6 \%)$ 


\begin{tabular}{|l|l|}
\hline \multicolumn{1}{|c|}{ Psychiatric } & \multicolumn{1}{c|}{ Physical } \\
\hline 1. Depression & 1. Enuresis \\
\hline 2. Anxiety & 2. Sleep Disorders \\
\hline 3. Bipolar Disorder (BD) & 3. Eczema \\
\hline 4. Conduct Disorder (CD) & 4. Allergies \\
\hline 5. Obsessive Compulsive Disorder (OCD) & $\begin{array}{l}\text { 5. Developmental Coordination Disorder } \\
\text { (Clumsy) }\end{array}$ \\
\hline 6. Substance Abuse & 6. Fibromyalgia \\
\hline 7. Tics and Tourette's Disorder (TD) & 7. Perthes Disease \\
\hline $\begin{array}{l}\text { 8. Pervasive Developmental Disorder } \\
\text { (PDD) }\end{array}$ & 8. Hypertension \\
\hline 9. Learning Disorder & 9. Height and Weight Problems \\
\hline 10. Developmental Dyslexia & 10. Epilepsy \\
\hline 11. Suicidality & \\
\hline
\end{tabular}

Table 1.

The core symptoms of ADHD are inattention, impulsivity and hyperactivity. Children with ADHD often have difficulty in concentration, are easily distractible, and are disorganized, hyper and impulsive. These symptoms of ADHD often are seen in comorbid conditions, as well. Various studies have looked into this overlapping of symptoms and provided evidence that ADHD is not artifact when shared with other psychiatric disorders and the comorbid conditions themselves are not artifact of overlapping symptoms and diagnostic criteria. (Farone2007)

There is a complex integrity between ADHD and commonly occurring comorbid conditions such as ODD, CD, etc. Comorbidity greatly influences presentation, diagnosis and prognosis, complicates treatment and significantly increases the morbidity and disease burden of ADHD. When ADHD is co-morbid with another psychiatric disorder, it is often the first disorder to develop. Children with severe ADHD symptoms have a higher chance of developing other psychiatric disorders.

When evaluating comorbidity, one should determine what the primary disorder and the symptoms present. If the primary condition fully explains the symptoms, a comorbid condition should not be diagnosed. If ADHD symptoms presents during episodes of bipolarity, ADHD would not be diagnosed. In practical life, which symptom causes the patient's impairment is difficult to determine when both disorders are chronic. If both conditions are contributing to patient's impairment, both ADHD and the comorbidity should be diagnosed and treated. This review will clarify how to diagnose and manage ADHD with comorbid psychiatric and other neuro-developmental disorders.

The term comorbidity first appeared in the psychological and psychiatry literature in the mid-1980s. Since that time, there has been a dramatic increase in the interest in this topic. In 1986, only 2 articles were published and by 1993, there were 243 articles. Since then, the number of articles has increased even further. It has been suggested that comorbidity has emerged as perhaps the single most important concept in psychiatry research. 


\section{Definition of comorbidity}

A variety of definitions of comorbidity has been offered from the medical epidemiological perspective. Dr. Feinisten (1970) has defined co-morbidity as any distinct additional entity that has existed or may occur during the clinical course of the patient who has the index disease. Blashfield $(2009,1994)$ has referred to the co-morbidity as a concordance of a different disease in the same individual. Likewise, Caron and Rutter have defined comorbidity as the simultaneous occurrence of 2 or more unrelated conditions.

As one can see from the definition, the term co-morbidity refers to the situation in which a person who has been diagnosed with one specific disorder is found to also meet the diagnostic criteria of one or more additional disorders. There has been a controversy regarding the use of this term, the primary reason highlighted by the definition of Carson and Rutter (1991). The word unrelated is the most relevant. One cannot be certain that an individual who meets diagnostic criteria for more than one psychiatric disorder actually has unrelated conditions. It has been suggested that what appears to be a separate disorder may actually be the result of overlapping diagnostic criteria or a different syndrome, like a variation of the underlying disorder, for example, anxiety disorders. What looks like a co-morbidity in younger children may reflect nonspecific expression of psychopathology associated with immature development of cognition and emotion, for example, anxiety and depression.

Both ADHD and co-morbidity disorders have a highly heterogeneous etiology, implying genetic and environmental risk factors. (Mick and Farone, 2008) Some authors have been advocated for distinct types of ADHD based on comorbid expressions (Barkley 2006) (Acosta, et al 2008).

Apart from the heterogeneousity of ADHD, the overlap between comorbidity and differential diagnosis renders both clinical and etiology research and ADHD and comorbidity difficult. Conduct disorders, emotional disorders, tic disorders, autism spectrum disorders, bipolar disorders, or specific developmental disorders have been comorbid with ADHD but all these psychiatric conditions also have to be considered in the diagnostic process as a differential diagnosis. Comorbid disorders of ADHD may result in ADHD like symptoms; depressive symptoms, for example, may comprise inattention distractibility, aggression, and irritability: symptoms that could mimic the phenotype of ADHD. On the other hand, ADHD may be accompanied with the depressive disorders or result in depressive mood due to constant or repetitive psychological failure and discouragement. With this knowledge of these conditions, a clinician must be aware of the developmental course over time.

Concerning the temporal course of occurrence, comorbidities may be present before evidence of ADHD symptoms (pre-morbidity). The onset of the comorbid disorder coincides with the time the ADHD symptoms reach clinically significant level is called simultaneous comorbidity. However, the majority of co-morbidities seems to occur later in the course of disorder called post-morbidity (Taurine-2010).

\section{Epidemiology}

The mental disorders that children develop are commonly divided into two groups: disruptive or externalizing behavior disorders (Ex. ADHD and Conduct Disorders) and 
emotional and internalizing behavior disorders (Ex. Anxiety and Depression). Concerning psychiatric comorbidity, externalizing disorders were found to be more frequently associated with ADHD; comorbidity rates range up to 90\% (Spencer T 1999, Wilens TE 2002) for externalizing and 50\% for internalizing disorders. (Jensen et al. 2001) A 5-year follow-up study in girls with ADHD ranged 6-18 years in comparison with girls without ADHD showed elevated rates of comorbidity associated with the ADHD females. (Biederman et al. 2008 ) In line with the formal reports (Stinhausen et al. 2006), a sample of 122 children and adolescents with ADHD between the ages of 6-18 years recruited by the Department of Child and Adolescent Psychiatry in the University of Wurzburg showed that $73 \%$ of affected individuals had one or more further psychiatric diagnosis according to a semi-structured interview. The most frequent comorbidity was oppositional defiant disorder (ODD) at $46.9 \%$, followed by mood disorder at $27.9 \%, \mathrm{CD}$ and elimination disorder (EID) at $18.5 \%$, dyslexia at $17.6 \%$, and anxiety at $16.7 \%$, and TD (Tourette disorders) at $9.5 \%$ (Taurin 2010). In adult ADHD patients, lifetime comorbidity with mood disorder was $57.3 \%$ and anxiety disorder 27.2\% (Spencer T. 1999), surpassing the already considerable rates following the pediatric population. Adults have a higher prevalence than children for substance abuse disorders, with a prevalence rate of $45.0 \%$ (Jacob et al. 2007). Personality disorders can develop throughout childhood and adolescent, but It is not until adulthood when personality disorders are able to be diagnosed in ADHD, for example antisocial PD (personality disorder). In childhood and adolescence, these potential personality disorders are known as conduct disorders. The presence of conduct disorders in children with ADHD has been found to be significant correlated with aggressive behavior or delinquency in adolescence and with the high road of antisocial personality in adult life. There has been awareness of a link between ADHD and emotional disorders (anxiety and depressive disorders), which has an important implication for a clinician assessing children with ADHD (that they don't miss the underlying internalizing symptoms because of the predominate presentation). Longitudinal research with children and ADHD and co-morbid major depression suggests that a concurrence of the two disorders may be associated with a particularly poor outcome. The high prevalence of developmental delay and neuropsychiatric disorders has long been established and may be indicative of an underlying developmental etiological pathway.

\section{Etiological consideration}

Many people assume that if the child is hyperactive, the brain must also be overactive. This is not the case, as seen in brain scan research by Dr. Zametkin. In his study, the glucose metabolism was measured in the brain, which is an indication of energy or mental activity. When given a problem to think about, the normal control subject brain indicates energy being used to think about the problem posed, but ADHD subjects' brains show little activity. Does these data show that ADHD is strictly a biological problem? No. ADHD appears to be influenced by environmental factors such as home environment, classroom structures and peers. Saying that ADHD is influenced by environmental factors such as home or classroom environment doesn't mean that ADHD is caused by faulty parenting or education. Rather it means that we have an opportunity to make things better or worse. The etiology to ADHD is complex and includes neuroanatomic abnormalities, neurobiological dysregulation, CNS insults, genetics and environmental factors. The neurobiological abnormalities are in the prefrontal cortex and anterior cingulate gyrus involvement has been 
demonstrated. The prefrontal cortex is involved in executive functioning, while the cingulate gyrus is involved in focusing attention and mediating response selection. Among individuals with ADHD, consistent findings have shown image regarding decreased dopaminergic transmission in these areas. A dopamine 4 (D4 receptor) is related to cognitive and emotional functioning is densely in prefrontal cortex of the brain. Genetics studies indicate the DRD4-7 repeat allele locus at higher rates among children with ADHD than among controls. (Leslie E. Parker 2010) Other numerous environmental factors predispose individuals to ADHD, including maternal smoking or alcohol abuse and low birth weight, which may have both genetic and environmental causes.

Other conditions must be ruled out before a diagnosis of ADHD is made because there are many other possibilities or explanations for hyperactive impulsive behavior. A few of the conditions are learning disability (that leads to poor school performance), frustrations, and a behavior pattern where the child stops paying attention or gives up. Attention lapses can be caused by partial complex seizure, obsessive thoughts, or silent impulsive rituals. A middle ear infection, causing an intermittent hearing problem, interferes with a child's ability to respond to an orally presented request. Disruptive unresponsive behavior can be due to anxiety, depression, or bipolar disorders. Environmental factors or stresses, such as divorce in the family, can lead to restlessness or inattention.

If executive dysfunction is a problem, the child often fails to give close attention to details or makes careless mistakes at school or during other activities, has difficulty sustaining attention in tasks or play, often does not follow thorough instructions, fails to finish school work or duties at the workplace, often has difficulty organizing tasks and activities, avoids/dislikes/is reluctant to engage in the task required to sustain mental effort like homework. In order to clarify the diagnostic issues, the neuropsychological assessment may be helpful. Although a variety of professionals including psychologists may be licensed to diagnose $\mathrm{ADHD}$, in my opinion, the diagnosis should be confirmed by a developmental pediatrician, board certified neurologist or adolescence psychiatrist who can rule out other medical problems that mimic the symptoms of ADHD and offer treatment.

\section{Clinical presentation}

ADHD has historically been identified primarily in boys, but a substantial number of girls suffer from ADHD as well. The male to female ratio is approximately 10:1 (Biederman J 1999). In a comparative study of boys and girls with ADHD, both sexes were comparable in respect to rate of inattention, impulsivity, hyperactivity and comorbidity. However, rates of conduct disorders varied between boys and girls (20\% in boys versus $8 \%$ in girls). Oppositional defiant disorders were manifested in approximately $62 \%$ of boys compared to $32 \%$ of the girls (Fredman 2001).

ADHD is highly comorbid with other psychiatric disorders. There has been controversy whether it exists as a primary disorder or only secondary to other psychiatric symptoms. The Nosological System advocates in the DSM that in the presence of two or more diagnoses, one should be considered primary and account for many of the symptoms observed in the secondary syndrome. Thus the mounting evidence of many conditions existing concurrently with ADHD each modifies the overall clinical presentation and treatment responses. The comorbid conditions should be considered as simultaneously present in order to broaden our understanding and maximizing the treatment. 
A depressed patient demonstrates diminished concentration, whereas an individual with bipolar disorder often manifests psychomotor agitation and destructibility. It may be difficult to differentiate these from the cardinal symptoms of ADHD. There are several ways to deal with the symptom overlap through research and clinical settings. Subtraction method requires that the same absolute number of symptoms be present to diagnose either disorder except shared symptoms are subtracted or removed from the diagnostic consideration. Some people think that the patient with comorbid depression and ADHD still retains the diagnosis of ADHD whether subtraction or proportional method was used. The longterm follow-up study had demonstrated the individual with ADHD and comorbid disorder had poor prognosis and greater hospitalization rate than those with ADHD alone.

Pharmacological studies demonstrated a clinical contribution of each of the comorbid disorders and treatment strategies. For example, an individual with a comorbid ADHD bipolar may be first treated with a stimulant. Alternatively, if a mood stabilizer is used, significant improvement may be expected. If symptoms of ADHD continue to persist after a mood stabilizer, this supports a diagnosis of co-occurring diagnosis. When a stimulant addition is indicated, therefore, both comorbid conditions are equally treated, the ADHD symptoms may not be remit.

\subsection{Identify comorbidity with handy tools}

There are many efficient tools to gather data in a structured manner that quickly pinpoint ADHD, which further helps your clinical acumen. In a busy primary care setting, a child behavior check list can be used as a handout or behavior assessment scale for children. Both of these generally have very similar type of output. These are very easy to score, and one can look to the parent as well as teachers to help with the scoring.

\section{Psychological problems associated with ADHD}

\subsection{Depression}

Does the person appear sad, blue or down and how can you tell? Is the person irritable, cranky, and moody? What does the person do in her spare time? Has the person been doing the activity once enjoyed? Does the person talk about suicide or about uselessness of life, has the person attempted suicide?

A total of $10-40 \%$ (Spencer, T 1999) of children and adolescents with ADHD show depression with symptomology of low or irritated mood, loss of interest and pleasure of usually enjoyable activities, sleep disturbances, and reduced appetite. Depressive disorders in youth with ADHD typically occur as a post comorbidity several years after the onset of ADHD. ADHD rates in depressed children and adults range up to 57\% (Angold, A 1993). Depression may be a reaction to unpredictable environmental stressors such as being rejected by peers, getting made fun of by others, or thinking that school is a negative and overwhelming place. In these cases, a separate diagnosis and treatment strategy is not necessary because depression is occurring in response to the ADHD and will likely decrease when ADHD symptoms are treated. In other instances, depression may run in the family or may be more directly linked to biological or genetic causes; therefore, a separate diagnosis and specific treatment for symptoms for depression would be more appropriate. Distinguishing between an emotional complication of ADHD and a separate depressive disorder may be very difficult. A therapist 
would look for an indication whether these depression or ADHD symptoms came first and whether there is a history of depression or ADHD in the family. It is very important to take depressive symptoms seriously, regardless of their cause. Children with ADHD and depression can have suicidal thoughts and statements/behavior should be monitored and addressed by a mental health clinician. Since these symptoms may attribute to normal childhood behavior, the diagnosis of depression may be missed for a long time. Family studies suggest there is some genetic link between depression and ADHD. This suggests genetics can contribute to solely ADHD, solely depression, or both disorders.

With regard to the treatment, most treatments that are effective for ADHD, like stimulants, do not significantly improve depression. In addition, treatments for mood disorders are generally not helpful for ADHD. In the presence of comorbid mood disorder, stimulants themselves are less effective for ADHD. Non-stimulant treatments that are noradrenergic but not serotonergic are effective for ADHD. In contrast, serotonergic medicine is effective for juvenile depression but not for ADHD.

Bupropion, an agent that affects both noradrenergic and dopaderenergic neurotransmitters, has been shown to be efficacious for adolescents with ADHD and comorbid depression. It is important that both disorders be treated simultaneously. Studies show using the combination of stimulant and SSRI for comorbid ADHD and severe depression are consistent with this observation. However, one should be cautious in using an SSRI in children with mild to moderate depression, due to a black box warning by the FD because SSRIs may increase suicidal tendencies. If an SSRI is used, the child should be monitored closely, and the patient and guardian should be aware of this warning.

\subsection{Suicidality}

Children with ADHD and depression are at an increased risk for suicide. In boys, major depression and social phobia are risk factors for suicide, as opposed to post-traumatic disorder in girls.

Early stimulant treatment of ADHD reduces the prevalence of depression as a comorbidity, which reduces the risk for future suicidality.

\subsection{Bipolar disorders}

Are there times where the person thinks he or she is able to do anything he or she want? Does the person appear unusually energetic at times or almost high without drugs? Does the person miss a lot of sleep at night but still acts energetic the next day? Does the person appear to have thoughts that appear so fast that it is impossible to keep up with them?

Bipolar disorder may occur with ADHD or may mimic its symptoms. Half of the boys and one-fourth of the girls with bipolar disorder also meet the criteria for ADHD. Children and adolescents with bipolar disorder often show strong emotional feelings, hyperactive behavior, overbearing manner, and difficulty waking up in the morning. Children and adolescents with severe bipolar symptoms may have excessive and lengthy temper tantrums that are destructive and often based on gross distortion of objective events. For example, when a friend wants to a play different game, bipolar children may think the friend is trying to purposefully be mean. The child gets angry at such mistreatment. This 
may result in a temper tantrum. Other symptoms include excessive talking, increased activity, inappropriate actions and verbal responses in social situations, lack of inhibition, chronic irritability, and distractibility. The prevalence is up to $20 \%$ (Singh, MK 2006) (Strober, M 1982). According to an Italian study, 24\% of 7-18 year old clinic attendees with bipolar disease had existing ADHD (Masi, G 2003).

The child behavior check list score better discriminates between children with ADHD, comania in context to pediatric bipolar disorders and control subjects.

For pharmacological treatment, mood stabilizers are the first line treatment for periodic bipolar disorders. However, when ADHD symptoms are present, subjects may benefit from short-term co-concomitant treatment with a stimulant or a co-medication of a non-stimulant.

The etiology of comorbid pediatric bipolar and ADHD have distinct characteristics. Neuro imaging studies suggest general changes in prefrontal areas in both disorders. However, there are a few primary differences between the two patient groups in the areas in indifference control, working memory, planning cognitive flexibility and fluency. Several authors reported that ADHD with comorbid pediatric bipolar disorder is its own distinct form of ADHD (Biederman 2008).

Fifty percent of the prepubescent depressed children in one sample manifest bipolar disorder within ten years of the onset of depression (Geller et al, 2002 a). Another study found $20 \%$ of depressed adolescents in another sample had revealed a bipolar disorder within 1-4 years (Kowatch RA 2005). When comparing to the children with ADHD without mania, the manic children have significantly higher rates of major depression, psychosis, multiple anxiety, conduct disorder, or oppositional defiant disorder, as well as significantly greater impairment of psychosocial functioning.

As with depression, bipolar must be treated effectively with symptoms of ADHD to resolve comorbidity affecting the individual. An atypical anti-psychotic agent appears to be effective in the elimination of juvenile mania. In an open study, Risperdal was found to be effective anti-manic but did not help ADHD symptoms. Among bipolar adults comobid for ADHD, Bupropion is effective for ADHD and depression but may lower the threshold for inducing mania.

\subsection{Oppositional defiant disorder}

Does the person defy you or the teacher by simply saying no or ignoring you? Does the person appear to be annoyed easily and bothered by trivial things? Does the person appear to annoy other people on purpose? When and where does this happen? Does the person appear angry, hot tempered, resentful, or full of spite?

ODD is most commonly associated with ADHD. Oppositional defiant disorder symptoms occur in as many as $21 \%$ to $60 \%$ (Cunningham 2002; Wilens, TE 2002) of children with ADHD. It is when the individual defies rules and in some cases acts out in anger and violence. ODD symptoms tend to occur more often with people whom the child is close to or knows well such as family or care givers. Children with comorbid ADHD and ODD may form an intermediate group between those who have ADHD alone and those with ADHD and conduct disorder. However, studies suggest that children with comorbid ODD and ADHD will always develop conduct disorder. It is just a matter of time before that happens. 


\subsection{Conduct disorder}

Does the person lie a lot? Does the person get into physical fights? Does the person try to hurt people? Has the person ever stolen or damaged people's property?

About $20-45 \%$ of children with ADHD also meet criteria for conduct disorders. It is very low in children without ADHD. This disorder involves aggression towards people or animals, property destruction, stealing, and violation of rules of society (i.e. missing school or running away from home).

Both stimulant and non-stimulant medicines will reduce aggressive behavior and antisocial acts, but a stimulant will work more rapidly. Mood stabilizers or atypical antipsychotics may be needed for highly aggressive cases. Besides pharmacotherapy, individual or family behavioral therapy may be required. Conduct disorder is a strong predictor for future substance abuse.

Medication just used for ADHD is also effective for treatment of comorbid ODD/CD as the first line of treatment. However, if ODD or CD persists, psychosocial treatment should be added. In clinical practice, it is not common to add atypical antipsychotics, but there is limited data to support this. (Kunwar, A 2007)

\subsection{Anxiety}

Does the person appear to be nervous and anxious? Are there times when the person appears panicked, stricken, or frozen by anxiety? Does the person appear very shy compared to others his same age? Does the person repeat certain actions over and over like a ritual?

Anxiety is a psychological and physiological state characterized by emotional, somatic, cognitive and behavioral issues. Anxiety is a well thought out, normal response to stress, but if the anxiety reaches its crest, it disturbs a person's normal routine. Anxiety with ADHD is a comorbidity with an estimated rate of $20-40 \%$. Often general anxiety is thought to be the most prevalent disorder followed by social phobia, separation anxiety. Some children with ADHD may present with more than one anxiety disorder. Anxiety and ADHD may inhibit impulsivity so children with ADHD and comorbid anxiety may have less impulsivity but more inattention. For this reason, it is possible to overlook ADHD in children with anxiety (especially the inattentive types). Children with anxiety are often preoccupied with a fear that impairs their ability to focus on the task at hand. For anxious children who do not have ADHD, when their anxiety improves, their inattention improves, whereas children with comorbid ADHD/anxiety will continue to struggle with inattention even in the absence of an anxious episode.

It has been suggested that anxiety associated with ADHD is a product of the inability to function in daily life because of the social and cognitive limitations associated with ADHD rather than typical phobic/fearful behavior. In this case, early recognition and treatment of ADHD may itself improve anxiety. In a sub-group of an ADHD person with comorbid anxiety disorder, various therapeutic measures have been proven to be effective in reducing both ADHD and anxiety symptoms. Psycho stimulants plus behavior therapy, atomoxetine alone, or atomoxetine with a combination of stimulants and antidepressants can help treat these symptoms. However, comorbid anxiety symptoms in children and adults with ADHD were linked to poor treatment response with a psycho-stimulant. There is some evidence 
that there is heritability between ADHD and comorbid anxiety disorders. There is also research suggesting ADHD and anxiety are independently transmitted in families (Freitag 2010).

It is very important to distinguish between the true anxiety disorders and those children who are experiencing anxiety in response to ADHD because treatment is very different. Stimulants, while helpful for ADHD symptoms, may actually worsen the symptoms of a true anxiety disorder.

\subsection{Learning disorders of various kinds}

Even when the person is paying attention, is learning difficult? Are there certain subtypes that the person has extreme difficulty with? How does this person do in reading, writing, and mathematic? Has the person ever been tested for a learning disability?

Over half of all children with ADHD also have learning disorders. ADHD, though it affects the ability to learn, is not a true learning disability. So treating the symptoms of ADHD will not correct the learning disorders that a child may have. A learning disability is a specific disorder that affects one of the four primary steps needed for learning. The steps are recording information (ex. input of a visual or auditory perception problem), understanding information (integration: ex. Sequencing and organization problem), storing information (placing information into memory), and retrieving information (memory: ex. Immediate recall of the recently learned information). Although ADHD may globally interfere with the success of these steps, it is the impulsivity, hyperactivity and distractibility that interfere with the learning process. ADHD does not specifically impact one of these four steps.

1. On IQ measurement, many studies have identified a fairly difficult profile of the neuropsychological test result in children with ADHD. This is seen commonly through certain attention loading subtests of commonly used IQ tests like the Coding Digit Span and the Wechsler scale. Two or more subtests on the Wechsler scale are often depressed relative to other subtests. Coding, digit response, arithmetic, and information subtests on the Wechsler scales are very often depressed related to the other subjects. Various tests of executive functions including Wisconsin card scoring test usually revealed moderate to major deficits. However, there are also those with ADHD who generally score low on all these tests without a particular learning disorder profile. 2. Overall decline in all IQ test values. 3. Higher than expected rate of subnormal intelligence in mental retardation/learning disability among children with ADHD 4. An increased rate of children with subnormal intelligence because the mistakenly diagnosed individual as suffering from ADHD.

Subnormal intelligence and ADHD: No formal study but clinical experience suggests that children with subnormal intelligence with no indices of specific subtest pattern of neuropsychological testing may show all typical symptoms of ADHD (particular symptoms of inattention is lack of persistence and forgetfulness).

Mental retardation/ learning disability and ADHD: Population studies show mental retardation may be 5-10 times as common in children with ADHD as compared children without ADHD. Though the rate of ADHD in mental retardation is rare, it is clear that relevance has increased beyond the level that is encountered in the population without 
learning disabilities. In one study in the US, at least $15 \%$ of individuals with a profound level of mental retardation may meet criteria for ADHD even the mental age has been taken into account (Fox 1998).

Reading disorder, written expression and dysgraphia: Reading disorders are common in ADHD. About $25-40 \%$ with ADHD have major reading and writing difficulties (Shaywitz, RA 1992). Some studies suggest ADHD and reading disorders combined may be a visual motor problem, more severe than would be expected on the basis of simply adding a basic learning disorder to another. Disorders of written expression are characterized by significant impairment in writing, grammatically correct sentences and paragraph organization and occasionally with dysgraphia.

Mathematic disorders: The overlay between ADHD and mathematic disorder is considerably more. It appears it is more associated with an inattentive type of ADHD. Correlation of ADHD with learning disorders and cognitive delay is common.

Developmental Dyslexia: Some developmental disorders, such as developmental Dyslexia may manifest as a simultaneous comorbidity with ADHD. Although some ADHD symptoms are generally presented in infancy, it is not until the school age group that a full extent of symptoms becomes prevalent because of the highly structured school environment. The developmental dyslexia and dyscalculia become apparent when the child attends school and is required to read, write, and do calculations. Developmental dyslexia, especially reading and spelling disorders, is characterized by difficulty with reading comprehension, reading decoding and reading fluency and spelling. Impairment in multiple cognitive functions, such as executive functions, is common in ADHD and developmental dyslexia. The symptoms may negatively affect the developmental course of ADHD and will lead to stagnation of the process of learning to read. In some cases, ADHD and dyslexia may not share an etiological factor. A dyslexic child may be inattentive in some classes because he or she will be impaired due to generalized reading problems. Both dyslexia and ADHD are considered a complex polygenetic disorder and pose a shared genetic risk factor exerting a unique effect on either ADHD or dyslexia.

\subsection{Pervasive developmental disorders/autism spectrum disorders}

It is commonly observed that children with ADHD frequently show symptoms of autism spectrum disorders and vice versa. Autism spectrum disorders are characterized by impairment in social interaction and communication and by restricted and repetitive behaviors (Frietag 2007). Intelligence is often impaired and the ability to learn and utilize new information is markedly restricted. Behavior symptoms may include hyperactivity, aggressiveness, impulsivity, short attention span and temper tantrums. Similarities between autism spectrum disorders and ADHD can lead to an early childhood diagnosis that suggest both are present. As this child grows up the difference becomes more distinct and one disorder often emerges as the true condition. Autism spectrum disorder children with additional ADHD symptoms showed exaggerated impairment in executive control and adaptive behavior. They show autistic traits as well as more severe oppositional defiance disorder type behavior than children with autism spectrum disorder only. For clarification of a possible etiological link between ADHD and autism spectrum disorders, well designed family based studies are required. 
Children with Asperger's syndrome have a very high rate of concomitant ADHD symptoms. A community-based study indicated that up to $80 \%$ of all people meeting criteria for Asperger's, according to Gillberg, also meet the ADHD criteria (Ehlers 1993). Autistic features in ADHD will benefit from interventions that are appropriate for autistic children without ADHD.

\subsection{Tics and Tourett's disorder}

Does the person have movement such as eye blinking, making an odd face, shrugging or moving an arm a lot that is not intentional? Does the person make noise without meaning to such as grunting, sniffling, or saying certain words? Do these symptoms get worse when person is under stress or anxiety and/or are these symptoms present while the person is sleeping?

Tic disorders including Tourett's disorder (TD) are neuro-developmental disorders characterized by waxing and waning motor and / or phonic tics. There is data that suggests that tics and Tourett's are associated with $47 \%$ of ADHD (Sapiro AK 1988). There is evidence that the children displaying both disorders (Tourett's and ADHD) are more likely to be referred for assessment and treatment rather than be referred to learn which disorder alone is prevalent.

The core symptoms of tics disorders are motor and vocal tics which wax and wane over time. Tourett's shows concomitant symptoms of multiple motor tics and one or more vocal tics. About $85 \%$ of patients with Tourett's show associated neuropsychiatric problems. These are often responsible for psychosocial impairment. Tics can be found more frequently as a comorbidity to ADHD when there is a family history of tic disorders and or there is an early onset of a tic disorder of high severity of symptomology. Children with Tourett's and ADHD suffer from more externalizing and internalizing behavior problem and low social adaption than children without Tourett's disorder.

Usually ADHD starts 2-3 years before tics, while a similar proportion of cases of ADHD can be only after a tic's onset. It is not fully clear if ADHD with early onset may be related to a greater presence of another disorder that will only become symptomatically striking months to years later. Early ADHD can be seen as a risk factor for further associated psychiatric problems like tic disorders.

The pathophysiological mechanism of co occurrence of ADHD and Tourett's is not yet clarified. The stimulant medication in ADHD patient possibly triggers tic occurrence. Alpha 2 agonists and atomoxetine significantly improve tic symptoms. There is evidence that supratherapeutic doses of dextroamphimine worsen the tics but methylphenidate does not worsen tic severity in the short term. If tics or TD are socially disabling then consider behavior therapy.

Patients with co-existing tic disorders may occasionally need, beside treatment with a stimulant for ADHD, additional medication with a dopaminergic agonist, like Risperdal.

\subsection{Obsessive compulsive disorders}

Obsessive-compulsive disorders are characterized by recurrent intrusive thoughts and images or repetitive behaviors that aim to reduce anxiety. Up to 30\% (Geller 1996) of children and adolescents with obsessive-compulsive disorders also present with ADHD 
symptoms. The rate of OCD among children with ADHD is 8-11\% (Arnold 2001), but that rate is higher among children with Tourette 's disorder. Patients with comorbid ADHD and OCD were characterized by early onset of OCD symptoms. Patients with comorbid OCD and ADHD symptoms seem to require special care and treatment because the longer those symptoms persist, the more they increase in severity. OCD can be treated with an SSRI, like Prozac, and behavior modification.

\subsection{Substance abuse and ADHD}

Do you suspect this person smokes, uses drugs or drinks alcohol? Why do you suspect this?

Individuals with ADHD have several characteristics that make them more vulnerable to substance abuse. These includes self-medication (i.e. teenagers taking Adderall), impulsive tendency to associate with other individuals who are not doing well in school, and social skill problems. Appropriately prescribed stimulant medication does not seem to increase the chance of later substance abuse. Several studies now show that stimulant treatment of ADHD does not increase the risk of later substance abuse and that if anything, such intervention considerably decreases the risk of substance use disorders.

Smoking is associated with ADHD. The presence of ADHD increases the risk he or she will be a cigarette smoker by at least 3 times the level of the general population. Smoking usually begins at an earlier age and persists throughout life. It is more difficult to give up in ADHD cohort. Alcohol another common type of substance abuse disorder in ADHD. In adolescence, alcohol abuse cases are seen in 1 in 3 individuals with ADHD in clinical based studies. In clinical practice, the suspicion of substance abuse warrants urine or blood screening to confirm the diagnosis. Adolescents with substance abuse disorders comorbid with ADHD are associated with a greater severity of substance abuse conduct problems and worse treatment outcomes (Sullivan 2001). Some have questions whether the long-term treatment of ADHD with a stimulant increases the risk for substance abuse in adolescents or adult. The most published study does not support this opinion. In fact, the study found that stimulant treatment in ADHD reduced the risk for substance abuse disorder by $50 \%$, bringing it to the level of the general population (Farone, SV 2003). This suggests the treatment of ADHD may itself reduce the long-term risk for development of substance use disorder and thus highlights the importance of early recognition and treatment of ADHD.

In patients with comorbid ADHD substance use disorder, specific substance abuse treatment should be instituted first and once the patient is substance free, medication can be used to treat the impairment associated with ADHD. Atomoxetine, a non-stimulant may be more suitable for treatment of ADHD symptoms in this population, though a stimulant can also be used effectively. I often encourage group therapy in dealing with the drug and encourage abstinence. Family therapy is also a good idea. The individual may benefit from a 12 step group. Bupropion (Solhkhah, R 2005) and tricyclics (Weiss, RD 1989) are typically considered the first line of pharmacotherapy for comorbid ADHD and substance abuse disorders in children and adolescents. Second line agents of the stimulant are generally thought to be a safe and effective treatment of the ADHD. If untreated, ADHD is associated with high rates of alcohol use during adulthood. A 15 year follow up study showed ADHD and alcohol abuse are highly comorbid among adults. Treatment of ADHD in children and adolescents may actually reduce the long-term risk for development of substance abuse. 


\subsection{Personality disorders}

In the adult age group, individuals with ADHD are often diagnosed as suffering from one or more of the so-called personality disorders. Even in late adolescence, there is a very high rate of individuals with ADHD with and without concomitant developmental coordination disorders who meet full symptomatic diagnostic for one or more personality disorders. However, in children under 18-years-old, these disorders are classified as conduct disorder rather than a personality disorder. These personality disorders could be of any type, but it appears that those involved in major social dysfunctions may be particularly common. These include, for example, schizoid, schizo typical, paranoid, avoidant or obsessive compulsiveness and borderline personality disorders. It is unclear to what extent it is helpful or not to make an additional diagnosis of personality disorders in a person who is suffering a neuro developmental/neuropsychiatric disorder such as ADHD. It is doubtful whether the personality disorder diagnosis contributes any in the way for further explicating the underlying nature of problem faced by the individual. It is however important for the adult psychiatrist to be aware that many of their patients who are meeting the criteria for one or more personality disorders are really suffering from ADHD.

\section{Non-psychological (physical) problems associated with ADHD}

There is substantial literature on the prevalence of psychiatric comorbidity in ADHD, but non-psychiatric disorders co-occurring with ADHD have recently gained wider attention. In addition to psychological issues, the person with ADHD may experience physical problems including recurrent headaches, muscle aches and pains, and abdominal pain, which may result from the bacterial infection, parasites, food allergies, neurotoxicins, or environmental toxicity. Severe fatigue allergic disorders are asthma and eczema, respiratory infections, and ear infections. Many adults suffer from Fibromyalgia or chronic fatigue syndrome. A problem may arise with the medication. Those medications that are given for comorbid may have an undesirable affect on ADHD and vice versa.

\subsection{Elimination disorders}

These include nocturnal enuresis, diurnal enuresis, and encopresis. According to one study, children with ADHD may have significant higher rates of incontinence, constipation, urgency, frequency voiding, nocturnal enuresis and dysuria than those without ADHD. For years, clinicians have anecdotally noted an increased incidence of enuresis in children with ADHD. Others have observed that their parents with enuresis have an increased incidence of ADHD. Because both conditions are fairly common, it is important to have more systemic studies that look at the relationship between enuresis and ADHD. An article in Southern Medical Journal published in 1997 compared a fairly large group of 6-year-old children with ADHD to a non-ADHD controlled group selected from a pediatric clinic population. That study found 6 year olds with ADHD had 2.7 times higher incidence of enuresis (Robson 1997). Sometimes enuresis may be more upsetting for children with ADHD. A non-ADHD child who successfully overcomes his fears may be able to accept his bedwetting more easily. However, a child with ADHD already feels different from his peers. His disorganization and impulsivity may lead to peer rejection and shame. Such children may cover his shame with appearance bravado. Some children with ADHD sleep deeply and have trouble waking up to go the bathroom when their bladder is full. A family study 
suggested that ADHD and this type of elimination disorders are etiologically independent psychiatric conditions.

To treat the child with both ADHD and enuresis, it is important to first do a complete physical examination and ask the individual with ADHD about current and past bedwetting problems. Don't neglect to ask adolescents about this too. They will rarely volunteer this information on their own. Also, ask what treatment has been tried in the past. Some children in their teens with ADHD have tried with different therapies in the past. Because of this, they already might expect the treatment to fail. One may modify behavior intervention to accommodate for the child's short attention span. One must prioritize symptoms. If the child has a myriad of behavior difficulties, the family cannot address all of them at once. When the family decides this is the right time to treat the enuresis, they may have to back off with some of their other behavior goals to avoid being overwhelmed. The child and the family should be made aware that there are several ways to treat the enuresis. If one does not work, you are not a failure. That should lead to making a different plan for the future.

\subsection{Sleep problems}

Studies suggest that children with ADHD are more likely to have sleep problems than children without ADHD. As many as 56\% of children with ADHD have problems falling asleep, compared to the $23 \%$ of normal children (Corkum 1998). Up to $39 \%$ of children with ADHD show a problem of waking up in the middle of the night. Resistance to go to bed and fewer total hours of sleep seems to be a major problem with many children with ADHD and likely adds to their difficulties in functioning at school. Studies on sleep pattern do not typically suggest specific difficulties with the nature of sleep itself in these children. Sleep related and involuntary movements appear to be more common in children, and these may be specifically linked to ADHD and coexisting disorders or treatment. One study suggested a link between ADHD and restless leg syndrome in children (Piccheietti 1998).

If sleep disorders are severe, polysomnography should be conducted. Treating sleep disorders may improve a child's attention at school. ADHD treatment with stimulant medication may cause insomnia. One should consider sleep hygiene before using medicine to reestablish new sleep-wake cycles.

\subsection{Allergies}

Minor allergic disorders have been implicated as a possible comorbid condition in ADHD. Most of the evidence runs to counter the notion that there is true association between allergies and ADHD. Despite various discussions in regards to the relationship between allergies and ADHD, studies on ADHD and allergic rhinitis and asthma have provided largely negative results, suggesting the relationship may be small, if it exists at all.

\subsection{Eczema}

A series evident immunological investigation reveals the strong association of ADHD and eczema (atopic eczema or atopic dermatitis). This eczema is a relevant cause of pediatric sleep disruption. Since disrupted sleep is a frequent feature of ADHD, this may constitute a putative causal link between both disorders. Even more interestingly, two independently 
longitudinal based birth cohort studies found a persisting effect of early detection of atopic eczema on mental health. Children who suffered from eczema during the first years of life and showing remnants of symptoms thereafter will have an increased risk for behavior problems at age 10. Even though the causal relation is still unclear, this data demonstrates that eczema during early childhood predisposes kids to developmental behavior problems later on. In a population based study on adult eczema, an association was observed with a wide range of psychiatric issues including depression and personality disorders, indicating that eczema may predispose individuals to age-dependent mental health problems. (Schmitt et al, 2009)

\subsection{Height and weight problems}

ADHD occurred in as many as $27 \%$ of children receiving treatment for obesity (Altfas, JR 2002). In addition, several studies suggest a negative effect on height and weight after years of treatment with central stimulant in ADHD. The evidence is equivalent and more recent studies say that a reduction of final height may be minimal or nonexistent. Although not related to the atopic mechanism, the assumption that nutritional components may cause or exacerbate ADHD symptoms further fuel various therapeutic diets and emphasizes the importance of dietary adjustments in ADHD children.

\subsection{Hypertension}

Blood pressure is significantly increased in individuals with ADHD treated with central stimulant and/or atomoxetine. It is unclear at this stage whether such treatment may have a lasting effect on blood pressure or increased risk for later hypertension.

\subsection{Fibromyalgia}

Fibromyalgia is a common condition in adults, particularly in women. Some adult psychiatrists with vast experience in this age group believe the rate of ADHD has increased the baseline population prevalence for this condition.

\subsection{Perthe's disease}

This hip disorder is typically encountered in children of preschool age who more or less start limping. It is listed here not because of there has been published empirical studies of possible connection between ADHD and Perthe's disease but because some pediatric orthopedics have suggested that many young patients with a hip problem may have ADHD.

\subsection{Clumsiness}

This occurs in $50 \%$ of children with clinical diagnosis of ADHD. Also, nearly half of children with clumsiness display ADHD. It is associated with attention, reading, and writing problems. Children with ADHD are more likely to experience injuries due to accidents than normal children.

Clumsiness improves over time, but $1 / 3$ of children will carry it into adult life, causing problems with fine and/or gross motor movements (Kadesjo, B 1999). 


\subsection{Epilepsy}

Three percent of children with ADHD have some kind of seizure disorder. Twenty five percent of children with epilepsy will have ADHD. Most children with ADHD and comorbidity will have staring episodes. It can be differentiated from any kind of seizure by asking the parents to make the child blink. If the child blinks, it is very unlikely that it is a seizure. If the staring is related to seizures, then there will be more autonomic nervous system related signs, such as increased heart rate and pupil dilation. Every child with the diagnosis of ADHD needs a routine EEG to rule out the most common disorder, childhood absence epilepsy. Though partial complex and generalized seizure disorders may exist, an EEG can confirm those findings.

Appropriate anti-epileptics should be used as treatment, in addition to treatment of ADHD. There is a $1-3 \%$ chance that stimulant medication may worsen the underlying seizure disorder.

\section{Discussion}

It can be challenging to identify and treat children with ADHD, especially in those with comorbidity. The high prevalence could be due to increased awareness by clinicians. Parents may refuse to accept their child's diagnosis of ADHD, and much less that of a comorbidity.

The high comorbidity rate between ADHD and other disorders have essentially created confusion regarding the definition of a true ADHD diagnosis. Since most children or adults with ADHD also have a second diagnosis where both sets of symptoms frequently overlap, critics suggest that the new scenes of ADHD have not been adequately described. Some even suggest that making an early ADHD diagnosis in a very young child is inappropriate. For instance, recently identified conditions in the DSM of early onset bipolar disorder mimics ADHD in the areas of impulsivity, mood inconsistencies and hyperactivity. It may not be clear whether a very young child suffers from ADHD, early onset bipolar disease or another condition that mimics ADHD symptoms. Obviously establishing an accurate ADHD diagnosis is very challenging for even the best clinician.

First point I would like to discuss is in regards to mental retardation. The diagnosis of ADHD should be given only when the symptoms of inattention or hyperactivity are present to the degree in excess of mental retardation of a child. However, there are no criteria for evaluating developmental inappropriateness in term of mental age. Some investigations consider it insufficient to establish deviation in terms of chronological age-appropriate behavior without special consideration to the mental age. Even with this difficult data determining developmental appropriateness, it is better to exclude the patient under mental age of 4 years and the patient of moderate to severe mental retardation with IQ under 50 . The cases of mental retardation and borderline intelligence together show the significance of concomitant delay and mental development. There are no reports of an ADHD patient with mental retardation and borderline intelligence. This finding makes it necessary to incorporate intelligence testing in ADHD diagnosis for evaluating whether the patient has maladaptive behavior arising not only from ADHD but from mental delay, as well.

It is clear that the issues of comorbidity have important implications for understanding assessment and treatment of the children with ADHD. Children with ADHD significantly 
display a learning disability, $O C D, C D$, anxiety disorder or depressive disorder while still others may show evidence of a co-occurring tic disorder or perhaps a bipolar disorder. Some show multiple comorbid disorders. The presence of comorbid conditions likely has a significant implication for long-term outcome. Children with comorbid features often show more serious levels of impairment, are more likely to have continuing problems and require a greater utilization of mental health services than do those without evidence of comorbidity. Assessing the presence of comorbid features complicate the clinical pictures essential in working with children with ADHD.

Giving the proper assessment should lead to optimal treatment. A treatment program for children with ADHD and comorbid conditions should address the full range of problems highlighted by assessment findings. For example, when a child not also only shows the feature of ADHD but also meets the diagnostic criteria for oppositional defiance disorder and learning disorders, treatment should focus on all the problems associated with each of these areas. This might involve pharmacological treatment for dealing with the child's hyperactivity, impulsivity, and inattentive behavior. Parents must be oriented to behavior management approaches to modify oppositional defiance behavior. Specially designed educational approaches are available to assist the child academically. Likewise, in the case of a child with ADHD and comorbid depression, it will be necessary to treat the child's depression as well as ADHD symptoms. With children displaying other patterns of comorbidity, other combinations or approaches to treatment may be suitable.

Simply treating symptoms of ADHD is not enough. Appropriate case management involves addressing the full range of clinical problem displayed. Indeed, effective treatment for children with ADHD and comorbid condition is likely to be multi-disciplinary in nature and more extensive and complex than the treatment for children with uncomplicated ADHD. More research is needed to guide treatment of children with ADHD who display a specific pattern of comorbidity.

Thus, ADHD is a complex condition, affecting at least $5 \%$ of children population and therefore, needs to be better identified and treated (Farone, S 2003). Those who seek medical or psychological help have at least one or more majorly associated disorders or problems. These problems need to be appropriately identified and treated, as well.

Co-existing disorders affect the outcomes of individuals with ADHD. It is not well known about the long-term outcome of children with ADHD and comorbidity. Some of these conditions like ODD and conduct disorders have a long-term negative prognosis in terms of later substance abuse and anti-social personality disorder. The clumsiness may affect long term academic problems.

A long-term follow study is needed to address the outcomes of these comorbidities with ADHD.

\section{References}

Acosta MT, et all (2008). Latent Class Subtyping of ADHD and Comorbid condition. J Am Acad Child Adolsc Psychiatry 47; 797-807

Altfas JR. (2002) Prevelence of Attention Devicit/Hyperavtivity disorder. Among Adults in Obesity Tretment. Bio Med Central Psychiatry 2 (1): 9. 
Angold A, Costello EJ, (1993) Depressive comorbidity in children and adolescents. Emperical, theoritical and methodological issues. Am J Psychiatry. 150, 17291791.

Arcelus Jon, Vostanis Panos. (2005) Psychiatric comorbidity in children and adolescents, current opinion in psychiatry. Volume 18(4) July 2007 429-434.

Arnold PD, Ickowicz A, Chen S, Schachar R (2005) Attention-deficit hyperactivity disorder with and without ovsessive-compulsive behaviours: clinical characteristics, cognitive assessment, and risk factors. Can J Psychiatry 50:59-66

Austin Margaret, Reiss Natalie Staats, Burgdorf Laura (2007) ADHD comorbidity mental help.net.

Barkley RA (2006). ADHD, a hand book for diagnosis and treatment, Guiford Pres, New York.

Biederman J, Ball SW, Monuteaux MC, Mick E, Spencer TJ, Mc-Creary M, Cote, Faraone SV (2008a) New insights into the comorbidity between ADHD and major depression in adolescent and young adult females. J Am Adad Child Adolesc Psychiatry 47:426434.

Biederman J, Faron SV, Mick E, et al. Clinical correlates of ADHD in female: finding from large group of girls ascertained from pediatric and psychiatric referral sources. J. Am Acad Adols C Psychiatry 1999: 38: 966-975.

Blashfield R, Keeley J, Burgers D. 2009. Classification In P.H. Blaney \& T.Millons (Eds) Oxford textbook of Psychopathology (35-57) N.Y. Oxford University Press.

Blashfield R-K, McElroy RA, Jr., Pfonl B, Blum N. (1994) Comorbidity and prototype model. Clinical Psychology: Science and Practice I, 96-99.

Caron C, Rutter M (1991), Comorbidity in Child Psychopathology: Concepts, issues and research strategies. J. child Psychology Psychiatry 32: 1063-1080.

Corkum P, Tanneck R, Moldofsky H. Sleep disturbances in children with attentiondeficit/hyperactivity disorder. J. Am. Acad. Child Adolesc. Psychiatry 1998; 37: 637-646

Ehlers S, Gillberg C (1993) The epidemiology of Aspergers syndrome. A total population study. J Child Psychol Psychiatry 34:1327-1350.

Farone S, Sergeant J, Gillberg C, Biederman J. (2003) The World Wide Prevelence of ADHD: Is it an American Condition? World Psychiatry. 2. 104-113.

Farone SV PhD, Kunwar, A.(2007) ADHD in children with Comorbid Conditions: Diagnosis, Misdiagnosis and keeping Tabs on Both. Medscape.org/viewarticle/555748

Farone SV, Wilen T, Does Stimulant Treatment Lead to Substance Use Disorder? J Clin Psychiatry. 2003: 64 (Suppl 11: 9-13.

Feinstein AR, The Pretherapeutic Classification of co-Morbidity in Chr.disease. J. Chron.Dis. 1970, 23:455-468.

Fredman Steffany J.MA, Korn Martin L., MD (2001) ADHD and comorbidity, medscape.org/viewarticle/418740.

Freitag CM, Rohde L, Lempp T, Romanos M (2010) Phenotypic and measurement influences on heritability estimates in childhood ADHD. Eur Child Adolesc Psychiatry 19:311323.

Fox RA, Wade EJ (1998) Attention deficit hyperactivity disorder among adults with severe and profound mental retardation. Res Devel Disabil 19:275-280. 
Geller DA, Biederman J, Griffin S, Jones J, Lefkowitz TR (1996) Comorbidity of juvenile ovsessive-compulsive disorder with disruptive behavior disorders. J Am Acad Child Adolesc Psychiatry 35: 1637-1646

Geller DA, Biederman J, Faraone SV, Cradock K, Hagermoser L, Zaman N, Frazier JA, Coffey BJ, Spencer TJ (2002b) Attention-deficit/hyperactivity disorder in children and adolescents with obsessive -compulsive disorder: fact and artifact? J Am Acad Child Adolesc Psychiatry 41:52-58.

Geller B, Zimerman B, Williams M et al (2002a) DSM-IV mania symptoms in a prepubertal and early onset bipolar disorder phenotype compared to attention-deficit hyperactivity and normal controls. J Child Adolesc Psychopharmacol 12:11-25.

Gillberg C (1991) Clinical and neurobiological aspects of Aspergers syndrome in six family studies. In: Frith U (ed) Autism and Asperger Syndrome. Cambridge, Cambridge University Press, pp 122-146.

Gillbert Christopher, Gillberg I. Carina, Rasmussen, Peder, et al (2004). Co-existing disorders in ADHD-implications diagnosis and intervention. European Child Adolescent psy. (Supl 1) 13:1/80-1/92.

Jacob CP, Ramanos J, Dempfle A, Heine M, Windemuth-Kieslbach C, Kruse a, Reif A, Walitza S. Romanos M, Strobel A, Brocke B, Schafer H, Schmidke A, Boning J, Lesch KP (2007) Co-Morbidity of adult attention-deficit/hyperactivity disorder with focus on personality traits and related disorders in a tertiary referral center. Eur Arch Psychiatry Clin Neruosci 257:309-317.

Jensen PS, Hinshaw SP, Kraemer HC, Lenora N, Newcorn JH, Abikoff HB et al (2001) ADHD comorbidity findings from the MTA Study Comparing comorbid subgroups. J AM Acad Child Adoles Psychiatry 40:14-158.

Jensen PS, Martin D, Cantwell DP, (1997) Comorbidity in ADHD; Implication for research, Practice and DSM-IV. J. Am. Acad Child Adoles Psychiatry 36: 1065-1079.

Johnson James H. (2009), Comorbidity and symptom mimic in ADHD, Current Management in Child Neurology 4 med.

Kadesjo B, Gillberg C. (1999). Developmental Coordination disorder in Swedish 7 year old children. J. Am Acad Child Adolsc Psychiatry 38: 820-828.

Kowatch RA, Fristad M, Birmaher B, Wagner KD, Findling RL, Hellander M. Child Psychiatric work group on Bipolar disorder. Treatment Guidelines for Children With Bipolar Disorder. J. Am Acad Child Adolsc Psychiatry 2005, 44: 213-235 abstract.

Kunwar A. Dewar M, Farone SV, Treating Common Psychiatric disorders associated with ADHD: Expert opinion pharmoco therapy 2007, 8: 555-562 (Abstract).

Larson K, PhD, Russ A Shirley, MD, Kahn, RS,MD, MPH, Halfen N, MD. Pattern of comorbidity functioning and services uses for U.S. children with ADHD, 2007 Pediatrics 2011 101:10.15421 Peds. 2010-0165.

Masi G, Toni C, Pergni G, Travierso MC, Millepiedi s, Mucci M, Akiskal HS, (2003). Externalizing disorders in consecutively referred children and adolescents with bipolar disorder. Comprehensive Psychiatry 44: 184-189.

McCracken, James T MD. Evolving Burden and diagnosis of ADHD, 2010

Mick E, Farone SV (2008) Genetics of AHDH, Child Adolsc Psych Cli N Am 17: 261-284. 
Mirsky AF, Duncan CC (2001). Anosology of disorder of Attention; Annals of the N.Y. Academy of sciences 931: 17-32.

Packer Leslie E., PhD (2010) ADHD: Differential diagnosis, Comorbidity Prognosis Tourette syndrome.net/disorders/attention/deficit-hyperactivity-disoder/adhd

Phend C, Tasmer Robert, Caputo D. Medical News: Comorbidities with ADHD in Pediatrics. www.medpagetoday.com/pediatircs/ADHD/ADD/24722.

Pichhiett DL, England SJ, Walters AS, Willis K, Verrico T, “Periodic Limb Movement disorder and restless legs syndrome in children with ADHD" Journal of child Neurology, 199813 (12) 588-94

Robson 1997, Enuresis in children with ADHD. Southern Medical Journal, 1997 May 90 (5) 503-505.

Schatz DB, Rostain AL. ADHD with comorbid anxiety: A review of Current literature. J. Atten Disorder. 2006, 10:141-149 abstract.

Schmitt and Ramanos M (2009), Lack of Studies investigating the association of childhood eczema, sleeping problem and ADHD. Pediatr Allergy Immunol.

Shapiro AK, Shapiro ES, Young JG, Feinberg TE, (1998) Gilles de la Tourette Syndrome. New York. Raven Press.

Shaywitz BA, Fletcher JM, Holahan JM, Shaywitz, SE (1992) Discrepancy compared to low achievement-definition of reading disability: results from Connecticut longitudinal study. J. Learning Disabil 25; 639-648.

Singh MK, DelBEllo MP, Kowatch RA, Strakowski JM. Co-occurrence of bipolar and attention-deficit hyperactivity disorder in children: Bipolar Disorder 8: 710-720.

Solhkhah R, Wilens TE, Daly J, Prince JB, Patten SL, Biederman J, Bupropion SR for the treatmrnt of substance-abusing outpatient adolescents with attentiondeficit/hyperactivity disorder and mood disorders. J child Psychopharmacol. 2005 oct;15(5)777-86.

Spencer T, Biederman J, Wilew J. Attention-Deficit/Hyperactivity disorder and comorbidity. Ped Clin North Am. 1999; 46, 915-927 VI abstract.

Spencer T, Biederman J, WIlens T. Attention-deficit/hyperactivity disorder and comorbidity. Pediatr Clin North Am. 1999;46:915-927, vii. Abstract

Spencer, Thomas J. (2006) ADHD and comorbidity in childhood. J. Clin Psychiatry 2006, 67. (suppl.8)

Steinhausen HC, Novik TS, Baldursson G, Curatolo P, Lorenzo MJ, Rodrigues Pereira R. Ralston SJ, ADORE RothenbergerA, Group Study (2006) Co-existing psychiatric problems in ADHD in the ADORE cohort. Eur Child Adolesc Psychiatry 15:i25i29.

Strober M, Carlson G. Bipolar illness in adolescents with major depression: clinical, genetic, and psycholpharmacologic predictors in three- to four year prospective follo-up investigation. Arch Gen Psychiatry. 1982 May;39(5);549-55.

Sullivan MA, Rudnik-Levin F (2001). Attention deficit and substance abuse. Diagnostic and therapeutic considerations. Ann N Y Acad Sci 931; 251-270

Taurin Regina, Schmitt, Jochen Renner Tobias, et al (2010) Developmental comorbidity in attention-deficit/hyperactivity disorder. ADHD (2010) 2:267-289).

Weiss RD, Mirin SM. Tricyclic antidepressants in the treatment of alcoholism and drug abuse. J clin Psychiatry 1989 Jul;50 Suppl:4-9; discussion 9-11. 
Wilens TE, Biederman J, Brown S, et al. Psychiatric comorbidity and functioning in clinically referred preschool children and school-age youths with ADHS. J Am Acad Child Adolesc Psychiatry. 2002;41:262-268. Abstract 


\title{
Comorbidity in ADHD: A Neuropsychological Perspective
}

\author{
Julio César Flores Lázaro and María Alejandra Salgado Soruco \\ ${ }^{1}$ SAP-Institutos Nacionales de Salud, Secretaría de Salud, \\ ${ }^{2}$ BUAP-Maestría en Neuropsicología, \\ México
}

\section{Introduction}

ADHD is commonly defined as a condition that affects attention, learning and behavior (American Psychiatric Association, 2001). Despite that over twenty years of scientific research has defined ADHD as a neurodevelopmental disorder, with the higher hereditability among psychiatric disorders; clinical-empirical taxonomy (DSM-IV) still prevail (McGough \& McCracken, 2006).

From an empirical point of view, other difficulties and also disorders that accompany this condition are considered "comorbid" (meaning: secondary). However several studies have found that quality of life in adolescents and adults with ADHD-child-diagnostic is not only related with attention deficits-severity, but also-mainly to comorbidity (Klassen, Miller \& Fine, 2004). Yang et al. (2007) studied a sample of 1000 children and adolescents with $\mathrm{ADHD}$, finding that comorbidity symptoms increases in relations to age, reaching the highest impact on behavior by the end of adolescence. Other studies are in agreement that in transition to adulthood comorbidity tends to: a) increase in intensity, and/or b) to appear for the first time (Kessler et al., 2006).

Particularly conduct and negativistic disorders are highly related with other types of comorbidity (Rhee, Willcutt, Hartman, Pennington \& Defries, 2007); some studies have found that comorbidity-type could be more important than attention-deficits-severity to produce and explain clinical behaviors: in general literature indicates that when ADHD and conduct disorder are clearly present and combined, the psychological impact is far more important than the effects produced separately by each disorder (Waschbusch, 2002); this impact is not found in the same magnitude for anxiety, depression or bipolar disorder (Pliszka, 2006). Flory et al. (2003) studied 481 adults with childhood-ADHD diagnosis, finding that subjects with higher characteristics for conduct disorder presented higher levels of drug consumption; in this same perspective Donohew et al. (1999) reported a relationship between the sensation seeking behavior profile and drug consumptions in adolescents (alcohol and marihuana). Harty et al. (2009) studied 87 children with ADHD, selecting from this sample subjects with oppositional defiant disorder diagnosis, and conduct disorder, applying diverse behavioral scales to set an initial evaluation during childhood (aggression, anger, and hostility), and a second evaluation teen years later. Finding that the ADHD- 
conduct disorder group presented higher levels of physical aggression in adolescence; both comorbid groups presented significant increment of anger (compared with adolescents with ADHD-only). Authors propose that emotional dysregulation could be an important component for ADHD during transition from childhood to adolescence.

Specific results on ADHD from the national comorbidity survey replication (Kessler et al., 2006) show a very important negative relation between years of school and symptoms prevalence: adults with ADHD profile presents fewer years of education, even adults with childhood onset without current full-symptoms presents a tendency to have mid to low education level (compared with the general population).

This report outlines that a diverse number of studies that have documented high societal costs for anxiety, mood and substance use disorders, have not included the role of comorbid ADHD. Indicating that only $10 \%$ of respondents diagnosed with ADHD reported having received treatment for adult $\mathrm{ADHD}$, this percentage is significantly lower than the reported rates for anxiety, mood, or substance use disorders. Revealing that many people with adultADHD are in treatment for other mental or substance use disorders but not for ADHD (Kessler et al., 2006).

ADHD symptoms overlap and are frequently prevalent among patients with others psychiatric disorder (mood, anxiety, substance use, and impulse control disorders). Metanalysis have shown that DSM-IV criteria for adult-ADHD has unclear validity, provoking underdiagnosis (Simon et al. 2009). This clinical under-recognition may negatively influence treatment effectiveness and outcome success (Barkley \& Brown, 2008).

McGough et al. (2009) interviewed 435 parents in 230 families with at least one affected children (present or past) with ADHD, using different rating scales based on DMSM-IV. Parents affected with ADHD (past or present affection) presented lower educational and occupational achievement, higher rates of lifetime comorbidity for depression, anxiety, disruptive behaviors, and substance use disorder. Higher risk for disruptive disorder was predicted only by male sex and ADHD; substance use disorder was mainly predicted by disruptive behavior disorders, male sex, and lower socioeconomic status.

Sex differences in comorbidity have also been found: in women drug consumption is mainly related to depression and anxiety (McGough et al., 2005). Ruhl et al. (2009) studied 2064 young-women (aged 18-25) with a structured interview according to DMS-IV criteria. Finding a $1.5 \%$ lifetime prevalence for ADHD, with $14 \%$ still suffering from ADHD since Childhood. Lifetime prevalence for conduct, somatoform, or posttraumatic stress disorder was significant higher in ADHD-women. Females with past or current ADHD-diagnosis were almost twice vulnerable to suffer from depressive or phobic disorder (than women from the general population). Authors also highlights that even when ADHD-diagnostic criteria is no longer fulfilled in adulthood, women with ADHD since childhood presents and increased prevalence for psychiatric comorbidity.

\section{Is comorbidity a psychological science?}

Drake and Wallach's (2007) article title is a perfect frame to develop this issue, like Stefanatos and Baron (2007) they propose scientific frames for conceptualizing and studying ADHD-comorbidity, instead of "clinical-empirical" criteria. 
Barkley (2009) and other scientists (i.e. Diamond, 2005) have demonstrated conceptual limitations in DSM-IV conceptualization for ADHD: according to Barkley diagnostic sensitivity to impulsivity symptoms is underrepresented in DSM-IV; pointing out that from a scientific-cognitive perspective, behavioral and cognitive control does not depend of attentional but on executive functions (these functions are mainly dependent on prefrontal cortex development -Diamond, 2001; van Leijenhorst et al., 2010-). They also highlights that others symptoms-behaviors-characteristics that could differentiate impulsivity like another possible category in ADHD are not appropriately represented in DSM-IV criteria.

Manassis et al. (2007) have found that children with anxiety disorders present an enhanced perception for negative emotions, specifically an incremented response in attention and memory processing to threatening stimuli; whereas ADHD-anxiety children didn't exhibited this profile. Authors consider that for each group anxiety may present different psychophysiological causes and characteristics.

Studies that compared children with ADHD-only vs children with ADHD-oppositional defiant disorder, reports attenuated electrophysiological responses (less P300 activation to cues) during attentional tasks (Continuous Performance Task: CPT) specifically for children with ADHD-only; indicating difficulties with anticipation and response preparation. This electrophysiological pattern is absent in ADHD-oppositional defiant disorder, indicating that comorbidity should not be viewed as a simple clinical-addition (Luman et al., 2009).

A consistent relationship between right hemisphere activation and a perceptual bias for negative emotions (fear, anxiety and sadness) has been frequently documented: more rightfrontal activity is found in shy-evitative children, and in subjects with depression and anxiety; in contrast more lef-frontal activity is found in sociable and low-social-fear children (McManis, Kagan, Snidman \& Woodwrad, 2000). By studying children with ADHD and their parents (affected and not affected), with electrophysiological recording during an attentional task (CPT), Hale et al. (2010) found that affected subjects (children and parents) presented an increased rightward asymmetry across frontal and central regions. An interesting relationship has been found: increased ADHD-familial loading correlated with increased rightward frontal asymmetry, instead increased rightward parietal asymmetry was correlated with reduced ADHD-family loading. Overall, this psychophysiological profile could predispose subjects with ADHD to be perceptually-biased to negativeemotional processing.

Schwartz et al. (2010) measured the orbito-frontal cortex density in normal subjects (18 years old) previously characterized in infancy as low-reactive or high-reactive subjects, finding that participants with low-reactive infant temperament presents greater thickness in the left orbito-frontal cortex, while subjects with high-reactive infant temperament present greater thickness in the right orbito-frontal cortex.

In literature it's been described that children with conduct disorder present mainly motivational problems, whereas children with ADHD present mainly attention deficits; Rubia et al. (2009) studied (by functional magnetic resonance imaging) 14 children with conduct disorder, and 18 children with ADHD; using a reward continuous performance task, and a sustained attention task. Results show brain activation differences between groups: conduct disorder subjects presented underactivation in paralimbic regions whereas ADHD subjects presented underactivation in diverse cerebral areas including prefrontal 
cortex. During the reward condition, conduct disorder subjects presented underactivation in right orbito-frontal cortex, while ADHD presented underactivation in posterior cingulated and precuneus; indicating neurocognitives differences among different clinical profiles.

Luman et al. (2009) found some neuropsychological differences among ADHD-children vs. ADHD-oppositional defiant disorder (ODD): children with ADHD-only presented difficulties in inhibition, timing estimation and reinforcement sensitivity, whereas children with ADHD-ODD presented an increased variability in timing estimation. Authors concluded that ADHD-ODD do not represent a more severe form of ADHD, instead a different one.

These studies indicate that neurocognitive/neuropsychological profiles are more specific and clinically illustrative for understanding comorbidity. Diverse authors has outlined that comorbidity is not only accompanying the disorder, and do not represent a clinical ambiguous variant for a given disorder. Instead the disorder itself and other -apparently no primarily related-clinical characteristics could depend on common cognitive, psychological and/or neuropsychological mechanisms.

Due that ADHD-comorbidity physiopathology is highly heterogeneous, it's been proposed that -scientific- models of comorbidity should be proposed. (Drake \& Wallach, 2007; Stefanatos \& Baron; 2007, Taurines et al. 2010). And although a neuropsychological approximation to understand the basic mechanisms of "psychological" disorders is continuously increasing, the neuropsychological profile for each disorder hasn't yet acquired a central role in diagnosis and treatment (Beblo, Sinnamon \& Baune, 2011: Neuropsychology Review). However with this approximation an optimal comprehension for this "attentional" disorder could be achieved (Diamond \& Anso, 2008).

\section{A neuropsychological perspective in ADHD}

From a neuropsychological perspective comorbidity is considered part of the same brain and cognitive mechanisms that produces attentional and or behavioral difficulties (Barkley, 2009; Diamond, 2005): children with attentional-variant presents a different (opposite) pattern of cognitive and behavioral profile than children with the hyperactive-variant. Inattentive profile includes social isolation, behavioral passivity, and cognitive slowness; in contrast hyperactive profile includes aggressive behavior, emotional instability, cognitive impulsivity and social disruptiveness (Flores Lázaro, 2009; Maedgen \& Carlson, 2000).

Attentional mechanisms do not represent a general-valid construct to explain: rejection to follow rules and to perform organized-structured activities, immediate seeking for gratification, low motivational drive, emotional lability, etc (Diamond, 2005). Instead of comorbidity summation, basics and common neuropsychological mechanism are proposed in order to explain from wider perspective attentional, behavioral and psychological profiles (Barkley, 1997, 2009; Stefanatos \& Baron, 2007).

The principal neuropsychological characteristics seen in ADHD could be explained by deficits in executive function, these functions depends on different brain circuits mainly located in prefrontal cortex (Barkley, 2010); for example children with combined-ADHD present more deficits in strategic memory -an executive function- than children with inattentive-ADHD type (Castel, Humpreys \& Moore, 2011). Executive functions permits 
complex cognitive and behavioral control, and are critical for success in school and daily life (Diamond, 2011).

\section{Attentional sub-type as an ADHD-cortical form}

Metanalysis of 19 developmental-functional neuroimaging studies in normal subjects has found that: fronto-parietal networks-interaction allows conscious-cognitive control of attentional processes. During brain development, functional integration between these and others networks is constructed; this phenomena also permits functional integration among several executive functions like inhibitory control, set switching and working memory (Fair et al., 2007). Inattentive predominant profile in children with ADHD includes difficulties in working memory, volume and processing speed, primary learning difficulties, and social isolation; the absence of behavioral difficulties as dominant profile, implicates neo-cortical mechanism that explains predominant cognitive consequences (Diamond, 2005).

Attentional control (focusing, stability, and alternation) and attentional processing (volume, processing speed) for cognitive academic and working environmental demands, depends mainly on cortical control -top-down regulation-; during child development, delay and inadequate integration between fronto-parietal networks leads to difficulties in attentional control and other executive functions (Fair et al., 2007). Stimulants like atomoxetin enhances prefrontal cortex functioning in ADHD-subjects -trough adrenergic and dopamine receptors- (Gamo et al., 2010); with positive psychophysiological results.

\section{Hyperactive sub-type as a ADHD-fronto-striatal form}

Hyperactivity, constant seek for gratification, motivational instability, cognitive impulsivity (works with hurry) and anxiety, has been the core symptoms in hyperactive sub-type (Rubia et al., 2009). Fronto-striatal circuits are highly replicated in literature for hyperactive children, pharmacological treatment with stimulants like Methylphenidate produces positives psychophysiological changes in cortical (cognitive) and fronto-striatal (dopaminereward systems) functioning (Zhu et al., 2011). It's been established that inhibitory control is the core mechanism for this variant (Barkley, 2009), this executive function permit optimal cognition and behavior regulation; metanalysis on 19 studies using Stroop interference measure (inhibitory control), indicates that this process is consistently compromised in ADHD (Lansbergen, Kenemans \& van Engeland, 2007).

During development dynamic interactions between brain regions changes, especially in fronto-estriatal circuits, as a result hyperactivity significantly diminishes during late childhood-adolescence; instead sensation seeking (for reward-goal oriented activities) develops in a $\cap$-shaped along childhood-adolescence-adulthood. Motivational cues for potential reward are specifically incremented in adolescence (striate hyper-responsiveness), and can lead to riskier choices during goal-oriented behavior (Somerville \& Casey 2010). Diverse studies that includes functional neuroimaging have found that only during adolescence a striate hyperactivation for reward expectation occurs (Van Leijenhorst et al., 2010), and although risk-detection capacity (in normal subjects) is adult-competent since 13 years old (Crone et al., 2005); in ADHD-adults is clinically diminished (Malloy-Diniz et al., 2007). 
Emotional dysregulation (e.g. high sensation seeking despite risk consequences) in ADHD could lead to greater risk for psychopathological comorbidity (Blaskey, Harris \& Nigg, 2008). Grall-Bronec et al. (2011) studied 85 adults with pathological gambling (this disorder is characterized by inadequate reward-regulation - Brevers et al., 2011-), finding that more than $25 \%$ of this sample presented history of ADHD, this subgroup presented elevated anxiety and impulsivity, more severe gambling problems, a higher frequency of psychiatric comorbidities and an elevated suicidal-risk.

\section{Impulsivity as a fronto-temporal-ADHD type}

Behavioral impulsivity, aggressiveness, conduct disorder, and emotional outburst; are the core symptoms in impulsivity variant (Harty et al., 2009). Fronto-temporal mechanisms are responsible for optimum regulation of emotional-based behavior (Gupta, Koscik, Bechara \& Tranel, 2011), adequate frontal regulation to emotional-based responses represent an important prerequisite for interpersonal and social interactions, allowing subjects to regulate verbal and behavioral interactions; these processes are fundamental for socially-adjusted behavioral and personality development during childhood (Damasio, 2005). Based on genetics studies low serotonin metabolism has been outlined as the key neurotransmitter system for behavioral-impulsivity and aggression in ADHD (Oades et al., 2008). By using magnetic resonance imaging Boes et al. (2008) studied the relationship between impulsive control and volume-size for diverse brain regions (including amygdala, anterior cingulate cortex and prefrontal cortex) in 61 normal-boys from 7 to 17 years old; finding that subjects with poorer impulsivity control presented (significantly different) less volume in the right ventro-medial -orbital- cortex.

Literature indicates that $\mathrm{ADHD}$ and conduct disorder combination represent a very important clinical condition (Waschbusch, 2002). Barkley and Fischer (2010) studied the influence of emotional impulsiveness over social, laboral and daily live activities, in 135 ADHD-young-adults; finding that if ADHD-symptoms are still persistent in adulthood, emotional impulsiveness severity contributes to significant impairment in occupational, educational, legal, and financial aspects. Authors highlight the importance of impulsivity in ADHD-criteria, considering it's at least as important as inattention/hyperactivity dimension for the development of adequate social, occupational and interpersonal functioning. Although highly important, impulsivity dimension is under-represented in DSM-IV criteria for ADHD (Barkley, 2009).

\section{Comorbidity classification by neuropsychological criteria}

In most cases in literature comorbidity is reported in relationship to prevalence (clinicalepidemiological approach), however ADHD-subtypes in adults are associated with different clinical comorbid correlates (Sprafkin et al., 2007), results coincide in describing predominant behavioral-psychological disorders in combined-ADHD, and predominant cognitive disorders at inattentive type (Murphy, Barkley \& Bush, 2002). To explore comorbid profiles from a neuropsychological point of view, a sample of 61 male-children with ADHD-diagnosis (DSM-IV criteria) were selected. Participants had between 6 and 10 years-old (mean: 7.6). Inclusion criteria were: not-medicated for ADHD (or any other neurodevelopemental/psychiatric disorder), no history of psychological treatment, normal I.Q. (WISC); exclusion criteria: others non-related neurodevelopmental disorder (epilepsy, 
cognitive deficit, etc). All subjects were evaluated with diverse neuropsychological tests, and evaluated by clinical-psychological criteria (see Flores Lázaro, 2009 for details). On the first analysis 20 subjects with ADHD-attentive type (ADD) (group one) were compared vs. 10 subjects with ADHD-combined (ADHD-C) type and 10 subjects with ADHD-impulsivity with or without hyperactivity-variant (ADHD-I-H) (group two: ADHD-C-I).

Based on Diamond (2005) proposal, comorbidity was divided in behavioral-psychological, and cognitive. Statistical analysis was performed by Fisher's exact test (FET). All results including tables are adapted from Flores Lázaro (2009)*

Data presented in tables 1 and 2, supports Barkley's and Diamond's basic division, illustrating that both groups are significantly different in behavioral-psychological and cognitive dimensions. These results coincide with other studies (Capdevila-Brophy, et al., 2005; Maedgen \& Carlson, 2000). ADHD-C-I group is mainly characterized by behavioralpsychological comorbidity; whereas cognitive comorbidity represents the main characteristic for ADD group.

\begin{tabular}{|l|c|c|c|}
\hline \multicolumn{1}{|c|}{ Behavioral-psychological comorbidity } & ADHD-C-I & ADD & FET \\
\hline Mood disorders & $43 \%$ & $10 \%$ & .017 \\
\hline Conduct disorder an/or agressivity & $39 \%$ & $8 \%$ & .040 \\
\hline Negativistic defiant disorder & $39 \%$ & $0 \%$ & .032 \\
\hline Anxiety/anxiety disorder & $35 \%$ & $8 \%$ & .040 \\
\hline Socialization difficulties & $35 \%$ & $40 \%$ & .432 \\
\hline Avoiding to follow rules-instructions & $39 \%$ & $0 \%$ & .001 \\
\hline Infantilization & $39 \%$ & $0 \%$ & .002 \\
\hline Low tolerance to frustration & $35 \%$ & $17 \%$ & .101 \\
\hline Insecurity (low self-concept) & $13 \%$ & $36 \%$ & .022 \\
\hline
\end{tabular}

Table 1. Percentage on behavioral-psychological comorbidity and differences between ADHD-C-I and ADD

\begin{tabular}{|l|c|c|c|}
\hline \multicolumn{1}{|c|}{ Cognitive comorbidity } & ADHD-C-I & ADD & FET \\
\hline Visoespatial difficulties & $39 \%$ & $70 \%$ & .014 \\
\hline Mathematics learning disorder & $17 \%$ & $40 \%$ & .032 \\
\hline Reading learning disorder & $26 \%$ & $35 \%$ & .356 \\
\hline General learning disorder & $4 \%$ & $35 \%$ & .001 \\
\hline Language difficulties/dysphasia & $4 \%$ & $30 \%$ & .012 \\
\hline
\end{tabular}

Table 2. Percentage on cognitive comorbidity and differences between ADHD-C-I and ADD

It's important to outlined that socialization difficulties are due to different causes in each group (evitative vs disruptive), reading learning disorder are also due to different neuropsychological causes (Roselli, Matute \& Ardila, 2006). Even when general-clinical-

* Partially/totally reproduced from "Características de comorbilidad en los diferentes subtipos de trastorno por déficit de atención con hiperactividad" de J.L. Flores Lázaro, 2009, Psicothema, 21(4), p. 594, 595. Copyright 2009 del Colegio Oficial de Psicólogos del Principado de Asturias. Reproducido con permiso" 
criteria coincide, psychological and neuropsychological analysis permits to scientifically differentiate specific differential-causes for the same criteria. Literature has progressively demonstrated that clinical-empirical dimensions without psychological, psychophysiological, or neuropsychological scientifically bases should not serve as professional taxonomic criteria (McGough \& McCracken, 2005; Stefanatos \& Baron, 2007).

\section{Hyperactive, inattentive and impulsive variants analysis}

During transition from childhood to adolescence, emotional regulation represents a central component for ADHD-mental health (Barkley \& Fisher, 2010), due that impulsivity variant is underrecognized in DSM-IV criteria (Barkley, 2009), and that emotional dysregulation is a core characteristic for impulsivity subtype (Harty et al., 2009); ADHD-Impulsivity variant still represents a very important clinical subgroup for scientific study.

Current knowledge on cognitive neuroscience and neuropsychology permits to validate the pertinence to investigate differences among hyperactive vs. impulsive subjects. Therefore a second analysis was performed with a three-group division: 20 children with inattentive variant (ADD), 20 children with combined variant (ADHD-C), and 21 children with impulsivity variant -with or without hyperactivity- (ADHD-I). Results are presented in tables 3 and 4, data shows a clear cognitive to behavioral-psychological comorbidity transition: inattentive-hyperactive-impulsive. As expected by neuropsychological criteria, impulsivity variant presents the highest significant-frequent behavioral-psychological comorbidity; Inattentive variant continues to present the highest significant-frequent cognitive comorbidity. Interestingly Hyperactive variant lies in the middle range on this group-division. Results illustrate Barkley's point of view that impulsivity-variant represents a sufficiently differentiated clinical-group.

Impulsivity-variant suggests fronto-temporal mechanisms as the main causes for comorbidity, differently to fronto-strital mechanism on hyperactive-variant. However specifics neuropsychological, psychophysiological, structural and functional neuroimaging studies should differentiate fronto-parietal, fronto-striatal and fronto-temporal mechanisms for each cases.

\begin{tabular}{|l|c|c|c|c|c|}
\hline Behavioral-psychological comorbidity & ADHD-I & FET & ADHD-C & FET & ADD \\
\hline Mood disorders & $47 \%$ & .202 & $31 \%$ & .118 & $10 \%$ \\
\hline Conduct disorder an/or agressivity & $42 \%$ & .191 & $26 \%$ & .204 & $10 \%$ \\
\hline Negativistic defiant disorder & $31 \%$ & .002 & $0 \%$ & & $0 \%$ \\
\hline Anxiety/anxiety disorder & $37 \%$ & .415 & $31 \%$ & .118 & $10 \%$ \\
\hline Socialization difficulties & $45 \%$ & .191 & $26 \%$ & .250 & $40 \%$ \\
\hline Avoiding to follow rules-instructions & $52 \%$ & .038 & $25 \%$ & .024 & $0 \%$ \\
\hline Infantilization & $26 \%$ & .329 & $42 \%$ & .002 & $0 \%$ \\
\hline Low tolerance to frustration & $31 \%$ & .585 & $35 \%$ & .137 & $15 \%$ \\
\hline Insecurity (low self-concept) & $5 \%$ & .284 & $15 \%$ & .137 & $36 \%$ \\
\hline
\end{tabular}

Table 3. Percentage on behavioral-psychological comorbidity and differences between groups 


\begin{tabular}{|l|c|c|c|c|c|}
\hline \multicolumn{1}{|c|}{ Cognitive comorbidity } & ADHD-I & FET & ADHD-C & FET & ADD \\
\hline Visoespatial difficulties & $70 \%$ & .100 & $42 \%$ & .222 & $26 \%$ \\
\hline Mathematics learning disorder & $40 \%$ & .250 & $25 \%$ & .081 & $5 \%$ \\
\hline Reading learning disorder & $34 \%$ & .500 & $40 \%$ & .065 & $15 \%$ \\
\hline General learning disorder & $34 \%$ & .022 & $5 \%$ & .488 & $0 \%$ \\
\hline Language difficulties/dysphasia & $30 \%$ & .118 & $10 \%$ & .232 & $0 \%$ \\
\hline
\end{tabular}

Table 4. Percentage of cognitive comorbidity and differences between groups

\section{Comorbid profiles according to ADHD-variants}

Comorbid characteristics among different ADHD-subtypes analyzed are specific, behavioral-psychological comorbidity is listed from more to less frequent:

- Inattentive profile: socialization (evitative -type), and low self-concept.

- Hyperactive profile: infantilization, low-tolerance to frustration, anxiety/anxiety disorder, mood disorders, conduct disorder/aggressiveness, socialization, and avoiding to follow rules and instructions,

- Impulsivity variant: following rules, limits and instructions. Mood disorders, socialization (disruptive-type), conduct disorder/aggressiveness, anxiety/anxiety disorder, negativistic defiant disorder, low-tolerance to frustration, and infantilization.

Cognitive comorbidity is listed from more to less frequent:

- Inattentive: visoespatial, mathematics, reading, and general learning difficulties, language/dysphasia.

- Hyperactive: visoespatial, reading, and mathematics learning difficulties.

- Impulsive: visoespatial, reading learning disorder.

\begin{tabular}{|l|c|}
\hline \multicolumn{1}{|c|}{ Behavioral-psychological comorbidity } & FET \\
\hline Mood disorders & .012 \\
\hline Conduct disorder an/or agressivity & .025 \\
\hline Negativistic defiant disorder & .003 \\
\hline Anxiety/anxiety disorder & .048 \\
\hline Socialization difficulties & .607 \\
\hline Avoiding to follow rules-instructions & .000 \\
\hline Infantilization & .014 \\
\hline Low tolerance to frustration & .181 \\
\hline Insecurity (low self-concept) & .015 \\
\hline
\end{tabular}

Table 5. Differences on behavioral-psychological comorbidity between extreme groups: ADD vs ADHD-I-H

\section{Extreme groups division}

As shown in tables 3 and 4, ADD and ADHD-I groups represent extreme comorbidity: behavioral-psychological vs cognitive. In order to analyze statistical differences among these groups FET has been applied. Results are presented in table 5 and 6, data indicate 
clearer/most frequent significant differences both comorbidity-types; inclusion of children with Hyperactive-variant (a transitional group) tends to diminish statistical differences (see tables 3 and 4 ).

\begin{tabular}{|l|c|}
\hline \multicolumn{1}{|c|}{ Cognitive comorbidity } & FET \\
\hline Visoespatial difficulties & .005 \\
\hline Mathematics learning disorder & .006 \\
\hline Reading learning disorder & .100 \\
\hline General learning disorder & .003 \\
\hline Language difficulties/dysphasia & .007 \\
\hline
\end{tabular}

Table 6. Differences on cognitive comorbidity between extreme groups: ADD vs ADHD-I

\section{Conclusions}

Neuropsychology permit to establish a conceptual bridge between clinical and basics sciences (psychology and neurosciences). Applied neuropsychological research posses high environmental validity in clinical settings (Beblo et al., 2011). Several researchers have criticized the reluctance of psychiatry-practice to move toward scientifically oriented pathophysiology -neurosciences- more common in actual days to general medicine and psychology (McGough \& McCracken, 2005); from a neuropsychological point of view ADHD is mainly a disexecutive disorder in which several cognitive, behavioral and psychological consequences are present, in different form, at diverse ontogenetic moments. In ADHD, attention is only one of these consequences; and for an important number of cases is not the most important. Diverse clinicians and researches in this field began to underline the need for systematic neuropsychological evaluation in order to objectify attention disorders (Chambry et al., 2011), interpretations on these results should be made within valid scientific models (Drake \& Wallach, 2007; Stefanatos \& Baron, 2007). Professionals should understand basic neuropsychological and psychological mechanism causing ADHD: attention is ONLY one of the cognitive processes involved, due to inadequate development in executive function control; in most cases comorbidity is ALSO explained by the same psychophysiological and/or neuropsychological mechanisms; based on this comprehension intervention programs will be more specific, and above all: more scientifically oriented.

Drake and Wallach's fundamental question for psychology may be better answered in this form: ADHD-comorbidity is -mainly-a neuropsychological science.

\section{References}

American Psychiatric Association (2001). Manual diagnóstico y estadístico de los trastornos mentales-IV-TR. Barcelona: Masson.

Barkley, R.A., Brown, T.E. (2008). Unrecognized attention-deficit/hyperactivity disorder in adults presenting with other psychiatric disorders. CNS Spectrums., 13,,977-84.

Barkley, R.A. (1997). Behavioral inhibition, sustained attention and executive functions: Constructing a unifying theory of ADHD. Psychologycal Bulletin, 121, 65-94. 
Barkley, R.A. (2009). Advances in the diagnosis and subtyping of attention deficit hyperactivity disorder: what may lie ahead for DSM-V. Revista de Neurología. 27,48, S101-6.

Barkley, R.A. (2010). Against the status quo: revising the diagnostic criteria for ADHD. J Am Acad Child Adolesc Psychiatry. 49,3,205-7.

Barkley R.A. \& Fischer, M. (2010). The unique contribution of emotional impulsiveness to impairment in major life activities in hyperactive children as adults. Journal of the American Academy of Child and Adolescent Psychiatry. 49,5,503-13.

Beblo, T., Sinnamon, G. \& Baune, B.T. (2011). Specifying the Neuropsychology of Affective Disorders: Clinical, Demographic and Neurobiological Factors. Neuropsychology Review. (Epub ahead of print). DOI: 10.1007/s11065-011-9171-0

Blaskey, L.G., Harris, L.J. \& Nigg, J.T. (2008). Are sensation seeking and emotion processing related to or distinct from cognitive control in children with ADHD?, Child Neuropsychology, 14,4,353-71.

Boes, A.D., Tranel, D., Anderson, S.W., Nopoulos, P. (2008). Right anterior cingulate: a neuroanatomical correlate of aggression and defiance in boys. Behavioral Neurosciences, 122,3.677-84.

Brevers, D., Cleeremans, A., Bechara, A., Laloyaux, C., Kornreich, C., Verbanck, P., Noël, X. (2011). Time course of attentional bias for gambling information in problem gambling. Psychology of Addictive Behaviors. doi: 10.1037/a0024201

Castel, A.D., Lee, S.S., Humphreys, K.L. \& Moore, A.N. (2001). Memory capacity, selective control, and value-directed remembering in children with and without attentiondeficit/hyperactivity disorder (ADHD). Neuropsychology. 25,1,5-24.

Chambry, J., Billard, C., Guinard, M., Lacaze, E., Idiart, M.E., Delteil-Pinton, F. Cohen de Lara, A. (2011). [Attention deficit disorder: Multidisciplinary observational study over 1 year. Encephale, 373, 180-190.

Capdevila-Brophy, C., Artigas-Pallares, J., Ramírez-Mallafré, A., López-Rosendo, M., Real, J., \& Obiols-Landrich, J.E. (2005). Fenotipos neuropsicológicos del trastorno por déficit atencional/hiperactividad, ¿existen diferencias entre los subtipos? Revista de Neurología, 40, 17-23.

Chambry, J., Billard, C., Guinard, M., Lacaze, E., Idiart, M.E., Delteil-Pinton, F. \& Cohen de Lara, A. (2011). Attention deficit disorder: Multidisciplinary observational study over 1 year. Encephale, 37, 3,180-190.

Crone, E.A., Bunge, S.A., Latenstein, H., van der Molen, M.W.(2005). Characterization of children's decision making: sensitivity to punishment frequency, not task complexity. Child Neuropsychology, 11,3,245-63.

Damasio, A. (2005). Human behavior: brain trust. Nature, 2,435,571-2.

Diamond, A. (2005). Attention deficit disorder (attention deficithyperactivity disorder without hyperactivity): A neurobiologycally and behaviorally distinct disorder Attention deficit-hyperactivity disorder (with hyperactivity). Developmental psychopathology, 17, 803-825.

Diamond, A. \& Amso, D. (2008). Contributions of Neuroscience to Our Understanding of Cognitive Development. Current Directions in Psychological Sciences, 17,2,136-141.

Diamond A. (2011). Biological and social influences on cognitive control processes dependent on prefrontal cortex. Progress in Brain Research, 189,319-39. 
Donohew, R.L., Hoyle, R.H., Clayton, R.R., Skinner, W.F., Colon, S.E. \& Rice, R.E. (1999). Sensation seeking and drug use by adolescents and their friends: Models for marijuana and alcohol. Journal of Studies on Alcohol, 60, 622-631.

Drake, R.E., y Wallach, M.A. (2007). Is comorbidity a Psychological Science? Clinical Psychology: Science and Practice, 14, 20-22.

Fair, D.A., Dosenbach, N.U., Church, J.A., Cohen, A.L., Brahmbhatt, S., Miezin, F.M., et al. (2007). Development of distinct control networks through segregation and integration. Proceedinga of the National Academy of Sciences U S A. 14,104,1350712.

Flores Lázaro J.C. (2009). [Comorbidity characteristics in the different subtypes of attention deficit-hyperactivity disorder. Psicothema, 21,4, 592-7.

Flory, K., Milich, R., Lynam, D.R., Leukefeld, C., y Clayton, R. (2003). Relation between childhood disruptive behavior disorders and substance use and dependence symptoms in young adulthood: Individuals with symptoms of attentiondeficit/hyperactivity disorder and conduct disorder are uniquely at risk. Psychology of Addictive Behaviors, 17, 151-158

Freitag, C.M., Hänig, S., Schneider, A., Seitz, C., Palmason, H., Retz, W. \& Meyer, J. (2011). Biological and psychosocial environmental risk factors influence symptom severity and psychiatric comorbidity in children with ADHD. Journal of Neural Transmission. 2011 May 31. [Epub ahead of print]

Gamo, N.J., Phil, M., Wang, M., \& Arnsten, A. (2010). Methylphenidate and Atomoxetine Enhance Prefrontal Function Through a2-Adrenergic and Dopamine D1 Receptors. Journal of the American Academy of Child and Adolescence Psychiatry, 49,10, 1011-1023.

Grall-Bronnec, M., Wainstein, L., Augy, J., Bouju, G., Feuillet, F., Vénisse, J.L. \& SébilleRivain, V. (2011). Attention Deficit Hyperactivity Disorder among Pathological and At-Risk Gamblers Seeking Treatment: A Hidden Disorder. European Addiction Research, 7,17,231-240.

Gupta, R., Koscik, T.R., Bechara, A., Tranel, D. (2011). The amygdala and decision-making. Neuropsychologia, 49,4,760-6.

Hale, T.S., Smalley, S.L., Walshaw, P.D., Hanada, G., Macion, J., McCracken, J.T., McGough, J.J., Loo, S.K. (2010). Atypical EEG beta asymmetry in adults with ADHD. Neuropsychologia, 48,12,3532-9.

Harty, S.C., Miller, C.J., Newcorn, J.H, \& Halperin JM. (2009). Adolescents with childhood ADHD and comorbid disruptive behavior disorders: aggression, anger, and hostility. Child Psychiatry and Human Development, 40,1,85-97.

Kessler, R.D, Adler,L., Barkley, R. Biederman, J. Conners, K., Demler, O. et al. 2006). The Prevalence and Correlates of Adult ADHD in the United States: Results From the National Comorbidity Survey Replication. American Journal of Psychiatry, 163, 716723).

Klassen, A.F., Miller, A., \& Fine, S. (2004). Health-related quality of life in children and adolescents who have a diagnosis of attention-deficit/hyperactivity disorder. Pediatrics, 114, 541-547.

Lahey, B.B. \& Willcutt, E.G. (2010). Predictive validity of a continuous alternative to nominal subtypes of attention-deficit/hyperactivity disorder for DSM-V. Journal of Clinical Child and Adolescent Psychology, 39,6,761-75. 
Lansbergen, M.M., Kenemans, J.L. \& van Engeland, H. (2007). Stroop interference and attention-deficit/hyperactivity disorder: a review and meta-analysis. Neuropsychology, 21,2,251-62.

Maedgen, J.W. \& Carlson, C.L. (2000). Social functioning and emotional regulation in the attention deficit hyperactivity disorder subtypes. Journal of Clinical Child Psychology, $29,30-42$.

Malloy-Diniz, L., Fuentes, D., Leite, W.B., Correa, H. \& Bechara, A. (2007). Impulsive behavior in adults with attention deficit/ hyperactivity disorder: characterization of attentional, motor and cognitive impulsiveness. Journal of the International Neuropsychological Society, 13,4,693-8.

McGough, J.J., Smalley, S.L., McCracken, J.T., Yang, M., Del'Homme, M., Lynn, D.E. \& Loo, S. (2005). Psychiatric Comorbidity in Adult Attention Deficit Hyperactivity Disorder: Findings From Multiplex Families. American Journal of Psychiatry, 162, 1621-1627.

McGough, J.J. \& McCracken, J.T. (2006). Adult Attention Deficit Hyperactivity Disorder: Moving Beyond DSM-IV. American Journal of Psychiatry, 163, 1673-1675.

McManis, M.H., Kagan, J., Snidman, N.C., Woodward, S.A. (2002). EEG asymmetry, power, and temperament in children. Developmental Psychobiology, 41,2, 169-77.

Manassis, K., Tannock, R., Young, A. \& Francis-John, S., Cognition in anxious children with attention deficit hyperactivity disorder: a comparison with clinical and normal children. Behavioral and Brain Functions, 3,4, 1-10.

Murphy, K.R., Barkley, R.A. \& Bush, T. (2002). Young adults with attention deficit hyperactivity disorder: subtype differences in comorbidity, educational, and clinical history. Journal of Nervous and Mental Disorders, 190,3,147-57.

Oades, R.D., Lasky-Su, J., Christiansen, H., Faraone, S.V., Sonuga-Barke, E.J., Banaschewski, T., Chen, W., et al. ( 2008). The influence of serotonin- and other genes on impulsive behavioral aggression and cognitive impulsivity in children with attentiondeficit/hyperactivity disorder (ADHD): Findings from a family-based association test (FBAT) analysis. Behavioral and Brain Functions, 20,4-48.

O'Brien, J.W., Dowell, L.R., Mostofsky, S.H., Denckla, M.B., Mahone, E.M (2010). Neuropsychological profile of executive function in girls with attentiondeficit/hyperactivity disorder. Archives of Clinical Neuropsychology; 25,7,656-70.

Pliszka, S.R. (2006). Subtyping ADHD based on comorbidity. The ADHD Report, 14,1-5.

Rosselli, M., Matute, E., y Ardila, A. (2006). Predictores neuropsicológicos de la lectura en español. Revista de Neurología, 42, 202-210.

Rhee, S.H., Willcutt, E.G., Hartman, C.A., Pennington, B.F., \& Defries, J.C. (2007). Test of alternative hypotheses explaining the comorbidity between attentiondeficit/hyperactivity disorder and conduct disorder. Journal of Abnormal Child Psychology, 35, 536-542.

Romine, C.B., Reynolds. C.R. (2005). A model of the development of frontal lobe functioning: findings from a meta-analysis. Applied neuropsychology, 12,4,190-201.

Rubia, K., Halari, R., Cubillo, A., Mohammad, A.M., Scott, S., Brammer, M.(2010). Disorderspecific inferior prefrontal hypofunction in boys with pure attentiondeficit/hyperactivity disorder compared to boys with pure conduct disorder during cognitive flexibility. Human Brain Mapping, 31,12,1823-33. 
Rubia, K., Halari, R., Cubillo, A., Mohammad, A.M., Brammer, M., Taylor, E. (2009). Methylphenidate normalises activation and functional connectivity deficits in attention and motivation networks in medication-naïve children with ADHD during a rewarded continuous performance task. Neuropharmacology, 57,7-8,640-52.

Ruhl, U., Rentsch, A., Bernardi, C., Türke-Teubner, V., Becker, E., Kirch, W., Margraf, J. \& Hach, I. (2009). Associations Between Childhood ADHD and Other Mental Disorders in Young Women. German Journal of Psychiatry, 12, 8-13).

Schwartz, C.E., Kunwar, P.S., Greve, D.N., Moran, L.R., Viner, J.C., Covino, J.M., Kagan, J., Stewart, S.E., Snidman, N.C., Vangel, M.G. \& Wallace, S. (2010). Structural differences in adult orbital and ventromedial prefrontal cortex predicted by infant temperament at 4 months of age. Archives of General Psychiatry, 67, 1,78-84.

Shaw, P., Gilliam, M., Liverpool, M., Weddle, C., Malek, M., Sharp, W., et al. (2011). Cortical development in typically developing children with symptoms of hyperactivity and impulsivity: support for a dimensional view of attention deficit hyperactivity disorder. American Journal of Psychiatry, 168,2,143-51.

Shuai, 1.C., Chan, 1.C. \& Wang, Y. (2011). Executive Function Profile of Chinese Boys with Attention-Deficit Hyperactivity Disorder: Different Subtypes and Comorbidity. Archives of Clinical Neuropsychology, 26, 2, 120-132.

Simon, V. Czobor, P., Balint, S., Mesza, A. \& Bitter, I. (2009). Prevalence and correlates of adult attention-deficit hyperactivity disorder:meta-analysis. The British Journal of Psychiatry, 194, 204-211.

Stefanatos, G.A., \& Baron I.S. (2007). Attention-deficit/hyperactivity disorder: A neuropsychologycal perspective towards DSM-IV. Neuropsychology Review, 17, 5-38.

Somerville, L.H. \& Casey, B.J. (2010). Developmental neurobiology of cognitive control and motivational systems. Current Opinion in Neurobiology, 20, 2, 236-241.

Sprafkin, J., Gadow, K.D., Weiss, M.D., Schneider, J. \& Nolan, EE. (2007) Psychiatric comorbidity in ADHD symptom subtypes in clinic and community adults. Journal of Attention Disorders, 11,2, 114-24.

Stuss, D.T., y Levine, B (2000). Adult clinical neuropsychology, lessons from studies of the frontal lobes. Annual Review of Psychology, 53,401-403.

Taurines, R., Schmitt, J., Renner, T., Conner, A.C., Warnke, A. \& Romanos, M. (2010). Developmental comorbidity in attention-deficit/hyperactivity disorder. Attention Deficit Hyperactive Disorders; 2,4,267-89.

Waschbusch, D.A. (2002). A meta-analytic examination of comorbid hyperactive-impulsiveattention problems and conduct problems. Psychologycal Bulletin, 128, 118-50.

Van Leijenhorst, L. Moor, B.G. Op de Macks, Z.A., Rombouts, S., Westenberg, P.M. \& Crone, E.A. (2010). Adolescent risky decision-making: Neurocognitive development of reward and control regions. Neuroimage, 15;51,345-55.

Yang, L., Ji, N., Guan, L.L., Chen, Y., Qian, Q.J., y Wang Y.F. (2007). Co- morbidity of attention deficit hyperactivity disorder in different age group. Beijing Da Xue Xue Bao, 39, 229-232

Zhu, J., Spencer, T.J., Liu-Chen, L.Y., Biederman, J. \& Bhide, P.G. (2011). Methylphenidate and $\mu$ opioid receptor interactions: A pharmacological target for prevention of stimulant abuse. Neuropharmacology, 61,1-2, 283-92. 


\title{
ADHD and Sleep Problems in Children
}

\author{
Elizabeth Hastings and Barbara T. Felt \\ Section of Developmental Behavioral Pediatrics, Division of Child Behavioral Health, \\ Department of Pediatrics and Communicable Diseases, \\ University of Michigan, \\ USA
}

\section{Introduction}

In the evaluation of Attention Deficit Hyperactivity Disorder (ADHD), medical and behavioral problems related to sleep are important to consider. A relationship between ADHD and sleep has been acknowledged for some time. Sleep disturbances were among criteria for ADHD diagnosis in the past and rating scales for ADHD included items related to sleep disturbance (American Psychiatric Association [APA], 1987; Conners, et al. 1998, 1998). While our knowledge about the relationship between ADHD and sleep problems has improved in recent years, it remains challenging to tease apart the effect of insufficient or poor quality sleep on attention, alertness, learning and behavior and what might be due to a primary ADHD, mood or behavior problem (Simonds, 1984). This interface of mental health disorder and sleep disorder is a challenge many clinicians face in practice. Medical, mental health, behavioral and psychosocial factors can directly affect the quality and duration of sleep. Poor sleep, in turn, can have effects on daytime functioning and health. In addition, such medical and mental health problems can have direct effects on attention, learning and behavior. Sorting out the important contributing factors during a comprehensive evaluation can be challenging due to sleep and ADHD problems commonly co-occurring. The primary purpose of this chapter is to highlight the importance of including the evaluation of sleep as a part of the assessment of disorders such as ADHD because the best management for the individual patient depends on the accuracy of the diagnostic evaluation.

As discussed in other chapters in this book, ADHD is a common disorder that presents in childhood. It is estimated to affect 5-10\% of school-age children and adolescents (Committee on Quality Improvement, 2000). Symptoms such as distractibility, forgetfulness and failure to complete work typify inattentive-type ADHD. Impulsivity, over activity and being fidgety are among symptoms that are common for individuals with hyperactive-impulsive type ADHD. Having symptoms that meet criteria for both subtypes is required for a diagnosis of combined-type ADHD. DSM-IV criteria require that symptoms have onset before age 7 years (APA, 2000); however, the symptoms of inattentive-type ADHD are often subtle and not recognized until later school years when the academic curriculum is more challenging and requires greater executive functioning skills to keep pace and do well. In addition, the symptoms of ADHD are non-specific on their own and can be related to other factors, contexts and diagnoses. Due to this, it can be challenging to determine if the pattern of an individual's behavior is best described by the diagnosis of ADHD. 
Sleep problems occur commonly, from infancy through childhood and adolescence and are related to daytime behavioral concerns. For instance, bedtime resistance and night waking are common among toddlers and preschoolers, affecting $30 \%$ or more. A significant relationship between sleep problems and parent-reported concerns about internalizing and externalizing behaviors has been demonstrated for this age group (Reid, 2009; Mindell, 2010). Among school-age children, 11 to $37 \%$ have problems with sleep and many describe feeling sleepy during the day (Sleep in America Poll Summary of Findings [SAP], 2004). Studies of adolescents and the 2006 National Sleep Foundation Poll of US adolescents nationwide also describe a high prevalence of sleep problems at this age (Owens, 2005; SAP, 2006). Forty-five percent of adolescents report short night time sleep durations and more than half report feeling tired or sleepy during the day. Many adolescents also report feeling unhappy and that they are not getting enough sleep.

In this chapter, we will discuss: the neurobiological aspects of ADHD and sleep; typical patterns of normal sleep for children and expected age-related changes; common sleep disorders and their effects on daytime functioning with particular attention to ADHD; sleep characteristics for children with ADHD and the effects of medications; and the importance of screening for sleep concerns in the evaluation and management of ADHD.

\section{Neurobiology}

\subsection{Neurobiology of ADHD}

To review our current understanding about the overlap of ADHD and sleep from a neurobiological perspective, we will briefly review key concepts for ADHD, and sleep in this section. Although the pathophysiology of ADHD is quite complex and likely results from a combination of multiple factors, genetic and environmental variables have been identified as possible contributing components (Faraone, 1998). Anatomical studies have isolated abnormalities in brain regions associated with cognition, executive functioning and behaviors consistent with ADHD (Dahl, 1996). Functional MRI (fMRI) studies have similarly demonstrated abnormal activation in these areas, including the prefrontal cortex, anterior cingulate gyrus and thalamus (Durston, 2003; Rubia et al., 2011). Gross anatomic studies have demonstrated a lower total brain volume in children with ADHD (Curatolo et al., 2009; Makris et al., 2009; Tripp, 2009). Additionally, decreased size of the right frontal lobe (RFL), corpus callosum, striatum and cerebellum have been observed in children and adolescents with ADHD (Curatolo et al., 2009; Makris et al., 2009; Tripp, 2009). The areas found to be different in ADHD are those which support emotion and higher brain functions including executive function and attention (Makris et al., 2009). Abnormalities observed in the cerebrum and cerebellum have been found to persist with advancing age, whereas, abnormalities in the caudate normalize with age (Curatolo et al., 2009; Konrad \& Eickhoff, 2010).

These anatomical studies and elucidating the mechanisms of stimulant medications used to treat ADHD, have helped to further our understanding of the neurobiological basis of this condition. ADHD is associated with catecholamine dysregulation, specifically related to dopamine and norepinephrine (Curatolo et al., 2009; Faraone, 1998, 2005; Gonon, 2009; Makris et al., 2009; Rosa-Neto et al., 2005; Swanson, 2000; Tripp, 2009). The dysregulation of dopamine in ADHD has been the focus of much inquiry. One neurophysiologic finding 
characteristic of ADHD, which is thought to underlie some ADHD symptoms, is an atypical response to reward (Tripp, 2009; Nigg, 2005). Dopamine is thought to be an important component of the neural system of reward (Tripp, 2009), as well as for ADHD. The brain regions believed to be important in children and adults with ADHD, in which gross anatomic differences have been demonstrated, are rich in dopamine signaling (Curatolo et al., 2009; Makris et al., 2009; Tripp, 2009). Additionally, genes associated with ADHD are involved in the regulation of extracellular dopamine (Faraone, 1998, 2005; Swanson, 2000). The therapeutic mechanism of stimulant treatment is related to dopamine and other catecholamines, and stimulant treatment is most often used in ADHD (Del Campo et al., 2011; Rosa-Neto et al., 2005; Rubia et al., 2011). Methylphenidate exerts a therapeutic effect for ADHD by increasing the extracellular availability of dopamine (and norepinephrine) by blocking the reuptake of dopamine via transporter binding (Del Campo et al., 2011; Tripp, 2009; Faraone, 1998). Amphetamines work similarly to methylphenidate, but also increase the release of dopamine and norepinephrine and decrease the catabolic activity of monoamine oxidase (Del Campo et al., 2011). PET imaging studies have shown acute increases in extracellular dopamine with methylphenidate administration (Rosa-Neto et al., 2005). Thus, the stimulant medications are thought to correct an underlying deficit related to dopamine regulation and availability.

Additional catecholamines, including norepinephrine and serotonin are thought to play a role in ADHD; however the roles of these neurotransmitters are less well understood (Zimmer, 2009; Del Campo et al., 2011). Norepinephrine neurons are mainly located in the locus cerulus, part of the reticular activating system, which is thought to be an important center in regulating attention and arousal (Owens, 2005). As discussed above, extracellular norepinephrine levels are increased with the use of psychostimulant medications (Del Campo et al., 2011). Non-stimulant medications, atomoxetine and alpha-agonists, effectively increase extracellular norepinephrine levels and have also been successfully used in the treatment of ADHD. A serotonin transporter has been associated with ADHD (Faraone, 2005), although serotonin's role in ADHD has not yet been clarified.

\subsection{Neurobiology of sleep}

The neurobiology of sleep has also been a focus of study in recent years, and has become much better understood. As reviewed below, sleep has two distinct stages, non-rapid eye movement (non-REM) and rapid-eye movement (REM) that alternate in cycles through the night. non-REM sleep is a period of relatively low brain activity, although the brain's regulatory capacity is still active, and body movements are preserved. non-REM sleep can be further divided into stages 1-3 by the 'deepness' of the sleep, stage 3 being the deepest. REM sleep is a period of desynchronized cortical activity, high metabolic rate, lack of muscle tone and episodic bursts of rapid eye movements (Mindell, 2010). Arousal opposes sleep in the sleep-wake cycle, and the daily shift between these states requires a fine-tuned system of neural interactions (Dahl, 1996). Multiple, different neural systems have been implicated in the control of the waking state (Lu, 2010; Espana, 2004).

A 2-process model has been proposed to explain the interactions of different biologic mechanisms of sleep. The homeostatic process, which regulates length and depth of sleep, interacts with the endogenous circadian rhythm system, which influences the internal sleep organization and the timing and duration of daily sleep-wake cycles (Owens, 2005; Pace- 
Schott \& Hobson, 2002). The homeostatic process or sleep drive is dependent upon the quality and quantity of previous sleep, as well as the period of time spent awake; the sleep drive increases with longer waking periods, and poorer sleep quality or duration (Owens, 2005). The circadian rhythm is synchronized with a 24 hour day, with predictable periods of increasing arousal and sleepiness, which are linked to ambient light exposure (Owens, 2005). The light-dark cycle is a powerful zeitgeber or cue that entrains the circadian clock to the 24-hour day. The suprachiasmatic nucleus of the hypothalamus responds to these cues by influencing the production of melatonin. Under typical circumstances, as one nears the usual dark period, melatonin production increases and facilitates sleep onset. In addition to environmental cues which help to keep the circadian clock on track, genetics is also thought to play a role. Individuals appear to have a genetic propensity to be a "lark" (sleep-wake cycle set earlier) or a "night owl" (sleep-wake cycle set later).

The ascending arousal system consists of multiple neurotransmitters and produces cortical arousal through two pathways. The dorsal route ascends through the thalamus, and the ventral route through the hypothalamus and basal forebrain (Fuller, et. al, 2006). The arousal system is opposed by the ventrolateral preoptic nucleus (VLPO), which is active during sleep (Fuller, et. al, 2006). The VLPO initiates sleep after input from the circadian rhythm, and homeostatic process (sleep drive) (Pace-Schott \& Hobson 2002).

Many neurotransmitters have been implicated in the regulation of behavioral state in the sleep-wake cycle. During wakefulness and rapid eye movement (REM) sleep, cholinergic neurons in thalamus, hypothalamus \& dorsal midbrain activate the thalamocortical signaling to produce fast cortical rhythms (Espana, 2004; Lu, 2010). In non-REM sleep, however, these neurons are relatively inactive (Espana, 2004; Lu,, 2010). Neurons in the locus ceruleus (mainly producing norepinephrine) are active during waking, less active during non-REM sleep and demonstrate almost no activity during REM sleep (Espana, 2004). Norepinephrine induces cortical activation \& wakefulness, especially in the setting of stress (Espana, 2004; Lu, 2010). Similarly, neuronal histamine is found in the tuberomammillary nucleus, which is active during wakefulness, with less activity during non-REM sleep and even less during REM sleep (Espana, 2004). Histamine promotes wakefulness, especially at the time of waking and during conditions that require high behavioral arousal (Espana, 2004). Serotonin has also been connected to the regulation of behavioral state. The dorsal raphe nucleus is an important source of serotonin, and has (similarly to other aminergic neurons) been found to be most active during wakefulness, less so during non-REM sleep and virtually inactive during REM sleep (Espana, 2004). Orexin/hypocretin neurons are most active during wakefulness, particularly during periods of increased arousal or locomotor activity (Espana, 2004; Lu, 2010). Orexin/hypocretin neurons appear to be inhibited by the amines, and are at their highest level at the end of the day, possibly working to oppose the increasing sleep drive (Espana, 2004).

Dopamine is thought to have wake-promoting properties, but its' role in the sleep-wake cycle has been difficult to understand due to the lack of obvious change in dopaminergic firing rate throughout the sleep-wake cycle (Espana, 2004; Lu, 2010). Yet, extracellular levels of dopamine are elevated during wakefulness and dopamine receptor agonists increase waking while reducing non-REM and REM sleep (Espana, 2004). It has also been noted that patients using dopamine antagonists, such as antipsychotics, and patients with Parkinson's Disease (deficient in dopamine) are often sleepy (Espana, 2004; Lu, 2010). This supports likely wake-promoting properties of dopamine. 
Recently, there has been increasing evidence regarding the neurobiological overlap of sleep and ADHD (Owens, 2005). A proposed relationship for sleep disruption and ADHD is an altered pattern of melatonin secretion or responses to environmental cues such as light. Alternatively, common anatomical pathways may provide the link between sleep and ADHD. The reticular activating system and prefrontal cortex have been implicated in the regulation of both attention/arousal and sleep (Owens, 2005). The thalamus is thought to play a role in working memory and behavioral inhibition abnormalities in ADHD, and in the regulation of non-REM sleep (Owens, 2005). Additionally, aberrations in dopamine and norepinephrine signaling have been identified in both sleep disturbances and ADHD (Owens, 2005; Espana, 2004). The role of other neurotransmitters important in sleep such as histamine and acetylcholine in ADHD, if any, have not yet been identified. Although we do not fully understand the relationship between sleep and ADHD, there appears to be substantial overlap. We will further explore this relationship throughout this chapter.

\section{Typical sleep in children and adolescents}

As noted above, sleep is a complex process described as a rapidly reversible state of reduced responsiveness to the environment. Through basic processes inherent in sleep, our memories are consolidated, growth is optimized, and the next day we hopefully feel less sleepy, have restored energy and attention and the prospect of better learning and mood. During childhood and adolescence, sleep durations and patterns and other aspects such as associated behaviors change with normal development. Here we briefly review the architecture of sleep and how the patterns of sleep-wake cycles change with age (Owens, 2005; Mindell, 2010).

The architecture of sleep involves ultradian rhythms or cycles that consist of two sleep stages, non-REM and REM sleep. The duration of these ultradian cycles changes with development, being about 50 minutes in infancy and lengthening to adult levels (90-120 minutes) by school-age. It is typical for individuals to have a brief arousal at the end of these cycles, about 4 to 6 times during the sleep period. Non-REM sleep includes stage 1 or transitional sleep from which a person can be readily aroused, stage 2 which accounts for the majority of non-REM sleep and stage 3 or slow wave sleep (SWS), from which it is more difficult to arouse. REM sleep is characterized by an active EEG pattern and bursts of eye movements are a hallmark of this stage that includes dreaming. Non-REM sleep is more predominant early in the sleep period, about the first third, and REM sleep accounts for a greater percentage of sleep in the last third. Important biological functions are attributed to each sleep stage, for instance growth and neuroendocrine function for non-REM sleep and memory consolidation and learning for REM sleep. The stages of non-REM sleep develop in infancy and the relative percentage of SWS peaks during early childhood and declines by adolescence. In infancy, REM sleep accounts for about $50 \%$ of sleep. This declines during childhood and reaches adult levels (about 25-30\% of sleep) by adolescence (Mindell, 2010).

The overall duration and pattern of sleep changes with age and development (Iglowstein et al., 2003). Other factors also influence sleep patterns including (among others) child health and temperament, activities and regularity of family routines and cultural expectations. Typically, infants and toddlers sleep 11-13 hours across day and nighttime sleep and this declines to 9-10 hours by school age. While adolescents might self-describe needing less 
sleep, typical adolescents do best with about 9 to 9.25 hours. Naps account for 3-4 hours of total sleep for infants, distributed as 2-4 a day and decline to 1 nap by about 18 months of age. Napping continues into toddler to preschool years; about $50 \%$ of children at age 3 years continue to nap, $25 \%$ at 4 years and $15 \%$ at 5 years. At school age, children are typically quite alert during the day and have little daytime sleepiness or napping unless driven by cultural preferences. At this age, daytime sleepiness would be a symptom deserving of further investigation, particularly in the context of learning, attention and behavioral problems. However, recent studies in this age group suggest that even in the absence of overt sleepiness, daytime behavioral concerns may indicate insufficient sleep duration or quality. By adolescence, the normal physiological phenomena of later sleep-wake cycles, called delayed sleep phase, typically predominates such that bedtimes and sleep onset times drift later, often by several hours. Concurrent early school start times set the stage for insufficient duration of night time sleep, typically 7 to 7.5 hours for many adolescents. In addition, teenagers have more variability in their sleep-wake patterns as they try to pay back their sleep debt on the weekend. Such irregular schedules add to the risk of sleep problems and chronic sleep deprivation affects several aspects of daytime functioning including attention, learning, and mood. Over half of adolescents report that they feel tired and sleepy during the day and about $25 \%$ reported falling asleep in school at least once a week (SAP, 2006). Sometimes caffeine will be used in the attempt to stay alert and result in further delay of sleep onset when consumed too close to bedtime.

It is also important to recognize that the ability of parents to report a sleep problem for their child also appears to be age-related. According to a recent national poll, about $16 \%$ of adolescents report having sleep problems; however, only about $7 \%$ of parents report problems with sleep for their teenagers (SAP, 2006). Parents of younger children may be more likely to be aware of the characteristics of their children's sleep and bring it up as a concern but not uniformly. Thus, it is important to inquire with children and adolescents directly as well as the parent when evaluating for sleep problems (Owens, 2000).

\section{Sleep disorders}

In the following section, we describe the medical and behavioral characteristics of several sleep disorders and review associated studies that examine daytime functioning, particularly related to attention, learning and behavior. The majority of pediatric research thus far has centered on the first two conditions described, sleep disordered breathing and restless legs or periodic limb movement disorder.

\subsection{Sleep Disordered Breathing}

The term sleep disordered breathing (SDB) represents a spectrum of disorders which includes at the severe end, obstructive sleep apnea (OSA). The SDB spectrum also includes other conditions such as, primary snoring (airflow causes airway vibration but no other observable abnormality), upper airway resistance syndrome (hypoventilation and increased intrathoracic pressure resulting in fragmented sleep but without frank apneas, or the cessation of breathing), and apnea due to central nervous system processes. OSA is marked by snoring and apnea, and accompanied by hypoxemia or oxygen desaturation. To determine whether the symptom of snoring is associated with periods of apnea or hypopnea (low airflow and associated oxygen desaturation) a polysomnogram or sleep study is 
needed. OSA is typically marked by repeated and sometimes prolonged periods of reduced or no airflow resulting in low oxygen delivery (hypoxia) to the brain and disrupted sleep (Mindell, 2010).

About $30 \%$ of children snore and about $3-4 \%$ have OSA. The parents of children with OSA might report hearing loud or heroic snoring and pauses in breathing, snorts or gasps. However, the symptoms of OSA may be less pronounced. Children with OSA may present with restless sleep, nocturnal sweating, unusual positions while sleeping (e.g., neck extended), morning headache and dry mouth. One risk for OSA emerges from processes of normal development. During preschool to early school age, children experience a relative increase of lymphoid tissue including tonsils and adenoids. When this increase outpaces the growth of the normal airway, a risk of airway obstruction occurs especially during periods of lower muscle tone, e.g., during sleep. Other factors also increase the risk of obstruction during breathing and include (among others), chronic nasal congestion, lower muscle tone due to medical conditions, oropharyngeal features, and obesity (Mindell, 2010; Hodges, 2011).

Growing concerns about SDB and the effect on day daytime functioning have fueled investigations about the relationship between ADHD and SDB in children presenting with one or the other condition or both. A number of methods have been used. Surveys have been conducted in general populations of children or in individuals suspected to have these specific disorders. Studies have also assessed for relationships between ADHD and SDB concurrently and prospectively as well as, before and after surgical intervention to remove tonsils and adenoids. Parents of children with an ADHD diagnosis were more likely to endorse symptoms of habitual snoring for their children than parents of non-snorers; $22 \%$ versus $12 \%$, respectively (Chervin, 2002). In addition, those children who were rated as having high SDB scores were more likely to describe ADHD symptoms of inattention and hyperactivity (Chervin, 1997). In a study of children ages two to thirteen years (at enrollment), those endorsed as habitual snorers at baseline were more likely to have hyperactive behavior four years later (Chervin, 2005). Early case studies reported ADHD symptoms in children referred for evaluation of SDB symptoms (Guilleminault C., 1981). In a study by Dillon et al, of children five to twelve years of age scheduled for adenotonsillectomy, nearly 37\% had evidence of ADHD and disruptive behavior disorder (including oppositional and conduct disorder) and about 28\% had ADHD (Dillon, 2007). This study also assessed behavioral outcomes after adenotonsillectomy. Fifty percent of the children who met criteria for ADHD at baseline before adenotonsillectomy no longer did so after surgery (Dillon, 2007). In a review of sixteen studies, parents who reported higher SDB symptoms for their children also reported they had higher inattention and hyperactivity symptoms (Hodges, 2008; Cortese, 2009). Thus, there is substantial evidence supporting a relationship between ADHD and SDB.

In children with SDB, excessive daytime sleepiness is a common symptom that affects functioning related to attention and activity. Whereas tired or sleepy adolescents may report more problems staying awake, younger sleepy children may present with hyperactive and agitated behaviors (Hodges, 2011). In school-age children with SDB, time to fall asleep on structured nap tests (mean sleep latency test) was associated with the severity of SDB before adenotonsillectomy. One year after surgery; however, when SDB symptoms had improved, this association was no longer observed (Chervin, 2006; Gozal, 2001). In a meta-analysis 
review of 16 studies, daytime sleepiness was among the variables associated with ADHD. A recent study also found a significant relationship between daytime sleepiness and inattentive type ADHD (Cortese, 2009; Mayes, 2009). Other studies have demonstrated similar neurobehavioral outcomes related to SDB and/or excessive daytime sleepiness including impaired attention, tests of executive functioning, behavioral regulation and scores of intellectual functioning in some studies (Beebe, 2005; Owens, 2005; Beebe, 2004; Gottlieb, 2004). In many children with SDB, adenotonsillectomy results in improvements in neurobehavioral outcomes, and improvement in sleepiness is felt to play a role.

There does not appear to be a clear "dose-response" relationship between SDB and ADHD. In a community-based study, parents rated their 5-7 year old children for ADHD symptoms and a subset of the children underwent polysomnogram. OSA was observed for $26 \%$ of the children with mild ADHD symptoms versus $5 \%$ for those with severe ADHD or no ADHD concerns. This study suggested that SDB might lead to mild ADHD symptoms but not explain more severe ADHD symptoms (O'Brien, 2003). Chervin suggests that the relationship between $\mathrm{ADHD}$ and SDB in at-risk children may be due to more subtle aspects of disordered breathing (Chervin, 2005). The mechanisms by which SDB and OSA in particular may relate to neurobehavioral sequelae have been proposed and involve the effect of sleep disturbance on the prefrontal cortex, sleep fragmentation, hypoxia, and hypercarbia (Beebe, 2005; Hodges, 2011) . The relationship of such core factors with individual variables of risk are under investigation. Important individual variables include resilience, environmental context and demands, and the progression of these over time with development.

As noted above, there is substantial overlap between brain areas that regulate sleep, arousal and attention raising the possibility that sleep disruption may have direct effects on central nervous system functioning. In addition, sleep disruption during development suggests the possibility that structures such as the prefrontal cortex may sustain longer term neuroanatomic or functional alterations as a result of early sleep problems. Prospective studies will provide important contributions regarding the relationship between ADHD symptoms and SDB and these are ongoing currently.

\subsection{Restless leg syndrome and periodic limb movement disorder}

Restless legs syndrome (RLS), periodic limb movements of sleep (PLMS) and periodic limb movement disorder (PLMD) are overlapping conditions that may occur separately or together and affect sleep quality. There is evidence of a genetic basis for RLS with PLMS involving chromosome 6p.(Picchietti, 2008) Restless legs syndrome is a diagnosis made based on presenting clinical symptoms such as uncomfortable sensations (e.g, feeling things crawling or tingling) and the urge to move, both typically involving the lower limbs. RLS symptoms usually occur while sitting or lying down, toward evening hours, and the symptoms are typically relieved by movement. In children, the presentation of symptoms may be more variable and described differently, such as "shark bites" or "growing pains" for some children (Rajaram, 2004). Specific criteria for RLS were published in 2003 by NIH (Allen, 2003). While first observed in adults, RLS has been recognized in children for over fifteen years and is estimated to occur in about 2\% of children (Walters, 1994; Picchietti, 1998; Pichietti, 2007; Garcia-Borreguero, 2006; Picchietti, 2008). 
PLMS are brief, repetitive and regular stereotyped jerks during sleep, typically occurring at the lower extremities and lasting up to 5 seconds. PLMD requires a polysomnogram to make a diagnosis which requires five or more characteristic movements per hour (criteria for children and adolescents), associated with sleep disturbance (e.g., arousal) or effects on daytime functioning (e.g., fatigue), and no other primary sleep disorder reason for the PLMD. The prevalence of PLMD in the general population is not known. However, based on survey information, PLM symptoms are thought to affect about $8-12 \%$ of children (Picchietti, 2008). There is evidence that altered dopaminergic central nervous system functioning underlies RLS and PLMD for many individuals. In addition, MRI and autopsy studies have identified lower iron stores in the striatum and other central nervous system areas for adults affected with RLS or PLMD. Further, serum ferritin $<50 \mathrm{ng} / \mathrm{ml}$ has been associated with RLS symptoms or findings of PLMD and iron supplementation has been demonstrated to improve symptoms. Therefore, iron deficiency may be a treatable medical risk factor for RLS, PLMS and PLMD particularly associated with growing children, women during pregnancy and for individuals with chronic medical conditions (renal disease and leukemia) (Konofal, 2007; Mindell, 2010).

RLS and PLMD have been associated with daytime symptoms of ADHD. In a study of 143, two to eighteen year-old children attending child psychiatry or general pediatric clinics, parents indicated PLMS might be related to ADHD symptoms (Chervin, 1997). In a questionnaire study of 830 children two to thirteen years of age, about $4 \%$ of parents endorsed PLM symptoms for their children (Chervin, 2001). Picchietti and others looked at the occurrence of PLMD for children with or without ADHD symptoms. In their study, 26\% of children with ADHD symptoms had PLMD, compared to 5\% of children who did not have ADHD symptoms (Picchietti, 1998). In a study comparing children referred for clinically defined ADHD versus two other groups, those identified as at risk for ADHD from a community survey and children without ADHD, those with clinically determined ADHD had higher PLMs than the other groups suggesting a greater risk for more active and disrupted sleep (O'Brien, 2003). In addition, in children with ADHD, serum ferritin less than $12 \mathrm{ng} / \mathrm{ml}$ was more prevalent in children with RLS symptoms (Oner, 2007). Thus, RLS, PLMS and PLMD appear to be related to daytime ADHD symptoms; however, but further work is needed to clarify the relationship and the role of iron specifically in the etiology and management.

\subsection{Other sleep disorders}

In the following section, other sleep disorders are described that affect sleep quality and/or duration but have fewer studies related to daytime behavioral consequences in children and adolescents. This is followed by a section describing the consequences of insufficient sleep in general.

\subsubsection{Behavioral insomnia of childhood}

Behavioral insomnia of childhood, the difficulty initiating and/or maintaining sleep due to a behavioral etiology, is very common, with a prevalence of $10-30 \%$. It is more often encountered in young children (Moore \& Meltzer, 2008). There are 2 types of Behavioral Insomnia of Childhood: Sleep Onset Association; and Limit Setting. Sleep Onset Association 
type of behavioral insomnia occurs when the onset of sleep is delayed in the absence of a certain item or circumstance. The child becomes dependent upon certain conditions being present during sleep onset, such as sucking on a pacifier or having another person close by. When the specific sleep onset conditions are met, sleep onset is quickly achieved. However, should the conditions not be met (i.e. the pacifier is on the floor, or mother is away) at sleep onset, then a struggle around getting to sleep may occur. In addition, when the child experiences a normal partial arousal during the night (commonly every 1-3 hours), the child who is used to certain conditions at bedtime will have difficulty returning to sleep if the conditions are no longer available. This circumstance of Behavioral Insomnia of Childhood, Sleep Onset Association type typically results in a night wakening and parent involvement to 'correct' or return the conditions for getting to sleep. Behavioral Insomnia of Childhood, Limit Setting type occurs when there are bedtime struggles. The child typically stalls or refuses to go to bed by requesting things such as a glass of water, or to go to the bathroom. When the caregiver has trouble setting and maintaining limits, the child's sleep onset is delayed as a consequence. However, when such limits are set consistently, the child is able to sleep quickly. Many children have a mixture of Sleep Onset Association and Limit Setting types. As children get older, they typically have less difficulty with Sleep Onset Association Insomnia as they are better able to get to sleep on their own and re-create their sleep onset conditions and return to sleep during the night. During those same developmental stages; however, children are working to become more independent and are testing limits set by their caregivers. Often this can result in greater difficulties with Limit Setting Insomnia with age (Moore \& Meltzer, 2008). Either type of behavioral insomnia can affect time to sleep onset, the continuity of nighttime sleep and the overall total amount of sleep achieved and therefore, the risk for daytime tiredness and associated sequelae for attention, learning and behavior.

\subsubsection{Delayed Sleep Onset in older children and adolescents}

\subsubsection{Delayed Sleep Phase Syndrome}

As children move toward adolescence, they typically begin to demonstrate a circadian preference for a later (delayed) sleep phase. If the preference for late sleep phase becomes problematic and interferes with the daytime functioning of the child or adolescent, it is referred to as having delayed sleep phase syndrome (DSPS). DSPS is common in adolescence with an estimated prevalence of $10 \%$ (Moore \& Meltzer, 2008). In DSPS, sleep onset and wake times are intractably later than socially acceptable (i.e., an adolescent who falls asleep at 1am, and wakes at 10am although school starts at 8am), occur at approximately the same time daily, and there is little or no difficulty maintaining sleep after sleep onset has occurred. In DSPS, the timing of the sleep-wake cycle is the problem rather than the quality of sleep. There are often complaints of 'insomnia' when the child or adolescent attempts to go to sleep at an early time; however not when attempting to sleep at a later, desired time. Typically the child or adolescent has a very difficult time waking up at a required time, such as for school, and has decreased alertness in the morning. This can lead to frequent tardiness and/or absence from school. The child or adolescent frequently attempts to 'catch-up' on lost sleep by sleeping in to the late morning or afternoon on weekends. DSPS can significantly shorten sleep duration, increasing the risk for daytime tiredness and associated sequelae. 


\subsubsection{Psychophysiologic insomnia}

Primary or psychophysiological insomnia can also be responsible for sleep problems in children and adolescents. Insomnia is broadly defined as difficulty with onset, maintenance or non-restorative sleep. The following consensus statement has been developed to more specifically define pediatric insomnia: 'repeated difficulty with sleep initiation, duration, consolidation or quality that occurs despite age-appropriate time and opportunity for sleep and results in daytime functional impairment for the child and/or family' (Mindell, 2010). Primary insomnia is characterized by tension and learned sleep-preventing associations. Children and adolescents with primary or psychophysiological insomnia tend to respond to stress with somatic symptoms, and while trying to downplay the stress, end up increasing the stressfulness of their insomnia. Primary insomnia is rare in pre-pubertal children, but has a prevalence of 9 to $13 \%$ in adolescents (Mindell, 2010).

\subsubsection{Partial arousal parasomnias}

Partial arousal parasomnias are behaviors during sleep that occur primarily related to nonREM or slow wave sleep. These include confusional arousals, sleep walking, and sleep terrors. These partial arousals typically occur within a few hours of sleep onset and are fairly common in childhood, when the percent of non-REM sleep is greater compared to adulthood. Individuals with such partial arousal parasomnias do not recall the events that occur during sleep. Family history appears to increase the likelihood for both sleep walking and sleep terrors if other family members also experienced it. Confusional arousals are episodes where individuals are aroused but disoriented and may not fully awaken for up to 30 minutes. These occur in at least $17 \%$ of children. Sleep walking on a regular basis is also common in childhood, observed in about 15 to $20 \%$. Both confusional arousals and sleep walking typically begin in the preschool years. Confusional arousals tend to dissipate by early adolescence, whereas, for about $20 \%$ of affected individuals, sleep walking persists into adulthood. Sleep terrors are often more significant and distressing but primarily to the observer because the affected child can appear frightened and agitated. Sleep terrors occur in about 1 to $6 \%$ of children, with onset between preschool and preteen years and they typically subside by adolescence. These parasomnias can be triggered by inadequate or irregular sleep, illness, stress and other sleep problems such as SDB or PLMD. Parent and child education about the disorders, what they can do to lessen the risks for occurrence and assuring safety during episodes are the most important interventions. These behaviors do not disrupt sleep continuity on their own and therefore, uncomplicated partial arousal parasomnias are not typically associated with daytime behavioral sequelae like ADHD. However, a 2006 study from Taiwan suggested an association based on parental ratings (Shur-Fen Gau, 2006). In general, the presence of these parasomnias might indicate another sleep disorder that has been related to ADHD symptoms and might be the clue to look further for problems such as SDB, PLMD, or inadequate sleep.

\subsubsection{Sleep-related Rhythmic Movement Disorder}

Rhythmic movement disorder (RMD) is common in childhood, usually having onset in the first year of life and waning by early school age. This disorder includes various combinations of movements of the head, limbs, and trunk resulting in head rocking, head or body rolling, and head banging, among others and typically occur on transition to or from 
sleep or during sleep. It is typical for the movements to have a regular, rhythmic pattern. To meet criteria for RMD, the behavior must interfere with normal sleep, impair daytime functioning or cause bodily injury, and not be due to another disorder or use of medication. Most individuals do not require treatment for RMD as the risk of injury is low and it dissipates with age; however, protective surfaces might be needed for more significant cases (Hoban, 2003). Case studies have suggested RMD in children with ADHD and ADHD in children with RMD but studies to date are small (Walters, 2008).

\subsubsection{Night-time fears and nightmares}

As children get older, nighttime fears and nightmares become more frequent sleeping problems. Most children have nighttime fears, with peak ages from 3-6 years for both sexes, and in girls at school-age (Moore \& Meltzer, 2008). Nighttime fears are usually short-lived developmental phenomena and quite benign. At the peak age of nighttime fears at preschool-age, children are becoming more aware of the consequences of their actions and that they can really be hurt or affected by occurrences around them (Mindell, 2010). Typical nighttime fears change with developmental level, with younger children being more afraid of imaginary creatures such as monsters and older children being afraid of more realistic dangers such as a natural disaster or a burglar. Nightmares are also common in childhood, with approximately $75 \%$ of children experiencing at least one nightmare (Moore \& Meltzer, 2008; Mindell, 2010). Similar to nighttime fears, nightmares are a normal part of a child's development, and the content tends to vary by age. Young toddlers worry about being separated from their parents, and as children get older they incorporate imaginary and then realistic fears into their nightmares. Different from nighttime fears, nightmares typically occur during REM sleep (usually in the last third of the sleep period when there is a greater percentage of REM sleep), and can be triggered by sleep deprivation. Both nighttime fears and nightmares can be triggered or exacerbated by stress and anxiety. Consequences of nighttime fears or nightmares include delayed sleep onset and prolonged night awakenings, respectively and either affect the degree to which sleep is restorative for the affected individual.

\subsubsection{Enuresis}

Sleep enuresis is another possible cause of sleep disturbance in children. Uncomplicated or monosymptomatic sleep enuresis is characterized by recurrent, involuntary voiding occurring during sleep. Enuresis can occur in any stage of sleep, although most occur in the first half of the night (Mindell, 2010). It, too, follows the developmental trajectory of the child, with a greater proportion of younger children experiencing sleep enuresis. The majority of sleep enuresis episodes are primary sleep enuresis, in which the child has not yet developed control over voiding at night, and has not had a succession of dry nights. These children typically fail to respond to the sensation of bladder fullness or inhibit bladder contractions during the night. Spontaneous remission of sleep enuresis is common as children age; the approximate remission rate is $15 \%$ of affected individuals per year. The current prevalence estimates are $30 \%$ in 4 -year-olds, $10 \%$ of 6 -year-olds, $5 \%$ of 10 -year-olds and $3 \%$ of 12 -year-olds. Sleep enuresis is typically not diagnosed until age 5, and not treated until age 7 or 8 years. Primary enuresis is more prevalent in children with a family history of sleep enuresis, and also, those with ADHD. Secondary enuresis, in which the child 
previously has had control over their bladder at night (and has had a previous dry period of at least 6 months) and then develops sleep enuresis, does not occur as a part of typical development, and usually requires further diagnostic evaluation. It can be triggered by psychosocial stressors, medical problems such as urinary tract infections, chronic constipation, diabetes mellitus, a neurologic disorder, or other sleep disorders, such as obstructive sleep apnea or PLMD. Children with complicated or non-monosymptomatic sleep enuresis have day- and nighttime symptoms.

\section{Consequences of insufficient sleep}

As noted above for SDB and RLS/PLMD, poor sleep can have a great impact on daytime function of the child and other family members. Adults with poor sleep tend to appear tired during the day, yawning and falling asleep easily in inappropriate settings. However, the symptoms of sleepiness in children are often 'paradoxical' behavioral manifestations such as emotional lability, irritability, neurocognitive deficits and behavioral inhibition. These difficulties can cause problems at home, in school and in other community settings. Multiple parental reports (and some teacher reports) show increased incidence and severity of behavioral difficulties in children with sleep problems than those without (Fallone, 2002; Sadeh, 2002). More severe behavioral problems have been shown in children with shorter sleep duration (Fallone, 2002). Increased sleepiness or decreased sleep duration within the previous day have been associated with increased risk of accidental injury in children from preschool-aged through adolescence (Fallone, 2002). Insufficient sleep and/or sleepiness in adolescents have been associated with increased risk-taking behaviors (Moore \& Meltzer, 2008). Children with behavior problems are also more likely to have sleep problems by parental report (Fallone, 2002). Studies have shown improvement in these behavioral problems with improved sleep; however, not across all settings (Fallone, 2002). Lower academic achievement has also been associated with poor sleep in children, when compared to children with unimpaired sleep (Fallone, 2002). Additionally, adolescents who reported poor sleep were more likely to report social problems as well (Owens, 2008). An association between acute sleep restriction and poorer memory or lower IQ has not been found (Fallone, 2002; O'Brien, 2004). However, longer-term sleep disturbance has been linked to impaired memory and intelligence ( $\mathrm{O}^{\prime}$ Brien, 2004).

Children's sleep problems can also cause a significant amount of distress in the family. The impact of childhood sleep problems is related to the parents' sleep, especially if the parents' poor sleep leads to daytime fatigue or mood problems (O'Brien, 2004). Alternatively, improving the child's sleep, and by extension that of the rest of the family, can improve the functioning of all family members involved.

\section{Sleep in children with ADHD}

As noted previously in this chapter, sleep difficulties were part of the diagnostic criteria for ADHD in previous versions of the Diagnostic Statistical Manual, indicating a long-standing belief that sleep is problematic in children with ADHD. However, although the relationship between sleep and ADHD has been studied extensively over the past few decades very few objective differences have been found between the sleep of children with ADHD and children without ADHD. Yet, many subjective differences have been uncovered. 


\subsection{Sleep and ADHD: subjective differences}

There is little disagreement in the literature that parents of children with ADHD report poorer sleep for their children than parents of children without ADHD. O'Brien et. al found that $77 \%$ of children with significant symptoms of ADHD, and $70 \%$ of children with mild symptoms of ADHD were reported to have sleep disturbances while only $43 \%$ of children without ADHD symptoms were reported to have sleep problems (O'Brien, 2003). Parents of children with ADHD report more of the following than parents of children without ADHD: bedtime resistance; sleep-onset delay; anxiety; parasomnias; night wakings; daytime sleepiness; and shorter duration of sleep (Owens, 2000; Corkum, 2001; O'Brien, 2003; Silvestri et al., 2009; Gruber, 2009). Parents also report more snoring in children with ADHD compared with controls (Cortese, 2009). Children with and without ADHD tend to have morning arousal at the same time, but children with ADHD have a shorter reported sleep duration thought to be due to delayed sleep onset (Owens, 2005).

There are several possible explanations for these increased subjective complaints in children with ADHD. One hypothesis suggests that some of these are not sleeping problems per se, rather, behavioral problems in the context of a difficult parent-child relationship (Cortese, 2009; Corkum, 2001). Another hypothesis is that families of children with ADHD are more likely to have poor sleep hygiene (i.e., bedtime routines, sleeping environment), leading to more difficulties at bedtime (Cortese, 2009). Alternatively, children with ADHD may have symptoms throughout the day and evening, which creates bedtime difficulties even in the appropriate environment (Cortese, 2009; Gruber, 2009). Some children with ADHD may have a disorder of their sleep-wake schedule (i.e., delayed sleep phase syndrome or other disorder of circadian rhythm), and aren't able to fall asleep when they are expected to, which can lead to bedtime resistance (Gruber, 2009). Children with ADHD may also have co-morbid psychiatric disorders which contribute to bedtime difficulties (see below). Owens et. al found that parents of children with ADHD recognize sleep-onset difficulties as different from bedtime refusal. Other studies have drawn similar conclusions; parents are able to recognize the difference between unwillingness and inability to sleep (Blader et al., 1997). Stimulant medication is frequently used to treat ADHD in children and may have effects on their sleep. This will be addressed in a later section of this chapter.

\subsection{Sleep and ADHD: objective differences}

There is little agreement in the literature regarding objective differences in sleep in children with ADHD. Sleep in children with ADHD has been frequently studied but with small populations, and with conflicting results. Higher rates of obstructive sleep apnea (higher apnea-hypopnea index) have been found in children with ADHD (O'Brien, 2003; Cortese, 2009; Golan, 2004). Children with mild symptoms of ADHD are more likely to have obstructive sleep apnea than those with significant or no symptoms (O'Brien, 2003). Children with ADHD have also been found to have an increased REM latency and smaller percentage of sleep spent in REM (O'Brien, 2003, 2003; Silvestri et al., 2009; Busby, 1981). $\mathrm{O}^{\prime}$ Brien et al found a positive correlation between length of REM latency and impulsivity, inattention, cognition and hyperactive behaviors, and a negative correlation between REM latency and attention and executive functioning ( $\mathrm{O}^{\prime}$ Brien, 2003). Yet, other studies have shown an increase in percentage of REM sleep in children with ADHD (Golan, 2004) or decreased REM latency (Khan, 1982; Kirov et al., 2004), and still others have shown no differences in REM latency or percentage (Golan, 2004; Cortese, 2009). Increased periodic 
limb movements have been discovered in children with ADHD (Busby, 1981; O'Brien, 2003; Silvestri et al., 2009). Others have found decreased sleep efficiency, increased arousal index and increased daytime sleepiness in children with ADHD (Goraya et al., 2009; Cortese, 2009; O'Brien, 2003; Silvestri et al., 2009; Gruber, 2009; Golan, 2004). The hyperactivity in children with ADHD has been hypothesized as a compensatory mechanism for the increased daytime sleepiness related to poor duration and/or quality sleep. Despite these many studies, there is much more to be discovered concerning the sleep of children with ADHD.

One theory which addresses these conflicting differences in the sleep of children with ADHD is that there are different subsets of ADHD, some of which are associated with more sleeping problems. O'Brien et. al compared the sleep of children with ADHD referred to their sleep clinic, children with ADHD symptoms from the community and children without ADHD, and found that while all children with ADHD had some differences compared to the controls, the children who were referred also had greater nightmares, enuresis and witnessed apneas compared to the children with ADHD symptoms from the community group (O'Brien, 2003). They proposed that the children who were referred represent a different subset of children with ADHD. Additionally, differences have been found, in some cases, between the sleep of children with ADHD inattentive subtype and combined subtype, with more differences in sleeping patterns found between children with combined subtype ADHD and typically developing children (Gruber, 2009; Mayes, 2009).

Co-morbid psychiatric disorders are common in children with ADHD, and are thought to contribute to problems with sleep. Children with ADHD and anxiety or depression were found to have more sleeping problems than those without, although children with comorbid oppositional defiant disorder (ODD) were not found to have increased sleeping problems (Mayes, 2009). Sleep problems have been shown to have a weaker relationship with ADHD after controlling for co-morbid anxiety, depression or disruptive behavior disorders (Mick, 2000). However, some of the studies previously discussed in this section found differences in sleep in children with ADHD without co-morbid psychiatric disorders compared to typically developing children.

\section{Medical treatment for ADHD and sleep problems}

\subsection{Psychostimulant medications for ADHD and effects on sleep}

Psychostimulant medication, the first-line treatment for ADHD, has often been cited as causing sleeping disturbances in this population. However, as discussed in previous sections, there is a complicated relationship between ADHD and sleep. Some have suggested that stimulant medication causes sleep disturbances in children and adolescents with ADHD. Some studies of stimulant medication have supported this, but others have shown no change or improvements in sleep in children and adolescents treated with psychostimulant medication, (Galland et al., 2010; Mick, 2000; Barkley et al., 1990; Giordani, 2008; O'Brien, 2003; Kim, 2010; Giblin \& Strobel, 2010).

Regarding studies where the use of psychostimulant medication has been associated with poor sleep, in a study by Mick et. al, there was a trend towards children with ADHD having more sleep disorders and when multiple confounding variables were accounted for, they found a relationship between the use of stimulant medication and poor sleep (Mick, 2000). Children with ADHD treated with psychostimulant medication were more likely than 
controls to be reported as a restless sleeper, waking during the night, talking in their sleep, afraid of sleeping in the dark and going to the bathroom during the night (Mick, 2000). Parents have also reported more difficulty with insomnia in children with ADHD treated with psychostimulants (Barkley et al., 1990). Several objective studies have also shown changes in sleep with psychostimulant medication. EEG studies have shown increased REM onset latency and of stage 1 and 2 sleep with a resultant decrease in time spent in REM and stage 3 sleep after nocturnal administration of a psychostimulant (Chatoor, 1983). Polysomnography studies showed no significant difference in sleep timing, duration or efficacy between children with ADHD off of medication for 48 hours and control children (Galland et al., 2010). However, when the children with ADHD had taken their dose of psychostimulant medication, they had a longer sleep latency and woke earlier in the morning, resulting in a shorter sleep duration in comparison to control children (Galland et al., 2010). Sleep architecture was not altered by the medication (Galland et al., 2010). Galland et. al observed that their study, and others that had found increased sleep onset latency on psychostimulant treatment, were designed such that the comparisons between methylphenidate and placebo were within-subject. They postulate that this may indicate that the individual differences of the effects of psychostimulant medication on sleep may be more important than larger group differences (Galland et al., 2010).

Another hypothesized factor is that the increased reported difficulties with sleep in children with ADHD treated with psychostimulant medication may be the result of the psychostimulant medication wearing off and/or causing a 'rebound' effect. This rebound effect can lead to an apparent increased arousal and hyperactivity. This led to the idea that some children may benefit from an additional late afternoon or early evening dose of psychostimulant medication. An additional late afternoon (4pm) dose was shown to improve behavior in 12 psychiatrically hospitalized children with ADHD (Kent et al., 1995). Low-dose $(10 \mathrm{mg})$ and high-dose $(15 \mathrm{mg})$ methylphenidate and placebo were given at $4 \mathrm{pm}$, with little effect on the sleep onset in the children (Kent et al., 1995). When receiving the high-dose methylphenidate, children did have an increased sleep onset latency (Kent et al., 1995). Notably, the increased sleep onset latency in children on the higher dose of methylphenidate was similar to that of children on the placebo (Kent et al., 1995). A similar study tested the effect of twice-daily dosing to thrice-daily dosing of methylphenidate in children with ADHD, and found that there were no differences in sleep duration between the two dosing regimens (Stein, 2001). However, there was a trend toward decreased sleep duration in the thrice-daily dosing compared to placebo on parental ratings and actigraphic measurements (Stein, 2001). Sleep onset latency did not differ significantly across the dosing protocols (including placebo) (Stein, 2001).

Other recent studies, have confirmed the relationship of ADHD with poor sleep, but demonstrated little association psychostimulant medication to poorer sleep in children with ADHD. Children with ADHD were found to have more sleep disturbances than those without ADHD, irrespective of their medication status (O'Brien, 2003). The use of psychostimulant medication was not found to have an association with poor sleep ( $\mathrm{O}^{\prime}$ Brien, 2003). Children with ADHD treated with psychostimulant medication tended to have longer REM onset latency, however this did not reach statistical significance ( $\mathrm{O}^{\prime}$ Brien, 2003).

Increased sleep disturbances have not been found in children treated with sustained release preparations of psychostimulants (Kim, 2010; Giblin \& Strobel, 2010). Osmotic-controlled 
release oral delivery system (OROS) methylphenidate was found to decrease the number of night arousals (parasomnias) in children with ADHD, but not alter sleep onset latency on either subjective or objective measures (Kim, 2010). However, children who reported subjective sleep difficulties during treatment with OROS methylphenidate had increased sleep onset latency, sleep onset delay and bedtime resistance when compared to those without subjective sleep complaints. This again, may indicate that the individual's response to stimulant medication may be the most important factor (Kim, 2010). Lisdexamfetamine was also shown to decrease nighttime arousals, and did not negatively impact sleep in children with ADHD (Giblin \& Strobel 2010). The decreased nighttime arousals may suggest more consolidated sleep in children treated with psychostimulants.

Therefore, the use of psychostimulant medications has been associated with poor sleep in children with ADHD in some studies; however the exact relationship remains unclear. Individuals with sleep complaints while on psychostimulant medications have been noted to have increased sleep disturbance. Perhaps it is best to consider each patient individually when assessing for adverse effects of treatment. It may be that for the majority of patients, treatment with psychostimulant medication does not interfere with sleep, but in those reporting worse sleep, the medication does play a role in sleep disturbances.

\subsection{Non-psychostimulant Medications for ADHD and effects on sleep}

In 2002, the US Food \& Drug Administration (FDA) approved the non-stimulant, atomoxetine for use in children with ADHD. Greater clinical improvement is typically attained with psychostimulant medication; however atomoxetine can be good alternative medication to use when a psychostimulant medication is not tolerated or cannot be used (Newcorn et al., 2008). When compared with thrice-daily dosing of methylphenidate, twicedaily atomoxetine was found to have decreased sleep onset latency, and increased sleep duration (Sangal, 2004). Treatment with twice-daily atomoxetine and thrice-daily methylphenidate resulted in decreased interrupted sleep; however, treatment with methylphenidate led to a larger decrease in interrupted sleep (Sangal, 2004). Children reported an easier time awakening in the mornings and falling asleep at night while taking atomoxetine (Sangal, 2004). The most common sleep-related adverse effect reported from atomoxetine is somnolence (Kratochvil et al., 2011; Cheng et al., 2007; Newcorn et al., 2008).

Alpha-agonists such a clonidine and guanfacine have been used to treat both the behavioral and sleep problems of ADHD. Clonidine has been helpful in treating sleeping problems in children with ADHD (Plizka, 2007). Trials of long acting clonidine (with and without the use of psychostimulants) have shown that somnolence and/or fatigue are the major sleeprelated adverse effect of treatment (Biederman et al., 2008; Kollins et al., 2011). However, Biederman et. al showed that this is usually a transient effect (Biederman et al., 2008).

\subsection{Medications for sleep problems in children with ADHD}

As discussed extensively in this chapter, sleeping disturbances are frequently associated with ADHD. Some of these sleep disturbances can be related to anatomic disorders, such as sleep-disordered breathing or perhaps an adverse effect of psychostimulant medication. However, in many cases, a modifiable cause is not identified that results in a medical treatment for the sleep disturbance. In fact, a large Netherlands study of children with 
ADHD on stimulant medication found that over $10 \%$ of the children were taking melatonin, presumably for sleep problems (Faber et al., 2006). Melatonin has been shown to decrease sleep onset latency in children with ADHD (not on medication) with chronic sleep-onset insomnia (Van der Heijden et al., 2006). Despite this improved sleep onset latency, there was not significant effect (positive or negative) on the children's behavior, cognition or quality of life (Van der Heijden et al., 2006). In follow-up of this study, approximately 3 years later, the majority of children remained on melatonin, and their parents thought it was an effective treatment (Hoebert et al., 2009). Parents reported that the melatonin improved their children's daytime behavior (Hoebert et al., 2009). A literature review confirmed that children with ADHD and insomnia showed improvement in regards to sleep onset latency when taking melatonin (Bendz \& Scates, 2010). However, these results are limited as there are few studies, and most of them have small sample sizes. Zolpidem has also been evaluated for treating sleep disturbances in children and adolescents with ADHD (Blumer et al., 2009). Zolpidem did not decrease sleep onset latency, nor improve sleep efficiency or nocturnal awakenings (Blumer et al., 2009). There was a trend toward improving behavioral symptoms, particularly in the adolescents; however, this did not reach statistical significance (Blumer et al., 2009).

\section{Clinical application}

In this section, we describe two clinical cases examples where ADHD evaluation might be complicated by sleep concerns. The purpose is to highlight several concepts regarding development and sleep.

\subsection{Younger child}

Alex, a 7-year-old boy, presented to his primary care physician's office with his mother due to problematic behavior at school. His mother notes that he's always been a busy and active child. Over the past few years, she's had some difficulty with his behavior at home, noting that he had frequent temper tantrums as a toddler and preschool-aged child, but the parents thought his behavior was manageable. However, last year in kindergarten Alex's teacher also expressed concern about his behavior and this has increased with his first grade teacher this year. He has been having difficulty staying seated with the rest of his class during story time and with staying in line with his class when they leave the classroom. He has also recently hit a classmate over a minor disagreement. He typically gets along with other children, and mother notes that he has two best friends, with whom he likes to ride bikes. There was no history of problems at birth or with development. He has no chronic medical concerns, has not had hospitalization or surgery and has not had significant injuries. He is involved in Boy Scouts and sports outside of school.

On review of systems, Alex's mother notes that his sleep schedule is typically uniform throughout the week. He goes to bed around 8 PM on weeknights, and wakes up at 7 AM for school. On the weekends, he tends to go to bed around 8:30 PM, and wakes at $7 \mathrm{AM}$ on his own. He tends to fall asleep approximately 30-45 minutes after his bedtime. He does not appear tired in the morning or throughout the day, and does not nap, nor fall asleep unintentionally. His mother does struggle to put him to bed most nights, noting that he seems "to do anything to avoid sleeping". He frequently asks for another story, and glass of water. Alex's mother also notes that he snores quite loudly; she can hear him snoring from 
her bedroom when his door is closed. She hasn't noted any pauses in breathing. He sweats during sleep frequently, requiring his mother to change the sheets during the night approximately once a week. He has been dry at night for the past three months, and prior to that he had sleep enuresis approximately once every two weeks. Alex's mother and older brother have ADHD, and his maternal grandfather was recently diagnosed with obstructive sleep apnea. He does not have any family history of learning disability or mood disorder. His physical exam was notable for mouth breathing and $3+$ tonsils.

Alex's sleep concerns raise questions about multiple sleep problems. One such problem is sleep disordered breathing, common in early school-age children. Alex is at a peak age for increased lymphoid hypertrophy, which can cause a relative narrowing of the airway during sleep. His bedtime resistance could suggest behavioral insomnia of childhood, limit setting type, with inadequate limit-setting by his mother or his negative behavior may be the result of tiredness made worse by the poor quality sleep due to sleep disordered breathing. Alex's duration of sleep is likely appropriate for his age; however the quality is likely disturbed and affecting his daytime functioning.

To evaluate further, an overnight polysomnography was performed, which demonstrated obstructive sleep apnea. Conners rating scales from both parents and his first grade teacher endorsed problems with attention, hyperactivity and impulsivity for age and gender.

Alex was evaluated by a pediatric otolaryngologist who recommended an adenotonsillectomy, which was subsequently performed. Three months after his surgery, Alex's mother noted significant improvements in his snoring, now only infrequently and that he no longer sweats at night, and has remained dry overnight. However, he has remained very active and 'on the go' and while his behavior problems at school had improved somewhat, he continued to have difficulties. His primary physician initiated a trial of short-acting stimulant medication, to which Alex responded quite well. Alex's presenting concerns seemed to be related to both his sleep problems and underlying ADHD.

\subsection{Early adolescent}

Susie, a 13-year-old female, presents to her primary doctor accompanied by her mother for concerns regarding poor school performance. Mother states Susie has had longer term concerns about being fidgety and distractible but had always been able to keep pace with her peers until 7th grade. There were no academic concerns in elementary school and grades continued to be adequate, although in retrospect, drifted downward in 6th grade. In 7th grade she had more trouble; she forgot to turn in assignments, seemed disorganized and didn't follow through on directions at school, as well as, at home. In 8th grade this year, she is close to failing. Teachers state her test performance has been good, but the frequently missing assignments and little engagement in classroom discussions have pulled down her grades. In addition, they report she has been prone to napping in the first-hour math class. There is no history of problems at birth or with development. She has no chronic medical concerns and has not had significant injuries. She started menses at about 10 years of age. She is active with sports and music for school, participating in swimming and playing cello throughout the year. Her group of friends has been stable. She describes a very busy school schedule and that she is concerned about doing well. 
On review of systems, Susie states that on weekends, she typically goes to sleep about midnight and will get up about 10 to $11 \mathrm{AM}$ on her own. During the school week, she tries to go to bed at 10 PM but "can't get to sleep for hours". She needs to be up by 6 AM for school. She describes feeling uncomfortable at sleep onset due to her legs feeling restless and thinking about the next school day. Mother has not had concerns about Susie snoring nor mouth breathing in the past. She recently slept in the same room on a vacation and did not hear audible mouth breathing but did notice that Susie seemed to toss a turn quite a bit during sleep. Susie had occasional sleep walking when younger but not in the last four years. Susie and her family are vegetarian and she doesn't usually take vitamins. Paternal family members have had ADHD and restless legs but no history of learning disability, anxiety or sleep disordered breathing. There were no significant physical exam findings.

Susie's sleep concerns raise a question about several sleep problems. One is delayed sleep phase syndrome, a common tendency among adolescents for the sleep-wake schedule to drift later. Susie's later sleep-wake cycle, when coupled with her school schedule, increases the risk for problems with sleep initiation and getting up in time for school. Her restlessness at bedtime and through the night, as well as the sensations at the lower extremities at bedtime suggest restless legs syndrome. She is at risk for iron deficiency, associated with RLS/PLMD, due to the less dietary iron, growth due to adolescence and losses due to early menarche. In addition, Susie's worries about doing well may add to her risk for insomnia. Altogether, Susie's duration and quality of sleep are likely disturbed and in turn, affecting daytime functioning through potentially direct effects related to RLS and indirectly through chronic sleep deprivation. Her daytime sleepiness is an indication that the sleep she is getting isn't enough. The parent has had a long-standing concern about some ADHD symptoms but the onset is later than typical.

To evaluate Susie further, a sleep log was obtained to assess sleep duration and the stability of sleep-wake pattern. Iron studies were obtained and showed a serum ferritin of 10, borderline low serum iron but no anemia. Conners rating scales from both parents and two teachers endorsed problems with attention and executive functioning but all other scales (including hyperactive-impulsive domains) were within normal limits for age and gender.

Susie was recommended to begin an iron supplement for the mild iron deficiency. In addition, Susie worked with the doctor to regularize her sleep-wake schedule between weekday and weekend. Susie also adjusted her daytime schedule so that she felt better able to meet her commitments for school, homework, practice times and friends. After three to four months, repeat iron studies showed correction of iron status and improved RLS symptoms. She was adhering to the new schedule and getting to sleep more easily. Conners rating scales and interview at follow up did not endorse ADHD concerns and teachers reported improved school performance. Taken together, Susie's presenting concerns seemed more likely related to sleep problems than ADHD at this time, but longer term follow up was recommended to assure she remained on track.

\section{Conclusion}

In this chapter, we have reviewed the relationship between ADHD and sleep disorders with the purpose of highlighting the importance of evaluating sleep problems when children and adolescents present for evaluation of ADHD. Studies have suggested that many 
practitioners do not routinely screen for sleep problems in general or for specific conditions like SDB in particular (Chervin, 2000; Chervin, 2001; Blunden, 2004). In one study in a general pediatric clinic, 830 parents of children ages two to thirteen years of age completed the Pediatric Sleep Questionnaire (PSQ), a validated survey of a number of medical and behavioral problems of sleep in children (Chervin, 2000). The parents of 86 children endorsed a sleep problem for their child and 44 of them (about $5 \%$ of the total number) endorsed symptoms of sleep disordered breathing. On a retrospective chart review of these children, less than $11 \%$ ( 5 of 44 ) had documentation that the symptoms were discussed (Chervin, 2001). Other studies have also called attention to the importance of screening about sleep problems in primary care settings (Blunden, 2004; Fallone, 2002).

\begin{tabular}{|c|c|c|c|}
\hline & $\begin{array}{l}\text { Toddler/preschool } \\
\text { (2-5 years) }\end{array}$ & $\begin{array}{l}\text { School-aged } \\
\text { (6-12 years) }\end{array}$ & $\begin{array}{l}\text { Adolescent } \\
\text { (13-18 years) }\end{array}$ \\
\hline $\begin{array}{l}\text { 1. Bedtime } \\
\text { problems }\end{array}$ & $\begin{array}{l}\text { Does your child have } \\
\text { any problems going to } \\
\text { bed? Falling asleep? }\end{array}$ & $\begin{array}{c}\text { Does your child have any } \\
\text { problems at bedtime? } \\
\text { (Parent) Do you have any } \\
\text { problems going to bed? } \\
\text { (Child) }\end{array}$ & $\begin{array}{c}\text { Do you have any } \\
\text { problems falling } \\
\text { asleep at bedtime? } \\
\text { (Child) }\end{array}$ \\
\hline $\begin{array}{l}\text { 2. Excessive } \\
\text { daytime } \\
\text { sleepiness }\end{array}$ & $\begin{array}{l}\text { Does your child seem } \\
\text { overtired or sleepy a lot } \\
\text { during the day? Does } \\
\text { she still take naps? }\end{array}$ & $\begin{array}{c}\text { Does your child have } \\
\text { difficulty waking in the } \\
\text { morning, seem sleepy } \\
\text { during the day or take } \\
\text { haps? (Parent) Do you feel } \\
\text { tired a lot? (Child) }\end{array}$ & $\begin{array}{l}\text { Do you feel sleep a } \\
\text { lot during the day? } \\
\text { In school? While } \\
\text { driving? (Child) }\end{array}$ \\
\hline $\begin{array}{l}\text { 3. Awakenings } \\
\text { during the } \\
\text { night }\end{array}$ & $\begin{array}{c}\text { Does your child wake } \\
\text { up a lot at night? }\end{array}$ & $\begin{array}{c}\text { Does your child seem to } \\
\text { wake up a lot at night? } \\
\text { Any sleepwalking or } \\
\text { nightmares? (Parent) Do } \\
\text { you wake up a lot at } \\
\text { night? Have trouble } \\
\text { getting back to sleep? } \\
\text { (Child) }\end{array}$ & $\begin{array}{l}\text { Do you wake up a } \\
\text { lot at night? Have } \\
\text { trouble getting back } \\
\text { to sleep? (Child) }\end{array}$ \\
\hline $\begin{array}{l}\text { 4. Regularity } \\
\text { and duration } \\
\text { of sleep }\end{array}$ & $\begin{array}{c}\text { Does your child have a } \\
\text { regular bedtime and } \\
\text { wake time? What are } \\
\text { they? }\end{array}$ & $\begin{array}{c}\text { What time does your child } \\
\text { go to bed and get up on } \\
\text { school days? Weekends? } \\
\text { Do you think he/she is } \\
\text { getting enough sleep? } \\
\text { (Parent) }\end{array}$ & $\begin{array}{l}\text { What time do you } \\
\text { usually go to bed on } \\
\text { school nights? } \\
\text { Weekends? How } \\
\text { much sleep do you } \\
\text { usually get? (Child) }\end{array}$ \\
\hline 5. Snoring & $\begin{array}{c}\text { Does your child snore a } \\
\text { lot or have difficulty } \\
\text { breathing at night? }\end{array}$ & $\begin{array}{c}\text { Does your child have loud } \\
\text { or nightly snoring or any } \\
\text { breathing difficulties at } \\
\text { night? (Parent) }\end{array}$ & $\begin{array}{l}\text { Does your teenager } \\
\text { snore loudly or } \\
\text { nightly? (Parent) }\end{array}$ \\
\hline
\end{tabular}

Source: Owens, J. and Dalzell, V. “Use of the 'BEARS' sleep screening tool in a pediatric continuity clinic: a pilot study." Sleep Medicine: 6 (2005) 63-69, used with permission

Table 1. BEARS Sleep Screening Algorithm 
A screening tool, the BEARS was developed by Owens et. al., and consists of five sleeprelated areas to screen at primary care visits. After implementing this instrument in a primary care setting, significantly more sleep issues were documented and sleep problems identified by the practitioners (Owens \& Dalzell, 2005).

The term “BEARS" stands for:

- $\quad$ B - Bedtime problems

- $\quad$ - Excessive daytime sleepiness

- A - Awakenings during the night

- $\quad \mathrm{R}$ - Regularity of sleep/wake cycles and average sleep duration

- $\quad$ S - Snoring

In conclusion, the relationship between ADHD and sleep disorders in children and adolescents can make evaluation of ADHD challenging. However, a methodical and thorough evaluation that recognizes expected and unexpected developmental features related to sleep will help to assure proper and timely identification and management of both ADHD and disorders of sleep.

\section{References}

2004 Sleep in America Poll Summary of Findings. 2004. Washington DC: National Sleep Foundation.

2006 Sleep in America Poll, Teens and Sleep, Summary of Findings. 2006. Washington DC: National Sleep Foundation.

Allen, R.P., Pichietti, D., Hening, W.A., et al. 2003. Restless legs syndrome: Diagnostic criteria, special considerations, and epidemiology. A report from the restless legs syndrome diagnosis and epidemiology workshop at the National Institutes of Health. Sleep Medicine 4:101-119.

Association, American Psychiatric. 2000. Diagnostic and statistical manual of mental disorders (4th ed., text rev.). 4 ed. Washington, DC.

Association", "American Psychiatric. 1987. Diagnostic and Statistical Manual of Mental Disorders, Third Edition, Revision. Washington DC: American Psychiatric Association.

Barkley, RA, McMurray MB, Edelbrock CS, Robbins K 1990. Side Effects of Metlyiphenidate in Children With Attention Deficit Hyperactivity Disorder: A Systemic, PlaceboControlled Evaluation. Pediatrics 86 (2):184-192.

Beebe, D. W. . 2005. Neurobehavioral effects of obstructive sleep apnea: an overview and heuristic model. Current Opinion in Pulmonary Medicine 11:494-500.

Beebe, D. W., Wells, C. T., Jeffries, J., Chini, B., Kalra, M., Amin, R. . 2004. Neuropsychological effects of pediatric obstructive sleep apnea. Journal of the International Neuropsychological Society 10:962-975.

Bendz, LM, Scates AC 2010. Melatonin Treatment for Insomnia in Pediatric Patients with Attention-Deficit/Hyperactivity Disorder. Ann Pharmacother 44 (1):185-191.

Biederman, J, Melmed RD, Patel A, McBurnett K, Konow J, Lyne A, Scherer N and for the SPD503 Study Group. 2008. A Randomized, Double-Blind, Placebo-Controlled Study of Guanfacine Extended Release in Children and Adolescents With Attention-Deficit/Hyperactivity Disorder. Pediatrics 121 (1):e73-e84. 
Blader JC, Koplewicz HS, Abikoff H, Foley C 1997. Sleep Problems of Elementary School Children: A Community Survey. Arch Pediatr Adolesc Med 151 (5):473-480.

Blumer JL, Findling RL, Shih WJ, Soubrane C, Reed, MD 2009. Controlled Clinical Trial of Zolpidem for the Treatment of Insomnia Associated With Attention-Deficit/ Hyperactivity Disorder in Children 6 to 17 Years of Age. Pediatrics 123 (5):e770e776.

Blunden, S. Lushington, K., Lorenzen, B., Ooi, T., Fung, F. Kennedy, D. . 2004. Are sleep problems under-recognised in general practice? Arch Dis Child 89:708-712.

Busby, K., Firestone, P., Pivik, R.T. 1981. Sleep Patterns in Hyperkinetic and Normal Children. Sleep 4 (4):366-383.

Chatoor, I., Wells, K., Conners, K., Seidel, W., Shaw, D. 1983. The Effects of Nocturnally Administered Stimulant Medication on EEG Sleep and Behavior in Hyperactive Children. Journal of American Academy of Child Psychiatry 22 (4):337-342.

Cheng J, Chen R, Ko J, Ng E 2007. Efficacy and safety of atomoxetine for attentiondeficit/hyperactivity disorder in children and adolescents-meta-analysis and meta-regression analysis. Psychopharmacology 194 (2):197-209.

Chervin, R. D., Archbold, K. H., Dillon, J. E., Panahi, P., Pituch, K. J., Dahl, R. E., Guilleminault, C. . 2002. Inattention, Hyperactivity, and Symptoms of SleepDisordered Breathing. Pediatrics 109 (3):449-456.

Chervin, R. D., Archbold, K. H., Panahi, P, Pituch, K. J. . 2001. Sleep Problems Seldom Addressed at Two General Pediatric Clinics. Pediatrics 107 (6):1375-1380.

Chervin, R. D., Dillon, J. E. Bassetti, C. Ganoczy, D. A., Pituch, K. J. . 1997. Symptoms of Sleep Disorders, Inattention, and Hyperactivity in Children. Sleep 20 (12):1185-1192.

Chervin, R. D., Hedger, K., Dillon, J. E. Pituch, K. J. . 2000. Pediatric Sleep Questionnaire (PSQ): validity and reliability of scales for sleep-disordered breathing, snoring, sleepiness, and behavioral problems. Sleep Medicine 1:21-32.

Chervin, R. D., Ruzicka, D. L., Archbold, K. H., Dillon, J. E. . 2005. Snoring Predicts Hyperactivity Four Years Later. Sleep 28 (7):885-890.

Chervin, R. D., Weatherly, R. A., Ruzicka, D. L., Burns, J. W., Giordani, B. J., Dillon, J. E., Marcus, C. L., Garetz, S. L., Hoban, T. F., Guire, K. E. 2006. Subjective sleepiness and polysomnographic correlates in children scheduled for adenotonsillectomy vs. other surgical care. Sleep 29 (4):495-503.

Chervin, R.D. 2005. Attention deficit, hyperactivity, and sleep disorders. In: Sheldon SH, Ferber R, Kryger Meir H, editors. Principals and Practice of Pediatric Sleep Medicine. 1 ed. Elsevier Saunders; . Principles and Practice of Pediatric Sleep Medicine: 161-169.

Committee on Quality Improvement, Subcommittee on Attention-Deficit/Hyperactivity Disorder. 2000. Clinical Practice Guideline: Diagnosis and Evaluation of the Child With Attention-Deficit/Hyperactivity Disorder. Pediatrics 105 (5):1158-1170.

Conners K, Sitarenios G, Parker JDA, Epstein JN 1998. The Revised Conners' Parent Rating Scale (CPRS-R): Factor Structure, Reliability, and Criterion Validity. Journal of Abnormal Child Psychology 26 (4):257-268.

Conners K, Sitarenios G, Parker JDA, Epstein JN 1998. Revision and Restandardization of the Conners Teacher Rating Scale (CTRS-R): Factor Structure, Reliability, and Criterion Validity. Journal of Abnormal Child Psychology 26 (4):279-291. 
Corkum, P., Tannock, R., Moldofsky, H., Hogg-Johnson, S., Humphries, T. 2001. Actigraphy and Parental Ratings of Sleep in Children with Attention-Deficit/Hyperactivity Disorder (ADHD). Sleep 24 (3):303-312.

Cortese, S., Faravone, S. V., Konofal, E., Lecendreux, M. 2009. Sleep in Children With Attention-Deficit/Hyperactivity Disorder: Meta-Analysis of Subjective and Objective Studies. American Academy of Child and Adolescent Psychiatry 48 (9):894908.

Curatolo P, Paloscia C, D'Agati E, Moavero R, Pasini A 2009. The neurobiology of attention deficit/hyperactivity disorder. European Journal of Paediatric Neurology 13 (4):299304.

Dahl RE 1996. The regulation of sleep and arousal: Development and psychopathology. Development and Psychopathology 8 (01):3-27.

Del Campo N, Chamberlain SR, Sahakian BJ, Robbins TW 2011. The Roles of Dopamine and Noradrenaline in the Pathophysiology and Treatment of AttentionDeficit/Hyperactivity Disorder. Biological Psychiatry 69 (12):e145-e157.

Dillon, J.E. Blunden, S. Ruzicka, D.L. Guire, K.E. Champine, D. Weatherly, R.A. Hodges, E.K. Giordani, B.J. Chervin, R.D. 2007. DSM-IV Diagnoses and Obstructive Sleep Apnea in Children Before and 1 Year After Adenotonsillectomy. American Academy of Child and Adolescent Psychiatry 46 (11):1425-1436.

Durston, S. Tottenham, N. Thomas K.M. Davidson, M.C. Eigsti, I. Yang, Y. Ulug, A.M. Casey, B.J. 2003. Differential Patterns of Striatal Activation in Young Children with and without ADHD. Society of Biological Psychiatry 53:871-878.

Espana, RA; Scammell TE. 2004. Sleep neurobiology for the clinician. In Sleep

Faber A, Kalverkijk LJ, de Jong-van den Berg LTW, Hugtenburg JG, Minderaa RB, Tobi H 2006. Parents Report on Stimulant-Treated Children in the Netherlands: Initiation of Treatment and Follow-Up Care. Journal of Child and Adolescent Psychopharmacology 16 (4):432-440.

Fallone, G., Owens, J. A., Deane, J. . 2002. Sleepiness in children and adolescents: clinical implications. Sleep Medicine Reviews 6 (4):287-306.

Faraone, S. V., Biederman, J. . 1998. Neurobiology of Attention-Deficit Hyperactivity Disorder. Society of Biological Psychiatry 44:951-958.

Faraone, S. V., Perlis, R. H., Doyle, A. E., Smoller, J. W., Goralnick, J. J., Holmgren, M. A., Sklar, P. . 2005. Molecular Genetics of Attention-Deficit/Hyperactivity Disorder. Society of Biological Psychiatry 57:1313-1323.

Fuller PM, Gooley JJ, Saper CB 2006. Neurobiology of the sleep-wake cycle: sleep architecture, circadian regulation, and regulatory feedback. Journal of Biological Rhythms 21 (6):482(12).

Galland BC, Tripp EG, Taylor BJ 2010. The sleep of children with attention deficit hyperactivity disorder on and off methylphenidate: a matched case-control study. Journal of Sleep Research 19 (2):366-373.

Garcia-Borreguero, D., Egatz, R., Winkelmann, J., Berger, K. 2006. Epidemiology of restless legs syndrome: The current status. Sleep Medicine Reviews 10:153-167.

Giblin JM, Strobel AL 2010. Effect of Lisdexamfetamine Dimesylate on Sleep in Children With ADHD. Journal of Attention Disorders.

Giordani, B., Hodges, E. K., Guire, K. E., Ruzicka, D. L., Dillon, J. E., Weatherly, R. A., Garetz, S. L., Chervin, R. D. 2008. Neuropsychological and behavioral functioning 
in children with and without obstructive sleep apnea referred for tonsillectomy. Journal of the International Neuropsychological Society 14:571-581.

Golan N, Shahar E, Ravid S, Pillar G 2004. Sleep disorders and daytime sleepiness in children with attention-deficit/hyperactive disorder. sleep 27 (2):261-6.

Gonon F 2009. The dopaminergic hypothesis of attention-deficit/hyperactivity disorder needs re-examining. Trends in Neurosciences 32 (1):2-8.

Goraya JS, Cruz M, Valencia I, Kaleyias J, Khurana DS, Hardison HH, Marks H, Legido A, Kothare SV 2009. Sleep Study Abnormalities in Children With Attention Deficit Hyperactivity Disorder. Pediatric Neurology 40 (1):42-46.

Gottlieb, D. J., Chase, C., Vezina, R. M., Heeren, T. C., Corwin, M. J., Auerbach, S. H., WeeseMayer, D. E., Lesko, S. M. 2004. Sleep-disordered breathing symptoms are associated with poorer cognitive function in 5-year-old children. Journal of Pediatrics 145:458-464.

Gozal, D., Wang, M., Pope Jr, D.W. 2001. Objective sleepiness measures in pediatric obstructive sleep apnea. Pediatrics 108 (3):693-697.

Gruber R, Xi T, Frenette S, Rober M, Vannasinh P, Carrier J 2009. Sleep Distrubances in Prepubertal Children with Attention Deficit Hyperactivity Disorder: A Home Polysomnography Study. Sleep 32 (3):343-350.

Guilleminault C, Korobkin R, Winkle R. . 1981. A review of 50 children with obstructive sleep apnea syndrome. . Lung 159:275-287.

Hoban T. 2003. Rhythmic Movement Disorder in Children. CNS Spectrums 8(2): 135-138.

Hodges, E., Felt, B., Giordani, B., Chervin, R. 2011. Sleep Disordered Breathing in Children: A Clinical Guide, Chapter: Behavioral Issues. 2011 (In Press)

Hodges, E.K., Bloomfied, E., Coulas, T., Giordani, B. 2008. Cognitive and behavioral change after adenotonsillectomy in children with sleep-disordered breathing: A review. Minerva Psichiatrica 49 (4):307-320.

Hoebert M, Van Der Heijden KB, Ban Geijlswijk IM, Smits MG 2009. Long-term follow-up of melatonin treatment in children with ADHD and chronic sleep onset insomnia. Journal of Pineal Research 47 (1):1-7.

Iglowstein I, Jenni OG, Molinari L, Largo RH 2003. Sleep Duration From Infancy to Adolescence: Reference Values and Generational Trends. Pediatrics 111 (2):302-307.

Kent JD, Blader JC, Koplewicz HS, Abikoff H, Foley CA 1995. Effects of Late-Afternoon Methylphenidate Administration on Behavior and Sleep in Attention-Deficit Hyperactivity Disorder. Pediatrics 96 (2):320-325.

Khan, AU. 1982. Sleep REM latency in hyperkinetic boys. Am J Psychiatry 139 (10):1358-1360.

Kim HW, Yoon, IY, Cho SC, Kim BN, Chung S, Lee H, Kim CW, Park SK, Yoo HJ 2010. The effect of OROS methylphenidate on the sleep of children with attentiondeficit/hyperactivity disorder. International Clinical Psychopharmacology 25:107-115.

Kirov R, Kinkelbur J, Heipke S, Kostanecka-Endress T, Westoff M, Cohrs S, Ruther E, Hajak G, Banaschewski T, Rothenberger A 2004. Is there a specific polysomnographic sleep pattern in children with attention deficit/hyperactivity disorder? Journal of Sleep Research 13 (1):87-93.

Kollins SH, Jain R, Brams M, Segal S, Findling RL, Wigal SB, Khayrallah M 2011. Clonidine Extended-Release Tablets as Add-on Therapy to Psychostimulants in Children and Adolescents With ADHD. Pediatrics 127 (6):e1406-e1413. 
Konofal, E., Cortese, S., Marchand, M., Arnulf, I., Lecendreux, M. 2007. Impact of restless legs syndrome and iron deficiency on attention-deficit/hyperactivity disorder in children. Sleep Medicine 8:711-715.

Konrad K, Eickhoff SB 2010. Is the ADHD brain wired differently? A review on structural and functional connectivity in attention deficit hyperactivity disorder. Human Brain Mapping 31 (6):904-916.

Kratochvil CJ, Vaughan BS, Stoner JA, Daughton JM, Lubberstedt BD, Murray DW, Chrisman AK, Faircloth MA, Itchon-Ramos NB, Kollins SH, Maayan LA, Greenhill LL, Kotler LA, Fried J, March JS 2011. A Double-Blind, Placebo-Controlled Study of Atomoxetine in Young Children With ADHD. Pediatrics 127 (4):e862-e868.

Lu B, Zee P. 2010. Neurobiology of Sleep. Clinical Chest Medicine 31:309-318.

Makris, N., J. Biederman, M. C. Monuteaux, and L. J. Seidman. 2009. Towards Conceptualizing a Neural Systems-Based Anatomy of Attention-Deficit/Hyperactivity Disorder. Developmental Neuroscience 31 (1-2):36-49.

Mayes, S. D., Calhoun, S. L., Bixler, E. O., Vgontzas, A. N., Mahr, F., Hillwig-Garcia, J., Elamir, B., Edhere-Ekezie, L., Parvin, M. 2009. ADHD Subtypes and Comorbid Anxiety, Depression, and Oppositional-Defiant Disorder: Differences in Sleep Problems. Journal of Pediatric Psychology 34 (3):328-337.

Mick, E., Biederman, J., Jetton, J., Faraone, S. V. . 2000. Sleep Disturbances Associated with Attention Deficit Hyperactivity Disorder: The Impact of Psychiatric Comorbidity and Pharmacotherapy. Journal of Child and Adolescent Psychopharmacology 10 (3):223231.

Mindell JA, Owens JA 2010. A Clinical Guide to Pediatric Sleep: Diagnosis and Management of Sleep Problems. Edited by S. Seigafuse. Second ed. Philadelphia: Lippincott Williams \& Wilkins.

Moore M, Meltzer LJ 2008. The sleepy adolescent: causes and consequences of sleepiness in teens. Paediatric Respiratory Reviews 9 (2):114-121.

Newcorn JH, Kratochvil CJ, Allen AJ, Casat CD, Ruff DD, Moore RJ, Michelson D, and Atomoxetine/Methylphenidate Comparative Study Group. 2008. Atomoxetine and Osmotically Released Methylphenidate for the Treatment of Attention Deficit Hyperactivity Disorder: Acute Comparison and Differential Response. Am J Psychiatry 165 (6):721-730.

Nigg, J. T. 2005. Neuropsychologic Theory and Findings in Attention-Deficit/ Hyperactivity Disorder: The State of the Field and Salient Challenges for the Coming Decade. Society of Biological Psychiatry 57:1424-1435.

O'Brien, L. M., Gozal, D. 2004. Neurocognitive dysfunction and sleep in children: from human to rodent. The Pediatric Clinics of North America 51:187-202.

O'Brien, L. M., Holbrook, C. R., Mervis, C. B., Klaus, C. J., Bruner, J. L., Raffield, T. J., Rutherford, J., Mehl, R. C., Wang, M., Tuell, A., Hume, B. C., Gozal, D. 2003. Sleep and Neurobehavioral Characteristics of 5- to 7-Year-Old Children With Parentally Reported Symptoms of

Attention-Deficit/Hyperactivity Disorder. Pediatrics 111 (3):554-563. O'Brien, L. M., Icanenko, A., Crabtree, V. M., Holbrook, C. R., Bruner, J. L., Klaus, C. J., Gozal, D. . 2003. Sleep Disturbances in Children with Attention Deficit Hyperactivity Disorder. Pediatric Research 54 (2):237-243. 
O’Brien, L. M., Tauman, R., Gozal, D. 2004. Sleep Pressure Correlates of Cognitive and Behavioral Morbidity in Snoring Children. Sleep 27 (2):279-282.

Oner, P., Dirik, E.B., Taner, Y., et al. 2007. Association between low serum ferritin and restless legs syndrome in patients with attention deficit hyperactivity disorder. Tohoku J Exp Med 213:269-276.

Owens, J. A., and V. Dalzell. 2005. Use of the 'BEARS' sleep screening tool in a pediatric residents' continuity clinic: a pilot study. Sleep Med 6 (1):63-9.

Owens, J. A., Maxim, R., Nobile, C., McGuinn, M., Msall, M. 2000. Parental and Self-report of Sleep in Children With Attention-Deficit/Hyperactivity Disorder. Arch Pediatr Adolesc Med. 154:549-555.

Owens, J. A., Spirito, A., McGuinn, M., Nobile, C. . 2000. Sleep Habits and Sleep Distrubance in Elementary School-Aged Children Developmental and Behavioral Pediatrics 21 (1):27-37.

Owens, J.A. 2005. The ADHD and Sleep Conundrum: A Review. Developmental and Behavioral Pediatrics 6 (4):312-322.

Owens, J.A. 2008. Classification and Epidemiology of Childhood Sleep Disorders. Primary Care: Clinics in Office Practice 35:533-546.

Pace-Schott E.F., Edward F., and Hobson A. 2002. The Neurobiology of Sleep: Genetics, cellular physiology and subcortical networks. Nat Rev Neurosci 3 (8):591-605.

Picchietti, D. L., England, S. J., Walters, A. S., Willis, K., Verrico, T. . 1998. Periodic Limb Movement Disorder and Restless Legs Syndrome in Children With Attentiondeficit Hyperactivity Disorder. Journal of Child Neurology 13:588-594.

Picchietti, M. A., Picchietti, D. L. 2008. Restless Legs Syndrome and Periodic Limb Movement Disorder in Children and Adolescents. Seminars in Pediatric Neurology 15:91-99.

Pichietti, D.L., et al. 2007. Restless legs syndrome: prevalence and impact in children and adolescents - the Peds REST study. Pediatrics 120:253-266.

Plizka S and AACAP Work Group on Quality Issues. 2007. Practice Parameter for the Assessment and Treatment of Children and Adolescents with AttentionDeficit/Hyperactivity Disorder. Journal of American Academy of Child and Adolescent Psychiatry 46 (7):894-921.

Rajaram, S., Walters, A.S., England, S.J., Mehta, D., Nizam, F. 2004. Some children with growing pains may actually have restless legs syndrome. Sleep 27:767-773.

Reid, G.J., Hong, R.Y., Wade, T.J. 2009. The relation between common sleep problems and emotional and behavioral problems among 2- and 3-year-olds in the context of known risk factors for psychopathology. Journal of Sleep Research 18:49-59.

Rosa-Neto P, Lou HC, Cumming P, Pryds O, Karrebaek H, Lunding J, Gjedde A. 2005. Methylphenidate-evoked changes in striatal dopamine correlate with inattention and impulsivity in adolescents with attention deficit hyperactivity disorder. NeuroImage 25 (3):868-876.

Rubia, K., R. Halari, A. M. Mohammad, E. Taylor, and M. Brammer. 2011. Methylphenidate normalizes frontocingulate underactivation during error processing in attentiondeficit/hyperactivity disorder. Biol Psychiatry 70 (3):255-62.

Sadeh, A., Gruber, R., Raviv, A. 2002. Sleep, Neurobehavioral Functioning, and Behavior Problems in School-Age Children. Child Development 73 (2):405-417. 
Sangal. R.B., Sangal, J.M. 2004. Rating scales for inattention and sleepiness are correlated in adults with symptoms of sleep disordered breathing syndrome, but not in adults with symptoms of attention-deficit/hyperactivity disorder. Sleep Medicine 5:133135.

Shur-Fen G, S. 2006. Prevalence of sleep problems and their association with inattention/hyperactivity among children aged 6-15 in Taiwan. Journal of Sleep Research 15:403-414.

Silvestri R, Gagliano A, Arico I, Calarese T, Cedro C, Bruni O, Condurso R, Germano E, Gervasi G, Siracusano R, Vita G, Bramani P 2009. Sleep disorders in children with Attention-Deficit/Hyperactivity Disorder (ADHD) recorded overnight by videopolysomnography. Sleep Medicine 10 (10):1132-1138.

Simonds, J.F., Parraga, H. 1984. Sleep Behaviors and Disorders in Children and Adolescents Evaluated at Psychiatric Clinics. Developmental and Behavioral Pediatrics 5 (1):6-10.

Stein, M.A., Mendelsohn, J., Obermeyer, W.H., Amromin, J., Benca, R. 2001. Sleep and Behavior Problems in School-Aged Children. Pediatrics 107 (4).

Swanson, J.M., Flodman, P., Kennedy, J., Spence, M.A., Moyzis, R., Schucka, S., Murias, M., Moriarity, J., Barr, C., Smith, M., Posner, M. 2000. Dopamine genes and ADHD. Neuroscience and Biobehavioral Reviews 25:21-25.

Tripp, G., Wickens, J.R. 2009. Neurobiology of ADHD. Neuropharmacology 57:579-589.

Van Der Heijden KB, Smits MG, Gunning WB. 2006. Sleep hygiene and actigraphically evaluated sleep characteristics in children with ADHD and chronic sleep onset insomnia. Journal of Sleep Research 15 (1):55-62.

Walters, A.S., Pichietti, D.L., Ehrenberg, B.L., Wagner, M.L. 1994. Restless legs syndrome in childhood and adolescence. Pediatric Neurology 11:241-245.

Walters, A.S., Silvestri, R., Zucconi, M., Chandrashedariah, R., Konofal, E. 2008. Review of the possibile relationship and hypothetical links between attention deficit hyperactivity disorder (ADHD) and the simple sleep related movement disorders, parasomnias, hypersomnias, and circadian rhythm disorders. Journal of Clinical Sleep Medicine 4:591-600.

Zimmer L 2009. Positron emission tomography neuroimaging for a better understanding of the biology of ADHD. Neuropharmacology 57 (7-8):601-607. 


\section{Part 2}

\section{Psychopharmacology: Mechanisms and Effects}





\title{
The Neuropsychopharmacology of Stimulants: Dopamine and ADHD
}

\author{
Paul E.A. Glaser and Greg A. Gerhardt \\ University of Kentucky \\ USA
}

\section{Introduction}

In this chapter we consider the neuropsychopharmacology of ADHD in general and dopamine and the stimulants more specifically. Attention will be given to the various neurotransmitter theories for ADHD. We will consider the theoretical mechanisms of actions for the various medicines used to treat ADHD. We will look at how the stimulants, although often assumed to be similar, actually show evidence of differential mechanisms of action. We will look at new data that utilizes the technique of reverse microdialysis to demonstrate how different the dose-response curves are for dopamine release in the striatum following local application of the different stimulants.

Throughout the text we will use ADHD (Attention-Deficit/Hyperactivity Disorder) without reference to the DSM-IV type, unless a specific reference pertains to combined, inattentive or hyperactive subtypes.

\section{Neuropsychopharmacology of stimulants}

The stimulant medications were discovered serendipitously with the indirect observation that amphetamines calmed and focused children who were given the medicine to try to treat headache that was caused by the technique of pneumoencephalography, a largely outdated procedure where the spinal fluid was drained and replaced with air in order to see the brain more clearly on X-ray (Bradley, 1937, Strohl, 2011). The form of amphetamine used by Bradley was Benzedrine, the racemic mixture, or 50/50 mixture of $d$ - and l-amphetamine. Because of research pointing to the dopamine releasing qualities of the stimulants, the earliest theory for ADHD was that it represented a hypodopaminergic state. This hypodopaminergic state theoretically led to alterations in reward sensitivity if it was in the nucleus accumbens, hyperactivity if lowered dopamine was in the striatum, and decreased inhibitory control if the lowered dopamine was in the frontal cortex. Although the collective data never supported such clean demarcations in brain structure and dependence solely on dopamine, the "hypodopaminergic" theory of ADHD is still a popular teaching in the clinical setting.

\subsection{Dopamine and ADHD}

Dopamine was not always considered a neurotransmitter. As details about the neurotransmitters were emerging dopamine was noted as the penultimate molecule in the 
synthesis of norepinephrine. The concept emerged of the monoamines being packaged into discrete vesicles that could be released when an action potential brought on an influx of calcium. Dopamine was transported into these synaptic vesicles by VMAT (Vesicular Monoamine Transporter). Then the enzyme Dopamine $\beta$-Hydroxylase inside the vesicle converted the dopamine to norepinephrine. Work by Carlsson and others in the 1950s showed that some regions of the brain, particularly the basal ganglia that includes the striatum and nucleus accumbens, were enriched in dopamine and had very little norepinephrine (Cooper et al., 2003). Following these discoveries, dopamine's importance in coordinating motor control, Parkinson's Disease, and reward were established. It was found that following release of dopamine from presynaptic vesicles that dopamine had specific receptors postsynaptically that could modulate the neurons function (both stimulatory and inhibitory modulation depending on the dopamine receptors and second messenger systems). Dopamine receptors were also found presynaptically and thought to allow for feedback mechanisms for precise regulation of dopamine release. Finally, dopamine's effects were terminated both through reuptake into the presynaptic cytoplasm by the dopamine transporter (DAT), and by metabolism either inside the neuron by MAO (monoamine oxidase) or extracellularly by COMT (catechol O-methyl transferase) (see Figure 1).

As discoveries about dopamine were evolving, stimulants were being used for many purposes in the mid to late 20th century. Bradley's observations on amphetamine's benefit for children with features of ADHD went largely ignored for several decades. The stimulants found use for their ability to keep people awake despite fatigue. Several militaries in World War Two used both amphetamine and methamphetamine for this purpose, although it was soon found that soldiers would "crash" following this use and need time to recover. Tolerance was also noted with increasing doses needed for effects such as euphoria. Abuse was reported for several decades before the FDA banned Benzedrine inhalers and limited amphetamines to prescription use only in 1959. Researchers in the 1970s and 1980s connected and clarified the stimulants function in increasing dopamine in the synaptic cleft, as well as its connection to treating ADHD and the role of both tonic and phasic levels of dopamine (Robbins \& Sahakian, 1979). Perhaps due to the ease of measurement and abudance of dopamine in the striatum and nucleus accumbens, dopamine research predominated over norepinephrine. In truth, amphetamines exert most of its CNS effects through dopamine and norephinephrine, with very little effects on serotonin. Methylphenidate is strongest at blocking dopamine and much less so norepinephrine, and even less so for serotonin (Gatley et al. 1996). Finally cocaine and methamphetamine seem to affect all three neurotransmitters, with their effect on serotonin theoretically leading to the greater euphoria. When this serotonin function is coupled to the reward function of dopamine release in the nucleus accumbens, it theoretically makes methamphetamine and cocaine have greater overall abuse potential compared to amphetamine and methylphenidate. To this day, stimulants are approved for use in ADHD, narcolepsy, and severe obesity; but with strict control by the FDA and other governmental agencies around the world.

\subsection{Other Neurotransmitters and ADHD}

As more intricacies have been revealed through animal models of ADHD and human research, other neurotransmitters have been implicated in ADHD. Perhaps the strongest case can be made for norepinephrine. Arnsten and colleagues have suggested that 
norepinephrine is as important as dopamine in attention and ADHD. Recent elegant work in non-human primates suggest that alpha-2 adrenergic input in the frontal cortex is critical in maintaining working memory in a visual attention task constructed by Arnsten's group (Wang et al., 2007). Interestingly, dopamine-1 receptor input is needed in the areas surrounding the circuitry of working memory to suppress areas of the frontal cortex that were not needed for that specific memory. One might say that norepinephrine was allowing for saliency and attention, and dopamine for signal-to-noise adjustment or inhibition of inappropriate information (Gamo et al., 2010).

Initially one might think that atomoxetine lends credence to just the norepinephrine theories of ADHD in that it is a NET (norepinephrine transporter) inhibitor. But research has shown that the NET transports dopamine as well as NE. Thus atomoxetine raises NE and DA in the prefrontal cortex. Since NET is primarily present in the frontal cortex and not the nucleus accumbens or striatum, the neurotransmitter modulating effects of atomoxetine are only in the frontal cortex. This accounts for its lack of abusability, and perhaps the fact that atomoxetine overall is a less efficacious medicine for ADHD compared to amphetamine and methylphenidate (Lile et al., 2006). The stimulants in blocking DAT (dopamine transporter) also create increases in both DA and NE, since like NET, DAT transports both DA and NE.

Other neurotransmitters implicated in ADHD include acetylcholine, histamine, adenosine receptors, and glutamate. Nicotinic receptors are involved in various tasks requiring attention and this has led to the speculation that the high rate of smoking seen in people with ADHD may be due in part to "self-medication". Although most nicotinic medications have targeted Alzheimer's, there use in memory may prove beneficial to ADHD. Several histamine-3-receptor antagonists are in the stages of being tested for ADHD and other cognitive disorder (Sander et al., 2008). Only a few studies have been reported thus far and their results using these histamine modulating drugs for ADHD have been mixed (Brioni et al., 2011). Caffeine, an adenosine receptor antagonist, can improve symptoms of ADHD in some animal models perhaps through interactions of adenosine receptors and dopamine systems. Caffeine is poorly studied in ADHD but appears to help alertness more than actual symptoms of ADHD (Smith 2002). Glutamate has recently been implicated from both neuroimaging and neuroscience. One open labeled trial has shown that glutamate modulating drugs, such as NMDA antagonist memantine shows some efficacy in treating ADHD (Findling et al., 2007). Interestingly a recent patch clamp study suggests that atomoxetine is also an NMDA antagonist at clinical levels (Ludolph et al., 2010).

\subsection{Heterogeneity amongst the stimulants}

Returning to the dopamine mechanisms of action involved in ADHD let us now focus on how the separate stimulants used in treating ADHD are different from each other. Methylphenidate has been shown to have a mechanism of action similar to cocaine in that it specifically blocks DAT (see Figure 1). D-amphetamine (the dextro-isomer of amphetamine) has been shown to have three potential mechanisms. The first is direct effect on the DAT by allowing reverse transport of DA from the cytoplasm presynaptically into the synapse, this is a calcium-independent DA release that is perhaps coupled to overall decrease in DA uptake. Secondly d-amphetamine inhibits MAO-B (Monoamine oxidase-B isoform) which catabolizes DA. Thirdly, d-amphetamine inhibits VMAT (vesicular monoamine transporter) leading to an increase in cytoplasmic DA that can be reverse transported out by DAT (see 
Figure 1) (Bergman et al. 1989; Cadoni et al. 1995). Although it is not known which of these three mechanisms is the most important of note is that all three are different than methylphenidate. This agrees with the clinically observed phenomena that responses to methylphenidate and d-amphetamine are not always equal in patients. Thus, if a patient is not doing well on one stimulant, say methylphenidate, then it is the recommended standard of care to then try an amphetamine preparation. Sonders et al. (1997) categorized pharmacological agents that act on the human dopamine transporter (hDAT) into two groups: substrates for DAT (including dopamine and amphetamine) and cocaine-like (including cocaine and methylphenidate). Thus, amphetamine can actually serve as a substrate for DAT, like dopamine itself; whereas methylphenidate is not a substrate for DAT.

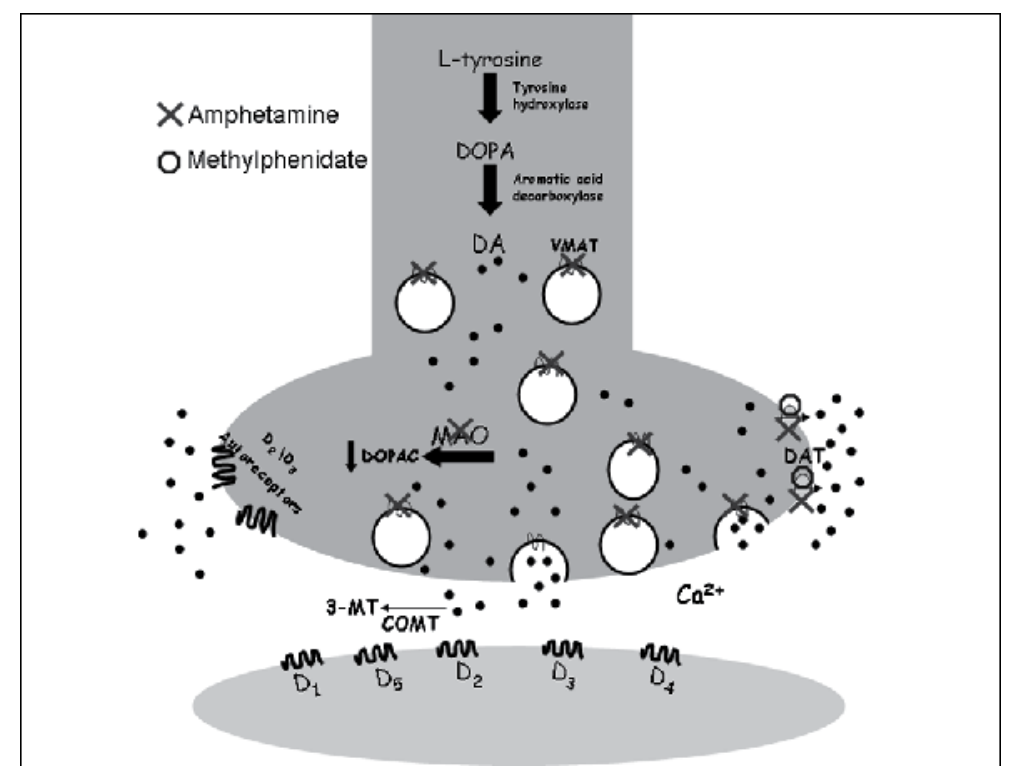

Fig. 1. Simplified Model of Dopamine synapse with putative mechanisms of action for amphetamine and metylphenidate.

But are all amphetamine preparations equivalent? What about the preparations such as Adderall that have some l-amphetamine (the opposite stereo isomer of d-amphetamine). In the 1990s the drug Adderall was introduced and marketed as a robust treatment for the symptoms of ADHD compared to other medications (Popper 1994; Patrick et al. 1997). One clinical study compared Adderall to D-amphetamine and found that Adderall decreased specific symptoms of hyperactivity slightly faster and over a longer time period than Damphetamine (James et al. 2001), but this was a minor difference. Other clinical trials support that Adderall is more effective than immediate-release methylphenidate on outcomes measured 4 to 5 hours after dosing (Pelham et al. 1999). A majority of data supports that population comparison of efficacy for stimulants in treating ADHD show little difference. It is only when you get to the individual patient that you find differences in the stimulants. For example, l-amphetamine alone has been tested and shown in a smaller study to be useful for some patients with ADHD, even a few which did not respond as well to damphetamine (Segal 1974). More recent comparison of controlled-release preparations of 
amphetamines and methylphenidate show little differences in overall efficacy. Previous in vivo voltammetry data in our laboratory showed differences in kinetics between amphetamine optical isomers (Glaser et al. 2005). In these studies, preparations with Lamphetamine evoked faster DA rise times and signal decay times compared to Damphetamine. Additionally, data collected by our group showed greater amplitudes and longer DA response signal kinetics following local applications of Adderall in comparison with D-amphetamine and D,L-amphetamine (Joyce et al. 2007) supporting different mechanistic effects of these drugs on DA release.

\subsection{Reverse microdialysis of stimulants in the rat striatum: hypothesis}

When comparing different stimulant medications and their effects on dopamine levels, several caveats have limited direct comparison. First of all, stimulants are often given by intraperitoneal injection, due in part to its ease and the fact that the rapid rise in blood levels makes dopamine easier to measure in brain regions. However variability in absorption and first pass effects of the liver make it difficult to compare concentrations between medications. Gavage or oral delivery of food, while simulating the clinical experience for $\mathrm{ADHD}$, has even more pharmacokinetic factors involved due to gut absorption factors as well. Finally, many injection and oral stimulant studies have to use larger, more abuse related dosing, because there are often little appreciable changes in dopamine at drug dosing similar to that used in ADHD, although a few studies have been able to accomplish this (Berridge et al, 2006). In order to circumvent some of these caveats, and yet still look at the in vivo effects of these drugs and their differences on striatum, we chose the technique of reverse microdialysis. This technology places the medication in the dialysate that goes directly to the striatum and allows for direct and sensitive dose-response curves for stimulant-evoked dopamine.

The technique of reverse microdialysis coupled with high performance liquid chromatography with electrochemical detection (HPLC-EC) was used to study local drugevoked increases in extracellular dopamine (DA) levels and changes in DA metabolites in the striatum of anesthetized rats. Purdom et al. (2003) showed data supporting that the order of administration of different concentrations of D-amphetamine significantly affected DA and DOPAC levels. These results were likely attributable to changes in the surface expression of DAT on DA nerve endings and/or DAT function. Other in vitro studies have shown substrate dependent trafficking of the DAT to and from the plasma membrane and subsequent changes in the ability to transport DA (Kahlig et al. 2005; Johnson et al. 2005; Saunders et al. 2000; Kahlig et al. 2004; Kahlig and Galli 2003). Therefore to have the most accurate dose-response curves the same animal should not be used to test several doses. For these experiments drug-naïve animals were used to circumvent issues regarding DAT trafficking and/or change in function following substrate exposure (Kahlig and Galli 2003; Kahlig et al. 2004; Purdom et al. 2003). We tested the hypothesis that stimulant concentration-response curves of DA and its metabolites will display differential patterns of DA overflow that correlate with their mechanistic properties at the level of DAT function. In addition, we tested a unique formulation of $25 \% \mathrm{D}$ - and $75 \% \mathrm{~L}$-amphetamine and termed this mixture "Reverse Adderall", to contrast it with Adderall that is $\sim 75 \% \mathrm{D}$ - and $25 \% \mathrm{~L}$ amphetamine. We hypothesized that the Reverse Adderall would also have a differential dose-response curve than the other amphetamine preparations. 


\subsection{Reverse microdialysis of stimulants in the rat striatum: methods}

Male Fischer 344 (F344) rats (3-6 months old) were anesthetized with urethane $(1.25 \mathrm{~g} / \mathrm{kg}$ i.p. in $0.9 \%$ saline). After placement into a stereotaxic frame (Kopf, Tujunga, CA, USA) with the incisor bar set at $-2.3 \mathrm{~mm}$, the rat striatum was prepared for study. Body temperature was maintained by use of an isothermal heating pad (Braintree Scientific, Braintree, MA, USA) at $37^{\circ} \mathrm{C}$ and periodically monitored by a rectal thermometer. After the retraction of the skin and tissue and exposure of the skull overlying the striatum, a small craniotomy $(2 \mathrm{x}$ $2 \mathrm{~mm}$ ) was carried out in the right hemisphere. The microdialysis probes were stereotactically placed with respect to bregma: $+1.0 \mathrm{~mm} \mathrm{AP}, \pm 2.2 \mathrm{~mm} \mathrm{ML}, \mathrm{DV}-6.0 \mathrm{~mm}$ ) (Paxinos and Watson, 1986). The 2-mm length membrane probes (CMA/11, CMA Microdialysis, Stockholm, Sweden) remained at this location for the duration of the experiment. All procedures were performed in accordance with the National Institutes of Health Guidelines for the Care and use of Mammals in Neuroscience and Behavioral Research (2003) and were approved by the Animal Care and Use Committee of the University of Kentucky.

Fluid flow through the microdialysis probes was achieved using a syringe pump (KDS230, KD Scientific, Holliston, MA) fitted with $1 \mathrm{ml}$ gastight syringes (1001 LTN, Hamilton USA, Reno, NV) containing dialyzing fluid. Dialysis probes were perfused at a flow rate of 1 $\mu \mathrm{l} / \mathrm{min}$. Syringes were connected to a liquid switch (CMA/110, CMA Microdialysis, Stockholm, Sweden) that allowed for alternation between treatments: artificial cerebral spinal fluid (aCSF) (in mM: $\mathrm{NaCl} 123, \mathrm{KCl} 3, \mathrm{CaCl} 2$ 1, MgCl2 1, NaHCO3 25, NaH2PO4 1 , and glucose 5.9) and aCSF + [drug]. Teflon tubing (FEP tubing, $0.12 \mathrm{~mm}$ i.d.) and tubing adapters (CMA Microdialysis, Stockholm, Sweden) were used to establish all connections. Samples were collected at twenty minute intervals into a $0.2 \mathrm{ml}$ microcentrifuge tube and manually injected into an HPLC-EC system. The order of administration for each of the drug solutions tested was as follows: samples 1-6 (0-120 minutes, aCSF), sample 7 (120-140 minutes, aCSF + stimulant drug solution), samples 8-12 (160-240 minutes, aCSF).

Probe recoveries were collected using a standard solution with known concentrations of DA, norepinephrine (NE), serotonin (5-HT), 3,4-dihydroxphenylacetic acid (DOPAC), homovanillic Acid (HVA) and 5-hydroxyindoleacetic acid (5-HIAA). In order for a probe to be used in these studies, in vitro recoveries of $10 \% \pm 1$ were required. Based on this exchange rate, seen for molecules similar in size to amphetamine such as DA, NE, and 5-HT, we were able to more accurately adjust the effective concentrations of stimulant drugs being studied.

Stimulant concentrations used for reverse microdialysis studies were chosen to represent a range that included clinically relevant levels and abuse levels that were normalized for the amount of D-amphetamine. Our prior studies support that D-amphetamine determines the amount of DA released in the presence of both enantiomers (Glaser et al. 2005). The following concentrations were used based on $\sim 10 \%$ exchange rate for the microdialysis probes: for D-amphetamine and methylphenidate, $0.1 \mu \mathrm{M}, 0.5 \mu \mathrm{M}, 1 \mu \mathrm{M}, 5 \mu \mathrm{M}, 10 \mu \mathrm{M}, 25$ $\mu \mathrm{M}, 50 \mu \mathrm{M}, 100 \mu \mathrm{M}, 400 \mu \mathrm{M}$ were studied; for Reverse Adderall, $0.1 \mu \mathrm{M}, 0.5 \mu \mathrm{M}, 1 \mu \mathrm{M}, 5 \mu \mathrm{M}$, $10 \mu \mathrm{M}, 25 \mu \mathrm{M}, 50 \mu \mathrm{M}, 100 \mu \mathrm{M}, 400 \mu \mathrm{M}$ and $533 \mu \mathrm{M}$; ; and for Adderall , $0.1 \mu \mathrm{M}, 0.5 \mu \mathrm{M}, 1$ $\mu \mathrm{M}, 5 \mu \mathrm{M}, 10 \mu \mathrm{M}, 25 \mu \mathrm{M}, 50 \mu \mathrm{M}, 100 \mu \mathrm{M}, 400 \mu \mathrm{M}$ and $539 \mu \mathrm{M}$ solutions were studied . For D,L-amphetamine (normalized to D-amphetamine/2), L-amphetamine, and cocaine, only a concentration of $400 \mu \mathrm{M}$ was tested for maximum effect comparisons. Prior to each 
experiment, $20 \mathrm{mM}$ ascorbic acid was added to each solution and solutions were aerated with $95 \% \mathrm{O} 2 / 5 \% \mathrm{CO}$. Solutions were immediately added to individual $1 \mathrm{ml}$ gastight syringes. Following each experiment, rats were intracardially perfused with $0.9 \% \mathrm{NaCl}$ solution followed by a $4 \%$ paraformaldehyde solution. They were then decapitated, and their brains were frozen, sliced on a cryostat, and sectioned stained with cresyl violet to verify probe placement in the striatum.

High-Performance Liquid Chromatography Coupled with Electrochemical Detection (HPLC-EC) analysis followed the methods previously described by Hall et al. (1989). The low level detections of DOPAC, DA, 5-HT, NE, 5-HIAA, and HVA were performed using an isocratic HPLC system (Beckman, Inc., Fullerton, CA) coupled to a dual-channel electrochemical array detector (model 5300A, ESA, Inc., Chelmsford, MA), E1 $=+0.35 \mathrm{mV}$ and E2 $=-0.25 \mathrm{mV}$, with an ESA model 5011A dual analytical cell. The compounds of interest were separated with reverse-phase chromatography, using a C18 column $(4.6 \mathrm{~mm} x$ 75 mm, $3 \mu \mathrm{m}$ particle size, Shiseido CapCell Pak UG120, Shiseido Co., LTD., Tokyo, Japan) with a $\mathrm{pH} 4.1$ citrate-acetate mobile phase, containing $4 \%$ methanol and $0.34 \mathrm{mM}$ 1-octanesulfonic acid delivered at a flow rate of $2.0 \mathrm{ml} / \mathrm{min}$. Peaks for the analytes were identified by retention times from known standards.

Data were collected from 5-6 animals per 10 drug concentrations (for Adderall, Damphetamine, Reverse Adderall, and methylphenidate). Data were collected for 5-6 animals for the highest drug concentration only for L-amphetamine, D,L-amphetamine, and cocaine. The raw microdialysis values were expressed as $\mathrm{nM}$ based on a $1 \times 10-7 \mathrm{M}$ mixed standard of known analytes and probe recoveries of $\sim 10 \%$. Outliers were excluded based on data falling outside of 2 standard deviations from the mean. Concentration-response curves were constructed based on the mean peak DA overflow concentration following the twenty minute reverse microdialysis of each drug concentration. GraphPad Prism statistical analysis software, version 4.0 (Prism, San Diego, CA, USA), was used to determine the appropriate nonlinear curve fit and Log half maximal effective concentration (EC50) of each drug. An initial one-way analysis of variance was used to determine significance of DA overflow from the aCSF control. A second one-way analysis of variance was used followed by post-hoc t-tests with Bonferroni's corrections to compare DA release produced following reverse microdialysis of clinically relevant drug concentrations and maximum concentrations. Potency measures were defined by the stimulant that reached its halfmaximal response on the concentration-response curve with the lowest effective concentration of stimulant. Efficacy measures were defined by the highest amount of DA overflow evoked when all stimulant concentrations were at maximal levels. Statistical significance was defined as $\mathrm{p}<0.05$.

\subsection{Reverse microdialysis of stimulants in the rat striatum: results}

Average baseline levels of DA $(<10 \mathrm{nM})$ were measured and found to be similar to previously collected data in the striatum of anesthetized and awake-behaving rats (Gerhardt and Maloney 1999; Ferguson et al. 2003; Garris et al. 1994; Kawagoe et al. 1992; Parsons and Justice 1992). Baseline DOPAC levels were determined to be $(\sim 800-1000 \mathrm{nM})$ in the rats used for the D-amphetamine and Adderall studies and are similar to previously reported levels (Ferguson et al. 2003). The DOPAC data are reported as percent of baseline due to increased variance in baseline samples collected from the rats used for the Reverse Adderall and 
methylphenidate studies (Fig. 3). Levels of the DA metabolite homovanillic acid (HVA), 5hydroxyindoleacetic acid (5-HIAA) and serotonin (5-HT) levels were measured and concentration-dependent effects were not detected (data not shown).

The twenty minute local tissue perfusions of drugs induced a concentration-dependent increase in DA overflow followed by a 60 minute time period to return to baseline supporting the DAT and DA uptake blocking effects of the tested stimulants (Wise and Hoffman 1992; Sulzer et al. 1993; Schweri et al. 1985). The resulting DA levels, at the highest concentration of drug, were similar to previous microdialysis measures of $150 \mathrm{nM}$ (Seeman and Madras 2002). The measures of DA were seen to decline over 2 more fractions post drug administration. Furthermore, applications of the lowest stimulant concentration resulted in DA levels that were not statistically different from those seen after reverse microdialysis of the artificial cerebral spinal fluid (aCSF) control.

The resulting D-amphetamine concentration-response curve for extracellular DA in rat striatum displayed an unexpected double-sigmoidal pattern with two plateaus. Plateaus in the amount of DA overflow occurred at the lower concentration (1 $\mu \mathrm{M} \mathrm{D}$-amphetamine) and at a higher concentration (100 $\mu \mathrm{M}$ D-amphetamine). At $0.1 \mu \mathrm{M}$ D-amphetamine, little or no

\section{D-amphetamine}

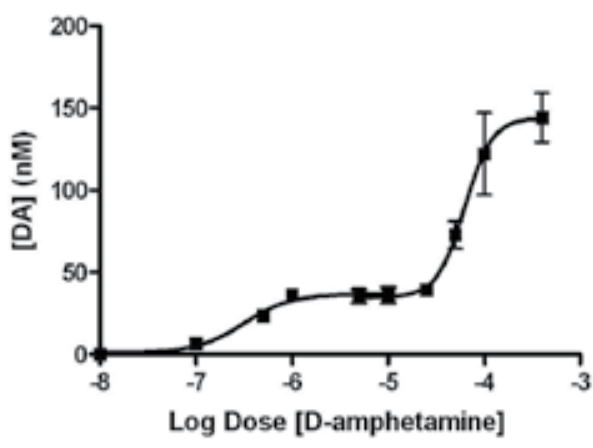

Adderall $^{\oplus}$

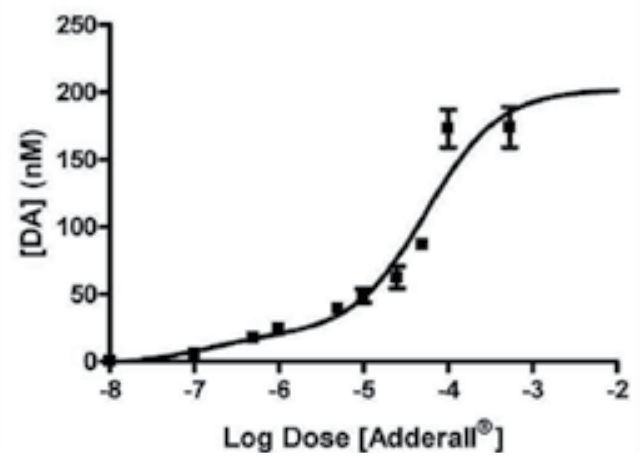

\section{Methylphenidate}

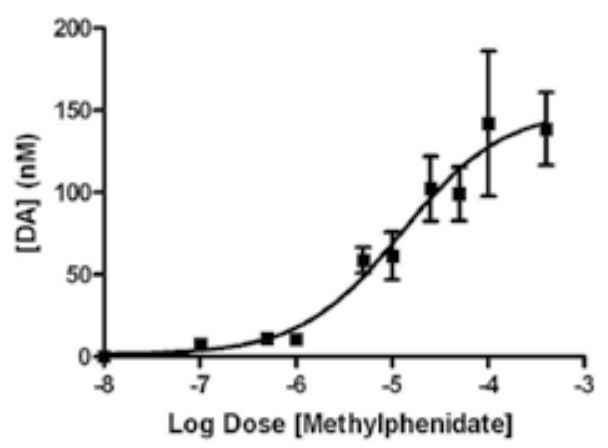

Reverse Adderall

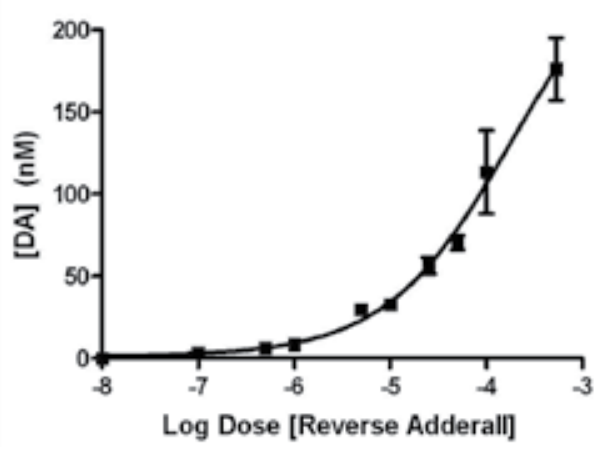

Fig. 2. Dose-Response curves for evoked overflow of dopamine in the rat striatum by various stimulants. 
increase in DA overflow resulted in comparison to aCSF control; and no significant differences were found between $100 \mu \mathrm{M}$ and $400 \mu \mathrm{M}$ D-amphetamine supporting an upper plateau in DA measures (Figure 2). Two half-maximal effective concentration (EC50) values are indicated for the higher potency (lower plateau) and lower potency (upper plateau) portions of this concentration-response curve (Table 1).

\begin{tabular}{|c|c|c|}
\hline Drug & $\begin{array}{c}\text { EC }_{50}[\text { Drug] }(\mu \mathbf{M}) \\
\text { For DA }\end{array}$ & $\begin{array}{c}\text { Maximum Response } \\
(\mu \mathbf{M}) \text { For DA }\end{array}$ \\
\hline Methylphenidate & 10 & $138.7 \pm 22.2$ \\
\hline Adderall & 25 & $184.6 \pm 12.3$ \\
\hline D-amphetamine II & 50 & $144.5 \pm 15.6$ \\
\hline Reverse Adderall & 50 & $176.7 \pm 19.1$ \\
\hline D-amphetamine I & 0.5 & N/A \\
\hline
\end{tabular}

Table 1. Stimulant Potency and Efficacy on DA Measures

D-amphetamine

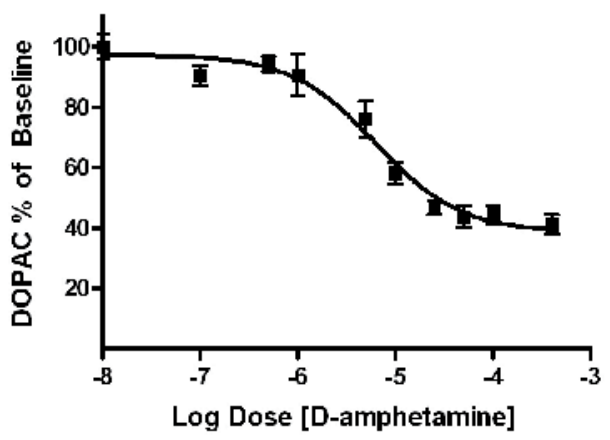

Adderall

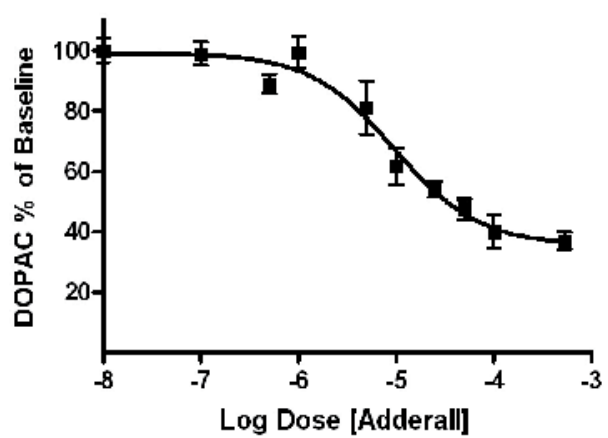

Methylphenidate

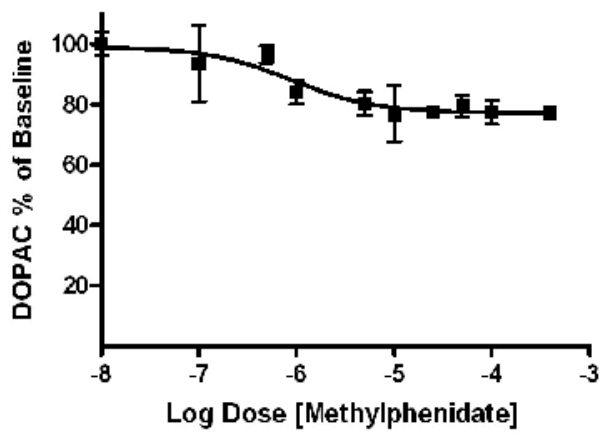

Reverse Adderall

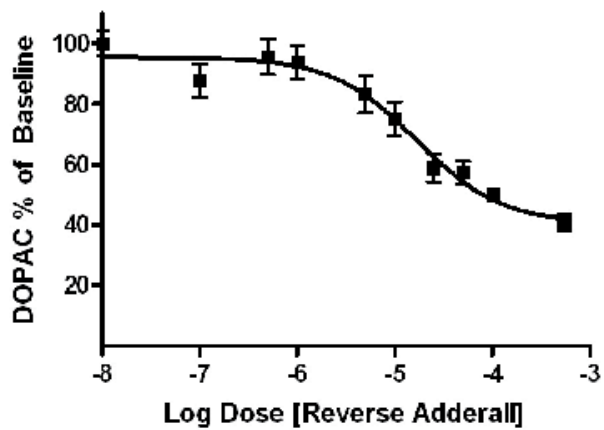

Fig. 3. Dose-Response curves for overflow of dopamine metabolite DOPAC in the rat striatum by various stimulants. 
The methylphenidate concentration-response curve for extracellular DA in the rat striatum also supports a concentration-dependent increase in DA levels (Figure 2). Since methylphenidate had previously been characterized as a DAT blocker and not a substrate that undergoes transport through the DAT, we hypothesized that we would see much lower levels of DA release in an anesthetized rat. It was therefore surprising to see that applications of $0.5-400 \mu \mathrm{M}$ methylphenidate increased DA concentrations significantly greater than aCSF control. However, in contrast to d-amphetamine, $0.1 \mu \mathrm{M}$ methylphenidate did not cause increased DA release that was significantly different from control. The two highest concentrations tested $(100$ and $400 \mu \mathrm{M})$ were not significantly different in the amount of DA release (Figure 2).

The Adderall concentration-response curve for extracellular DA measured in the rat striatum demonstrated a similar range of evoked DA overflow, although the dose-response curve was closer to a single sigmoidal curve (Figure 2). An upper plateau in DA levels occurred at $100 \mu \mathrm{M}$ Adderall, as $100 \mu \mathrm{M}$ and $400 \mu \mathrm{M}$ Adderall were not significantly different in response. At $0.1 \mu \mathrm{M}$, Adderall did not produce DA levels that were significantly different from local application of aCSF control.

Finally, the Reverse Adderall (75\% L-amphetamine, 25\% D-amphetamine) concentrationresponse curve for extracellular DA showed a concentration-dependent increase in evoked DA at all concentrations tested except for $0.1 \mu \mathrm{M}$; which was not significantly different from aCSF control (Figure 2). While Reverse Adderall was predominantly made of L-amphetamine, it did not increase DA levels to the extent of Adderall at some concentrations (Table 1). The highest two concentrations of Reverse Adderall tested were significantly different supporting that a plateau of DA measures will likely occur at a higher concentration.

Figure 3 shows the individual tracings of detected DOPAC levels (represented as \% of baseline) following reverse microdialysis of D-amphetamine at multiple concentrations. Damphetamine, Adderall, and Reverse Adderall inhibited DOPAC levels in a similar manner following local perfusion of drug at 120 minutes and continued to decrease DOPAC production up to one hour when DOPAC levels returned to baseline. While methylphenidate caused increased DA levels similar to the other stimulants, it did not affect DOPAC levels in a consistent manner and was similar in this aspect to the effects of cocaine. DOPAC production was less affected by methylphenidate and cocaine in comparison to Adderall ( $p<0.001)$, and Damphetamine $(\mathrm{p}<0.01, \mathrm{p}<0.05)$. Reverse Adderall, L-amphetamine, and $\mathrm{D}$, L-amphetamine all caused significantly greater effects on DOPAC levels in comparison to cocaine $(p<0.001)$. An initial increase in DOPAC was seen following application of $100 \mu \mathrm{M}$ and $400 \mu \mathrm{M}$ methylphenidate followed by a decrease similar to that of other concentrations without a pronounced concentration-dependent pattern (data not shown).

\subsection{Reverse microdialysis of stimulants in the rat striatum: implications}

These data represent novel findings regarding the effects of various stimulants across a range of concentrations on dopamine release in the striatum. The concentration-response curve for D-amphetamine displayed a double-sigmoidal pattern that supported dualfunctionality properties of the DAT and/or differential mechanisms by which high and low levels of D-amphetamine affect DA efflux. In addition, these data show for the first time that local applications of methylphenidate increased DA levels in a concentration-dependent 
pattern and even demonstrated a greater EC50 (DA) when compared to other stimulants. These data are in agreement with our previous in vivo high speed chronoamperometric data that support the robust local activity of Adderall compared to other ADHD medications (Joyce et al. 2007). Decreased DA levels caused by cocaine compared to higher DA levels after local application of methylphenidate suggest dissociation between the local effects of methylphenidate and cocaine. DOPAC levels in these studies showed significant decreases following additions of any of the amphetamine preparations in a dose- dependent fashion, whereas cocaine and methylphenidate were less effective in inhibiting DOPAC production.

The data shown here are consistent with the known DA releasing properties of amphetamine predominantly due to DAT reversal of normal reuptake into the presynaptic terminal (Giros et al. 1996). Likewise, amphetamine has been shown to impair DA reuptake, inhibit MAO activity, and affect vesicular conditions that lead to emptying of vesicular stores via the vesicular monoamine transporter 2 (VMAT2) (Horn et al. 1971; Sulzer et al. 1995; Dubocovich et al. 1985; Heikkila et al. 1975; Uretsky and Snodgrass 1977; Green and El Hait 1978; Cadoni et al 1995). Our previous data support amphetamine enantiomeric differences that could not be accounted for across multiple concentrations due to technical limitations; particularly with the current difficulties of studying the effects of low levels of these drugs on DA release using in vivo electrochemical methods (Glaser et al., 2005). We chose to carry out these studies in this manner based on information supporting the dynamic changes that occur in DA neuronal systems in response to DAT substrates and inhibitors. Purdom et al. (2003) showed data supporting that the order of administration of different concentrations of D-amphetamine significantly affected DA and DOPAC levels. These results were likely attributable to changes in the surface expression of DAT on DA nerve endings and/or DAT function. Other in vitro studies have shown substrate dependent trafficking of the DAT to and from the plasma membrane and subsequent changes in the ability to transport DA (Kahlig et al. 2005; Johnson et al. 2005; Saunders et al. 2000; Kahlig et al. 2004; Kahlig and Galli 2003). Therefore we used stimulant-naïve animals for these studies, an important but often neglected consideration in many mechanistic studies of stimulant medications.

While we have described the use of voltammetric studies to investigate the properties of stimulants at low levels, it is difficult to accurately predict what the resulting effective concentrations were in these studies. Voltammetry affords the ability to study neurotransmission with high temporal and spatial resolution; however, we lose a magnitude of sensitivity that is available using microdialysis coupled with HPLC-EC. Using HPLC-EC to analyze samples collected during reverse microdialysis (local application) of stimulant drugs allows for studies to be carried out with lower drug concentrations. These studies were designed to complement our previous studies and mimic longer administration (over 20 minutes) in converse to the rapid pressure ejection used earlier (20 seconds). As a final rationale of this work, we proposed to investigate complete concentration-response studies using reverse microdialysis coupled with HPLC-EC. Investigations of concentration-response patterns were intended to increase our understanding of ADHD drug mechanistic activity by looking at their effects on DA and metabolite levels.

Although we did measure norepinephrine (NE) with our HPLC methods, the peak was not consistently measurable due to its proximity to the solvent edge. In addition, NE is not a 
common neurotransmitter in the striatum. Therefore, one caveat of our study is that it does not investigate the possibility that NE elevation, and not DA, in the PFC (and not the striatum) is most important for clinical efficacy in ADHD. This theory goes on further to state that DA is elevated in the synapse only at higher doses of stimulants and that this leads more to the rewarding symptoms and drug-abuse potential (the downward slope of the theoretical inverted-U of stimulant action). Our data would suggest that this may be true for methylphenidate, but that d-amphetamine does involve appreciable DA at lower-doses that may work in concert with NE in the PFC. This would also give credence to the fact that the two main stimulants are both tried on patients with ADHD because some will respond well to one and not the other, where as other patients respond to both. Obviously, a repeat of this study using microdialysis in the PFC and an HPLC method to pick up the lower levels of $\mathrm{DA}$ and NE in the PFC would be needed to answer this mechanism of action question.

One possible mechanism for the D-amphetamine double-sigmoidal concentration-response curve involves targeting of specific DA pools and amphetamine concentration-dependent effects. Some data support contribution of both cytosolic and vesicular stores to the released DA following exposure to amphetamine (Pifl et al. 1995); while other data indicate a predominant vesicular DA contribution (Jones et al. 1998). Jones et al. (1998) measured DA released following electrical stimulation and amphetamine perfusion of striatal brain slices and noticed a delay in DA release with amphetamine, supporting that DA had to be redistributed to the cytosol prior to being released from the cell. Based on these different contributions to amphetamine-evoked DA increases, we suggest that lower concentrations of D-amphetamine release "newly synthesized" DA pools in the cytosol, and higher concentrations contribute to the emptying of vesicular stores. Together this produces a biphasic pattern and a marked increase in the amount of DA released at the higher concentrations (Seiden et al. 1993; Langeloh and Trendelenburg 1987; Sulzer et al. 1993,2005).

An alternative mechanism for the D-amphetamine concentration-response curve might be explained by an upregulation of DAT levels caused by stimulation of D2R autoreceptors leading to second messenger regulation. Others have reported a link between stimulation of D2R autoreceptors and levels of membrane DATs (Parsons et al. 1993; Cass and Gerhardt 1994; Rothblat and Schneider 1997; Dickinson et al. 1999; Hoffman et al. 1999; Mayfield and Zahniser 2001). For example, decreased DA clearance in the striatum, prefrontal cortex, and nucleus accumbens after administration of the D2R agonist raclopride has been demonstrated (Cass and Gerhardt 1994). In addition, acute amphetamine stimulation caused increased synaptosomal DAT surface expression that occurred within 30 seconds (Johnson et al. 2005) indicating the rapid trafficking of the DAT and supporting that these changes would have occurred during the time frame we were sampling (Saunders et al. 2000). Due to the comparatively increased sensitivity of D2R autoreceptors, low levels of extracellular DA are sufficient to stimulate these autoreceptors that would result in increased DA clearance (Cooper et al. 2003) (Fig. 4). The small amounts of released DA required to stimulate these autoreceptors would be taken up quickly through increased levels of membrane DATs, supporting the effects we see with the first plateau of the D-amphetamine concentrationresponse curve. At higher concentrations of D-amphetamine, increased DA clearance will likely be followed by autoreceptor desensitization caused by the high levels of DA released after such a robust concentration of drug (Khoshbouei et al. 2004; Gorentla and Vaughan 


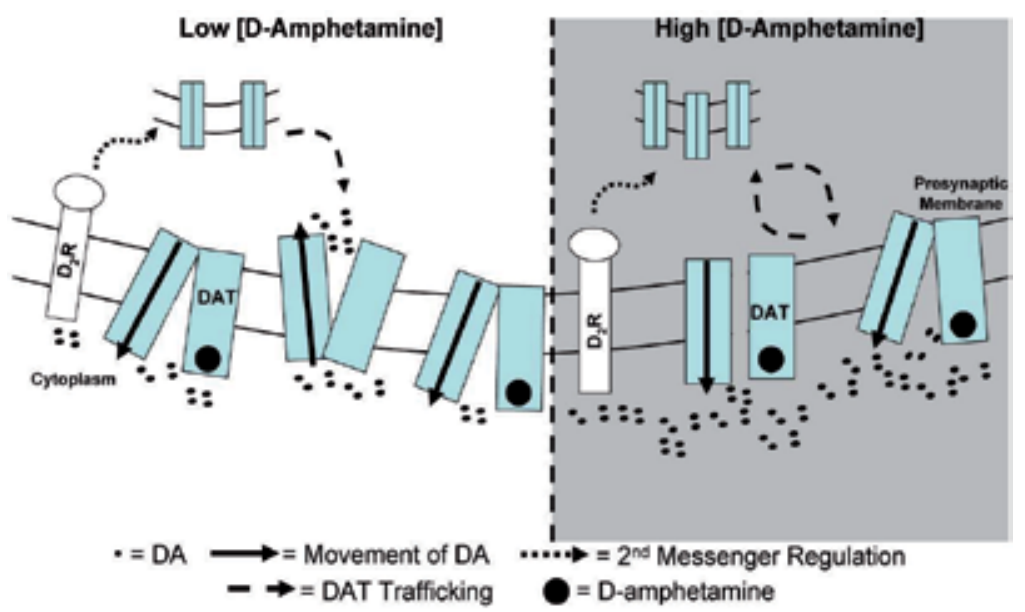

Fig. 4. Theoretical model of activity describing the double Plateaus of the D-amphetamine concentration-response curve for DA: Plateau I Low [D-amphetamine]: Lower concentrations of D-amphetamine cause reverse transport of low levels of DA through the DAT. In addition, DA sensitive D2R autoreceptors are stimulated. Due to the increased clearance of DA, the result is the first plateau of the concentration-response curve. Plateau II High [D-amphetamine]: Amphetamine has been shown to interact with DATs and facilitate DA release followed by DAT internalization. Higher concentrations of Damphetamine will likely cause increased DA release and DAT internalization. D2R autoreceptor desensitization is likely to occur and interrupt DAT expression. Higher levels of extracellular DA and decreased DA clearance likely cause the second plateau.

2005; Kim et al. 2001; Namkung and Sibley 2004; Ferguson et al. 1996; Tang et al. 1994) (Fig. 4). Finally, data support that interactions of amphetamine and the DAT lead to DAT internalization via phosphorylation of target residues in the C- and N-termini (Khoshbouei et al. 2004; Kahlig et al. 2006; Fog et al. 2006), that may contribute to the effects we see in the second plateau of the D-amphetamine concentration-response curve. We propose that the amount of D-amphetamine in Adderall leads to a similar but slightly different concentration-response curve. Previous in vivo electrochemical data support the faster kinetics of the effects of L-amphetamine in combination with the slower kinetics of Damphetamine could allosterically modulate DAT trafficking rendering a concentrationresponse with less apparent plateaus (Glaser et al. 2005; Joyce et al. 2007). Another possible correlation is the similar EC50 for both the second D-amphetamine sigmoidal curve and the DOPAC decrease consistent with MAO inhibition.

While the double plateaus we note here are in regards to increasing concentrations of Damphetamine, other reports suggest biphasic effects of catecholamine transporters over different parameters. Johnson et al. (2005) described the effects of amphetamine on DAT surface expression in rat synaptosomes. They described initial amphetamine upregulation of DATs to the plasma membrane leading to DA efflux followed by amphetamine induced internalization of DATs after repeated doses of amphetamine. Jayanthi et al. (2005) described mechanisms that contribute to a biphasic regulation of endogenous serotonin transporters (SERTs) expressed in platelets. Protein Kinase C (PKC) activation in platelets resulted in the initial reduction of functional SERTs followed by enhanced endocytosis of SERTs. 
Intraperitoneal administration of methylphenidate in freely-moving rat has been shown to cause increases in DA levels measured in dialysates and some argue that the greatest effects were seen in the prefrontal cortex (Hurd and Ungerstedt 1989; Berridge et al. 2006). In particular, Hurd and Ungerstedt found that amphetamine and methylphenidate caused similar increases in DA levels; however, methylphenidate caused these levels over a longer time period correlating with the robust effects of methylphenidate we present here. This study also reported that methylphenidate had less of an effect on decreasing DOPAC levels compared to the more pronounced decrease caused by amphetamine (Hurd and Ungerstedt 1989). In terms of behavioral effects, D-amphetamine and methylphenidate have been shown to induce locomotor activity at low doses and cause stereotypies at higher doses (Fessler et al. 1980; Hughes and Greig 1976; Scheel-Kruger 1971). Additionally, methylphenidate has also been found to be reinforcing in regards to drug abuse potential in humans, and it has been self-administered in animal models (Stoops et al. 2005; Rush et al. 2001; Risner and Jones 1975). In general, cocaine and methylphenidate are thought to work in a similar manner by predominantly acting as competitive inhibitors of the DAT (Wu et al. 2001) and increases in extracellular DA result predominantly from this blockade after impulse-dependent release of DA. However, the robust extracellular effects of methylphenidate in this study argue against this concept as the effects observed mirror d-amphetamine and not cocaine.

While the argument can be made that the studies herein that involve local applications of drugs fail to account for pharmacokinetic differences between these stimulants, we propose that this is a particular strength of our study. For these experiments, drugs were applied over a range of levels, including clinically relevant concentrations (10-50 $\mu \mathrm{M})$ and potentially drug abuse levels $(>400 \mu \mathrm{M})$ (West et al. 1999; Shader et al. 1999; Kuczenski and Segal 2001; Solanto et al. 2001; Grilly and Loveland 2001). The low concentrations were projected to simulate potential levels of drug that would be present in brain tissue following systemic or oral administration. Finally, administering the drugs via reverse microdialysis eliminated pharmacokinetic issues from the study allowing for more of the pure effects of the drugs on DA nerve terminals.

In summary, we have shown that the D-amphetamine concentration-response curve of DA displayed a double plateau pattern indicating effects on DA stores and/or rapid regulation of DAT trafficking and/or function. These data support that methylphenidate may cause DA release in addition to acting as a DA uptake inhibitor. Taken together, these data explain the effects of clinically available stimulants on DA levels over a range of concentrations and confirm that methylphenidate, $\mathrm{d}$-amphetamine, and combinations of amphetamine isomers have potent, yet different, effects on dopamine in the striatum.

\subsection{Future directions in the neuropsychopharmacology of ADHD}

The data presented herein demonstrates the translational aspect of how the currently available stimulants are different from each other, and therefore backs up the current practice of trying different stimulants on patients to maximize efficacy and minimize side effects. It is also suggests that other percentages of l-amphetamine may be useful to test in the future for some patients with ADHD may respond better to them. Yet, this data does little to address some of the larger problems that face us in understanding ADHD and in finding superior treatments for ADHD. Some might argue that the stimulants are largely effective and safe already. But with the risk of abuse and diversion, the fact that many 
adolescents and adults do not like how they feel on them and many other factors such as whether or not they truly decrease a person's subsequent risk for substance abuse, there is still room for improved medications. Perhaps one goal may be to find a medication that truly helps only those with ADHD, since stimulants actually can be "performance enhancing" drugs that can give people such as college students without ADHD benefits in studying or taking tests with little knowledge of their possible dangers or ethical implications.

In the future it will be useful to more fully understand not only the neurotransmitters involved in the various aspects of ADHD, but the way that circuitry and brain region interact to lead to dysfunction. Neuroimaging may contribute greatly to this as it obtains greater resolution and ways to measure separate neurotransmitter systems. Our lab and others have begun to use neurotransmitter specific probes to measure more accurately second by second changes in neurotransmitters. We are finding the milieu is much more heterogeneous than previously understandable by microdialysis. As microelectrode technology improves and gets more compact, real-time recordings of multiple brain areas while the animal is awake will answer more questions and allow for more precise drug development. Finally, pharmacogenomics is starting to yield some benefit and may help in more targeted use of the right stimulants for the right patient instead of the trial and error method now employed.

\section{Conclusion}

In this chapter we have reviewed the case for dopamine's role in ADHD especially as it pertains to the mechanisms of action of the stimulants methylphenidate and the various amphetamines. We have also shown how many other neurotransmitters are involved in ADHD and alternative medications for ADHD. No doubt as the neuropsychopharmacology of ADHD evolves, we will discover more intricate details about the relative contributions of the neurotransmitters and how they relate to the genetic and neurocircuitry levels of our understanding. The ultimate goal of this knowledge is to improve treatment and maximize safety for people of all ages with ADHD.

\section{Acknowledgment}

Special thanks to B. Matthew Joyce, Ph.D., Garretson D. Epperly, M.S., Theresa Currier Thomas, Ph.D for their contributions in conducting the experiments in this chapter. These studies were supported by USPHS grants MH066393, MH01245, DA14944, and NS39787. Pure substance Adderall@ was provided by Shire Pharmaceuticals, Hampshire, Chineham, England.

\section{References}

Bergman J., Madras BK., Johnson SE., \& Spealman RD. (1989). Effects of cocaine and related drugs in nonhuman primates. III. Self-administration by squirrel monkeys. J Pharmacol Exp Ther, Vol. 251, No. 1, (October 1989), pp. 150-155

Berridge CW., Devilbiss DM., Andrzejewski ME., Arnsten AF., Kelley AE., Schmeichel B., Hamilton C., \& Spencer RC. (2006). Methylphenidate preferentially increases catecholamine neurotransmission within the prefrontal cortex at low doses that enhance cognitive function. Biol Psychiatry, Vol. 60, No. 10, (November 2006), pp. 1111-1120. 
Brioni JD., Esbenshade TA., Garrison TR., Bitner SR., \& Cowart MD. (2011). Discovery of Histamine H3 Antagonists for the Treatment of Cognitive Disorders and Alzheimer's Disease. J Pharmacol Exp Ther, Vol 336, No. 1, (January 2011),pp. 38-46.

Bradley C. (1937). The Behavior of Children Receiving Benzedrine. Am J Psychiatry, Vol. 94, (November 1937), pp. 577-581.

Cadoni C., Pinna A., Russi G., Consolo S., \& Di Chiara G. (1995). Role of vesicular dopamine in the vivo stimulation of striatal dopamine transmission by amphetamine: evidence from microdialysis and Fos immunohistochemistry. Neuroscience, Vol. 65, No.4, (April 1995), pp. 1027-1039

Cass WA., Gerhardt GA. (1994). Direct in vivo evidence that D2 dopamine receptors can modulate dopamine uptake. Neurosci Lett, Vol.176, No.2, (August 1994), pp.259-63.

Cooper JR., Bloom FE., \& Roth RH. (2003). The Biochemical Basis of Neuropharmacology (8 $8^{\text {th }}$ ed.), Oxford University Press, New York.

Dickinson SD., Sabeti J., Larson GA., Giardina K., Rubinstein M., Kelly MA., Grandy DK., Low MJ., Gerhardt GA., Zahniser NR. (1999). Dopamine D2 receptor-deficient mice exhibit decreased dopamine transporter function but no changes in dopamine release in dorsal striatum. J Neurochem, Vol. 72, No. 1, (January 1999), pp.148-56.

Dubocovich ML., \& Zahniser NR. (1985). Binding characteristics of the dopamine uptake inhibitor ${ }^{3} \mathrm{H}$-nomifensine to striatal membranes. Biochem Pharmac, Vol. 34, No. 8, (April 1985), pp.1137-1144.

Ferguson SA., Felipa HN., Bowman RE. (1996). Effects of acute treatment with dopaminergic drugs on open field behavior of adult monkeys treated with lead during the first year postpartum. Neurotoxicol Teratol, Vol. 18, No.2, (March 1996), pp.181-8.

Ferguson SA., Gough BJ., Cada AM. (2003). In vivo basal and amphetamine-induced striatal dopamine and metabolite levels are similar in the spontaneously hypertensive, Wistar-Kyoto and Sprague-Dawley male rats. Physiol Behav. Vol. 80, No.1, (October 2003), pp.109-14.

Fessler RG., Sturgeon RD., \& Meltzer HY. (1980). Effects of phencyclidine and methylphenidate on D-amphetamine-induced behaviors in reserpine pretreated rats. Pharmacol Biochem Behav, Vol.13, No. 6, (December 1980), pp. 835-884.

Findling RL., McNamara NK., Stansbrey RJ., Maxhimer R., Periclou A., Mann A., \& Graham SM. (2007). A pilot evaluation of the safety, tolerability, pharmacokinetics, and effectiveness of memantine in pediatric patients with attention-deficit/hyperactivity disorder combined type. J Child Adolesc Psychopharmacol, Vol. 17, No. 1 (February 2007), pp.19-33.

Fog JU., Khoshbouei H., Holy M., Owens WA., Vaegter CB., Sen N., Nikandrova Y., Bowton E., McMahon DG., Colbran RJ., Daws LC., Sitte HH., Javitch JA., Galli A., Gether U. (2006). Calmodulin kinase II interacts with the dopamine transporter $C$ terminus to regulate amphetamine-induced reverse transport. Neuron, Vol. 51, No.4, (August 2006), pp.417-29.

Gamo NJ., Wang M., \& Arnsten AF. (2010). Methylphenidate and atomoxetine enhance prefrontal function through a2-adrenergic and dopamine D1 receptors. J Am Acad Child Adolesc Psychiatry. Vol. 49, No.10 (October 2010), pp.1011-23.

Garris PA., Ciolkowski EL., Wightman RM. (1994). Heterogeneity of evoked dopamine overflow within the striatal and striatoamygdaloid regions. Neuroscience. Vol. 59, No.2 (March 1994), pp.417-27.

Gatley SJ., Pan D., Chen R., Chaturvedi G., \& Ding YS. (1996). Affinities of methylphenidate derivatives for dopamine, norepinephrine and serotonin transporters. Life Sci, Vol. 58, No. 12, (February 1996), pp. 231-239. 
Gerhardt GA., Maloney RE. (1999). Microdialysis studies of basal levels and stimulusevoked overflow of dopamine and metabolites in the striatum of young and aged Fischer 344 rats. Brain Res. Vol. 16 (January 1999) pp. 68-77.

Giros B., Jaber M., Jones SR., Wightman RM., \& Caron MG. (1996). Hyperlocomotion and indifference to cocaine and amphetamine in mice lacking the dopamine transporter. Nature, Vol. 379, (February 1996), pp. 606-612.

Glaser PEA., Thomas TC., Joyce BM., Castellanos FX., \& Gerhardt GA. (2005). Differential effects of amphetamine isomers on dopamine release in the rat striatum and nucleus accumbens core. Psychopharmacology, Vol. 178 ( March 2005), pp. 250-258.

Gorentla BK., Vaughan RA. (2005). Differential effects of dopamine and psychoactive drugs on dopamine transporter phosphorylation and regulation. Neuropharmacology, Vol. 49, No. 6, (November 2005), pp.759-68.

Green AL., \& El Hait MJ. (1978). Inhibition of mouse brain monoamine oxidase by (+)amphetamine in vivo. J Pharm PHarmac, Vol. 30, (April 1978), pp. 262-263.

Grilly DM., \& Loveland A. (2001). What is a "low dose" of d-amphetamine for inducing behavioral effects in laboratory rats? Psychopharmacology (Berl), Vol. 153, No. 2 (January 2001), pp. 155-169.

Hall ME., Hoffer BJ., \& Gerhardt GA. (1989). Rapid and sensitive determination of catecholamines in small tissue samples by high performance liquid chromatography coupled with dual-electrode coulometric electrochemical detection. LC-GC, Vol. 7, No. 3, pp. 258-265.

Heikkila RE., Orlansky H., \& Cohen E. (1975). Studies on the distinction between uptake inhibition and release of ${ }^{3} \mathrm{H}$-dopamine in rat brain tissue slices. Biochem Pharmac, Vol 24, No. 8, (April 1975), pp. 847-852.

Hoffman AF., Zahniser NR., Lupica CR., Gerhardt GA. (1999). Voltage-dependency of the dopamine transporter in the rat substantia nigra. Neurosci Lett, Vol. 260, No.. 2 (January 1999). pp.105-8.

Horn AS., Coyle JT., \& Snyder SH. (1971). Catecholamine uptake by synaptosomes from rat brain. Structure-activity relationships of drugs with differential effects on dopamine and Norepinephrine neurons. Mol Pharmacol, Vol. 7, No. 1, (January 1971), pp. 66-80.

Hughes RN., \& Greig AM. (1976). Effects of caffeine, methamphetamine and methylphenidate on reactions to novelty and activity in rats. Neuropharmacology, Vol.15, No. 11, (April 1976), pp.673-676.

Hurd YL., \& Ungerstedt U. (1989). In vivo neurochemical profile of dopamine uptake inhibitiors and releasers in rat caudate-putamen. Eur J Pharmacol, Vol.166, No. 2, pp.251-260.

James RS., Sharp WS., Bastain TM., Lee PP., Walter JM., Czarnolewski M., \& Castellanos FX. (2001). Double-blind, placebo-controlled study of single-dose amphetamine formulations in ADHD. Journal of the American Academy of Child and Adolescent Psychiatry, Vol. 40, No. 11, (November 2001), pp. 1268-1276.

Jayanthi S., Deng X., Ladenheim B., McCoy MT., Cluster A., Cai NS., Cadet JL. (2005). Calcineurin/NFAT-induced up-regulation of the Fas ligand/Fas death pathway is involved in methamphetamine-induced neuronal apoptosis. Proc Natl Acad Sci USA, Vol. 102, No. 3, (January 2005), pp.868-73.

Johnson LA., Furman CA., Zhang M., Guptaroy B., \& Gnegy ME. (2005). Rapid delivery of the dopamine transporter to the plasmalemmal membrane upon amphetamine stimulation. Neuropharmacology, Vol. 49, No. 6, (November 2005), pp.750-758. 
Jones SR., Gainetdinov RR., Wightman RM., \& Caron MG. (1998). Mechanisms of amphetamine action revealed in mice lacking the dopamine transporter. J Neurosci, Vol. 18, No. 6, (March 1998), pp.1979-1986.

Joyce BM., Glaser PEA., \& Gerhardt GA. (2007). Adderall produces increased striatal dopamine release and a prolonged time course compared to amphetamine isomers. Psychopharmacology (Berl), Vol. 191, No.3, (April 2007), pp. 669-677.

Kahlig KM., \& Galli A. (2003). Regulation of dopamine transporter function and plasma membrane expression by dopamine, amphetamine, and cocaine. European Journal of Pharmacology, Vol. 479, (October 2003), pp.153-158.

Kahlig KM., Javitch JA., \& Galli A. (2004). Amphetamine regulation of dopamine transportcombined measurements of transporter currents and transporter imaging support the endocytosis of an active carrier. Journal of Biological Chemistry, Vol. 279, No.10, (March 2004),pp. 8966-8975.

Kahlig KM., Binda F., Khoshbouei H., Blakely RD., McMahon DG., Javitch JA., \& Galli A. (2005). Amphetamine induces dopamine efflux through a dopamine transporter channel. Proc Natl Acad Sci USA, Vol. 102, No. 9, (March 2005), pp.3495-3500.

Kahlig KM., Lute BJ., Wei Y., Loland CJ., Gether U., Javitch JA., Galli A. (2006). Regulation of dopamine transporter trafficking by intracellular amphetamine. Mol Pharmacol, Vol. 70, No. 2, (August 2006), pp.542-8.

Kawagoe KT., Garris PA., Wiedemann DJ., Wightman RM. (1992). Regulation of transient dopamine concentration gradients in the microenvironment surrounding nerve terminals in the rat striatum. Neuroscience, Vol. 51, No.1, (November 1992), pp.55-64.

Khoshbouei H., Sen N., Guptaroy B., Johnson L., Lund D., Gnegy ME., Galli A., Javitch JA. (2004). N-terminal phosphorylation of the dopamine transporter is required for amphetamine-induced efflux. PLoS Biol, Vol. 2, No.3, (March 2004). pp.387-93.

Kim KM., Valenzano KJ., Robinson SR., Yao WD., Barak LS., Caron MG. (2001). Differential regulation of the dopamine D2 and D3 receptors by $\mathrm{G}$ protein-coupled receptor kinases and beta-arrestins. J Biol Chem, Vol. 276, No. 40, (October 2001), pp.37409-14.

Kuczenski R., Segal DS. (2001). Locomotor effects of acute and repeated threshold doses of amphetamine and methylphenidate: relative roles of dopamine and norepinephrine. J Pharmacol Exp Ther, Vol. 296, No. 3, (March 2001), pp.876-83.

Langeloh A., \& Trendelenburg U. (1987). The mechanism of the 3 H-noradrenaline releasing effect of various substrates of uptake 1: role of monoamine oxidase and of vesicularly stored 3H-noradrenaline. Naunyn Schmiedebergs Arch Pharmacol, Vol. 336, No. 6, (December 1987), pp. 602-610.

Lile JA., Stoops WW., Durell TM., Glaser PE., \& Rush CR. (2006). Discriminative-stimulus, selfreported, performance, and cardiovascular effects of atomoxetine in methylphenidatetrained humans. Exp Clin Psychopharmacol, Vol. 14, No.2 (May 2006), pp. 136-47.

Ludolph AG., Udvardi PT., Schaz U., Henes C., Adolph O., Weigt HU., Fegert JM., Boeckers TM., \& Föhr KJ. (2010). Atomoxetine acts as an NMDA receptor blocker in clinically relevant concentrations. Br J Pharmacol. Vol. 160, No. 2 (May 2010), pp. 283-91.

Namkung Y., Sibley DR. (2004). Protein kinase C mediates phosphorylation, desensitization, and trafficking of the D2 dopamine receptor. J Biol Chem, Vol. 279, No. 47, (November 2004), pp. 49533-41.

Mayfield RD., Zahniser NR. (2001). Dopamine D2 receptor regulation of the dopamine transporter expressed in Xenopus laevis oocytes is voltage-independent. Mol Pharmacol, Vol. 59, No. 1, (January 2001), pp.113-21. 
Parsons LH., Justice JB Jr. (1992). Extracellular concentration and in vivo recovery of dopamine in the nucleus accumbens using microdialysis. J Neurochem, Vol.58, No.1, (January 1992), pp.212-8.

Parsons LH., Schad CA., Justice JB Jr. (1993). Co-administration of the D2 antagonist pimozide inhibits up-regulation of dopamine release and uptake induced by repeated cocaine. J Neurochem, Vol.60, No.1, (January 1993), pp.376-9.

Patrick KS., \& Markowitz JS. (1997). Pharmacology of methylphenidate, amphetamine enantiomers and pemoline in attention-deficit hyperactivity disorder. Human Psychopharmacology-Clinical and Experimental, Vol. 12, No. 6, (November/December 1997), pp.527-546.

Paxinos G., \& Watson C. (1986). The rat brain in stereotaxic coordinates (2nd ed.), Academic, Sydney.

Pelham WE., Gnagy EM., Chronis AM., Burrows-MacLean L., Fabiano GA., Onyango AN., Meichenbaum DL., Williams A., Aronoff HR., \& Steiner RL. (1999). A comparison of morning-only and morning/late afternoon Adderall to morning-only, twice-daily, and three times-daily methylphenidate in children with attention-deficit/hyperactivity disorder. Pediatrics, Vol. 104, No.6, (December 1999), pp. 1300-1311.

Pifl C., Drobny H., Reither H., \& Hornykiewicz OEAS. (1995). Mechanisms of the dopaminereleasing actions of amphetamine and cocaine: plasmalemmal dopamine transporter versus vesicular monoamine transporter. Mol Pharmacol, Vol.47, No. 2, (February 1995), pp. 368-373.

Popper CW. (1994). The story of four salts. J Child Adolesc Psychopharmacol, Vol. 4, pp. 217-223.

Purdom MS., Stanford JA., Currier TD., \& Gerhardt GA. (2003). Microdialysis studies of Damphetamine-evoked striatal dopamine overflow in young versus aged F344 rats: effects of concentration and order of administration. Brain Research, Vol. 979, No.12, (July 2003), pp. 203-209.

Risner ME., Jones BE. (1975). Self-administration of CNS stimulants by dog. Psychopharmacologia, Vol. 43, No. 3, (September 1975), pp.207-13.

Robbins TW., \& Sahakian BJ. (1979). "Paradoxical" effects of psychomotor stimulant drugs in hyperactive children from the standpoint of behavioural pharmacology. Neuropharmacology, Vol. 18, No. 12, (December 1979), pp. 931-950.

Rothblat DS., Schneider JS. (1997). Regionally specific effects of haloperidol and clozapine on dopamine reuptake in the striatum. Neurosci Lett, Vol. 228, No. 2, (June 1997), pp.119-22.

Rush CR., Essman WD., Simpson CA., Baker RW. (2001). Reinforcing and subject-rated effects of methylphenidate and d-amphetamine in non-drug-abusing humans. J Clin Psychopharmacol, Vol. 21, No. 3, (June 2001), pp.273-86.

Sander K., Kottke T., \& Stark H. (2008). Histamine H3 Receptor Antagonists Go to Clinics. Biol. Pharm. Bull, Vol. 31, No. 12, (December 2008), pp. $2163-2181$.

Saunders C., Ferrer JV., Shi L., Chen JY., Merrill G., Lamb ME., Leeb-Lundberg LMF., Carvelli L., \& Javitch JA, Galli A. (2000). Amphetamine-induced loss of human dopamine transporter activity: an internalization- dependent and cocaine-sensitive mechanism. Proc Natl Acad Sci USA. Vol. 97, No.12, (June 2000), pp. 6850-6855.

Scheel-Kruger J. (1971). Comparative studies of various amphetamine analogues demonstrating different interactions with the metabolism of the catecholamines in the brain. Eur J Pharmacol, Vol. 14, No.1, pp. 47-59.

Schweri MM., Skolnick P., Rafferty MF., Rice KC., Janowsky AJ., Paul SM. (1985). $[3 \mathrm{H}]$ Threo-(+/-)-methylphenidate binding to 3,4-dihydroxyphenylethylamine uptake sites in corpus striatum: correlation with the stimulant properties of ritalinic acid esters. J Neurochem, Vol.45, No.4, (October 1985), pp.1062-70. 
Seeman P., Madras B. (2002). Methylphenidate elevates resting dopamine which lowers the impulse-triggered release of dopamine: a hypothesis. Behav Brain Res, Vol.130, No.1, (March 2002), pp.79-83.

Seiden LS., \& Sabol KE. (1993). Amphetamine-effects on catecholamine systems and behavior. Annu Rev Pharmacol Toxicol, Vol. 33, pp.639-677.

Segal DS. (1974). Behavioral characterization of d- and l- amphetamine: neurochemical implications. Science, Vol. 190, (October 1974), pp. 475-477.

Shader RI., Harmatz JS., Oesterheld JR., Parmelee DX., Sallee FR., Greenblatt DJ. Population pharmacokinetics of methylphenidate in children with attention-deficit hyperactivity disorder. J Clin Pharmacol, Vol. 39, No. 8, (August 1999), pp.775-85.

Smith A. (2002). Effects of caffeine on human behavior. Food Chem Toxicol. Vol. 40, No. 9, (September 2002), pp. 1243-55.

Solanto MV., Arnsten AFT., \& Castellanos FX. (2001) Stimulant drugs and ADHD: basic and clinical neuroscience, Oxford University Press, New York.

Sonders MS., Zhu SJ., Zahniser NR., Kavanaugh MP., \& Amara SG. (1997). Multiple ionic conductances of the human dopamine transporter: the actions of dopamine and psychostimulants. J Neurosci, Vol. 17, No. 3, (February 1997), pp. 960-974.

Stoops WW., Lile JA., Fillmore MT., Glaser PE., Rush CR. (2005). Reinforcing effects of methylphenidate: influence of dose and behavioral demands following drug administration. Psychopharmacology (Berl), Vol. 177, No. 3, (January 2005), pp.349-55.

Strohl MP. (2011). Bradley's Benzedrine studies on children with behavioral disorders. Yale J Biol Med, Vol. 84, No. 1, (March 2011), pp. 27-33.

Sulzer D., Maidment NT., \& Rayport S. (1993). Amphetamine and other weak bases act to promote reverse transport of dopamine in ventral midbrain neurons. J Neurochem, Vol. 60, No. 2, (February 1993), pp.527-535.

Sulzer D., Chen TK., Lau YY., Kristensen H., Rayport S., Ewing A. (1995). Amphetamine redistributes dopamine from synaptic vesicles to the cytosol and promotes reverse transport. J Neurosci, Vol. 15, No.5, (May 1995), pp.4102-8.

Sulzer D., Sonders MS., Poulsen NW., \& Galli A. (2005). Mechanisms of neurotransmitter release by amphetamines: a review. Progress in Neurobiology, Vol. 75, No.6, (April 2005), pp. 406- 433.

Tang L., Todd RD., O'Malley KL. (1994). Dopamine D2 and D3 receptors inhibit dopamine release. J Pharmacol Exp Ther, Vol. 270, No. 2, (August 1994), pp.475-9.

Uretsky NJ., \& Snodgrass SR. (1977). Studies on the mechanism of stimulation of dopamine synthesis by amphetamine in striatal slices. J Pharmac Exp Ther, Vol. 202, No.3, (September 1977), pp.565-580.

Wang M., Ramos BP., Paspalas CD., Shu Y., Simen A., Duque A., Vijayraghavan S., Brennan A., Dudley A., Nou E., Mazer JA., McCormick DA., Arnsten AF. (2007). Alpha2Aadrenoceptors strengthen working memory networks by inhibiting cAMP-HCN channel signaling in prefrontal cortex. Cell. Vol. 129, No.2, (April 2007), pp.397-410.

West CH., Boss-Williams KA., Weiss JM. (1999). Motor activation by amphetamine infusion into nucleus accumbens core and shell subregions of rats differentially sensitive to dopaminergic drugs. Behav Brain Res, Vol. 98, No. 1, (January 1999), pp.155-65.

Wise RA., Hoffman DC. (1992). Localization of drug reward mechanisms by intracranial injections. Synapse, Vol. 10, No.3, (March 1992), pp.247-63.

Wu Q., Reith ME., Kuhar MJ., Carroll FI., \& Garris PA. (2001). Preferential increases in nucleus accumbens dopamine after systemic cocaine administration are caused by unique characteristics of dopamine neurotransmission. J Neurosc, Vol. 21, No. 16, (August 2001), pp. 6338-6347. 


\title{
Trends in the Prescribing and Adverse Drug Reactions Patterns of Psychostimulants Among Danish Children and Adolescents
}

\author{
Lise Aagaard and Ebba Holme Hansen \\ Department of Pharmacology and Pharmacotherapy, Section for Social Pharmacy, \\ FKL-Research Centre for Quality in Medicine Use, \\ Danish Pharmacovigilance Research Project (DANPREP), \\ Faculty of Pharmaceutical Sciences, \\ University of Copenhagen \\ Denmark
}

\section{Introduction}

The use of psychostimulants, particularly methylphenidate, to treat Attention Deficit/Hyperactivity Disorder (ADHD) symptoms in children and adolescents has increased rapidly since the 1990s (Schubert et al. 2010). In the 2000s serious reports on cardiovascular adverse drug reactions (ADRs), sudden deaths and psychiatric disorders have led the regulatory agencies in Europe and the United States (US) to warn against use of psychostimulants in the paediatric population (Schubert et al., 2010). In July 2007, class labels were implemented in the product information to reflect more specific information about cardiovascular and psychiatric adverse events and long-term suppression of growth (European Medicines Agency, 2007, US Food and Drug Administration, 2007). Despite these warnings, the number of children treated as well as the amount of psychostimulants prescribed per child has steadily increased (Kalverdijk et al., 2008).

Studies have shown that the use of psychostimulants in children in the Netherlands increased 8 times from 1996 to 2006 (Trip et al., 2009) and in Germany with 96\% from 2000 to 2007 (Schubert et al., 2010). From 1994 to 2004 the use of psychostimulants in children increased 5 times in Norway (Asheim et al., 2007), and from 1987 to 1996 the use of psychostimulants increased 10 times among US children (Zuvekas et al., 2006).

Information about ADRs from psychostimulants has been reported in several clinical studies of short duration, primarily conducted in 6 to 12-year olds boys, particularly in the US (Bloch et al., 2009). The majority of ADRs reported in these studies were of the type gastrointestinal disorders as well as nervous- and psychiatric disorders, and only few serious ADRs were reported (Bloch et al., 2009). In spite of pharmacological treatment of children with ADHD being common, this practice has been highly debated among health care professionals due to the dilemma of treating children with substances potential for abuse, where information about long-term safety aspects is very limited (Bloch et al., 2009). The 
concerns about safety issues from use of psychostimulants in the paediatric population are also due to the many anecdotal reports of serious psychiatric ADRs and sudden deaths that have been submitted to the regulatory agencies over recent years (Pringsheim \& Steeves, 2011). Systematic analyses of ADRs reported to national databases are necessary, as these databases constitute an important, though underestimated, source of data, especially about new, serious and rarely occurring ADRs (Hansen, 1992; Aagaard \& Hansen, 2009a). We did not locate any studies which systematically have analysed spontaneous reports for psychostimulants submitted to national databases compared to data on medicine use in the paediatric population.

The objective of this chapter was to 1) describe trends in prescribing of psychostimulants in the Danish paediatric population and 2) characterise spontaneous ADR reports submitted to the Danish Medicines Agency (DKMA) over a decade with respect to occurrence, seriousness, type and age and gender of the child, reported for psychostimulants.

\section{Methods}

\subsection{Design}

We conducted a retrospective analysis of all spontaneous ADR reports for 0-17-year old children from 2000 to 2009. Data were obtained from the Danish ADR database and placed at the disposal of this study in anonymous form with encrypted person identification. The unit of analysis was one ADR. Data on medicine use from each of the stimulants were extracted on individual level from the national Danish medicines registry as defined daily doses (DDD) per 1000 inhabitants per day and users (number of treated persons per 1000 inhabitants).

\subsection{Setting}

\subsubsection{Danish registry of medicine use}

In only a few countries information about medicine use at the individual level is available, Denmark being one of these countries. The Registry of Medicinal Product Statistics is a national database covering all outpatient pharmacy-dispensed prescriptions in Denmark.

The registry was established in 1994 to provide complete statistics on the use of medicines in Denmark. Data on prescriptions are registered for each patient via the civil registration number. Each prescription record contains the date of purchase, the dispensing pharmacy, the prescribing physician and detailed information on the drug dispensed, including product name, anatomical therapeutic classification (ATC) system name, dosage, package size and formulation. Medicine use is recorded in different ways. In this study we apply the defined daily doses (DDD) per 1000 inhabitants and number of users per 1000 inhabitants for our analysis. The DDD is defined at the assumed average maintenance dose per day for a drug used for its main indication in adults (WHO, 2011).

\subsubsection{Definition of psychostimulants}

A psychostimulants is an agent that causes an increase in functional activity, usually of the central nervous system (Howard et al., 2010). Examples of these kinds of effects may 
include enhanced alertness, wakefulness, and locomotion, among others. Due to their effects typically having an "up" quality to them, stimulants are also occasionally referred to as "uppers". Stimulants (analeptics) produce a variety of different kinds of effects by enhancing the activity of the central and peripheral nervous systems (Howard et al, 2010).

In this study we focus on the type of stimulants most frequently prescribed in Denmark, i.e. methylphenidate, atoxometine, modafinil and amphetamine derivates in the paediatric population. According to the official summary of product information methylphenidate and atomoxetine are licensed for treatment of ADHD in children and adolescents from 6 and 8 years of age respectively. Modafinil and amphetamine derivates are not licensed for use in children (Danish Medicines Agency, 2011).

\subsubsection{ADR reporting system}

The reporting of ADRs has been obligatory in Denmark since 1 May 1968. Initially, only physicians were covered by the obligation; however, in 1972, dentists and veterinary surgeons were also required to report ADRs (Aagaard et al., 2008b). Since 1995, drug manufacturers have been obliged to keep registers of suspected and demonstrated ADRs and to make these available to the regulatory authorities. Since July 2003, consumers and other health care professionals have been able to report ADRs directly to the authorities (Aagaard et al., 2008b). An ADR report is defined by the following four criteria, which must be included in all reports: (i) information about the patient; (ii) the suspected medicine(s); (iii) the presumed $\mathrm{ADR}(\mathrm{s})$; and (iv) information about the person making the report. An ADR report may contain one or more ADR terms (Aagaard et al., 2008b).

After receiving the ADR reports, professional staff at the DKMA code and categorize the ADRs by degree of seriousness and type of reaction according to the Medical Dictionary for Regulatory Activities (MedDRA) terminology (Aagaard et al., 2008b).

ADR reports are forwarded electronically to the respective pharmaceutical companies, which periodically assess the ADR reports received. The results of their assessments are reported to the DKMA via periodic safety update reports (PSURs). The Danish ADR database contains all spontaneous ADR reports in Denmark, including those reported directly to the pharmaceutical companies (Aagaard et al., 2008b).

\subsubsection{Adverse drug reaction (ADR)}

An ADR is defined as "any noxious and unintended response to medicines that occurs at doses normally used in humans for the prophylaxis, diagnosis or therapy of diseases" (Volume 9, 2009).

\subsubsection{Criteria for seriousness}

Severity of reported ADRs was classified according to the criteria defined in volume 9 of the rules governing medicinal products in the European Union guideline (Volume 9, 2009).

Here serious ADRs are divided into: resulting in death, life-threatening, requiring hospitalization or prolongation of existing hospitalization, resulting in persistent or 
significant disability/incapacity in the reporter's opinion, a congenital anomaly/birth defect and other medically important conditions (Volume 9, 2009).

Other reactions were classified as being non-serious.

\subsubsection{Anatomical therapeutic chemical (ATC) classification groups}

The ATC system classifies medicinal products according to the primary constituent, organ or system on which they act and their chemical, pharmacological and therapeutic properties. Medicines are divided into 14 main groups (first level), with one therapeutic subgroup (second level). The third and fourth levels are chemical/pharmacological/therapeutic subgroups and the fifth level is the chemical substance (WHO ATC index, 2011).

The extract from the ADR database only provides information according to the trade name of those medicinal products that have been reported as causing ADRs. Therefore it was necessary to translate manually trade names into generic names at ATC level 5 in the national medicines register, and then run the generic form of the medicine name against the ADRs reported.

\subsection{Data extraction}

Prescription data were extracted from the Danish registry of dispensed medicine. We extracted information about DDDs per 1000 inhabitants per day and number of persons per 1000 inhabitants prescribed for psychostimulants among children from 0 to 17-years of age. Information about gender was also extracted from the registry. ADR reports submitted to the DKMA were placed at the disposal of this study in anonymous form with encrypted identification of the medicine user. Data were extracted from the ADR database on Microsoft Excel files and the material comprised all ADR reports on children from birth to 17 years of age reported to the Danish Medicines Agency from 2000 to 2009 for the psychostimulants. The unit of analysis was one ADR.

The Danish ADR database defines five categories of persons who may submit data to the database. This article applies this official designation for category of person submitting reports:

- Lawyer: patient injury insurers and/or law firms

- Pharmacist: community or hospital pharmacists

- Physician: general practitioners, physicians and dentists

- Other health care professional: nurses, pharmaceutical companies, social and health care assistants

- Consumers: patients, patients' relatives, other members of the public

Because consumers have had the opportunity to report ADRs in Denmark since 2003, results from this category only cover data from the last 6.5 years of the study period.

\section{Results}

\subsection{Prescribed psychostimulants, 2000-2009}

Figure 1 displays the annual number of DDD/1000 inhabitant/day and no. of persons/1000 inhabitants/day for all types of psychostimulants prescribed for children from 0 to 17-years of age. 
From 2000 to 2009 the number of prescribed DDDs/1000 inhabitants/day increased with 1629 \% (from 10.87 to $187.97 \mathrm{DDD} / 1000 /$ inhabitants/day) and the number of treated persons/1000 inhabitants/day increased with $775 \%$ (from 21 to 183.67 DDD/1000/inhabitants/day).

From 2000 to 2004 the level of prescribing was rather constant, however from 2005 and onwards large increases in the prescribing of psychostimulants were observed.

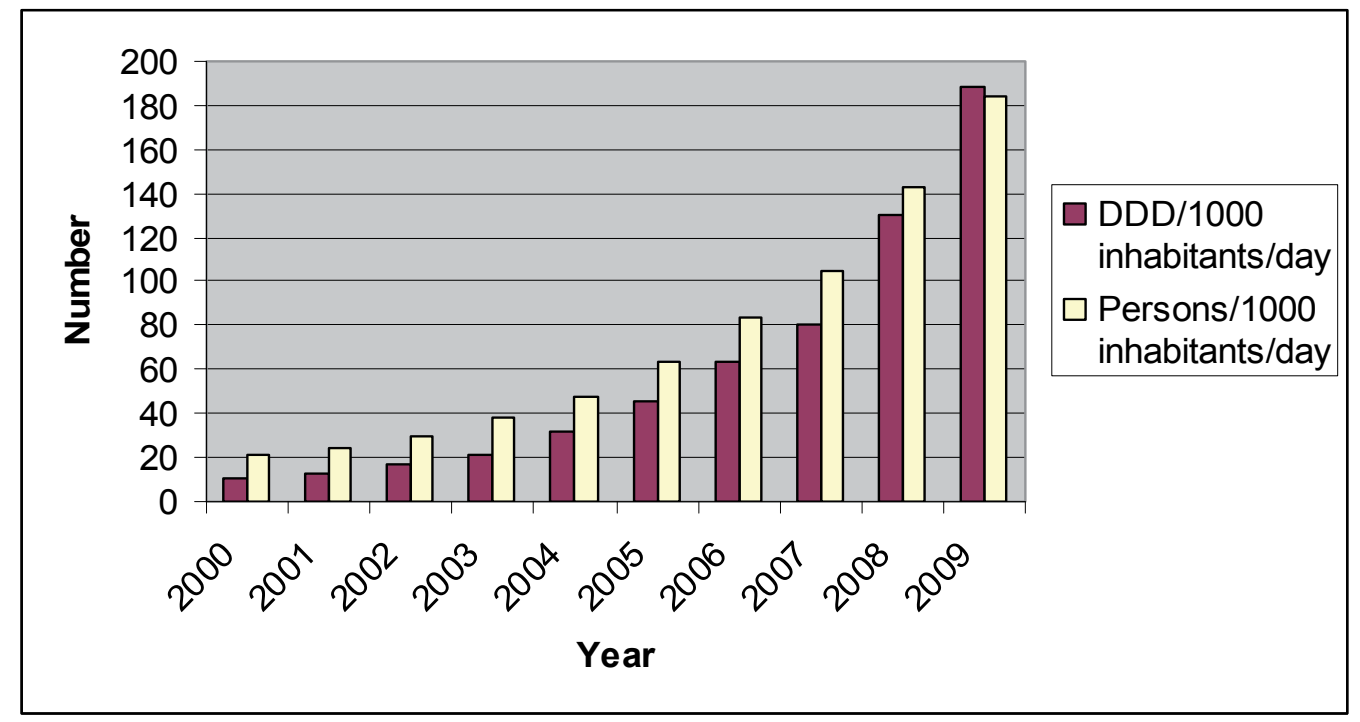

Fig. 1. Annual number of DDDs/1000 inhabitants/day and number of persons/1000 inhabitants/day for psychostimulants for children from 0 to 17-years of age, (2000-2009)

Table 1 displays DDDs per 1000 inhabitant/year per category of psychostimulant medications from 2000 to 2009. Methylphenidate was the dominant prescribed psychostimulant followed by atomoxetine. Only few children and adolescents were prescribed products containing amphetamine derivates and modafinil.

From 2000 to 2009 the number of prescribed DDDs/1000 inhabitants/day increased with $1557 \%$ for methylphenidate (from 10.69 to 177.16 DDD/1000/inhabitants/day), and for atomoxetine an increase in DDDs of $1274 \%$ was observed (from 0.74 to 10.17 DDD/1000/ inhabitants/day).

\begin{tabular}{lllllllllll}
\hline Year & $\mathbf{2 0 0 0}$ & $\mathbf{2 0 0 1}$ & $\mathbf{2 0 0 2}$ & $\mathbf{2 0 0 3}$ & $\mathbf{2 0 0 4}$ & $\mathbf{2 0 0 5}$ & $\mathbf{2 0 0 6}$ & $\mathbf{2 0 0 7}$ & $\mathbf{2 0 0 8}$ & $\mathbf{2 0 0 9}$ \\
\hline $\begin{array}{l}\text { Amphetamine } \\
\text { derivates }\end{array}$ & 0.17 & 0.18 & 0.15 & 0.18 & 0.3 & 0.3 & 0.36 & 0.44 & 0.41 & 0.3 \\
Atomoxetine & 0 & 0 & 0 & 0 & 0 & 0 & 0.74 & 4.03 & 7.17 & 10.17 \\
$\begin{array}{l}\text { Methylphenidate } \\
\text { Modafinil }\end{array}$ & 0 & 0.06 & 0.22 & 0.32 & 0.33 & 0.25 & 0.18 & 0.24 & 0.31 & 0.25 \\
\hline
\end{tabular}

Table 1. Annual number of DDD/1000 inhabitants/day by psychostimulants for children from 0 to 17-years of age, (2000-2009) 
Figure 2 displays the annual distribution of prescribed DDDs/1000 inhabitants/day for methylphenidate by gender. The majority, $80 \%$ of DDDs were prescribed in boys.

From 2000 to 2009 the use of psychostimulants in boys increased with a factor 16 (from 18.92 to 294.45) and in girls with a factor 32 (from 2.39 to 76.03 DDD/1000 inhabitants/day).

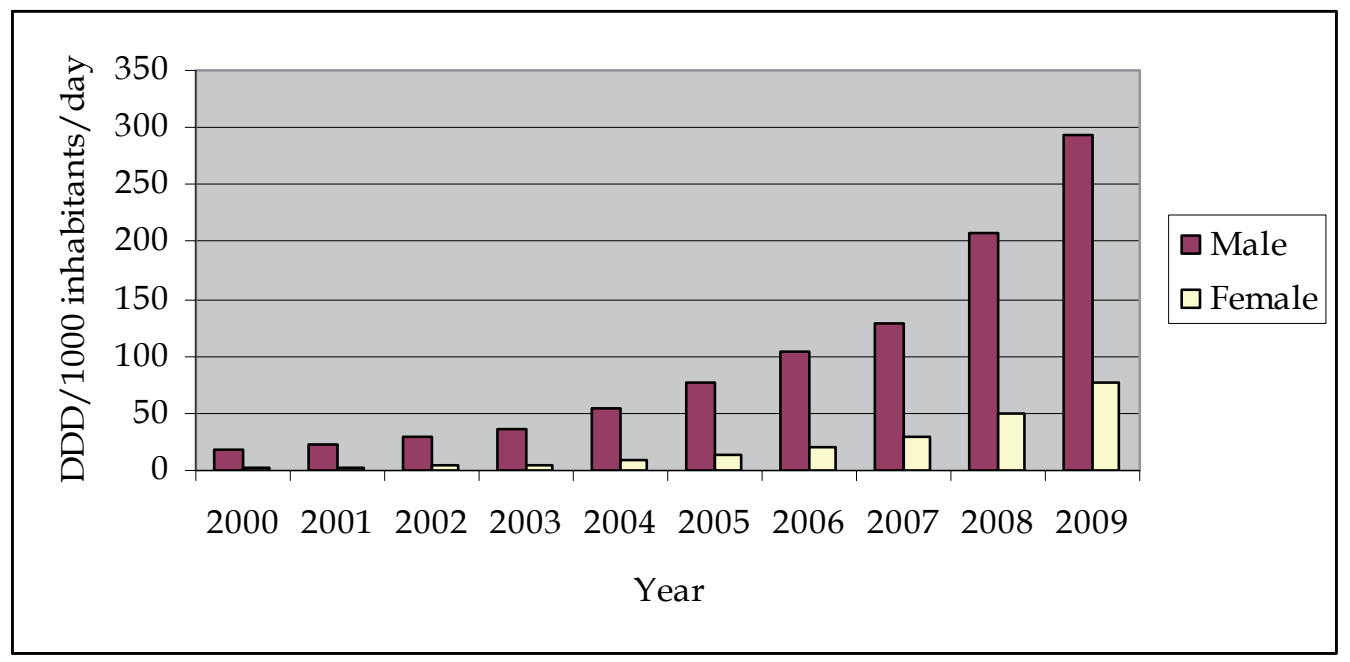

Fig. 2. Annual distribution of DDDs/1000 inhabitants/day for methylphenidate by gender, 2000 - 2009.

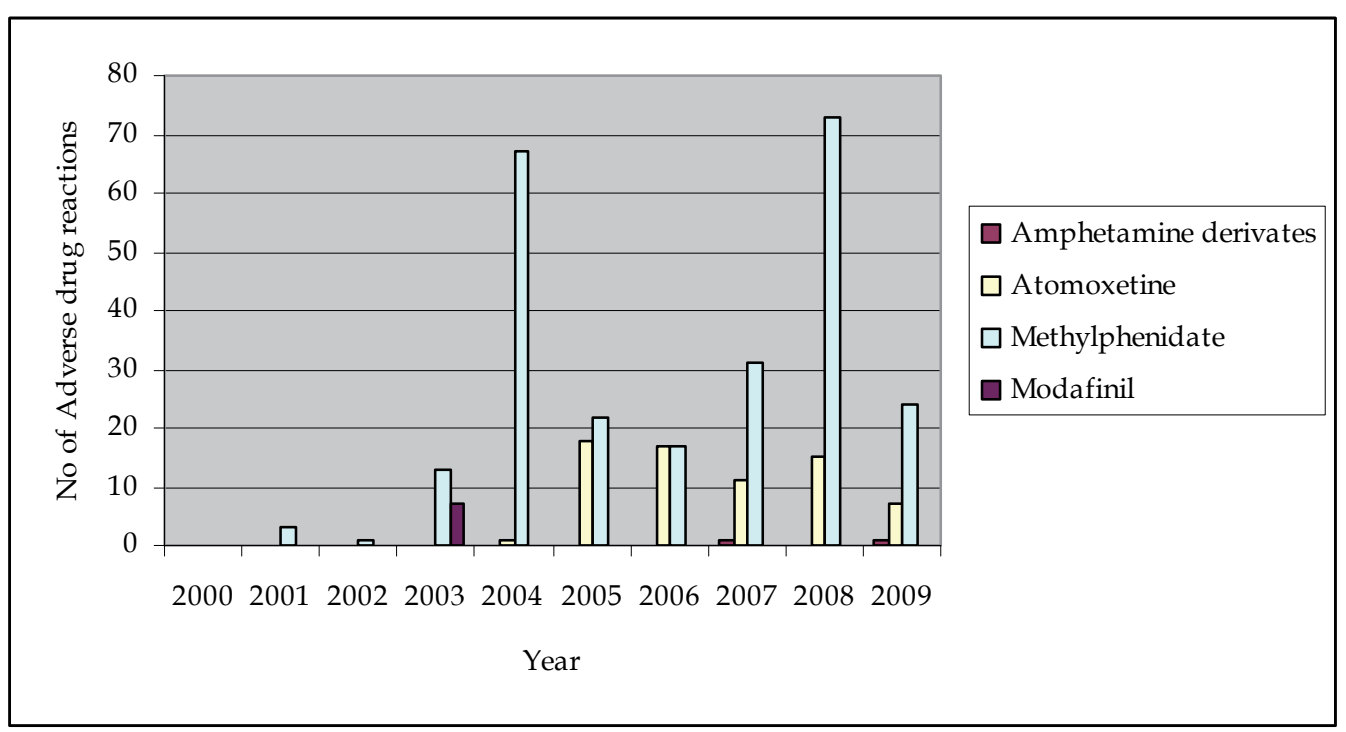

Fig. 3. Annual number of adverse drug reactions (ADRs) reported for psychostimulants in children, $2000-2009$ 


\subsection{Reported adverse drug reactions for psychostimulants, 2000-2009}

From 2000 to 2009 a total of 130 ADR reports including 329 ADRs were reported for psychostimulants for individuals from birth to 17 years of age. One half of ADRs were serious $(\mathrm{N}=165)$, and no fatal cases were reported.

Figure 3 displays the annual number of reported ADRs by type of psychostimulant. The majority of the ADRs were reported for methylphenidate $(\mathrm{N}=251)$ and atomoxetine $(\mathrm{N}=69)$. Only few ADRs were reported for amphetamine derivates $(\mathrm{N}=2)$ and modafinil $(\mathrm{N}=7)$.

\subsubsection{Adverse drug reactions by type of reporter}

The distribution of ADRs reported for psychostimulants by seriousness and type of reporter is displayed in table 2.

Seventy-six percent of ADRs were reported by physicians, $13 \%$ of ADRs by consumers and $10 \%$ of ADRs were reported by other health care professionals.

\begin{tabular}{lrrrr}
\hline Type of reporter & Physician & OHCP* & Consumer & \multicolumn{2}{c}{ Total } \\
\hline Amphetamine derivates & $2(1)$ & 0 & 0 & $2(1)$ \\
Atomoxetine & $68(31)$ & 0 & $1(0)$ & $69(31)$ \\
Methylphenidate & $190(71)$ & $31(25)$ & $30(30)$ & $251(126)$ \\
Modafinil & $7(7)$ & 0 & 0 & $7(7)$ \\
\hline Total & $267(110)$ & $31(25)$ & $31(30)$ & $329(165)$ \\
\hline
\end{tabular}

*: OHCP: other health care professionals

Table 2. Total number of adverse drug reactions (ADRs) distributed by type of reporter, medication and criteria of seriousness (in parentheses), 2000-2009

\subsubsection{Adverse drug reactions by age and gender}

Table 3 shows the distribution of ADRs reported for psychostimulants by age, gender and seriousness in the paediatric population, 2000-2009.

Totally, $80 \%$ of ADRs were reported for boys and $20 \%$ of ADRs for girls.

The largest number of ADRs ( $\mathrm{N}=75$ ) were reported in 9 to 10 -year-olds, followed by 16 to 17 -year olds $(\mathrm{N}=33$ ) and 15 to 16 -year olds $(\mathrm{N}=32)$. Less than one percent of ADRs was reported in children up to 4 years of age.

The largest number of ADRs was reported in 9 to 10-year old boys ( $\mathrm{N}=68$ ). In 16-17 -year olds girls, a larger number of ADRs $(\mathrm{N}=20)$ were reported than in boys. In the age group a number of ADR reports were submitted without information about gender.

Approximately $50 \%$ of ADRs reported for both boys and girls were serious, however large variations were observed within age groups.

Table 4 shows the distribution of reported ADRs by gender and type of psychostimulant. 


\begin{tabular}{lrrrr}
\hline Age groups (Y) & Boys & Girls & NA & Total \\
\hline$<1$ & 0 & 0 & 0 & 0 \\
$1<2$ & 0 & 0 & 0 & 0 \\
$2<3$ & 0 & 0 & 0 & 0 \\
$3<4$ & 0 & 0 & 0 & 0 \\
$4<5$ & $1(0)$ & 0 & 0 & $1(0)$ \\
$5<6$ & $6(2)$ & 0 & 0 & $6(2)$ \\
$6<7$ & $18(13)$ & 0 & 0 & $18(13)$ \\
$7<8$ & $10(5)$ & $4(0)$ & $3(0)$ & $17(5)$ \\
$8<9$ & $21(7)$ & $4(3)$ & 0 & $25(10)$ \\
$9<10$ & $68(42)$ & $7(0)$ & 0 & $75(42)$ \\
$10<11$ & $14(1)$ & 0 & 0 & $14(1)$ \\
$11<12$ & $20(6)$ & $1(0)$ & 0 & $21(6)$ \\
$12<13$ & $19(9)$ & $1(0)$ & 0 & $20(9)$ \\
$13<14$ & $23(16)$ & $3(0)$ & 0 & $26(16)$ \\
$14<15$ & $16(5)$ & $5(3)$ & 0 & $21(8)$ \\
$15<16$ & $24(10)$ & $8(6)$ & 0 & $32(16)$ \\
$16<17$ & $13(8)$ & $20(15)$ & 0 & $33(23)$ \\
$17<18$ & $18(10)$ & $2(0)$ & 0 & $20(10)$ \\
\hline Total & $271(134)$ & $55(31)$ & $3(0)$ & $329(165)$ \\
\hline & & & &
\end{tabular}

*: ADR reports without information about gender.

Table 3. Total number of reported ADRs by age group and gender, serious ADRs in parentheses, 2000 - 2009

\begin{tabular}{lrrrr}
\hline Medicines & Boys & Girls & NA & Total \\
\hline Amphetamine derivates & $1(1)$ & 1 & 0 & $2(1)$ \\
Atomoxetine & $48(22)$ & $21(9)$ & 0 & $69(31)$ \\
Methylphenidate & $221(110)$ & $34(23)$ & $3(0)$ & $258(133)$ \\
Modafinil & $7(7)$ & 0 & 0 & 0 \\
\hline Total & $271(134)$ & $55(31)$ & $3(0)$ & $329(165)$ \\
\hline
\end{tabular}

Table 4. Distribution of total number of reported ADRs $(\mathrm{N})$ by gender and medication (numbers of serious ADRs in parentheses), 2000 - 2009

Approximately $85 \%$ of ADRs were reported in boys, the majority for methylphenidate and atomoxetine. All ADRs reported for modafinil occurred in boys and they were all serious. For amphemine derivates one out of 2 reported ADRs occurred in boys.

Approximately $45 \%$ of ADRs occurring in boys reported for atomoxetine and methylphenidate were serious. 


\subsubsection{Adverse drug reactions by type and seriousness}

Table 5 displays the characteristics of ADRs reported for psychostimulants by type, system organ class, seriousness and medication.

The largest share of reported ADRs was of the type "psychiatric disorders" (21\% of total ADRs) followed by the SOCs "general disorders" (19\% of total ADRs) and "nervous system disorders" (16\% of total ADRs).

For ADRs of the type nervous- and psychiatric disorders the share of serious ADRs ranged from approximately 50 to $70 \%$ of total ADRs. For the medications atomoxetine and methylphenidate approximately $45 \%$ of all reported ADRs were serious, and for modafinil all reported ADRs were serious.

\begin{tabular}{|c|c|c|c|c|c|}
\hline $\begin{array}{l}\text { System Organ Class (SOC) } \\
\text { (alphabetical order) }\end{array}$ & $\begin{array}{c}\text { Amphetamine } \\
\text { derivates }\end{array}$ & Methylphenidate & Atomoxetine 1 & Modafinil & Total \\
\hline $\begin{array}{l}\text { Blood and lymphatic system } \\
\text { disorders }\end{array}$ & 0 & $2(2)$ & 0 & 0 & $2(1)$ \\
\hline Cardiac disorders & 0 & $11(4)$ & $6(3)$ & 0 & $17(7)$ \\
\hline $\begin{array}{l}\text { Congenital, familial and genetic } \\
\text { disorders }\end{array}$ & 0 & 0 & $1(1)$ & 0 & $1(1)$ \\
\hline Ear and labyrinth disorders & 0 & 1 & 0 & 0 & $1(0)$ \\
\hline Endocrine disorders & 0 & 0 & 2(2) & 0 & $2(2)$ \\
\hline Eye disorders & 0 & 6 & 0 & 0 & $6(0)$ \\
\hline Gastrointestinal disorders & 1 & $22(12)$ & $10(2)$ & $1(1)$ & $34(15)$ \\
\hline General disorders & 0 & $55(15)$ & $5(2)$ & 0 & $60(17)$ \\
\hline Infections and infestations & 0 & 0 & $1(1)$ & 0 & $1(1)$ \\
\hline Investigations & 0 & $13(10)$ & $7(3)$ & 0 & 20(13) \\
\hline Metabolism and nutrition disorders & 0 & $7(1)$ & 3 & 0 & 10(1) \\
\hline Musculoskeletal disorders & 0 & $6(3)$ & 1 & 0 & $7(3)$ \\
\hline Nervous system disorders & 0 & $48(33)$ & $4(3)$ & $3(3)$ & $55(39)$ \\
\hline Psychiatric disorders & $1(1)$ & $48(32)$ & $21(12)$ & $3(3)$ & $73(48)$ \\
\hline Renal and urinary disorders & 0 & $3(1)$ & 1 & 0 & $4(1)$ \\
\hline $\begin{array}{l}\text { Reproductive system and breast } \\
\text { disorders }\end{array}$ & 0 & 1 & 0 & 0 & $1(0)$ \\
\hline Respiratory disorders & 0 & $4(2)$ & 1 & 0 & $5(2)$ \\
\hline $\begin{array}{l}\text { Skin and subcutaneous tissue } \\
\text { disorders }\end{array}$ & 0 & $19(5)$ & $3(1)$ & 0 & $22(6)$ \\
\hline Surgical and medical procedures & 0 & 0 & 1 & 0 & $1(0)$ \\
\hline Vascular disorders & 0 & $5(2)$ & $2(1)$ & 0 & $7(3)$ \\
\hline Total & $2(1)$ & $251(126)$ & $69(31)$ & $7(7)$ & $329(165)$ \\
\hline
\end{tabular}

Table 5. Total number of reported ADRs (N) for children distributed by System Organ Class (SOC), medication (serious ADRs in parentheses), 2000-2009 
Table 6 shows characteristics of ADRs reported for the SOC "psychiatric disorders" by type of medication and seriousness.

\begin{tabular}{|c|c|c|c|c|c|}
\hline $\begin{array}{l}\text { Adverse drug reaction (ADR) } \\
\text { (alphabetical order) }\end{array}$ & $\begin{array}{c}\text { Amphetamine } \\
\text { derivates }\end{array}$ & Methylphenidate & Atomoxetine & Modafinil & Total \\
\hline Affect lability & 0 & $3(3)$ & 0 & 0 & $3(3)$ \\
\hline Agitation & 0 & $2(1)$ & 0 & 0 & $2(1)$ \\
\hline Aggression & 0 & 1 & 2 & $1(1)$ & $4(1)$ \\
\hline Anger & 0 & 0 & $2(1)$ & 0 & $2(1)$ \\
\hline Animal phobia & 0 & $1(1)$ & 0 & 0 & $1(1)$ \\
\hline Anxiety & 0 & $6(2)$ & $2(2)$ & 0 & $8(4)$ \\
\hline Asocial behaviour & 0 & 1 & 0 & 0 & 1 \\
\hline Communication disorder & 0 & $1(1)$ & 0 & 0 & $1(1)$ \\
\hline Confusional state & 0 & $2(1)$ & 0 & 0 & $2(1)$ \\
\hline Depression & 0 & $4(3)$ & $4(1)$ & 0 & $8(4)$ \\
\hline Drug abuse & 0 & $2(2)$ & 0 & 0 & $2(2)$ \\
\hline Emotional disorder & 0 & 1 & 0 & 0 & 1 \\
\hline Euphoric mood & 0 & $2(2)$ & 0 & 0 & $2(2)$ \\
\hline Food aversion & 0 & 0 & $1(1)$ & 0 & $1(1)$ \\
\hline Hallucination & 0 & $2(2)$ & 0 & $1(1)$ & $3(3)$ \\
\hline Impulse-control disorder & 0 & 0 & 1 & 0 & 1 \\
\hline Insomnia & 0 & $3(2)$ & 0 & 0 & $3(2)$ \\
\hline Intentional self-injury & 0 & 0 & $1(1)$ & 0 & $1(1)$ \\
\hline Mood swings & 0 & 0 & $1(1)$ & 0 & $1(1)$ \\
\hline Nightmare & 0 & 0 & 1 & $1(1)$ & $2(1)$ \\
\hline Obsessive thoughts & 0 & 0 & $1(1)$ & 0 & $1(1)$ \\
\hline Psychotic disorder & $1(1)$ & 0 & $1(1)$ & 0 & $2(2)$ \\
\hline Personality change & 0 & $1(1)$ & 0 & 0 & $1(1)$ \\
\hline Restlessness & 0 & $1(1)$ & 0 & 0 & $1(1)$ \\
\hline Sleep disorder & 0 & $7(5)$ & $1(1)$ & 0 & $8(6)$ \\
\hline Social avoidant behaviour & 0 & $3(3)$ & 0 & 0 & $3(3)$ \\
\hline Suicidal ideation & 0 & $1(1)$ & $3(2)$ & 0 & $4(3)$ \\
\hline Tic & 0 & 2 & 0 & 0 & 2 \\
\hline Total & 1(1) & $46(33)$ & $21(12)$ & $3(3)$ & $73(48)$ \\
\hline
\end{tabular}

Table 6. Total number of reported ADRs of the type "psychiatric disorders" by medication, and seriousness (in parentheses), 2000-2009.

Two-third of ADRs was reported for amphetamine derivates and methylphenidate, and the most frequently reported ADRs were anxiety, depression, and sleep disorders.

Table 7 displays the characteristics of ADRs reported for the SOC "nervous system disorders" by type of medication and seriousness.

Within this SOC approximately $90 \%$ of ADRs were reported for methylphenidate, the majority of reactions being dizziness, headache and dyskinesia. 


\begin{tabular}{lrrrrr}
\hline Adverse drug reaction (ADR) & $\begin{array}{c}\text { Amphetamine } \\
\text { derivates }\end{array}$ & Methylphenidate & Atomoxetine & Modafinil & Total \\
\hline Akathisia & 0 & $1(1)$ & 0 & 0 & $1(1)$ \\
Atypical benign partial epilepsy & 0 & 0 & $1(1)$ & 0 & $1(1)$ \\
Burning sensation & 0 & 2 & 0 & 0 & 2 \\
Cataplexy & 0 & 0 & 0 & $1(1)$ & $1(1)$ \\
Crying & 0 & $2(1)$ & 0 & 0 & $1(1)$ \\
Demyelination & 0 & $2(2)$ & 0 & 0 & $2(2)$ \\
Disturbance in attention & 0 & $1(1)$ & 0 & 0 & $1(1)$ \\
Dizziness & 0 & $8(5)$ & $1(1)$ & $1(1)$ & $10(7)$ \\
Dyskinesia & 0 & $2(2)$ & 0 & 0 & $2(2)$ \\
Dystonia & 0 & $1(1)$ & 0 & 0 & $1(1)$ \\
Epilepsy & 0 & $2(1)$ & 0 & 0 & $2(1)$ \\
Headache & 0 & $12(9)$ & $2(1)$ & 0 & $14(10)$ \\
Hemiparesis & 0 & $2(2)$ & 0 & 0 & $2(2)$ \\
Hypoaesthesia & 0 & 0 & 0 & $3(2)$ \\
Hypotonia & 0 & 0 & 0 & $1(1)$ & $1(1)$ \\
Loss of consciousness & 0 & $2(1)$ & 0 & 0 & $2(1)$ \\
Nystagmus & 0 & 1 & 0 & 0 & 1 \\
Psychomotor hyperactivity & 0 & $2(1)$ & 0 & 0 & $2(1)$ \\
Restless legs syndrome & 0 & 1 & 0 & 0 & 1 \\
Somnolence & 0 & $2(1)$ & 0 & 0 & $1(1)$ \\
Speech disorder & 0 & 0 & 0 & $2(2)$ \\
Syncope & 0 & $4(1)$ & 0 & 0 & $1(1)$ \\
Total & 0 & $4(3)$ & $3(3)$ & $55(39)$ \\
\hline & & & & & \\
\end{tabular}

Table 7. Total reported ADRs of the type "nervous system disorders" by medication, and seriousness (in parentheses), 2000-2009.

Table 8 shows the characteristics of ADRs reported for the SOC "general disorders and administration site conditions" by type of medication and seriousness.

Within this SOC almost all ADRs were reported for amphetamine derivates, and no ADRs were reported for atomoxetine and modafinil. Many ADRs relating to the efficacy of the medications were reported.

A large number of ADRs within the SOC "gastrointestinal disorders" were also reported (see table 5). These reactions were predominantly reported for methylphenidate, and were of the type's abdominal pain, diarrhoea and dry mouth (data not shown).

\section{Discussion}

This is the first study from a national ADR database that has examined the characteristics and occurrence of ADRs from use of psychostimulants in the paediatric population. The study showed that over a decade the prescribing of psychostimulants in the Danish paediatric population has increased dramatically, and that methylphenidate was the predominant psychostimulant. Two third of ADRs were reported by physicians, and approximately $80 \%$ of all ADRs were reported for boys from above 5 years of age. The 


\begin{tabular}{lrrrrr}
\hline Adverse drug reaction (ADR) & $\begin{array}{c}\text { Amphetamine } \\
\text { derivates }\end{array}$ & Methylphenidate & Atomoxetine Modafinil & Total \\
\hline Asthenia & 0 & $2(2)$ & 0 & 0 & $2(2)$ \\
Chest pain & 0 & $5(2)$ & 0 & 0 & $5(2)$ \\
Chills & 0 & 1 & 0 & 0 & 1 \\
Drug effect decreased & 0 & $17(1)$ & 0 & 0 & $19(1)$ \\
Drug effect increased & 0 & 1 & 0 & 0 & 1 \\
Drug ineffective & 0 & $14(2)$ & 0 & 0 & $14(2)$ \\
Drug interaction & 0 & $1(1)$ & $1(1)$ & 0 & $2(2)$ \\
Face oedema & 0 & 1 & 0 & 0 & 1 \\
Feeling hot & 0 & 1 & 0 & 0 & 1 \\
Fatigue & 0 & $7(6)$ & $3(1)$ & 0 & $10(7)$ \\
Irritability & 0 & 0 & 1 & 0 & 1 \\
Pain & 0 & $1(1)$ & 0 & 0 & $1(1)$ \\
Pyrexia & 0 & 1 & 0 & 0 & 1 \\
Therapeutic response unexpected & 0 & 3 & 0 & 0 & $3(0)$ \\
with drug substitution & 0 & $55(15)$ & $5(2)$ & 0 & $60(17)$ \\
\hline Total & 0 & & & & \\
\hline
\end{tabular}

Table 8. Total number of reported adverse drug reactions of the type "general disorders and administration site conditions" by medication, and seriousness (in parentheses), 2000-2009.

largest shares of reported ADRs was of the type "general disorders and administration conditions"; "psychiatric and nervous system disorders".

From 2000 to 2009 large increases in number of prescribed dosages and users were observed, and these findings were in line with results from other countries (Asheim et al. 2007; Kalverdijk et al., 2008; Trip et al., 2009; Schubert et al, 2010; Zuvekas et al., 2006). The majority of users were boys; but we found a large increase in use of psychostimulants among girls, and this pattern was expected (Hodgkins et al, 2011). From 2000 to 2004 the use of psychostimulants were at a stable level, however from 2005 the use has increased dramatically. Explanations of this increase could be the marketing pressure, change in diagnostic criteria and the development of new types of psychostimulants such as atomoxetine and modafinil for treatment of ADHD. In the same period of time the pharmaceutical companies have marketed long-term release formulations of methylphenidate for school use (van den Ban et al, 2010). Use of psychostimulants in Danish children has increased more over the last decade than in other countries; however this may be explained by the fact that use of psychostimulants in Denmark was lower at the beginning of the period than in other countries.

This is a descriptive study, and therefore we cannot conclude whether ADRs are reported more commonly for methylphenidate than the other products, neither whether girls experience more ADRs than boys. The number of ADR reports does not reflect the use of psychostimulants, and the question is where in the lifecycle of medicinal products ADRs are reported. However, since the issue is long-term treatment of children and adolescents ADR signals are extremely important. A high number of reports on decreased drug effect and/or ineffective drugs from use of methylphenidate were reported. Whether this signal is due to problems with the quality of generic products or that real life is different from the testing conditions (Hansen, 1992).

The majority of reports were submitted by physicians, however, we would also have expected a larger number of reports from parents, as they should be concerned about administration 
medications to their children, especially medications for long-term use, where information about long-term effects are limited. The explanation of the large number of reports from physicians could be that the parents have become aware of the ADRs, and asked their physician to report them. To increase knowledge about ADRs from long term use of psychostimulants the number of reports submitted to the Danish Medicines Agency and probably other medicines agencies should be increased and the regulatory authorities must focus on increasing ADR reporting rates from both health care professionals and consumers.

An empirical study showed that the licensing material for methylphenidate provided minimal evidence on efficacy and safety in children (Aagaard et al. 2009b), and no information about long-term safety aspects was reported. Spontaneous reports are an important source of information about new and previously unrecognized ADRs, and the value of spontaneous reporting schemes lies in their ability to act as hypothesis-generating signals. Due to the low number of ADRs reported for psychostimulants in Denmark it is necessary to conduct further studies to explore data about rare and serious ADRs in larger databases, such as the EU database EudraVigilance and the international WHO-ADR database Vigibase (Aagaard et al, 2010). These databases contain large amounts of data submitted from European and global populations, and are therefore suitable for studying specific ADRs or new signals.

The strength of our study is that data comprised psychostimulant prescriptions and all ADRs reported in one country over a decade. The purpose was to analyse information reported to the Danish ADR database on ADRs in the paediatric population from use of psychostimulants in the treatment of ADHD, and not to calculate the incidence of ADRs in this population as this is not feasible in material based on spontaneous reporting.

A major limitation to this study is that we do not know to which extent the causality of these ADRs can be confirmed, and this has implications for the interpretation of the results. Spontaneous reporting systems suffer from different barriers, such as incomplete recognition of ADRs, administrative barriers to reporting, and low data quality, all of which may result in the underreporting of important serious and rare events. Nonetheless, the study provides information on reported ADRs, and this information contributes to broadening the knowledge base on psychostimulants safety.

\section{Conclusion}

In Denmark, a large increase in the use of psychostimulants in the paediatric population has been observed. The large number of serious reported ADRs indicate that psychostimulants should be prescribed with caution of that greater care is needed in relation to prescribing these medications for children. The Danish Medicines Agency should monitor prescribing patterns more tightly to identify potential risks in the paediatric population in relation to the evolving utilisation of use of psychostimulants among children and adolescents and related risks.

\section{References}

Aagaard, L.; Stenver, DI.; Hansen, EH. (2008b). Structures and processes in spontaneous reporting systems: a comparative study of Australia and Denmark. Pharmacy World and Science, Vol. 30, pp. 563-70. 
Aagaard, L.; Hansen, EH. (2009a). Information about ADRs explored by pharmacovigilance approaches: a qualitative review of studies on antibiotics, SSRIs and NSAIDs. BMC Clinical Pharmacology, Vol. 3; No. 9: pp. 4.

Aagaard, L.; Thirstrup, S.; Hansen, EH. (2009b). Opening the white boxes: the licensing documentation of efficacy and safety of psychotropic medicines for children. Pharmacoepidemiology and Drug Safety, Vol.18, pp. 401-411.

Aagaard L, Christensen A, Hansen EH. (2010). Information about adverse drug reactions reported in children: a qualitative review of empirical studies. British Journal of Clinical Pharmacology, Vol. 70, pp. 481-491.

Asheim, H. ; Nilsen, KB ; Johansen, J ; Furu, K. (2007). Prescribing of stimulants for ADHD In Nordland County. Tidsskrift for den Norske Laegeforening, Vol. 127, pp. 2360-2362. [In Norwegian]

Bloch, MH.; Panza, KE.; Landeros-Weisenberger, A.; Leckman, JF. (2009). Metaanalysis:treatment of attention-deficit/hyperactivity disorder in children with comorbid tic disorders. Journal of American Adolescent Psychiatry, Vol. 48, pp. 884-893.

European Medicines Agency. Meeting highligths from the Committee for Medicinal Products for Human Use, Ref. EMEA/431407/2007 London, (2007).

Food and Drug Administration. Ritalin-Ritalin SR. (2007).

Hansen, EH. (1992). Technology assessment in a user perspective - experiences with drug technology. International Journal of Technology Assessment in Health Care, Vol. 8, pp. 150-165.

Hodgkins, P.; Sasane, R.; Meijer, WM. (2011). Pharmacologic treatment of attentiondeficit/hyperactivity disorder in children: incidence, prevalence, and treatment patterns in the Netherlands. Clinical Therapeutics, Vol. 33, pp. 188-203.

Howard, P.; Shuster, J.; Twycross, R.; Mihalyo, M.; Wilcock, A. (2010). Psychostimulants. Journal of Pain and Symptomatic Management. Vol. 40, pp. 789-795.

Kalverdijk, LJ.; Tobi, H.; van den Berg, PB.; Buiskool, J.; Wagenaar, L.; Minderaa, RB.; de Jong-van den Berg, LT. (2008). Use of antipsychotic drugs among Dutch youths between 1997 and 2005. Psychiatric Services; Vol. 59, pp. 554-560.

Pringsheim, T.; Steeves, T. (2011). Pharmacological treatment for Attention Deficit Hyperactivity Disorder (ADHD) in children with comorbid tic disorders. Cochrane Database Systematic Reviews, Vol. 13, CD007990.

Schubert, I. ; Köster, I. ; Lehmkuhl, G. (2010). The changing prevalence of attentiondeficit/hyperactivity disorder and methylphenidate prescriptions. Deutsches Ärtzeblatt International, Vol. 107, pp. 615-621.

Trip, A-M.; Visser, ST.; Kalverdijk, L.; de Jong-van den Berg, LTW. (2009). Large increase of the use of psycho-stimulants among youth in the Netherlands between 1996 and 2006. British Journal of Clinical Pharmacology; Vol. 67, pp. 466-468.

Van den Ban, E.; Souverein, PC.; Swaab, H.; van Engeland, H.; Egberts, TC.; Heerdink, ER. (2010). Less discontinuation of ADHD drug use since the availability of long-acting ADHD medication in children, adolescents, and adults under the age of 45 in the Netherlands. Attention Deficit Hyperactivity Disorder, Vol. 2; pp. 213-220.

World Health Organization (WHO) Collaborating Centre for Drug Statistics Methodology, 2011. Volume 9: ICH guideline for medicinal products for human use and veterinary products (2009).

Zuvekas, SH. ; Vitiello, B. ; Norquist, GS. (2006). Recent trends in stimulant medication use among U.S. children. American Journal of Psychiatry, Vol. 163, pp.579-585. 


\title{
Do Stimulant Medications for Attention-Deficit/Hyperactivity Disorder (ADHD) Enhance Cognition?
}

\author{
Claire Advokat and Christine Vinci \\ Louisiana State University, \\ USA
}

\section{Introduction}

Characteristic symptoms of Attention-Deficit/Hyperactivity Disorder (ADHD) have been recognized in the medical literature for over 200 years. The earliest known clinical description is found in the book by the Scottish physician Sir Alexander Crichton, entitled An Inquiry into the Nature and Origins of Mental Derangement (Crichton, 1798, as cited in Baumeister et al., 2011). In the chapter, "Attention, and its Diseases" (p. 254), Crichton notes that these conditions make people "incapable of attending with constancy to any one object of education" (p. 271), cause "mental restlessness", "walking up and down", and the "fidgets" (p. 272). Although Crichton was clearly describing disorders of attention, George Still, a British pediatrician, is usually credited with being the first person to describe the syndrome that has since been recognized as ADHD. Dr. Still gave a series of lectures on Some Abnormal Psychical Conditions in Children in 1902, in which he noted that even some children with normal intelligence may exhibit a "lack of attention which is very noticeable... [and which] no doubt accounts to a considerable extent for backwardness in school acquirements" (Still, 1902b, p. 1081 as cited in Baumeister et al., 2011).

Since those original descriptions, the diagnostic characterization of ADHD has been revised numerous times. The three core symptoms are presently considered to be Inattention, Impulsivity and Hyperactivity (Baumeister et al., 2011; Biederman \& Faraone, 2005; Davidson, 2008). Current prevalence estimates in the United States are 6-9\% for children and adolescents and 3-5\% in adults (Dopheide \& Plizka, 2009). In the most recent National Comorbidity Survey, in which nearly 3200 adults, 19 to 44 years of age, were screened, the prevalence was $4.4 \%$ (Kessler et al., 2006).

Unlike the childhood presentation, symptoms of hyperactivity and impulsivity are less prominent in ADHD-diagnosed adults than problems resulting from inattention, distractibility and disorganization (Dopheide \& Plizka, 2009). The psychosocial difficulties caused by these symptoms have been well-documented, including marital and relationship problems, poor job performance and employment histories, and lower socioeconomic status (Biederman et al., 2011a; Fischer et al., 1990; Hechtman et al., 1984; Hechtman \& Greenfield, 2003; Ingram et al., 1999; Mannuzza et al., 1993; Spencer et al., 2007). Many adults with ADHD report frequent employment changes, difficulty in organizing finances, and 
household and parental management responsibilities, dangerous driving, and unstable social relationships or social isolation (Weiss \& Murray, 2003). Adults with ADHD are less likely to attain the same educational (and occupational) level as those without the diagnosis relative to what would be predicted based on their IQ, even with pharmacotherapy (Biederman et al., 2006; Biederman et al., 2008a; Mannuzza et al., 1993). Moreover, cognitive deficits of adults with ADHD, relative to adults without the diagnosis, do not change across the lifespan (Biederman et al, 2010). For example, although 84\% of ADHD-diagnosed adults were statistically expected to be college graduates, only $50 \%$ reached this level of education (Biederman et al., 2008a).

In adults, as with youth, first-line treatment options include the stimulant drugs, usually one of the many formulations of either methylphenidate (MPH) or amphetamine (AMPH) (Adler et al., 2007; Berman et al., 2009; Dopheide \& Plizka, 2009; Dodson, 2005; Faraone et al., 2004; Paterson et al., 1999; Wender et al., 2011). These agents are the most efficacious drug treatments for ADHD, with large effect sizes, as measured by standardized rating scales in clinical trials. Methylphenidate and amphetamine formulations are considered similar in efficacy, with between $55-75 \%$ of drug-treated patients (compared with $4-30 \%$ of placebo - treated patients) showing "clinically significant" improvement for up to 4 to 6 weeks (Berman et al., 2009; Dopheide \& Plizka, 2009). Available information indicates that on standard efficacy measures, amphetamine is at least equivalent may be superior to methylphenidate, and, that individuals with ADHD who don't respond to methylphenidate will show significant improvement on amphetamine (Berman et al., 2009). When both drugs are tried, response rates may be as high as 85\% (Dopheide \& Plizka, 2009).

Given their substantial and reliable clinical benefit for treatment of attention disorders, it is not surprising that prescriptions for stimulants have increased dramatically in the last few years. Between 1998 and 2005, there was a 133\% increase in amphetamine product prescriptions and a 52\% increase in methylphenidate products, for teenagers and preteenagers in the US (Setlik et al., 2009). According to US government data, from 1998 to 2007, total amphetamine prescriptions increased by about 11.7 million, or 463\% (Stix, 2009).

The increase in stimulant prescriptions has resulted in a corresponding escalation of illicit use, particularly in college students, confirmed by numerous survey results (Advokat et al., 2008; Arria \& DuPont, 2010; Hall et al., 2005; McCabe et al., 2005; Rabiner et al., 2008; 2009a, 2009b; Rabiner et al., 2010; Teter et al., 2003; Teter et al., 2005; Teter et al., 2006; Weyandt et al., 2009; White et al., 2006; Wilens et al., 2008). Wilens and colleagues (2008) report lifetime rates of diversion ranging from 16 to $29 \%$, with medical prescriptions being given, sold or traded by students. Studies consistently show that most students report using stimulant medications, legally or illicitly, to improve academic performance, specifically to increase concentration, organization, and the ability to stay up longer and study. Because the rationale for illicit stimulant use in undergraduates is usually stated to be improvement of academic performance, rather than recreational, it is not always considered to be as problematic as other types of drug abuse. Unfortunately, this is not necessarily the case, and the medical and legal consequences of illicit stimulant use may be underappreciated (Arria \& DuPont, 2010; Arria et al., 2008; Arria et al., 2011).

The current escalation in stimulant diversion and misuse has initiated debate about the moral implications of using drugs to improve academic performance. Ethical discussions about taking drugs for 'cognitive enhancement,' have been the subject of several editorials 
and commentaries (Farah et al., 2004; Greely et al., 2008; Harris, 2009; Monastersky, 2008), which confirmed the widespread use of these agents, especially among college students, professionals and academics. Often the term is used very broadly to include drugs "...that improve memory, concentration, planning and reduce impulsive behavior and risky decision-making..."(Sahakian \& Morein-Zamir, 2007, p. 1157). Thoughtful proposals for the 'responsible use of cognitive-enhancing drugs' are espoused, calling for the scientific study of the expected risks and the benefits to be gained as well as the moral consequences of allowing broad access to pharmacological enhancement of mental capacities.

These developments led the Ethics, Law and Humanities Committee of the American Academy of Neurology (AAN) to release a special report, "Responding to requests from adult patients for neuroenhancements," (Larriviere et al., 2009). According to lead author, Dan Larriviere, "A growing number of patients without illness believe they can improve their memory, cognitive focus and attention span by taking neuroenhancement drugs and are asking for prescriptions." For the most part, these 'neuroenhancers' consist of stimulant drugs. "The drugs most commonly used for cognitive enhancement at present are stimulants, namely Ritalin (methylphenidate) and Adderall (mixed amphetamine salts), and are prescribed mainly for the treatment of attention deficit hyperactivity disorder (ADHD)." One of the strongest endorsements was expressed at the 60th Annual Conference of the Canadian Psychiatric Association in 2010 by Dr. Derryck Smith who presented a workshop on the subject and stated that psychiatrists should not hesitate to prescribe stimulants for neuroenhancement, if they wish. "We know they work....I think the effects of these medications are the same whether you have a medical diagnosis or not - they make everybody better" (Johnson, 2010).

These developments illustrate the fact that, because stimulants have been used effectively for decades to reduce hyperactivity, impulsivity and inattention in children, and now adults, with ADHD, it has understandably been assumed that the drugs enhance long-term intellectual performance. Although that would seem to be a reasonable conclusion, it turns out that the scientific evidence for this conclusion is less than compelling. Recent reviews (Advokat, 2010; de Jongh et al., 2008; Repantis et al., 2010; Smith \& Farah, 2011) provide very little experimental support for stimulant-induced cognitive enhancement. deJongh (2008) cites a few research studies that found some improvement in acute memory task performance with amphetamine in individuals with a low memory baseline, "...while high[memory]span subjects are either not affected or get worse" (p. 763). Similar results were summarized for methylphenidate, "With regard to MPH [methylphenidate], we were not able to provide sufficient evidence of positive effects in healthy individuals from objective tests" (Repantis et al., 2010, p. 204). A more detailed analysis of the scientific research on stimulant-induced cognitive effects in adults with and without ADHD (Advokat, 2010) also found little support for 'cognitive neuroenhancement' with these drugs. And recent articles in the New Yorker (Talbot, 2009) and Scientific American (Stix, 2009), describing the current resurgence of these agents confirm the modest intellectual benefit derived from their use in the 'real world.'

Accordingly, this selective review will discuss the evidence regarding cognitive effects of the two major stimulant medications, amphetamine (AMPH) and methylphenidate (MPH). We will emphasize information related to academic outcomes, incorporating some results of our own research on the neuropsychological and cognitive effects of stimulant medications 
in college undergraduates, which show that these drugs do not reduce the academic disparity between ADHD-diagnosed and nonADHD-diagnosed students. We will discuss explanations proposed to account for the lack of cognitive improvement with stimulant drugs. Our goal is to shed some light on the apparent paradox of stimulant medications, namely: Why do drugs that acutely increase attention and concentration produce so little long-term intellectual benefit?

\section{Academic achievement of children and adolescents with ADHD}

The beneficial effect of stimulant drugs for classroom manageability of behavior-disordered children was first reported by Bradley (1937). During short, weekly, treatment periods he described increased productivity, comprehension and accuracy of the children, particularly in output of arithmetic problems. Since then, a vast literature has confirmed similar shortterm benefits. These medications have been shown to acutely increase the quality of notetaking, scores on quizzes and worksheets, writing output and homework completion. The drugs"... reduce overactivity, restlessness and distractibility, enhance attention span or concentration and reduce impulsivity in responding to various tasks. Since a child who is attentive and better able to concentrate would presumably learn more from his classroom experiences, it should follow that these stimulant drugs would facilitate the scholastic performance of hyperkinetic children" (Barkley \& Cunningham, 1978; p. 85). Nevertheless, it has been recognized for over 30 years that there is little evidence that these drugs improve the long-term academic achievement of ADHD diagnosed children.

Barkley and Cunningham (1978) reported in the first review of the topic, that in long term studies lasting at least one year (and as long as 5 to 10 years) the drugs had little impact on academic outcome. A substantial proportion of ADHD-diagnosed children were in special schools or classes, had failed one or more grades, had reading or arithmetic difficulty and were having problems sitting still and studying. The authors concluded that, in spite of various procedural differences among the published studies, the outcomes were the same stimulant drugs had little impact on the "...long-term academic outcome or adjustment of hyperkinetic children. If the drugs contribute positively, they appear to reduce disruptive behavior rather than improve academic performance" (p. 89-90, italics added). The same conclusion was reached in a subsequent review by Gadow (1983) and almost 8 years later, another group (Swanson et al., 1991) acknowledged that "Even though it has been established that stimulants do improve productivity, it is still unclear whether stimulants alone improve longterm academic achievement, and, that... whether this widespread clinical practice has a long term beneficial effect on learning or academic achievement is still an open question" (p. 220). Carlson and Bunner (1993), incorporating the studies previously discussed by Gadow and Swanson concurred that stimulants facilitated acute academic performance of children with ADHD, but that long term treatment did not improve outcomes measured by the Wide Range Achievement Test (WRAT), the Peabody Individual Achievement Test (PIAT), the Stanford Achievement Test (SAT), and failed grades.

There is now substantial evidence for persistent academic underachievement and poor educational outcome in children and adolescents diagnosed with ADHD (Loe \& Feldman, 2007). Children with ADHD have a consistently lower full-scale IQ than normal controls. They score significantly lower on reading and arithmetic achievement tests, use more remedial academic services, are more likely to be placed in special education classes, more 
likely to be expelled, suspended or repeat a grade, compared with controls. By the time they reach adolescence, individuals with ADHD fail more grades have lower report card scores, lower class rankings, and worse scores on standardized achievement tests than "matched normal controls." They take more years to complete high school, and have lower rates of college attendance and graduation. Subsequent investigations of long-term outcomes of children with ADHD have only confirmed these conclusions and verified the modest academic impact of stimulant medications (Advokat, 2009; Barbaresi et al., 2007a; 2007b; Galéra et al., 2009; Scheffler et al., 2009; Van der Oord et al., 2008).

Many investigators have considered possible reasons for this negative result, including the possibility that the stimulants might not affect the underlying cause of the academic dysfunction (Barkley \& Cunningham, 1978). Gadow (1983) raised several issues in regard to the clinical use of the drugs. He discussed the possibility that doses required for behavioral control might be greater than needed to improve (and might actually worsen) cognition. He suggested that short-acting agents might wear off during a typical school day, such that information presented in the morning would be experienced while the child was under the influence of medication, while material presented in the afternoon might not be. He noted that the duration of treatment might not have been long enough to provide benefit for performance on achievement tests, because such tests assess concepts taught over several grade levels. He pointed out that previous studies did not take into account the contribution of co-morbid diagnoses, especially learning disabilities, the inclusion of different ADHD subtypes, or of non-responders. (However, as noted above, most patients do respond to stimulant medications if efforts are made to determine which drug would be most effective. A meta-analysis of the five studies in children that compared MPH to AMPH in blind crossover conditions found that about $37 \%$ of patients had a clearly better outcome on an amphetamine preparation, and $26 \%$ had a clearly better response to methylphenidate. The other $37 \%$ of stimulant responders could use either molecule with equal benefit, Greenhill et al., 1996).

\section{Academic achievement of college students with ADHD}

During the last 30 years, special education and disability laws have been passed enabling a variety of qualified students with disabilities to graduate from college preparatory programs in high schools and enter colleges and universities. Specifically, the Americans with Disabilities Act (1990), the Individuals with Disabilities Education Act (1975) and Section 504 of the Rehabilitation Act (1973) mandated educational accommodation for students with disabilities, and more students with disabilities are now successfully completing high school and attending college. Students with "hidden disabilities," which includes ADHD, have represented the greatest increase. Because these students don't have to report to disability offices it is difficult to determine the prevalence of ADHD, but the best estimate is that $25 \%$ of students getting disability services do so because of ADHD and that $2 \%$ to $8 \%$ of the undergraduate population "self-report" ADHD symptoms (Weyandt \& DuPaul, 2006; Wolf, 2001).

Because most ADHD-diagnosed adults do not obtain a college degree, it is possible that those who do successfully progress through a college curriculum might differ from those who do not attend, or do not complete college. In other words, adults with ADHD who meet admission criteria for postsecondary education might be less cognitively impaired 
than those who don't. As noted by Frazier et al., (2007), not all ADHD-diagnosed individuals have academic deficits. Moreover, college students with ADHD might have more intellectual ability, better academic preparation and be able to compensate better than their non-collegiate cohort (Frazier et al., 2007). Such students might have developed a way to use stimulant medications more effectively, or to apply successful learning strategies, or both. If these individuals were able to benefit academically from stimulants it would be important to know how they did it. On the other hand, if the drugs are no more effective for this population than they were for elementary and high school students, it would be important to try to understand why they don't provide the expected intellectual advantage.

Moreover, the undergraduate population provides an excellent opportunity to evaluate some of the pharmacological explanations offered, in the past, for why stimulants did not improve academic outcome in children and adolescents. As noted above, one hypothesis was that the stimulant doses required to control the hyperactivity of ADHD-diagnosed children might be greater than doses that are most effective for improving cognition. However, unlike children, college students are less likely to be characterized as hyperactive, and more commonly diagnosed with, or to self-report, the symptom of inattention (Frazier et al., 2007; Norwalk et al., 2009; Rabiner et al., 2008; Schwanz et al., 2007) even without a specific diagnosis of ADHD (Lewandowski et al., 2008). Therefore, undergraduates should be able to determine the amount of stimulant medication that would presumably improve their attention and concentration without having to control hyperactivity as well.

Previous reviews of children had also speculated that perhaps the short-acting agents didn't provide sufficient coverage during a standard school day, and that daily variability in blood levels made it difficult to benefit from the intellectual advantages of the drugs. But duration of coverage is also less of a problem in undergraduate populations since long-acting formulations are now available, and regardless, college classes are usually not scheduled all day long. These considerations make arguments about dosage and variability of blood levels less persuasive, and would predict greater efficacy in the college population.

Furthermore, therapeutic use of stimulants in children usually involves administration primarily during the school day, so that drug effects wear off in the evening, perhaps while homework is being done, to allow for sufficient sleep. This is not necessarily how college students, or other adults, routinely use stimulants. Surveys report that undergraduates often use the drugs to stay up at night to study or complete other projects. That is, adults are able to choose when they take the drugs, which might also promote more effective cognitive outcomes.

The first review to describe the general academic functioning of college students with ADHD appeared only a few years ago and summarized results from 23 studies (Weyandt \& DuPaul, 2006). They found that ADHD-diagnosed college students did not differ in IQ from those without ADHD, and were able to meet the demands of college courses. Nevertheless, they had significantly lower grade point averages (GPAs), reported more "academic problems," and were less likely to graduate from college. Students who self-reported high levels of ADHD symptoms used significantly fewer coping strategies compared with those who did not (see also Reaser et al., 2007). They were less organized and 'methodical,' they had less self-control and discipline, and they procrastinated more. On laboratory 
administered neuropsychological tests they showed significant deficits in attention, but were not different from normal students on other measures, such as the ability to be flexible and to maintain performance as task demands were varied. There was also no difference between those with and without ADHD on computerized tasks that assessed divided attention. However, the role of medications in these outcomes was not determined "...it is unclear what effects medications have on academic, interpersonal and psychological outcomes among college students" (Weyandt \& DuPaul, 2006, p. 14).

Since that review, numerous studies have reached similar conclusions. Some (Advokat et al., 2008; Advokat et al., 2011; Blase et al., 2009) have found significantly lower GPAs in ADHDdiagnosed college students relative to non-ADHD controls. Not surprisingly, higher levels of ADHD symptomatology are consistently associated with poor study habits, skills and academic adjustment, and greater self-reports of attention deficits (Norwalk et al., 2009; Schwanz et al., 2007). Recent surveys (Rabiner et al., 2008; 2009a; 2009b) show no difference between the ADHD-diagnosed undergraduates who used stimulant medications and those who didn't, in regard to self-reported concerns with their academic performance, problems of inattentiveness, hyperactivity, depression or their social life. In other words medication had no discernible effect in the transition to college of students with ADHD (Blase et al., 2009).

For the last several years, our laboratory has been conducting research in undergraduates with ADHD to try to understand the cognitive effects of stimulant medications (Advokat et al., 2007; Advokat et al., 2008; Advokat et al., 2011; Advokat \& Luo, unpublished; Barrilleaux \& Advokat, 2009). Given evidence that suggests there is a positive relationship between the Grade Point Average of undergraduates and their working memory (Gropper \& Tannock, 2009) our efforts to clarify the cognitive actions of these drugs include studies of both, neuropsychological and academic performance of adult ADHD-diagnosed undergraduates.

\subsection{Neuropsychological assessment of ADHD-diagnosed college students}

Because inattention is a core symptom of ADHD, Barrilleaux and Advokat (2009) tested the effect of stimulant medications on attention with a repeated measures design, using the computerized, "Standard" version of the Conner's Continuous Performance Test (CPT). In this version, letters of the alphabet are presented one at a time for $250 \mathrm{~ms}$ and the respondent is instructed to press the space bar for every letter except the letter X. ADHDdiagnosed undergraduates $(n=13)$, and those without ADHD $(n=17)$, were tested twice on the CPT. For the ADHD-diagnosed participants one test was administered after they had taken their medication and the other when they were not on their medication.

The results are summarized in Figure 1, which shows the average number of commission errors, that is, responses made when they should not have been (when the letter $X$ appeared). This kind of mistake is often viewed as a measure of impulsivity. The left side of the figure shows the mean number of commission errors for the Control Group, on the first session (open bar), second session (dark bar) and the average of the two sessions (light bar). The right side of the figure shows the mean number of commission errors for the ADHD Group when they were Medicated (dark bar) and Non-Medicated (light bar). Nonmedicated ADHD-diagnosed adults made significantly more commission errors than 


\section{Commission Errors}

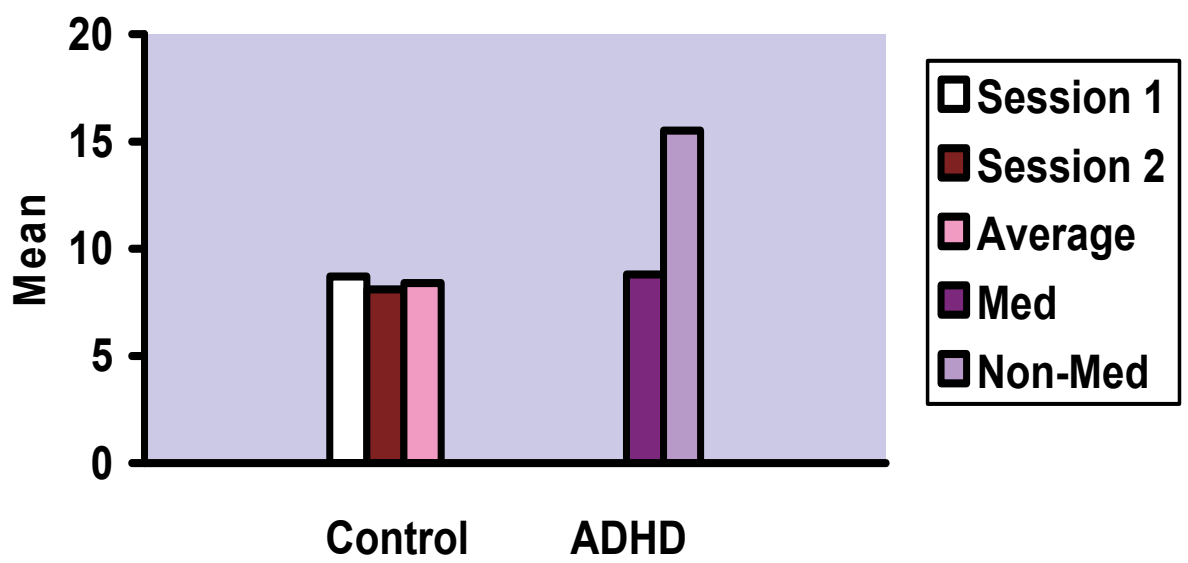

Fig. 1. Mean number of Commission Errors of Non-ADHD (Control) and ADHD-diagnosed undergraduates on the Continuous Performance Test (CPT).

controls; when medicated, they performed as well as controls and significantly better than when they were unmedicated. These data illustrate the typical, classic impairment of attention found in numerous studies of ADHD diagnosed individuals, and the improvement produced by stimulant medications.

In subsequent studies we assessed the effect of stimulant medications on several other neuropsychological tests. Unlike the CPT, performance on these tasks is influenced by practice; therefore, all of our other studies used a between-subject procedure. That is, in each experiment, three groups of undergraduate students were tested, one group without an ADHD diagnosis (Control), one group of ADHD-diagnosed students tested without medication (Off Meds) and one group of ADHD-diagnosed students tested while on their medication (On Meds). Several types of tests were administered, ranging from assessments of motor dexterity, verbal fluency, acquisition and retention of word lists, distractibility and problem-solving.

Motor dexterity was tested with a mirror-tracing task. Each participant was asked to trace the outline of a star shape, which was presented on a computer screen. Tracing direction was set to mirror-reversed, such that the participant had to move the cursor in the opposite direction to that of the pattern lines in order to trace the pattern. That is, the participants had to learn over successive trials to trace the star shape as if it was being shown in a mirror.

The results, summarized in Figure 2 below, showed no overall difference in latency among the three groups; all participants completed the task in the same amount of time. However, while the control group showed a significant decrease in latency across the five trials $(p=.002)$ the two ADHD groups did not (Advokat \& Vinci, unpublished data). That is, unlike nonADHD students, the performance of ADHD-diagnosed students did not improve significantly regardless of whether or not they were on stimulant medication. 


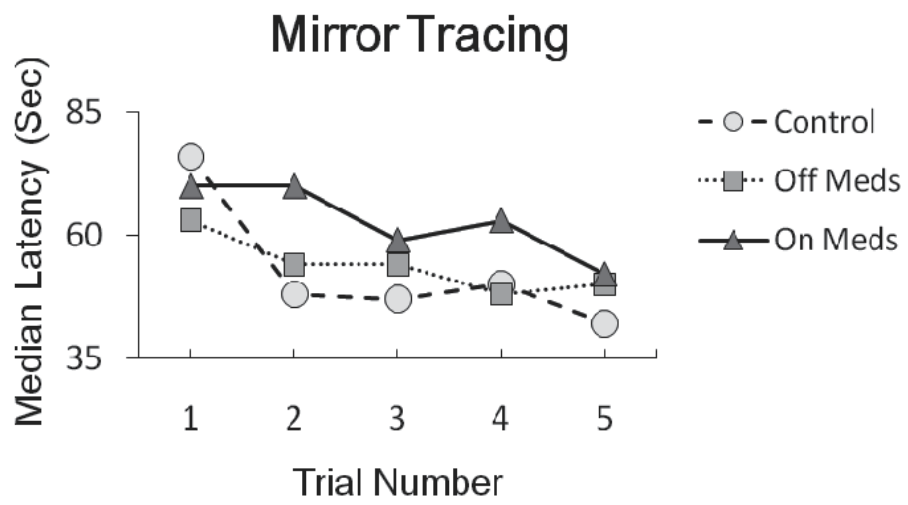

Fig. 2. Median latency of mirror-tracing across 5 successive trials, in 3 groups of college students: nonADHD (Controls) ( $\mathrm{n}=19)$, ADHD-diagnosed students, tested without medication (Off Meds; $\mathrm{n}=22$ ) and ADHD-diagnosed students tested when on their medication (On Meds; $\mathrm{n}=22$ ).

Our result is reminiscent of a report by Tucha \& Lange (2004) that medication worsened some aspects of handwriting in ADHD-diagnosed children. In that situation, the handwriting impairment was attributed to a drug-induced enhancement of attention. That is, the results were interpreted to mean that, when they were on their medication, children with ADHD paid so much attention to the writing process that it impaired the fluency of their handwriting movements. It is tempting to speculate that a similar phenomenon occurred in our ADHD-diagnosed undergraduates.

These observations show that although a decrease in behavioral activity is the most reliable effect of stimulant medications in ADHD, all types of behavioral activity are not reduced or improved by these drugs. ADHD-diagnosed children often show impairment in rudimentory motor function, including postural stability (Jacobi-Polishook et al., 2009), gait (Leitner et al., 2007), motor timing (Rubia et al., 2003) and other neurological reflexes (Stray et al., 2009), which is generally alleviated by stimulants. However, improvement is not always complete, or evident under all conditions. For example, Pelham et al., (1990) evaluated methylphenidate in boys with ADHD while they were playing a series of softball games. Although the drug improved the children's attention during the games, it did not affect their actual performance or skill.

There is a vast literature describing experimental efforts to determine the neurocognitive deficits associated with ADHD, and how they might be affected by stimulant drugs. While the technology and the conceptual models have become more sophisticated, progress has been modest. Recent analyses of stimulant effects on neurocognitive impairments in ADHD children (Doyle, 2006; Gualtieri \& Johnson, 2008; Swanson et al., 2011) have essentially confirmed early observations (Robbins \& Sahakian, 1979): stimulants are most likely to improve performance of ADHD diagnosed individuals in the domains of reaction time and processing speed, rather than in more complex functions requiring "...inhibition, working memory, strategy formation, planning and set-shifting" (Swanson et al., 2011, p. 211).

Several reviews have assessed neuropsychological functions in the adult population with ADHD (Frazier et al., 2004; Hervey et al., 2004; Schoechlin \& Engel, 2005; Woods et al., 2002) 
and shown modest, but inconsistent impairment. Furthermore, these deficits are also not always eliminated by stimulant drugs. Turner et al., (2005) tested ADHD adults, before and after methylphenidate, on attention tests and one memory task, and did not find improvement. Müller et al., (2007) reported that medicated ADHD adults were still impaired, relative to controls, on several neuropsychological measures such as the Tower of London test of 'planning ability,' and the Stroop test of 'distractibility.' Kurscheidt et al., (2008) reported retrospective results of 34 patients on chronic methylphenidate. Compared to baseline, the drug significantly improved attention and 'verbal memory performance' after months of treatment - while other tasks were not affected. Tucha et al., (2011) reported differences between nonADHD and ADHD-diagnosed adults on the Tower of London task and one that measured verbal fluency. In this case, MPH did improve performance of ADHD adults on the Tower of London, but not on the verbal fluency task. Biederman et al., (2008b) administered a battery of tests to non-ADHD subjects and separate groups of ADHD patients who were either on or off medication. They found the largest beneficial effects on sustained attention (vigilance) and verbal learning, whereas stimulants did not significantly improve measures of interference (i.e. distractibility) or processing speed (on the Stroop test).

We recently conducted a study of the most commonly used neuropsychological tasks in our undergraduate population, including a verbal fluency measure, the Tower of London planning task and the 'distractibility task,' the Stroop test. The only significant difference among the groups was on the Stroop test. This test involved three sets of stimuli, presented on a computer screen. The word reading stimuli consisted of three color words (blue, red, and green) in black ink, which the participant read aloud. In the color naming test, the stimuli were a series of five Xs (i.e., XXXXX) in all blue, all red, or all green ink, and the participant read aloud the ink color. Finally, in the incongruent color naming stimulus set (interference condition), stimuli consisted of the color words blue, red, and green printed in an incongruent color. The participant had to name the color of the ink in which the word is printed, not the word color.

We found modest, but statistically significant differences on two measures of the Stroop test. As shown in the top half of Figure 3, below, on the Stroop Interference test, the Control $(n=35)$ and ADHD (On Med) groups $(n=36)$ reacted significantly faster than the ADHD (Off Med) group ( $n=33)$. Part B shows that the Control group made slightly, but significantly, fewer mistakes (was more accurate) than the ADHD (Off Med) group, while the ADHD (On Med) group did not differ from the other two groups.

These data are consistent with other studies showing first, that adults (Johnson et al., 2001; King et al., 2007; Murphy et al., 2001; Rapport et al., 2001) as well as children with ADHD (Bedard et al., 2002; Prehn-Kristensen et al., 2011), are impaired on the Stroop interference measure compared to control populations. Second, Biederman et al., (2008b) showed that medication did not normalize Stroop interference control in young adults with ADHD. In our study, the Interference RT of the On Med group was normalized. Yet, the Interference accuracy of the On Med group, although improved, was still not significantly different from either of the other two groups. Considering the small absolute difference in magnitude, it is surprising that the drugs did not fully eliminate the accuracy deficit along with the RT deficit. One possibility is that the reduction in RT increased impulsivity, which might have impaired a corresponding improvement in accuracy. 

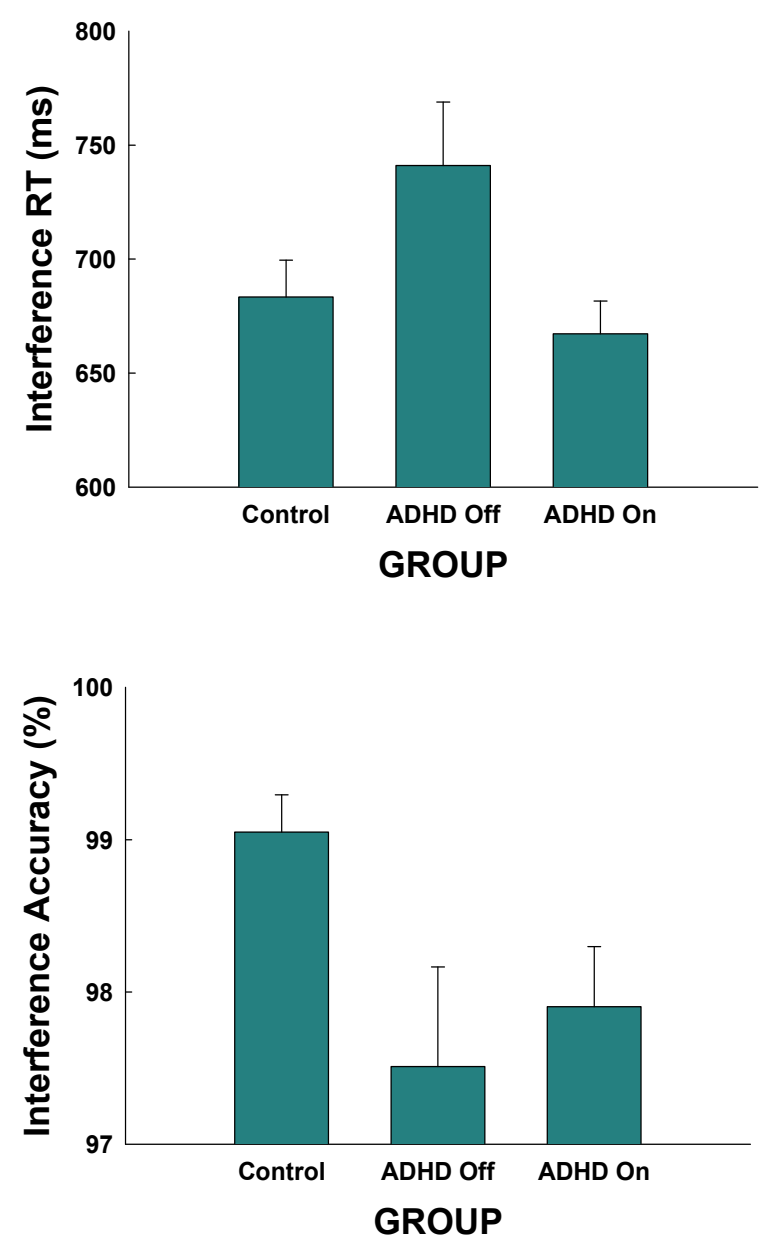

Fig. 3. Top: Stroop Interference Reaction Time (RT - milliseconds). The Control group $(n=35)$ and ADHD (On Med) group ( $n=36)$ reacted faster than the ADHD Off Med group, $\mathrm{p}<0.05(\mathrm{n}=33)$. Bottom: Stroop Interference Accuracy. The Control group made fewer mistakes than the ADHD (Off Med) group in interference, $\mathrm{p}<0.05$, and the ADHD On Med group did not differ from the other two groups. Bars represent standard error of the mean.

In brief, our results showed that undergraduate students with ADHD did not show significant deficits in verbal fluency or planning ability, compared to normal students. The only statistical effects occurred in the Stroop test, and the absolute differences were extremely small. Even so, while medications improved the performance of ADHD students they did not eliminate the deficit, nor did they help ADHD students perform better than normal students in domains of functions where no deficits were found. It is possible that more differences might have been seen in measures other than the Stroop test, if the groups were larger. However, our group sizes were comparable to those typically used. A more likely possibility is that our ADHD population may have had less severe symptomatology than participants in other studies, cited above, who appeared to be clinic patients. 
Although research shows practically no cognitive benefit of either methylphenidate or amphetamine on acquisition (Advokat, 2010), there is some evidence that stimulants might improve retention of previously acquired information (Izquierdo et al., 2008; Soetens et al., 1993; 1995; Zeeuws \& Soetens, 2007). In a series of experiments by Soetens and colleagues, nonADHD adult males received placebo and amphetamine, in a within-subject experimental procedure. After each drug, participants were asked to study word lists and were tested at various times afterward to assess how many words they could recall. The first test took place immediately after presentation of each of 10 word lists, the next test was given at the end of each daily session (after all the lists), and other tests were given after 1 hour, after 1 day, and after 3 days.

There was no drug effect after immediate recall or at the end of the daily session, that is, no effect of amphetamine on acquisition of the words. But there was a significant effect of amphetamine on recall 1 hour after the end of the session, and at 1 and 3 days later. These results were interpreted to mean that although amphetamine did not improve learning (acquisition), it facilitated the consolidation of information that had already been learned, and that the beneficial effect on consolidation was responsible for the improved retention of the words at 1 hour and 1 and 3 days later. The term 'consolidation' refers to the process by which memory undergoes a change from the short-term, labile, form to a long-term, stable form.

We attempted to replicate this phenomenon in our undergraduates. Although we could not administer stimulant drugs to nonADHD participants, it was possible that ADHD students might have a memory impairment that could be reduced by stimulants. In our study, participants from each of the three experimental groups viewed five sets of ten words. After each list of 10 words the participants were asked to write down as many words as they could remember (Test 1). After the fifth set of words was presented, participants were 'distracted' by performing a second behavioral task (the mirror tracing task, discussed above). Following the mirror tracing task, participants were asked to write down as many of the 50 words as they could remember (Test 2). Last, participants were contacted on the next day and asked a third time to recall as many words as they could remember from the word lists (Test 3). As shown in Figure 4, below, there was no difference among the groups. ADHD students did not differ from nonADHD students in regard to acquisition or retention of the word lists at any time point, regardless of whether or not they were on medication during the test.

In summary, our assessment of neuropsychological function in undergraduates with ADHD showed the expected impairment on the classic, CPT test of attention, which was normalized with stimulants. We also observed a previously unreported motor deficit (on the mirror tracing task), which was not improved by stimulants. We saw two types of deficit in the Stroop test of 'distractibility,' namely, a slower reaction time, corrected by stimulants, and a very slight impairment in accuracy, which was not eliminated by stimulants. These results are consistent with the literature, and illustrate the counterintuitive nature of stimulant effects in ADHD. That is, although cognitive processing speed and basic aspects of attention are normalized, even slight deficits in 'distractibility' are not eliminated. Finally, although we did not replicate the memory improvement that had been shown in nonADHD adults with amphetamine, we also did not see any deficits in that task in our ADHD students. It is appreciated that because they were college students, our ADHD population might have had very mild symptoms and were perhaps not representative of the typical ADHD adult. We could not confirm their diagnoses. However, if that was the case, we would not expect them to 


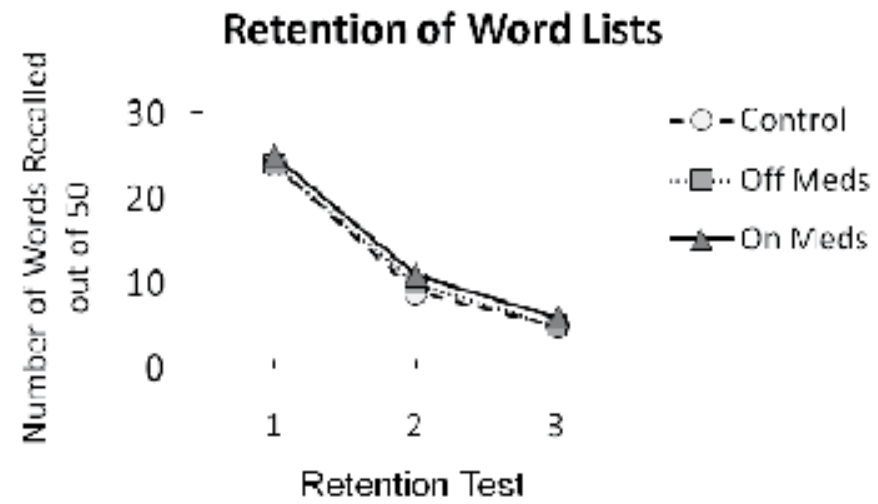

Fig. 4. Retention of word lists in nonADHD students (Control), ADHD students, who were tested when they were not on medication (Off Meds) and ADHD students who were tested when they were on their medication (On Meds). Retention was tested immediately after each list of 10 words (Test 1), about 20 minutes after the 5 lists (Test 2) and 1 day after the 5 lists (Test 3 ). There was no difference among the groups in the number of words recalled on any test.

be academically impaired, relative to their nonADHD counterparts, especially considering that the stimulants exert the same types of effects in those with or without the diagnosis. The data obtained regarding academic performance, discussed below, show that that is not the case.

\subsection{Academic performance of ADHD-diagnosed college students}

The ADHD-diagnosed undergraduates we tested showed very little neurocognitive impairment. Perhaps the fact that they had successfully entered college meant either that they had less severe symptomatology or that they were able to gain some academic benefit from the stimulants (or both). If the latter, it would be important to find out how, so that their strategies could be broadly implemented. On the other hand, in view of growing concerns about escalating abuse and diversion of stimulant drugs, it would be equally important to know if these medications did not provide any academic advantage for ADHD-diagnosed undergraduates.

In these investigations we compared the self-reported drug use (both licit and illicit), study habits and strategies of ADHD-diagnosed and non-diagnosed undergraduates. We administered questionnaires to find out if there was a substantial difference in how these two groups approached their schoolwork, and, if there was any corresponding difference in their respective Grade Point Averages (GPA) and other measures of academic achievement.

In the first study (Advokat et al., 2008) we asked about the legal use of prescription stimulants by undergraduates diagnosed with ADHD, and compared that with illicit use by students without the diagnosis.

A total of 1550 undergraduate students completed at least part of the survey, with 163 $(10.5 \%)$ of these students reporting a diagnosis of ADHD (the ADHD Group). Among the remaining 1387 respondents, $591(43 \%)$ reported that they used stimulant medications without a prescription (the No ADHD, Illicit Use group) while $794(57 \%)$ stated they did not use stimulant medications illicitly (the No ADHD, No Illicit Use group). 
As shown in Figure 5, the three groups differed significantly in response to the question: "Do ADHD medications help academic performance," in that a significantly greater proportion of the ADHD, and No ADHD Illicit Use groups, endorsed this statement compared with the No ADHD No Illicit Use group. The fact that a majority of illicit users endorsed this statement supports the conclusion that the drugs were primarily used as study aids. For that matter, a surprising $12 \%$ of illicit users in that study reported that they believed they also had ADHD. That might even be true, but we have no way of verifying that assumption.

\section{Do ADHD Medications Help Academic Performance? *}

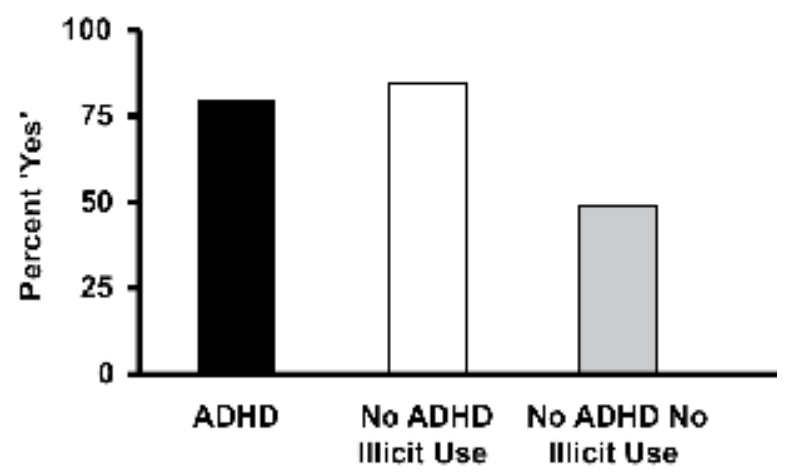

Fig. 5. Percent of undergraduates who agreed with the statement "Do ADHD Medications Help Academic Performance." *Significantly fewer students in the No ADHD No Illicit Use group endorsed this statement compared to each of the other two groups.

Figure 6 shows that the same groups also differed significantly in GPA, in that the GPA of the ADHD group (3.05, out of a possible 4.0) was significantly lower than that of the No ADHD Illicit Use group (3.15) and the No ADHD No Illicit group (3.19).

\section{Grade Point Averages (GPA) *}

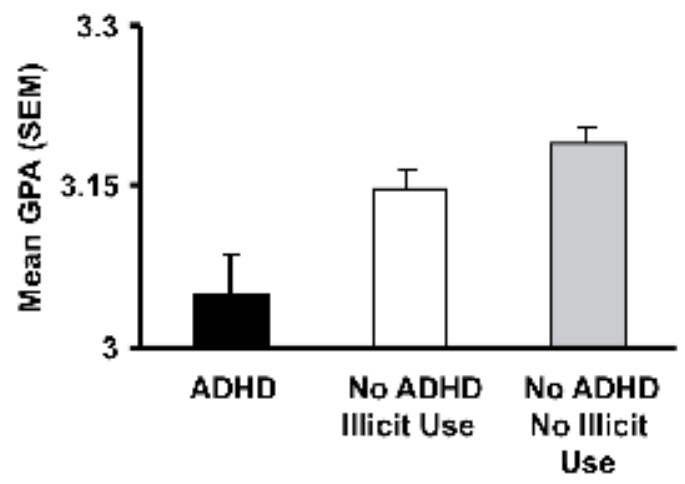

Fig. 6. Mean Grade Point Average (GPA) of each of the three groups. * The GPA of the ADHD group (3.05, out of a possible 4.0) was significantly lower than that of the No ADHD Illicit Use group (3.15) and the No ADHD No Illicit group (3.19). 
It should be noted that we cannot tell from these data how many students in the ADHD group actually used stimulant medications. Nor can we tell if the GPA of both groups that used stimulant drugs would be even lower if those students didn't use the drugs. That is, we can't tell if the drugs were effective, either because they worked or because the students believed the drugs worked.

Last, Figure 7, below, summarizes reported recreational drug use of Tobacco, Alcohol, and Marijuana of these same groups. In each case there was a significant difference among the groups in frequency of drug use. For each drug, post-hoc comparisons indicated that a greater percent of the No ADHD Illicit Use and ADHD groups used these drugs compared with the No ADHD No Illicit Use group. Only in the case of alcohol did the ADHD group use less than the illicit stimulant users, although they still drank significantly more frequently than those who didn't use stimulants illicitly.

Overall, recreational drug use in our student sample was very modest. Nevertheless, the significant difference between ADHD-diagnosed and Non-diagnosed-non-stimulant users was unexpected, and we can only speculate about the reasons for this result. First, perhaps, believing that their symptoms are effectively controlled by stimulant medications, these students feel they are able to use other drugs responsibly. Second, because of the impulsiveness that often characterizes ADHD, these young adults might not be able to inhibit other drug use, especially if the drugs are offered in the social context of a party, or other nonacademic environment, when students might not be using their stimulants "medically." Third, considering that alcohol and marijuana are sedating, ADHD students might use them to counteract the stimulatory effect of the medications, that is, to help them relax or go to sleep. It is also possible that those with ADHD (and nonADHD, illicit users) have comorbid conditions (such as conduct disorder) or other risk factors that increase use.

These data show that, although the GPA of the ADHD students was significantly lower than that of the nonADHD-Illicit users, both groups endorsed significantly greater recreational drug use than students who did not use stimulant drugs illicitly. Unfortunately, we can't tell from these data if perhaps ADHD students were more vulnerable than nonADHD students to a detrimental effect of recreational drugs on academic performance, or if both groups might have had a better GPA without such use.

Our results showed that a majority of ADHD diagnosed students (and many without the diagnosis) believed that stimulants improved academic performance. And although the GPAs of ADHD students were statistically lower than those of nonADHD students that did not mean the stimulants didn't help. The aim of our next study was to find out more about the influence of stimulant medications on academic outcome. We surveyed the self-reported study habits and strategies of ADHD-diagnosed and non-diagnosed undergraduates, to determine if they differed in response to the academic demands of the college curriculum (Advokat et al., 2011).

A total of 143 students without ADHD (Control group) and 92 students with an ADHD diagnosis (ADHD group) completed the survey. The average age of the two groups, approximately 21 years, did not differ, and most participants in each group were Caucasian (81.8\% and $89.1 \%$ for Control and ADHD, respectively), although there were significantly more males in the ADHD group (38\% compared to $17.5 \%$ ). 


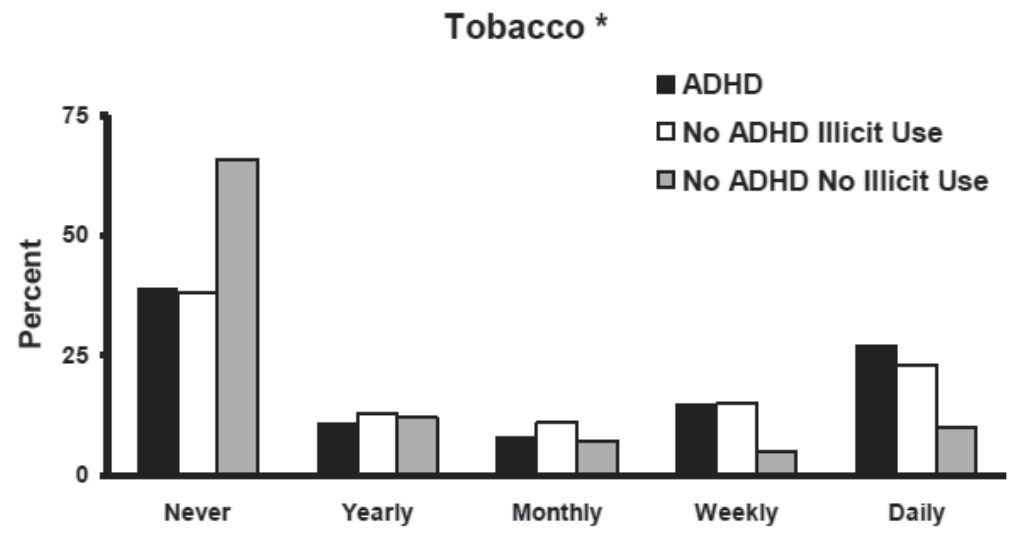

Alcohol *

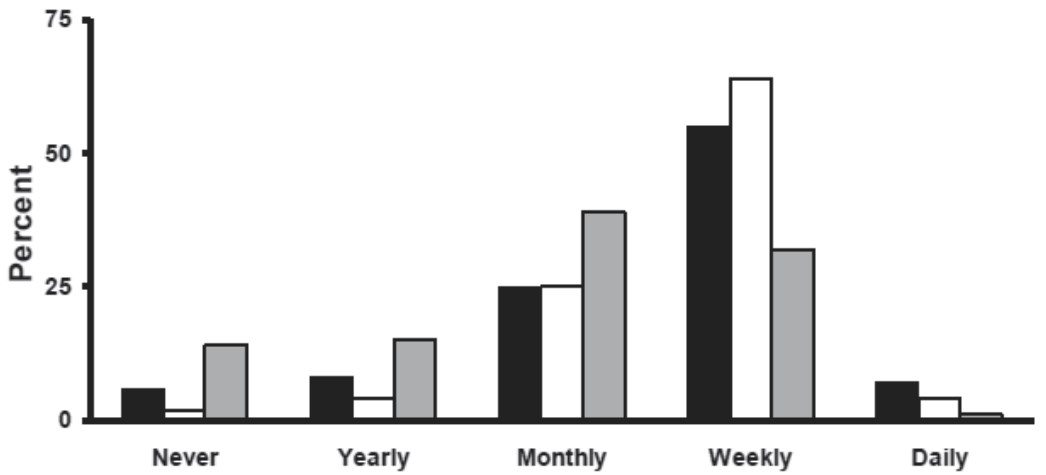

Marijuana *

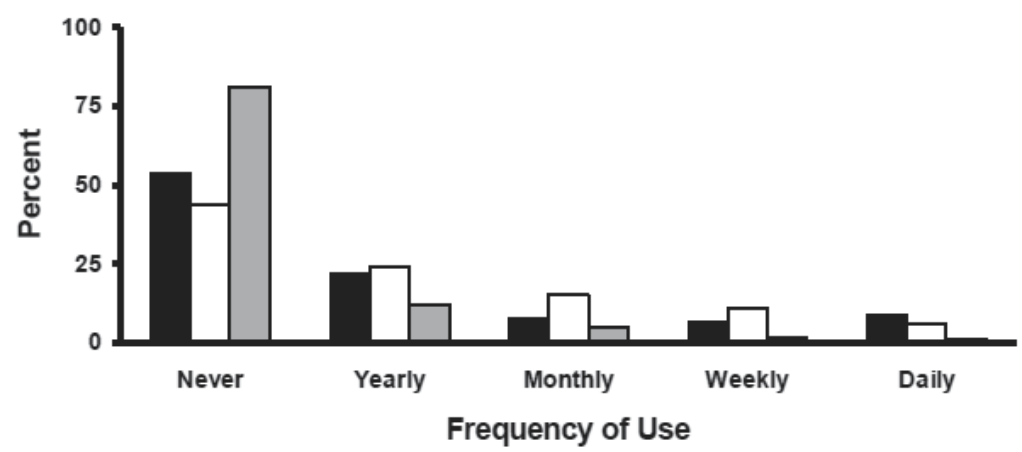

Fig. 7. Percent of respondents in each group as a function of the frequency with which they used tobacco, alcohol and marijuana. *In each case, the ADHD and the No ADHD Illicit Use groups endorsed significantly more use than the No ADHD, No Illicit Use group. The ADHD group also used alcohol significantly less than the No ADHD Illicit Use group. 
The average age at which the ADHD participants received the diagnosis was between 15 and 16 years, about 5 years before entering college. While nearly $98 \%$ of this group had taken ADHD medication, only $78.3 \%$ (72 respondents) were currently using the drugs, while $19.6 \%$ (18 respondents) stated that they were not currently taking the medications. When asked why they might not be taking stimulant medications, the majority cited the problem of side effects. Although specific side effects were not always mentioned ("I did not like the way it made me feel; ...make me feel crappy; the negative side effects outweigh the positives"), some individuals cited headaches, irritability, temporary heart rate elevation, nausea, sleep interference and 'antisocial' feelings as examples of undesirable reactions. Five in this subgroup also stated that they either didn't need the drugs anymore, or, they wanted to see if they didn't need them anymore.

Similar to the previous study, more than $90 \%$ of the students with ADHD endorsed the statement that medications helped them academically. Most of them stated that the drugs helped them to focus or to concentrate better i.e. pay attention, stay awake, and organize their studying. However, relatively few students $(n=6)$ with ADHD specifically stated that they took medication to avoid distractions.

With regard to academic performance, Control and ADHD students took the same amount of Advanced Placement Credits (an average of 8.1 for the Controls and 6.7 for ADHD students). For those few in each group who took the SAT (Scholastic Aptitude Test, a national exam sometimes required for college admission), there was no difference in their self-reported SAT scores. They did not differ in the number of scholarships awarded, were enrolled for the same number of semesters (between 6 and 7), took the same number of credits per semester (about 14.5), and studied the same number of hours per week (about 9.5 to 10.5).

These two groups also did not differ statistically on many of their answers to questions about study habits. The same proportion of both groups believed that they studied "about the same as" other students and that the quality of their class notes was either "better" or "the same" as those of other students. The same proportion in each group did not review their notes either before or after class, and only "sometimes" read assigned reading before class. About three-quarters of each group stated that they were "somewhat accurate" at predicting how well they did after exams. The fact that there was no statistical difference between nonADHD-diagnosed students, and those with ADHD in response to these questions suggests that the diagnosis did not seem to promote better study habits in the ADHD group.

However, the Control and ADHD groups did differ on several academic variables, as shown in Table 1. (Although the values of ADHD students who stated that they did not take stimulant medications are shown for some of the variables, the statistical results are based on a comparison between Control and all ADHD students).

As in our previous report, the college GPAs of ADHD students were statistically lower than that of the Controls. In this study we also found that ADHD students had a significantly lower high school GPA and ACT score than Controls. Although small, this difference was detected in spite of the fact that a minimum high school GPA and ACT score were required for admission to the university. The table also shows that ADHD students were significantly more likely to withdraw from a class, to say that they were worse than other students at planning for and completing class assignments, frequently taking class notes, studying ahead of time for exams and avoiding distractions. 


\begin{tabular}{|c|c|c|c|c|}
\hline & $\begin{array}{l}\text { Control (n) } \\
(\mathrm{N}=143)\end{array}$ & $\begin{array}{l}\text { ADHD (n) } \\
(\mathrm{N}=92)\end{array}$ & $\begin{array}{l}\text { ADHD No Meds } \\
\quad(\mathrm{n}=18)\end{array}$ & $\mathrm{P} * *$ \\
\hline High School GPA & $3.55 \pm 0.47(141)$ & $3.38 \pm 0.43(89)$ & & 0.006 \\
\hline College GPA & $3.12 \pm 0.49(142)$ & $2.94 \pm 0.44(91)$ & $2.90 \pm 0.46$ & 0.006 \\
\hline ACT Score & $25.45 \pm 5.2(124)$ & $24.1 \pm 3.0(80)$ & $24.6 \pm 2.61(16)$ & 0.032 \\
\hline Times Withdrawn from a Class & $1.6 \pm 1.7(139)$ & $2.3 \pm 2.4(89)$ & $3.28 \pm 3.89(18)$ & 0.008 \\
\hline Planning for class assignment & & & & $<0.001$ \\
\hline Better & $32.9(47)$ & $26.1(24)$ & $27.8(5)$ & \\
\hline Worse & $13.3(19)$ & $35.9(33)$ & $38.9(7)$ & \\
\hline \multirow[t]{2}{*}{ About the same } & $53.8(77)$ & $38.0(35)$ & $33.3(6)$ & \\
\hline & & & & $<0.001$ \\
\hline \multicolumn{5}{|l|}{ Completing class assignment } \\
\hline Better & $33.6(48)$ & $30.4(28)$ & $33.3(6)$ & \\
\hline Worse & $2.1(3)$ & $19.6(18)$ & $22.2(4)$ & \\
\hline About the same & $62.9(90)$ & $47.8(44)$ & $44.4(8)$ & \\
\hline Frequency of note-taking & & & & 0.002 \\
\hline Nearly every lecture & $82.5(118)$ & $63.0(58)$ & & \\
\hline Sometimes & $16.1(23)$ & $27.2(25)$ & & \\
\hline Read someone else's notes & $1.4(2)$ & $5.4(5)$ & & \\
\hline \multirow[t]{2}{*}{ Neither take notes nor read other's notes } & 0 & $4.3(4)$ & & \\
\hline & & & & 0.011 \\
\hline \multicolumn{5}{|l|}{ When you study for an exam do you } \\
\hline Study well before the exam & $39.2(56)$ & $25(23)$ & $16.7(3)$ & \\
\hline Study in the day or two before the exam & $60.1(86)$ & $69.6(64)$ & $83.3(15)$ & \\
\hline Both situations could happen & $0.7(1)$ & $5.4(5)$ & & \\
\hline Avoiding distractions while studying & & & & $<0.001$ \\
\hline Better & $16.8(24)$ & $16.3(15)$ & $11.1(2)$ & \\
\hline Worse & $33.6(48)$ & $64.1(59)$ & $66.7(12)$ & \\
\hline About the same & $49.0(70)$ & $18.5(17)$ & $22.2(4)$ & \\
\hline
\end{tabular}

* Unless otherwise indicated, scores are percent; ** $\mathrm{p}$ values are for Control vs all ADHD respondents GPA and ACT scores are all self-reported

\section{Table 1. Academic Variables That Differed Between Controls and ADHD-Diagnosed College Students *}

These results show that Control and ADHD-diagnosed students differ in some of their selfreported study habits. But these data alone don't tell us if these endorsements are relevant to the academic achievement of either group. In other words, we didn't know if the statistically significant differences in professed study habits were related to the respective GPAs. We 
therefore analyzed the answers to some of the questions on which the two groups differed as a function of GPA. The results are summarized in Table 2.

There were significant differences within the groups on several measures. Control students who stated they were 'better' at planning and completing assignments, took 'frequent' notes in class and 'avoided distractions,' had higher GPAs than those who said they weren't, suggesting that their judgments were accurate. However, the GPA of Control students did not differ as a function of whether or not they said that they studied 'ahead of time' or just days before an exam. In other words, Control students did not 'pay a price' in GPA for waiting until a few days before exams to study. In contrast, the GPA of ADHD students who stated that they studied 'well before' an exam, was significantly higher than the GPA of those (the majority of this group) who said they studied in the 'day or two' before an exam. Unlike Control students, ADHD students did 'pay a price' for waiting until the exam was imminent before they began to study. This difference seemed to epitomize a fundamental behavioral impairment in ADHD, so we examined it further.

\begin{tabular}{lll}
\hline Variables & Control & ADHD \\
\hline Planning for class assignment & $*$ & $*$ \\
Better & $3.26 \pm 0.43(46)$ & $3.09 \pm 0.39(23)$ \\
Worse & $2.92 \pm 0.52(19)$ & $2.79 \pm 0.41(33)$ \\
About the same & $3.06 \pm 0.50(77)$ & $2.93 \pm 0.48(35)$ \\
Completing class assignment & $*$ & $*$ \\
Better & $3.30 \pm 0.44(48)$ & $3.04 \pm 0.42(28)$ \\
Worse & $2.49 \pm 0.82(3)$ & $2.67 \pm 0.39(18)$ \\
About the same & $3.02 \pm 0.47(90)$ & $2.97 \pm 0.46(43)$ \\
Frequency of note-taking & $*$ & $\mathrm{NS}$ \\
Nearly every lecture & $3.13 \pm 0.46(117)$ & $3.0 \pm 0.46(57)$ \\
Sometimes & $3.06 \pm 0.57(23)$ & $2.86 \pm 0.42(24)$ \\
Read someone else's notes & $2.25 \pm 0.35(2)$ & $2.76 \pm 0.39(5)$ \\
When you study for an exam do you & $\mathrm{NS}$ & $*$ \\
Study well before the exam & $3.12 \pm 0.48(56)$ & $3.16 \pm 0.35(22)$ \\
Study in the day or two before the exam & $3.10 \pm 0.50(86)$ & $2.86 \pm 0.47(64)$ \\
Avoiding distractions while studying & $*$ & $\mathrm{NS}$ \\
Better & $3.30 \pm 0.51(24)$ & $3.0 \pm 0.44(15)$ \\
Worse & $2.91 \pm 0.50(48)$ & $2.88 \pm 0.46(58)$ \\
About the same & $3.18 \pm 69(69)$ & $3.07 \pm 0.40(17)$ \\
\hline
\end{tabular}

${ }^{*} \mathrm{p}<0.05$, for GPAs within each of the 2 groups, as a function of their answers to each question

Table 2. Factors Affecting College GPA \pm SD (n) of Control and ADHD-Diagnosed Students

Because we had asked the students if they were taking ADHD medications, we could distinguish between those ADHD students who said they did $(n=72)$ from the smaller group, who said they didn't $(n=18)$. The existence of these subgroups allowed us to compare the GPA of ADHD students who did or did not take the drugs, as a function of 
whether or not they studied ahead of time for exams. This resulted in 4 groups, with the following GPAs ( \pm Standard Errors): Those who took the medications, and did not study ahead of time ( $\mathrm{n}=47), 2.88 \pm 0.48$; those who used the drugs and did study early $(\mathrm{n}=19)$ $3.15 \pm 0.35$; those who did not take the medications, and did not study ahead of time $(\mathrm{n}=$ 15), $2.84 \pm 0.47$; and those few who did not take the drugs but did study early $(n=3) 3.19 \pm$ 0.36. A two-way analysis of these GPAs found no effect of medication, but a statistically significant effect of study interval, $(\mathrm{F}=4.06, \mathrm{p}=.047)$.

This preliminary analysis showed that, if ADHD students utilized the well-known strategy of studying ahead of time for exams, they could overcome their achievement deficit, even if they didn't take stimulant medications. In spite of the fact that only 3 students 'studied ahead of time' without using the drugs, their GPA was comparable to that of the 19 undergraduates who did take the drugs in addition to using good study habits. Obviously this outcome needs to be validated in a larger group of individuals. But, the data suggest the optimistic implication that the GPA disparity between ADHD and nonADHD students could be eliminated if ADHD students were able to develop well-established study habits.

Unfortunately, it is not clear from these data alone if taking stimulant medications actually helps ADHD students to develop beneficial study habits. That is, do the stimulant drugs help students to plan ahead, e.g., to begin studying ahead of time so that they can compensate for their cognitive deficit? If so, why didn't this behavior occur in more of the ADHD students?

These data provide preliminary empirical evidence that, as with elementary and high school students, adult college students with ADHD are less likely to reach the same academic level as their non-ADHD counterparts, even when they use stimulant medications. Stimulants do not necessarily normalize academic achievement, even when they can be administered at the most appropriate doses and durations for maximum efficacy, and, even in a population that is considered to be less intellectually impaired than the typical adult with ADHD.

It should be noted that even those ADHD students who did not engage in good study habits were not failing. Their average GPA was just above a ' $C$,' which means they were able to progress towards graduation at a normal pace. On the other hand, the absolute difference between the GPAs of ADHD students and Control students was not very large, only about a third of a grade. Yet this difference was consistent, and it is surprising that the stimulants were not more effective in narrowing the gap. Why aren't the stimulant medications more cognitively effective?

\section{Discussion}

In their consideration of this question, Barkley and Cunningham (1978) raised the possibility that the problem with stimulant medication might be that it made hyperactive children less aware of their environment, perhaps more intellectually 'constricted' and rigid and less inquisitive or interested in learning. Similarly, Gadow noted that stimulant drugs might produce 'cognitive perseveration,' akin to amphetamine-induced stereotypies (that is, the repetitive performance of an invariant behavioral sequence). Subsequently, Carlson and Bunner (1993) proposed the term 'cognitive toxicity' to describe this same hypothesized phenomenon. 
Robbins and Sahakian (Robbins \& Sahakian 1979; Sahakian \& Robbins, 1977) provided an insightful discussion of this point, proposing that stimulant-induced stereotypy might play a role in the behavioral effect of the stimulants. They noted that, “...although stereotypy may improve performance in certain situations, perhaps by focusing or channeling "attention," it may also lead to impairments when behavioral flexibility is required" (p. 944). Their review summarized results of numerous studies in ADHD-diagnosed children, including activity measures and complex motor tasks, cognitive tasks, intelligence tests, verbal fluency and language tests, and attention and vigilance tests. Across all these categories, the greatest improvement from stimulants was seen in activity tests (measured with photo cells, actometers and stabilimetric apparatus') and the least amount of improvement occurred in the problem-solving test category.

Subsequently, these researchers obtained evidence for stimulant-induced 'cognitive perseveration'. They conducted a study in children who had been on methylphenidate for 4 to 29 months (Dyme et al., 1982). Each child was tested 3 times, first, under a 'no drug' practice condition, then on two trials of either placebo or drug $(1 \mathrm{mg} / \mathrm{kg})$. The tasks included a test of 'flexibility of thinking,' the Wisconsin Cart Sort Test (WCST), involving a set of response cards which could be sorted according to color form or number. Subjects were not told which variable was correct for sorting, only when the choice was right or wrong; they had to figure it out for themselves. After 10 cards were sorted correctly the examiner changed the sorting variable without telling the subject who then had to determine the new 'correct' sort. Subjects were scored on the number of errors as well as correct cards sorted. 'Perseverative Errors' were mistakes in which the subjects sorted according to a prior category after they had already been told that sort was "wrong."

The results showed that although all children improved their speed or performance on measures of attention, none improved on this flexibility measure and most got worse. This result has since been replicated (Tannock \& Schachar, 1992), although not in all cases (Swartwood et al., 2003). Moreover, Tannock and Schachar (1992) found that this impairment recovered rapidly (that is, tolerance developed).

Nevertheless, consistent with the WCST data, Schroeder et al., (1987) found that methylphenidate impaired performance of 15 normal male volunteers, 18 - 40 years old, on a unique procedure. "The task was an arcade-like game called Telekinesis Star Wars, which was highly engaging.., as opposed to the more monotonous tasks commonly used in these neuropsychological studies." Several measures were obtained from the subject's performance in this game, which reflected the strategies used by each player, and indicated if they were improving or not. In each case, the data showed that subjects given methylphenidate, at doses of 0.15 or $0.30 \mathrm{mg} / \mathrm{kg}$, were poorer than controls at improving their performance throughout the test session. Drug-treated subjects did not develop "adaptive problem-solving strategies selected by controls."

Similarly, Burns et al., (1967) also described a learning task that was worsened by amphetamine. Subjects were seated in front of 8 lights, each placed above a key that controlled one of the lights. The subjects "had to learn which single key was the correct response to each [of 8] light[s] when the keys were randomly assigned, except that the correct button was never in front of the light associated with it." The session consisted of 840 trials balanced for frequency of light/key pairings. In this situation, the d-amphetamine group showed a significantly slower rate of learning than the placebo group. Such results 
suggest that, due to their profound effects on attention, stimulants may impair adaptive problem-solving ability, perhaps by inducing perseverative behavior.

Similarly, because attentional 'overfocusing' and creativity may be inversely related, there has been concern that stimulants might decrease creativity in people using the drugs for cognitive enhancement. Several studies have explored this possibility, mostly in children, with mixed results. Swartwood et al., (2003) found that MPH actually worsened the scores of ADHD-diagnosed children on a battery of tests assessing 'divergent thinking,' relative to their nondrug tests. Funk et al., (1993) saw no effect of methylphenidate on a test battery of 'creative thinking,' in young boys, relative to a nonADHD comparison group. Solanto and Wender (1989) also reported that methylphenidate maintained 'divergent thinking' in ADHD-diagnosed children when the children were tested over several days, relative to the decline that occurred on nondrug days. And Douglas et al., (1995) found that "... MPH doses up to $0.9 \mathrm{mg} / \mathrm{kg}$ had an increasingly positive effect on measures of mental flexibility and other cognitive processes" in 17 ADHD children.

To our knowledge, there are no studies of stimulant effects on creativity in ADHD adults. However, Farah et al., (2008) examined the effect of amphetamine in 16 nonADHD adults on four tests of creativity, "two tasks requiring divergent thought and two requiring convergent thought (p. 542)." Only performance on the convergent tasks was affected, and the effect depended on baseline performance. The results suggested that people who are already very creative might be unaffected or even impaired, whereas those who were not very creative to begin with might improve with stimulants. It remains to be seen however whether ADHD-diagnosed adults (or even children for that matter) are actually less 'creative' than their nonADHD cohorts.

Stimulant-induced perseveration and impairment of cognitive flexibility (creativity) have been the most commonly proposed reasons for why these drugs produce so little long-term cognitive benefit. But, stimulants are also sympathomimetics, that is, they produce the same effects as the sympathetic transmitters that mediate arousal and alertness. This physiological action is rarely discussed in regard to ADHD, perhaps because stimulants are used therapeutically to decrease rather than increase behavioral activity. But arousal is known to have significant effects on memory; moderate levels of arousal enhance memory while too much arousal impairs memory.

Recent studies of Brignell and colleagues (Brignell et al., 2006; 2007) illustrate the possible relevance of this approach. In one study, separate groups of subjects were given either placebo or methylphenidate while undergoing classical conditioning of a skin conductance response. Methylphenidate did not impair conditioning; the subjects who received the drug did acquire the conditioned response. However, the drug increased the number of responses to both, the non-conditioned stimulus and conditioned stimulus, compared to the placebo group. This was described as a "general pattern of methylphenidate-induced arousal increasing propensity to respond." It was concluded "methylphenidate decreased responses to highly arousing stimuli and increased responses to the less arousing stimuli (512)."

A second study suggests that this phenomenon might be relevant to the issue of stimulants and cognition. In this case, subjects were presented with a series of slides that were designed to tell a story that included a very 'emotional' component. One week later the participants 
returned to answer multiple choice questions about the story. Placebo-treated subjects showed the expected enhancement of memory for the emotional slides, relative to the neutral slides. But methylphenidate-treated subjects did not show this increase in memory of emotional material, even though the drug had increased their pulse and blood pressure. Unlike placebo-treated subjects, those given the drug showed comparable retention across all phases of the story. In other words, methylphenidate eliminated the preferential retention of the information on the emotional slides. These data suggest that the physiological arousal produced by stimulant drugs may be relevant to the fact that they do not seem to provide lasting cognitive benefit.

\section{Conclusion}

There is a paradox in regard to the use of stimulant medications indicated for the treatment of ADHD. On the one hand, there is much recent medical, legal and ethical concern about escalating use of these drugs, both licit and illicit, primarily to enhance cognition. On the other hand, there is surprisingly little evidence that the stimulant drugs truly are 'cognitive enhancers.' Results from neuropsychological studies confirm that while stimulants apparently increase attention in adults diagnosed with ADHD, the drugs produce very modest and inconsistent improvement on a variety of other neuropsychological tasks. Although responses may be faster, inaccuracy and errors persist, especially on tests of 'distractibility.' Intuitively, it would seem logical that drugs that improve attention and concentration should promote learning and academic achievement. Yet, for more than 30 years data have shown that this is not the case in regard to children and adolescents. Evidence presented here supports the same conclusion for adult college students. Whether this lack of effectiveness is due to drug-induced perseveration, inflexibility, arousal or some other factor(s) remains to be determined.

\section{References}

Adler, L.A.; Newcorn, J.H. \& Faraone, S.V. (2007). The Impact, Identification, and Management of Attention-Deficit /Hyperactivity Disorder in Adults. CNS Spectrums, Vol.12, Suppl. 23, (December 2007), pp. 1-17, ISSN1092-8529

Advokat, C.; Martino, L., Hill, B. \& Gouvier, W. (2007). Continuous Performance Test (CPT) of College Students with Attention-Deficit-Hyperactivity-Disorder (ADHD), Psychiatric Disorders, Cognitive Deficits, or No Diagnosis. Journal of Attention Disorders, Vol.10, No. 3, (February 2007), pp. 253-256, ISSN1087-0547

Advokat, C. (2009). What Exactly are the Benefits of Stimulants for ADHD? Guest Editorial for Journal of Attention Disorders, Vol.12, No.6, (May 2009), pp. 495-498, ISSN10870547

Advokat, C.; Lane, S. \& Luo, C. (2011). College Students with and Without ADHD: Comparison of Self-Report of Medication Usage, Study Habits and Academic Achievement. Journal of Attention Disorders, OnlineFirst, published on August 2, 2010 as doi: $10.1177 / 1087054710371168$

Advokat, C. (2010). What are the Cognitive Effects of Stimulant Medications? Emphasis on Adults with Attention-Deficit/Hyperactivity Disorder (ADHD). Neuroscience and Biobehavioral Reviews, Vol.34, No.8, (July 2010), pp.1256-1266, ISSN0149-7634 
Advokat, C. \& Luo, C. (2011). Do the Stimulant Medications Improve Neuropsychological Performance of College Students with Attention-Deficit/Hyperactivity Disorder (ADHD)? Submitted, Journal of Attention Disorders ISSN1087-0547

Advokat, C.; Martino, L. \& Guidry, D. (2008). Licit and Illicit Use of Attention-Deficit Hyperactivity (ADHD) Medication by College Students. Journal of American College Health, Vol.56, No.6, (May 2008), pp. 601-606, ISSN0744-8481

Arria, A.M.; Caldeira, K. M., O'Grady, K. E.,Vincent, K. B., Johnson, E. P. \& Wish, E. D. (2008). Nonmedical Use of Prescription Stimulants Among College Students: Associations with Attention-Deficit-Hyperactivity Disorder and Polydrug Use. Pharmacotherapy, Vol.28, No.2, (February 2008), pp.156-169, ISSN0277-0008

Arria, A.M. \& DuPont, R. L. (2010). Nonmedical Prescription Stimulant Use Among College Students: Why We Need to Do Something and What We Need to Do. Journal of Addictive Diseases, Vol.29, No.4, (September 2010), pp. 417- 426, ISSN1055-0887

Arria, A.M.; Garnier-Dykstra, L. M., Caldeira, K. M.,Vincent, K.B., O'Grady, K. E. \& Wish, E. D. (2011). Persistent Nonmedical Use of Prescription Stimulants Among College Students: Possible Association With ADHD Symptoms. Journal of Attention Disorders, Vol.15, No. 5, (July 2011), pp. 347-356, ISSN1087-0547

Barbaresi, W.J.; Katusic, S.K., Colligan, R.C., Weaver, A.L. \& Jacobsen, S.J. (2007a). LongTerm School Outcomes For Children With Attention-Deficit/Hyperactivity Disorder: A Population-Based Perspective. Journal of Developmental $\mathcal{E}$ Behavioral Pediatrics, Vol.28, No.4, (August 2007), pp. 265-273, ISSN0196-206X

Barbaresi, W.J.; Katusic, S.K., Colligan, R.C., Weaver, A.L. \& Jacobsen, S.J. (2007b). Modifiers of Long-Term School Outcomes For Children With Attention-Deficit/Hyperactivity Disorder: Does Treatment With Stimulant Medication Make A Difference? Results From a Population-Based Study. Journal of Developmental $\mathcal{E}$ Behavioral Pediatrics, Vol.28, No.4, (August 2007), pp. 274-287, ISSN0196-206X

Barkley, R.A. \& Cunningham, C. (1978). Do Stimulant Drugs Improve the Academic Performance of Hyperkinetic Children? Clinical Pediatrics, Vol.17, No.1, (January 1978), pp. 85-92, ISSN0009-9228

Barrilleaux, K. \& Advokat, C. (2009). Attribution and Self-Evalation of CPT Task Performance in Medicated and Unmedicated Adults with AttentionDeficit/Hyperactivity Disorder. Journal of Attention Disorders, Vol.12, No. 4, (January 2009), pp. 291-298, ISSN1087-0547

Baumeister, A.A.; Henderson, K., Pow, J.L. \& Advokat, C. (2011). The Early History of the Neuroscience Of Attention- Deficit/Hyperactivity Disorder. In Press, Journal of the History of the Neurosciences ISSN0964-704X

Bedard, A.; Ickowicz, A. \& Tannock, R. (2002). Methylphenidate Improves Stroop Naming Speed, But Not Response Interference, in Children With Attention Deficit Hyperactivity Disorder. Journal of Child and Adolescent Psychopharmacology, Vol.12, No. 4, (December 2002), pp. 301-309, ISSN1044-5463

Berman, S.M.; Kuczenski, R., McCracken, J.T. \& London, E.D. (2009). Potential Adverse Effects of Amphetamine Treatment on Brain and Behavior: A Review. Molecular Psychiatry, Vol.14, No.2, (February 2009), pp. 123-142, ISSN1359-4184

Biederman, J. \& Faraone, S.V. (2005). Attention-Deficit Hyperactivity Disorder. Lancet, Vol.366, No. 9481, (July 2005), pp. 237- 248, ISSN0140-6736 
Biederman, J.; Faraone, S.V., Spencer, T.J., Mick, E., Monuteaux, M.C. \& Aleardi, M. (2006). Functional Impairments in Adults With Self-Reports of Diagnosed ADHD: A Controlled Study of 1001 Adults in the Community. Journal of Clinical Psychiatry, Vol.67, No.4, (April 2006), pp. 524-540, ISSN0160-6689

Biederman, J.; Fried, R., Petty, C.R., Wozniak, J., Doyle, A.E., Henin, A., Corkum, L., Claudat, K. \& Faraone, S.V. (2010). Cognitive Development in Adults With Attention-Deficit/Hyperactivity Disorder: A Controlled Study in Medication-Naïve Adults Across the Adult Life Cycle. Journal of Clinical Psychiatry, Vol. , No. , ( ), pp. , ISSN0160-6689

Biederman, J.; Petty, C.R., Clarke, A., Lomedico, A. \& Faraone, S.V. (2011a). Predictors of Persistent ADHD: An 11-Year Follow-Up Study. Journal of Psychiatric Research, Vol.45, No 2, (February 2011), pp. 150-155, ISSN0022-3956

Biederman, J.; Petty, C.R., Fried, R., Kaiser, R., Dolan, C.R., Schoenfeld, S., Doyle, A.E., Seidman, L.J. \& Faraone, S.V. (2008a). Educational and Occupational Underattainment in Adults With Attention-Deficit/Hyperactivity Disorder: A Controlled Study. Journal of Clinical Psychiatry, Vol.69, No. 8, (August 2008), pp. 1217-1222, ISSN0160-6689

Biederman, J.; Seidman, L.J., Petty, C.R., Fried, R., Doyle, A.E., Cohen, D.R., Kenealy, D.C. \& Faraone, S.V. (2008b). Effects of Stimulant Medication on Neuropsychological Functioning in Young Adults With Attention Deficit/Hyperactivity Disorder. Journal of Clinical Psychiatry, Vol.69, No. 7, (July 2008), pp. 1150-1156, ISSN 01606689

Biederman, J.; Mick, E., Fried, R., Wilner, N., Spencer, T.J. \& Faraone, S.V. (2011b). Are Stimulants Effective in the Treatment of Executive Function Deficits? Results From a Randomized Double Blind Study of OROS-Methylphenidate in Adults With ADHD. European Neuropsychopharmacology, Vol.21, No. 7, (July 2011), pp. 508-515, ISSN

Blasé, S.L.; Gilbert, A.N., Anastopoulos, A.D., Costello, E.J., Hoyle, R.H., Swartzwelder, H.S. \& Rabiner, D.L. (2009). Self- Reported ADHD and Adjustment in College: CrossSectional and Longitudinal Findings. Journal of Attention Disorders, Vol.13, No. 3, (November 2009), pp. 297-309, ISSN1087-0547

Bradley, C. (1937). The Behavior of Children Receiving Benzedrine. American Journal of Psychiatry, Vol.94, No. 3, (November 1937), pp. 577-585, ISSN0002-953X

Brignell, C.M. \& Curran, H.V. (2006). Drugs, Sweat and Fears: A Comparison of the Effects of Diazepam and Methylphenidate on Fear Conditioning. Psychopharmacology, Vol.186, No. 4, (July 2006), pp. 504-516, ISSN0033-3158

Brignell, C.M.; Rosenthal, J. \& Curran, H.V. (2007). Pharmacological Manipulations of Arousal and Memory For Emotional Material: Effects of a Single Dose of Methylphenidate or Lorazepam. Journal of Psychopharmacology, Vol.21, No. 7, (September 2007), pp. 673-683, ISSN0269-8811

Burns, J.T.; House, R.F., Fensch, F.C. \& Miller, J.G. (1967). Effects of Magnesium Pemoline and Dextroamphetamineon Human Learning. Science, Vol.155, No. 3764, (February 1967), pp. 849-851, ISSN0036-8075

Carlson, C.L. \& Bunner, M.R. (1993). Effects of Methyphenidate on the Academic Performance of Children With Attention Deficit Hyperactive Disorder and 
Learning Disabilities. School Psychology Review, Vol.22, No. 2, ( ), pp. 184-198, ISSN0279-6015

Davidson, M. A. (2008). Literature Review: ADHD in Adults: A Review of the Literature. Journal of Attention Disorders, Vol.11, No. 6, (May 2008), pp. 628-641, ISSN1087-0547

deJongh, R.; Bolt, I., Schermer, M. \& Olivier, B. (2008). Botox For The Brain: Enhancement of Cognition, Mood and Pro-Social Behavior and Blunting of Unwanted Memories. Neuroscience and Biobehavioral Reviews, Vol.32, No. 4, ( ), pp. 760- 776, ISSN0149-7634

Dodson, W.W. (2005). Pharmacotherapy of Adult ADHD. Journal of Clinical Psychology/In Session, Vol.61, No. 5, (May 2005), pp. 589-606, ISSN0021-9762

Dopheide, J.A. \& Pliszka, S.R. (2009). Attention-Deficit-Hyperactivity Disorder: An Update. Pharmacotherapy, Vol.29, No. 6, (June 2009), pp. 656-679, ISSN0277-0008

Douglas, V.I.; Barr, R.G., Desilets, J. \& Sherman, E. (1995). Do High Doses of Stimulants Impair Flexible Thinking in Attention-Deficit Hyperactivity Disorder? Journal of the American Academy of Child and Adolescent Psychiatry, Vol. 34, No. 7, (July 1995), pp. 877-885, ISSN0890-8567

Doyle, A. (2006). Executive Functions in Attention-Deficit Hyperactivity Disorder. Journal of Clinical Psychiatry, Vol. 67, No. Suppl. 8, (), pp. 21-26, ISSN0160-6689

Dyme, I.Z.; Sahakian, B.J., Golinko, B.E. \& Rabe, E.F. (1982). Perseveration Induced by Methylphenidate in Children: Preliminary Findings. Progress in NeuroPsychopharmacology \& Biological Psychiatry, Vol.6, No. 3, ( ), pp. 269-272, ISSN02785846

Farah, M.J.; Haimm, C., Sankoorikal, G. \& Chatterjee, A. (2008). When we Enhance Cognition With Adderall, do we Sacrifice Creativity? A Preliminary Study. Psychopharmacology, Vol.202, No. 5, (July 2008), pp. 541-547, ISSN0269-8811

Farah, M.J.; Illes, J., Cook-Deegan, R., Gardner, H., Kandel, E., King, P., Parens, E., Sahakian, B. \& Wolpe, P.R. (2004). Neurocognitive Enhancement: What Can We Do And What Should We Do? Nature Reviews Neuroscience, Vol.5, No. 5, (May 2004), pp. 421-425, ISSN1471-003X

Faraone, S.V.; Spencer, T., Aleardi, M., Pagano, C. \& Biederman, J. (2004). Meta-Analysis of the Efficacy of Methylphenidate for Treating Adult Attention Deficit Hyperactivity Disorder. Journal of Clinical Psychopharmacology, Vol.24, No.1, (February 2004), pp. 24-29, ISSN02710749

Fischer, M.; Barkley, R.A., Edelbrock, C.S. \& Smallish, L. (1990). The Adolescent Outcome of Hyperactive Children Diagnosed by Research Criteria: II. Academic, Attentional, and Neuropsychological Status. Journal of Consulting and Clinical Psychology, Vol.58, No.5, (October 1990), pp. 580-588, ISSN0022-006X

Frazier, T.W.; Demaree, H.A. \& Youngstrom, E.A. (2004). Meta-Analysis of Intellectual and Neuropsychological Test Performance in Attention Deficit/Hyperactivity Disorder. Neuropsychology, Vol.18, No. 3, (July 2004), pp. 543-555, ISSN0894-4105

Frazier, T.W.; Youngstrom, E.A., Glutting, J.J. \& Watkins, M.W. (2007). ADHD and Achievement: Meta-Analysis of the Child, Adolescent, and Adult Literatures and a Concomitant Study With College Students. Journal of Learning Disabilities, Vol.40, No. 1, (February 2007), pp. 49-65, ISSN0022-2194

Funk, J.; Chessare, J.B., Weaver, M.T. \& Exley, A.R. (1993). Attention Deficit Hyperactivity Disorder, Creativity, and the Effects of Methylphenidate. Pediatrics, Vol.91, No. , ( ), pp. 816-819, ISSN0031-4005 
Gadow, K. (1983). Effects of Stimulant Drugs on Academic Performance in Hyperactive and Learning Disabled Children. Journal of Learning Disabilities, Vol.16, No. 5, (May 1983), pp. 290-299, ISSN0022-2194

Galéra, C.; Melchoir, M., Chastang, J.-F., Bouvard, M.-P. \& Fombonne, E. (2009). Childhood and Adolescent Hyperactivity- Inattention Symptoms and Academic Achievement 8 Years Later: The GAZEL Youth Study. Psychological Medicine, Vol.39, No. 11, (November 2009), pp. 1895-1906, ISSN0033-2917

Greely, H.; Sahakain, B., Harris, J., Kessler, R.C., Gazzaniga, M., Campbell, P. \& Farah, M.J. (2008). Towards Responsible Use of Cognitive Enhancing Drugs by the Healthy. Nature, Vol. 456, No.7223, (December 2008 ), pp. 702-705, ISSN0028- 0836

Greenhill, L.; Abikoff, H., Arnold, L.E., Cantwell, D., Conners, C.K., Elliott, G., Hechtman, L., Hinshaw, S.P., Hoza, B., Jensen, P.S., March, J.S., Newcorn, J., Pelham, W.E., Severe, J.B., Swanson, J.M., Vitiello, B. \& Wells, K. (1996). Medication Treatment Strategies in the MTA Study. Journal of the American Academy of Child and Adolescent Psychiatry, Vol.35, No. 10, (October 1996), pp. 1304-1313, ISSN 0890-8567

Gropper, R. \& Tannock, R. (2009). A Pilot Study of Working Memory and Academic Achievement in College Students With ADHD. Journal of Attention Disorders, Vol.12, No.6, (May 2009), pp. 574-581, ISSN1087-0547

Gualtieri, C.T. \& Johnson, L.G. (2008). Medications Do Not Necessarily Normalize Cognition In ADHD Patients. Journal of Attention Disorders, Vol.11, No. 4, (January 2008), pp. 459-469, ISSN1087-0547

Hall, K.M.; Irwin, M.M., Bowman, K.A., Frankenberger, W. \& Jewett, D.C. (2005). Illicit Use of Prescribed Stimulant Medication Among College Students. Journal of American College Health, Vol.53, No. 4 , ( ), pp. 167-174, ISSN0744- 8481

Harris, J. (2009). Is it Acceptable for People to Take Methylphenidate to Enhance Performance? Yes. BMJ, Vol.338, No. (June 2009), pp. b1955, ISSN09598138

Hechtman, L. \& Greenfield, B. (2003). Long-Term Use of Stimulants in Children With Attention Deficit Hyperactivity Disorder. Pediatric Drugs, Vol.5, No.12, (December 2003), pp. 787-794, ISSN 1174-5878

Hechtman, L.; Weiss, G. \& Perlman, T. (1984). Young Adult Outcome of Hyperactive Children Who Received Long-Term Stimulant Treatment. Journal of the American Academy of Child Psychiatry, Vol.23, No. 3, (May 1984), pp. 261-269, ISSN0890-8567

Hervey, A.S.; Epstein, J.N. \& Curry, J.F. (2004). Neuropsychology of Adults With Attention Deficit/Hyperactivity Disorder: A Meta-Analytic Review. Neuropsychology, Vol.18, No. 3, (July 2004), pp. 485-503, ISSN0894-4105

Ingram, S.; Hechtman, L. \& Morgenstern, G. (1999). Outcome Issues in ADHD: Adolescent and Adult Long-Term Outcome. MRDD Research Reviews, Vol.5, No. 3, ( ), pp. 243250, ISSN1080-4013

Izquierdo, I.; Bevilaqua, L.R., Rossato, J.I., Lima, R.H., Medina, J.H. \& Cammarota, M. (2008). Age-Dependent and Age- Independent Human Memory Persistence is Enhanced by Delayed Posttraining Methylphenidate Administration. Proceedings of the National Academy of Sciences, Vol.105, No. 49, (December 2008), pp. 19504-19507, ISSN0027-8424

Jacobi-Polishook, J.; Shorer, Z. \& Melzer, I. (2009). The Effect of Methylphenidate on Postural Stability Under Single and Dual Task Conditions in Children With Attention Deficit Hyperactivity Disorder - A Double Blind Randomized Control 
Trial. Journal of the Neurological Sciences, Vol.280, No. 1-2, (May 2009), pp. 15-21, ISSN0022-510X

Johnson, D.E.; Epstein, J.N., Waid, L.R., Latham, P.K., Voronin, K.E. \& Anton, R.F. (2001). Neuropsychological Performance Deficits in Adults With Attention Deficit/Hyperactivity Disorder. Archives of Clinical Neuropsychology, Vol.16, No. 6, (August 2001), pp. 587-604, ISSN0887-6177

Johnson, K. (2010). Medications for Cognitive enhancements in the Healthy: Psychiatrists Dilemma. Medscape Medical News

Kessler, R.C.; Adler, L., Barkley, R., Biederman, J., Conners, C.K., Demler, O., Faraone, S.V., Greenhill, L.L., Howes, M.J., Secnik, K., Spencer, T., Ustun, T.B., Walters, E.E. \& Zaslavsky, A.M. (2006). The Prevalence and Correlates of Adult ADHD in the United States: Results From The National Comorbidity Survey Replication. American Journal of Psychiatry, Vol.163, No. 4, (April 2006), pp. 716-723, ISSN0002$953 X$

King, J.A.; Colla, M., Brass, M., Heuser, I. \& Cramon, D.Y. (2007). Inefficient Cognitive Control in Adult ADHD: Evidence From Trial-By-Trial Stroop Test and Cued Task Switching Performance. Behavioral and Brain Functions, Vol.3, No. , (August 2007), pp. 42-60, ISSNISSN1744-9081

Kurscheidt, J.G.; Peiler, P., Behnken, A., Abel, S., Pedersen, A., Suslow, T. \& Deckert, J. (2008). Acute Effects of Methylphenidate on Neuropsychological Parameters in Adults With ADHD: Possible Relevance for Therapy. Journal of Neural Transmission, Vol.115, No. 2, (February 2008), pp. 357-362, ISSN0300-9564

Larriviere, D.; Williams, M.A., Rizzo, M. \& Bonnie, R.J. (2009). Responding to Requests From Adult Patients for Neuroenhancements. Guidance of the Ethics, Law and Humanities Committee. Neurology, Vol.73, No.17, (October 2009), pp. 1406-1412, ISSN 0028-3878

Leitner, Y.; Barak, R., Giladi, N., Peretz, C., Eshel, R., Gruendlinger, L. \& Hausdorff, J.M. (2007). Gait in Attention Deficit Hyperactivity Disorder. Effects of Methylphenidate and Dual Tasking. Journal of Neurology, Vol.254, No. 10, (October 2007), pp.13301338, ISSN0340-5354

Lewandowski, L. J.; Lovett, B. J., Codding, R. S. \& Gordon, M. (2008). Symptoms of ADHD and Academic Concerns in College Students With and Without ADHD Diagnoses. Journal of Attention Disorders, Vol.12, No.2, (September 2008), pp. 156-161, ISSN1087-0547

Loe, I. \& Feldman, H. (2007). Academic and Educational Outcomes of Children With ADHD. Ambulatory Pediatrics, Vol.7, No. 1, (January 2007), pp. 82-90, ISSN1530-1567

Mannuzza, S.; Klein, R.G., Bessler, A., Malloy, P. \& La Padula, M. (1993). Adult Outcome of Hyperactive Boys. Educational Achievement, Occupational Rank, and Psychiatric Status. Archives of General Psychiatry, Vol. 50, No.7, (July 1993), pp. 565- 576, ISSN0003-990X

McCabe, S.E.; Knight, J.R., Teter, C.J. \& Wechsler, H. (2005). Non-Medical Use of Prescription Stimulants Among US College Students: Prevalence and Correlates From a National Survey. Addiction, Vol.100, No. 1, (January 2005), pp. 96-106, ISSN0965-2140 
Monastersky, R. (2008). Some Professors Pop Pills for an Intellectual Edge; Scientists Say Drugs Help Concentration. Chronicle of Higher Education, Vol.54, No.32, (April 2008), pp. 33- , ISSN0009-5982

Müller, W.B.; Gimbel, K., Keller-Pliessnig, A., Sartory, G., Gastpar, M. \& Davids, E. (2007). Neuropsychological Assessment of Adult Patients With Attention Deficit/Hyperactivity Disorder. European Archives of Psychiatry and Clinical Neuroscience, Vol.257, No. 2, (March 2007), pp. 112-119, ISSN0940-1334

Murphy, K.R.; Barkley, R.A. \& Bush, T. (2001). Executive Functioning and Olfactory Identification in Young Adults With Attention Deficit-Hyperactivity Disorder. Neuropsychology, Vol.15, No.2, (April 2001), pp. 211-220, ISSN0894-4105

Norwalk,K.; Norvilitis, J.M. \& MacLean, M.G. (2009). ADHD Symptomatology and its Relationship to Factors Associated With College Adjustment. Journal of Attention Disorders, Vol.13, No. 3, (November 2009), pp. 251-258, ISSN1087-0547

Paterson, R.; Douglas, C., Hallmayer, J., Hagan, M. \& Krupenia, Z. (1999). A Randomized, Double-Blind, Placebo-Controlled Trial of Dexamphetamine in Adults With Attention Deficit Hyperactivity Disorder. Australian and New Zealand Journal of Psychiatry, Vol.33, No.4, (August 1999), pp. 494-502, ISSN1440-1614

Pelham, W.E.; McBurnett, K., Harper, G.W., et al. (1990). Methylphenidate and Baseball Playing in ADHD Children: Who's On First? Journal of Consulting and Clinical Psychology, Vol.58, No. 1, (February 1990), pp. 130-133, ISSN0022-006X

Prehn-Kristensen, A.; Krauel, K., Hinrichs, H., Fischer, J., Malecki, U., Schuetze, H., Wolff, S., Jansen, O., Duezel, E. \& Baving, L. (2011). Methylphenidate Does Not Improve Interference Control During a Working Memory Task in Young Patients With Attention-Deficit Hyperactivity Disorder. Brain Research, Vol.1388, No. , (May 2011), pp. 56-68, ISSN0006-8993

Rabiner, D.L.; Anastopoulos, A.D., Costello, E.J., Hoyle, R.H. \& Swartzwelder, H.S. (2008). Adjustment to College in Students With ADHD. Journal of Attention Disorders, Vol.11, No.6, (May 2008), pp. 689-699, ISSN1087-0547

Rabiner, D.L.; Anastopoulos, A.D., Costello, E.J., Hoyle, R.H., McCabe, S.E. \& Swartzwelder, H.S. (2009a). The Misuse and Diversion of Prescribed ADHD Medications by College Students. Journal of Attention Disorders, Vol.13, No. 2, (September 2009), pp. 144-153, ISSN1087-0547

Rabiner, D.L.; Anastopoulos, A.D., Costello, E.J., Hoyle, R.H., McCabe, S.E. \& Swartzwelder, H.S. (2009b). Motives and Perceived Consequences of Nonmedical ADHD Medication Use by College Students. Are Students Treating Themselves For Attention Problems? Journal of Attention Disorders, Vol.13, No.3, (November 2009), pp. 259-270, ISSN1087-0547

Rabiner, D.L.; Anastopoulos, A.D., Costello, E.J., Hoyle, R.H. \& Swartzwelder, H.S. (2010). Predictors of Nonmedical ADHD Medication Use by College Students. Journal of Attention Disorders, Vol.13, No. 6, (May 2010), pp. 640-648, ISSN1087- 0547

Rapport, L.J.; VanVoorhis, A., Tzelepis, A. \& Friedman, S.R. (2001). Executive Functioning in Adult Attention-Deficit Hyperactivity Disorder. The Clinical Neuropsychologist, Vol.15, No. 4, ( ), pp. 479-491, ISSN1385-4046

Reaser, A.; Prevatt, F., Petscher, Y. \& Proctor, B. (2007). The Learning and Study Strategies of College Students With ADHD. Psychology in the Schools, Vol.44, No. 6, (July 2007), pp. 627-638, ISSN0033-3085 
Repantis, D.; Schlattmann, P., Laisney, O. \& Heuser, I. (2010). Modafinil and Methylphenidate for Neuroenhancement in Healthy Individuals: A Systematic Review. Pharmacological Research, Vol.62, No. 3, (September 2010 ), pp. 187-206, ISSN1043-6618

Robbins, T.W. \& Sahakian, B.J. (1979). „Paradoxical“ Effects of Psychomotor Stimulant Drugs in Hyperactive Children From the Standpoint of Behavioral Pharmacology. Neuropharmacology, Vol.18, No. 12, (December 1979), pp. 931-950, ISSN0028-3908

Rubia, K.; Noorloos, J., Smith, A., Gunning, B., Sergeant, J. (2003). Motor Timing Deficits in Community and Clinical Boys With Hyperactive Behavior: The Effect of Methylphenidate on Motor Timing. Journal of Abnormal Child Psychology, Vol.31, No. 3, (June 2003), pp. 301-313, ISSN00910627

Sahakian, B. \& Morein-Zamir, S. (2007). Commentary: 'Professor's Little Helper.' Nature, Vol.450, No. 7173, ( December 2007), pp. 1157-1159, ISSN0028-0836

Sahakian, B.J. \& Robbins, T.W. (1977). Are the Effects of Psychomotor Stimulant Drugs on Hyperactive Children Really Paradoxical? Medical Hypotheses, Vol.3, No.4, (JulyAugust 1977), pp. 154-158, ISSN0306-9877

Scheffler, R.M.; Brown, T.T., Fulton, B.D., Hinshaw, S.P., Levine, P. \& Stone, S. (2009). Positive Association Between Attention-Deficit/Hyperactivity Disorder Medication Use and Academic Achievement During Elementary School. Pediatrics, Vol.123, No. 5, (May 2009), pp. 1273-1279, ISSN0031-4005

Schoechlin, C. \& Engel, R.R. (2005). Neuropsychological Performance in Adult Attention Deficit Hyperactivity Disorder: Meta-Analysis of Empirical Data. Archives of Clinical Neuropsychology, Vol.20, No.6, (August 2005), pp. 727-744, ISSN0887-6177

Schroeder, S.R.; Mann-Koepke, K., Gualtieri, C.T., Eckerman, D.A. \& Breese, G.R. (1987). Methylphenidate Affects Strategic Choice Behavior in Normal Adult Humans. Pharmacology, Biochemistry and Behavior, Vol.28, No. 2, (October 1987), pp. 213-217, ISSN0091-3057

Schwanz, K.A.; Palm, L.J. \& Brallier, S.A. (2007). Attention Problems and Hyperactivity as Predictors of College Grade Point Average. Journal of Attention Disorders, Vol.11, No.3, (November 2007), pp. 368-373, ISSN1087-0547

Setlik, J.; Bond, R. \& Ho, M. (2009). Adolescent Prescription ADHD Medication Abuse is Rising Along With Prescriptions for These Medications. Pediatrics, Vol.124, No. 3, (September 2009), pp. 875-880, ISSN0031-4005

Smith, M. E. \& Farah, M.J. (2011). Are Prescription Stimulants "Smart Pills?" The Epidemiology and Cognitive Neuroscience of Prescription Stimulant Use by Normal Healthy Individuals. Psychological Bulletin, Vol.137, No.5, (September 2011), pp.717-741, ISSN 0033-2909

Soetens, E.; Casaer, S., Dhooge, R. \& Hueting, J. (1995). Effect of Amphetamine on LongTerm Retention of Verbal Material. Psychopharmacology, Vol.119, No. 2, (May 1995), pp. 155-162, ISSN0033-3158

Soetens, E.; Dhooge, R. \& Hueting, J. (1993). Amphetamine Enhances Human-Memory Consolidation. Neuroscience Letters, Vol.161, No.1, (October 1993), pp. 9-12, ISSN0304-3940

Solanto, M.V. \& Wender, E.H. (1989). Does Methylphenidate Constrict Cognitive Functioning? Journal of the American Academy of Child and Adolescent Psychiatry, Vol.28, No.6, (November 1989), pp. 897-902, ISSN0890-8567 
Spencer, T.J.; Biederman, J. \& Mick, E. (2007). Attention-Deficit/Hyperactivity Disorder: Diagnosis, Lifespan, Comorbidities, and Neurobiology. Journal of Pediatric Psychology, Vol.32, No. 6, (July 2007), pp. 631-42, ISSN0146-8693

Stix, G. (2009). Turbocharging the Brain. Scientific American, Vol.301, No. , (October 2009), pp. 46-55, ISSN0036-8733

Stray, L.L.; Stray, T., Iversen, S., Ruud, A. \& Ellertsen, B. (2009). Methylphenidate Improves Motor Functions in Children Diagnosed With Hyperkinetic Disorder. Behavioral and Brain Functions, Vol.5, No. 22, (May 2009), pp. 21-33, ISSN1744-9081

Swanson, J.; Baler, R.D. \& Volkow, N.D. (2011). Understanding the Effects of Stimulant Medications on Cognition in Individuals With Attention-Deficit Hyperactivity Disorder: A Decade of Progress. Neuropsychopharmacology, Vol.36, No. 1, (January 2011), pp. 207-226, ISSN0893-133X

Swanson, J.M.; Cantwell, D., Lerner, M., McBurnett, K. \& Hanna, G. (1991). Effects of Stimulant Medication on Learning in Children With ADHD. Journal of Learning Disabilities, Vol.24, No. 4, (April 1991), pp. 219-230, ISSN0022-2194

Swartwood, M.O.; Swartwood, J.N. \& Farrell, J. (2003). Stimulant Treatment of ADHD: Effects on Creativity and Flexibility in Problem Solving. Creativity Research Journal, Vol.15, No. 4, ( ), pp. 417-419, ISSN1040-0419

Talbot, M. (2009). Brain Gain: The Underground World of Neuroenhancing Drugs. New Yorker April 27, pp. 32-43.

Tannock, R. \& Schachar, R. (1992). Methylphenidate and Cognitive Perseveration in Hyperactive Children. Journal of Child Psychology and Psychiatry, Vol.33, No. 7, (October 1992), pp. 1217-1228, ISSN0021-9630

Teter, C.J.; McCabe, S.E., Boyd, C.J. \& Guthrie, S.K. (2003). Illicit Methylphenidate Use in an Undergraduate Student Sample: Prevalence and Risk Factors. Pharmacotherapy, Vol.23, No. 5, (May 2003), pp. 609-617, ISSN0277-0008

Teter, C.J.; McCabe, S.E., Cranford, J.A., Boyd, C.J. \& Guthrie, S.K. (2005). Prevalence and Motives for Illicit Use of Prescription Stimulants in an Undergraduate Student Sample. Journal of American College Health, Vol.53, No. 6, ( ), pp. 253-262, ISSN07448481

Teter, C.J.; McCabe, S.E., LaGrange, K., Cranford, J.A. \& Boyd, C.J. (2006). Illicit Use of Specific Prescription Stimulants Among College Students: Prevalence, Motives, and Routes of Administration. Pharmacotherapy, Vol.26, No. 10, (October 2006), pp. 15011510, ISSN0277-0008

Tucha, O.; Mecklinger, L., Laufkotter, R., Klein, H.E., Walitza, S. \& Lange, K.W. (2006). Methylphenidate-Induced Improvements of Various Measures of Attention in Adults With Attention Deficit Hyperactivity Disorder. Journal of Neural Transmission, Vol.113, No. 10, (October 2006), pp. 1575-1592, ISSN0300-9564

Tucha, O. \& Lange, K.W. (2004). Handwriting and Attention in Children and Adults With Attention Deficit Hyperactivity Disorder. Motor Control, Vol.8, No. 4, (October 2004), pp. 461-471, ISSN1087-1640

Tucha, L.; Tucha, O., Sontag, T.A., Stasik, D., Laufkotter, R. \& Lange, K.W. (2011). Differential Effects of Methylphenidate on Problem Solving in Adults With ADHD. Journal of Attention Disorders, Vol.15, No.2, (February 2011), pp. 161-173, ISSN10870547 
Turner, D.C.; Blackwell, A.D., Dowson, J.H., McLean, A. \& Sahakian, B.J. (2005). Neurocognitive Effects of Methylphenidate in Adult Attention Deficit/Hyperactivity Disorder. Psychopharmacology, Vol.178, No. 2-3, (March 2005), pp. 286-295, ISSN0033-3158

Van der Oord, S.; Prins, P.J.M., Oosterlaan, J. \& Emmelkamp, P.M.G. (2008). Efficacy of Methylphenidate, Psychosocial Treatments and Their Combination in School-Aged Children With ADHD: A Meta-Analysis. Clinical Psychology Reviews,Vol.28, No. 5, (June 2008), pp. 783-800, ISSN0272-7358

Weiss, M. \& Murray, C. (2003). Assessment and Management of Attention-Deficit Hyperactivity Disorder in Adults. Journal of the Canadian Medical Association, Vol.168, No. 6, (March 2003), pp. 715-722, ISSN0008-4409

Wender, P.H.; Reimherr, F.W., Marchant, B.K., Sanford, M.E., Czajkowski, L.A. \& Tomb, D.A. (2011). A One-Year Trial of Methylphenidate in the Treatment of ADHD. Journal of Attention Disorders, Vol.15, No.1, (January 2011), pp. 36-45, ISSN1087-0547

Weyandt, L.L. \& DuPaul, G. (2006). ADHD in College Students. Journal of Attention Disorders, Vol.10, No. 1, (August 2006), pp. 9-19, ISSN1087-0547

Weyandt, L.L.; Janusis, G., Wilson, K.G., Verdi, G., Paquin, G., Lopes, J., Varejao, M. \& Dussault, C. (2009). Nonmedical prescription Stimulant Use Among a Sample of College Students: Relationship With Psychological Variables. Journal of Attention Disorders, Vol.13, No.3, (November 2009), pp. 284-296, ISSN1087-0547

White, B.P.; Becker-Blease, K.A. \& Grace-Bishop, K. (2006). Stimulant Medication Use, Misuse, and Abuse in an Undergraduate and Graduate Student Sample. Journal of American College Health, Vol.54, No. 5, (March 2006), pp. 261-268, ISSN0744-8481

Wilens, T.E.; Adler, L.A., Adams, J., Sgambati, S., Rotrosen, J., Sawtelle, R., Utzinger. L. \& Fusillo, S. (2008). Misuse and Diversion of Stimulants Prescribed for ADHD: A Systematic Review of the Literature. Journal of the American Academy of Child $\mathcal{E}$ Adolescent Psychiatry, Vol.47, No. 1, (January 2008), pp. 21-31, ISSN0890-8567

Wolf, L.E. (2001). College Students With ADHD and Other Hidden Disabilities. Annals of the New York Academy of Sciences, Vol.931, No. 1, (June 2001), pp. 385-395, ISSN00778923

Woods, S.P.; Lovejoy, D.W. \& Ball, J.D. (2002). Neuropsychological Characteristics of Adults With ADHD: A Comprehensive Review of Initial Studies. The Clinical Neuropsychologist, Vol.16, No. 1, (February 2002), pp. 12-34, ISSN1385-4046

Zeeuws, I. \& Soetens, E. (2007). Verbal Memory Performance Improved via an Acute Administration of D-Amphetamine. Human Psychopharmacology, Vol.22, No. 5, (July 2007), pp. 279-287, ISSN0885-6222 


\title{
Motor Skills in Children with ADHD: Comparative Study from the Farmacological Treatment
}

\author{
Jose Armando Vidarte Claros ${ }^{1}$ and Consuelo Velez Alvarez ${ }^{2}$ \\ ${ }^{1}$ Universidad Autónoma de Manizales, Department of Human Movement, \\ Body-Movement Research Group, \\ ${ }^{2}$ Universidad de Caldas, Department of Public Health, \\ Promotion and prevention Research Group, \\ Colombia
}

\section{Introduction}

(Gaillard et al., 2004) affirm that the term Hyperactivity is about a type of disturbing behavior that was always stigmatized by the terms agitation, psychomotor instability and hiperkinesia. And the terms "psychomotor" and "hiperkinesia" refer to a motor behavior resulting from attention and excitation problems becoming an outer presence, on the other hand, (Vidarte et al., 2009) on the Psychomotor profile of the children clinically diagnosed with TDAH in the city of Manizales, concluded how the mobility factors of the diagnosed children presented normal rank values which were significantly worse when comparing them with the healthy children in all the ages, this chapter tries to demonstrate the results obtained in a research process, that shows the differences happened in the psychomotor profile in children diagnosed with the TDAH that receives pharmacological treatment with two variables of analysis (sex and the type of TDAH) as a basis.

The neurochemical hypothesis which poses a dysfunction of fronto striatal connections either of anatomical or functional origin, has been the justification for the use of pharmacotherapy (including methylphenidate or Ritalin, a stimulant that activates the inhibitory network behavior). This hypothesis remains attractive from a heuristic point of view, it tends to consider all the cortex as a reflecting organ of frontal lobe, rather than seeing it as the control center of social behavior, which also addresses environmental responses (Pliszka et al., 1996 y Tannock, 1998) and where it is considered the expression of the motor skills as a socializing element.

In this paper the authors try to provide elements that show the motor skills behaviors in children diagnosed with ADHD and taking medications and also this paper allows new hypotheses to the scientific evidence for this relationship. For this reason, the objective of this paper is to establish the differences in the behavior of the psychomotor profile of children clinically diagnosed with ADHD and taking phramochological treatment in the city of Manizales, Colombia. 
The development of this paper has primarily a theoretical and conceptual approach of the disorder from the scientific evidence established by different authors where issues as the definition, prevalence, etiology, comorbidities and therapeutic implications arise, making emphasis on motor skill and comorbidity; secondly the results obtained in the study show the relationship between the psychomotor profile of children diagnosed from pharmachological treatment.

\section{Attention Deficit Disorder and hyperactivity}

The attention deficit disorder with hyperactivity is an important health problem, for its own characteristics, and for its alterations that are associated or triggered as a result of inattention or hyperactivity. People with this syndrome, not only suffer the direct effects of the disorder, but also the academic, social or labor impact involved, and it often persists even after you have submitted the disorder. The attention deficit disorder with hyperactivity (ADHD) is, along with dyslexia, the most important cause of school failure.

Disorder Attention Deficit is known by the acronyms: ADD (Attention Deficit Disorder), ADS (Attention Deficit Syndrome), AD (Attention Deficit), ADHD (Atention Deficit Hiperactivity Disorder), and more commonly ADHD (Attention Deficit Hyperactivity Disorder). The (American Psychiatric Association, 2002) characterizes it as "a persistent pattern of inattention and / or hyperactivity-impulsivity that is stronger and severe than typically observed in individuals of a similar level of development". These three dimensions: inattention, hyperactivity and impulsivity are the axes of the disorder and they are characterized as follows:

Attention disorders are the inability to maintain the standard care for long periods of time, characterized by fleeting attention and inhibited impulses, in the waking state is one of the manifestations of this disorder. People who are inattentive have difficulty concentrating on one thing and become bored with a task after only a few minutes. Lack of attention can be inferred from the observation of behavior, as, for example, if a child is not performing a task for the required time, a task that is available to other children with the same age, intelligence and schooling, from which is different (Barkley, 1991b).

The diagnostic criteria for attention deficit disorder in DSM-IV version (1995) and DSM-IVTR (2002) have often not enough attention to details or careless mistakes done in schoolwork, in the work or other activities. It often has difficulty sustaining attention in tasks or playing activities. It often does not seem to listen when spoken to directly. It often does not follow instructions and fails to finish schoolwork, chores or duties in the workplace (not due to oppositional behavior or failure to understand instructions). It often has difficulty organizing tasks and activities. It often avoids, dislikes or is reluctant to engage in tasks that require sustained mental effort (such as schoolwork or household). It often loses things necessary for tasks or activities (e.g. toys, school assignments, pencils, books or tools). It is often distracted by irrelevant stimuli. It is often forgetful in daily activities.

Hyperactivity is the second component of ADHD and typically manifested by excessive and continuous movement when it is inappropriate to do so, restlessness, nervousness and inability to sit without getting up, to "be running" continuously, as having an internal engine; and talking too much. The problems of children with ADHD include both an excess of activity and inappropriate activity depending on their age and circumstances (Barkley, et 
al., 1990b). It is also said to be desobidient, who do not stop tapping their fingers, moving in their seats or to annoy their classmates, (Whalen, 1986; Whalen y Henker, 1991a; Arnold, et al., 1997b).

Hyperactive children seem to have problems regulating their actions according to the wishes of others or the demands of context, (Teichner, et al., 1996; Solanto, et al., 2001; Slusarek, et al., 2001). Hyperactive behavior is usually extended to a group of behaviors such as aggression, constant activity, distractibility, impulsiveness, inability to concentrate and difficulty in participating in "silent" activities such as reading and activities that require a similar behaviors, although they may relate to each other, they do not always correspond with hyperactivity itself.

The diagnostic criteria for hyperactivity in the version of the DSM-IV (1995) and DSM-IV-TR (2002) are: He often fidgets with hands or feet or squirms in seat. He often leaves seat in classroom or in other situations in which remaining seated is expected. He often runs about or climbs excessively in situations in which it is inappropriate (in adolescents or adults it may be limited to subjective feelings of restlessness). He often has difficulty playing or engaging in leisure activities quietly. He often "is ongoing." He often talks excessively.

Impulsivity is the third component of ADHD, this behavior turns into impatience, inability to postpone a reply, to answer before the question has been made in full, and to stop frequently, causing problems in social situations. Also, individuals can make inappropriate comments, display a lack of attention to understanding the rules, buffoonery, etc.. That is, children are unable to control themselves, to adapt their behavior to environmental demands and delay gratification. (Barkley, 1997) described the multidimensional nature of these symptoms, according to it, including cognitive and behavioral aspects. Behaviors associated with hyperactivity and impulsivity are: social disinhibition, lack of caution in dangerous situations and impulsively breaking social norms, so that these children suffer continuous accidents and are easily rejected by equal groups.

The diagnostic criteria for impulsivity in the DSM-IV version (1995) and DSM-IV-TR (2002) are: It often blurts out answers before questions have been completed. It often has difficulty awaiting a turn. It often interrupts or intrudes on the activities of others (eg, butts into conversations or games.

From these components ADHD has been classified: predominantly hyperactive, predominantly combined and predominantly inattention. For many authors, ADHD is the most common disorder in childhood (Shaywitz y Shaywitz, 1991; Barkley, et al., 1971; Biederman et al., 1996a; Wolraich et al., 1996) and it appears to be persistant into adolescence and adulthood (Barkley, et al., 1990b; Ferguson, et al., 1993). The prevalence of ADHD ranges between 3\% and 7\% in general population and between $10 \%$ and $15 \%$ in clinical population (Fischer, et al., 1990).

The prevalence of ADHD symptoms varies depending on who reports (parents or teachers), age and sex of children and the evaluation criteria used (Amador y Forns, 2001). Epidemiological studies indicate that the prevalence of ADHD varies by age, subtype of the disorder (ADHD inattentive type or hyperactive-impulsive type) and gender. In this sense, ADHD hyperactive-impulsive type is four times more common in boys than girls (4:1), and ADHD inattentive type is in 2:1 ratio. Both subtypes are more common between eight and ten years, (Wolraich et al., 1996; Bathia et al., 1991). 
In Colombia, there has been several prevalence studies, such as one involving children of 5 to 7 years in the city of Manizales, in which individual interviews were used based on DSMIV. The prevalence of the disorder was $8.2 \%$. These data comfirmed those provided by different literature sources, finding that the disorder was more common in children who were 6 years old, the male / female ratio was 5 to 1, the most common age of onset of symptoms, according to parents was at 5 years and according to teachers at 6 years (Vidarte y Vélez, 1999).

Subsequently, these authors developed a second phase with children 8 to 12 years, it was found that the prevalence was $7.1 \%$. Comparatively, the prevalence was lower than the one found in the first study, with the differential variable between the age of the subjects. Both studies also suggest the importance of the problem of ADHD in this city of Colombia and the need to continue to advance knowing about the problem (Vidarte y Vélez, 2001). Another study found a prevalence of $16.1 \%$. The diagnostic criteria used a list of symptoms that corresponded exactly to the 18 items of DSM-IV criterion for diagnosis of ADHD. Data were obtained from parents of children and adolescents from 4 to 17 years in the city of Manizales (Pineda et al., 2001).

In the city of Cali, a study on the neuropsychological and behavioral profiles of children with ADHD (Bará et al., 2003) was done. In addition to describing the neuropsychological profiles of children, a prevalence of ADHD of $16 \%$ was showed. In 2005, it was established from the study on the prevalence of ADHD in Colombian adolescents from Sabaneta town (Antioquia) a prevalence according to DSM-IV 15.86\% (Cornejo et al., 2005).

Previous studies show that in Colombia there is a high prevalence of ADHD, much higher than in studies elsewhere. This discrepancy could be explained as a result of several factors which include the use of more or less restrictive criteria in establishing the diagnosis, or the existence of psychosocial risk factors of the environment from which population samples are extracted. Added to this, there are other important elements as follows: from the total population diagnosed, only $7.4 \%$ received a diagnosis confirmed by a structured psychiatric interview, and only $6.6 \%$ of patients have treatment which could indicate that there could be a subregister in the diagnosis in developed countries.

Although the DSM itself in its different versions indicates the absence of evidence to suggest organic disorders in the etiology of $\mathrm{ADHD}$, recent studies have been providing relevant information in this area. For example, there is strong evidence that genetic factors are important in causing the disorder, but also other factors are considered: biochemical, neurological, environmental, viruses, problems during pregnancy, working and others that alter brain development.

Since genetic factors, research in this field in the etiology of ADHD are based on studies of twins and families. The studies results with families have shown a higher incidence of ADHD or symptoms thereof, in biological parents of these children (Shaywitz y Shaywitz, 1991; Biederman, et al., 1992; Faraone, 1996). Also, a higher proportion of psychiatric disorders in biological parents of children with ADHD was found, a higher incidence of anxiety disorders and depression in mothers and antisocial personality disorders and alcoholism in parents was found (Biederman, et al., 1990; Biederman, et al., 1991; Faraone, et al., 1998a; Schaill, et al., 1999). 
A study shows that the biological parents of children with ADHD have higher risk of attention deficit (Frick, 1994). However, some studies have shown the existence of environmental factors involved in the presentation and severity of ADHD symptoms and their association with other pathologies such as aggressive behavior (Shaywitz y Shaywitz 1991; Biederman, et al.,1991; August, et al., 1996a; Biederman, et al., 1997). In a study conducted to evaluate the association between ADHD and disorders of first-degree biological parents and adoptive parents of children with ADHD, it is supported in the hypothesis that ADHD has a significant biological component (Sprich, et al., 2000). The data found in twins studies support the hypothesis of a genetic component in the manifestation of ADHD. A greater concordance between monozygotic twins than in dizygotic twins was found. These results support the idea of a genetic influence in the etiology of the disorder (Gillis, et al., 1991; Sherman, et al., 1997b).

It has been found then the manifestation of ADHD is explained in $50 \%$ by genetic factors. It was also found a concordance for ADHD of $81 \%$ in monozygotic twins and dizygotic twins 29\% (Goodman y Stevenson, 1989; Sherman, et al., 1997a; Silberg, et al., 1998). Similarly it was found a genetic influence in the a etiology of ADHD in childhood, while the emergence in adolescence with aggressive behavior explained would preferably be in an environmental influence (Silberg, et al. 1996 p.803). According to the data above, it could argue that the results obtained from studies of twins and families support the hypothesis of polygenic inheritance in the transmission of ADHD.

From the neurochemical factors, recent findings suggest a neurochemical disorder in the etiology of ADHD, finding changes in cerebral blood flow and metabolism (Teeter y Semrud-Clikeman, 1995). These changes produce less activation in the frontal, temporal, and limbic areas. After conducting various researches, it has been conjectured an underlying genetic predisposition to the etiology of ADHD, which carries a hypofunctional of dopaminergic pathways in the prefrontal and limbic system, involved in the mechanism responsible for the manifestation of symptoms ADHD (Barkley, et al., 1992).

From a behavioral point of view, the deficiency of dopamine in the prefrontal region results in an inability to control impulses and a difficulty in planning and carrying out a sequence of actions aimed at achieving a goal, the difficulty in delaying gratification and excessive motor skill activity, key features of attention deficit disorder with hyperactivity. Both norepinephrine and epinephrine, and dopamine have been related to the pathophysiology of ADHD (Pliszka, et al., 1996). These chemical mediators are responsible for transmitting stimuli from neuron to neuron and they are involved in explaining many psychopathologic changes. The authors suggested that these three neurotransmitters had some kind of problem, namely one in neurotransmitters dopamine hypofunctional.

Other studies (Castellanos, et al., 1996b; Arnstein, et al., 1996) have reformulated the catecholamine hypothesis by emphasizing the role played by dopamine and norepinephrine in the pathophysiological explanation for ADHD. These authors propose the existence of a subactivación and overactivation in two dopaminergic regions as mechanisms in the genesis of ADHD. The subactivación would be located in the cortical region (e.g. anterior cingulate) and it would be responsible for cognitive deficits of these children, and overactivation in the region would be subcortical (e.g. caudate nucleus) and it would explain the excessive neural activity (Swanson, et al., 1991b), noting that it seems unlikely that ADHD is associated with just one hypofunctional of the dopaminergic system. 
In fact, the selective dopamine agonist administration has not shown improvement in symptoms of ADHD (Navarro y Manzanaque, 1998). Add to that the complex web of connections available to the dopamine neurotransmitter. Today, it has been discovered up to five different subtypes of these neurotransmitters, each one with different routes and different roles or behavioral circuits (Navarro, 2000). It might be concluded that in the results of research on the neurochemical components in explaining the pathophysiology of ADHD there are many questions to be answered, as a failure to demonstrate a direct relationship between one type of neurotransmitter and its effects on the child's behavior.

From the neuroanatomical factors, studies using neuroimaging techniques have found differences, although discordant in brain morphology of children with ADHD (Castellanos et al., 1996b; Castellanos et al., 1996a). Early studies with these techniques had numerous methodological biases, (Casey et al., 1997; Swanson et al., 1998). A hypothesis currently supported in a neuroanatomical study of ADHD is involving the prefrontal lobe in explaining the symptoms of this disorder, (Catellanos et al., 1996a; Colby, 1991; Catellanos et al., 1994). This area, in particular, the frontal lobe, is responsible for planning, directing, making decisions and evaluating the results of our actions, what has been referred to as the general system of self-regulation of behavior (Barkley et al., 1992).

The relationship between dysfunction in the prefrontal cortex areas and deficits in response inhibition in visual-motor tasks in a sample of 13 children with ADHD and 10 normal (Ross et al., 1994) has been examined. Children with ADHD showed significant differences compared with the control group, in the execution of response inhibition tasks, but not in visuospatial memory tasks or response latency. The authors associated these deficits to a dysfunction in the dorsolateral cortex, other authors, based on studies of neuronal circuits interconnected identifies five anatomical structures that may represent an important pathophysiological model in explaining the genesis of ADHD (Ross et al., 1994). These structures have connections with motor and sensory cortex.

Studies using MRI indicate that some regions of the frontal lobe (anterior, superior and inferior) and basal ganglia (caudate nucleus and globus pallidus) are lower in children with ADHD than in normal children. Thus, it has found a total brain volume $5 \%$ smaller in children with ADHD than in normal ones, particularly in the area of the caudate nucleus, a deficit in prefrontal neural processing in children with ADHD compared to the control group and a smaller volume of white matter in the right frontal lobe (Silberstein et al., 1998; Semrud-clikeman e $t$ al., 2000). These authors suggest that morphological changes in the frontal lobe and the caudate nucleus are inversely correlated with measures of inhibition and externalizing behavior of children with ADHD. The results of these studies are consistent with theoretical models of the altered function of fronto-striate and parietal lobes.

With regard to risk factors, several studies have indicated the existence of risk factors in the explanation of ADHD (Johnston y Pelham, 1986;Burnley, 1993; Milberger et al, 1997; Max et al., 1998). Among the proposed risk factors include: smoking in pregnant women, complications during pregnancy and childbirth, exposure to chemicals and brain injury (Wozniak et al., 1999). Ultimately, and based on the checked results on etiology, it is likely to be a neuroanatomical and neurochemical basis in the explanation of ADHD. The findings in family studies of twins and genetics support the hypothesis of a polygenic inheritance in the transmission of ADHD. The results in neurochemistry and neuroanatomy of the etiology of 
this disorder pose many questions to be answered, as the failure to demonstrate a direct relationship between one type of neurotransmitter and its effects on behavior. Although the mechanisms responsible are unknown in the genesis of ADHD, studies on pathophysiology point to the existence of neuroanatomical, genetic and environmental factors as well as in the expression and severity of ADHD (Kasdejo et al., 2001).

\subsection{Therapeutic implications}

At present, the treatment of subjects with ADHD moves in three directions: first, a reeducation orientation which has several variants depending on the emphasis given to academics or cognitive-behavioral disturbances; the second one is an interdisciplinary approach in which different professionals (physician, psychologist, educator, physical educator, etc.). have their respective areas in the various manifestations of this disorder, and the third one that is pharmacological in nature, not without controversy, but it offers research on results, not only more numerous but also more accurate than the others.

In general, neuropharmacological treatment of ADHD, aims to improve the nerve impulses that act on certain frontostriatal circuits. Dopaminergic and noradrenergic mediation between nerve impulses and the optimal functioning of these circuits would result in a substantial reduction in symptoms of ADHD, especially in regard to executive functions. Medication treatment was started in 1937 when Bradley described the effects of benzedrine in the behavior of children. Since then it has clearly documented the role and efficacy of stimulants and it has been demonstrated their effectiveness in the treatment of motor skill activity and lack of attention. Methylphenidate (MPH) and dextroamphetamine (DA) are the medications most commonly used stimulants. Alternatively, the tricyclic antidepressants have benefited some patients.

Although treatment of the hyperactive child is based on four therapeutic pillars, the medical pharmachologic approach has a special relevance. For years, alternative treatments for ADHD have been confined to the use of psychostimulants, and their favorable effects in reducing the symptoms and manifestations of ADHD are the medications of first-line treatment. These medications improve the behavior between 70 and $90 \%$ of children older than 5 years (Barkley, 1988). The efficacy of these stimulants has been shown from placebocontrolled double-blind study in children and adults, a $65-85 \%$ of ADHD patients had a clinical response to methylphenidate, compared to $4-30 \%$ with placebo (Wilens y Spencer, 2000). Scientific evidence suggests that methylphenidate and amphetamine salts are equally effective in the treatment of ADHD in children (Vitiello et al., 2001; Montañes-Rada et al., 2009).

The new formulations of these compounds have demonstrated long-acting, at least, similar efficacy to the immediate release (Faraone e $t$ al., 2006), which is similar throughout the different stages of life, including preschool, children, adolescents and adults (Jensen et al., 2001; Ramos-Quiroga et al., 2006; Conners et al., 2001). Despite the high efficiency, approximately $20-35 \%$ of patients do not respond to these treatments (Conners et al., Barkley, 1977).

The benefit with the use of stimulants is $75 \%$ to $90 \%$. subjects receiving this treatment not only are less impulsive, restless and distracted and they internalize the information better, have better relationships, establish commitments in a better way and thus they autocontrol 
more efficiently, and they let them be more accepted and liked by their peers and receive less punishment, improving self-esteem. However, this type of pharmacological intervention has some restrictions, such as misuse of medication, the pharmacodependency that occurs in some people and the misconception that the pharmacological treatment is sustained as the only alternative to the problem of attention, ignoring other alternatives such as integral treatment (Fernández Jaen, 1999).

Recently there have been results referred to a Multimodal Study of Children with Attention Deficit / hiperactivity Disorder (MTA Cooperative Group, 2003). This study has shown that stimulant medication had clearly superior results to those obtained with behavioral treatment and environmental care. In addition, combined therapy (behavioral and pharmacological) was not significantly superior to medical therapy alone. The use of stimulants often produces an immediate improvement in behavior, it also improves selfcontrol, attention, aggression, and interpersonal relationships, especially at family and school level. It states that it is important for teachers to know that medication do not control the child, they simply help filter distractions allowing them to concentrate on the tasks they perform, helping to reduce impulsiveness and facilitate better decision-making (Arias, 2003).

Concerning the effects of some medication used in ADHD on the motor skill, studies are scarce. A study on the effect of methylphenidate in children with ADHD and DCD on fine motor skills of these two groups and a control group. The results showed that children with ADHD-DCD, performed the motor tasks with greater motor skill deficiency than children in the control group. In particular, the activities of manual dexterity subtests had poorer quality of handwriting, and drew more quickly but less accurately than healthy graphomotor tasks. Applying methylphenidate, manual dexterity and improved quality of the handwriting and the beats in the graphomotor task were less rapid and more accurate (Houwe y Schoemaker, 2006). In short, the most widely used medication to treat ADHD is methylphenidate. Its effectiveness is supported by numerous studies over five decades (Conners et al., 2001; Artigas-Pallarés, 2004; Rapport et al., 1994; Whalen et al., 1979). The action of methylphenidate is a selective inhibition of dopamine reuptake and acts to improve both academic performance, such as social interaction, and a general behavior, since it reduces hyperactivity and keeps attention. Under research there are medicines derived from nicotine, which use improves dopaminergic neurotransmission, memory and executive functions (Faraone et al., 1997).

From the known components or integrated multimodal treatments, two issues should be highlighted: first, variations in the number of professionals integrated into the program, and the second, in terms of objectives and methods that are oriented primarily to reduce or prevent dysfunctions associated with ADHD symptoms (aggression, negativism, antisocial behavior, etc.). In this regard, it is noted the effectiveness of treatments made from conduct modification, when recognizing the child working with parents and educators in parallel. Physical activity, and TDH-oriented therapeutic modality could be a route of considerable interest, when in advance, accurately described motor abnormalities in these patients have been found.

In adulthood, about 15 to $20 \%$ of children with attention deficit disorder and hyperactivity continue to experience symptoms of this disorder; another third have symptoms of antisocial personality disorder. A significant percentage shows medication abuse $(16 \%)$, especially when two are provided before adolescence behavioral problems and academic 
partners are corrected. If this is done, a group formed by the most severe cases will struggle in adulthood with persistent symptoms of the disorder. (Conners et al., 2001). Literature refers to only $20 \%$. The children who do not receive a treatment or it is incomplete, an $80 \%$ has a bad long-term prognosis with emergence of explosive conduct disorders in adolescence, disocial conduct disorder, criminal-type personality disorder, alcoholism, drug abuse, job instability and difficulties in personal relationships.

It is generally considered that $80 \%$ of children with ADHD have a favorable long-term prognosis, when prior to adolescence behavioral problems are corrected and academic partners. If this is done, the literature speaks of only $20 \%$, a group formed by the most severe cases will struggle in adulthood with persistent symptoms of the disorder (Conners et al., 2001). Of the children who did not receive any treatment or it is incomplete, $80 \%$ have a poor long-term, explosive emergence of behavioral disorders in adolescence, conduct disorder, personality type of crime, alcoholism, drug abuse, job instability and difficulties in personal relationships.

In adulthood, about 15 to $20 \%$ of children with attention deficit disorder and hyperactivity continue to experience symptoms of this disorder and another third have symptoms of antisocial personality disorder. it shows a significant percentage of medication abuse $(16 \%)$, especially when there are two. Evidence has been compiled in order to indicate that a high percentage of cases, ADHD is accompanied by other psychopathologic dirorders (Zarin et al., 1998; Bennett, 2000). This makes the clinical study of hyperactivity difficult and raises the inevitable question of differential diagnosis between ADHD and various disorders that may coexist, such as patterns of major depression, anxiety disorders, oppositional defiant disorder, the dissocial and learning disorders (August et al., 1996a; Epstein et al., 1992; Abikoff y Klein, 1992).

Comorbidity refers to a high probability of association of these diseases in one person, and it does not necessarily imply a causal relationship or interdependence between different coexisting disorders. (Artigas-Pallares 2003) states that at least two conditions to give a useful meaning to the term comorbidity are required.

These conditions are that the presence of comorbidity conditions a presentation, a prognosis and a therapeutic approach for each comorbidity process. The condition of the frequency with which one appears when the other is present is higher than the isolated prevalence in the general population isolated.

Approximately 45\% and 65\% of children with ADHD have other behavioral and emotional problems, such as conduct disorder, oppositional-defiant disorder, anxiety-dipression disorders, etc. (Abikoff y Klein, 1992). This has led to consider ADHD as a heterogeneous disorder, usually seen as a disorder, which usually occurs with other psychopathological problems, which interact and modify the diagnosis significantly in the clinical phenomenology, not only in the psychological characteristics but also in the psychosocial consequences, in the clinical course, in the prognosis and in response to the treatment of this complex association (McBurnett et al., 1999).

From the clinical perspective, children with ADHD associated with other disorders are of greater severity, they are affected in several domains of child development (social, academic, emotional and physical interaction) and they continue to develop more favorably 
than children with ADHD without comorbidity (Abikott y Klein, 1992; Jensen et al., 1996; Rapport et al 1999). The studies establish the following associated disorders: Comorbidity with conduct problems, comorbidity with learning disorders, comorbidity with anxiety disorders, comorbidity with mood disorders with changes of the motor skills.

It is clear that one of the comorbidity disorders of ADHD is showed in the motor skill. These motor skill dysfunctions seem to affect many individuals with ADHD, to the point that the quality of motor skill performance during the first 5-6 years could be a predictor of subsequent onset of symptoms of ADHD, and even the association between clumsy motor skill and ADHD is a worse prognosis of the pattern (Kroes et al., 2002; Pascual-Castro Viejo, 2004; Kasdejo y Gillberg, 1999).

Some studies that have compared the fine motor skills of individuals with ADHD to a control group have found that the first ones showed less motor skill ability than the latter and the type of difficulties observed differed by the subtypes of the disorder (Piek et al., 1999; Steger et al., 2001). In this sense, the authors found that men affected by attention deficit and corresponding to the combined type (ADHD amd Hyperactivity) displayed less skill than those attached to the hyperactive-impulsive type and the control group. Also, in general, all children with ADHD had poorer fine motor skill performance than controls. These deficiencies are reflected in both the clumsiness to be with their body to occupy a space and move in it with a willful and symbolized motility fluid enough (Mazet y Houzal, 1981).

However, some studies disagree with these results, noting the absence of differences among people motor skills with ADHD and controls (Leung y Connolly, 1998). Based on motor skills characteristics prevalent in children with ADHD, it shows the existence of a high proportion of children with such deficits who have difficulties in gross motor skills development (Bauermeister, 2002). As a result of these deficiencies, subjects may appear awkward in their movements, expressing difficulty when running and jumping. With regard to fine motor skill development, the difficulties are manifest in tasks that involve grasping objects (such as a fork and a knife), buttoning clothes, playing with a ball, coloring within the limits of the figure, write lines or write in a uniform size, or run the script with an acceptable calligraphy and complete the written work in the classroom.

On the other hand, hyperactivity in children has been associated with motor coordination problems, and ot is stated that, currently, there are enough data to argue that hyperactive children have visual motor skill deficits, higher motor skill reaction times (they need more time to respond a motor skill at the onset of a stimulus) and make more errors (Orjales, 2002).

The study aimed to compare the performance of fine motor skill development, gross motor skill, visual motor skill in children of 6 years, the study worked with 49 children with ADHD and 48 normal children comparing their performances. The results showed significant differences between groups, indicating that the motor skill development of children with ADHD was significantly low compared to other children, all perceptual-motor skill measures. They also showed the existence of significant correlations among all variables considered motor skills in relation to hyperactive and inattentive children. Also, regression analysis indicated that the total visual motor skill and motor skill outcomes were significant predictors of group classification with and without ADHD. These results confirm the importance of early assessment and treatment of the disorder (Yochman et al., 2006). 
The authors cited above indicate that, while performing a neurological examination, it is usually not complete and also it does not outline the motor skills abnormalities that could be seen. The low importance attached to psychomotor development, and more specifically to the motor skills within the ADHD symptoms, with the prevalence of these disorders in patients affected by this syndrome, constitutes a gap in the knowledge of ADHD. Overcoming this ignorance could be a concern from the theoretical and applied point of view. Another study on the influence of disorder of the development coordination and attention deficit disorder in children associated movements, aimed to determine the relationship between associated movements (AMs) and the level of motor performance of children. It was researched whether children with Development Coordination Disorder (DCD), those with ADHD and healthy subjects differed in the severity of associated movements. The total sample was 10 children with DCD, 10 with ADHD and 10 belonged to a control group, it was found that two groups with limited motor skill had AMs significantly more severe than the healthy group. These results suggest that the level of motor performance should be considered in future research, trying to understand individual differences in the severity of AMs, as well as a function of motor learning, as well as a deficit linked to ADHD (Licari et al., 2006).

In general, it is estimated that over $50 \%$ of children with ADHD may have motor skill problems (Arnstein et al., 1996; Yochman et al., 2006). However it also noted that the diagnosis and clinical monitoring of patients (including neurologic examination) is mostly very superficial (Yochman et al, 2006; Licari et al., 2006). These authors explain that this situation could result from the fact that those who made the diagnosis and treatment tend to be doctors and psychologists that focus primarily on the symptoms of their field and, they are usually unaware of the nuances of the motor skill.

The study on the relationship between ADHD and the ability for the sport (Yochman et al., 2006), the study sample was 100 men with ADHD, aged 6 to 16 years with normal IQ, that is on the 85 percentile, motor skill was evaluated by the views of parents regarding their children's skill for drawing, writing, and problems for walking, jumping and playing; children answered a questionnaire of 8 questions related to their love of sport, type of sport, the position they played football if they practiced it, the assessment of sports performance (both staff and parents and peers), the frequency and discipline in sport practice and also if sport was practicedi or instead of football what other physical activity was practiced. The results showed that the most common neurological involvement was hypotonia, it was expressed in all joints in the form of hyperextension and hyper-flexibility in the feet, where hypotonia was more evident and it was present in plano-valgus feet, forcing an awkward walk, in general, the excessive shoulder and trunk elasticity coexisted with a significant global muscle power.

A $67 \%$ said they play sports and a $20 \%$ chose football, followed by cycling with a $12 \%$. Swimming, taekwondo, basketball, etc., were the less chosen by children. A $42 \%$ of those who conistently practiced sport expressed their motivation for this activity, while $58 \%$ acknowledged that they continued practicing sports at the behest of their parents. Almost all considered to have a satisfactory performance in sports, but the parents of half of them recognized that the implementation was poor compared with other children. In general, this study is interested in reseraching the possible relationship between the motor skill efficiency of children with ADHD and sports practice. However, the evaluation of the motor skills based solely on parental opinions and sports performance, measured only by the opinions 
of children themselves and their parents is far from meeting the methodological requirements of a rigorous motor skill assessment.

The therapeutic potential value of motor skills in the treatment of ADHD has been the subject of various studies in recent years. In most of them, it is considered within a multimodal treatment, which hampers the assessment of the effects of exercise in isolation. In other cases, the effect of the activity is evaluated (exercise and others) on various manifestations of the syndrome. For example, it has been shown that motor skill practice on cognitive functioning, and especially to vigorous physical activity can benefit children suffering from attention deficit disorder and hyperactivity (Lemura et al., 2000).

In another study, aerobic exercise was used (walking on treadmill) in children with ADHD and it was found that exercise could have a positive effect on the typical behaviors of the disorder, it may provide the child with ADHD the following benefits: On a physical level: correct laterality problems or coordination and to work gross and fine motor skill. At level of education: to promote motor skill learning, facilitate the understanding of body structure and promote understanding of the movement. At the social level: sharing a group activity, learn to respect rules, to accept others and be respected by the group (Tantillo et al., 2002).

However, this work does not specify the results obtained after an aerobic exercise program, merely proposing the supposed benefits that could result of the program. Some of these benefits, such as the ones the authors propose at a social level, would be difficult to get through the treadmill walk, as this activity does not imply, in principle, social relations nor the development of respect for self and others. Nor it is easy to understand how lateral or coordination problems could be corrected, nor improving the fine motor skills by working in the treadmill, and so on. In addition, studies of this nature, far from clarifying the relationship between physical exercise and ADHD, may even lead to confusion.

In relation to the exercise as part of treatment, (Barkley, 2003) presents some case studies of ADHD patients whose treatment included physical exercise and/or sports, mainly intense aerobics, affirming to have seen a clear benefit. In any of these studies, the type of exercise prescribed to the subjects is not made operative, nor the improvements obtained are quantified. What the author offers is a brief description of the treatment, indicating the exercise prescription and adding an anecdotal comment on the effects obtained.

Studies of children with ADHD taking stimulants and noted that the performance of these children in terms of fitness and gross motor skills was below average, when it is compared with the standards established for children of this age and gender. They also showed that athletic incompetency and academic failure could contribute to these children maintain a low self-concept, which caused them a feeling of worthlessness and frustration with social activities (Winnick, 2004).

Motor skills can provide a large field of learning to improve social skills in individuals with ADHD. If there are different causes and forms of ADHD, there must be several ways to teach children with this disorder. It is suggested specific instructional strategies for teachers to help children with ADHD to maintain focus and concentration in different school settings (Winnick, 2004). These strategies involve a highly structured approach and consistent routines, establishing rules, the use of behavior management programs, clearly express the expectations expected, choose activities that involve slow, controlled movements to reduce 
hyperactivity and impulsivity, to highlight relevant commands, to encourage the child permanently, to change the motor skill tasks permanently, to minimize competitive games and activities to encourage cooperation.

In the same way, physical activity is recommended to children with ADHD, as far as possible. It is considered that at least an hour a day of aerobics, karate do, swimming, dancing, gymnastics and other sports should be provided. These authors consider that energy expenditure made by the child to exercise will allow higher levels of stillness. They also argue that higher-level of exercise of the child requires more concentration, which generalize to intellectual tasks. Although both arguments may be reasonable, to reduce hyperactivity by increasing energy expenditure, which would achieve in all cases, a temporary reduction and not a modification of the neuropsychological mechanisms that underlie the etiology of the disorder. Also, to expect a transfer from the alleged concentration generated by exercise to intellectual tasks suggests a very superficial knowledge of the functioning of attention processes.

\subsection{Development disturbances in ADHD}

Although initially motor skill difficulty was used as a catch-all room where all the paintings were more heterogeneous, now it tends to rigorously define its scope. For example, lesions and neurological symptoms are excluded and the concept of motor skill difficulty for motor impairments of the child whose etiology is of another nature is reserved. These shortcomings are reflected in both the awkwardness to be with their body to occupy a space and to move in it with a symbolized and intentional mobility (Barkley, 2003).

The interest in studying motor skill functioning in ADHD and in discriminating children with ADHD from those with neurological disorders is not new (Konrad et al., 2000). This interest has been revitalized in part by research on DAMP (Gillberg, 2003; Kasdejo y Gilbert, 1998) conducted in Sweden and also by other contributions on neuroimaging studies related disorders, indicating that both clinical experience and experimental evidence suggest the role of motor skill factors in ADHD.

A variety of neurological tests have shown that children with ADHD are different from the control group in motor size skills. This includes repetitive movements (Carte y Hinshaw, 1996; Denckla y Rudel, 1978), difficulties in fine motor skills associated with hyperactiveimpulsive or disantención symtomps (Pitcher et al., 2003); coordination failures (Jucaite et al., 2003), problems in controlling the movements and especially when there is no visual feedback (Eliasson et al., 2004); balance deficiencies (Raberger y Wimmer, 2003); maladjustment in rhythmic beating sequential tasks (Lemura et al., 2000); excessive unnecessary movement (Mostofsky et al., 2003); difficulty with motor skills Acquisitions (Karatekin et al., 2003); and slow central motor skill processing (Ucles et al., 2000); lower motor skills and intervals and number of errors increase (Tantillo et al., 2002).

In general, it is estimated that over $50 \%$ of children with ADHD may have motor skill problems (Barkley et al., 1990b; Yochman et al., 2006). The low importance attached to psychomotor development, and more specifically to the motor skills within the ADHD symptoms, with the prevalence of these disorders in patients affected by this syndrome constitute a gap in knowledge of ADHD. Overcoming this ignorance could be of interest from the theoretical and applied point of view. 
The differentiation between subtypes of ADHD have shown that the "mixed" subtype has greater difficulties in gross motor skills, while the "inattentive" subtype is less in fine motor skills and manual skill tests (Piek y Skinner, 1999). Meanwhile, clinical studies have highlighted the presence of motor skill dysfunction in ADHD, but it does not indicate what specific process is ADHD involved: motor skill programming, preparation, adjustment, etc.

\section{Motor skills in children with ADHD: Comparative study from a pharmacological treatment}

This research work aims to show the practical elements, as evidenced by the results presentation that seek a statistical approach to the existing theoretical claim that affirms that children with ADHD who are undergoing medication therapeutically present a different psychomotor profile compared to children with ADHD who are not receiving medication. This established a methodology process that starts with a descriptive correlational study. Sampling frame was used as 422 children between 5 and 12 years old from Manizales Colombia with a medical diagnosis of ADHD, which exceeded the representative sample size of 397 subjects, calculated by using the Epilnfo program , v. 6.04, designed by the Center for Disease Control in Atlanta, in its Spanish version, and considering a total population of 46,387 children, with $95 \%$ confidence and a margin of error of $5 \%$. Their distribution by age and gender was 306 male (72,5\%) and 116 female (27,5\%).

Also 308 children from the total sample did not use methylphenidate and 114 did not use methylphenidate if consumed. Regarding the subtype of ADHD it was found that $84(20.6 \%)$ were diagnosed with predominantly inattentive, $184(43,6 \%)$ predominantly hyperactive and 151 (35.8\%) with combined dominance. An age range between 5 and 12 was chosen, because 5 years is usually set when first diagnosed ADHD and because in previous studies (Vidarte and Velez, 1999, 2001) it had worked with these ages and they had shown elevated levels of disorder prevalence. Inclusion criteria for healthy children were: being aged between 5 and 12 years, belonging to any genre, being physically fit to develop the respective evaluations and having the corresponding informed consent. For children diagnosed with the disorder, in addition to the above requirements, an ADHD diagnosis is required by a certified physician.

Children who had any other pathology such as cerebral palsy, mental disorders, language disorders and anxiety disorders, among others, or who have had traumatic processes that alter the mobility during the last month were excluded from the sample. Once all administrative authorizations were obtained in order to know in which schools were children with ADHD. The integral service unit was (interdisciplinary unit under the Ministry of Education of the city, which is made up of physicians, psychologists, speech therapists and occupational therapists, and who are responsible for therapeutic intervention in children with different problems and to provide pedagogical support to teachers and parents).

The sociodemographic variable data were obtained through a questionnaire of open and closed questions and the corresponding psychomotor variables by using the psychomotor observation battery (POB) proposed by Da Fonseca. It is a system for observing the various components of the motor system, and the data obtained allow to reflect the degree of 
neurological organization of the child, allowing the identification of alterations. The theoretical basis on which the BPM based its proposal consists of contributions from Luria, reformulated and repurposed by Da Fonseca provide justification for the neuropsychological data.

The assessment was conducted individually with each of the children in their own school and in their study schedule. In the case of the only hospital care center, the procedure was similar and the children were assessed during the time they went to receive their treatments. Except in this case, and in order to avoid information bias due to convenience, a blind process of measurement was condected: the evaluators did not know which were children diagnosed with ADHD. Finally, the physician of integral service unit reported which of the children assessed were diagnosed with the disorder, allowing the review of medical records to prove the diagnosis of ADHD and to confirm the inclusion and exclusion of children.

The study results are described first through a univariate analysis then a bivariate analysis that shows the relationship between psychomotor profiles and variable subtypes and gender.

\begin{tabular}{|c|c|c|}
\hline Psyichomotor profile & Frequency & Percentage \\
\hline Apraxic (weak) & 1 & 0,2 \\
\hline Dispraxico(Satisfactory) & 73 & 17,3 \\
\hline Eupraxic (Good) & 336 & 79,6 \\
\hline Hiperpraxic (Excellent) & 12 & 2,8 \\
\hline Total & 422 & 100,0 \\
\hline
\end{tabular}

Table 1. Psychomotor profile of children clinically diagnosed with ADHD.

The psychomotor profile of 422 children was $79.6 \%$ eupraxic, it maens normal, while $17.3 \%$ was dispraxic or performed with difficulty.

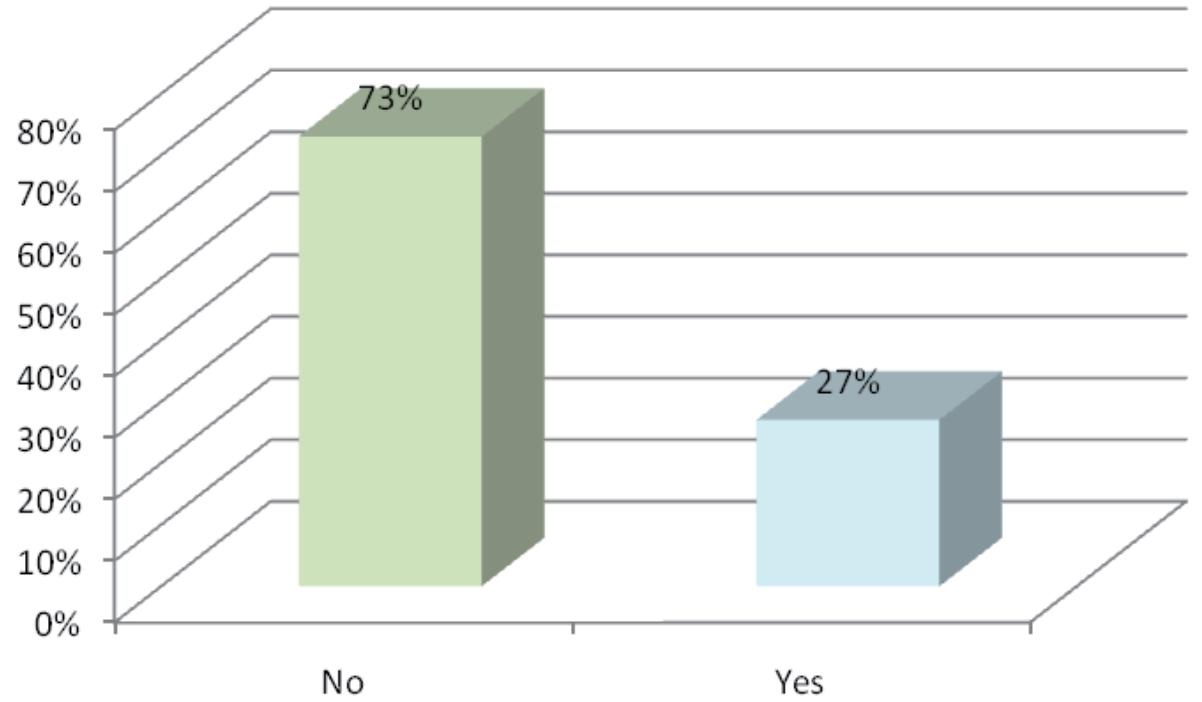

Fig. 1. Results obtained in the participant sample by taking medications (methylphenidate) 
It is remarkable how $100 \%$ of the sample participating in the study only $27 \%$ use methylphenidate.

\begin{tabular}{|l|c|c|c|c|}
\hline \multirow{2}{*}{ Psyichomotor profile } & \multicolumn{2}{|c|}{$\begin{array}{c}\text { Use } \\
\text { medication }\end{array}$} & \multicolumn{2}{c|}{$\begin{array}{c}\text { No use } \\
\text { medication }\end{array}$} \\
\cline { 2 - 5 } & Fcia & $\mathbf{0}$ & Fcia & \% \\
\hline Apraxic (weak) & 1 &, 9 & 0 & 0 \\
\hline Dispraxic(Satisfactory) & 18 & 15,8 & 55 & 17,9 \\
\hline Eupraxic (Good) & 89 & 78,1 & 247 & 80,2 \\
\hline Hiperpraxic (Excellent) & 6 & 5,3 & 6 & 1,9 \\
\hline Total & 114 & 100,0 & 308 & 100,0 \\
\hline
\end{tabular}

Table 2. Results of psychomotor profile in children who use medication compared with those who do not use it.

In this study was found that from 114 children taking medication, 83.3\% (95) are male and $16.7 \%$ (19) female. Depending on the level of schooling, the largest proportion was located in the third grade of school (28 boys), 26 in the first grade and 22 in fourth grade and according to the age of children medicated, the greater proportion was 7 and 10 years (21 children) and 8-9 and 11 years (14 children).
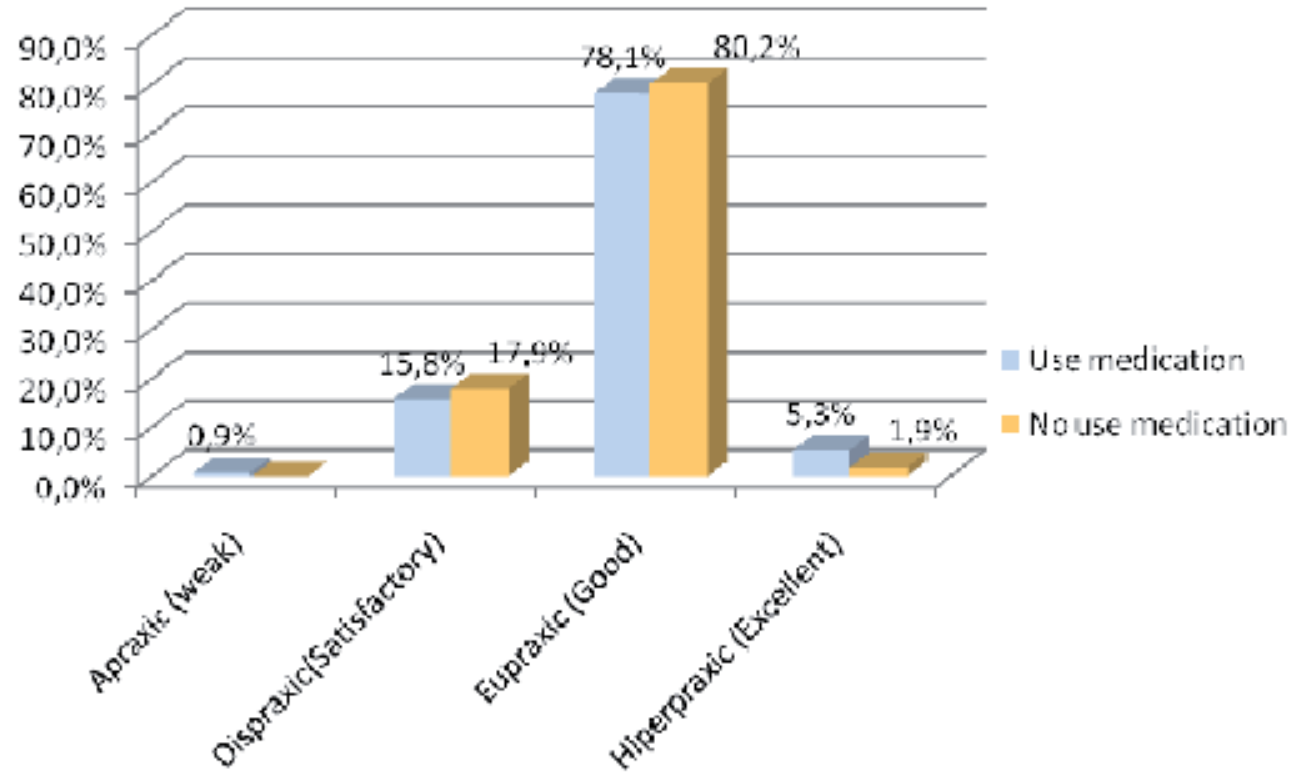

Fig. 2. Psychomotor profile results in children who use medication compared with those who do not use it.

Notice although the mean difference for each of the motor skills factors assessed in clinically diagnosed children is higher in children who use medication compared to those who do not use it when comparing these differences, it was found that their differences are not statistically significant. 


\begin{tabular}{|c|c|c|c|c|}
\hline Variable & $\begin{array}{c}\text { X children who } \\
\text { use medication }\end{array}$ & $\begin{array}{c}\text { X children who do } \\
\text { not use medication }\end{array}$ & U & Sig. Bilat. \\
\hline Tone & 3,06 & 3,00 & 16834,000 &, 490 \\
\hline Balance & 2,96 & 2,83 & 16000,500 &, 124 \\
\hline Laterality & 1,22 & 1,21 & 17411,000 &, 854 \\
\hline Body notion & 2,70 & 2,56 & 15791,000 &, 079 \\
\hline Estructuration espacial & 1,96 & 1,86 & 16339,500 &, 235 \\
\hline Global Praxia & 1,89 & 1,78 & 16221,500 &, 189 \\
\hline Fine Praxia & 2,50 & 2,42 & 16308,500 &, 218 \\
\hline
\end{tabular}

Table 3. Comparison between groups of children with ADHD from obtained results in factors of psychomotor profile

\begin{tabular}{|c|c|c|c|c|}
\hline Variable & $\begin{array}{c}\text { X children who use } \\
\text { medication }\end{array}$ & $\begin{array}{c}\text { X children who do } \\
\text { not use medication }\end{array}$ & U & Sig. Bilat. \\
\hline Tone & 3,02 & 3,00 & 486,000 &, 903 \\
\hline Balance & 2.91 & 2,78 & 452,000 &, 547 \\
\hline Laterality & 1,24 & 1,17 & 460,500 &, 538 \\
\hline Body notion & 2,60 & 2,56 & 473,000 &, 756 \\
\hline Estructuration espacial & 1,89 & 1,78 & 464,000 &, 756 \\
\hline Global Praxia & 1,91 & 1,61 & 403,000 &, 203 \\
\hline Fine Praxia & 2,45 & 2,50 & 476,000 &, 792 \\
\hline
\end{tabular}

Table 4. Comparison between groups of children with ADHD predominantly hyperactive from results obtained in the factors of psychomotor profile

Comparing the motor skill factors among children diagnosed with ADHD predominantly hyperactive differences in the means are not statistically significant. It was found from the psychomotor profile 55 children use methylphenidate of which $69.1 \%$ have a normal profile (Eupraxic), 5.5\% hyperpraxic and $23.6 \%$ dispraxic.

\begin{tabular}{|c|c|c|c|c|}
\hline Variable & $\begin{array}{c}\text { X children who use } \\
\text { medication }\end{array}$ & $\begin{array}{c}\text { X children who do } \\
\text { not use medication }\end{array}$ & U & Sig. Bilat. \\
\hline Tone & 3,20 & 3,09 & 2358,500 &, 346 \\
\hline Balance & 3,06 & 2,84 & 2189,000 &, 102 \\
\hline Laterality & 1,20 & 1,19 & 2593,500 &, 943 \\
\hline Body notion & 2,74 & 2,62 & 2462,000 &, 566 \\
\hline Estructuration espacial & 2,00 & 1,85 & 2351,500 &, 320 \\
\hline Global Praxia & 1,94 & 1,83 & 2365,500 &, 341 \\
\hline Fine Praxia & 2,49 & 2,43 & 2472,500 &, 599 \\
\hline
\end{tabular}

Table 5. Comparison between groups of children with ADHD predominantly inattention of the obtained results of the psychomotor profile factors

Comparing the motor skill factors among children diagnosed with ADHD and predominantly inattention, the differences in the means are not statistically significant. It is noteworthy that in relation to the psychomotor profile, it did not occur in these apraxic profile children and a higher proportion of children with predominantly inattentive profile were normal. 


\begin{tabular}{|c|c|c|c|c|}
\hline Variable & $\begin{array}{c}\text { X children who } \\
\text { use medication }\end{array}$ & $\begin{array}{c}\text { X children who do } \\
\text { not use medication }\end{array}$ & U & Sig. Bilat. \\
\hline Tone & 3,00 & 2,91 & 1338,000 &, 651 \\
\hline Balance & 2,91 & 2,78 & 1287,000 &, 443 \\
\hline Laterality & 1,23 & 1,24 & 1400,500 &, 895 \\
\hline Body Notion & 2,91 & 2,46 & 918,000 &, 004 \\
\hline Estructuration. Espacial & 2,09 & 1,84 & 1142,000 &, 117 \\
\hline Global Praxia & 1,77 & 1,77 & 1396,000 &, 895 \\
\hline Fine Praxia & 2,56 & 2,36 & 1148,500 &, 117 \\
\hline
\end{tabular}

Table 6. Comparison between groups of children with ADHD predominantly combined of the obtained results of the factors of psychomotor profile.

Comparing the motor skill factors among children diagnosed with ADHD predominantly combined, the mean differences were not statistically significant in the body notion variable. It is important to highlight in these children the apraxic profile and in a higher proportion the children with combined prevalence obtained an excellent or hiperpraxic profile.

\begin{tabular}{|c|c|c|c|c|}
\hline \multirow{2}{*}{ Pharmachological Treatment } & \multicolumn{3}{|c|}{ Type of TDAH } & Total \\
\cline { 2 - 5 } & Inattention & Hyperactivity & Combined & \\
\hline YES & 57 & 35 & 22 & 114 \\
\hline NO & 30 & 149 & 129 & 308 \\
\hline
\end{tabular}

\begin{tabular}{|l|c|c|r|}
\hline & Value & gl & Sig. asintotic (bilateral) \\
\hline Chi-square of Pearson & $83.237 \mathrm{a}$ & 2 & .000 \\
\hline Reason of verosimilitude & 75.874 & 2 & .000 \\
\hline Lineal per lineal association & 60.599 & 1 & .000 \\
\hline N of valid cases & 422 & & \\
\hline
\end{tabular}

Table 7. Relation between consumption of medication and subtype of ADHD

The table above shows that there seems to be a relationship between the consumption of medication and subtype of ADHD $(p<0.00)$.

The data above show differences in mean scores achieved in the development of motor skills developed by children clinically diagnosed with ADHD who use medication in relation to the ones who do not, but these differences were statistically significant only for children with Combined ADHD predominant factor in the notion of body $(p<0.004)$, the other factors and each of the subtypes were not found differences statistically significant.

(Pelham et al., 1990) examined the effectiveness of methylphenidate to improve the performance of hyperactive children in the game of baseball. Although medication did not enhance skills for the game, it produced significant effects on attention, so that involvement in the activity was higher. In short, while psychostimulant medication did not make children more skilled players, their teammates thought that they showed a better disposition towards the game.

These results have a positive nature because, as suggested by Pelham et al (Pelham et al., 1990), peers are more benevolent in judging a child who strives and makes mistakes for his 
clumsiness, than when a fellow is judged by errors made due to his uninterest for the game. Papers published in recent years by the Multimodal Treatment Study of Children with Attention Deficit / Hyperactivity Disorder (MTA Cooperative Group, 1999 confirm the efficacy of psychostimulants in the treatment of ADHD.

In fact, a number of reports have shown that methylphenidate decreases in children with ADHD their disobedience, their verbal aggression and their antisocial behaviors (Hinshaw et al., 1993; Klein et al., 1997), it facilitates the standardization of their behavior in class (Rapport et al., 1994) and it favors social interactions with classmates (Whalen y Henker, 1991). Similarly, other research has found improvements in the performance of children with ADHD / C on homework such as math problems, word reading (Smith et al., 1998) and quality of writing (Tucha y Klaus, 2001) the administration of methylphenidate.

\section{Conclusion}

In attempting to solve the objective of the study, it can be concluded that the psychomotor profile of children diagnosed with ADHD is eupraxic and it is classified in the same category as children who consume and don't consume medication, and they are located in the same profile for eupraxic healthy children, although their score is lower and the performance levels of each factor are worse. Exceptionally, there were no differences in the psychomotor profile of different factors, but in this case, it is also deficient in healthy children. (Vidarte et al., 2009).

In general, studies of motor skills in children with ADHD are overly simplistic and marred by methodological shortcomings. This makes it difficult to know precisely: motor behavior of children with ADHD, the most appropriate type of exercise in improving such symptoms, neurological and biochemical pathways through which explain the improvements made and its effectiveness compared to other traditional treatments traditional, or as part of a multimodal treatment.

It is therefore recommended to initiate a systematic process that allows the collection of information on an ongoing basis and thereby make permanent monitoring to processes conducted with multimodal intervention in children diagnosed with ADHD. The inclusion of this condition in the policy of early childhood at national level, given its high prevalence and the possibility of effective management in the beginning of school age.

\section{Study limitations}

The study limitations were: the absence of stratified sampling by sex and the low participation of children studying in private institutions, this variable would have enable to identify possible differences by socioeconomic status.

\section{References}

Abikoff, H. y Klein, R. (1992). Attention-deficit hyperactivity and conduct disorder, comorbidity and implications for treatment. Journal of Consulting Clinical Psychology, $60,881-892$. 
Amador, J.A., Forns, M. y Martorell, B. (2001). Síntomas de desatención e hiperactividadimpulsividad: análisis evolutivo y consistencia entre informantes. Anuario de Psicología; 32: 51-66.

American Psychiatric Association. (1995). DSM-IV. Manual Diagnóstico y Estadístico de los Trastornos Mentales. Barcelona: Masson (Edición original, 1994).

American Psychiatric Association. (2002). DSM-IV-TR. Manual Diagnóstico y Estadístico de los Trastornos Mentales. Barcelona: Masson (Edición original, 2000).

Arias, B.G. (2003). La evaluación y el diagnóstico educativo y psicológico y el enfoque histórico-cultural. Perspectiva Psicologica, 3-4, 17-34.

Arnold AC, Conners EG, Hectman H, Hoza J, Kraemer M, Newcorn P, Richters SS, Swanson V, y Wells. (1997b). National Institute of Mental Health colaborative multimodal treatment study of children with ADHD (the MTA). Archives of General Psychiatry; 54: 865-870.

Arnstein, A., Steere, J. y Hunt, R. (1996). The contribution of D2- noradrenergic mechanisms to prefrontal cortical cognitive function. Potential significance for attention-deficit disorder. Archives General of Psychiatry, 53, 448-455.

Artigas-Pallarés, J. (2004). Nuevas opciones terapéuticas en el tratamiento del trastorno por déficit de atención/ hiperactividad. Revista de Neurologia, 38, S117-S123.

August, G.J., Realmulto, G., MacDonald, A.W., Nugent, S. y Grosby, R. (1996a). Prevalence of ADHD and comorbid disorders among elementary school children screened for disruptive behavior. Journal of Abnormal Child Psychology, 24, 571-595.

Bará S, Henao P, Jiménez DA, Pineda B, y Vicuña GC. Perfiles neuropsicológicos y conductuales de los niños con trastorno por déficit de atención / hiperactividad de Cali, Colombia. Revista de Neurología, (2003); 37: 608-615.

Barkley RA. (1997). ADHD and the nature of self-control. Nueva York: Guilford Press.

Barkley RA. (1991).The ecological validity of laboratory and analogue assessment methods of ADHD symptoms. Journal of Abnormal Child Psychology; 19: 149-178.

Barkley RA, Fischer M, Edelbrock GS, y Samllish L. (1990b) The adolescent outcome of hyperactivity children diagnosed by research criteria, I. An 8-year prospective follow-up study. Journal of the American Academy of Child \& Adolescent Psychiatry; 29: 546-557.

Barkley RA, Anastopoulos AA, Guevremont DC y Fletcher KE. (1991). Adolescents with ADHD, patterns of behavioral adjustment, academic functioning and treatment utilization. Journal of the American Academy of Child \& Adolescent Psychiatry, 1971; 30: 752-761.

Barkley RA. (1977). A review of stimulant drug research with hyperactive children. J Child Psychol Psychiatry; 18: 137-65.

Barkley RA, Fischer M, Edelbrock GS y Samllish L. (1990b) The adolescent outcome of hyperactivity children diagnosed by research criteria, I. An 8-year prospective follow-up study. Journal of the American Academy of Child E Adolescent Psychiatry; 29: 546-557.

Barkley, R.A., Grodzinsky, G. y DuPaul, G.J. (1992). Frontal lobe functions in attention deficit disorder with and without hyperactivity: A review and research report. Journal of Abnormal Child Psychology, 20, 163-188. 
Barkley RA. (1988)The effects of methylphenidate on the interactions of preschool ADHD children with their mothers. J Am Acad Child Adolesc Psychiatry; 27: 336-41

Barkley R.A. (2003) Issues in the diagnosis of attention-deficit/hyperactivity disorder in children. Brain Develpment, 25, 77-83.

Bathia MS, Nigam VR, Bohra N, y Malik SC. (1991).Attention deficit disorder with hyperactivity among pediatric outpatients. Journal of Child Psychology and Psychiatry; 32: 297-306.

Bauermeister, J.J. (2002). Hiperactivo, impulsivo, distraído, ¿me conoces?. Guía acerca del déficit atencional para padres, maestros y profesionales. Nueva York: Guilford.

Bennett, K. (2000). Screening for externalizing behavior problems. Journal of the American Academy of Child \& Adolescent Psychiatry, 39, 1341-1343.

Biederman J, Faraone SV, Milberger S, Curtis S, Chen L, Marrs A, Ouellette C, Moore P y Spencer T. (1996a) Predictors of persistence and remission of ADHD into adolescence. Results from a four-year prospective follow-up study. Journal of American Academy of Child and Adolescent, (35): 343-351.

Biederman J, Faraone SV, Keenan K, Benjamin J, Krifcher B, Moore C, Sprich-Buckminster S, Ugaglia K, Jellinek MS, Steingard R, Spencer T, Biederman J, Faraone SV, Keenan K, Knee D, y Tsuang MT. (1990)Family-genetic and psychosocial risk factors in DSM-III attention deficit disorder. Journal of the American Academy of Child $\mathcal{E}$ Adolescent Psychiatry; 29; 526-533.

Biederman J, Nwecorn J, y Sprich S. (1991). Comorbidity of attention deficit hyperactivity disorder with conduct, depressive, anxiety and other disorders. American Journal of Psychiatry; 148: 564-577.

Biederman, J., Wilens, T., Mick, E., Faraone, S.V., Weber, W., Curtis, S., Thornell, A., Pfister, K., Garcia, J. y Soriano, J. (1997). Is ADHD a risk factor for psychoactive substance use disorder?. Findings from a four-year prospective follow-up study. Journal of the American Academy of Child \& Adolescent Psychiatry, 36, 21-29

Burnley, (1993). A team approach for identification of an attention deficit hyperactivity disorder child. The School Counselor, 40, 228-230.

Castellanos, F.X., Giedd, J.N., Eckburg, P., Marsh, W.L., Vaituzis, C., Kaysen, D., Hamburger, S.D. y Rapoport, J.L. (1994). Quantitative morphology of the caudate nucleus in attention deficit hyperactivity disorder. Neurology, 151, 1791-1796.

Castellanos, F.X., Giedd, J.N., Marsh, W.L., Hamburger, S.D., Vaituzis, C., Kickstein, D.P., Sarfatti, S.E., Vauss, C., Snell, J.W., Rajapake, J.C. y Rapoport, J.D. (1996b). Quantitative brain magnetic resonance imaging in attention-deficit hyperactivity disorder. Archives of General Psychiatry, 53, 607-616.

Castellanos, F.X., Giedd, J.N., Hamburger, S.D, Marsh, W.L. y Rapoport, J.L. (1996a). Brain morphometry in Tourette Syndrome, the influence of comorbid attentiondeficit/hyperactivity disorder. Neurology, 47, 1581-1583.

Carte, E., Nigg, J., Hinshaw S.P. (1996). Neuropsychological functioning motor speed and language processing in boys with and without ADHD. Journal of Abnormal Child Psychology, 24, 481-498.

Casey, B., Castellanos, F., Giedd, J., Marsh, W., Hamburger, S., Schubert, A., Vauss. Y., Vaituzis, C., Dicksteins, D., Sarfatti, S. y Rapoport, J. (1997). Implication of right frontostriatal circuitry in response inhibition and attention-deficit/ hyperactivity 
disorder. Journal of the American Academy of Child \& Adolescent Psychiatry, 36, 374383.

Colby, C.L. (1991). The neuroanatomy and neurophysiology of attention. Journal of Child Neurology, 16, S90-S118.

Conners CK, Epstein JN, March SS, Angold A, Wells KC, Klaric J, et al. (2001). Multimodal treatment of ADHD in the MTA; an alternative outcome analysis. J Am Acad Child Adolesc Psychiatry 40: 159-67.

Cornejo JW, Osio O, Sánchez, Y, Carrizosa J, Griosales H, Castillo H y Holguín J. (2005). Prevalencia del trastorno por déficit de la atención e hiperactividad en niños y adolescentes colombianos. Revista de Neurología, 40: 716-722.

Da Fonseca, V. (1998). Manual de observación psicomotriz. Barcelona: INDE.

Denckla. M. y Rudel, R. (1978). Anomalies of motor development in hyperactive boys. Annual Neurology, 8, 231-233.

Eliasson, A.C., Rosblad, B. y Forssberg, H. (2004). Disturbances in programming goaldirected arm movements in children with ADHD. Developmental Medicine $\mathcal{E}$ Child Neurology, 46, 19-27.

Epstein, M.A., Shaywitz, S.E., Shaywitz, B.A. y Woolston, J.L. (1991). The boundaries of attention deficit disorder. Journal of Learning Disabilities, 24, 78-86.

Faraone SV (1996). Discussion of "Generic influence on parent-reported attention-related problems in a norwegian general population twin sample. Journal of the American Academy of Child \& Adolescent Psychiatry; 35: 596-599.

Faraone, S.V., Biederman, J., Mennin, D., Wozniak, J. y Spencer, T. (1997). Attention deficit hyperactivity disorder with bipolar disorder, a familial subtype? Journal of the American Academy of Child \& Adolescent Psychiatry, 36, 1378-1387.

Faraone SV, Biederman J, Mennin D, Russell R, y Tsuang MT.(1998a) Familial subtypes of attention deficit hyperactivity disorder, a 4 year follow-up study of children from antisocial ADHD families. Journal of Child Psychiatry Psychology; 39: 1045-1053.

Faraone SV, Biederman J, Spencer TJ, Aleardi M. (2006).Comparing the efficacy of medications for ADHD using meta-analysis. Med Gen Med; 8: 4

Fergusson DM, Horwood J, y Lynskey MT. (1993). Prevalence and comorbidity of DSM-IIII$\mathrm{R}$ diagnoses in a birth cohort of 15 year olds. Journal of the American Academy of Child $\mathcal{E}$ Adolescent Psychiatry, 32: 1127-1134.

Fernández Jaén, A. (1999). Trastorno de déficit de atención con hiperactividad e hipomelanosis de Ito. Revista de Neurología, 31, S680-S681.

Fischer M, Barkley R, Edelbrock C, y Smallish L. The adolescent of hyperactive children diagnosed by research criteria, academic, attentional and neuropsychological status. Journal of Consulting and Clinical Psychology, 1990; 58: 580-588.

Frick, P.J. (1994). Family disfunction, the disruptive behavior disorders. A review of recent empirical findings. En Advances In Clinical Child Psychology. Ollendick, T.H. y Prinz R.J. (Eds.). pp. 203-227. New York: Plenum Press.

Gaillard F, Quartier v, y Besozzi G. (2204). Más allá de la hiperactividad: Un detallado análisis retrospectivo de 30 casos estudiados en la Clínica de Psicología de la Universidad de Lausanne. Revista Argentina de Neuropsicología; (2): 15-25.

Gillberg, C. (2003). Deficits in attention, motor control and perception: a brief review. Archives of Disability Child, 88, 904-910. 
Gillis, J.J., Gilber, J.W., Pennington, B.F. y Defries, J.C. (1991). Attention deficit disorder in reading disabled twins, evidence for a genetic etiology. Journal of Abnormal Child Psychology, 4, 303-315.

Goodman, R. y Stevenson, J. (1989). A twin study of hyperactivity II. The etiological role of genes, family relationship and perinatal adversity. Journal of Child Psychology and Psychiatry, 30, 691709.

Hinshaw SP, Heller T, McHale JP. (1993). Covert antisocial behavior in boys with attention deficit hyperactivity disorder: External validation and effects of methylphenidate. J Consul Clin Psychol; 60: 274-81.

Houwe, J. y Schoemaker, T. (2006). Kijk op het imago. Deventer: Van Loghum Slaterus.

Jensen PS, Hinshaw SP, Swanson JM, Greenhill LL, Conners CK, Arnold LE. (2001). Findings from the NIMH Multimodal Treatment Study of ADHD (MTA): implications and applications for primary care providers. J Dev Behav Pediatr; 22: 60-77.

Jensen, P.S., Watanabe, H.K., Richters, J.E., Roper, M., Hibbs, E.D., Salzberg, A.D. y Liu, S. (1996). Scales, diagnoses, and child psychopathology: II. Comparing the CBCL and the DISC against external validators. Journal of Abnormal Child Psychology, 24, 151168

Johnston, C. y Pelham, W. (1986). Teacher rating predict peer of aggression at 3 year followup in boys with attention deficit hyperactivity disorder. Journal of Consulting and Clinical Psychology, 54, 571-572.

Jucaite, A., Fernell, E., Forssberg, H. y Hadders-Algra, M. (2003). Deficient coordination of associated postural adjustments during a lifting task in children with neurodevelopmental disorders. Developmental Medicine \& Child Neurology, 45, 731742.

Kadesjo, B. y Gilbert, C. (1988). Attention deficit and clumsiness in Swedish 7 year old children. Developmental Medicine \& Child Neurology, 40, 796-804.

Kadesjo, B. y Gillberg, C. (1999). Developmental coordination disorder in Swedish 7-yearold children. Journal of the American Academy of Child $\mathcal{E}$ Adolescent Psychiatry, 38, 820-828.

Kadesjo, C., Kadesjo, B., Hagglof, B. y Gillberg, C. (2001). ADHD in Swedish 3 to 7 year old children. Journal of the American Academy of Child \& Adolescent Psychiatry, 40, 10211028.

Karatekin, C., Markiewicz, S.W. y Siegel, M. (2003). A preliminary study of motor problems in children with attention-deficit/hyperactivity disorder. Perceptual and Motor Skills, 97, 1267-1280.

Konrad, K., Gauggel, S.A., Manz A. y Scholl, M. (2000). Lack of inhibition: A motivational deficit in children with attention deficit/hyperactivity disorder and children with traumatic brain injury. Child Neuropsychology, 6, 286-296.

Klein R, Abikoff H, Klass E, Ganales D, Seese L, Pollack S. (1997). Clinical efficacy of methylphenidate in conduct disorder with and without attention deficit hyperactivity disorder. Arch Gen Psychiatry; 54: 1073-80.

Kroes, M., Kessels, A.G.H., Kalff, A.C., Feron, F.J.M., Vissers, Y.L.J., Jolles, J. (2002). Quality of movement as predictor of ADHA: results from a prospective 
population study in 5- and 6-year-old children. Developmental Medicine $\mathcal{E}$ Child Neurology, 44, 753-760.

Lemura, L. M., Von Duvillard, S.P. y Mookerje, S. (2000). The effects the physical training of functional capacity in adults, ages 66 to 90: A meta-analysis. Journal of Sport Medicine and Physical Fitness, 40, 1-10.

Leung, P.W.L. y Connolly, K.J. (1998). Do hyperactive children have motor organization and/or execution deficits?. Developmental Medicine \& Children Neurology, 40, 600607.

Licari, M., Larkin, D. y Miyahara, M. (2006). The influence of developmental coordination disorder and attention deficits on associated movements in children, Human Movement Science, 25, 90-99.

Max, J.E., Arndt, S., Castillo, C.S., Bokura, H., Robin, D.A., Lindgren, S.D., Smith, W.L., Sato, Y. y Mattheis, P.J. (1998). Attention deficit hyperactivity symptomatology after traumatic brain injury, a prospective study. Journal of Abnormal Child and Adolescent Psychiatry, 37, 841-847

Mazet, P. y Houzal, A. (1981) Le sommeil de l'enfant et ses troubles. París: Presses Universitaires de France.

Milberger, S., Biederman, J., Faraone, S.V, Chen, L. y Jones, J. (1997). ADHD is associated with early initiation of cigarette smoking in children and adolescent. Journal of the American Academy of Child \& Adolescent Psychiatry, 36, 37-44.

McBurnett, K., Pfiffner, L.J., Willcutt, E., Tamm, K., Lerner, M., Ottolini,. Y.L. y Furmna, M.B. (1999). Experimental cross validation of DSM-IV types of attention deficit hyperactivity disorder. Journal of the American Academy of Child $\mathcal{E}$ Adolescent Psychiatry, 38, 17-24.

MTA Cooperative Group. (1999). A 14-month randomized clinical trial of treatment strategies for attention-deficit/hyperactivity disorder. Arch Gen Psychiatry; 56: 1073-86.

MTA Cooperative Group. (2003). Moderators and mediators of treatment response for children with ADHD. Archive General of Psychiatry, 56, 1088-1096.

Navarro, J.F. y Manzanaque, J.M. (1998). Actualización de los receptores dopaminérgicos. Psiquiatría Biológica, 5, 205-209.

Montañés-Rada F, Gangoso-Fermoso AB, Martínez-Granero MA. (2009).Fármacos para el trastorno por déficit de atención/hiperactividad. Rev Neurol; 48: 469-81.

Mostofsky, S.H., Reiss, A.L., Lockhart, P. y Denckla, M.B. (2003). Evaluation of cerebellar size in attention deficit hyperactivity disorder. Journal of Child Neurology, 9, 434439.

Navarro, J.F. (2000). Bases biológicas de las psicopatologías. Madrid: Pirámide

Norman D, Kolodny R, Kraus I, Perrin J, Keller M, y Tsuang M. Futher evidence for familygenetic risk factors in attention deficit hyperactivity disorder. Archives of General Psychiatry, 1992; 49: 728-738.

Orjales, I. (2002). Programa de intervención cognitivo-conductual para niños con déficit de atención con hiperactividad. Madrid: CEPE.

Pascual Castroviejo, I. (2004). Síndrome de déficit de atención con hiperactividad y capacidad para el deporte. Revista de Neurología, 38, 1001-1005. 
Pelhan WE, McBurnett K, Harper GW, Milich R, Murphy DA, Clinton JE, et al. (1990). Methylphenidate and baseball playing in ADHD children: Who's on first? J Consult Clin Psychol; 58: 130-3.

Piek, J.P., Pitcher, T.M. y Hay, D.A. (1999). Motor coordination and kinaesthesis in boys with Attention Deficit Hyperactivity Disorder. Developmental Medicine \& Child Neurology, 41, 159-165.

Piek, J.P. y Skinner, R.A. (1999). Timing and force control during a sequential tapping task in children with and without motor coordination problems. Journal of the International Neuropsychological Society, 5, 320-329.

Pineda DA, Lopera GC, Palacio JD, y Henao GC. (2001). Prevalencia del trastorno por déficit de atención en una comunidad colombiana. Revista de Neurología; 33: 2-17.

Pitcher, T.M., Piek, J. y Hay, D. (2003). Fine and gross motor ability in males with ADHD, Developmental Medicine \& Child Neurology, 45, 525-535.

Pliszka S., McCracken J. y Maas, J. (1996). Catecholamines in attention deficit hyperactivity disorder, current perspectives. Journal of the American Academy of Child $\mathcal{E}$ Adolescent Psychiatry, 35, 264-272.

Raberger, T. y Wimmer, H. (2003). On the automaticity/cerebellar deficit hypothesis of dyslexia: Balancing and continuous rapid naming in dyslexic and ADHD children, Neuropsychologia, 41, 1493-1497.

Ramos-Quiroga JA, Bosch-Munsó R, Castells-Cervelló X, Nogueira- Morais M, GarcíaGiménez E, Casas-Brugué M. (2006).TDAH en adultos: características clínicas y terapéuticas. Rev Neurol; 42: 600-6

Rapport, M.D., Denney, C., Dupaul, G.J. y Gardner, M.J. (1994). Attention Deficit Disorder and methylphenidate, normalization rates, clinical effectiveness, and response prediction in 76 children. Journal of the American Academy of Child $\mathcal{E}$ Adolescent Psychiatry, 33, 882-893.

Rapport, M.D., Scanlan, S.E. y Denney, C.B. (1999). Attention deficit hyperactivity disorder and scholastic achievement, a model of dual developmental pathways. Journal of Child Psychology and Psychiatry, 40, 1169-1183.

Ross, R.G., Hommer, D., Breiger, D., Varley, C. y Radant, A. (1994). Eye movement task related to frontal lobe functioning in children with attention deficit disorder. Journal of the American Academy of Child \& Adolescent Psychiatry, 33, 869-874.

Schaill, L., Schwab-Stone, M., Merikangas, K.R., Leckman, J.F., Zhang, H. y Kasl, S. (1999). Psychosocial and clinical correlates of ADHD in a community sample of school-age children. Journal of the American Academy of Child \& Adolescent Psychiatry, 38, 976984 .

Semrud-Clikeman, M., Steingard, R., Filipek, P., Biederman, J., Bekken, K. y Renshaw, P. (2000). Using MRI to examine brain-behavior relationships in males with attention deficit disorder with hyperactivity. Journal of the American Academy of Child $\mathcal{E}$ Adolescent Psychiatry, 3, 477-484.

Sherman, D.K, Iacono, W.G y McGue, M.K. (1997b). Attention deficit hyperactivity disorder dimensions, a twin study of inattention and impulsivity hyperactivity. Journal of the American Academy of Child \& Adolescent Psychiatry, 36, 745-753.

Shaywitz SE y Shaywitz BA. (1991). Introduction to the special series on attention deficit disorder. Journal of Learning Disabilities; 24: 68-77. 
Silberg, J., Rutter, M., Meyer, J., Maes, H., Hewitt, J., Simonoff, E., Pickles, A., Loeber, R. y Eaves, L. (1996). Genetic and environmental influences on the covariation between hyperactivity and conduct disturbance in juvenile twins. Journal of Child Psychology and Psychiatry, 37, 803-816.

Sherman, D.K., McGue, M.K. y Iacono, W.G. (1997a). Twin concordance for attention deficit hyperactivity disorder, a comparison of teachers' and mothers' reports. American Journal Psychiatry, 154, 532-535.

Silberstein, R.B., Farrow, M., Levy, F., Pipingas, A., Hay, D.A. y Jarman, F.C. (1998). Functional brain electrical activity mapping in boys with attention deficit hyperactivity disorder. Archives of General of Psychiatry, 55, 1105-1112.

Slusarek M, Velling S, Bunk D, y Eggers C. (2001). Motivational effect on inhibitory control in children with ADHD. Journal of the American Academy of Child $\mathcal{E}$ Adolescent Psychiatry; 40: 355-363.

Smith BDH, Pelham WE, Gnagy E, Yudell R. (1998).Equivalent effects of stimulant treatment for attention deficit hyperactivity disorder during childhood and adolescence. J Am Acad Child Adolesc Psychiatry; 37: 314-21.

Solanto MV, Abikoff H, Sonuga-Barke E, Schachar R, Logan GD, Wigal T, Hechtman L, Hinshaw S, y Turkel, E. (2001). The ecological validity of delay aversion and response inhibition as measures of impulsivity in ADHD: a supplement to the NIMH multimodal treatment study of ADHD. Journal of Abnormal Child Psychology; 29: 215-228.

Sprich, S., Biederman, J., Crawford, M. H., Mundy, E. y Faraone, S. V. (2000). Adoptive and Biological families of children and adolescents with ADHD. Journal of the American Academy of Child \& Adolescent Psychiatry, 39, 1432-1437.

Steger, J., Imhof, K., Gundelfinger, R., Steinhausen, H.C. y Brandeis, D. (2001). Attentional and neuromotor deficits in ADHD. Developmental Medicine $\mathcal{E}$ Child Neurology, 43, 172-179.

Swanson, J.M., Posner, M.I., Potkin, S., Bonforte, S., Youpa, D., Fiore, C., Cantwell, D. y Crinella, F. (1991b). Activating tasks for the study of visuo-spatial attention in ADHD children: a cognitive anatomical approach. Journal of Child Neurology, 6, 119127

Swanson, J.M., Wigal, S., Greenhill, L.L., Browne, R., Waslid, B., Lerner, M., Williams, L., Flynn, D., Agler, D., Crowley, K., Fineberg, E., Baren, M. y Cantwell, D.P. (1998). Analog classroom assessment of Adderall in children with ADHD. Journal of Abnormal Child and Adolescent Psychiatry, 37, 519-526.V

Tannock R. (1998). Attention Deficit Hyperactivity Disorder. Advance in Cognitive, Neurobiological and Genetic Research. Journal of Child Psychology and Psychiatry, (39): 65-99.

Tantillo, M., Keisck, C.M., Hynd G.H., y Dishman, R.M. (2002). Physical education and behavior. Exploring the relationship between physical education and enhancing behavior in pupils with emotional behavioral difficulties. Support for Learning, 21, 169-174.

Teichner, Ito, Glod y Barber. (1996). Objective measurement of hyperactivity and attentional problems in ADHD. Journal of the American Academy of Child \& Adolescent Psychiatry; 35: $334-342$ 
Teeter, P.A. y Semrud-Clikeman, M. (1995). Integrating neurobiological, psychosocial, and behavioral paradigms, a transactional model for the study of ADHD. Archives of Clinical Neuropsychology, 10, 433461.

Tucha O, Klaus WL. (2001). Effects of metilphenidate on kinematic aspects of handwriting in hyperactive boys. J Abnorm Child Psychol; 29: 351-6.

Ucles, P., Serrano J.L. y Rosa, F. (2000). Central conduction time of magnetic brain stimulation in attention-deficit hyperactivity disorder, Journal of Child Neurology, 15, 723-728.

Vidarte JA y Vélez .C.. (1999). Caracterización del TDAH en los niños entre 5 y 7 años escolarizados de la ciudad de Manizales. Trabajo de Grado. Universidad Autónoma de Manizales, Colombia.

Vidarte JA y Vélez C. (2001). Características del TDAH en los niños entre 5 y 7 años escolarizados de la ciudad de Manizales. Trabajo de Grado. Universidad Autónoma de Manizales, Colombia.

Vidarte JA, Ezquerro M, y Giraldez, M. (2009). Perfil psicomotor de niños de 5 a 12 años diagnosticados clínicamente de trastorno por déficit de atención/hiperactividad en Colombia. Rev. Neurol ; 49 (2): 69-75

Vitiello B, Severe JB, Greenhill LL, Arnold LE, Abikoff HB, Bukstein OG, et al. (2001). Methylphenidate dosage for children with ADHD over time under controlled conditions: lessons from the MTA. J Am Acad Child Adolesc Psychiatry; 40: 18896.

Whalen CK, y Henker B. (1991). Therapies for hyperactive children, comparisons, combinations and compromises. Journal of Consulting and Clinical Psychology; (39): 126-137.

Whalen CK, Henker B, y Hinshaw SP. (1985). Cognitive behavioral therapies for hyperactive children, premises, problems, and prospects. Journal of Abnormal Child Psychology; (13): 391-410.

Whalen, C.K., Henker, B., Collins, T., Mc Auliffe, M.L. y Vaux, J. (1979). Peer interaction in a structured communication task: Comparisons of normal and hyperactive boys and of methylphenidate (Ritalin) and placebo effects. Child Development, 50,388401.

Whalen CK, Henker B. (1991). Social impact of stimulant treatment for hyperactive children. J Learn Disabil; 4: 231-41.

Wilens TE, Spencer TJ. (2000).The stimulants revisited. Child Adolesc Psychiatr Clin N Am; 9: 573-603

Winnick, J.P. (2004). Educação física e esportes adaptados. Barueri: Manole.

Wolraich M, Hannah J, Pinnock T, Baumgaertel A, y Brown J. (1996). Comparison of diagnostic criteria for attention deficit hyepractivity disorder in a country-wide sample. Journal of the American Academy of Child \& Adolescent Psychiatry; (35): 319324.

Wozniak, J., Grawford, M.H., Biederman, J., Farone, S.V., Spencer, T.J., Taylor, A. y Blier, H.K. (1999). Antecedents and complications of trauma in boys with ADHD, findings from a longitudinal study. Journal of the American Academy of Child $\mathcal{E}$ Adolescent Psychiatry, 38, 48-55. 
Yochman, A., Ornoy, A. y Parush, S. (2006). Co-occurrence of developmental delays among preschool children with attention-deficit-hyperactivity disorder. Developmental Medicine E Child Neurology, 48, 483-488.

Zarin, D.A., Suárez, A.P., Pincus, H.A., Kupersanin, E. y Zito, J.M. (1998). Clinical and treatment characteristics of children with attention deficit/hyperactivity disorder in psychiatric practice. Journal of Abnormal Child and Adolescent Psychiatry, 37, 12621270. 


\title{
Methylphenidate and Dyslipidemia
}

\author{
Gideon Charach, Nechemia Kaysar, \\ Alexander Rabinovich, Ori Argov and Moshe Weintraub \\ Department of Internal Medicine "C", Tel Aviv Sourasky Medical Center, \\ Sackler Faculty of Medicine, Tel Aviv University, Tel Aviv,
}

Israel

\section{Introduction}

Methylphenidate is one of the drugs that has been shown to elicit behavioral sensitization (1). Methylphenidate was synthesized by Ciba chemist Leandro Panizzon. His wife, Marguerite, had low blood pressure and would take the drug as a stimulant before playing tennis. He named the substance Ritaline, after his wife's nickname, Rita (2).

Its use has increased rapidly over the years and currently the drug is often prescribed for a large span of ages from childhood through adolescence and up to adulthood, thus facilitating the study of its effect on human physiology. Therefore, it there is an abundance of opportunities to study its various effects on the human organism.

Methylphenidate is a piperidine derivative, structurally related to amphetamines and acts as a CNS stimulant. Methylphenidate has been widely used since 1937 for numerous indications including attention deficit hyperactivity disorder (ADHD), narcolepsy, cataplexy (3) and conduct disorder (4) in children and adolescents as well as adults (4). Although it has been indicated for ADHD since 1957 it has gained widespread use during the last two decades (5). Methylphenidate was found to affect brain sterol metabolism in mice by inhibition of the incorporation of its precursors, acetate and glucose, into the brain and by reduction of the brain's sterol levels (6). This reduction was found to occur within 24 hours in the neuronal cellular membrane, the site of methylphenidate's action (6).

\section{Risk factors of atherosclerosis and cardiovascular disease}

Atherosclerosis is a slowly progressive process starting at a young age (7). Therefore, many effective measures are taken early in life to prevent future cardiovascular disease worldwide, the most important being the awareness of a proper lifestyle, including physical activity, a proper diet, and abstaining from drugs and habits that may increase the likelihood of atherosclerosis through the development of different risk factors (e.g., hyperlipidemia).

Several reports (8-9) found no association between prolonged administration (1 to 4 years) of methylphenidate to hyperactive boys regarding hematopoietic, endocrine (including blood glucose levels), hepatic or cardiovascular function. This is unlike the hypercholesterolemic effect of various psychotropic medication (see below) that can cause 
hypercholesterolemia secondary to cholestasis(10). It is known that cholestasis can cause hypercholesterolemia (10). Serum cholesterol appeared to be dominantly affected by lipoprotein X. Intra-hepatic cholestasis leading to reflux of bile lipids into the blood stream and subsequent formation of lipoprotein $X$ appears to be the mechanism (11) underlying this phenomenon .

\subsection{Methylphenidate's toxicity}

The therapeutic use of methylphenidate for the management of ADHD in children is constantly increasing. As therapeutic use this increases the risk of unintentional overdoses, medication errors, and intentional overdoses caused by abuse, misuse, or suicide attempts. Side effects, which include nervousness, headache, insomnia, anorexia, and tachycardia increase linearly with dose (3). Clinical manifestations of overdose include agitation, hallucinations, psychosis, lethargy, seizures, tachycardia, dysrhythmias, hypertension, and hyperthermia. Hepatotoxicity was reported in rodents (11). A possible mechanism is inhibition of cytochrome p-450 (12). There are few reports about different organ failure. These were manifested by abnormal liver function enzymes, poor urine output, hypotension, tachypnea, tachycardia, abnormal blood gases, rising serum BUN and creatinine, and hyperactive deep-tendon reflexes (13). Despite its abuse potential, there is disagreement regarding the extent to which methylphenidate is being diverted from legitimate use to abuse in children and adolescents.

Gontkovsky et al (14) reported a decrease of $26 \%$ in serum glucose values after methylphenidate initiation in a patient post- cerebellar tumour resection.

Being a neurostimulant, the possible linkage of methylphenidate to the cardiovascular system was investigated. The data are controversial. Samuels et al. (8) investigated the effect of methylphenidate on blood pressure. Their study provided evidence for a possible negative cardiovascular effect of the stimulant medication on children with ADHD. Vitiello (15) did not find clinically significant changes in cardiovascular function in the majority of cases investigated. However, Langendijk et al (16) in their review show an increase in blood pressure and heart rate among adults treated with methylphenidate as being a risk factor for cardiovascular event, and Rapport et al (17) described a transient increase in blood pressure and heart rate among children treated by methylphenidate for ADHD. This was dose dependent and easily rectified with dosage adjustment. Spivak et al (18) reported a thrombocythopenia and decreased levels of norepinephrine, dopa and serotonine in children treated for three months with methylphenidate which means inhibitory impact on platelets activation.

Heart failure due to idiopathic dilated cardiomyopathy (DCM) is uncommon in young people. Cocaine and amphetamines are known to have caused dilated cardiomyopathy (19). There are very few reports linking methylphenidate therapy to DCM. Two reports from Norway described serious cardiomyopathy in young patients treated with methylphenidate (20-21). One of them was severly obese (BMI-40). An obesity-linked susceptibility to the toxic effect of methylphenidate could, therefore, play a role in the development of DCM in this patient. This is remarkable especially with regards to the short (one year) duration of the treatment. With a BMI of 40 the above mentioned patient had suffered from extreme obesity. The hyperdynamic circulation, with increased cardiac output, thought to be a 
compensatory adaptation to increased adipose tissue may, at the expense of left ventricular hypertrophy and remodeling, lead to non ischemic dilated cardiomyopathy in severe obese subjects $(22,23)$. This is, however, unlikely to be the only cause. Human obesity is also characterized by sympathetic nervous activation (23). The concern is that methylphenidate is the responsible agent. Although the number of patients treated with this drug is high, and the reported cardiovascular side effects are few, scientists were concerned about the serious long term results this side effect could have on children and young adults. No guidelines are available to help identify individuals prone to cardiomyopathy due to central stimulating drugs.

Cardiac adverse effects from methylphenidate have been shown to affect myocardial ultrastructure in rats. This effect was irreversible after 12 weeks $(24,25)$. Methylphenidate is a drug which stimulates the central nervous action and produce similar effects as amphetamines. Both drugs increase synaptic and intracellular norepinephrine and dopamine in rodents and baboons $(25,26)$. For amphetamine the mechanism has been shown to trigger both an increased release of catecholamines and blocking their synaptic reuptake and degradation (26-27). It is the increase in adrenergic action that is believed to be cardiotoxic over time and promote cardiomyopathy $(24,28)$. This can be understood by the observation that, in transgenic mice, myocardial over expression of beta-adrenergic receptors was associated with myocyte apoptosis and the development of dilated cardiomyopathy $(27,29)$.

\section{Methylphenidate and dyslipidemia}

In many cases methylphenidate is prescribed for extended periods of time, usually starting from childhood or adolescence. Therefore, in the long run any metabolic consequence of the treatment might be deleterious, especially when atherosclerosis is considered. From this perspective, it seemed essential to substantiate the potential of methylphenidate to affect lipid profile. In order to evaluate this, we first looked into the possibility that other psychotropic drugs have an impact on cholesterol and tryglicerides.

Hyperlipidemia is one of the major risk factors for atherosclerosis and cardiovascular diseases $(30,31)$. The causes of lipid metabolism abnormalities are mainly genetic. Established causes for secondary dyslipidemia include inappropriate lifestyle, liver disease, renal disease and thyroid disease $(7,9)$. The various drugs that are known to affect lipid metabolism include hormones (glucocorticoids, estrogens and androgens), beta-blockers, and diuretics (3, 7, 32-40). Several psychotropic drugs were reported to cause secondary hyperlipidemia, especially carbamazepine, phenobarbital, tricyclic antidepresants (in particular amitriptyline), secondgeneration antipsychotic medication, mainly olanzapine but also risperidone and ziprasidone. In their extensive review Ruetsch and co-writers elaborate on weight gain induced by psychotropic medications (38). According to this and many other reports weight gain is the mainstay of the psychotropic pharmacological induced hyperlipidemia. Weight gain was found to induce dyslipidemia in correlation with dosage and duration of treatment (38). In addition to increased total cholesterol and low-density lipoprotein cholesterol (LDL-c) there was also a tendency to elevate blood pressure levels and thus enhance the impact on the development of atherosclerosis (31-34)., These can aggravate health risks, including higher rate of coronary heart desease, ischemic stroke due to impaired glucose tolerance, diabetes mellitus, dyslipidemia, respiratory problems. 
Weight gain appears to be most prominent with some mood stabilizers (e.g. lithium, valproate, 34-38). According to current concepts, appetite and feeding are regulated by a complex of neurotransmitters, neuromodulators, cytokines and hormones interacting with the hypothalamus, including leptin and tumor necrosis factor system (37-39). The pharmacologic mechanisms underlying weight gain are presently poorly understood: possibly the various activities at some receptor systems may induce it, but also genetic predisposition plays an important role. In addition the insulin-like effect of lithium is well known.

Weight gain has been described since the discovery and the use of the first psychotropic drugs, but seems to intensify especially with some of the second generation antipsychotic medications. Understanding of the side effects of psychotropic drugs, including their metabolic consequences (weight gain, diabetes, dyslipidemia) is essential in order to avoid, firstly, a risk of lack of compliance with the ensuing risk of relapse and re-hospitalization, and secondly, the acute, life threatening events (diabetic ketoacidocetosis and non ketotic hyperosmolar coma) and long term risk complications of diabetes and overweight $(31,37,39)$.

Many psychotropic drugs (especially methylphenidate) were designed to be started early in childhood in order to improve various target organ, cognitive and neuropsychiatric functions. These medications appeared to be very potent and significantly changed life quality, which led to their widespread use all over the world (5). This medications are often continued for a long periods (years) and may cause unfavorable metabolic effects. Rader and Hobbs (40) reported that some of these drugs affect lipid and lipoprotein metabolism and increase or decrease atherogenicity.

Methylphenidate is the most abundantly used medication for the treatment of ADHD in all ages worldwide (5). It is well known that methylphenidate has an effect on intracellular cholesterol in the brain, but to date its effect on plasma lipid metabolism has been studied to a limited extent in clinical setting $(6,41)$. We recently published the results of a randomized study that examined the effect of methylphenidate on blood lipid levels (42). A total of 42 outpatients with an established diagnosis of ADHD were studied. The baseline characteristics of the study group are presented in Table 1 . There were 22 males and 20 females whose median age was 16 years (range 11-31). Table 2 displays the differences in the examined parameters before and after a 3 month treatment by methylphenidate. BMI didn't change during the study period. Significant decrease was found in total cholesterol, LDL- c and tryglicerides levels. Non significant changes were seen in HDL-c, apolipoprotein A and apolipoprotein B levels. The changes in Lp (a) unexpectedly turned out to be statistically significant. There were no gender- based differences in any of these parameters after adjustment according to age, nor any correlations between the lipid parameters.

This is the first investigation into the impact of methylphenidate on plasma lipid profile and atherosclerosis. The results of the current study showed that methylphenidate has a significant and positive impact on the lipid and lipoprotein profile with regard to atherosclerosis. It significantly decreases total cholesterol, triglycerides and the main atherosclerotic lipoproteins, LDL-c and Lp(a). There is no good explanation of lipid lowering mechanism. However, in contrast to other psychotropic medication it didn't cause weight gain. The possible explanation: amphetamine related agents-(including methylphenidate) increase synaptic dopamine by stimulating presynaptic release of the last 


\begin{tabular}{lccc}
\hline Variable & Median & $\begin{array}{c}\text { Lower } \\
\text { quartlile }\end{array}$ & $\begin{array}{c}\text { Upper } \\
\text { quartlile }\end{array}$ \\
\hline Age (years) & 16 & 14 & 22 \\
Males & $52 \%$ & & \\
BMI & 22 & 20 & 24 \\
T-Chol(mg/dl) & 157 & 142 & 179 \\
HDL-C(mg/dl) & 51 & 45 & 64 \\
LDL-C(mg/dl) & 93 & 74 & 114 \\
Trigl(mg/dl) & 76 & 58 & 100 \\
Apo A(mg/dl) & 122 & 109 & 137 \\
Apo B(mg/dl) & 71 & 56 & 83 \\
Lp(a)(mg/dl) & 23 & 9 & 40 \\
\hline
\end{tabular}

BMI,body mass index ; HDL-C, high density lipoprotein;

LDL-C, low density lipoprotein; Apo A, apolipoprotein A

Apo B, apolipoprotein B; Lp (a), lipoprotein (a).

Table 1. Patient characteristics $(n=42)$

\begin{tabular}{lcccc}
\hline Variable & Median & Lower quartle & Upper quartle & $p^{*}=$ \\
\hline$\Delta$ BMI & 0 & 0 & 0.3 & $\mathbf{0 . 2 4}$ \\
$\Delta$ T-Chol $(\mathrm{mg} / \mathrm{dl})$ & $\mathbf{- 9}$ & -15 & -3 & 0.0002 \\
$\Delta$ HDL-C $(\mathrm{mg} / \mathrm{dl})$ & 2 & 0 & 4 & 0.1 \\
$\Delta$ LDL-C $(\mathrm{mg} / \mathrm{dl})$ & -5 & -8 & 1 & 0.016 \\
$\Delta$ Trigl $(\mathrm{mg} / \mathrm{dl})$ & -8 & -15 & -3 & 0.016 \\
$\Delta$ Apo A $(\mathrm{mg} / \mathrm{dl})$ & -4. & -12 & 7 & 0.16 \\
$\Delta$ Apo B $(\mathrm{mg} / \mathrm{dl})$ & -2 & -5. & 5 & 0.6 \\
$\Delta$ Lp $(\mathrm{a})(\mathrm{mg} / \mathrm{dl})$ & -2 & -5 & 0 & 0.0007 \\
$\Delta$ nonHDLC $(\mathrm{mg} / \mathrm{dl})$ & $\mathbf{- 1 1}$ & -18 & -2 & 0.0001 \\
\hline
\end{tabular}

$\Delta$-difference

* Based on Wilcoxson test.

Table 2. Differences in lipid profile and BMI parameters before and after the treatment by methylphenidate.

and cause an anorexigenic effect by changes in hypothalamic monoaminergic activity and in the anorexigenic cocaine-amphetamine -regulated transcript neuropeptide ( CART) expressed in the paraventricular nucleus and hypothalamic perifornical nucleus $(43,44)$.

Conclusion: the presented data support some positive effects on lipid profile by decreasing total cholesterol, triglycerides, LDL-c and Lp (a). No conclusions could be reached concerning atherosclerosis.

\section{Limitations}

These conclusions are tentative because of the large rate of early drop outs from the studies, which may limit the validity of the results. The study was performed on a relatively small number of patients (42 patients) mostly teenagers. There is not enough data about impact of methylphenidate on adult population. The reported short term study, conducted for three 
months, showed positive effects on lipid profile. However it doesn't allow us to predict whether this effect will persist and whether atherosclerosis will develop.

Further, large, well-designed trials are necessary to establish the different metabolic effects of methylphenidate

\section{References}

[1] Yang P, Swann A, Dafny N. Valproate prevents the induction and expression of MK-801 sensitization. Brain Res 2002; 954: 151-159.

[2] Mayers RL Methylphenidate (Ritalin). In "The 100 most important chemical compounds: a reference guide". pp-178-180. Greenhood publishing group 2007.

[3] Leonard B, McCartan D,White J,King D. Methylphenidate: a review of its neuropharmacological, neuropsychological and adverse clinical effects. Hum Psychopharmacol Clin Exp.2004; 19: 151-180.

[4] Gerardin P, Cohen D, Mazet P, Flament M. Drug treatment of conduct disorder in young people. Eur Neuropsychopharmacol 2002; 12: 361-370.

[5] Gonzalez de Dios J, Cardo E, Servera M. Methylphenidate in the treatment of attention deficit/hyperactivity disorder: are we achieving an adequate clinical practice? Rev Neurol 2006; 43:705-714.

[6] Kabara JJ. Brain cholesterol XVIII: EFFECt of methylphenidate (Ritalin) on [U-14C] glucose and $[2-3 \mathrm{H}]$ acetate incorporation. Proc Soc Epidemiol Biol Med 1975; 150: 525-528.

[7] Botton J, Heude B, Kettaneh A, Borys JM, Lommez A, Bresson JL, Ducimetiere P, Charles MA; FLVS Study Group. Cardiovascular risk factor levels and their relationships with overweight and fat distribution in children: the Fleurbaix Laventie Ville Sante 2 study. Metabolism 2007;56: 614-622.

[8] Samuels JA, Eranco K, Wan F, Sorof JM Effect of stimulants on 24 ambulatory blood pressure in children with ADHD Ped Nephrol. 2006; 21(1): 92-5.

[9] Satterfield JH, Scell AM , Barb SD Potential risk of prolonged administration of stimulant medication for hyperactive children. Dev Behav Pediatr. 1980; 1(3): 102-107.

[10] Zidan H, Lo S, Talano J, Alemzadeh R. Severe hypercholesterolemia mediated by lipoprotein $X$ in a pediatric patient with chronic graft-versus-host disease of the liver. Pediatr Blood Cancer 2008; 50(6):1280-1.

[11] Roberts S, Harbison R, Roth L, James R. Methylphenidate-induced hepatotoxicity in mice and its potentiation by beta-adrenergic agonist drugs. Life Sci 1994; 55 (4)269281.

[12] Le Nedelec M, Rosengren R, Methylphenidate inhibits cytochrome P450 in Swiss Web mouse. Hum Exp Toxicol 2002 May; 21(5): 273-80.

[13] Stechyc O, Loludice T,Demeter S, Jacobs J. Multiple organ failure resulting from intravenous abuse of methylphenidate hydrochloride. Ann of Emerg. Med. 1985;14:597-599.

[14] Gontokovsky SR, Nevel R, McDonald NB, Winkelman MH. Decreased serum glucose levels after initiation of methylphenidate in a patient status post- cerebellar tumour resection: a potential interaction with glipizide. Clin Drug Investig. 2007;217: 719725 . 
[15] Vitiello B . Understanding the risk using medications for attention deficit hyperactivity disorder with respect to physical growth and cardiovascular function Child Adoles Psychiatr Clin N am 2008; 17(2): 459-74, xi.

[16] Langendijk PN, Wilde AA Medication for ADHD and the risk of cardiovascular mortality Ned Tijdschr Geneeskd 2006; 150(31) : 1713-4.

[17] Rapport MD, Moffitt C. Attention deficit / hyperactivity disorder and methylpenidate . A review of height/weight, cardiovascular, and somatic complaints side effects. Clin Psychol Rev 2002 ;22(8): 1107-31.

[18] Spivak B, Veref Y, Yoran-Hegesh R, Graff E, Averbuch E Vinokurow S, Weitzman A, Mester R. The influence of three months of methylphenidate treatment on platelepoor biogenic amine levels in boys with attention deficit hyperactivity disorder. Hum Psychopharmacol. 2001 ;16(4): 333-337.

[19] Lange RA, Hillis LD. Cardiovascular complications of cocaine use. N E J Med. 2001; 345: 351-358.

[20] Tollofsrude C,Hoel T. A young man with acute dilated cardiomyopathy. Tidsskr Nor Laegeforen. 2006; 126: 1338-1339.

[21] Nymark T, Hovland A, Bjernstadt H, Nielsen E. A young man with dilated cardiomyopathy associated with methylphenidate. Vasc Health risk Manag 2008; 4(2): 477-9.

[22] McGavock JM, Victor RG, Unger RH, et al. Adiposity of the Heart, Revisited. Ann Intern Med. 2006; 144: 517-24.

[23] Eikelis N, Esler M. The neurobiology of human obesity. Exp Physiol. 2005; 90: 673-82.

[24] Henderson TA, Fischer VW. Effects of methylphenidate (Ritalin) on mammalian myocardial ultrastructure. Am J Cardiovasc Pathol.1995; 5:68-78.

[25] Schiffer WK, Volkow ND, Fowler JS, et al. Therapeutic doses of amphetamine or methylphenidate differentially increase synaptic and extracellular dopamine. Synapse. 2006; 59: 243-51.

[26] Knepper S, Grunewald G, Rutledge C. Inhibition of norepinephrine transport into synaptic vesicles by amphetamine analogs. J Pharmacol Exp Ther 1988; 247: 487-94.

[27] Singh K, Xiao L, Remondino A, et al. Adrenergic regulation of cardiac myocyte apoptosis. J Cell Physiol. 2001; 189: 257-65.

[28] Pitts WR, Vongpatanasin W, Cigarroa JE, Hillis LD, Lange RA. Effects of the intracoronary infusion of cocaine on left ventricular systolic and diastolic function in humans. Circulation 1998;97:1270-

[29] Viles-Gonzalez JF, Anand SX, Valdiviezo C, Zafar MU, Hutter R, Sanz J, Rius T, Poon M, Fuster V, Badimon JJ. Update in atherothrombotic disease. Mt. Sinai J Med 2004; 71:197-208

[30] Ruetsch O, Viala A, Bardou H, Martin P, Vacheron M. Psychotropic drugs induced weight gain: a review of the literature concerning epidemiological data, mechanisms and management. Encephale 2005; 31:507-516.

[31] Zeitlhofer J, Doppelbauer A, Trible G, Leitha T, Deecke L. Changes of serum lipid patterns during long-term anticonvulsive treatment. Clin Investig.1993; 71(7): 574-8

[32] Yilmaz E, Dosan Y, Gurgoze M, Gungor S. Serum lipid changes during anticonvulsive treatment serum lipids in epileptic children. Acta Neurol Belg. 2001 Dec;101(4):21720. 
[33] Yalcin E,Hassanzadeh A, Mawlud K. The effect of long term anticonvulsive treatment on serum lipid profile. Acta pediatr Jpn. 1997; 39(3):342-345

[34] Pita-Calandre E, Rodrigez-Lopez C, Cano M, Pena-Bernal M. Serum lipids. lipoproteins, and apolipoproteins in adult epileptics treated with carbamazepine, valproic acid, or phenytoin. Rev Neurol.1998: 27(159):785-9

[35] Lee E, Chow LY, Leung CM. Metabolic profile of first and second generation antipsychotics among Chinese patients. Psychiatry Res. 2011; 185(3):456-8.

[36] Komossa K, Rummel-Kluge C, Hunger H,Schmid F, Schwarz S,Duggan L, Kissling W, Leucht S. Olanzapine versus other atypical antipsychotics for schizophrenia Cochrane Database Syst Rev. 2010 Mar 17;(3):

[37] Newcorner J.Second-generation (atypical) antipsychotics and metabolic effects: a comprehensive literature review. CNS Drugs. 2005;19 Suppl 1:1-93.

[38] Ruetsch O, Viala A, Bardou H, Martin P, Vacherone M. Psychotropic drugs induced weight gain: a review of the literature concerning pidemiological data, mechanisms and management. Encephale 2005;31(4 Pt 1):507-16.

[39] Chang H, Chou C, Chen p, Gean P, Huang H, Lin C, Yang Y, Lu R. High prevalence of metabolic disturbances in patients with bipolar disorder in Taiwan. J Affect Disord. 2009 Sep;117(1-2):124-9. Epub 2009 Feb 4.

[40] Rader D, Hobbs H. 2005. Disorders of lipoprotein metabolism, in Harrison s Principles of Internal Medicine, 16 th ed. L. Kasper, E. Braunwald and A. Fauci, editors. Mc Graw-Hill, New -York.2286-2298.

[41] Klein-Schwartz W. Abuse and toxicity of methylphenidate Curr Opin Pediatr. 2002 14(2); 219-223

[42] Charach G, Kaysar N, Grosskopf I, Rabinovich A, Weintraub M. Methylphenidate has positive hypocholesterolemic and hypotriglyceridemic effects: new data. J Clin Pharmacol. 2009; 49(7): 848-51.

[43] Serano A, Pavon F, Tovr S, Casanueva F, Senaris R, Dieguez C, de Fonseca F. Oleolethanolaminde: effects on hypothalamictransmitters and gut peptides regulating food intake. Neuropharmacology 2011;60(4): 593-601.

[44] Adamson T, Corll C, Syec F, Porter J. Role of the perifornical hypothalamic monoamine neurotransmitter system in anorectic effects of endotoxin. 2010; 91(1): 48-55. 


\section{Part 3}

Non-Medication Interventions 



\title{
ADHD and Stress: The Role of Meditation to Reduce Stress, and Improve Brain Function and Behavior Regulation
}

\author{
Sarina J. Grosswald ${ }^{1}$ and Fred Travis ${ }^{2}$ \\ 1SJ Grosswald \& Associates \\ ${ }^{2}$ Center for Brain, Consciousness and Cognition \\ United States
}

\section{Introduction}

The number of children worldwide diagnosed with ADHD increases every year. As information about the medical risks associated with ADHD medication is becoming more widely understood, it is not surprising that large numbers of parents are seeking alternative therapies as a response to concerns about the physiological and psychological effects of the drugs.

A growing number of clinicians also support alternative therapies, arguing that an emphasis on medical therapy alone focuses only on control of symptoms, rather than attending to the need for children to develop important behavioral and social skills (Zametkin, \& Ernst, 1999).

While the use of alternative approaches such as yoga, meditation, exercise, special diets, and nutritional supplements for treating children with ADHD is becoming widespread, little is known about the effectiveness of many of these approaches. There is a growing body of research on the beneficial effects of meditation on health risks, psychosocial factors, and on brain development. However there is little published research about the use of meditation for ADHD.

\section{Stress, anxiety and ADHD}

ADHD is associated with impaired executive function, specifically brain circuitry governing behavior (Bush, et al., 2005; Durston, et al., 2004; Zametkin, et al., 1993). Dysfunction of these circuits leads to impulsivity and lack of normal social inhibition. It also leads to impaired working memory, inability to focus attention, and impaired temporal organization.

Stress interferes with executive function and behavior regulation (McEwen, 1998). Stressimpaired executive function is associated with impaired working memory, impaired impulse control, and lack of mental flexibility and coping strategies. Stress also dramatically compromises selective attention and the ability to sustain attention (Lupien, et al., 2005). 
In comparing symptoms of stress and symptoms of ADHD, there is a striking similarity. Recent research sheds light on the relationship between stress and ADHD. Vance, et al., demonstrated dysfunction of the right prefrontal regions of the brain in ADHD children (2007). This region is responsible for developing coping strategies, influencing the ability to handle stress. Chronic acute stress damages the body's ability to return to non-stress levels, leading to chronically elevated levels of cortisol, a biochemical marker of stress. In children with ADHD high cortisol levels impair executive function, self-regulation, and letter knowledge (Blair, Grange, \& Razza, 2005).

Chronic stress can originate not only from emotional causes but can also result from physical origins. Studies of premature birth and birth weight show an association between physical stress and risk of ADHD. Danish researchers found that babies born prematurely had up to 70\% greater risk of ADHD. Similarly, babies born of low birth weight had 50-90\% greater risk of ADHD, depending on the weight at birth (Linnet, Wisborg, Agerbo, Secher, Thomsen, \& Henriksen, 2006).

Early experiences of stress are believed to affect the level of responsiveness of the hypothalamic-pituitary-adrenal axis and the autonomic nervous system. Young children exposed to chronic stress can become conditioned to higher levels of adrenaline. This raises the "set point," making these children more vulnerable to stress, resulting in situations more easily triggering stress responses.

\section{Pharmaceutical treatment}

The United States Centers for Disease Control and Prevention estimates that over $66 \%$ of children diagnosed with ADHD are taking medication (CDC, 2008). The agency states, however, that concern persists regarding the side effects and long-term health outcomes associated with these drugs (CDC, 2005).

Further, the long term effectiveness of pharmaceutical treatment is mixed. The largest study conducted on ADHD treatment was the Multimodal Treatment Study of Children with ADHD, referred to as the MTA, published in 1999, which reported effectiveness of drug treatment (MTA Cooperative Group, 1999). However follow-up studies at three years and eight years, reported no significant differences in symptoms among the children who received the intensive drug treatments and those who did not (Molina, et al. 2009; Jensen, et al. 2007). Additionally, those on the drug regimens had significantly higher rates of delinquency and substance use (Molina, Flory, Hinshaw, Greiner, Arnold, \& Swanson, 2007).

Persistent and negative side effects of the drugs include sleep disturbances, reduced appetite, weight loss, suppressed growth, and mood disorders. The long-term effects are not fully known at this time; however evidence suggests risks of cardiac disorders and sudden death, liver damage, and psychiatric events. The side-effects and long-term health outcomes associated with the drug treatment have potentially important health implications for the millions of children who are currently taking medication for ADHD (CDC, 2005). Consequently, there is a growing interest in alternative treatment options, such as nutritional approaches, meditation, and yoga. 


\section{Meditation}

There are many systems of meditation, which differ widely from one another in their procedures, content, beliefs, and goals. Research has shown that the different techniques have different subjective and objective effects. Meditation types have generally been classified into two categories: techniques of concentration or techniques of contemplation (Shapiro, 1982). Each of these different techniques uses different processes and thus has different effects (Orme-Johnson, \& Walton, 1998; Shear, 2006).

Most recently, Travis and Shear (2010) identified three types of meditation practices, classified according to their EEG signatures and the corresponding cognitive processes. The three classifications are focused attention, open monitoring, and automatic selftranscending.

Techniques of focused attention are concentration techniques, and are associated with voluntary sustained control of attention to keep it focused on the object of meditation, such as an event, image, or sound. The brain activity during concentration meditations is characterized by EEG in the beta- $2(20-30 \mathrm{~Hz})$ and gamma $(30-50 \mathrm{~Hz})$ frequency bands. Open monitoring or mindfulness-based techniques, involve dispassionate non-evaluative monitoring of ongoing experience. These techniques are characterized by frontal theta (5-8 $\mathrm{Hz})$ EEG, and perhaps occipital gamma $(30-50 \mathrm{~Hz})$ EEG. Automatic self-transcending meditation is defined as effortless transcending of the meditation process itself (Travis \& DuBois, 2004; Travis \& Shear, 2010). EEG activity of an automatic self-transcending technique is associated with alpha-1, characteristic of reduced mental activity and relaxation.

Research has shown improvements in attention and flexibility among a study group that was using various different forms of meditation (Rutschman, 2004), and in ADHD symptoms using Sahaja yoga meditation, a form of contemplative or open monitoring meditation (Harrison, Manocha, \& Rubia, 2004).

The Transcendental Meditation technique falls into the category of automatic selftranscending. Concentration and open monitoring meditations both require some mental effort (i.e., holding attention on its object or maintaining a stance of open monitoring, respectively). The Transcendental Meditation technique is said to automatically lead to the experience of "consciousness itself," awareness without any objects of awareness, a lowstress state called transcendental or pure consciousness (Travis \& Pearson, 2000).

It is described as a simple and effortless, non-religious mental technique that is easy to learn, and does not require the ability to concentrate or control the mind. Regular practice of the technique creates a state referred to as "restful alertness." The term reflects a combination of markedly decreased metabolism, heart rate, respiration rate, and blood flow to the limbs, similar to deep rest or sleep; while at the same time mental alertness is maintained, as measured by EEG (Dillbeck, \& Orme-Johnson, 1987; Jevning, Wallace, \& Beidebach, 1992; Orme-Johnson, 1973; Travis, \& Wallace, 1999; Wallace, 1970).

EEG measurements also show increases in brain coherence both during the practice of the Transcendental Meditation technique and afterwards in activity (Travis and Shear 2010; Dillbeck \& Araas-Vesely, 1986; So \& Orme-Johnson, 2001). The primary areas of brain 
activated during the practice are the frontal and prefrontal executive areas responsible for attention, executive function, emotional stability, and anxiety (Dixon, et. al., 2005; So, 2001; Fergusson, 1993; Gaylord, Orme-Johnson, \& Travis, 1989).

Positive effects of the Transcendental Meditation technique extend to the noradrenergic networks (Jevning, Wallace, \& Beidebach, 1992; Jevning, Wilson,\& Davidson, 1978; MacLean, Walton, Wennenberg, et. al., 1997; Walton, Gelderloos, \& MacRae, 1995). The practice of the technique results in a decrease in the stress hormone cortisol, both during meditation and longitudinally, outside meditation during activity. Practice of the technique increases serotonin availability, improving mood and reducing the activation of the brain centers for fear, anxiety, and anger.

The use of the TM technique for stress reduction in adolescents has resulted in improvement in school behavior, decreases in absenteeism and rule infractions, and reduction in suspensions due to behavior-related problems (Barnes, Bauza, \& Trieber, 2003). Students practicing the TM technique show higher performance on scales of self-actualization (Alexander, Rainforth, \& Gelderloos, 1991), increased emotional regulation, and improved well-being (Rosaen \& Benn, 2006), as well as improved academic performance.

This chapter describes two research studies examining the effects of the Transcendental Meditation technique in reducing stress, improving the symptoms of ADHD, and improving brain coherence and executive function.

\section{Pilot study}

The Transcendental Meditation technique was selected for an exploratory study to evaluate whether regular practice of the technique by children ages 11 to 14 with ADHD would result in improvements in stress, behavioral symptoms, and executive function.

\subsection{Subjects}

The study was conducted at an independent school for children with language-based learning disabilities. Participation in the study was restricted to students with pre-existing diagnoses of ADHD. The cohort was 10 students ages 11-14, nine boys and one girl. All had a diagnosis of ADHD, six with inattentive-type and four with combined-type. Six students had comorbidities including general anxiety disorder, dysthymia, obsessive compulsive disorder, pervasive developmental disorder, sleep disorders, and tics. Eight students were taking stimulants (e.g. Concerta, Ritalin); three of those were also on other medications such as antidepressants (e.g. Zoloft, Welbutrin) and atypical antipsychotics (e.g., Risperdal). By virtue of attending the school, all students had language-based learning disabilities.

\subsection{Measures}

Data collection was divided into two categories to correspond to the primary and secondary hypotheses: 1) measures of stress, anxiety, ADHD symptoms as reported by parent, teacher, and student inventories, 2) measures of executive function as measured by parent and teacher inventories, and by performance tests. Anecdotal information was collected by informal video interviews at pre- and post test times. 


\subsubsection{Stress, anxiety, and ADHD symptoms}

Teachers and parents completed the Achenbach Child Behavior Checklist (CBCL) inventory, which is validated as diagnostic of ADHD based on criteria defined in the Diagnostic and Statistical Manual of Mental Disorders, fourth edition (DSM-IV). The CBCL is one of the most widely-used measures in child psychology, assessing behavior and social competency. It is composed of 112 items, each differentiating clinical from non-clinical children. Summarized in two categories: Syndrome Scales and DSM-Oriented Scales, the CBCL provides eight symptom subscale scores and a Total Problem score.

Students completed the self-administered Achenbach Youth Self-Report (YSR) and the Revised Children's Manifest Anxiety Scale (RCMAS) to assess overall behavioral and emotional functioning. The YSR is a companion to the CBCL, providing parallel measures from the teachers, parents, and students. Like the CBCL the YSR has 112 items measuring eight subscale symptoms categorized under Syndrome Scales and DSM-Oriented Scales, and a Total Problem score. The RCMAS is a 37-item self-report instrument assessing the level and nature of anxiety in children and adolescents.

\subsubsection{Executive function}

Teachers and parents completed the Behavior Rating Inventory of Executive Function (BRIEF). The BRIEF consists of 86 items divided into eight subscales. These scales form two broader indices: Behavioral Regulation and Metacognition. These two indices are combined to create an overall Global Executive Composite score. The BRIEF assesses behavior regulation and executive functioning.

Four performance tests were used to measure different aspects of executive function. The Cognitive Assessment System (CAS) Expressive Attention, Delis-Kaplan Executive Function System (D-KEFS) Verbal Fluency test, Tower of London (TOL), and Connor's CPT II.

The CAS Expressive Attention subtest is a color-word interference test. It measures higher level complex attention, and ability to inhibit. Names of colors are presented in ink colors of either the name of the color or a different color. When the color of ink is different from the color name the task requires attention, inhibiting the reflexive response, and cognitive processing (switching) to say the correct color.

The D-KEFS Verbal Fluency subtest measures the ability to generate words fluently, generate words in specific categories, and shift attention by switching from one category to another. The student is asked to list as many words as possible starting with a certain letter, to name as many items in a category, and to name items switching from one category to another.

The TOL measures higher order problem-solving, and is used to evaluate difficulties in executive function. Subjects are shown a configuration of colored balls stacked on pegs. The subject executes a sequence of moves that transforms his or her board to match the displayed configuration with the balls arranged on the designated pegs.

The Connors' Continuous Performance Test (CPT) II is a computerized test that measures sustained attention. The subject is presented stimuli on a computer screen (e.g., a letter or asterisk) and is asked to respond by pressing a button only when detecting a specified letter after seeing another specified letter. 


\subsection{Procedure}

Six weeks before the start of the intervention, two classroom teachers and two administrators at the school were instructed in the Transcendental Meditation technique, in the same way the students would be (see below). The instruction in Transcendental Meditation is standardized and all practitioners around the world are instructed in the identical way, thus assuring consistency in instruction and in practice of the technique.

Following completion of baseline assessments, students were provided the standard 7-Step course of instruction in the Transcendental Meditation technique (see 5.4 below). All 10 children in the group were able to learn the TM technique, and practice it successively as evidenced during the morning and afternoon TM sessions and by responses to course assessment questionnaires.

Over the 3-month study period, subjects participated in customary follow-up checks of correct meditation, which is standard with the instruction of the technique. Students meditated at school in a group for 10 minutes twice each day, once in the morning and once in the afternoon. The classroom teachers who had also learned TM led the students in twice daily meditation sessions at the school, telling students when to begin and end meditation, and timing the meditation. The Certified TM teachers monitored the group meditation two or three times a month to assure correct group meditation.

Parents were encouraged to have students continue regular meditation at home on weekends and holidays. Home compliance was not rigorously monitored.

\subsection{Intervention: The Transcendental Meditation technique}

The Transcendental Meditation technique is a mental technique practiced for 10-20 minutes twice each day, sitting in a chair with eyes closed. The technique is taught by certified Transcendental Meditation teachers. The 7-Step course of instruction involves two informational lectures (Steps 1 and 2), a brief interview with the TM instructor (Step 3), individual personal instruction (Step 4), which is followed by three days of verification of practice and additional information (Steps 5-7). The interview is about 10 minutes, while the remaining steps are approximately one hour each day. Each step can be conducted in a group except Step 4, which is conducted individually, one-on-one. Periodic meetings with the student assures correct practice and reinforces regularity of the practice.

During the course of instruction, the student learns how to let the mind move from active focused levels of thinking to silent, expanded levels of wakefulness at the source of thought, without concentration or effort (Travis, 2001).

After the personal instruction, students in the study meditated in a group at the beginning of the first period in the morning and the beginning of the last period in the afternoon. Meditation sessions were led by a school faculty member also instructed in the TM technique. A certified TM teacher periodically monitored group meditations, and met with students individually as needed to assure correct practice as described above. Three months after instruction, post testing was administered.

\subsection{Results}

Variables were grouped into two conceptual categories to correspond to the hypotheses. One grouping, stress, anxiety, and ADHD symptoms, included the associated variables from 
the YSR, RCMAS, and CBCL. The second grouping, executive functioning, included the responses from teachers on the BRIEF, and the data from the performance tests. Analysis was conducted on measures for which there was complete data $(\mathrm{N}=10)$.

Parent inventories and CPT scores were not included in the analysis because fifty percent of the parent post test inventories were not returned, and thirty percent of students had already left school for summer break at the time of CPT post testing.

Raw scores were used for all analyses except performance tests, which were converted to standard scores using the tables provided in the test manuals. Repeated measure MANOVAs were used to test for significant pre-posttest differences.

\subsection{Stress, anxiety, and ADHD symptoms}

From the YSR, the variables selected for analysis were Anxious/Depressed, Withdrawn/ Depressed, Affective Problems, Anxiety Problems, Attention Problems, ADHD Problems, and Total Problems (Table 1). Added to these variables was the single result of the RCMAS. The analysis yielded significant improvement of symptoms from pretest to post test $\mathrm{F}(1,9)=4.7, \mathrm{p}=.0285$.

The same measures analyzed from the YSR were selected for analysis of the teacher reported CBCL. Teacher results corroborated student reported significant reductions in symptoms, $\mathrm{F}(1,9)=6.5, \mathrm{p}=.0155$.

\begin{tabular}{|c|c|c|c|c|c|c|c|c|c|c|}
\hline \multicolumn{6}{|c|}{ Student } & \multicolumn{5}{|c|}{ Teacher } \\
\hline & \multicolumn{2}{|c|}{ Pretest } & \multicolumn{2}{|c|}{ Post Test } & \multirow[b]{2}{*}{ ES } & \multicolumn{2}{|c|}{ Pretest } & \multicolumn{2}{|c|}{ Post Test } & \multirow[b]{2}{*}{ ES } \\
\hline Symptom & Mean & SD & Mean & SD & & Mean & SD & Mean & SD & \\
\hline $\begin{array}{l}\text { Anxious/ } \\
\text { Depressed }\end{array}$ & 5.7 & 3.6 & $2.7^{*}$ & 3.7 & .8 & 10.2 & 6.4 & $5.6^{*}$ & 3.6 & .7 \\
\hline $\begin{array}{l}\text { Withdrawn/ } \\
\text { Depressed }\end{array}$ & 4.8 & 2.7 & $3.9^{* *}$ & 3.0 & .3 & 4.3 & 3.8 & 3.6 & 4.0 & .2 \\
\hline $\begin{array}{l}\text { Affective } \\
\text { Problems }\end{array}$ & 5.8 & 3.4 & $4.1^{*}$ & 2.5 & .5 & 5.0 & 3.7 & 3.6 & 3.6 & .4 \\
\hline $\begin{array}{l}\text { Anxiety } \\
\text { Problems }\end{array}$ & 3.2 & 2.0 & $1.6^{* *}$ & 1.9 & .8 & 3.7 & 3.5 & 2.1 & 2.4 & .5 \\
\hline $\begin{array}{l}\text { Attention } \\
\text { Problems }\end{array}$ & 7.0 & 4.0 & $5.2^{*}$ & 3.5 & .5 & 23.6 & 10.5 & 22 & 11.0 & .2 \\
\hline $\begin{array}{l}\text { ADHD } \\
\text { Problems }\end{array}$ & 5.4 & 2.9 & 4.3 & 2.7 & .4 & 12.4 & 5.7 & 11.7 & 5.7 & .3 \\
\hline $\begin{array}{l}\text { Total } \\
\text { Problems }\end{array}$ & 52.5 & 25.6 & $40^{*}$ & 27.4 & .5 & 63.1 & 24.8 & $56^{*}$ & 31.2 & .3 \\
\hline
\end{tabular}

${ }^{*} \mathrm{p} \leq .05 .{ }^{* *} \mathrm{p}<.005 \mathrm{ES}=$ Effect Size

Table 1. Achenbach Youth Self Report, Stress, Anxiety, and ADHD Symptoms

\subsection{Executive function}

The BRIEF includes 3 composite measures: Behavioral Regulation Index, Metacognition Index, and General Executive Composite. The Global Executive Composite is composed of 
the Behavioral Regulation Index and the Metacognition Index. A repeated measures MANOVA of the General Executive Composite indicated significant improvement in executive function from pretest to post test, $F(1,9)=5.5, p=.022$. Repeated measures MANOVA on the scales comprising the two indices also were statistically significant: Behavioral Regulation Index (Inhibit, Shift, and Emotional Control), F(1,9) = 23.7, p <.00001; and Metcognition Index (Initiate, Working Memory, Planning, Organize Material, and Monitoring), $\mathrm{F}(1,9)=13.7, \mathrm{p}=.0025$. Table 2 presents the pre- and post test means and standard deviations for the individual scales associated with each index.

The Tower of London was omitted from analysis because the assessment has been found to be less consistent than other measures in distinguishing specific effects associated with ADHD from those related to learning disorders (Sergeant, Geurts, \& Oosterlaan, 2002).

\begin{tabular}{|l|c|c|c|c|}
\hline & \multicolumn{2}{|c|}{ Pre-Test } & \multicolumn{2}{c|}{ Post Test } \\
\hline \multicolumn{1}{|c|}{ Scale } & Mean & SD & Mean & SD \\
\hline $\begin{array}{l}\text { Behavioral Regulation } \\
\text { Index (BRI) }\end{array}$ & 54.1 & 16.9 & $46.4^{*}$ & 13.2 \\
\hline Inhibit & 19.0 & 6.9 & $17.1^{*}$ & 6.5 \\
\hline Shift & 18.5 & 5.3 & 15.5 & 3.9 \\
\hline Emotional Control & 16.6 & 6.7 & $13.8^{*}$ & 4.3 \\
\hline Metacognition Index (MI) & 94.8 & 32.1 & 85.3 & 23.2 \\
\hline Initiate & 15.1 & 3.7 & $13.2^{*}$ & 3.2 \\
\hline Working Memory & 22.8 & 4.2 & $20.1^{*}$ & 5.3 \\
\hline Plan/Organize & 21.8 & 4.4 & 20.6 & 5.5 \\
\hline Organization of Materials & 14.6 & 4.9 & 13.2 & 5.0 \\
\hline Monitor & 20.4 & 5.9 & $18.2^{*}$ & 4.5 \\
\hline $\begin{array}{l}\text { General Executive } \\
\text { Composite (GEC) }\end{array}$ & 151.6 & 37.7 & $131.7^{*}$ & 33.1 \\
\hline
\end{tabular}

* $\mathrm{p} \leq .05$

Table 2. Teacher Behavior Rating Inventory of Executive Function (BRIEF)

The performance measures analyzed were the CAS and the D-KEFS (Table 3). The CAS scores of Expressive Attention and Accuracy Ratio were combined with the D-KEFS scores for Category Fluency and Category Switching. A repeated measures MANOVA yielded a significant improvement in scores on these performance measures from pre to posttest, $\mathrm{F}(1,9)=5.0, \mathrm{p}=.026$.

\begin{tabular}{lccccc}
\hline \multirow{2}{*}{ Measure } & \multicolumn{2}{c}{ Pre-Test } & & Post Test \\
\cline { 2 - 3 } & Mean & SD & Mean & SD \\
\hline CAS Expressive Attention & 36.10 & 10.63 & & $44.70^{*}$ & 13.59 \\
CAS Accuracy & 37.70 & 2.21 & $39.40^{*}$ & \\
D-KEFS Category Fluency & 25.10 & 7.83 & 28.70 & 7.61 \\
D-KEFS Category Switching & 8.30 & 3.19 & 9.60 & 2.63 \\
\hline
\end{tabular}

${ }^{*} \mathrm{p} \leq .05$.

Table 3. Performance Measures 


\section{Randomized controlled trial of brain function}

To further understand the effects of the Transcendental Meditation technique on children with ADHD, a second study explored improvements in brain coherence and brain development. The purpose was to provide insight into the underlying mechanisms of observed changes. A randomized controlled trial examined the EEG coherence, theta/beta ratio, and executive function.

EEG studies report decreased activation in ADHD populations in parietal areas of the brain that weave sensory input into concrete perception (Silk et al., 2005), higher density and amplitude of theta activity (di Michele, Prichep, John, \& Chabot, 2005; Janzen, Graap, Stephanson, Marshall, \& Fitzsimmons, 1995), and lower density and amplitude of alpha and beta activity (Barry, Clarke, \& Johnstone, 2003). Theta/beta power ratios are highly correlated with severity of ADHD symptoms (Monastra, Lubar, \& Linden, 2001; Monastra et al., 1999).

During memory tasks, theta EEG is generated in the hippocampus and is thought to block out irrelevant stimuli during memory processing. In ADHD subjects, greater theta activity may block out relevant as well as irrelevant information.

Another brain marker of ADHD is EEG coherence, a measure that reflects the number and strength of connections between different brain areas (Thatcher, Walker, \& Giudice, 1987). In children diagnosed with ADHD, coherence in all frequencies is reported lower (Barry, Clarke, McCarthy, \& Selikowitz, 2007, 2009). Alpha coherence is thought to play an important role in attention and consciousness.

\subsection{Subjects}

As in the previous study, the subjects were students at an independent school for children with language-based learning differences. Eighteen students were stratified by age, and randomly assigned to learn TM immediately (TM Group: 6 boys, 3 girls, average age $12.9 \pm$ 1.3) or learn TM in three months (Delayed-Start Group: 7 boys, 2 girls, average age $13.0 \pm$ 1.6). Co-morbidities included General Anxiety Disorder (3 subjects), Obsessive Compulsive Disorder (1 subject), and Autism (3 subjects). In each group, five of the nine subjects were on ADHD medication.

\subsection{Measures}

\subsubsection{Executive function}

The Delis-Kaplan Executive Function System (D-KEFS) Verbal Fluency and the Tower of London were used to replicate the previous study. Inventories included the BRIEF, the Achenbach Child Behavior Checklist, and the Spielberger State Trait Anxiety Scale.

EEG was recorded during a computer-administered paired choice reaction-time task to calculate theta/beta ratios and patterns of EEG coherence. The task began with a display on a computer screen of a one- or two-digit number, followed by a blank screen, then another one- or two-digit number. Subjects were asked to press a left- or right-hand button to indicate which number was larger in value. 


\subsection{Results}

The primary analysis tested differences from baseline to the 3-month posttest. The Transcendental Meditation group practiced the TM technique for three months along with the curriculum designed for children with language-based learning disabilities; the delayedstart comparison group received only the curriculum at the school. In this analysis two repeated measures MANOVAs were conducted-psychological and performance variables in one, and coherence in the other-and an ANCOVA of theta/beta ratio differences, covarying for pretest scores.

A secondary analysis of the delayed-start group compared differences from baseline to the 3-month posttest, when these subjects were not yet meditating, to differences from the 3month to the 6-month posttest, when these subjects were meditating.

\subsubsection{Theta/beta ratios}

The ANCOVA of theta/beta differences, covarying for pretest scores yielded significant decreases in theta/beta ratios in the $\mathrm{TM}$ group $(\mathrm{F}(1,17)=4.7, \mathrm{p}=.05)$. The average theta/beta ratio seen in normal populations is 3 . At pretest, both groups were well above average $(\mathrm{TM}=9$, delayed-start=10.2). At the 3-month post test, theta/beta ratios increased in the delayed-start (11.9) group, while the TM subjects moved closer to normal values (7.6). At the 6-month post test, after both groups were practicing the TM technique, theta/beta ratios decreased in both groups (TM=4.9, delayed-start=7.6).

For the delayed start group, theta/beta ratios also significantly decreased from the 3-month to 6-month posttest $(-4$. 3) compared to baseline to 3-month posttest $(1.3)(\mathrm{F}(1,8)=5.1, \mathrm{p}=$ $.053)$, representing the results of three months practice of the technique.

\subsubsection{Coherence maps}

Coherence maps during pretest, 3-month, and 6-month posttests were averaged within each group and subtracted: coherence at 3-month posttest minus coherence during pretest for the delayed-start and TM group who had been meditating for 3-months; and 6-month posttest minus 3-month posttest in the delayed-start group after they had been meditating for 3months. These coherence difference maps present coherence in theta (5.0-7.5 Hz), alpha (8.0$12 \mathrm{~Hz})$, beta1 $(13-20 \mathrm{~Hz})$, and gamma bands $(20.5-50 \mathrm{~Hz})$.

Coherence maps (Figure 1) show few sensors with higher coherence in the delayed-start group at the 3-month posttest compared to their pretest values (top row). In contrast, there were many frontal and parietal areas with higher coherence in the TM group at 3-month posttest compared to pretest values (middle row); and there were many frontal and parietal areas with higher coherence in the delayed-start group at the 6-month posttest compared to the 3-month posttest values (bottom row).

\subsubsection{Executive function}

The Delis-Kaplan Executive Function System (D-KEFS) Verbal Fluency and the Tower of London were used to replicate the previous study. Inventories included the BRIEF, the Achenbach Child Behavior Checklist, and the Spielberger State Trait Anxiety Scale. However not enough inventories were returned to provide sufficient complete data for analysis. 
Control Group: Coherence during 3-mon Posttest - Coherence during Pretest
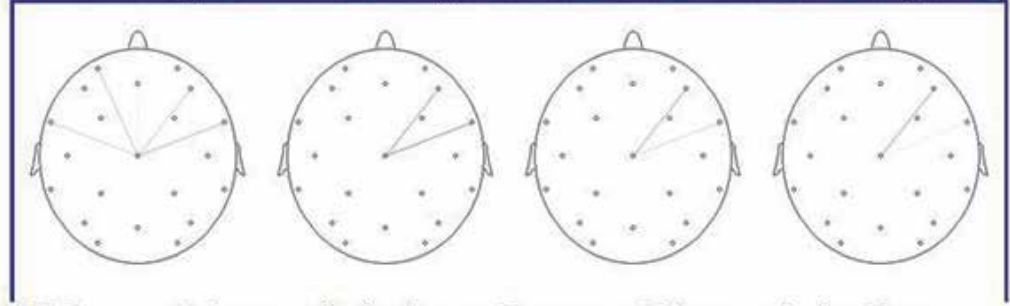

TM Group: Coherence during 3-mon Posttest - Coherence during Pretest

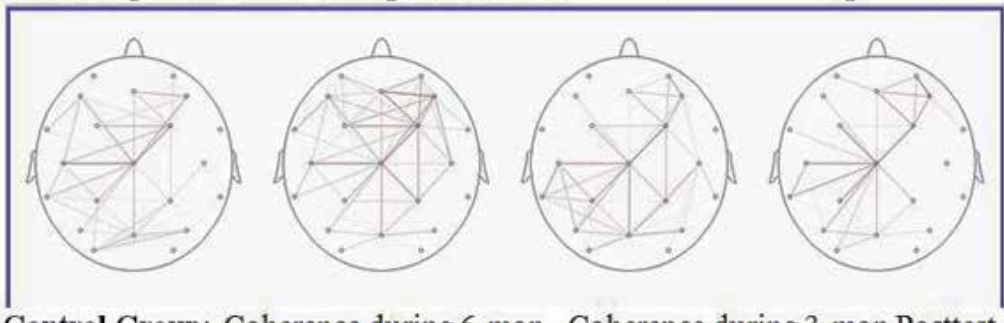

Control Group: Coherence during 6-mon-Coherence during 3-mon Posttest

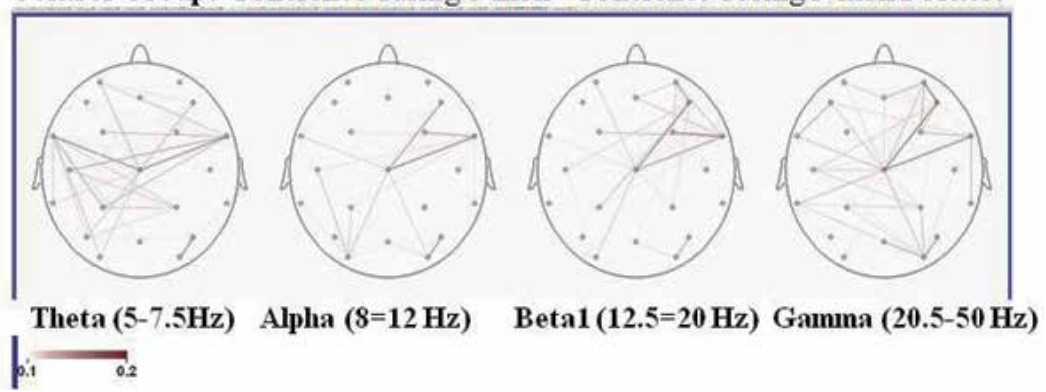

Three coherence-difference maps are presented in this figure. In the top row is coherence during the 3month posttest minus baseline coherence for the delayed-start subjects. The middle row is coherence during the 3-month posttest minus baseline coherence for the TM subjects. The bottom row is coherence during the 6-month posttest minus 3-month posttest for the delayed-start subjects, who had been meditating over this time.

Fig. 1. Coherence maps

\subsubsection{Tower of London}

There were no significant differences between groups from pretest to post test for the Tower of London. There appears to have been significant learning effects in subjects in both groups on this test. As mentioned above, The Tower of London has been found to be less consistent than other measures in distinguishing specific effects associated with ADHD from those related to learning disorders. Consequently this may not be a useful measurement for a population that also has established learning disorders, as was the case in these studies.

\subsubsection{D-KEFS verbal fluency}

There were significant increases from pretest to 3-month posttest in Letter Fluency for the TM group $(F(1,15)=7.7, p=.017)$, and no significant group differences on other components of the Verbal fluency test. 
There were also significant increases in D-KEFS in the delayed-start group after they learned TM compared to the time from baseline to the 3-month posttest $(F(1,8)=7.8, p=0.024)$.

\section{Discussion}

Stress and anxiety can overwhelm and lessen the effectiveness of the individual. Anxiousness contributes to poor impulse control, inability to sustain attention, impaired working memory, and lack of cognitive flexibility. This raises the question as to whether, in some cases, it is the symptoms of stress and anxiety that are being diagnosed as ADHD. Whether these problems are a result of ADHD or are the cause of ADHD-like symptoms, these studies suggest that reducing stress and anxiety by the Transcendental Meditation technique can potentially reduce ADHD symptoms and improve executive function.

Compared to other meditation techniques, the Transcendental Meditation technique may be particularly suited to children with ADHD. It is easy to learn and is learned in less than an hour. The technique does not involve controlling the mind; and once learned requires no other time investment than the 10-15 minute sessions twice a day. Yet changes in the brain are seen within a short time.

The Transcendental Meditation technique can be done anywhere one can sit and close the eyes. Therefore it is portable, and a personal means of self-regulation for ADHD children. To maximize the benefits, the recommended routine is one meditation session in the morning and one in the late afternoon, every day. Incorporating the TM program in a school setting, as was used for these studies, provides an excellent means of maintaining regularity, benefiting not only the students but also the school. Teachers' anecdotal comments revealed that teachers were able to teacher more; and the students were able to learn more because they were less stressed and anxious.

With recent research showing reduced ability to cope with stress and slower brain development in ADHD children, a technique that can positively affect both of these factors can be a valuable resource for treatment of ADHD. The technique does not conflict with other cognitive or behavioral approaches, or with medication. With less stress and anxiety, the child may be more responsive to other interventions, potentially increasing their effectiveness.

Both studies were small, therefore we cannot infer strong cause and effect relations between TM practice and reduction in ADHD symptoms. However, the first study included a number of objective performance tests of attention and executive function to support selfreport inventories that are commonly used to diagnosis ADHD. This allowed for withinstudy corroboration of results. The second study provided a strong study design of a randomized controlled trial.

Both the psychosocial improvements and improved brain function seen in these studies are consistent with previous research on the Transcendental Meditation technique. Psychosocial research shows reduced anxiety, increased emotional regulation, and improved social behavior in non-ADHD students. Studies of the effect of the TM technique on executive function show increased frontal brain coherence, associated with more effective executive functioning, improved attention, greater emotional stability, and decreased anxiety. 
These results suggest that further research is warranted to assess the impact of Transcendental Meditation practice as a potential treatment option for ADHD, either alone or as adjunct treatment. It would be valuable to replicate the results with larger groups, and further study improvements in brain coherence and brain development to gain insight into the underlying mechanisms of observed changes.

\section{Conclusions}

The initial pilot study represents the first systematic study of the effects of the Transcendental Meditation technique on the symptomatology of ADHD. The results indicate that Transcendental Meditation practice 1) can be learned and successfully practiced by children with ADHD, 2) has the potential to reduce stress, anxiety, and stress related ADHD symptoms within three months, and 3) can result in improvements in executive function.

In the random assignment study, three months practice of the TM technique resulted in 1) significant decreases in theta/beta ratios, 2) significant increases in theta coherence, and trends for increases in alpha and beta coherence during tasks, and 3) significant increases in Letter Fluency. The single-group within analysis yielded similar decreases in theta/beta ratios and increases in Letter Fluency after the delayed-start group learned TM.

\section{Acknowledgements}

The authors would like to thank their co-investigator William Stixrud, Ph.D., Department of Psychiatry, George Washington University School of Medicine and Health Sciences.

\section{References}

Alexander, C.N., Rainforth, M.V., Gelderloos, P. (1991), Transcendental Meditation, selfactualization, and psychological health: a conceptual overview and statistical metaanalysis, Journal of Social Behavior and Personality, 6(5), 189-247.

Barnes, V. A., Bauza, L. B., \& Trieber. F, A. (2003). Impact of stress reduction on negative school behavior in adolescents. Health Quality of Life Outcomes, 1(10). Retrieved from http://www.hqlo.com/content/1/1/10.

Barry, R. J., Clarke, A. R., \& Johnstone, S. J. (2003). A review of electrophysiology in attention-deficit/hyperactivity disorder: I. Qualitative and quantitative electroencephalography. Clinical Neurophysiology, 114(2), 171-183.

Barry, R. J., Clarke, A. R., McCarthy, R., \& Selikowitz, M. (2007). EEG coherence in children with attention-deficit/hyperactivity disorder and comorbid oppositional defiant disorder. Clinical Neurophysiology, 118(2), 356-362.

Blair C., Grange G. R., \& Razza, R. P. (2005). Cortisol reactivity is positively related to executive function in preschool children attending head start child development. Child Development, 76(3), 554-567.

Bush, G., Valera, E. M., \& Seidman, L. J. (2005). Functional neuroimaging of attentiondeficit/hyperactivity disorder: a review and suggested future directions. Biological Psychiatry, 7(11), 1273-1284. 
Centers for Disease Control and Prevention. (2008, July). Diagnosed Attention Deficit Hyperactivity Disorder and Learning Disability: United States, 2004-2006. Vital and Health Statistics , 10 (237).

Centers for Disease Control and Prevention. (2005). Morbidity and Mortality Weekly Report, 54, 842-847.

Dillbeck, M.C., \& Araas-Vesely, S. (1986). Participation in the Transcendental Meditation program and frontal EEG coherence during concept learning. International Journal of Neuroscience, 29, 45-55.

Dillbeck, M. C., \& Orme-Johnson, D. W. (1987). Physiological differences between Transcendental Meditation and rest. American Psychologist, 42, 879-881.

di Michele, F., Prichep, L., John, E. R., \& Chabot, R. J. (2005). The neurophysiology of attention-deficit/hyperactivity disorder. International Journal of Psychophysiology, 58(1), 81-93.

Durston S., Hulshoff Pol H. E., Schnack, H. G., Buitelaar, J. K., Steenhuis, M, P., Minderaa, R. B., et. al. (2004). Magnetic resonance imaging of boys with attentiondeficit/hyperactivity disorder and their unaffected siblings. Journal of the American Academy of Child and Adolescent Psychiatry, 43(3), 332-340.

Fergusson, L. C. (1993). Field independence, Transcendental Meditation and achievement in college art: a reexamination. Perceptual and Motor Skills, 77, 1104-1106.

Gaylord, C., Orme-Johnson, D., \& Travis, F. (1989). The effects of the transcendental meditation technique and progressive muscle relaxation on EEG coherence, stress reactivity, and mental health in black adults. International Journal of Neuroscience, 46(1-2), 77-86.

Harrison, L. J., Ramesh, M., \& Rubia, K. (2004). Sahaja yoga meditation as a family treatment programme for children with attention deficit-hyperactivity disorder. Clinical Child Psychology and Psychiatry, 9(4), 479-497.

Janzen, T., Graap, K., Stephanson, S., Marshall, W., \& Fitzsimmons, G. (1995). Differences in baseline EEG measures for ADD and normally achieving preadolescent males. Biofeedback Self Regul, 20(1), 65-82.

Jensen, P. S., Arnold, E., Swanson, J. M., Vitiello, B., Abikoff, H. B., Greenhill, L. L., et al. (2007). 3-Year Follow-up of the NIMH MTA Study. Journal of the American Academy of Child and Adolescent Psychiatry , 46 (8), 989-1002.

Jevning, R., Wallace, R., \& Beidebach, M. (1992). The physiology of meditation: a review. A wakeful hypometabolic integrated response. Neuroscience and Biobehavioral Reviews, $16,415-424$.

Jevning, R., Wilson, A. F., \& Davidson, J. M. (1978). Adrenocortical activity during meditation. Hormones and Behavior, 40, 603-606.

Linnet, K. M., Wisborg, K., Agerbo, E., Secher, N. J., Thomsen, P. H., \& Henriksen, T. B. (2006). Gestational age, birth weight, and the risk of hyperkinetic disorder. Archives of Disease in Childhood, 91, 655-660.

Lupien, S. J., Fiocco, A., Wan, N., Maheu, F., Lord, C., Schramek, T., et al. (2005). Stress hormones and human memory function across the lifespan. Psychoneuroendocrinology, $30(3), 225-242$.

MacLean, C. R. K., Walton, K. G., Wennenberg, S. R., Levitsky, D. K., Mandarino, J. P., Waziria, R., et al. (1997). Effects of the Transcendental Meditation program on 
adaptive mechanisms: changes in hormone levels and responses to stress after 4 months of practice. Psychoneuroendocrinology, 22(4), 277-295

McEwen, B. S. (1998). Protective and damaging effects of stress mediators. New England Journal of Medicine, 338(3), 171-179.

Molina, B. S., Hinshaw, S. P., Swanson, J. M., Arnold, E. L., Epstein, J. N., Hoza, B., et al. (2009). The MTA at 8 years: prospective follow-up of children treated for combined-type ADHD in a mulitsite study. Journal of the American Academy of Child and Adolescent Psyciatry, 48 (5), 484-500.

Molina, B., Flory, K., Hinshaw, S., Greiner, A., Arnold, L., \& Swanson, J. (2007). Delinquent behavior and emerging substance use in the MTA at 36 months: Prevalence, course, and treatment effects. Journal of the American Academy of Child and Adolescent Psychiatry , 46 (8), 1028-1040.

Monastra, V. J., Lubar, J. F., \& Linden, M. (2001). The development of a quantitative electroencephalographic scanning process for attention deficit-hyperactivity disorder: reliability and validity studies. Neuropsychology, 15(1), 136-144.

Monastra, V. J., Lubar, J. F., Linden, M., VanDeusen, P., Green, G., Wing, W., et al. (1999). Assessing attention deficit hyperactivity disorder via quantitative electroencephalography: an initial validation study. Neuropsychology, 13(3), 424-433.

MTA Cooperative Group. (1999). A 14-month randomized clinical trial of treatment strategies for attention-deficit/hyperactivity disorder. The MTA Coorperative Group. Modality Treatment Study of Children with ADHD. Archives of General Psychiatry , 56 (12), 1073-86.

Orme-Johnson, D.W. (1973). Autonomic stability and Transcendental Meditation. Psychosomatic Medicine, 35, 341-349.

Orme-Johnson D., \& Walton, K. G. (1998). All approaches to preventing or reversing effects of stress are not the same. American Journal of Health Promotion, 12(5), 297-299.

Rosaen, C, \&. Benn, R. (2006). The experience of Transcendental Meditation in middle school students: a qualitative report. Explore, 2(5), 422-425.

Rutschman, J. R. (2004). Effects of techniques of receptive meditation and relaxation on attentional processing. Canadian Undergraduate Journal of Cognitive Science, 6-16.

Sergeant, J. A., Geurts, H., \& Oosterlaan, J. (2002). How specific is a deficit of executive function for attention deficit/hyperactivity disorder? Behavioral Brain Research 130, 3-28.

Shapiro, D. H. (1982). Clinical and physiological comparison of meditation and other selfcontrol strategies. American Journal of Psychiatry, 139, 267-274.

Shear, J. (2006). The Experience of Meditation: Experts Introduce the Major Traditions. Paragon House, St. Paul, MN.

Silk, T., Vance, A., Rinehart, N., Egan, G., O'Boyle, M., Bradshaw, J. L., et al. (2005). Frontoparietal activation in attention-deficit hyperactivity disorder, combined type: functional magnetic resonance imaging study. British Journal of Psychiatry, 187, 282283.

So, K. T., \& Orme-Johnson, D.W. (2001). Three randomized experiments on the longitudinal effects of the Transcendental Meditation technique on cognition. Intelligence, 29, 419-440.

Thatcher, R. W., Walker, R. A., \& Giudice, S. (1987). Human cerebral hemispheres develop at different rates and ages. Science, 236(4805), 1110-1113. 
Travis, F., \& Shear, J. (2010). Focused attention, open monitoring and automatic selftranscending:Categories to organize meditations from Vedic, Buddhist and Chinese. Consciousness and Cognition. In press. Retrieved from doi:10.1016/j.concog.2010.01.007

Travis, F. T., \& DuBois, D. (2004). Psychological and physiological charactieristics of a proposed object-referral/self-referral continuum of self-awareness. Consciousness and Cognition , 12 (2), 401-420.

Travis, F. (2001). Transcendental Meditation Technique. In E. W. Craighead \& C. B. Nemeroff (Eds.), Corsini Encyclopedia of Psychology and Neuroscience (pp. 17051706). New York: John Wiley and Sons.

Tavis, F. T., \& Pearson, C. (2000). Pure consciousness: distinct phenomenological and physiological correlates of "consciousness itself". International Journal of Neuroscience, 100 (1-4), 77-89.

Travis, F., \& Wallace, R. K. (1999). Autonomic and EEG patterns during eyes-closed rest and Transcendental Meditation (TM) practice: A basis for a neural model of TM practice. Consciousness and Cognition, 8, 302-318.

Vance, A., Silk, T. J., Casey, M., Rinehart, N. J., Bradshaw, J. L., Bellgrove, M. A., et al. (2007). Right parietal dysfunction in childen with atention deficit hyperactivity disorder, combined type: Afunctional MRI study. Molecular Psychiatry , 12 (9), 826-832.

Wallace, R. K. (1970). Physiological effects of Transcendental Meditation. Science, 167, 17511754.

Walton, K. G., Pugh, B. S., Gelderloos, P., \& MacRae, P. (1995). Stress reduction and preventing hypertension: Preliminary support for a psychoneuroendocrine mechanism. The Journal of Alternative and Complementary Medicine, 1(3), 263-283.

Zametkin, A. J., \& Ernst, M. (1999). Problems in the management of attention-deficithyperactivity disorder. New England Journal of Medicine, 7, 340(1), 40-46.

Zametkin, A.J., Liebenauer, L. L., Fitzgerald, G. A., King, A. C., Minkunas, D. V., Herscovitch, P., et. al.(1993). Brain metabolism in teenagers with attention-deficit hyperactivity disorder. Archives of General Psychiatry, 50(5), 333-340. 


\title{
The Value of Coached Behaviour Modification in the Effective Management of Attention Deficit Hyperactivity Disorder (ADHD)
}

\author{
Tilla Olivier* and Ana Gomes \\ Nelson Mandela Metropolitan University, \\ South Africa
}

\section{Introduction}

Attention Deficit Hyperactivity Disorder (also referred to as ADHD) depicts a very common, chronic, neurobiological, medical, brain-based, behavioural disorder (Olivier, Gomes \& Greyling, 2009:237), with psychiatric co-morbidities, such as depression, anxiety, substance abuse and behavioural disorders (Gooding, 2007a:40), often continuing through childhood and adolescence, into adulthood (Sherman, Rasmussen \& Baydala, 2008:347; Weyandt \& DuPaul, 2008:311; Young \& Amarasinghe, 2009:116).

It is thought to be caused by an imbalance of two neurotransmitters to the brain: dopamine and serotonin (Gooding, 2007a:40). According to Parker \& Boutelle (2009:204), ADHD is "now understood as pervasive impairment in the self-regulation of behaviour". The name ADHD is frequently employed as a marker, which Graham (2008a:85) refers to as a "lexical label", for people who face significant difficulties, such as lack of concentration, impulsiveness, extreme levels of activity and lack of control (Barkley, 2005:36).

ADHD prevails in every country and ethnic group (Barkley, 2005:91). Occurrence in South Africa corresponds with that in the USA and Europe, where it is estimated that up to $12 \%$ of school-age children (Sandler, Glesne \& Gellert, 2008:111; Webb, Amend, Webb, Goerss, Beljan, \& Olenchek, 2005:42), up to 8\% of college students (Weyandt \& DuPaul, 2008:312), and up to 5\% of adults (Plumer \& Stoner, 2005:290; Swartz, Prevatt \& Proctor, 2005:647) suffer from ADHD.

In the latter group it manifests in "inattention, impulsivity, disorganisation and a lack of self-regulation" (Swartz, et al., 2005:647). The number of new cases reported each day, known as the incidence of ADHD, also seems to be on the increase, but this may be due to more media attention of late (Gomes, 2008:39). However, Gooding (2007b:37) claims that currently " $80 \%$ of adults with ADHD are undiagnosed and untreated".

ADHD has impairing consequences for the everyday psychological, academic and social functioning (Weyandt \& DuPaul, 2008:312), as well as the behaviour of the individual; these, in turn, further influence the individual's growing experiences (Sherman, et al., 2008: 347; 
Plumer \& Stoner, 2005:290; Webb, et al., 2005:36). The symptoms of ADHD are thought to be aggravated by the individual's experiences, neurological and nutritional factors and environmental variables (Sonna, 2005:36).

The problems experienced include cognitive, emotional, social, behavioural and motivational deficits (Rief, 2005:4; Steenkamp, 2001:72; Swartz, et al., 2005: 647). These problems result in learning disabilities, self-regulation problems, external locus of control, perception, speech and sleep problems, disorganisation, lack of time-management skills, the inability to prioritise tasks and poor stress-management skills (Barkley, 2005:2005; Gooding, 2007b:37; Olivier, et al., 2009:238) The manifesting behaviour problems are related to the individual's inability to maintain the level of attention necessary to internalise information; a higher level of impatience; the inability to control movement and activity; and boredom.

According to Swartz, et al. (2005:647), adult ADHD-sufferers are prone to "academic underachievement, drug abuse, poor occupational adjustment, anti-social behaviour, relationship difficulties, mood and affective disorders, and personality disorders". This is confirmed by Weyandt and DuPaul (2008:312), who maintain that the "...risk for lower achievement scores, poor academic coping skills in general, but especially in writing", are the prevalent characteristics of ADHD-diagnosed college students. They also find it difficult to adjust to college life and to form social relationships.

In order to counteract the dilemmas faced by ADHD, the presence of ADHD needs to be established through proper diagnosis. The American Psychiatric Association's DSM-IV RT (2000) differentiates between the following three sub-types: ADHD-I: the predominantly inattentive type; ADHD-HI: the predominantly hyperactive-impulsive type; and ADHD-C: the combined type. They also provide clear indicators of further symptoms for diagnosis.

These are clustered around the three cores of: poor sustained attention, poor impulse control and apathy (Gomes, 2008:27). The diagnosis must be done professionally by an expert and is not a simple matter. It is believed that in some cases parents contribute to the diagnosis - in order to alleviate their own responsibility and blame for the child's behaviour, and to gain access to school support services (Graham, 2008b:7).

Numerous definitions of ADHD in the literature also refer to the specific syndrome characteristics of inattention, hyper-responsiveness, impulsivity, poor inhibition and tediousness (Rief, 2005: 4). Furthermore, the literature mentions challenges such as emotional outbursts, enthusiasm shortages, low self-esteem, and difficulty with problemsolving (Aberson, Shure \& Goldstein, 2007: 291; Olivier, et al., 2009: 237).

However, in this chapter we argue that a definition that focuses on the child's differences as strengths, rather than as deficiencies, indicates that ADHD is the result of high responsiveness and strong alertness. In turn, the individual's creativity and expression are enhanced, which may be further complemented by increased motor movement, intensified hearing capacity, tactile sensitivity, above-average peripheral vision and vulnerable emotional balance (Gomes, 2008:31).

The diagnosis can also shed light on the specific strengths of the individual to direct and reinforce during the management of ADHD. Seen from such a viewpoint, ADHD then becomes more manageable than when it is perceived as a deficiency (Webb, et al., 2005:100). This is precisely what will determine and direct the management approach. 
The following pertinent question, therefore, becomes relevant:

How can ADHD-related behaviour be effectively managed?

\section{Management of ADHD}

Against the background discussed above, it becomes apparent that the individual diagnosed with ADHD experiences major challenges, which make it difficult to manage the individual's behaviour (Aberson, Shure \& Goldstein, 2007; Barkley, 2005; Rief, 2005; Olivier, et al., 2009: 237). The individual's regular unacceptable and/or inappropriate conduct often results in further aggravated predicaments for which solutions must constantly be found (Johnston \& Mash, 2001; Khamis, 2006).

ADHD is a continuing, chronic disorder, which is not curable; but it is treatable and manageable - through the right intervention and management approach (Jacklyn \& Ravichandran, 2009:1057; Gooding, 2007a:40). Many different strategies for intervention and approaches to the treatment of ADHD exist. When referring to the management of ADHD, this implies, "not only finding the best possible method of intervention, but also the implementation and monitoring thereof" (Gomes, 2008:55; Rief, 2005: 29).

Programmes for managing ADHD should be flexible, to allow for frequent adjustment to the individual's specific needs. A programme's purpose and effectiveness should also be assessed and improved continuously, in order to ensure that it remains effective and relevant (Gomes, 2004:126).

No single approach exists that can solve the difficulties of ADHD (Heriot, Evans \& Foster, 2007:121). According to Graham (2008b:17), “ADHD comes to be understood through a biopsycho-social theoretical framework". The management of ADHD should therefore be multimodal in nature (Graham, 2008b: 20; Sherman, et al., 2008:348; Gooding, 2007a:40; Heriot, et al., 2007:121), because a combination of approaches seems to be more effective with the biological, as well as the social-emotional aspects of ADHD. It includes the following:

\subsection{Physical assessment}

Before any management of the disorder can commence, the individual has to undergo a thorough physical check-up by a clinician, to ascertain the individual's general health condition in comparison with the norm, and to further ensure that the symptoms being experienced are not caused by other illnesses or deficiencies (Young \& Amarasinghe, 2009:116).

\subsection{Medical/pharmacological approaches}

It is usually believed that ADHD can best be managed by means of biochemical treatment, depending on the needs, mass and age of the individual involved (Hallowell \& Ratey, 1995:238). This is referred to as the "first-line approach" (Graham, 2008a:92; Graham, 2008b:19). Medication is consequently often prescribed to improve attention and to reduce hyperactivity. However, medication treatment is usually more effective when coupled with coached behaviour modification. 
Based on the "medical model" (Graham 2008a: 85; Graham, 2008b:19) and because it is believed that the medication will "increase the level of dopamine and norepinephrine between the synapses or neurotransmitters" of the brain, prescriptions for stimulants, such as Ritalin and Concerta, have increased over time (Graham, 2008a:86.87). Criticism against the medical model is that it appears to "accept the 'disordered' children as having little or no control over their actions" (Graham, 2008b: 21).

Many voices of concern are springing up against the use of medication (Lakoff, 2000: 150; Sandler \& Bodfish, 2008:105; Weyandt \& DuPaul, 2008:316) and the overuse of stimulant therapy (Sandler et al., 2008:111), as it is not guaranteed to benefit all cases in a similar or a positive way (Plumer \& Stoner, 2005:290). In this regard, (2008a:90) refers to the "(dis)illusion" and the "paradoxical effect" of medicines.

The long-term positive effects of medication have not been indisputably proven (Heriot, et al., 2007:121; Sandler, et al., 2008:111; Young \& Amarasinghe, 2010:127), and are not necessarily long-lasting, as they cease without the medication, often resulting in more agitation and anxiety (Gomes, 2008:94). Furthermore, in the case of the abuse of medicine, addiction becomes a danger (Cowan, 2002). These stimulants also have reported nominal treatment-emergent side-effects (Sandler, et al., 2008:111), such as sleep disturbance, appetite suppression and weight changes, emotional instability, growth retardation and headaches (Barkley, 2005:269; Graham, 2008:90).

Yet, despite all the concern, the consumption of medicine for ADHD-related symptoms is still increasing (Graham, 2008a: 86). Some physicians suggest that the medication should not be used continuously, in order for the individual to learn to manage ADHD without it (Mehl-Madrona, 2005: 1). This contributes to the growing use of complementary and alternative approaches in the treatment of ADHD (Sandler, et al., 2008a:111)

Neurotherapy, also known as EEG biofeedback or neurofeedback training, is an attempt to normalise ADHD brainwave responses to stimuli; and it is seen as, "an effective alternative to stimulant medication" (Steenkamp, 2001:95). It teaches the individual to focus on his/her brainwave motions, applying swift computers, which make available both auditory and visual feedback, unfortunately at high cost, because of the expensive equipment used (Sonna, 2005:143).

Dietary intervention is also an attempt to help decrease the symptoms of ADHD, especially the aspect of restlessness (D'Adamo, 2001:133). Furthermore, multivitamins are used to supply what the brains needs for proper functioning (Sonna, 2005:41). Homeopathic medication is also utilized in an attempt to control the symptoms of ADHD, but it takes a long time to show effects, and is therefore less popular (Barkley, 2005: 269). Furthermore, essential fatty acids, such as fish oil, can contribute to the individual's wellbeing (Sonna, 2005:39), while specially coloured eyeglasses can improve the visual perception (Gomes, 2008:104) of the individual diagnosed with ADHD.

It is a well-known fact that medication alone is insufficient in the management of ADHD, and has "failed to provide a solution to the 'problem' it was meant to solve" (Graham, 2008a:92). Each individual is a unique being with unique needs and strengths. That is why a multimodal approach, namely a combination of approaches by a multidisciplinary team of specialists, usually provides the best results (Young \& Amarasinghe, 2009:116). 


\subsection{Remedial and educational measures}

Based on the "within-the child deficit-model" (Graham, 2008a:91), school-going learners diagnosed with ADHD are often assisted in dealing with school-related challenges, such as adjusting to school, or working out homework schedules, through remedial teaching and the teaching of learning and coping strategies (Rief, 2005:57).

Psycho-educational management refers to the emphasis of the learner's learning abilities in relation to his/her overt behaviour (Riding \& Rayner, 2005:100). Classroom accommodations include measures, such as "recorded textbooks" and "note-taking services", as well as support groups and individual counselling (Swartz, et al., 2005:648). The assistance of a remedial specialist, speech therapist and an occupational therapist can be valuable with regard to perceptual and co-ordination difficulties (Rief, 2005:233; Sonna, 2005:15), again confirming the significance of a multidisciplinary team and a multimodal approach for the management of ADHD (Heriot, et al., 2007:121; Gooding, 2007a:40; Graham, 2008a:92).

\subsection{Psychological management}

The point of departure of the "psychological model" is that the child "can exert or learn selfcontrol" (Graham, 2008b: 21). A professional psychologist usually undertakes a proper assessment to establish the individual's emotional stability and perception. Therapy and counselling are often used in an attempt to alleviate the problems related to the disorder (MacGraw, 2005:15).

This would include family therapy (Hallowell \& Ratey, 1995:136), as well as individual therapy for the diagnosed individual and other individuals involved in the case, such as the parents. Emotional and social support are crucial for the proper understanding of ADHD, good communication, healthy relationships and building the individual's self-esteem, by focusing on positive, rather than negative aspects, as well as uncovering and practising existing strengths (Hallowell \& Ratey, 1995:144; Lawlis, 2004:249; Rief, 2005:84; Sonna, 2005:72).

Cognitive behavioural therapy (Graham, 2008a:92), such as rational-emotive therapy (RET), can be done - in order to manage the symptoms of ADHD. This involves the examination and improving of behaviours and interaction through skills teaching and positive reinforcement (MacGraw, 2005:64; Rief, 2005:61). Cognitive therapy is especially useful for adult sufferers of ADHD, because not all people can learn effective strategies to overcome their challenges (Young \& Amarasinghe, 2009:126). However, the success of cognitive therapy is doubtful when applied by itself, because of its focus on cognitive, rather than educational interventions (Graham, 2008a:93).

After consideration of all the above-mentioned approaches, this chapter strongly argues for behaviour modification to be dominant in the multimodal approach to ADHD, to enable the individual to learn new socially approved behaviours - implemented during therapy and reinforced at home, in the school and in the social environment, as will be discussed in the next section.

\section{Behaviour modification as a way of managing ADHD}

The challenges experienced by the individual diagnosed with ADHD are a reality that must be faced on a daily basis, by all people involved with the individual. Behaviour cannot 
easily be altered, because it can be determined by heredity, or strongly engrained, but should be modified to render it more acceptable, in order to help the individual with ADHD to live a fulfilled life in society, as an accepted human being (Gomes, 2008:98). Consequently, behaviour modification is often applied to amend the unacceptable and/or inappropriate behaviour (Sonna, 2005:153).

\subsection{What is behaviour modification?}

Behaviour modification is a therapeutic intervention practice, involving therapeutic techniques based on the theory of operant conditioning of Skinner and other behaviourlearning theories (Gates, Newell \& Wray, 2001:86). These theories are grounded in the truth that learning brings about change in behaviour, by means of the methodical and conditioned utilization of positive or negative reinforcement.

When a specific stimulus-response pattern is contingently reinforced, the individual is conditioned to respond (Hukamdad, Shahzad, Ali, Quadeer \& Khan, 2011:904). Reinforcement is accomplished by means of anything that will strengthen the desired response, or weaken the undesired response.

This approach entails a technique whereby improper and inappropriate conduct is substituted by a more acceptable alternative (Gomes, 2008:99). The four basic principles of the theory are: "positive reinforcement, negative reinforcement, punishment and extinction" (Hukamdad, et al., 2011:904). Behaviour modification can be distinguished from other techniques by the fact that it focuses only on observable, describable and measurable behaviours (Smith, 2011:1).

Through this approach systematic attempts are made to modify or strengthen the probability of the occurrence of behaviour, by reinforcing desired behaviour, and discouraging or ignoring undesirable behaviour. All attention is considered to be reinforcing, whether positive or negative. However, praise and rewards are more often used than punishment and ignoring - to accomplish the goals of behaviour modification. Punishment refers to an unpleasant stimulus after undesirable behaviour; while a reward refers to a pleasant stimulus upon desirable behaviour.

\subsection{Why is behaviour modification needed?}

Behaviour modification is effectively used to treat many disorders and can be very valuable in managing ADHD (Davies \& Witte, 2000:135). It often happens that people, such as those diagnosed with ADHD, are branded as worthless or lesser individuals (Lawlis, 2004:55). As a result, they develop a low self-concept and self-perception of being unable to function properly and being incompetent and pathetic, further aggravating their deficient characteristics (Levine, 2002:327).

Instead, their strengths should be uncovered, focused on and further reinforced, by means of behaviour-modification techniques. For that reason behaviour modification can be used effectively.

Proper behaviour and interaction can be taught (Sonna, 2005:153). People involved with the ADHD-diagnosed individual should learn to respond to the ADHD-diagnosed individual differently (Barkley, 2005:106). For example, positive verbal praise, when justified and 
consistently given, can be surprisingly effective. According to Plumer and Stoner (2005:291), the teaching of appropriate social skills will advance the individual's general social functioning.

\subsection{How is behaviour modification done?}

Behaviour modification can be used in conjunction with any other means for the management of ADHD-related behaviour, and it can be reinforced at home, school or in other social settings (Barkley, 2005:242; Lawlis, 2004:262; Rief, 2005:165; Sherman, et al., 2008:348). It entails certain measures are used to both reinforce and strengthen the appropriate behaviour, or to reduce and discourage any behavioural difficulties. Examples of positive reinforcements are rewards, such as praise, approval, modelling, and shaping.

This does not only apply to the ADHD-diagnosed individual, but also to the parents, siblings and other people involved with the individual (Neethling, Rutherford \& Schoeman, 2005:32).

The natural tendency to focus only on the positive reward, can be balanced by a combination of rewards and punishments, known as "response cost" (Hukamdad, et al., 2011:904), which will assist in decreasing the individual's impulsive inclinations. Rewards should be tangible, especially for younger ADHD-sufferers; they should be granted frequently, and the feedback should immediately follow the behaviour (the closer, the better), in order to allow the individual to make the necessary associations (Young \& Amarasinghe, 2009:117). Hukamdad, et al., (2011:908), also emphasize that rewards or punishment should immediately follow the behaviour; and they should be consistent and fair.

Parent training forms a crucial part of the approach (Young \& Amarasinghe, 2009:121). This should enhance parenting skills and assist parents in dealing with their challenges by means of appropriate behavioural techniques and intervention skills. They need relevant information and behavioural strategies to implement at each level of the child's development (Young \& Amarasinghe, 2009:118).

This would include the identification and manipulation of behaviours, the targeting and monitoring of problematic behaviour, rewards for appropriate behaviour, such as praise, positive attention, tangible rewards, or a decrease in unwanted behaviours through "timeout", and effective commands, appropriate rewards, immediate feedback and positive reinforcement (Hukamdad, et al., 2011:905). Parents should also guard against risk factors, such as depression, marital turmoil, and lack of support, by means of open discussion, appropriate information, basic coping strategies, support groups and counselling (Young \& Amarasinghe, 2009:118; Gomes, 2008).

In the school environment, well-structured routines, rules and expectations are required by the ADHD-diagnosed individual, as well as the careful attention of the teacher (Rief, 2005:165). Realistic academic targets and behaviour goals can serve as motivating factors (Young \& Amarasinghe, 2009:118). Approval can be as simple as a nod of the head, or a verbal sound, such as 'Mmmm', or a comment, such as 'well done'. Environmental manipulations can also be implemented, for example, seating the child away from any source of distractions. 
The co-operation between school and home is important, and regular contact should be scheduled to assess the specific needs, progress and intervention procedures. Where possible and necessary, individualised approaches will be more successful and a "contingency contract" programme can be applied (Hukamdad, et al., 2011:906). It is possible for the individual to learn to apply skills, such as making eye contact, giving compliments, taking turns, listening, problem-solving, verbal instruction ('thinking out loud'), obeying rules, teamwork, using time schedules, self-monitoring, emotional control, and self-reinforcement (Young \& Amarasinghe, 2009:119-120).

Behaviour that is strengthened will recur, while behaviour that is not rewarded will probably fade away. According to Sherman, et al. (2008:347), gestures used by people involved with the ADHD-diagnosed individual, can influence the individual's performance, for example, with academic puzzles. People with more tolerance and acquaintance with intervention techniques, who can work well and co-operate within a multi-disciplinary team of specialists and who have a positive mindset, can also influence the development of a more positive outcome for the individual diagnosed with ADHD (Sherman, et al., 2008:347).

Many skilled specialists consider behaviour modification as being the most appropriate instrument in the management of ADHD-related behaviour (Davies \& Witte, 2000:135). People involved with ADHD-diagnosed individuals should assemble as much information as possible about behaviour modification - in order to increase their focus on any existing strengths, rather than on the deficiencies (Rief, 2005:14). Positive attention can be used effectively as reinforcement, for example, by praising well-meant attempts and refocusing or redirecting any failed efforts (Gomes, 2008:100).

\section{Coaching as a way of managing ADHD}

ADHD coaching is based on the coaching model used in athletics and life-skills coaching (Swartz, et al., 2005:648). Coaching is an "important and relatively new profession" (Gates, et al., 2001:87; Murphy, Ratey, Maynard, Sussman \& Wright, 2010:546; Parker \& Boutelle, 2009:205). Parents, caregivers and teachers have been coaching ADHD-diagnosed children for a long time, in order to manage their behaviour, but no-one has ever thought of this action in terms of a structured strategy, until recently, when the term was coined with regard to psychological supervision, support and goal achievement.

In 2002, a task force of coaches organized by the Attention Deficit Disorder Association (ADDA) wrote: "The Guiding Principles for Coaching Individuals with Attention Deficit Disorder" (Jaksa \& Ratey, 1999). This document suggests elements that should be considered as essential to ADHD coaching; it establishes standards and outlines the ethical principles required to help people better understand ADHD coaching. Appropriate coaching not only provides supervision and support, but it also gives a sense of accountability and consistency.

The topic of ADHD coaching currently enjoys increased favour, even though it has not yet been researched extensively; and the effectiveness has not proven by many empirical research studies (Murphy, et al., 2010:546). For the latter reason, some scientists remain sceptical about this approach (Swartz, et al., 2005:648). However, several research studies (Hollin \& Palmer, 2006; Jones \& McCaughey, 1992; Jordan, Singh \& Repp, 1989; Stevenson, Whitmont, Bornholt, Livesey \& Stevenson, 2002; Zwart and Kallemyn, 2001;) have produced clear evidence of success with coaching. 


\subsection{What is coaching?}

Dawson and Guarre's coaching model (2000) is a promising, innovative approach to ADHDrelated difficulties (Plumer \& Stoner, 2005:292; Swartz, et al., 2005:648). Young and Amarasinghe (2010:126) see coaching as a "derivative of cognitive behavioural paradigms", based on a "collaborative mentoring partnership, which draws on an individual's personal strengths, and aims to provide structure, support and feedback".

This would entail the necessary emphatic and gentle psychological support and supervision (Carroll, 2006:4) for skills development, problem-solving and the management of own time and actions, by means of significant "complex dyadic interactions" (Gates, et al., 2001:87). This approach should help to manage the symptoms of ADHD, as well as in providing the encouragement to independently develop internal and external structures for taking responsibility for reaching specifically set goals (Jaksa \& Ratey, 1999; Parker \& Boutelle, 2009:205).

Plumer and Stoner (2005:292) contend that coaching is based on "correspondence training", focusing - as it does - on the reinforcement of behaviour that corresponds with the set goals. According to Swartz, et al. (2005:648), this process of "observational learning" strengthens the individual's ability to independently counteract any ADHD symptoms. At the same time, the individual gains self-efficacy, as well as the increased self-confidence needed to reach his/her goals.

Coaching, with reference to ADHD, involves a "collaborative alliance" (Young \& Amarasinghe, 2010:127). It involves the supervision of behaviour, strategies and tasks, by a person to whom the ADHD-diagnosed individual must be accountable - whether the ADHD-diagnosed individual be an adult or a child. Clearly, the coaching principles must remain the same; it is the strategies and tasks that have to be age-appropriate, and needspecific (ADDA, 2002; Quinn, Ratey \& Maitland, 2000; Wallace, 1998).

The basis of coaching is that the coach and the ADHD-diagnosed individual should work together to set goals and develop the tools, strategies and confidence necessary to help the individual overcome the behaviour difficulties encountered, and to replace any unacceptable behaviour with the correct behaviour for the specific situation (Hallowell \& Ratey, 1994; Jaksa \& Ratey, 1999; Swartz, et al., 2005:648).

\subsection{Why is coaching needed?}

According to the Diagnostic Criteria from DSM-IV-RT (2000) and the Diagnostic and Statistical Manual of Mental Disorders (1994), executive functioning issues, such as time management, prioritization, procrastination, and realistic views of a task or situation are among the many aspects which cause behavioural difficulties for those diagnosed with ADHD (American Psychiatric Association, 2000; American Psychiatric Association, 1994).

Others in the list provided by the DSM-IV include differences in learning styles between parents and children, learning disabilities resulting from the inability to process and retain information, the inability to pay attention for lengthy periods of time, the inability to obtain and organise information, the inability to self-regulate and self-monitor own behaviours and tendencies, the lack of accountability, the inability to form and maintain lasting relationships (American Psychiatric Association, 2000; American Psychiatric Association, 1994). 
These areas of concern are typically addressed by means of behaviour- modification coaching.

Coaching is an excellent tool for parents of small children who have been diagnosed with ADHD, and also for the adult struggling with organisational and time-management aspects, both in the private, as well in the professional sectors of daily life (Jaksa \& Ratey, 1999; Plumer \& Stoner, 2005:292; Swartz, et al., 2005:648). Coaching helps both children and adults suffering from ADHD-related difficulties, to focus on the various aspects of their task, without the feeling that they are being reprimanded or 'bossed' around, as in the case of children, and criticized and picked on, as in the case of adult sufferers (Chronis, Chacko, Fabiano, Wymbs \& Pelham, 2004).

Coaching helps individuals to concentrate better on specific aspects and personal strengths, often not obvious. Frequently, in such instances, incorrect behaviours are learned as mechanisms for coping with perceived difficult situations. Coaching can then re-direct and correct incorrect behaviour habits (Quinn, et al., 2000). Often the order of importance of a task's steps eludes an individual completely, resulting in errors, accidents and frustrating delays.

Coaching helps in the elimination of the frustration of task re-direction, and it teaches the ADHD-diagnosed how to develop the ability to set goals and keep to the aims required to complete the task. Bear in mind that the major problems of ADHD are the lack of organizational skills and consistency, mutual respect, trust and compatibility. These are key aspects of the coaching process designed to foster accountability and responsibility, and to minimise fear of failure, lack of self-esteem and lack of self-confidence on the part of the ADHD-diagnosed individual (Jaksa \& Ratey, 1999).

Coaching helps individuals of all ages to make positive, lasting changes that will impact their lives long after they leave school and enter adult life. Chronis, et al. (2004), confirms that coaching teaches individuals diagnosed with ADHD how to develop the necessary correct skills that they will need to employ throughout life. Through coaching, the individual gains the responsibility to function independently (Plumer \& Stoner, 2005:298) and develops the self-regulating skills needed for self-determination and the selfmanagement of ADHD-related challenges (Parker \& Boutelle, 2009:205). It can also effectively assist the individual with problems, such as procrastination, lack of concentration, poor planning, anxiety, social incompetence or time management (Swartz, et al., 2005:648).

\subsection{How is coaching done?}

Coaching normally involves pairing an individual (child or adult) diagnosed with ADHD with another person, who then serves as his/her coach (Plumer \& Stoner, 2005:292). Through their "mindful involvement" (Carroll, 2006:4) they finalise goals, suggested by the individual diagnosed with ADHD, after which behaviour monitoring, supervision, encouragement and support, provision of feedback, as well as contingency management follows, with the aim of learning new and appropriate behaviour.

ADHD coaches are usually those closest to the ADHD-diagnosed individual, i.e. the parents, older siblings, peers and teachers, although there may also be some therapists and 
other professionals involved in the individual's coaching. The most important point is, that however many different people coach any one individual at different times of the daily routine, that they should all be synchronised and in agreement on the type of coaching, as well as on the requirements and the strategies being used. This will prevent confusion and frustration of all concerned (Gomes, 2008; Jaksa \& Ratey, 1999; Swartz, et al., 2005:648).

Together, the coach and the individual diagnosed with ADHD undertake difficulties, such as time-management and problem-solving (Swartz, et al., 2005:648).

Precisely how ADHD coaching is done will depend on who is being coached and who is doing the coaching. When dealing with children diagnosed with ADHD, parents, teachers and sometimes older siblings, peers or caregivers can serve as coaches; and the coaching work should be done on a daily basis, in the form of guidance, reminders and supervision of tasks, such as routines, habits, homework, or household chores. Coaching, in this instance, is an ongoing strategy that serves as guidance for the ADHD-diagnosed child to learn the correct techniques with which to work, both at that moment in time, as well as in the future (National Resource Centre on ADHD, 2003). Coaching entails pertinent questions that stimulate reflective thinking, in pursuit of planning to reach one's goals (Parker \& Boutelle, 2009:205).

This cognitive element assists the individual to acquire awareness of his/her own behaviour and the required behaviour changes (Swartz, et al., 2005:648). It enables the individual to apply the behaviour without the presence of the coach - in the end.

Coaching children is a little more involved than coaching adults, but the basic strategy remains the same. Although the ADHD coaching partnership is still flexible and designed to meet the needs of the ADHD adult sufferer, coaching at this level allows more freedom of thought, action and decision- making, while at the same time, requiring a higher level of commitment and accountability.

Coaching serves as a support system to assist the ADHD-diagnosed adult in learning how to manage time, develop organisational skills, determine priority of task performance, and it provides the perfect platform for the development of personal accountability and responsibility (National Resource Centre on ADHD, 2003).

\subsection{Who does the coaching and who gets coached?}

According to the International Coach Federation (2003) (ICF) - an international association of personal and business coaching that is evolving as the principal governing body for this field - there is currently no specialised schooling or licensing required in becoming a coach or a coach who specialises in ADHD (www.coachfederation.org). Yet, Murphy, et al. (2010:546), explain that a specialist coach must undergo meticulous training at an institution, in order to gain certification.

Quite a number of professional institutions are now training coaches to work with ADHDdiagnosed individuals, such as the Association for Professional Executive Coaching and Supervision (APECS) (Carroll, 2006:4; Murphy, et al., 2010:546). This is important as regards the quality control and reliability of this new profession. This is vital in developing the appropriate coaching skills, such as "listening, reflection, questioning and empathy" (Carroll, 2006:4; Swartz, et al., 2005:651). 
The designated coach should be committed and consistent in his/her coaching approach. A good knowledge of the needs and difficulties faced by the ADHD-diagnosed individual is required. Knowledge of ADHD-related issues, such as in the case of parents acting as coaches, is also needed in order to coach someone who has been diagnosed with ADHD (Carroll, 2006:4; Swartz, et al., 2005:648).

Coaching is, generally speaking, a two-people process. Although the parents are the primary 'coaches' for their ADHD-diagnosed children, they may need outside help, in the form of professional coaching, from a counsellor, psychologist or from marriage and family therapists better versed in the more intricate aspects of the effects of ADHD behaviour on the family in general (Carroll, 2006:4; Murphy, et al., 2010:546).

When we speak of coaching children diagnosed with ADHD, the coach may frequently be a different person in different settings, i.e. the parents could take turns coaching the child at home and/or in social settings, according to the situation, the need, the place and/or the task being dealt with. At school, the teacher would be the child's coach. On the playground, the coach could be an appointed more mature peer or friend, to whom the child looks up and admires, or a student teacher, specifically appointed to coach play-time behaviour (Jaksa \& Ratey, 1999).

ADHD coaching may be helpful to adults suffering from the effects of ADHD, but only if they recognise the importance of their commitment to the process. Children are reliant on their parents to know what is best for them and will willingly follow coaching directions, without being self-conscious about it. Adults, however, need to admit that they have a problem that they are unable to overcome alone. To do this, they have to entrust the managing of their problem-solving strategies to another; thereby, relinquishing their control over the process, something that adults often struggle with (National Resource Centre on ADHD, 2003).

If ADHD-diagnosed adults need assistance in dealing with these practical challenges in daily life, a coach may be a good source of help (Carroll, 2006:4; Murphy, et al., 2010:546). However, if the adult needs assistance with emotional, psychiatric, or interpersonal problems, then a professionally licensed therapist should be consulted. When both types of assistance are needed, as is often the case, it may be helpful to select a coach and a therapist who will agree to work together - thereby forming a team group to provide the necessary support and accountability to the sufferer (Gomes, 2008; National Resource Centre on ADHD, 2003).

\section{Coached behaviour modification in the management of ADHD}

In the majority of cases, especially those involving adults suffering from the effects of ADHD, coached behaviour modification is the most effective route, because it requires daily feedbacks, adjustments and reorganisation. It not only provides much-needed support and encouragement, but it also requires accountability, commitment and trust, from both the ADHD sufferer, as well as from the chosen coach. As a general rule, coaches deal with problems in everyday living, such as organization, time management, memory, followthrough, and motivation. 
Their focus is on what, when and how, but not so much on the why (National Resource Centre on ADHD, 2003).

All cases of ADHD are unique and the ADHD coaching involves the use of different and unique intervention skills to suit each particular individual (Gooding, 2007a:41; Murphy, et al., 2010:549). Although the symptoms may be similar, the frequency, duration, location and content will differ for each individual (Swartz, et al., 2005:649). Through coaching, behaviour modification can be done more effectively. It is a 'gentler' way of re-directing behaviour than simply issuing a set of instructions (Gates, et al., 2001: 86).

Like all coaching, ADHD-coached behaviour modification focuses on the specific needs of the individual whose behaviour is being adjusted and modified (Young \& Amarasinghe, 2010:128).

The most common question asked by ADHD sufferers is: 'How long will I need coaching?' The answer to this question is once again different for each unique individual. The duration of ADHD coaching depends on the difficulties being experienced by the individual, as well as the individual's goals and rate of progress, amongst other things. The individual's determination and commitment also play an important role in the length, progress and conclusion of the coaching process (Carroll, 2006:4; Murphy, et al., 2010:546).

For most parents, their child's behaviour modification coach may last as long as their child lives at home, which would probably be about the first $20+$ years of their child's life. Thereafter, depending on their personal relationship, coaching by the parents could go on for even longer than that. The choice belongs to both parties equally. Some young adults enjoy the time they spend with their parents, especially same-sex parents, and choose to continue being accountable for their progress to that parent (Hyde Park, Ratey, \& Jaksa, 2002).

Many however, learn to cope by themselves and choose to ask for help only when absolutely necessary. Frequently, the individual's response to coaching is affected by their own sense of self-esteem and personal value. The higher their self-esteem, the more often they will resort - by themselves - to finding the appropriate coach for their need (Hyde Park, et al., 2002).

Coached behaviour modification usually progresses according to the following format:

An in-depth discussion takes place during the initial meeting (Swartz, et al., 2005:650) or first session, where the ADHD-diagnosed adult and the assigned coach get to know each other, the process is explained and the coaching relationship is defined in terms of needs, difficulties, requirements, strategies to be used, accountability and responsibility. Both individuals discuss what the ADHD-diagnosed individual wants to achieve, and what his/her present symptoms, difficulties and strengths are.

The first important aspect of coaching is to build up a good collaborative relationship and to collect a proper case history. Thereafter, one would compile an acceptable schedule for the coaching process. Small steps at a time and different coaching goals then become the order of the day. Initially, one basic routine is striven for per week, and then gradually more goals are added. Thereafter, the different techniques, tools and actions for accomplishing the goals will be brainstormed. These have to be implemented consistently. Furthermore, the 
measures for supervision, monitoring and support need to be agreed upon. Throughout the process, readjustments will need to be made.

Key aspects of the process are explained, such as personal phone calls, or e-mails as reminders and means of encouragement, as well as future face-to-face contact sessions. Not more than three long-term goals and tangible, measurable, observable, specific, reasonable and possible achievements should be identified at this stage; and additionally, a plan of action, suggested by die ADHD-diagnosed individual and agreed upon by both parties, is set up (Swartz, et al., 2005:650).

The ADHD-diagnosed individual usually determines the details of the discussion, with the coach's encouragement, but both the coach and the ADHD-diagnosed individual talk about what's working and what can be improved, and/or changed. The achievement of the long term goals can extend past the coaching process of about eight weeks. This first discussion session can last about 90 minutes (Swartz, et al., 2005:650), but this time limit is usually agreed upon at the start of the appointment.

Thereafter, regular sessions last approximately 30 minutes per week, or bi-weekly, to assess goal-accomplishment (Swartz, et al., 2005:650). It is during these shorter sessions that shortterm goals or easily attainable smaller stepping stones are set, while long-term goals are adjusted, and further structures and strategies are developed, with the aim of building more self- confidence. During these sessions, the action plan for the following week is also determined and agreed upon (Jaksa \& Ratey, 1999; Swartz, et al., 2005:650) then it should be written down and signed by both parties.

Rewards and punishment (referred to as "consequences" by Swartz, et al. (2005:651), initially suggested by the ADHD-diagnosed individual and agreed upon, form an important part of the process of coaching, in order to manage the ADHD difficulties. These guidelines serve the purpose of reinforcement of the appropriate behaviour; and they will ensure the client's commitment to the coaching process. The outcome is to get the ADHD-diagnosed individual to eventually monitor his/her own behaviour.

One very interesting aspect of coaching that is introduced at a higher level of responsibility than that found in the coaching of small children, is the importance of check-ins (National Resource Centre on ADHD, 2003). These are a crucial part of the coaching process and require that the ADHD-diagnosed individual send the coach a brief status update on each planned action - either by e-mail or by telephone. This fosters accountability and responsibility on the part of the ADHD-diagnosed individual, and also contributes to eliminating any procrastination, and helps to keep the individual on track between sessions.

The coach and the individual agree on how often these 'check-ins' should occur. Although such 'check-ins' should form part of the daily process; in time, these should have longer time lapses in between, depending on the individual's progress, commitment and trustworthiness (Swartz, et al., 2005:650).

Through coaching, the individual is helped to focus on the task at hand; to manage time more effectively; to prioritise responsibilities and manage tension; and to control unacceptable behaviour (Gooding, 2007a:40). This can be done through repeated monitoring, the provision of regular feedback and praise. Tangible success always has a powerful effect (Murphy, et al., 2010:550). Accomplishments and establishing a good habit serve to gradually build more confidence, self-awareness and self-management. 
Ultimately, the goal of coaching is to provide support - until the individual learns the necessary skills to be able to function independently (Plumer \& Stoner, 2005: 292), and stay on track over time - be that individual an adult or a child. The purpose of behaviour modification coaching is to provide the resources, strategies and skills needed to equip the individual for life (National Resource Centre on ADHD, 2003; Quinn \& Ratey, 2002).

\subsection{Does coaching take the place of therapy?}

No, coaching is not a substitute for therapy. Both coaching and therapy are based on a wellness model (Parker \& Boutelle, 2009:205); and additionally, based on trust. This involves complex relationships and change, giving feedback, and learning new skills (Summerfield, 2006:24). However, the coach's task is "neither counsellor nor advisor, but a collaborator" (Swartz, et al., 2005:648).

Coaching aims to improve performance and to make the ADHD-diagnosed individual more independent and responsible for his/her own behaviour and actions through supervision (Murphy, et al., 2010:547). It is important that coaching be approached with the full cooperation of the individual needing help. ADHD-diagnosed individuals need to understand the importance of working with a behavioural coach for the coaching process to be successful and effective (Hyde Park, et al., 2002; Shintel \& Keysar, 2007).

The ADHD-diagnosed individual should be relatively healthy, psychologically speaking, as coaches are not expected to give therapy for serious psychiatric or mental disturbances. The individual should also accept that he/she is suffering from ADHD-related problems and needs assistance and coaching to manage these problems. The individual also needs to commit him/herself to the coaching intervention (Murphy, et al., 2010:551).

Therapy is based on the premise that remedial change and emotional healing are needed (Murphy, et al., 2010:547). Where emotional problems, such as depression or other psychological issues hinder daily functioning, these need to be addressed by professionals, such as the family doctor, the psychologist or the psychiatrist (Hallowell \& Ratey, 1994; Murphy, et al., 2010:551; Quinn \& Ratey, 2002). If these issues remain untreated, coaching will not be successful. In such cases, it is advisable for the coach to work in tandem with the individual's various healthcare providers to overcome these obstacles (Wallace, 1998).

The purpose of coaching is to support the development of methods to manage the day-to-day ADHD-related challenges (Gooding, 2007a:40). It is based on "action learning" (Murphy, et al., 2010:547), that is to say, learning that supports the individual up to the point where he/she can manage by him/herself. Coaching is a form of verbal reminder, a guiding of the methods for carrying out a task, or of adjusting behaviour, especially where children are concerned. For the adult sufferer it assists with the personal and professional growth and development, based on the pursuit of specific outcomes. These, in turn, are linked to personal or professional success (Gomes, 2008; Quinn \& Ratey, 2002; Quinn, et al., 2000; Wallace, 1998).

The main aim of coaching is to show the individual how to achieve these goals in the most practical manner possible (Quinn \& Ratey, 2002; Quinn, et al., 2000). In coaching the emphasis is on the individual's ability to "take action on life goals in a balanced and fulfilling way" (Parker \& Boutelle, 2009:205). The ADHD-diagnosed individual is seen as resourceful 
and creative. The individual is helped to develop his/her own systems of effective functioning in pursuit of his/her goals.

Therapy, on the other hand, deals with the healing of past experiences of pain that have resulted from the stigma attached to the ADHD diagnosis (Murphy, et al., 2010:547). Whether in a family situation or outside it in social settings, such as school or work situations, ADHD is hugely misunderstood (Hallowell \& Ratey, 1994; Jaksa \& Ratey, 1999). This failure to understand, coupled with the resulting dysfunction and conflict within an individual, can so easily cause pain and insecurity. Such trauma is best addressed in therapy (Gomes, 2008).

According to the International Coach Federation (2003), there are several issues that can interfere with the coaching process. These will often require referral to a medical or mental health professional, for example, in cases where the ADHD-diagnosed individual is not able to use simple self-management or organizational strategies to achieve the desired goals, despite the coach's support, encouragement and reminders; in cases where the ADHDdiagnosed individual has a co-existing psychiatric condition, such as depression, bipolar disorder, anxiety disorder, substance abuse, or personality disorder; where the ADHDdiagnosed individual has stressful life circumstances, such as marital problems, divorce, or the death of a loved one, or where the ADHD-diagnosed individual has a serious physical illness or other chronic medical condition (Hallowell \& Ratey, 1994; Quinn \& Ratey, 2002; Wallace, 1998).

Under such circumstances, coaching should be carried out in collaboration with the medical or mental health professionals involved in the ADHD-diagnosed individual's care. In such cases, the ADHD-diagnosed individual may benefit from the addition of traditional treatments, such as medication and psychological therapy, as is often the case where a multimodal approach to treatment would work best (Hallowell \& Ratey, 1994).

The focus of therapy is often on resolving difficulties arising from the past which hamper an individual's emotional functioning in the present. Coaching, on the other hand, is forwardmoving and future-focused, providing support, hope and encouragement (Hyde Park, et al., 2002; Shintel \& Keysar, 2007). While positive feelings/emotions may be the natural outcome of coaching, the primary focus is on creating workable strategies for achieving specific goals in the individual's work or personal life. Coaching emphasises action, accountability and follow-through. Therapy, on the other hand provides a platform for reflection, and working through difficult emotional feelings that result from the conflict associated with ADHD (Hallowell \& Ratey, 1994).

\section{A possible coached behaviour-modification programme}

The Coping Skills Programme of Behaviour Modification (Gomes, 2008) is a valuable programme to use in a coaching situation, as it provides many flexible alternatives for all ages and situations. The Coping Skills Programme guides the designated coach through a process of initial investigation of the presenting difficulties, in order to define the most appropriate way to deal with and resolve problematic situations and behaviours. Used correctly, systematically and consistently, the Coping Skills Programme is one of the most practical and effective programmes to use. Its flexibility makes it ideal for all age groups, situations and needs (Gomes, 2008). 
Some of the strategies used by the Coping Skills Programme include an initial investigation into the specific needs of the family or individual faced with ADHD-related issues and problems. This investigation is done by means of observation and dialogue; and it is not just based on the existing information available via the media and other resources. This method of investigation encourages parents and other designated coaches to observe behaviour based on ability and strengths, rather than simply to focus on deliberate misbehaviour. It requires that the parent really observe the child's behaviour and determine what it is that provokes such behaviour. Once this fact has been established, strategies may be set in place to replace the undesirable behaviour with a more desirable alternative (Gomes, 2008, Hukamdad, et al., 2011).

Thereafter, other strategies and skills can be set in place, such as coaching contracts, where applicable, the setting of reasonable and attainable goals, accountability and responsibility, positive feedback from the parents and designated coaches, the selection of tools and strategies to assist with executive functioning issues, such as calendars, timers, planners, study buddies/coaches, journaling for older children and adult individuals, and time management.

A learning styles inventory is sometimes also implemented, to show parents why their children so frequently have difficulty in paying attention in class and during homework sessions at home. Often the conflicting learning styles of teachers and learners, as well as those of parents and their children are all that stand between doing well and struggling through the school day (Gooding, 2007a:40; Graham, 2008a:92; Heriot, et al., 2007:121).

Classroom arrangements are also discussed and adjusted, where possible, in conjunction with teachers and other scholastic staff, in order to improve the focus and decrease distractibility, and also the establishing of consistent daily routines for all members of the family, extended family and care givers (Gomes, 2008, Rief, 2005:57).

Periodical adjustments of learned skills have to be carried out as soon as each new skill has been mastered; that is to say, a new skill should remain in place, in its original form, only as long as it still serves its purpose for task execution. As soon as the skill stops being effective, it needs to be upgraded or adjusted, to help with the next difficulty. For example, a baby learns how each type of food 'behaves' in his/her plate, by handling it with his/her fingers first (skill 1) - a natural skill that all babies have. Soon, mommy places a spoon in the baby's hand and teaches him/her how to use the spoon (skill 2). This is not a natural skill; and it will, therefore, take a little longer to master.

The spoon may, at first, be used as an addition to feeding with the fingers; it may even be used as a toy, but it will eventually be used properly, at which point the skill needs to be upgraded to using a fork, and so on. It is still the same skill, but is being upgraded continuously to the next level, in small steps one at-a-time (Gooding, 2007a:40; Graham, 2008a:92).

If periodical adjustments are carried out regularly and efficiently, the child will internalise the 'skill', and later learn to adapt it to adult life situations, having learned how to exhaust all the possibilities in one specific skill, changing it and then using the adaptation for a different difficulty. Non-ADHD-diagnosed people do it automatically all the time; it is how humans survive; they adapt to their surroundings and their needs. However, this is not a 
natural behaviour mechanism for ADHD-diagnosed individuals; perhaps, because early on in their lives their mothers tried to compensate for their every difficulty.

For ADHD-diagnosed children, skills have to be learned and integrated. When skill adjustment is carried out speedily, the child's confidence in his/her ability to adapt to changes - the biggest difficulty for ADHD sufferers - is boosted, and ADHD-related difficulties become less prominent and problematic. This is the function of coaching: to help find, integrate, monitor and adjust the appropriate skills being learned and used (Hyde Park, et al., 2002).

Chronis, Chacko, Fabiano, Wymbs and Pelham (2004) discovered in their research that behavioural parent training (BPT), in which parents are guided in how to coach their children's behaviour modification, is one of the most effective behaviour-modification processes in the treatment of ADHD-related misbehaviour. They found that, because so many aspects influence the child's behaviour and affect the parent's reactions to that behaviour, continuous programme adjustments are needed in the way parents teach their children, the style that they use, and how the children interpret what is being taught.

This is true in adult coaching also, in that adults have a tendency to assume various permutations in the outcomes of their actions. It is this aspect that results in the difficulties faced by adults with ADHD-related difficulties, the 'what-if-factor'. For example: 'What if I do the work required, but nothing changes? If that is so, then why do the work? I might as well carry on as I have before'. Children don't question therapy in this way. Adults are the ones who will do this more frequently (Gomes, 2008).

Programme follow-up periods should take place from time to time, after the completion of the Coping Skills Programme, (Gomes, 2008), in order to make any necessary adjustments and deal with any difficulties that the parents might experience along the way, and which might not have been previously covered by the Coping Skills Programme. The parents, as and when they feel it is necessary, usually initiate these follow-up periods; and in some instances, there may be a short succession between follow-up periods, and then a longer gap until help is requested again. In other instances, follow-up periods may be less frequent and fewer in number (Gomes, 2008).

Programme follow-up periods are important for both the ADHD-diagnosed individual and the parents, and/or designated coach, as these provide the opportunity to make adjustments to the programme from time to time. Likewise, the parents/coaches benefit, because the up-date sessions serve as a form of support system where their questions may be answered, reassurance provided and new information obtained (Gomes, 2008).

\section{Conclusion}

The management of the challenging and difficult behaviour associated with ADHD presents a problem that causes stress for all persons involved, in the home, school and society at large. The management of ADHD-related problem behaviour is therefore a well-researched topic, and refers to an array of approaches.

In this chapter we have, however, argued for the use of a gentler approach, namely coachedbehaviour modification, which is "extremely powerful" (Scoular \& Linley, 2006:11) in modifying unacceptable or inappropriate behaviour. This is done through a mutual- 
mentoring partnership, grounded on an individual's personal strengths. It involves the provision of emphatic psychological supervision, support, encouragement and feedback. It aims at confidence, internal structure, goal-directed action, task persistence and responsible functioning of the ADHD-diagnosed individual.

Our contention is aptly summed-up by Parker and Boutelle (2009:212): The suggested approach is appropriate to help individuals diagnosed with ADHD to "develop selfregulation skills for managing challenges caused by their executive function difficulties through methods that emphasise self-determination". It is important to constantly bear in mind that it is the behaviour that is undesirable and not the person.

\section{References}

Aberson, B.; Shure, M.B. \& Goldstein, S. (2007). Social problem-solving intervention can help children with ADHD. Journal of Attention Disorders, 12(5) March, 391-393.

ADDA: Attention Deficit Disorder Association (2002).

American Psychiatric Association, (1994). Fourth Edition. Diagnostic and Statistical Manual of Mental Disorders. Washington, DC: American Psychiatric Association, USA.

American Psychiatric Association (APA) (2000). Diagnostic and statistical manual of mental disorders $-4^{\text {th }}$ edition, revised text (DSM-IX-RT). Arlington, VA: APA.

Barkley, R.A. (2005) Revised Edition. Taking charge of AD/HD: The complete authoritative guide for parents. New York, Guilford.

Carroll, M. 2006. Key issues in coaching psychology. The Coaching Psychologist, 2(1):4-8.

Cowan, D. (2002)a. Treatment Options for AD/HD. The ADD Information Library. http://www.newideas.net/attention_deficit/treatment.htm [Retrieved April 2011].

Chronis, A.M. Chacko, A.; Fabiano, G.A.; Wymbs, B.T. \& Pelham, W.E. (2004). Enhancements to the behavioural parent training paradigm for families of children with ADHD. Review and future directions. Clinical Child and Family Psychology Review, 7(1), March: 1-27.

D'Adamo, P.J. (2001). Live right for your type. London: Penquin Books Ltd.

Davies, S. \& Witte, R. (2000). Self-management and peer monitoring within a group contingency to decrease uncontrolled verbalisations of children with attention deficit/hyperactivity disorder. Psychology in the Schools, 37(2): 135-147.

Dawson, P. \& Guarre, R. (2000). Coaching the ADHD student. New York: Guilford.

Gates, B.; Newell, R. \& Wray, J. (2001). Behaviour modification and gentle teaching workshops: management children with learning disabilities exhibiting challenging behaviour and implications for learning disability nursing. Journal of Advanced Nursing, 34(1), April: 86-95.

Gomes, A.M. 2004. An Investigation of the Nature and Design of a Family- Focused Group Training Programme for the Management of Attention-Deficit Hyperactivity Disorder. Unpublished MA dissertation. Port Elizabeth: University of Port Elizabeth. RSA.

Gomes, A.M. (2008). A Supplementary Coping Skills Programme for Parents of Children Diagnosed with Attention Deficit Hyperactivity Disorder. Unpublished Doctoral Thesis. Port Elizabeth: University of Port Elizabeth. RSA.

Gooding, A.D. (2007)a. Coaching niches. Part 2. Annals of the American Psychotherapy Association, 10(3), Summer: 40-41. 
Gooding, A.D. (2007)b. Coaching niches. Part 3. Annals of the American Psychotherapy Association, 10(3), Fall: 37.

Graham, L.J. (2008)a. Drugs, labels and (p)ill-fitting boxes: ADHD and children who are hard to teach. Discourse: Studies in the Cultural Politics of Education, 29(1):85-106.

Graham, L.J. (2008)b. From ABCs to ADHD: The role of schooling in the construction of behaviour disorder and production of disorderly objects. International Journal of Inclusive Education, 12(1):7-33.

Hallowell, E.M. \& Ratey, J.J. (1995). Driven to Distraction: Recognizing and Coping with Attention-Deficit Disorder from Childhood through Adulthood. New York: Simon and Schuster. Pantheon Books. USA.

Heriot, S.A.; Evans, I.M. \& Foster, T.M. (2007). Critical influences affecting response to various treatments in young children with ADHD: a case series. Child Care Health and Development, 34(1):121-133.

Hollin, C.R. \& Palmer, E.J. (2006). Offending behaviour programmes: controversies and resolutions. In Hollin, C.R. and Palmer, E.J. (Eds.) Offending behaviour programmes: Development, application and controversies. Chichester: John Wiley \& Sons.

Hukamdad; Shahzad, S.; Ali, R.; Quadeer, M.Z. \& Khan, M.S. (2011). Comparison of Teachers' and Students' Views on the Use of Behaviour Modification Techniques at Secondary Level. International Journal of Academic Research, 3(10), January: 904-908.

Hyde Park, I.L.; Ratey, N. \& Jaksa, P. (Eds.). (2002). ADDA Subcommittee on ADD Coaching. The ADDA Guiding Principles for Coaching Individuals with Attention Deficit Disorder.

International Coach Federation, (2003). www.coachfederation.org

Jacklyn, H.Q. \& Ravichandran, S. (2009). Motivating Children with attention deficiency Disorder using certain Behaviour Modification Strategies. 13 th International Conference on Biomedical Engineering IFMBE Proceedings 23(3):1057-1060.

Jaksa, P. \& Ratey, N. (1999). 25 Stupid Mistakes Parents Make. Lowell House Press/NTC Contemporary. USA.

Jones, R.S.P. \& McCaughey, R.E. (1992). Gentle teaching and applied behaviour analysis: a critical review. Journal of Applied Behaviour Analysis, 25: 853-867.

Jordan, J.; Sing, N.N. \& Repp, A.C. (1989). An evaluation of gentle teaching and visual screening in the reduction of stereotypy. Journal of Applied Behaviour Analysis, 22: 922.

Lakoff, A. (2000). Adaptive will: the evolution of attention deficit disorder. Journal of the History of the Behavioural Sciences, 36(2), Spring: 149-169.

Lawlis, F. (2004). The ADD answer: how to help your child now. New York: Viking Penguin Group Inc.

Levine, M. (2002). A Mind at a Time: How Every Child Can Succeed. London: Simon and Schuster.

MacGraw, P. (2005). Family first. London, Simon \& Schuster, UK Ltd.

Mehl-Madrona, L. (2005). Traditional healing in modern medicine. Innovative healing. USA. Retrieved on 16 May 2011 from

http://ns2.whygee- dns.com/shop/bookstore/teleseminartranscripts/transcripts2006/174

Murphy, K.; Ratey, N.; Maynard, S.; Sussman, S. \& Wrights, S.D. (2010). Coaching for ADHD. Journal of Attention Disorders, 13(5):546-552. 
National Resource Centre on ADHD. (2003). Retrieved in June 2011 from www.help4adhd.org

Neethling, K., Rutherford, R. \& Schoeman, R. (2005). Very Smart Parents: 21 Building Blocks for Growing up Creatively in the $21^{\text {st }}$ century. Solution Finding. Halfway House. RSA

Olivier, M.A.J.; Gomes, A.M. \& Greyling, A.J. (2009). Attention Deficit Hyperactivity Disorder (ADHD) - dilemmas and solutions. Journal of Psychology in Africa, 19(2): 237-241.

Olivier, M.A.J. \& Steenkamp, D. (2004). Attention Deficit Hyperactivity Disorder (ADHD) and intrinsic motivation. International Journal for Advancement of Counselling. 26(1) March: 47-63.

Parker, D.R. \& Boutelle, K. (2009). Executive function coaching for college students with learning disabilities and ADHD: a new approach for fostering self-determination. Learning Disabilities Research and Practice, 24(4), Nov: 204-215.

Plumer, P.J. \& Stoner, G. (2005). The relative effects of classroom peer tutoring and peer coaching on the positive social behaviours of children with ADHD. Journal of Attention Disorders, 9(1), August: 290- 300.

Quinn, P.O., Ratey, N.A. \& Maitland, T.L. (2000). Coaching college student with ADHD. Silver Springs, MD, Advantage Books.

Quinn, P.O. \& Ratey, N.A. (2002). Life coaching for adult ADHD. In Goldstein, S. \& Ellison, A.T. (Eds.). Clinician's Guide to Adult ADHD: Assessment and Intervention. London: Academic Press.

Riding, R. \& Rayner, S. (2005). Cognitive styles and learning strategies: understanding style differences in learning and behaviour. London: David Fulton Publishers Ltd., UK.

Rief, S.F. (2005). Second Edition. How to reach and teach children with ADD/ADHD: practical techniques, strategies and interventions. Jossey-Bass: Wiley Imprint.

Sandler, A.D. \& Bodfish, J.W. (2008). Open-label use of placebos in the treatment of ADHD: a pilot study. Child Care, Health and Development, 34(1):104-110.

Sandler, A.D.; Glesne, C. \& Gellert, G. (2008). Children's and parents' perspectives on openlabel use of placebos in the treatment of ADSD. Child Care, Health and Development, 34(1):111-120.

Scoular, A. \& Linley, P.A. (2006). Coaching, goal-setting and personality type: what matters? The Coaching Psychologist, 2(1):9-11.

Sherman, J.C.; Rasmussen, C. \& Baydala, L. (2008). The impact of teacher factors on achievement and behavioural outcomes of children with attention deficit/Hyperactivity Disorder (ADHD): a review of the literature. Educational Research, 50(4): 347-360.

Shintel, H. \& Keysar, B. (2007). You Said It Before and You'll Say It Again: Expectations of Consistency in Communication. Journal of Experimental Psychology: Learning, Memory, and Cognition. 33(2) March: 357-369.

Smith, K. (2011). Intervention Tip Sheet. Institute on Community Integration, College of Education, University of Minnesota, Minneapolis, USA.

http://www.cehd.umn.edu/ceed/publications/tipsheets/preschoolbehaviortipsh eets/behmod.pdf)

Sonna, L. (2005). The everything parent's guide to children with ADD/ADHD. Avon: Adams Media F \& W Publications Inc. sel? Thoughts on the 'emotional life' of a coaching session. The Coaching Psychologist, 2(1):24-27. 
Steenkamp, D.S. (2001). Attention Deficit Hyperactivity Disorder and Motivational deficits in Early Adolescence. Unpublished D Ed Thesis. Port Elizabeth: University of Port Elizabeth.

Stevenson, C.S.; Whitmont, S.; Bornholt, L.; Livesey D. \& Stevenson, R.J. (2002). A cognitive remediation program for adults with attention deficit hyperactivity disorder. Australian and New Zealand Journal of Psychiatry, 36: 610-616.

Summerfield, J. (2006). Do we coach or do we counsel? Thoughts on the 'emotional life' of a coaching session. The Coaching Psychologist, 2(1):24-27.

Swartz, S.L.; Prevatt, F. \& Proctor, B.E. (2005). A coaching intervention for college students with Attention Deficit/Hyperactivity Disorder. Psychology in Schools, 42(6):647-656.

Wallace, I. (1998). You and Your ADD Child: Practical Strategies for coping with everyday problems. Sydney: Harper Collins Publishers. Australia.

Webb, J.T.; Amend, E.R.; Webb, N.E.; Goerss, J.; Beljan, P. \& Olenchek, F.R. (2005). Misdiagnosis and dual diagnosis of gifted children and adults. Scotsdale: Great Potential Press, Inc.

Young, S. \& Amarasinghe, J.M. 2010. Practitioner review: non-pharmacological treatments for ADHD: a lifespan approach. Journal of Child Psychology and Psychiatry, 51(2):116133.

Zwart, L.M. \& Kallemyn, L.M. (2001). Peer-based coaching for college students with ADHD and learning disabilities. Journal of Postsecondary Education and Disability, 15:1-15. 


\title{
Distractor or Noise? The Influence of Different Sounds on Cognitive Performance in Inattentive and Attentive Children
}

\author{
Göran Söderlund ${ }^{1,2^{*}}$ and Sverker Sikström ${ }^{3}$ \\ ${ }^{1}$ Department of Pedagogics, Sogndal University College, \\ ${ }^{2}$ School of Psychology, University of Southampton, \\ ${ }^{3}$ Department of Psychology, Lund University, \\ ${ }^{1}$ Norway \\ ${ }^{2}$ England \\ ${ }^{3}$ Sweden
}

\section{Introduction}

It is a well known and certified fact that noise under most circumstances interfere with cognitive processing of various kinds, e.g. vigilance (e.g. Broadbent, 1951), arithmetic's (Broadbent, 1958), and response speed (Broadbent, 1957). This effect is assumed to be due to the competition of attentional resources between the target and the distracting stimuli. This finding is often replicated and found valid among different tasks and participant populations (Belleville, Rouleau, Van der Linden, \& Collette, 2003; Boman, 2004; Klatte, Meis, Sukowski, \& Schick, 2007; Rouleau \& Belleville, 1996). Most research since Broadbent's days has dealt with the negative effects of noise and different kinds of auditory distraction. In line with this earlier research has demonstrated that inattentive persons, such as children with ADHD (attention deficit/hyperactivity disorder) are even more susceptible to distraction as compared with their attentive peers. This has been shown in numerous of studies (e.g. Corbett \& Stanczak, 1999; Geffner, Lucker, \& Koch, 1996; Rickman, 2001).

However, in contrast to the main body of evidence, there have been a few reports of contradictory findings. Specifically, it has been shown that under certain circumstances, children with attentional problems, rather than being distracted, actually benefit from environmental noise presented with the concurrent target task. Until recently, this facilitating effect of non-task related environmental auditory stimulation has been limited to the effects of background music on arithmetic task performance by children with ADHD (Abikoff, Courtney, Szeibel, \& Koplewicz, 1996; Gerjets, Graw, Heise, Westermann, \& Rothenberger, 2002). In addition, road traffic noise was found to improve episodic memory among children from households with low socio-economic status, a group that is likely to be distinguished by attentional problems and academic under-achievement (Matheson et al., 2010; Stansfeld et al., 2005). However, these studies have not provided a satisfactory theoretical account for why noise, under certain circumstances, can be beneficial for cognitive performance. 
There are some early studies that provide a theoretical account for noise enhancement. In these studies, hyperactive children improved their performance in demanding attention tasks where noise was introduced by visual stimulation (Zentall, 1986; Zentall \& Dwyer, 1989; Zentall, Falkenberg, \& Smith, 1985), or auditory stimulation (Zentall \& Shaw, 1980). In these experiments the positive effect was attributed to a general increase of arousal, formulated in a theoretical framework named "the optimal stimulation theory" (Zentall \& Zentall, 1983). However, this optimal stimulation theory has not been explored or developed further.

The aim with the present chapter is to present a plausible theoretical explanation as to why, when, and how noise can improve executive functions and cognitive performance in various tasks. Our research has recently extended these findings and for the first time will here be suggested a theoretical framework for understanding which conditions are necessary for noise induced cognitive enhancement to occur. We have shown that auditory noise has different effects on the memory performance of children with an ADHD diagnosis compared to normally developed children (Söderlund, Sikstrom, \& Smart, 2007). These effects have been replicated, and found valid in further studies comprising sub-clinical, inattentive participants (Söderlund, Marklund, \& Lacerda, 2009; Söderlund, Sikström, Loftesnes, \& Sonuga-Barke, 2010). In the following section we introduce a model and findings that demonstrate a link between noise stimulation and cognitive performance. This has been named the Moderate Brain Arousal (MBA) model (Sikström \& Söderlund, 2007), which suggests a link between attention, dopamine transmission, and external auditory noise (white noise) stimulation.

\section{The phenomenon of Stochastic Resonance}

Perceptual stochastic resonance (SR) is the counterintuitive phenomenon by which weak sensory signals that cannot be detected because they are presented below the detection threshold, become detectable when additional random (stochastic) noise is added (Moss, Ward, \& Sannita, 2004). Signaling in the brain is characterized by noisy inputs and outputs. The crucial task of the central nervous system is to distinguish between the signal, the information-carrying component, and noise that constitute meaningless neural inputs. The paradox is that the brain can actually use noise to differentiate the signal in the targeted stimuli from noise, so noise actually improves or increases the signal-to-noise ratio. The requirement for this phenomenon to occur is the introduction of non-linearity in the response, for example through a threshold function. This is shown in Figure 1, where the noise and the signal interact. The noise adds to the signal and brings the neuron over the activation threshold, and elicits a neural response (action potential), giving the auditory system a representation of the signal (a sinus tone).

SR is well established across a range of settings, and exists in any threshold-based system. The concept of SR was originally introduced to explain climate changes (Benzi, Parisi, Sutera, \& Vulpiani, 1982), but has been identified in a number of naturally occurring phenomena, some examples are: in bi-stable optical systems (Gammaitoni, Hänggi, Jung, \& Marchesoni, 1998); in mechanoreceptors of the crayfish (Douglass, Wilkens, Pantazelou, \& Moss, 1993); and in the feeding behavior of the paddlefish (Russell, Wilkens, \& Moss, 1999). $\mathrm{SR}$ is in particular found in the nervous system, distinguished by its all-or-none nature of action potentials. 


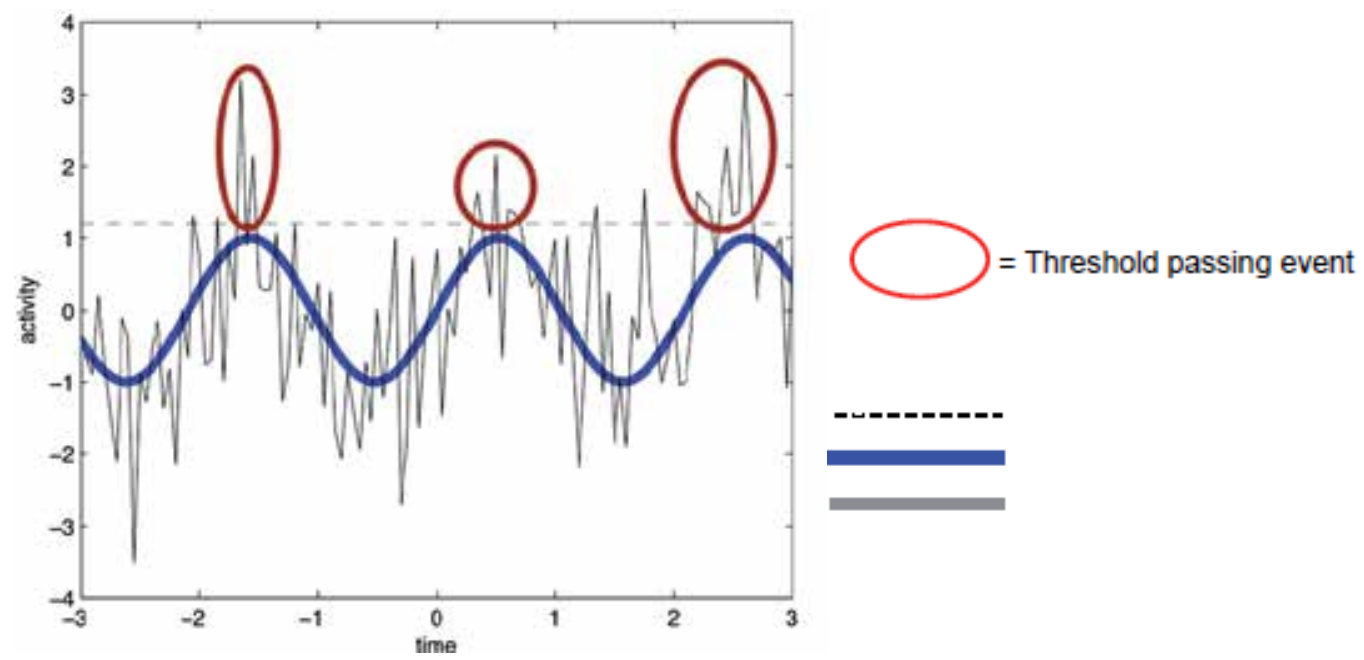

Fig. 1. Stochastic resonance where a weak sinusoidal signal goes undetected as it does not bring the neuron over its activation threshold. With added noise, the same signal results in action potentials.

In humans SR has been found in different modalities: in touch, where tactile random stimulation made skin receptors more sensitive (Wells, Ward, Chua, \& Timothy Inglis, 2005); in audition, where white noise improves auditory detection in a group with normal hearing (Zeng, Fu, \& Morse, 2000), and in participants with cochlear implants (Behnam \& Zeng, 2003); in vision, where visual (flickering) noise improved detection of weak signals (Simonotto et al., 1999). Interestingly, cross modal SR has been found, where weak visual signals became detectable when participants where exposed to loud auditory white noise (Manjarrez, Mendez, Martinez, Flores, \& Mirasso, 2007). SR can improve motor control and balance as well. Elderly, diabetics, and Parkinson patients' performance was enhanced through stochastic noise transmitted by vibrating soles (Novak \& Novak, 2006; Priplata, Niemi, Harry, Lipsitz, \& Collins, 2003; Priplata et al., 2006). In neurodegenerative disorders galvanic stimulation of the vestibular organs improved motor control considerably (Pan, Soma, Kwak, \& Yamamoto, 2008; Yamamoto, Struzik, Soma, Ohashi, \& Kwak, 2005). To sum up, SR is present in the entire nervous system in all modalities, and it seems that the nervous system can take advantage of noise both in sensory discrimination and motor control. SR is usually quantified by plotting detection of a weak signal, or cognitive performance, as a function of noise intensity. This relation exhibits an inverted U-curve, where performance peaks at a moderate noise level. That is, moderate noise is beneficial for performance, whereas too much, or too little noise attenuates performance.

While less known, empirical evidence also suggest that SR improves central processing in the brain and thus improves cognitive performance. For example a facilitating effect of cognitive SR has been found where auditory noise improved the speed of arithmetic computations in a normal group of school pupils (Usher \& Feingold, 2000). In a visual task, face recognition, response times got shorter when the vestibular organs where stimulated by a weak stochastic galvanic current (Wilkinson, Nicholls, Pattenden, Kilduff, \& Milberg, 2008) finally, figure copying became more accurate when exposed to galvanic stimulation 
(Wilkinson, Zubko, Degutis, Milberg, \& Potter, 2009). This indicates that also higher cognitive processing is susceptible for SR.

\section{Individual differences in SR and the Moderate Brain Arousal Model (MBA)}

Most of the above-referred references of the SR-effect are made with normal populations, and the revealed effects of noise are found to be valid for the entire population. Our research group has focused on cognitive effects of SR in particular groups with attentional problems, like in ADHD, where we have found differential effects of noise on cognitive performance. Some groups of participants improve their performance, whereas the performance other groups deteriorate when exposed to noise. The question is how these differentiations can be explained. We propose the Moderate Brain Arousal model (MBA) which is developed to address and explain these differences (Sikström \& Söderlund, 2007). The MBA model was developed to respond to the limitation of standard psychophysical models in explaining the noise facilitating effect in children with attention problems. The model is based on established facts concerning SR; first, that the SR phenomenon is highly sensitive to the intensity of the signal and; second, the intensity of the noise, where the cognitive or perceptual performance shows an inverted U-shaped curve when plotted against noise intensity (e.g. Moss, et al., 2004). Thus, a moderate level of noise is beneficial for performance. Too little noise does not add sufficient input to bring the signal over the activation threshold, and too much noise overpowers the signal - in both cases leading to deterioration in attention and performance. The crucial and innovative insight of the MBA model is that there are individual differences in the benefit of noise; some people need just a small amount of noise and some need a lot of noise to achieve optimal performance (see Figure 2). This is because individuals differ in internal levels of background noise and signal levels in their neural systems. That is, where noise levels are low, external noise has to be added to reach an optimal performance, and to achieve a moderate brain arousal level. Furthermore, required noise levels are linked to neurotransmitter function and in particular to dopamine. A hypo-functioning dopamine system is linked to inattention, and recent research suggests that ADHD possess low levels of extracellular dopamine (Solanto, 2002; Volkow et al., 2009; Volkow et al., 2007). The MBA model proposes that noise, as an alternative to stimulant medication, can compensate for low dopamine levels (Sikström \& Söderlund, 2007).

In summary, the MBA model posits that cognitive performance in ADHD and inattentive children benefits from noisy environments because the dopamine system modulates the SR phenomenon. It suggests that the stochastic resonance curve is right shifted in persons with a ADHD diagnose due to lower gain or lower dopamine. levels The MBA model predicts that for a given cognitive task ADHD children and inattentive children require more external noise or stimulation compared to control children, in order to reach optimal (i.e. moderate) brain arousal level (see Figure 2). This prediction has been tested and confirmed in several different settings with various participant groups and tasks. Word recall tests in children with ADHD (Söderlund, et al., 2007), non-clinical, inattentive school children (Söderlund, et al., 2010), and low performing school children (Söderlund \& Sikström, 2008). The effect has also been found in a dichotic listening task, and in a visuo-spatial working memory task in a normal student population, where half of the participants rated themselves as inattentive (Söderlund, et al., 2009). At the moment we have preliminary data 
showing significant effects of noise on three different cognitive tasks in parity with, or even larger, than the effects of stimulant medication (Söderlund et al., in progress).

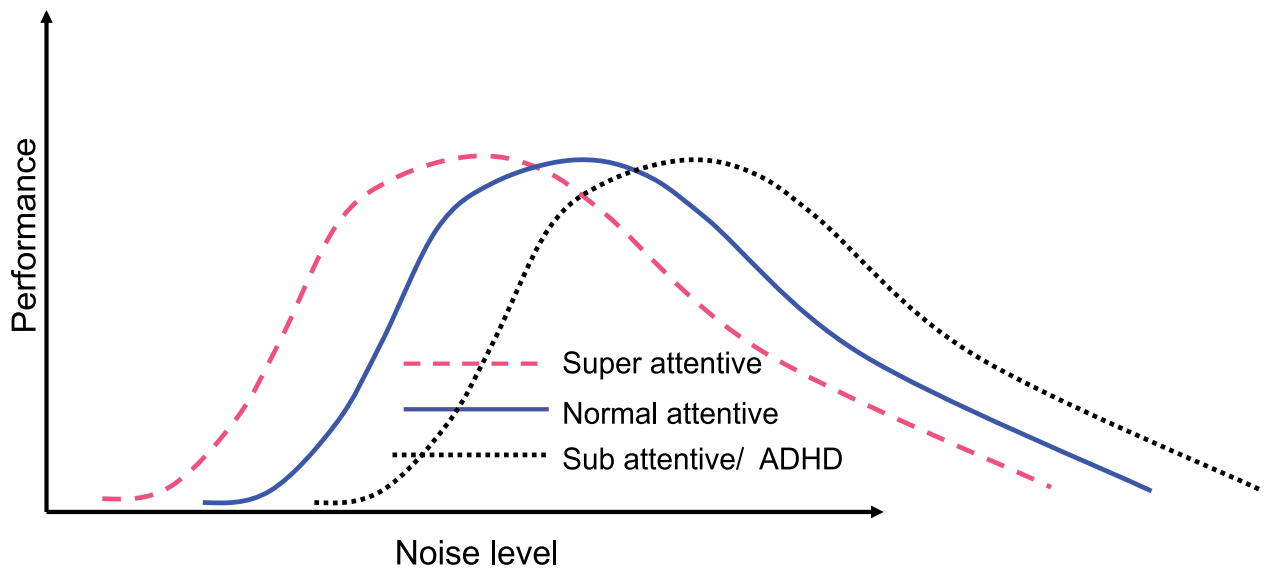

Fig. 2. The relationship between noise levels, attention ability, and cognitive performance. Sub-attentive participants (e.g. ADHD) require more noise for maximal performance according to the MBA model.

\section{The difference between distractors and noise}

As shown in numerous experiments, environmental auditory noise exerts a negative influence on schoolwork and on cognitive performance in general. Comparisons have been made with various sounds that are proposed to have a negative impact on different kinds of intellectual work. Both irrelevant meaningful speech and road traffic noise have been found ta have a detrimental effect on both semantic and episodic memory recall in adults (Hygge, Boman, \& Enmarker, 2003). Also school children were susceptible to these kinds of distractors when performing mathematical computations (Ljung, Sörqvist, \& Hygge, 2009). Aircraft noise seems to be detrimental during most kinds of work that require attention (Hygge, Evans, \& Bullinger, 2002; Matheson, et al., 2010; Stansfeld, et al., 2005), even the day after the noise exposure (Stansfeld, Hygge, Clark, \& Alfred, 2010). Semantically meaningful irrelevant information is found to be distracting, but does also interact with working memory capacity; persons that possess a high working memory capacity were less distracted by irrelevant speech than peers with lower capacity (Sörqvist, 2010a, 2010b; Sörqvist, Ljungberg, \& Ljung, 2010).

In the present study we further investigated different environmental soundscapes that have ecological relevance out of a school perspective, and their impact on a demanding working memory task. For this purpose we created four different background noises or soundscapes that could occur in a classroom setting: 1) speech or classroom noise; 2) white noise; 3) a mix of speech + white noise; and finally 4) a silent condition. We posed the question whether pure noise is the best way of introducing cognitive enhancement in inattentive children, or whether ecologically valid soundscapes could produce similar cognitive enhancement.

We predicted that auditory environmental stimuli would have a positive effect on inattentive persons and be detrimental to the attentive persons. In particular, based on previous data, we 
predicted this effect to occur for white noise. However, we posed no direct prediction on whether speech might produce cognitive enhancement effects on inattentive children.

\section{Methods}

\subsection{Participants}

Twenty-two primary school children between 7 and 10 years old $(\mathrm{M}=8.3 \mathrm{yrs})$ participated in the present study (14 boys and 8 girls). The twenty-two participants were screened and selected out of a group of 33 participants according their attention ability as reported by their teachers. The eleven that scored lowest on the attention scale where selected for the inattentive group while the ones that scored high on attention formed the attentive group. What was considered normal or above average in attention was decided according to their teacher's judgments. For this purpose a SNAP score with 18 questions were used (Swanson et al., 2007). Mean score for the inattentive group was 28.8 and for the attentive 1.5. 28 points is slightly below the cut off point for ADHD diagnosis (36). None of the participants were consequently diagnosed with ADHD or any other neuropsychiatric diagnoses. Participants were also considered to be within a normal range with regard to general school performance.

\subsection{Materials}

A visuo-spatial working memory (vsWM) test was used (spanboard; Westerberg, Hirvikoski, Forssberg, \& Klingberg, 2004). This test is a sensitive measure of cognitive deficits in ADHD. The test determines working memory capacity without being affected by previous skills or knowledge. The visuo-spatial WM task consists of red dots (memory stimuli) that are presented one at a time at a computer screen in a four by four grid. Interstimulus-intervals were 4 seconds, target is shown for $2.225 \mathrm{sec}$ and a $1.725 \mathrm{sec}$ pause is given before the next target turns up. Participants are asked to recall location, as well as the order in which the red dots appear. The working memory load increases after every second trial, and the working memory capacity is estimated based on the number of correctly recalled dots. Dependent variable was total number of correctly recalled dots.

All noise conditions were recorded and reproduced on a CD player. The speech part of the speech and noise condition was recorded at a café at Stockholm University, where five students discussed films, books, and what they did over the weekend. The equivalent continuous sound level of the white noise was set to $78 \mathrm{~dB}(\mathrm{~A})$ in the three noise conditions, in accordance with findings from earlier studies (Söderlund, et al., 2007; Söderlund, et al., 2010).

\subsection{Design}

We used a $2 \times 4$ design, where sound environment (silence vs. white noise; silence vs. speech; silence vs. speech + white noise) was the within group variable. The between group variable was teacher rated classroom attention level (attentive vs. inattentive)

\subsection{Procedure}

The testing was conducted at the children's school, following permission from parents and children. The regional ethic board in Stockholm approved the study. The participants were tested individually in a room during the school day. 
The participants were tested individually in a room during the school day. All participants used the same 15' laptop PC for the visuo-spatial test (span-board). Headphones provided the noise, and $\mathrm{dB}$ levels where checked for all participants ahead of every session. Before starting the experiment proper, two practice trials were conducted. The time taken to complete each test was approximately 5 minutes, depending on the performance level (the better performance the longer time). Altogether, the testing sessions lasted approximately 30 minutes including instructions and test trials. The noise conditions were presented in random order so each condition appeared equally many times in each position (first, second, third, and forth).

\section{Results}

A $2 \times 4$ mixed ANOVA was conducted including all noise conditions. No main effect of noise was found, but a significant overall interaction was found between noise and group $\left(\mathrm{F}(18,3)=3.44, \mathrm{p}=.039\right.$, eta $\left.^{2}=.365\right)$. The difference between groups was also significant, where the attentive group outperformed the inattentive group in all conditions $(F(20,1)=$ $12.63, \mathrm{p}=.002$, eta $\left.^{2} .387\right)$

Thereafter we conducted three separate $2 \times 2$ mixed ANOVA's, one for each noise condition. It comprised one between-subject factor, group (attentive vs. inattentive) and one withinsubjects factor, encoding stimulation condition (silence vs. white noise; silence vs. speech; silence vs. speech + white noise). The data from these tests are presented in the three graphs below. In neither of the three noise conditions we found a main effect of noise, but in two out of three conditions we found a robust noise $x$ group interaction.

In the first ANOVA, (silence vs. white noise, Figure 3) we found an interaction between group and noise. The inattentive group improved its performance whereas the attentive

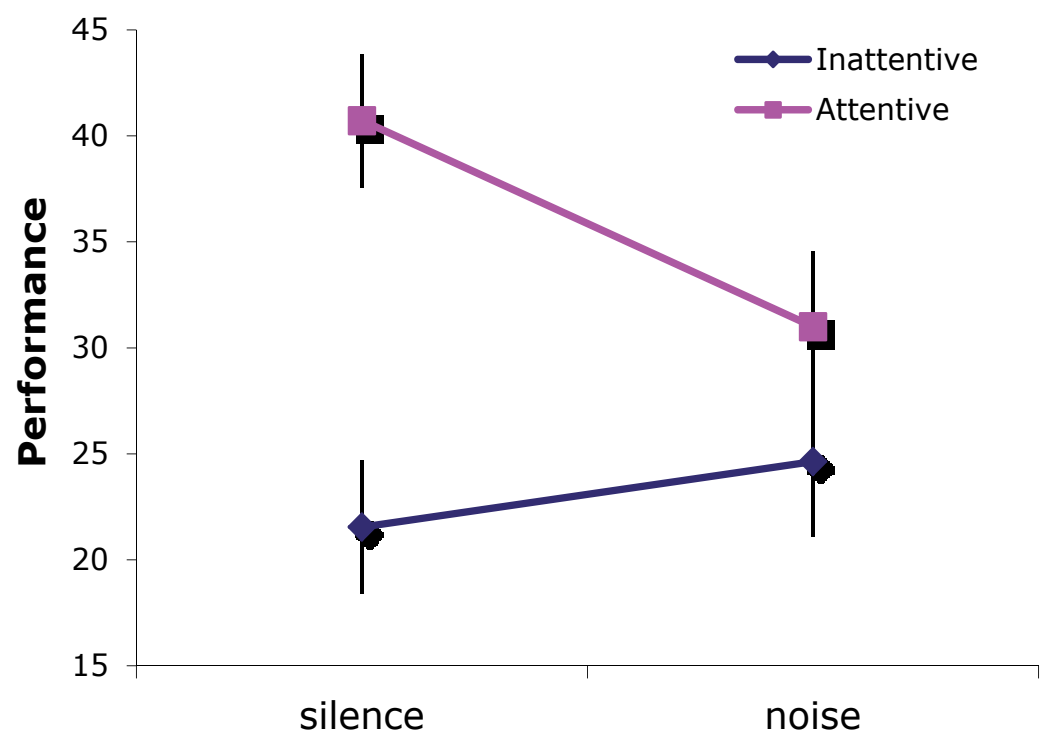

Fig. 3. Number of correctly recalled items in a visuo-spatial working memory task as a function of noise condition; silence vs. white noise in two groups: attentive ( $N=11)$ and inattentive $(\mathrm{N}=11)$. 
group declined under the white noise condition $\left(F(20,1)=8.17, p=.010\right.$, eta $\left.{ }^{2}=.290\right)$. A oneway ANOVA showed that the difference between groups in the silent condition disappeared in the noise condition $(p<.001$ vs. $p=.222)$

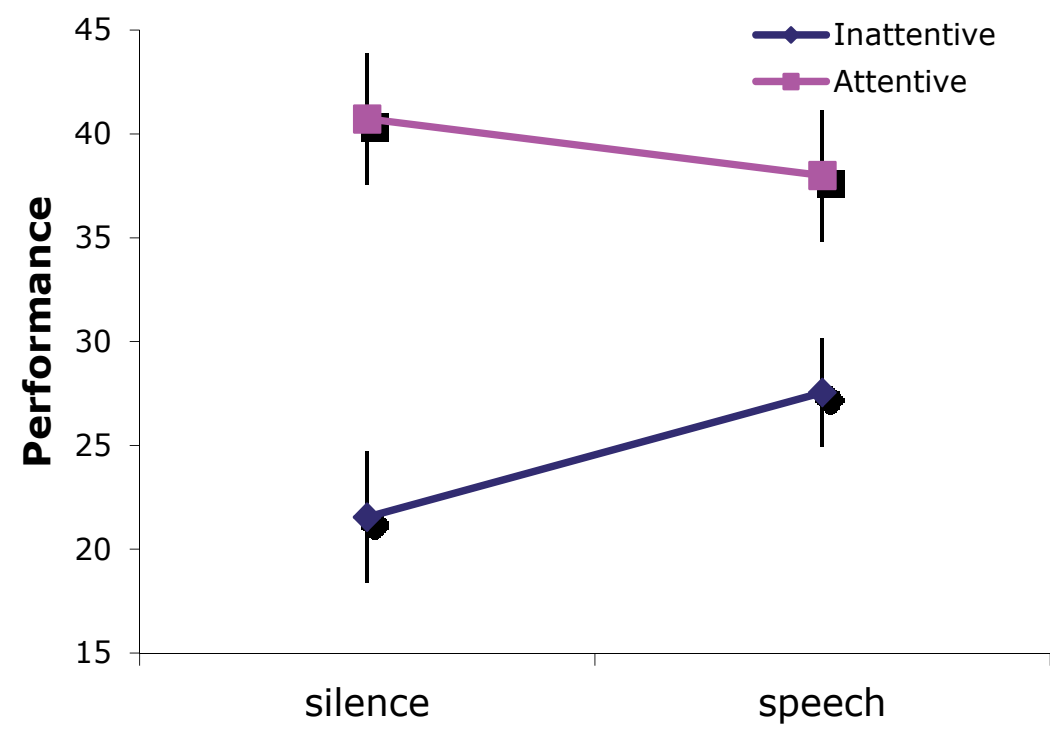

Fig. 4. Number of correctly recalled items in a visuo-spatial working memory task as a function of noise condition; silence vs. speech noise in two groups: attentive $(\mathrm{N}=11)$ and inattentive $(\mathrm{N}=11)$.

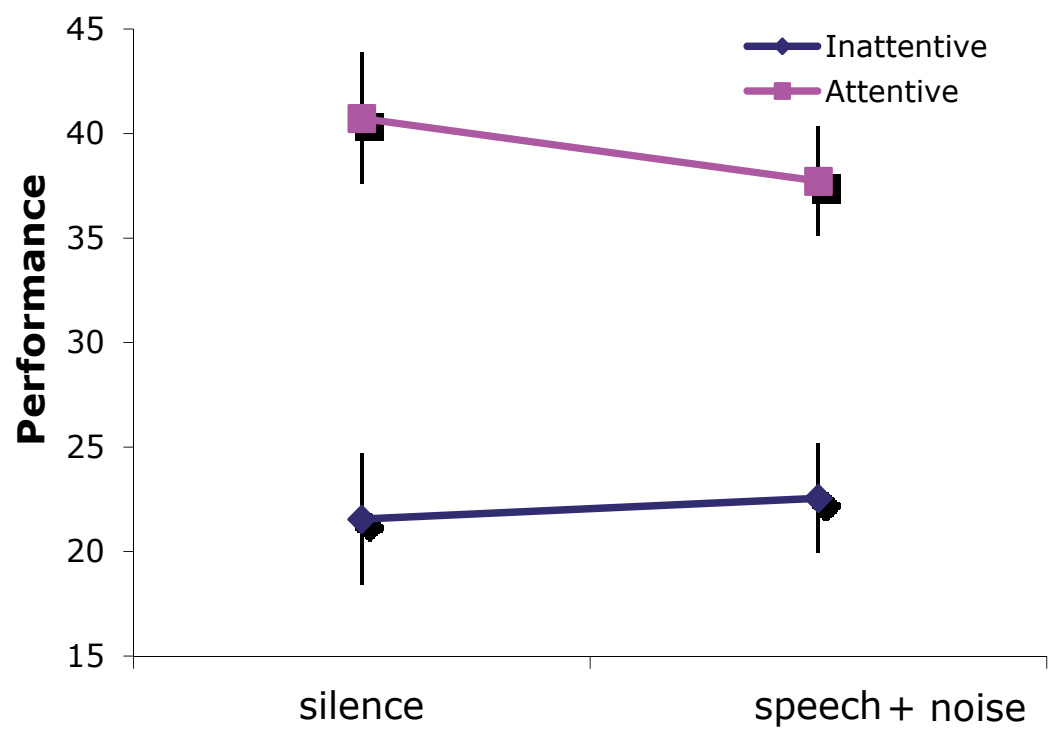

Fig. 5. Number of correctly recalled items in a visuo-spatial working memory task as a function of noise condition; silence vs. speech noise in two groups: attentive $(\mathrm{N}=11)$ and inattentive $(\mathrm{N}=11)$. 
A paired samples test, testing groups separately, revealed that the decrease for the attentive group was significant $(t(10)=-2.95, p=.015)$, whereas the increase in performance for the inattentive group did not reach significance $(t(10)=1.02, p=.333$; Figure 3$)$.

The second ANOVA (silence vs. speech, Figure 4) also showed a significant interaction, in this case, between group and speech $\left(\mathrm{F}(20,1)=6.15, \mathrm{p}=.019\right.$, eta $\left.{ }^{2}=.246\right)$. The inattentive group performed better and the attentive group performed worse in the speech condition as compared to performance in the silent condition.

A paired samples test, testing groups separately, revealed that the increase for the inattentive group was significant in the speech condition $(t(10)=3.01, p=.013)$ whereas the decrement for the attentive group was not $(t(10)=.981, p=.350)$. Finally, the one-way ANOVA showed that, despite the improvement for the inattentive group, in the speech condition the difference between groups remained significant $(F(20,1)=5.43, p=.030)$.

In the last ANOVA (silence vs, speech + noise, Figure 5) there was no interaction between the group and noise condition The robust different between groups remained in the speech + noise condition $(\mathrm{F}(20,1)=16.94, \mathrm{p}=.001)$. Neither did a paired sample t-test reveal a difference between groups as an effect of noise, both groups performed at the same level in silence as in the speech + noise condition $(p=.766$ vs. $p=.368)$

\section{Conclusions and future challenges}

As predicted, the results shown above confirm earlier findings showing different effects of white noise on attentive and inattentive children selected from a normal population. The sub-clinical inattentive group did indeed benefit from noise, while their attentive peers did not. Interestingly, the speech (classroom noise) condition did not lead to any detrimental effects for the inattentive group, but improved their performance as well. These results suggest that the beneficial effects of auditory environmental stimulation on inattentive people are found not only in pure noise conditions, as has been found previously, but generalize to broader sets of environmental sounds. In particular, this study demonstrates that noise enhancement can be found for speech. To what extent noise enhancement effects generalize to other auditory stimuli is still unexplored. However, these results suggest that we need to be open to the idea that wider sets of environmental stimulation may serve the benefit of cognitive enhancement in inattentive people. The cafeteria/classroom noise condition improved working memory performance for the inattentive group, whereas the attentive participants' performance decreased.

The reviewed literature has found inconsistent results regarding the effect of noise on performance in cognitive tasks. Studies have shown detrimental effects, no effects, and that noise interacts with other variables such as gender or time of the day (Baker \& Holding, 1993; Baker, Holding, \& Loeb, 1984; Belleville, et al., 2003; Boman, Enmarker, \& Hygge, 2005; Rouleau \& Belleville, 1996). It is plausible that controlling for participant characteristics such as age, attention ability and working memory capacity would provide other results. Differential effects of noise can be hidden in group means, were some participants improve while the performance of others is impaired.

Our findings suggest a need for further studies of psychoacoustics on different soundscapes. Previous research has focused on testing different noise levels (amplitudes in $\mathrm{dB}$ ) over larger samples of participants. However, the data presented here propose that different 
sounds need to be investigated in relation SR. White noise might be tiring to listen to for extended periods. Future research needs to address the question of whether sounds from waterfalls, shivering leafs or sounds from bamboo grass could be beneficial as well. These noise-like sounds possibly include sufficient variability in both amplitude and frequencies to induce the required increase of variability into the nervous system.

Future studies should investigate the neurophysiological traces set by noise by EEG measures. Earlier studies have shown that ADHD patients display elevated relative theta power, theta/alpha, and theta/ beta ratios during rest (Barry, Clarke, \& Johnstone, 2003). We have reason to believe that noise exposure could normalize these anomalies, and increase the level of beta and gamma activity particularly. Beta and gamma activity is crucial for higher mental activities, such as focused attention. Furthermore, the expanding field of neuro-feedback is providing / investigating interesting tools to improve attention; however, small effect sizes have been shown this far (Arns, de Ridder, Strehl, Breteler, \& Coenen, 2009; Gevensleben et al., 2009). The outcome effects of neuro-feedback might get boosted if combined with noise exposure and may, in particular, shorten the time needed to obtain robust and long-lasting effects. The field of noise-induced improvement is still in its infancy, and a lot of research is needed to get a good picture of the potential contributions from this new field. Nevertheless, we find the results very promising this far, and foresee a growing field of possible applications. In Swedish education and elsewhere, school failures increase. Current figures show that about $25 \%$ do not achieve a complete exam from compulsory or upper secondary school (Skolverket, 2005). Individually adapted study environments, utilizing the benefits of noise, may be one possibility to turn this downward trend.

\section{References}

Abikoff, H., Courtney, M. E., Szeibel, P. J., \& Koplewicz, H. S. (1996). The effects of auditory stimulation on the arithmetic performance of children with ADHD and nondisabled children. Journal of Learning Disabilities, 29(3), 238-246.

Arns, M., de Ridder, S., Strehl, U., Breteler, M., \& Coenen, A. (2009). Efficacy of neurofeedback treatment in ADHD: the effects on inattention, impulsivity and hyperactivity: a meta-analysis. Clin EEG Neurosci, 40(3), 180-189.

Baker, M. A., \& Holding, D. H. (1993). The effects of noise and speech on cognitive task performance. J Gen Psychol, 120(3), 339-355.

Baker, M. A., Holding, D. H., \& Loeb, M. (1984). Noise, sex and time of day effects in a mathematics task. Ergonomics, 27(1), 67-80.

Barry, R. J., Clarke, A. R., \& Johnstone, S. J. (2003). A review of electrophysiology in attention-deficit/hyperactivity disorder: I. Qualitative and quantitative electroencephalography. Clinical Neurophysiology, 114(2), 171-183.

Behnam, S. E., \& Zeng, F. G. (2003). Noise improves suprathreshold discrimination in cochlear-implant listeners. Hearing Research, 186(1-2), 91-93.

Belleville, S., Rouleau, N., Van der Linden, M., \& Collette, F. (2003). Effect of manipulation and irrelevant noise on working memory capacity of patients with Alzheimer's dementia. Neuropsychology, 17(1), 69-81.

Benzi, R., Parisi, G., Sutera, S., \& Vulpiani, A. (1982). Stochastic resonance in climatic change. Tellus, 34, 10-16. 
Boman, E. (2004). The effects of noise and gender on children's episodic and semantic memory. Scandinavian Journal of Psychology, 45(5), 407-416.

Boman, E., Enmarker, I., \& Hygge, S. (2005). Strength of noise effects on memory as a function of noise source and age. Noise Health, 7(27), 11-26.

Broadbent, D. E. (1951). Noise, paced performance and vigilance tasks. Medical Research Council, Applied Psychology Research Unit Report, 165(51), 8.

Broadbent, D. E. (1957). Effects of noises of high and low frequency on behaviour. Ergonomics, 1, 21-29.

Broadbent, D. E. (1958). Effect of noise on an "intellectual" task. Journal of the Acoustical Society of America, 30, 824-827.

Corbett, B., \& Stanczak, D. E. (1999). Neuropsychological performance of adults evidencing Attention-Deficit Hyperactivity Disorder. Archives in Clinical Neuropsychology, 14(4), 373-387.

Douglass, J. K., Wilkens, L., Pantazelou, E., \& Moss, F. (1993). Noise enhancement of information transfer in crayfish mechanoreceptors by stochastic resonance. Nature, 365(6444), 337-340.

Gammaitoni, L., Hänggi, P., Jung, P., \& Marchesoni, F. (1998). Stochastic resonance. Reviews of Modern Physics, 70(1), 223-287.

Geffner, D., Lucker, J. R., \& Koch, W. (1996). Evaluation of auditory discrimination in children with ADD and without ADD. Child Psychiatry \& Human Development, 26(3), 169-180.

Gerjets, P., Graw, T., Heise, E., Westermann, R., \& Rothenberger, A. (2002). Deficits of action control and specific goal intentions in hyperkinetic disorder. II: Empirical results/Handlungskontrolldefizite und störungsspezifische Zielintentionen bei der Hyperkinetischen Störung: II: Empirische Befunde. Zeitschrift für Klinische Psychologie und Psychotherapie: Forschung und Praxis, 31(2), 99-109.

Gevensleben, H., Holl, B., Albrecht, B., Vogel, C., Schlamp, D., Kratz, O., . Heinrich, H. (2009). Is neurofeedback an efficacious treatment for ADHD? A randomised controlled clinical trial. J Child Psychol Psychiatry, 50(7), 780-789. doi: JCPP2033 10.1111/j.1469-7610.2008.02033.x

Hygge, S., Boman, E., \& Enmarker, I. (2003). The effects of road traffic noise and meaningful irrelevant speech on different memory systems. [Research Support, Non-U.S. Gov't]. Scandinavian journal of psychology, 44(1), 13-21.

Hygge, S., Evans, G. W., \& Bullinger, M. (2002). A prospective study of some effects of aircraft noise on cognitive performance in schoolchildren. [Clinical Trial Research Support, Non-U.S. Gov't Research Support, U.S. Gov't, P.H.S.]. Psychological science, 13(5), 469-474.

Klatte, M., Meis, M., Sukowski, H., \& Schick, A. (2007). Effects of irrelevant speech and traffic noise on speech perception and cognitive performance in elementary school children. Noise Health, 9(36), 64-74.

Ljung, R., Sörqvist, P., \& Hygge, S. (2009). Effects of road traffic noise and irrelevant speech on children's reading and mathematical performance. Noise $\mathcal{E}$ health, 11(45), 194198. doi: $10.4103 / 1463-1741.56212$

Manjarrez, E., Mendez, I., Martinez, L., Flores, A., \& Mirasso, C. R. (2007). Effects of auditory noise on the psychophysical detection of visual signals: cross-modal stochastic resonance. Neurosci Lett, 415(3), 231-236. 
Matheson, M., Clark, C., Martin, R., van Kempen, E., Haines, M., Barrio, I. L., . . Stansfeld, S. (2010). The effects of road traffic and aircraft noise exposure on children's episodic memory: the RANCH project. [Comparative Study Multicenter Study

Research Support, Non-U.S. Gov't]. Noise E health, 12(49), 244-254. doi: 10.4103/14631741.70503

Moss, F., Ward, L. M., \& Sannita, W. G. (2004). Stochastic resonance and sensory information processing: a tutorial and review of application. Clinical Neurophysiology, 115(2), 267-281.

Novak, P., \& Novak, V. (2006). Effect of step-synchronized vibration stimulation of soles on gait in Parkinson's disease: a pilot study. J Neuroeng Rehabil, 3, 9.

Pan, W., Soma, R., Kwak, S., \& Yamamoto, Y. (2008). Improvement of motor functions by noisy vestibular stimulation in central neurodegenerative disorders. J Neurol, 255(11), 1657-1661. doi: 10.1007/s00415-008-0950-3

Priplata, A. A., Niemi, J. B., Harry, J. D., Lipsitz, L. A., \& Collins, J. J. (2003). Vibrating insoles and balance control in elderly people. Lancet, 362(9390), 1123-1124.

Priplata, A. A., Patritti, B. L., Niemi, J. B., Hughes, R., Gravelle, D. C., Lipsitz, L. A., . . . Collins, J. J. (2006). Noise-enhanced balance control in patients with diabetes and patients with stroke. Ann Neurol, 59(1), 4-12.

Rickman, D. L. (2001). The effect of classroom-based distraction on continuous performance test scores of ADHD and nonADHD children. Dissertation Abstracts International: Section B: The Sciences \& Engineering, 61(10-B), 5578.

Rouleau, N., \& Belleville, S. (1996). Irrelevant speech effect in aging: an assessment of inhibitory processes in working memory. The Journals of Gerontology. Series B, Psychological Sciences and Social Sciences, 51(6), P356-363.

Russell, D. F., Wilkens, L. A., \& Moss, F. (1999). Use of behavioural stochastic resonance by paddle fish for feeding. Nature, 402(6759), 291-294.

Sikström, S., \& Söderlund, G. B. W. (2007). Stimulus-dependent dopamine release in attention-deficit/hyperactivity disorder. [Review]. Psychological review, 114(4), 10471075. doi: 10.1037/0033-295X.114.4.1047

Simonotto, E., Spano, F., Riani, M., Ferrari, A., Levero, F., Pilot, A., . . Moss, F. (1999). fMRI studies of visual cortical activity during noise stimulation. Neurocomputing: An International Journal. Special double volume: Computational neuroscience: Trends in research 1999, 26-27, 511-516.

Skolverket. (2005). Education Results National level. . Report, 257. (Part 1), 1-172.

Solanto, M. V. (2002). Dopamine dysfunction in AD/HD: integrating clinical and basic neuroscience research. Behavioral Brain Research, 130(1-2), 65-71.

Stansfeld, S., Berglund, B., Clark, C., Lopez-Barrio, I., Fischer, P., Ohrstrom, E., . . Berry, B. F. (2005). Aircraft and road traffic noise and children's cognition and health: a cross-national study. Lancet, 365(9475), 1942-1949.

Stansfeld, S., Hygge, S., Clark, C., \& Alfred, T. (2010). Night time aircraft noise exposure and children's cognitive performance. [Multicenter Study]. Noise $\mathcal{E}$ health, 12(49), 255262. doi: $10.4103 / 1463-1741.70504$

Swanson, J. M., Schuck, S., Mann, M., Carlson, C., Hartman, K., Sergeant, J., . . McCleary, R. (2007). Categorical and dimensional definitions and evaluatios of ADHD: The SNAP and the SWAN rating scales. Retrieved December 2007 from http://www.ADHD.net. 
Söderlund, G. B. W., Marklund, E., \& Lacerda, F. (2009). Auditory White Noise Enhances Cognitive Performance Under Certain Conditions: Examples from Visuo-Spatial Working Memory and Dichotic Listening Tasks. Proceedings from FONETICS 2009, Dept of Linguistics, Stockholm University, Stockholm, Sweden.

Söderlund, G. B. W., Sikstrom, S., \& Smart, A. (2007). Listen to the noise: Noise is beneficial for cognitive performance in ADHD. [Controlled Clinical Trial]. Journal of child psychology and psychiatry, and allied disciplines, 48(8), 840-847. doi: 10.1111/j.14697610.2007.01749.x

Söderlund, G. B. W., \& Sikström, S. (2008). Positive effects of noise on cogntive performance: Explaining the Moderate Brain Arousal Model. Proceedings from ICBEN, International Comission on the Biological Effects of Noise.

Söderlund, G. B. W., Sikström, S., Loftesnes, J. M., \& Sonuga-Barke, E. (2010). The effects of background white noise on memory performance in inattentive school children. Behavioral and Brain Functions, 6(55). doi: doi:10.1186/1744-9081-6-55

Sörqvist, P. (2010a). High working memory capacity attenuates the deviation effect but not the changing-state effect: further support for the duplex-mechanism account of auditory distraction. [Research Support, Non-U.S. Gov't]. Memory \& cognition, 38(5), 651-658. doi: 10.3758/MC.38.5.651

Sörqvist, P. (2010b). The role of working memory capacity in auditory distraction: a review. [Research Support, Non-U.S. Gov't Review]. Noise E health, 12(49), 217-224. doi: 10.4103/1463-1741.70500

Sörqvist, P., Ljungberg, J. K., \& Ljung, R. (2010). A sub-process view of working memory capacity: evidence from effects of speech on prose memory. [Research Support, Non-U.S. Gov't]. Memory, 18(3), 310-326. doi: 10.1080/09658211003601530

Usher, M., \& Feingold, M. (2000). Stochastic resonance in the speed of memory retrieval. Biological Cybernetics, 83(6), L11-16.

Volkow, N. D., Wang, G. J., Kollins, S. H., Wigal, T. L., Newcorn, J. H., Telang, F., . . . Swanson, J. M. (2009). Evaluating dopamine reward pathway in ADHD: clinical implications. JAMA, 302(10), 1084-1091. doi: 302/10/1084 [pii] 10.1001/jama.2009.1308

Volkow, N. D., Wang, G. J., Newcorn, J., Fowler, J. S., Telang, F., Solanto, M. V., . . Pradhan, K. (2007). Brain dopamine transporter levels in treatment and drug naive adults with ADHD. Neuroimage, 34(3), 1182-1190.

Wells, C., Ward, L. M., Chua, R., \& Timothy Inglis, J. (2005). Touch noise increases vibrotactile sensitivity in old and young. Psychological Science, 16(4), 313-320.

Westerberg, H., Hirvikoski, T., Forssberg, H., \& Klingberg, T. (2004). Visuo-spatial working memory span: a sensitive measure of cognitive deficits in children with ADHD. Child Neuropsychology, 10(3), 155-161.

Wilkinson, D., Nicholls, S., Pattenden, C., Kilduff, P., \& Milberg, W. (2008). Galvanic vestibular stimulation speeds visual memory recall. Exp Brain Res, 189(2), 243-248. doi: 10.1007/s00221-008-1463-0

Wilkinson, D., Zubko, O., Degutis, J., Milberg, W., \& Potter, J. (2009). Improvement of a figure copying deficit during subsensory galvanic vestibular stimulation. $J$ Neuropsychol. doi: jnp194 [pii] 10.1348/174866409X468205 
Yamamoto, Y., Struzik, Z. R., Soma, R., Ohashi, K., \& Kwak, S. (2005). Noisy vestibular stimulation improves autonomic and motor responsiveness in central neurodegenerative disorders. Ann Neurol, 58(2), 175-181.

Zeng, F. G., Fu, Q. J., \& Morse, R. (2000). Human hearing enhanced by noise. Brain Research, 869(1-2), 251-255.

Zentall, S. S. (1986). Effects of Color Stimulation on Performance and Activity of Hyperactive and Nonhyperactive Children. Journal of Educational Psychology, 78(2), 159-165.

Zentall, S. S., \& Dwyer, A. M. (1989). Color Effects on the Impulsivity and Activity of Hyperactive Children. Journal of School Psychology, 27(2), 165-173.

Zentall, S. S., Falkenberg, S. D., \& Smith, L. B. (1985). Effects of color stimulation and information on the copying performance of attention-problem adolescents. Journal of Abnormal Child Psychology, 13(4), 501-511.

Zentall, S. S., \& Shaw, J. H. (1980). Effects of classroom noise on performance and activity of second-grade hyperactive and control children. Journal of educational psychology, 72(6), 830-840.

Zentall, S. S., \& Zentall, T. R. (1983). Optimal stimulation: A model of disordered activity and performance in normal and deviant children. Psychological Bulletin, 94(3), 446471. 


\section{Part 4}

EEG Biofeedback 



\title{
QEEG Characteristics and Biofeedback Modalities in Children with ADHD
}

\author{
Nada Pop-Jordanova \\ Department for Psychophysiology, University Pediatric Clinic, Skopje, \\ R. Macedonia
}

\section{Introduction}

Attention Deficit Hyperactivity Disorder (ADHD) is one of the most frequent neuropsychiatric diagnoses during childhood, varying between 2 and $10 \%$ depending on country or cultural area. In our country (R. Macedonia), the incidence of ADHD is about $2 \%$. The other labels of the disorder introduced in the past are hyperactivity, hyperkinesis, hyperkinetic syndrome, minimal brain dysfunction and minimal brain damage. According to DSM - IV (American Psychiatric Association, 1994) there are three main clinical forms of this disorder: inattentive, hyperactive/impulsive and combined. European diagnostic criteria for hyperkinetic disorder as defined by the ICD-10 (International Classification of Diseases, $10^{\text {th }}$ revision, 1993) include children displaying developmentally inappropriate levels of attention, hyperactivity and impulsivity that begin in childhood and cause impairment to school performance, intellectual functioning, social skills, driving and occupational functioning. Generally, ICD-10 criteria are more restrictive than DSM-IV diagnosis because they need a greater degree of symptom expression.

The overlapping of all three forms with learning disabilities is very high (up to $70 \%$ ), as well as with conduct problems. The inattentive (ADD) form mainly overlaps with anxiety disorders and learning disability, while hyperactive form (ADHD) mostly overlaps with conduct disorder. Some children with ADHD have movement disorders or tics and occasionally they may have seizure disorders (Barkley, 1990). Strong genetic component related to defect in chromosome 11 is showed in some studies (Anokhin et all. 2006). Concerning the involvement of specific neurotransmitter systems in the pathophysiology of ADHD some authors suggest that the catecholaminergic dysregulations are centrally involved.

This chapter is mainly devoted to the QEEG characteristics of ADHD in children, connected to endophenotypes, event related potentials, brain rate and biofeedback treatment. Firstly, it will be explained why QEEG recording is important for the exact diagnostics and the therapy planning for ADHD children. Based on QEEG differentiation, the endophenotypes clustering enables more precise diagnostics. In addition, it will be shown that event related potentials (ERP's) are related to the possible dysfunction of executive system, as a part of the brain the most involved in this disorder. The applied calculation of brain rate is our original proposal for the evaluation of general mental activation level, considering under or overarousal states, so that the planning of the treatment protocols becomes more easy. 
Finally, biofeedback will be offer as the non-pharmaceutical treatment for ADHD, with long-term effects.

\section{QEEG recording}

The EEG recording is a noninvasive, painless, and safe measurement using digital technology of electrical patterns at the surface of the scalp which primarily reflect cortical electrical activity or brainwaves. The Quantitative EEG procedure uses multi-electrode EEG recording where the data are processed with various algorithms and than statistically analyzed comparing values with normative database reference values. The processed EEG could be also converted into color maps of brain functioning called brain maps.

The placement of electrodes from the first recording (introduced in 1924) was strongly precise and the system was called the10-20 International System of Electrode Placement. This system of recording is used until now. The "10" and "20" refer to the fact that the actual distances between adjacent electrodes are either $10 \%$ or $20 \%$ of the total front-back or rightleft distance of the skull (Fig.1).

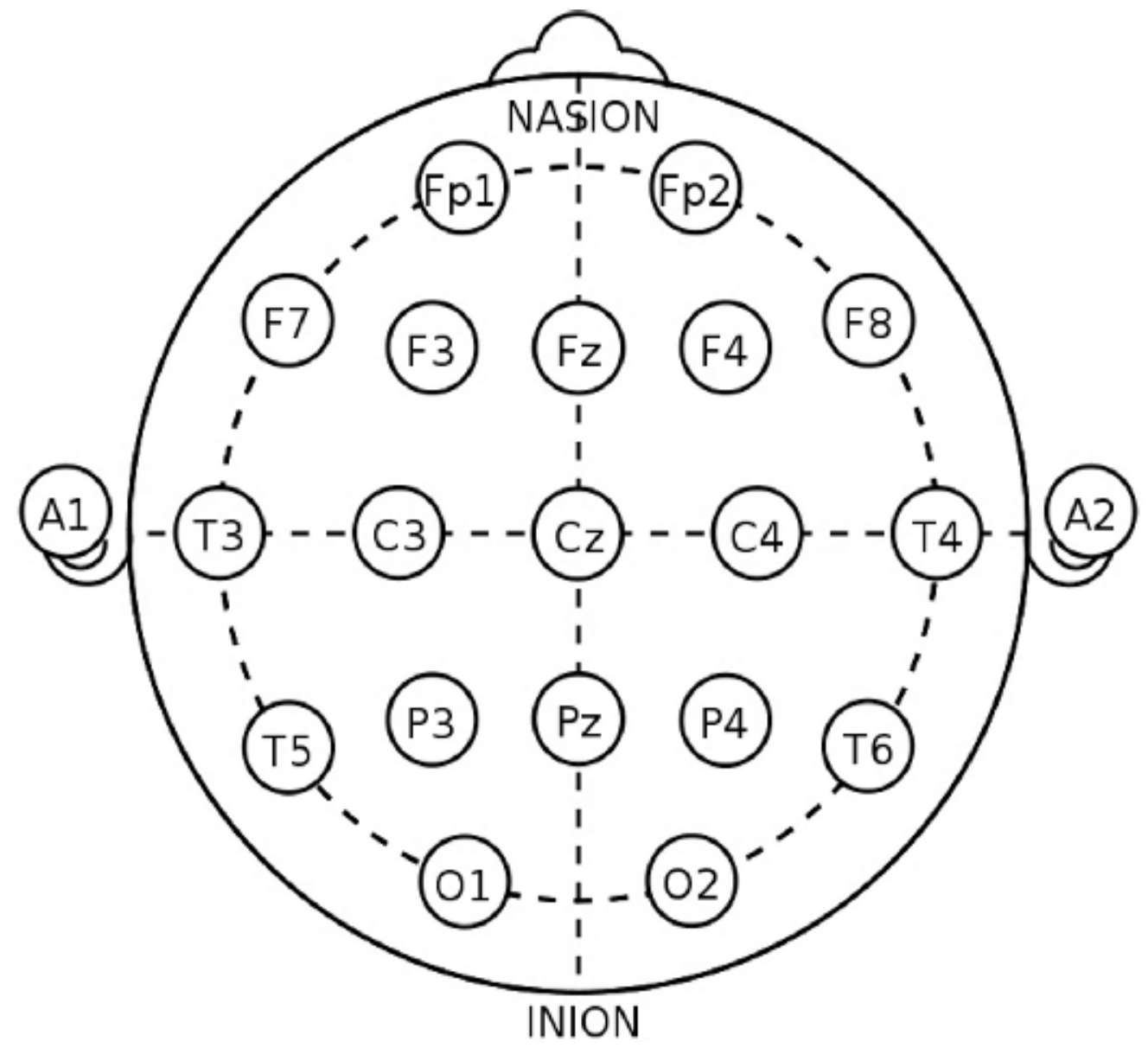

Fig. 1. The 10-20 International System 
Each site has a letter to identify the lobe and a number to identify the hemisphere location. The letters F, T, C, P and O stand for Frontal, Temporal, Central, Parietal, and Occipital, respectively. Note that in fact there exists no central lobe, the " $\mathrm{C}$ " letter is only used for identification purposes only. The letter "z" (zero) refers to an electrode placed on the midline. Even numbers $(2,4,6,8)$ refer to electrode positions on the right hemisphere, whereas odd numbers $(1,3,5,7)$ refer to those on the left hemisphere.

One important element of the EEG signal is its rhythmicity. Rhythms differ in frequency, location, mechanism of generation and functional meaning. They evolve in time, so that a representative EEG recording usually takes three or more minutes (in our patients we use five minute recording). For compressing the information about rhythmicity over time, as a most powerful method, the Fourier analysis is used. The parameters of Fourier analysis can be adjusted to the goal of a specific task.

When EEG is recorded from many electrodes that cover the whole cortex, it is possible to compute a 2D representation of a measured EEG characteristic. The characteristics could be either potential or power taken at a particular frequency.

Computerized analysis of EEG signals involve a number of factors: frequency distribution, voltage (as amplitude of the signal), locus of the phenomena, wave shape morphology, inter-hemispheric symmetries, character of waveform occurrence and reactivity (changes in an EEG parameter with changes in state).

The pattern of neuronal oscillations plays an important role in the evaluation and treatment of children (and adult as well) with ADHD. These patients are characterized with QEEG abnormalities in up to $80 \%$. In this population, frontal regions are most likely to show deviations from normal development, with disturbed thalamo-cortical and septalhippocampal pathways, altogether named as executive system.

The term "executive functions" refers to the coordination and control of motor and cognitive actions to attain specific goals. In neuropsychology, the term "executive functions" has long been used as a synonym for frontal lobe function. A modern view postulates several sub-components in the hypothetical executive mechanism. In a frequently cited classification, Smith and Jonides (1999) distinguished between mechanisms relating to (a) attention and inhibition, (b) task management, (c) planning, (d) monitoring and (e) coding. There is, however, no consensus on the number and the precise nature of functional subcomponents.

As we said previously, it is supposed that the main brain system impaired in ADHD is the executive system. The executive system is characterized by two parameters: general activation of the system (arousal $=\mathrm{A}$ ) and the response associated with different operations such as working memory, action selection, action inhibition and action monitoring (focused activation $=\mathrm{At})$.

It is well known that EEG recorded in eyes open (EO) and eyes closed (EC) resting state is good indicator of metabolic activity in the brain cortex. Low metabolic activity in the area that generates the corresponding EEG is characterized by increase of slow activities (delta and theta waves) and decrease of beta activities. It means that in this condition, the level of activation is low (low A), as well as the amplitude of responses we named focused activity (At) is also low. 
The early studies of EEG abnormalities in children with minimal brain dysfunction are made in the period 1930-1950. Basically, EEG studies showed the increase of delta and theta bands in central and frontal regions during EEG recording. This finding was confirmed by quantitative EEG analysis in the 1970s and later. These studies are supported by recent PET (positron emission tomography) and SPECT (single photon emission computerized tomography) scan studies, which also indicate abnormalities in cerebral metabolism in these particular brain areas.

The most studies of QEEG in ADHD population confirm elevated levels of slow wave power in frontal region indicating frontal lobe deactivation, in comparison to normal children. These children experience decreased metabolism in prefrontal regions of the brain and have been good candidates for the use of stimulant medication or neurofeedback. Other children show deficits in limbic system activity and are characterized as having oppositional behavior, emotional outbursts, and impulsiveness. These children also show significant decreased metabolism in the prefrontal lobes and the anterior cingulated gyrus. They are good candidates for the use of tricyclic antidepressants. The third subgroup comprises individuals who have increased activity in the medial superior frontal gyrus. Although these children experience ADD they are often characterized as having an attention deficit with obsessive-compulsive disorder. They have a very short attention span and are often impulsive and oppositional. These patients sometimes respond to clomipramine. By far the most common group of children with ADD consists of those with excessive slow activity in frontal and central brain regions.

Having in mind that the absolute values of EEG spectra depend on some brain unrelated features, such as thickness of the skull, a relative parameter defined as the theta-beta ratio is introduced. Multi-centric studies in USA (Monastra et al. 2001; Lubar 1991, 1997; Mann et al 1992) used the theta-beta ratio as an index of inattention. This so called inattention index is defined as a ratio of theta EEG power (measured within the 4-8 Hz frequency band) and beta EEG power (measured within 13-21 Hz frequency band). Usually this index is calculated by EEG recording at a single place $\mathrm{Cz}$ in reference to linked ears. It was found that this index is three times higher in inattentive and combine types of ADHD children at the age of 6-10 years compared with normal group. Monastra et al. (2001) found that the sensitivity of this index was $86 \%$ and his specificity is $98 \%$.

Opposite to these findings Russian scientists from the Human Brain Institute in St Petersburg (Kropotov, 2009) showed that this index is a good measure only for a part of ADHD population. Mapping this index in normal population showed that the location of the maximum of this index changes significantly with age. For example, the maximum of theta-beta index move from central-parietal location at 7-8 years old children to frontalcentral location in adults. The conclusion was that for better results in discriminating the ADHD population from healthy subjects this index must be measured in different electrode position depending on age.

Generally, we can infer that QEEG recording is needed for more precise diagnostics of ADHD, in addition to the clinical criteria. The method is a non invasive, relatively easy for manipulation with standardized locations of the electrodes over the scalp. The recording is performed in different conditions: eyes open, eyes closed and during some cognitive tasks (Go/ NoGo paradigm, visual or auditive performance tasks, reading or math tasks). QEEG is useful if neurofeedback treatment is planned to be applied. 


\section{Endophenotypes}

Endophenotypes are characteristics indicating biological markers of the brain disease. Endophenotype must obey some requirements: a) to be stable and reproducible in time intervals during which behavioral patterns associated with the state of the brain remain unchanged; b) endophenotype must reflect a function of a certain brain system that in a specific way determine the human behavior; and c) it must be inherited. EEG spectra, or amplitude of the background EEG in certain frequency bands, obey these requirements and consequently can be considered as endophenotypes.

We said in the previous text that EEG oscillations (expressed as e.g. alpha or theta rhythm) wane and wax in time. The degree of variability of the basic oscillation depends on the frequency band and the state (eyes open, eyes closed, task etc) of the subject. For example, alpha spindles in the posterior regions vary with periods of few seconds, and burst of the frontal midline theta appear with interburst periods of few deco-seconds. However, if averaged over significantly long time intervals the resulting spectra become quite stable characteristics of the brain. So, these characteristics of EEG spectra could be considered as a reliable and stable estimation of the brain functional state.

Different oscillations reflect different mechanisms: alpha rhythms reflect the state of thalamocortical pathways; frontal midline theta rhythms reflect functioning of the limbic system; beta rhythms are more local reflecting state of specific cortical areas. So, defining abnormal rhythmic activities in EEG and associating these abnormalities with distinct systems fit the second requirement for endophenotypes as the biological markers of disease. Finally, there is strong experimental evidence that spectral characteristics of EEG are inherited.

Generally, it can be said that endophenotype is becoming an important concept in the study of different mental disorders such as ADHD, schizophrenia etc. The term was coined in 1966 and applied in psychiatry by Gottesman and Shileds in 1972. It is gradually substituting some similar terms such as "biological markers", "vulnerability marker", "subclinical traits" or "intermediate phenotype". An endophenotype may be neurophysiological, biochemical, endocrinological, neuroanatomical, or neurophychological in nature. Endophenotype represents simpler clue to genetic mechanism than the behavioral symptoms.Thereby, endophenotypes can help to define subtypes of a particular disorder and can be used as a quantitative trait in genetic analyses of probands and families.

For example, some researchers suggest a biological rationale for lack of inhibition as an endophenotype for ADHD. Inhibitory tasks activate the prefrontal cortex and basal ganglia, regions in which the dopamine system is associated with executive functioning. Tests of inhibitory function, such as the stop signal task, often consists of two concurrent tasks (a "Go" task and "NoGo" task) in which the subject is signaled to produce a particular response. An individual with a poor inhibitory system will have a long reaction time. This deficit, being replicated several times, is concerned to be specific to ADHD. Imaging studies have shown that this inhibitory task activates the prefrontal cortex and basal ganglia. Inhibition also correlates with family history, so that $48.1 \%$ subjects with poor inhibition have a family history of ADHD compared with only 7.7\% of normal controls. (Crosbie, Schachar, 2001).

Endophenotypes may also be useful in exploring different pathways leading up to the disorder. Patients having the same diagnosis may differ strongly in the number and severity of symptoms they portray, suggesting heterogeneity in the causal pathways. Creating more 
homogeneous subgroups of patients based on their endophenotypic functioning, may facilitate unraveling these differential causal pathways.

Recently, a QEEG spectrum classification of ADHD population has been developed defining four main subtypes: I subtype (abnormal increase of delta-theta frequency range centrally or centrally-frontally), II subtype (abnormal increase of frontal midline theta rhythm), III subtype (abnormal increase of beta activity frontally), and IV subtype (excess of alpha activities at posterior, central, or frontal leads).

In the following, I will show (on Figs. 2-8) some our examples of QEEG spectra from different subtypes of ADHD children (Pop-Jordanova 2007, 2009; Zorcec 2007, 2008).

The first and second subtypes are characterized clinically with inattention, while in the third subtype mainly hyperactivity, impulsivity and social inadaptation are prevalent. The low attention span is also the main complain of children with alpha excess.

Among the investigated Macedonian ADHD children (over 250), very slow alpha excess (subtype 4 ) was showed in $25 \%$ of children, and high theta/beta ratio in frontal-central cortex (subtype 1) in other $25 \%$ of children. The majority of $48 \%$ belong to the combined 1 and 2 subtypes. Very rarely (under $2 \%$ ) we found subtype III were overactive cortex is typical finding (Pop-Jordanova et al. 2007; Zorcec et al. 2007, 2008).
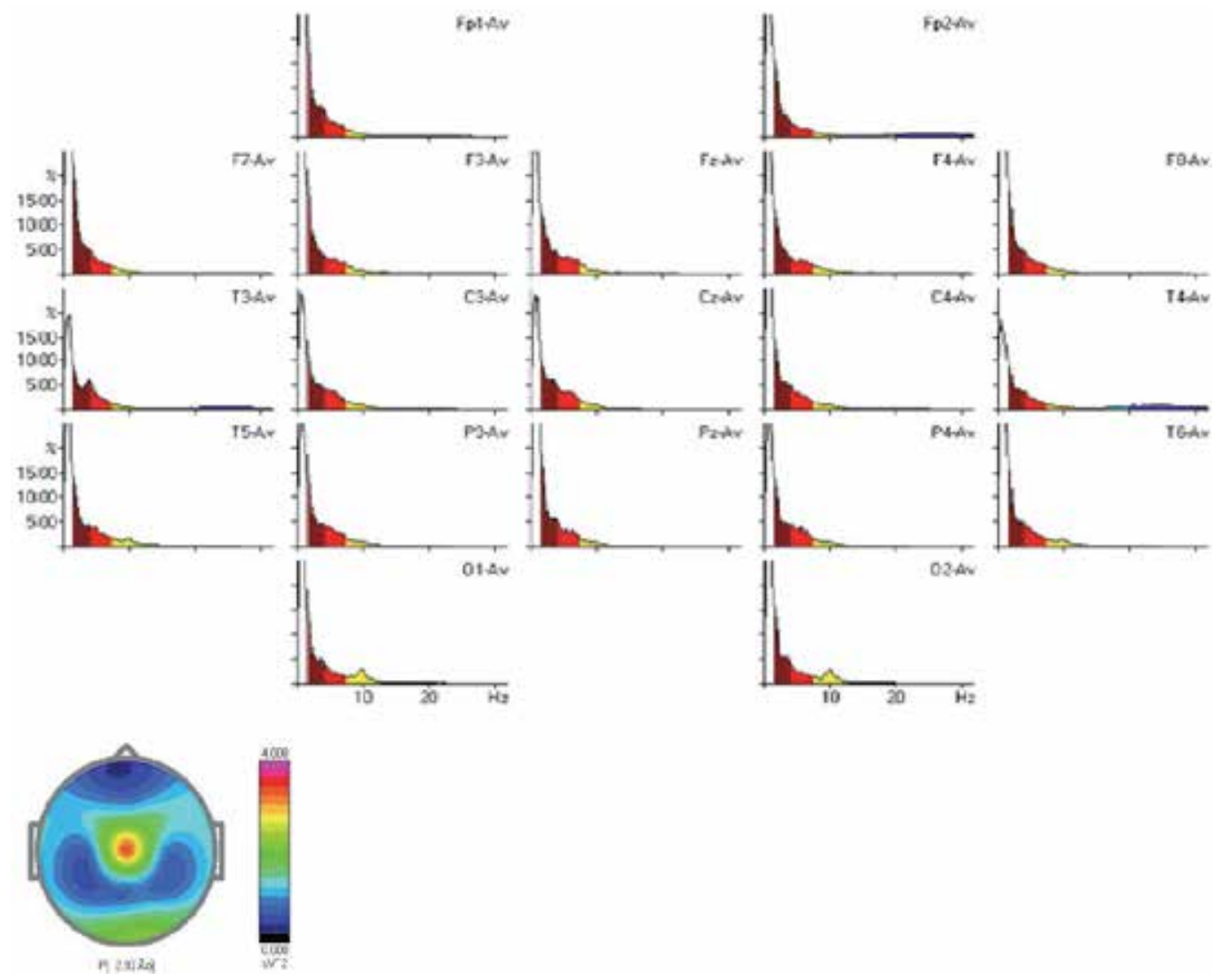

Fig. 2. Subtype I - High delta and theta amplitudes in frontal-central cortex 
As can be seen on Fig. 1, in frontal and central region of brain cortex the dominant frequencies are in the range of delta and theta waves, while alpha and beta waves are practically absent.

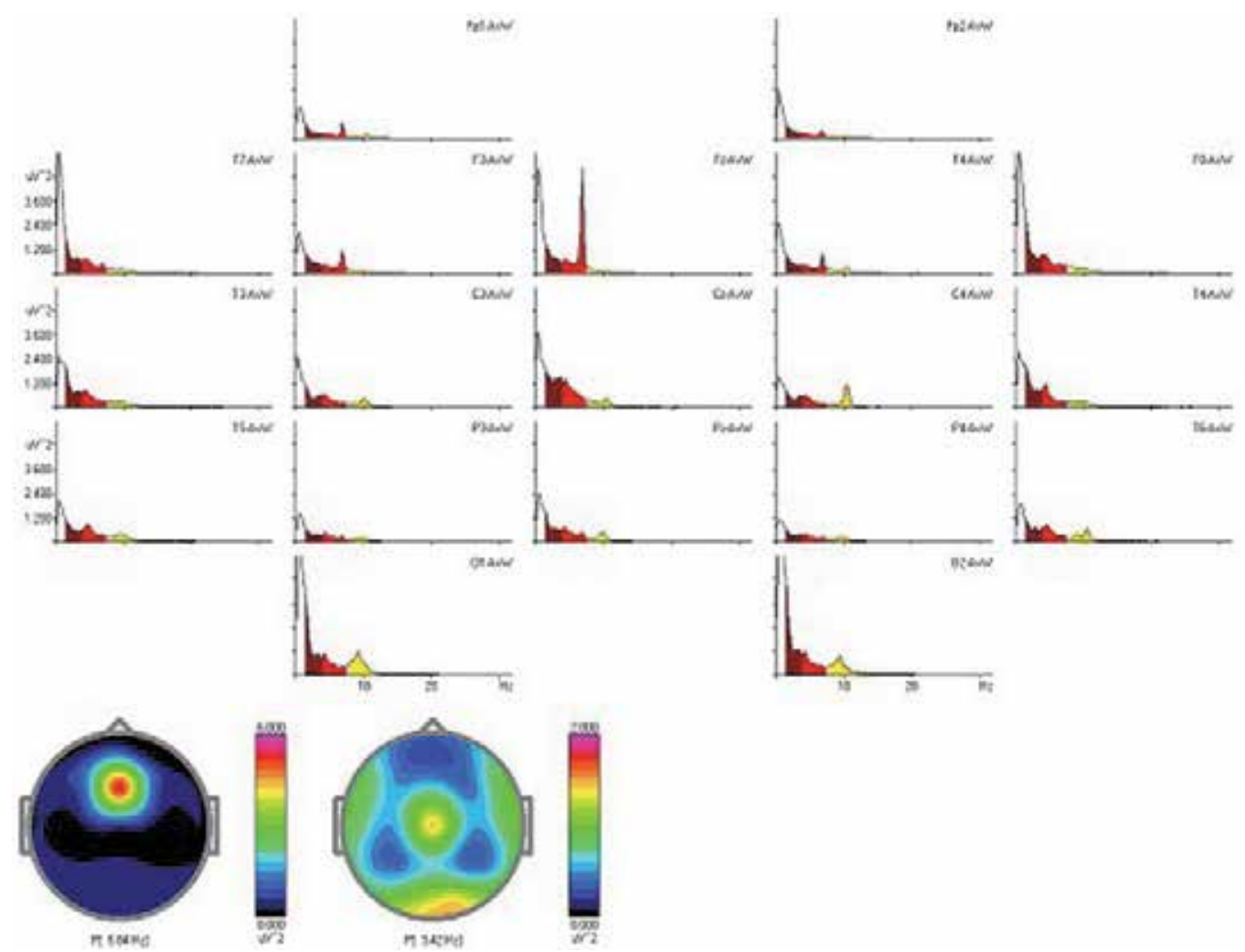

Fig. 3. Subtype II Excess of frontal-midline theta

On Fig.3 we can see a pick of theta activity (P $6.54 \mathrm{~Hz}$ ) in frontal-midline area as the most important finding. It is also combined with generally slow activity (lack of alpha and beta activity). The same is visible on the row EEG record shown in Fig. 4.

Both Fig.5 and 6 show over activation of the cortex expressed by the pronounced beta brain waves.

In addition to the QEEG, SPECT (Amen, 1997, Amen et al. 1998) shows the corresponding specifics in ADHD children which can be summarized as:

- frontal lobe deactivation (presented clinically as ADD, which usually respond to therapy with Ritalin)

- temporal lobe dysfunction (very like temporal epilepsy, respond to therapy with anticonvulsant)

- homogenous cortical suppression (respond to combination antidepressives + Ritalin)

- increased activity in the anterior medial aspects of the frontal lobes - gyros rectus (responds to alpha adrenergic blockers like clonidine)

- hypofrontality at rest, but normal frontal activity in intellectual stress (respond to Ritalin) 


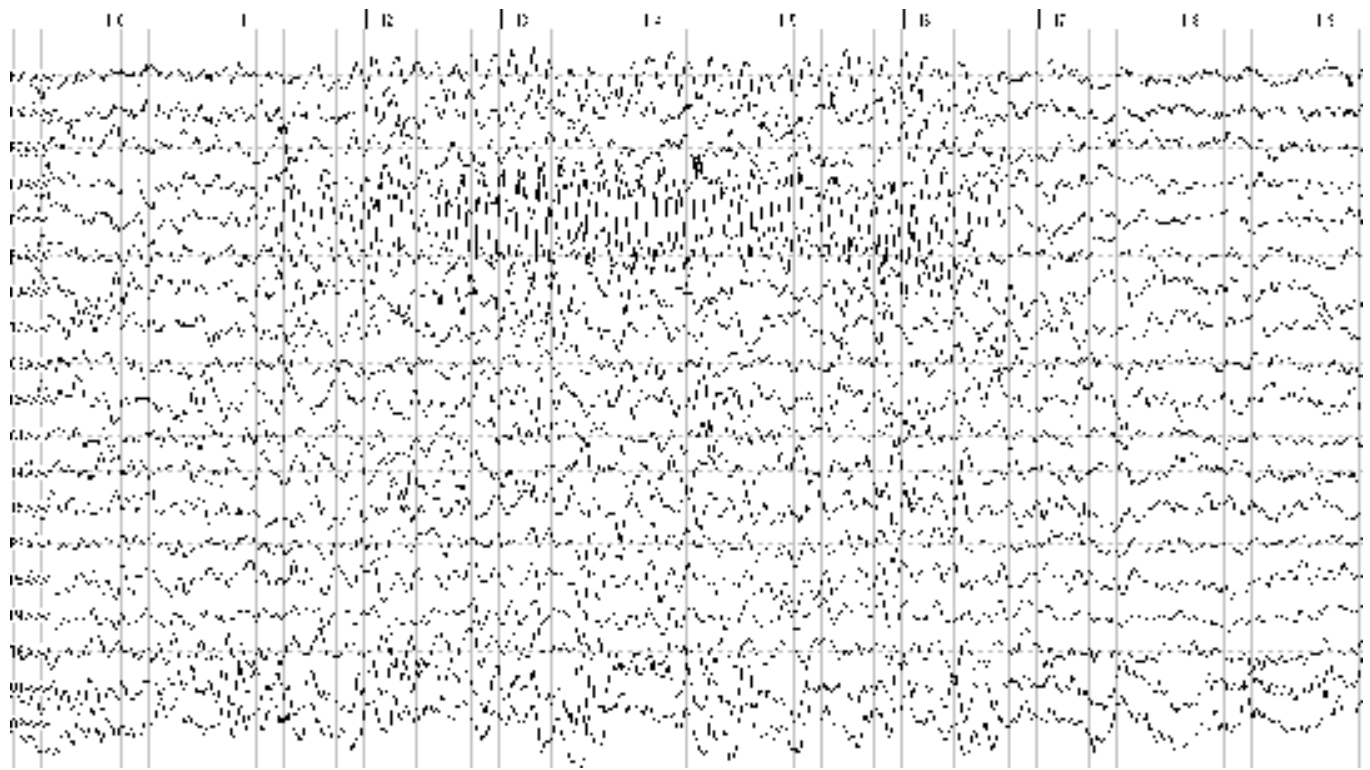

(Note slow delta/theta waves in frontal and central area)

Fig. 4. Subtype II - row EEG recording

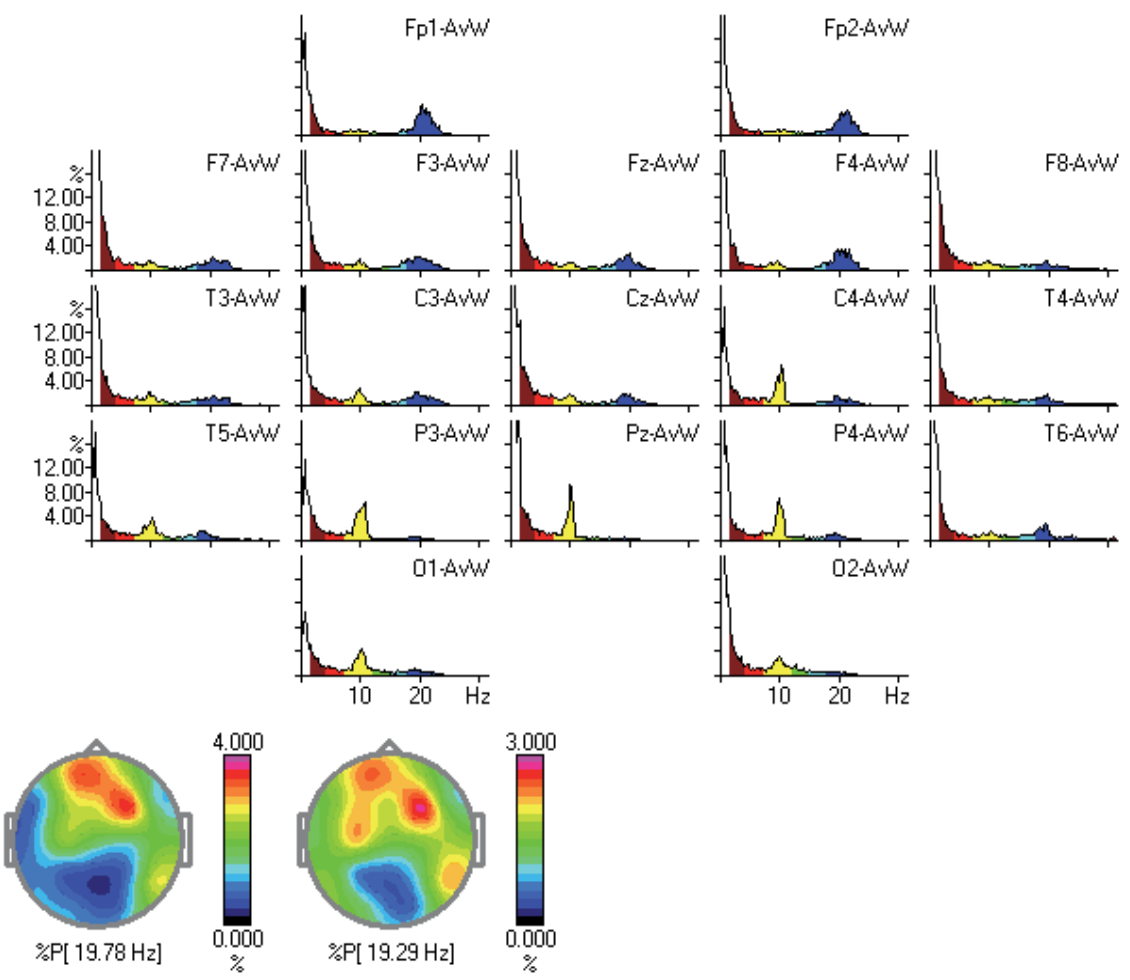

Fig. 5. Subtype III - over activated beta in frontal, central and parietal cortex 


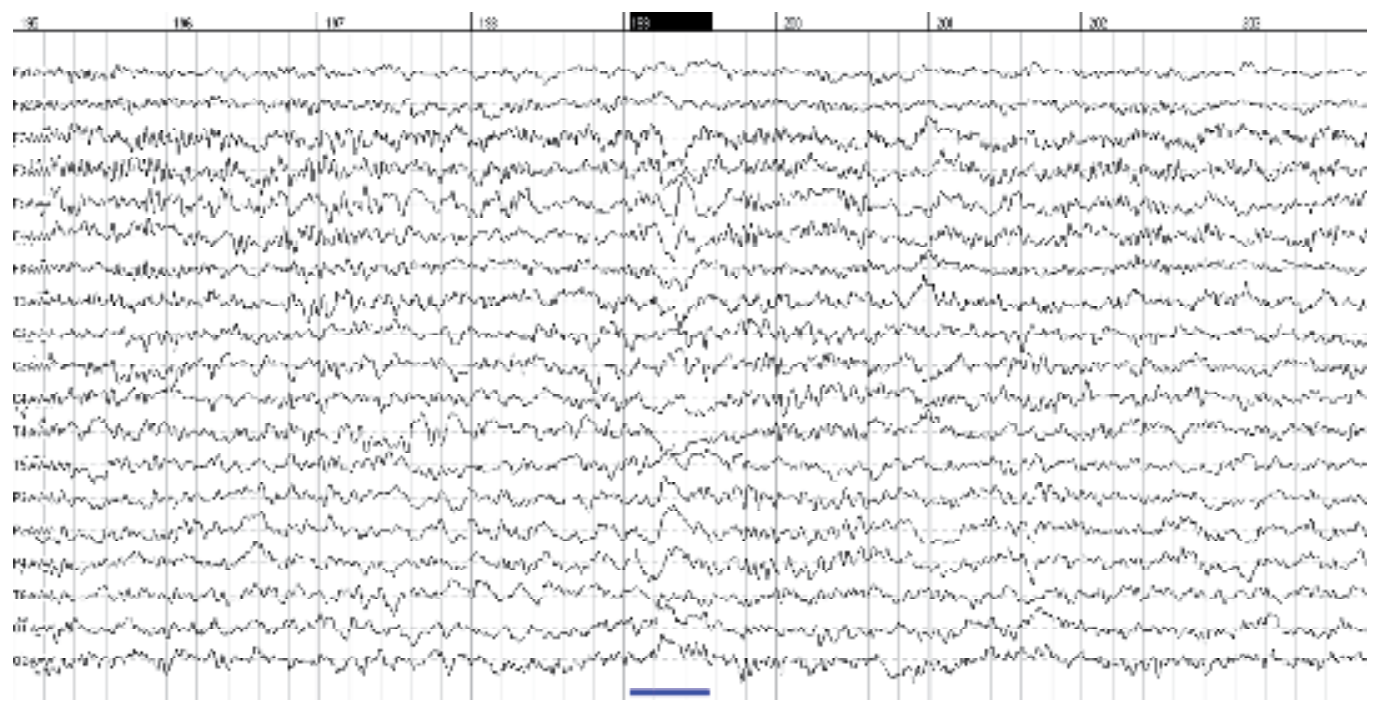

(Note fast brain waves over all cortex)

Fig. 6. Row EEG recording - overactive cortex

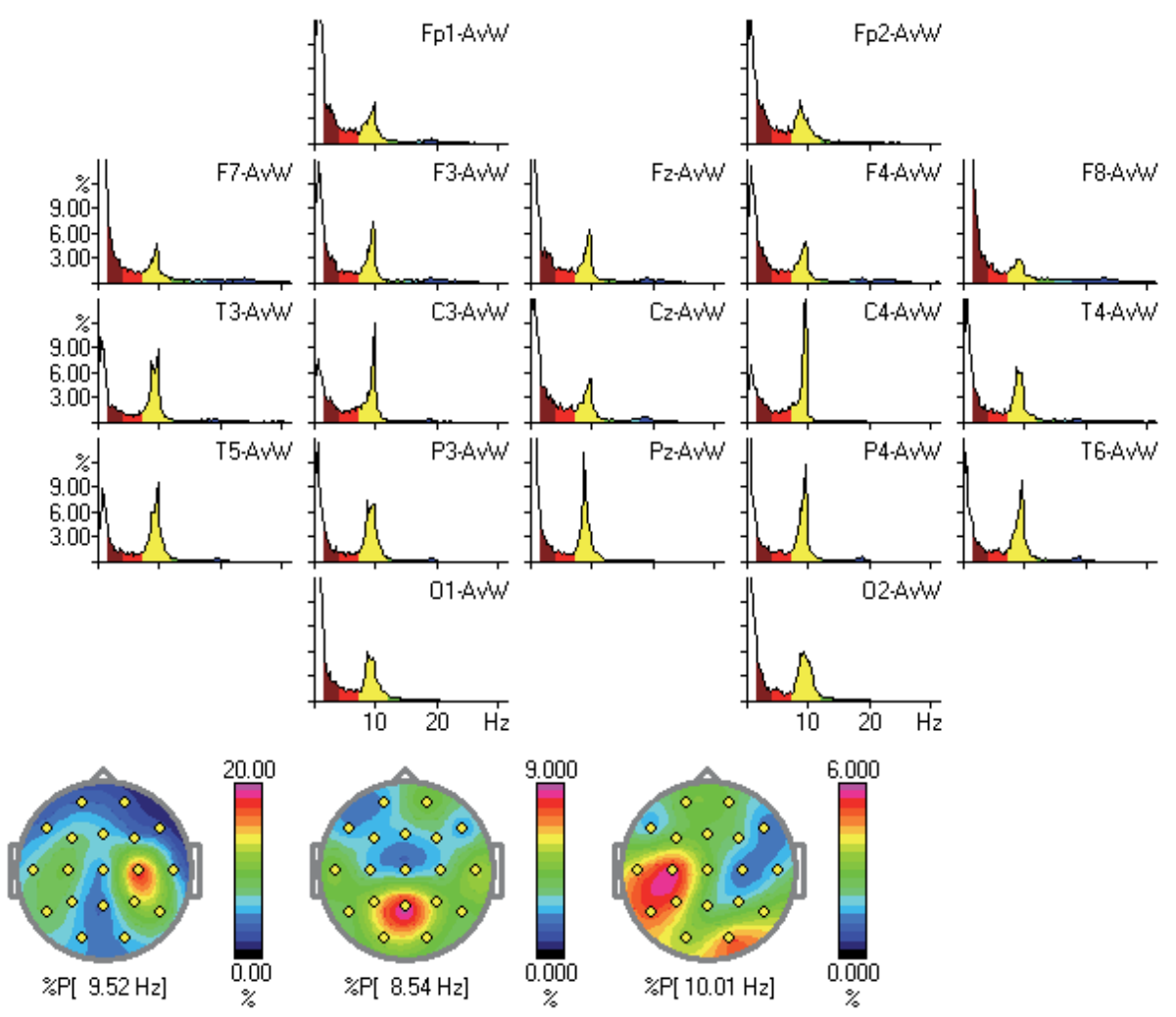

Fig. 7. Subtype IV - alpha excess 


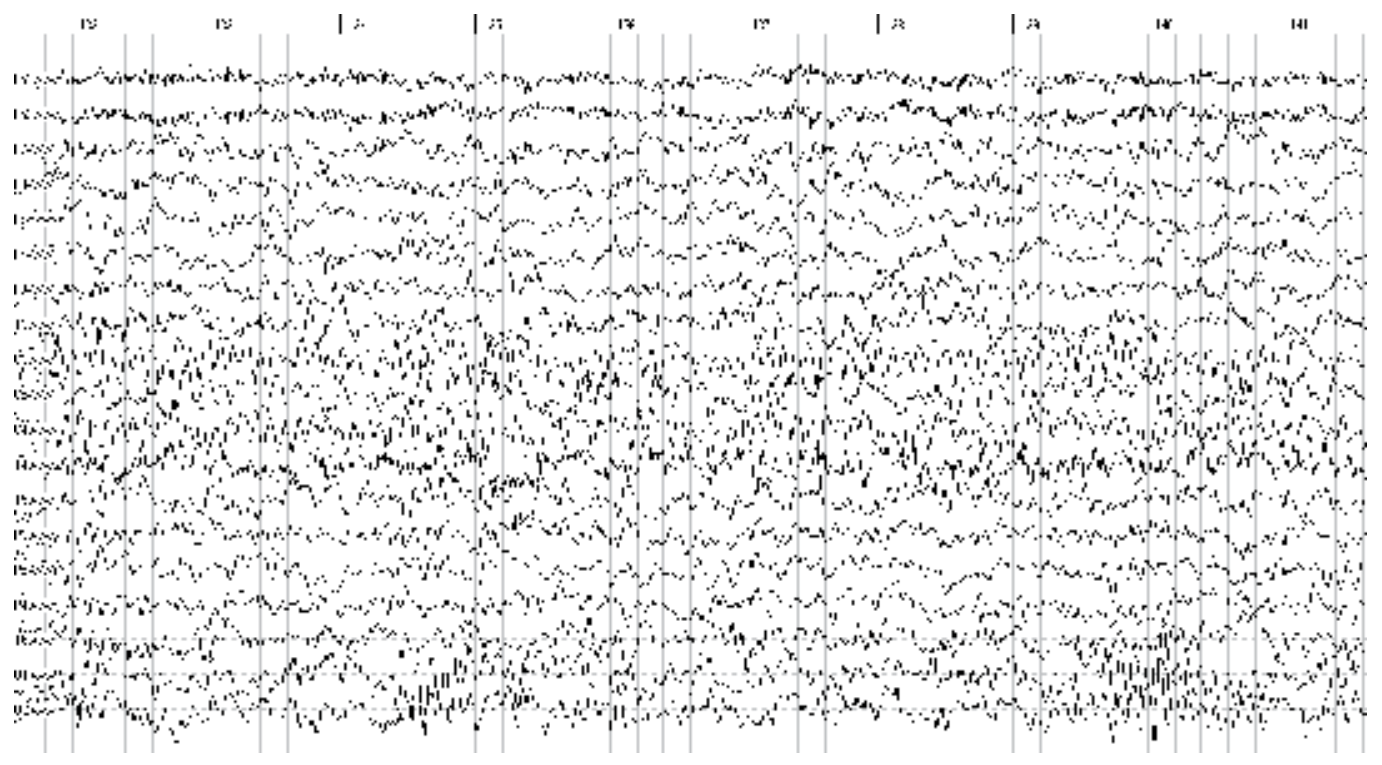

Fig. 8. Subtype IV- Row recording alpha excess

The evidence is not sufficient to permit conclusions about the benefits of SPECT imaging in the diagnosis and treatment of ADHD. A significant number of published studies are focused on investigating differences in regional cerebral perfusion in response to drug therapy and on serotonin and dopamine receptor and transporter activity. These studies are only preliminary. On the other side, the risks associated with SPECT imaging include exposure to low-dose radiation which is not recommendable in children.

\section{Event related potentials}

As we said previously, the term "executive functions" refers to the coordination and control of motor and cognitive actions to attain specific goals. The executive control is needed for optimizing behavior. The need for an executive control mechanism has been postulated for non-routine situations requiring a supervisory system (e.g. selection of appropriate action from variety of options, inhibition of inappropriate actions, and keeping in working memory the plan of the action as well as the outcome).

The executive system comprises a complex brain system such as several cortical and sub cortical structures interconnected with each other. The cortical structures include the prefrontal areas interconnected with the corresponding thalamic nuclei. The striatum is the most important in the subcortical circuits and it is considered as a cognitive map of cortical representations of actions. Together with basal ganglia the prefrontal cortex performs executive functions associated with engagement, disengagement, monitoring operations and working memory.

These operations of executive system are reflected in event related potentials (ERPs) evoked in different paradigms like Go/NoGo, oddball and working memory tasks. In our practice with ADHD children, we use the Human Brain Institute (HBI) normative database for comparing the obtained ERPs from the patients and norms. 
The following executive components in the Go/NoGo stimulus tasks could be obtained:

- the motor and action suppression components associated with frontal negativivities at 200 millisecond (the conventional N2 inhibition component)

- the engagement component associated with parietal positivity at 300 millisecond (the conventional P3b component)

- the monitoring component associated with frontal-central positivity at 400 millisecond (P400).

The N2 motor inhibition component is generated in the ipsi-lateral premotor cortex, the P3b component is generated in the parietal cortical area, and the P400 monitoring component is generated in the cingulated cortex. It is supposed that dopamine is the main mediator of the executive system.

For the psychometric assessment of the executive functions in ADHD patients most frequently we use the Stroop Color Word Task - SCWT (Stroop 1935), and Wisconsin Card Sorting Test - WCST (Berg 1948).

The obtained results for a group of 30 children diagnosed as combined form of ADHD, aged 7-14 years are presented on Table 1 and Table 2 (Zorcec and Pop-Jordanova, 2010).

\begin{tabular}{|lccccc|}
\hline WCST & T-score & $\begin{array}{c}\text { Significance } \\
\text { of the test }\end{array}$ & $\begin{array}{c}\text { T-score } \\
\text { controls }\end{array}$ & $\begin{array}{c}\text { Significance } \\
\text { of the test }\end{array}$ & $\mathrm{P}$ \\
N categories & ADHD & Low average & 55 & average & 0,32 \\
$N$ perseverations & 31 & below average & 51 & average & $0,00001^{*}$ \\
$N$ mistakes & 32 & below average & 50 & average & $0,00000^{*}$ \\
carts total & 30 & below average & 52 & average & $0,00001^{*}$ \\
$M$ categories & 31 & below average & 51 & average & $0,00001^{*}$ \\
\hline
\end{tabular}

* statistical significance

Table 1. T-score and statistical significance for WCST obtained for ADHD children compared with healthy control

\begin{tabular}{|c|c|c|c|c|c|}
\hline SCWT & $\begin{array}{l}\text { T-score } \\
\text { ADHD }\end{array}$ & Significance of the test & $\begin{array}{c}\text { T-score } \\
\text { controls }\end{array}$ & Significance of the test & $\mathrm{P}$ \\
\hline Mistakes (St) II & 50 & average & 55 & average & 0,1 \\
\hline Mistakes III & 29 & Very low & 50 & average & $0,00001^{*}$ \\
\hline Mistakes III/II & 28 & Very low & 53 & average & $0,00000^{*}$ \\
\hline St III-St II & 50 & average & 53 & average & 0,02 \\
\hline
\end{tabular}

*statistical significance

Table 2. T- score and statistical significance for SCWT obtained for ADHD children compared with healthy control

In the electrophysiological evaluation of ADHD children we used VCPT (visual cognitive performance task) with two stimulus Go/NoGo task developed specifically for the HBI database. The task consisted of 400 trials. The duration of stimuli is equal to $100 \mathrm{~ms}$. Trials consisted of presentation of a pair of stimuli with inter stimulus interval of $1.1 \mathrm{sec}$. Interval between trials is equal to 3,100 ms and response interval from 100 to 1,000 ms. Subjects were 
instructed to press a button with index finger of their right hand as fast as possible every time when animal or angry face was followed by an animal or angry face (Go condition), respectively, and to withhold the suppressing on the other three trials (NoGo condition).

The VCPT during QEEG for ADHD children showed very high ommission and commission errors, shorter reaction time (RT) and higher variation of the reaction time (var RT), compared with the results obtained for control healthy children.(Table 3 )

\begin{tabular}{|lcccc|}
\hline VCPT & ADHD & Norm & $t$-test & P \\
omission errors (Go) & 32,25 & 4 & 15,65 & $0,00001^{*}$ \\
commission errors (NoGo) & 4,75 & 1 & 7,58 & $0,0000^{*}$ \\
$R T(m s)$ Go & 456,89 & 486 & $-9,17$ & $0,00001^{*}$ \\
var $R T$ & 18,97 & 11,7 & 8,78 & $0,00000^{*}$ \\
\hline
\end{tabular}

*statistical significance

Table 3. Statistical significance for VCPT forADHD children compared with tests norms

For the P3Go component (activation processes) we did not obtained significant differences for the latency as well as for the amplitude, while for P3NoGo component (inhibition processes) we obtained not significant differences for the latency, but significant differences concerned to the amplidude. (Tabl. 4)

\begin{tabular}{|lcccc|}
\hline & ADHD & norm & $t$-test & $\mathrm{p}$ \\
P3Go $(m s)$ & 327,15 & 327,89 & $-0,12$ & 0,9 \\
P3Go $(\mathrm{mV})$ & 9,73 & 8,55 & 0,77 & 0,44 \\
P3NoGo $(\mathrm{ms})$ & 402,05 & 415,78 & $-0,69$ & 0,49 \\
P3NoGo $(\mathrm{mV})$ & 4,67 & 6,23 & $-2,89$ & $0,006^{*}$ \\
\hline
\end{tabular}

*statistical significance

Table 4. Statistical significance for P3Go and P3NoGo for ADHD children compared with tests norms

Generally, our research (Zorcec and Pop-Jordanova 2010) dedicated to the psychometric and electrophysiological evaluation of children diagnosed as ADHD showed significant presence of the perseverative mistakes and difficulties in the mental flexibility. The results obtained for VCPT showed significantly higher ommission and commission errors, lower reaction time (RT) as well as higher variation of time reaction (var RT) compared to the tests norms. The P3Go component values in tle latency and amplitude did not differ from the norms, but the P3NoGo component showed significant difference in the amplitude.

\section{Brain rate evaluation}

Reviewing the EEG studies of patients with ADHD it can be concluded that most of them have generalized or intermittent spectrum shift. It is the reason that we introduced the brain rate calculation (Pop-Jordanova and Pop-Jordanov, 2005) in addition to theta/beta ratio for both aims, in the assessment procedure and as a neurofeedback parameter. 
The brain rate (EEG spectrum weighted frequency) can be considered as an integral state attribute correlated to brain electric, mental and metabolic activity. In particular, it can serve as a preliminary diagnostic indicator of general mental activation (i.e. consciousness level), in addition to heart rate, blood pressure or temperature as standard indicators of general bodily activation.

In our research it was shown that brain rate can be used to discriminate between the groups of under-arousal (UA) and over-arousal (OA) disorders, to assess the quality of sleep, as well as to indicate the IQ changes caused by some environmental toxins (Pop-Jordanova 2009; Pop-Jordanov and Pop-Jordanova 2009, 2010). Brain rate is also suitable to reveal the patterns of sensitivity/rigidity of EEG spectrum, including frequency bands related to permeability of corresponding neuronal circuits. Based on all this findings, the individually adapted neurofeedback protocols can be elaborated.

The main characteristic of the integral (polychromatic) EEG spectrum is its mean frequency, weighted over the whole spectrum (brain rate $\left.-f_{b}\right)$, defined as

$$
\begin{aligned}
& f_{b}=\frac{1}{V} \int f V(f) d f, \quad V=\int V(f) d f \\
& o r \\
& f_{b}=\sum_{i} f_{i} P_{i}=\sum_{i} f_{i} \frac{V_{i}}{V} ; \quad V=\sum_{i} V_{i}
\end{aligned}
$$

where, $i$ denotes the frequency band (for delta $i=1$, theta $i=2$, etc.), and $V_{i}$ - the corresponding mean amplitude of the electric potential. (Pop-Jordanova N., Pop-Jordanov J., 2005)

In the following I will present some results obtained for brain rate (Demerdzieva, 2011) calculated for a group of 50 patients diagnosed as ADHD (age 119. 98; SD = 25.32 months,

\begin{tabular}{|c|c|c|c|c|c|c|c|}
\hline & & Frontal & Central & Posterior & Left & Midline & Right \\
\hline 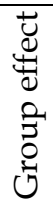 & $\begin{array}{l}\text { Normal's vs. } \\
\text { ADHD }\end{array}$ & & 0.000000 & 0.000000 & 0.000000 & 0.029686 & 0.000082 \\
\hline \multirow{5}{*}{ 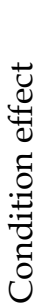 } & EC vs. EO & 0.033569 & 0.015872 & 0.000000 & 0.000083 & 0.000000 & 0.000014 \\
\hline & EO vs. VCPT & 0.000001 & & & & & \\
\hline & EC vs. VCPT & 0.000000 & 0.007530 & 0.000000 & 0.014954 & 0.000000 & \\
\hline & EC vs. ACPT & 0.000005 & 0.000196 & 0.000000 & 0.000004 & 0.000000 & 0.000089 \\
\hline & VCPT vs.ACPT & 0.000005 & & & & & \\
\hline
\end{tabular}
two females and 48 males) compared with a group of 50 healthy controls (mean age 117.84; $\mathrm{SD}=24.89$ months, and the same gender ratio as the ADHD group) (Table 5).

Table 5. Summary of significant interactions between groups, conditions and regions for brain rate results (evaluated with post hoc Bonferroni test) 
Maximum values of $f_{b}$ for sagittal topography were obtained in central and posterior regions, which is statistically significant, $F(3,390)=24.849, p=.00000$ (Fig. 9, left panel). Anyway these results are lower than those in healthy controls. According to the different conditions, obtained results were also statistically significant, $F(9,949.31)=72.294, p=0.0000$. Maximum values for $f_{b}$ were obtained in the posterior region during EC and minimum values in the frontal region again during EC condition for both ADHD and healthy groups..
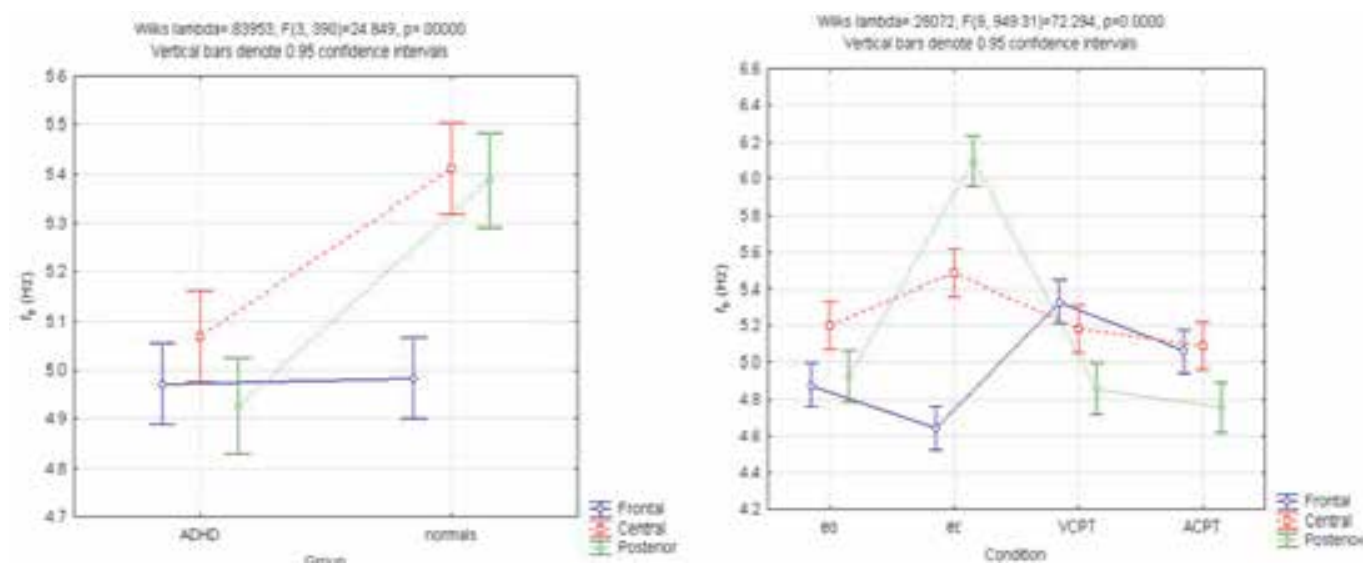

Fig. 9. Results for $\mathrm{f}_{\mathrm{b}}$ values for sagittal topography according groups-left panel and according conditions-right panel (EC-eyes closed, EO-eyes opened, VCPT- visual continuous performance test, ACPT- auditory continuous performance test)

Maximum values of $f_{b}$ for lateral topography are obtained in the left and right sides (Fig.10, left panel), which indicates higher excitability of lateral regions. The results are again significantly higher in normal group which indicates under arousal (UA) in children with ADHD (corresponding to subtype I prevalence). According different conditions in lateral topography the lower $f_{b}$ values were obtained in midline for all four conditions (Fig.10, right panel).
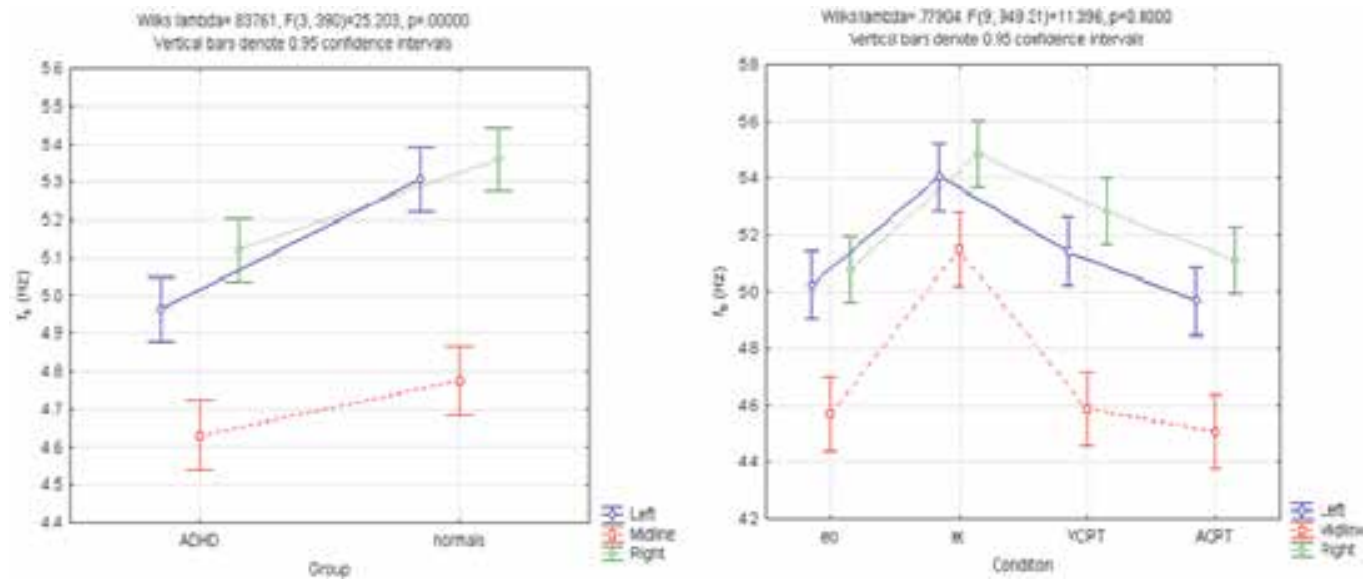

Fig. 10. Results for $f_{b}$ values for lateral topography according groups - left panel and according conditions - right panel (EC-eyes closed, EO-eyes opened, VCPT-visual continuous performance test, ACPT-auditory continuous performance test) 
The brain rate cocept is shown to be useful in the case of ADHD adults as well (MarkovskaSimoska and Pop-Jordanova, 2011). Maximum values of $f b$ for sagittal topography are obtained in central region, while the minimum in frontal region, corresponding to increased arousal (which is in agreement with the neurophysiological considerations). Maximum values of $f b$ for lateral topography are obtained in the left and right sides, while the minimum in the midline region, which indicates higher excitability of lateral regions. As expected, a positive correlation between $f b$ values and the QEEG spectra subtypes was obtained. Lower values were found for first and second subtype, and higher for the third and fourth subtype. This can be explained by the comparable arousal level for first and second subtype, and higher arousal in the third and fourth subtype. On the other hand, there was no correlation between behavioral symptoms (obtained with Barkley's scale) and $f b$ values (i.e. spectrum gravity), as well as between QEEG ADHD subtypes and behavioral symptoms, illustrating the heterogeneous and multifactorial character of ADHD.

Generally we can summarize that through different clinical experiments and pediatric practice concerning brain rate it was shown that:

- Brain rate may serve as an indicator of general mental arousal level, similar to heart rate (Kaniusas et al, 2007), blood pressure and temperature as standard indicators of general bodily activation.

- By comparing eyes closed and eyes open brain rate values the diagnoses of inner arousal can simply be achieved (Pop-Jordanov and Pop-Jordanova, 2009).

- As a measure of arousal level, brain rate can be applied to discriminate between subgroups (clusters) of "mixed" disorders (e.g. ADHD, OCD, headache) (PopJordanova, 2007, 2008, 2009).

- Brain rate can be more useful for selecting patients which need neurofeedback training (Pop-Jordanova, 2009).

\section{Biofeedback treatment}

Most prevalent approaches in the treatment of ADHD involve the use of stimulants, occasionally supplemented by tricyclic antidepressants, alpha-blockers and, in rare cases, antipsychotic drugs or selective serotonin reuptake inhibitors. In addition, the extensive behavior management, cognitive-behavioral therapy, individual psychotherapy and family system approaches have been applied. In the last two decades, biofeedback modalities have been offered in the treatment of different conditions and diseases (Schwartz 1987).

Biofeedback modalities can be divided into peripheral (based on electromyography, electrodermal response, heart rate, temperature, blood volume pulse) or central i.e. neurofeedback (based on electroencephalography). In what follows, we will concentrate on electrodermal response and neurofeedback.

Electrodermal response (EDR) is a complex reaction with a number of control centers in CNS. Three systems related to arousal, emotion and locomotion are responsible for the control of electrodermal activity (Bouscin 1992). The reticular formation controls EDR in connection to states of arousal, the limbic structures (hypothalamus, cingulated gyres and hippocampus) are involved in EDR activity related to emotional responses and thermoregulation, while the motor cortex and parts of the basal ganglia are involved in locomotion. In particular, skin potential and skin conductance used as parameters in EDR 
biofeedback are related to both sympathetic and parasympathetic arousal (Andreassi 2000; Mangina 1996).

Treatment by EDR biofeedback is generally based on training patients in strategies for lowering arousal and maintaining a healthful sympathetic/parasympathetic tone. Consequently, EDR biofeedback modality is a first choice for introvert persons, where high inner arousal is a typical finding and biofeedback training is supposed to lower sympathetic activity. Changes in electrodemal activity can be reliably detected within one second of stimulus presentation, often following a single event. It is important to know that electrodermal conductance precede any other signals related to neuroimaging such a positron emission tomography (PET), blood oxygen level-dependent functional magnetic resonance (BOLD), single photon emission computerized tomography (SPECT) etc. In other words, the changes of electroderamal activity can be registered before the changes obtained by the other neuroimaging techniques.

Neurofeedback (NF) i.e. EEG biofeedback refers to a specific operant-conditioning paradigm where an individual learns how to influence the electrical activity (frequency, amplitude or synchronization) of his brain. It involves teaching skills through the rewarding experience of inducing EEG changes reflected in a perceivable signal (light or sound). Neurofeedback has been shown to be particularly useful in reference to pathologies characterized by dysfunctional regulation of cortical arousal, such as epilepsy and attention deficit hyperactivity disorder (Lubar 1991, 1997; Birbaumer 1999; Mann et al. 1992; Monastra 2001; Pop-Jordanova et al 2005). Our team also used EEG biofeedback in anorectic girls (PopJordanova 2000, 2003), posttraumatic stress disorder - PTSD (Pop-Jordanova 2004), headaches (Pop-Jordanova 2008), as well as for optimal school (Pop-Jordanova, Cakalaroska, 2008) and music performance (Markovska-Simoska et al. 2008).

As we explained previously, the application of spectral analysis to EEG shows that in some brain dysfunctions the EEG amplitude in certain frequency bands significantly differs from the EEG amplitude of healthy subjects. For example, a relatively large group of children with ADHD reveals an excess of the theta/beta ratio i.e. decrease of $f b$ in central - frontal leads. This EEG abnormality is associated with hypo activation of frontal lobes. Neurofeedback provides a best tool for correcting such deviations from normality.

Neurofeedback is based on three scientific facts. First, EEG parameters reflect brain dysfunction in a particular disease (in the case of specific subgroup of ADHD children this means the corresponding changes of theta beta ratio and of $f b$ ). Second, subject can voluntarily change the state of his/her brain so that changes can be associated with increasing or decreasing the relevant parameter. Third, the brain can memorize this new state and keep it for longer time not only in lab conditions but also in other environments, such as school, home etc.

For applying neurofeedback therapy, QEEG evaluation of each patient is needed. In our studies the QEEG is obtained by standard MITSAR EEG recordings (21 electrodes), with the administration of standardized tests: eyes-open (EO), eyes-closed (EC), visual continuous performance (VCPT), auditory continuous performance (ACPT), reading test and math test. EEG data are analyzed for frequency content using the fast Fourier transformation. Statistical analysis compares subject's data with a normative database corrected for time-ofday variations. Data are also evaluated for percentage change across states and compared 
with a normative database for state modulation. The obtained topographic maps show covariance between all sites at relevant frequencies compared with a normative database, illustrating functional cortical interactions.

Many studies of QEEG for the ADHD group confirmed an increased theta activity predominantly in frontal regions, and a decreased beta activity in comparison to normal children (Mann et al 1992). In this context increased theta/beta ratio is reported as a typical finding in ADHD children (Lubar 1991; Monastra et al. 2001; Muller, 2006). So, the typical ADHD finding can be underarousal. As we said previously, the recent cluster analysis identified several subgroups of ADHD based on different EEG topographies. As a consequence, manly four distinct subgroups of ADHD with regard to electrophysiology are defined (Kropotov, 2009).

Neurofeedback treatment is particularly indicated in ADHD patients who show excessive EEG slowing in the superior frontal cortex or the midline central cortex (i.e. the first two subtypes) The most relevant neurological EEG correlate in these ADHD cases is usually assumed in the place where the highest ratio of theta/beta activity or lowest $f b$ is seen, so that placement of the electrode between $\mathrm{Cz}$ and $\mathrm{Fz}$ is the best for training. We followed two consecutive treatment protocols. (1) Training to increase the SMR EEG rhythm $(11-13 \mathrm{~Hz})$ and at the same time, starting to inhibit (decrease) slow activity in the theta range $(4-8 \mathrm{~Hz})$; this approach is primarily used for the hyperactive component of ADHD. (2) Training to focus attention aiming at increasing higher beta activity $(16-20 \mathrm{~Hz})$, while training for decreasing the slow activity continued. The training is performed with 40 sessions, 60 min duration per session, one per week. To obtain stress diminishing, before neurofeedback we use peripheral biofeedback for all children.

Practically all neurofeedback interventions can be roughly reduced to the need of mastering flexibility in increasing or decreasing the general mental activation, i.e. mental arousal (which is somehow coupled with metabolic activity). Thereby, in practice, whenever a certain band is trained, the other bands are affected too (it may even appear that e.g. "...the changes that occurred as a result of stimulating in the alpha frequency were not in alpha but were in beta..."(Lubar 1997). Therefore, the introduced brain rate $f_{\mathrm{b}}$ could be employed as a complementary biofeedback parameter, characterizing the whole EEG spectrum (as distinct from e.g. theta-beta ratio). The rationale is that, according to the mentioned empirical results, the EEG frequency shifts are related to mental activation / deactivation, as the main objective of the treatment.

Using brain rate as a neurofeedback parameter for a group of ADHD children ( $N=50$ mean age 11.11 years) Pop-Jordanova et al. (2005 and 2008) obtained the shifting of the spectrum from under-arousal to normal mental arousal, and it corresponded to improved attention and cognition as well as better school performance. Thereby obtained change of brain rate (i.e. arousal level) appeared to be more realistic in respect to the changes of psychological state of children than the drastic reduction of theta/beta ratio, which appeared to be even halved.

If we introduce brain rate as a general indicator of mental arousal in ADHD example, we can see that the first two subtypes are correlated with lower brain rate, (underarousal-UA) the third subtype with higher brain rate (overarousal-OA), while the four subtype is related to excess of alpha activity and "normal" arousal state. In the first three subtypes of ADHD the protocol for UA and OA is clear, while for the "normal" arousal it is not. 
The detailed analysis of QEEG after the neurofeedback training with brain rate as a parameter could detect which bands have been most changed. For instance, in some cases shifting the brain rate to higher values could result in increasing high alpha or beta frequencies; in other, the same change can appear due to diminishing the power of theta or delta bands. As a result, the QEEG comparison before and after the brain rate training can be informative for assessing the individual spectrum band sensitivity.

\section{Conclusions}

The frontal regions are most likely to show deviations from normal development in the case of ADHD, with disturbed thalamo-cortical and septal-hippocampal pathways, altogether named as executive system.

Application of spectral analysis to EEG shows that the EEG amplitude in certain frequency bands significantly differs from the EEG amplitude of healthy subjects. For example, a relatively large group of children with ADHD reveals an excess of the theta/beta ratio i.e. decrease of brain rate in central - frontal leads. This EEG abnormality is mostly associated with under activation of frontal lobes.

For the exact diagnosis of ADHD, it is recommendable QEEG to be combined with DSM-IV (or ICD-10) behavioral based approach.

There are four main endophenotypes in ADHD population: I subtype (abnormal increase of delta-theta frequency range centrally or centrally-frontally), II subtype (abnormal increase of frontal midline theta rhythm), III subtype (abnormal increase of beta activity frontally), and IV subtype (excess of alpha activities at posterior, central, or frontal leads). Thereby, we consider the second subtype as a subgroup of the first subtype.

The dysfunction of the executive system can be evaluated by event related potentials (ERP's) as well. Children with ADHD showed significant presence of the perseverative mistakes and difficulties in the mental flexibility. The results obtained for visual cognitive performance test (VCPT) showed significantly higher ommission and commission errors, lower reaction time (RT) as well as higher variation of time reaction (var RT) compared to the tests norms.

Distribution of brain rate values for sagittal and lateral topographies reflects the arousal levels in the corresponding conditions. There is a positive correlation between brain rate values and the QEEG spectra subtypes.

Neurofeedback provides a tool for correcting deviations from normality, especially for the subtypes I, II and III. For obtaining better therapeutic results, before neurofeedback, the use of peripheral biofeedback (such as electrodermal activity) aiming to obtain stabilization of sympathetic/parasympathetic system is recommended for all patients.

Brain rate can be used as a multiband biofeedback parameter in mediating the under arousal or over arousal states, complementary to few-band parameters and the skin conduction

Follow-up research is needed in order to determine more precisely the specificity and sensitivity of QEEG and brain rate approach related to neurophysical substrates of ADHD. 


\section{References}

Amen, DG. (1997) Brain SPECT imaging: Implication for EEG biofeedback. Keynote address presented at 28th annual meeting of the Association for Applied Psychophysiology and Biofeedback, San Diego, CA.

Amen DG, Hanks C, Prunella J. (2008) Preliminary evidence differentiating ADHD using brain SPECT imaging in older patients, J Psychoactive Drugs. 40(2):139-46.

Andreassi, JL. (2000) Psychophysiology. Human behavior and physiological response. LEA, London.

Anokhin, AP, Heath AC, Myers E. (2006) Genetic and environmental influences on frontal EEG asymmetry: A twin study. Biol. Psychol. 71 (3): 289-295

Barkley, R. (1990) Attention Deficit Hyperactivity Disorder: A Handbook for Diagnosis and Treatment, Guilford Press, New York

Berg, A. (1948) A simple objective technique for measuring flexibility in thinking, J. Gen. Psychol. 39: 15-22

Birbaumer, N, Roberts LE, Lutzenbrger W, Rockstroh B, Elbert T. (1999) Area-specific selfregulation of slow cortical potential on the sagittal midline and its effects on behavior. Electroencephalogr Clin Neurophysiol 84:352-361

Boucsein, W. (1992) Electrodermal activity. Plenum, New York.

Crosbie J, Schachar R. Deficient inhibition as a marker for familial ADHD. Am J Psychiatry. 2001; 158:1884-1890.

Culbert, TC, Kajander RL, Reaney JB. (1996) Biofeedback with children and adolescent: clinical observations and patient perspectives. J Dev Pediatr 17(5):342-350

Demerzieva, A. (2011) EEG spectra power characteristics of Attention Deficit Hyperactivity Disorder in childhood, Epilepsija: 124-136

Gottesman I I. and Shields J., (1972) Schizophrenia and Genetics: A Twin Study Vantage Point. Personality and Psychopathology Series, New York and London, Academic Press.

Kaniusas, E, Varoneckas G, Alonderis A, Podlipsky A. (2007) Heart rate variability and EEG during sleep using spectrum-weighted frequencies - a case study, COST B27. EU/ESF, Brussels.

Kropotov, J. (2009) Quantitative EEG, ERP's and neurotherapy. Elsevier, Amsterdam

Lubar JF. (1991) Discourse on development on EEG diagnostics and biofeedback treatment for attention deficit/hyperactivity disorders. Biofeedback Self-Regul 16: 201-225

Lubar JF. (1997) Neurocortical dynamics: Implications for understanding the role of 388 neurofeedback and related techniques for the enhancement of attention. Appl Psychophysiol Biofeedback 22(2):111-126

Mann, C., Lubar J., Zimmerman A., Miller C., and Muenchen R. (1992) Quantitative analysis of EEG in boys with attention-deficit/hyperactivity disorder: A controlled study with clinical implications. Paediatric Neurology, 8: 30-36

Mangina CA, Beuzeron-Mangina JH. (1996) Direct electrical stimulation of specific human brain structures and bilateral electrodermal activity. Int J Psychophysiol 22:1-8

Markovska-Simoska S, Pop-Jordanova N, Georgiev D. (2008) Simultaneous EEG and EMG biofeedback for peak performance in musician. Prilozi 1:239-253

Markovska-Simoska S., Pop-Jordanova N. (2011) Quantitative EEG Spectrum-weighted Frequency (Brain Rate) Distribution in Adults with ADHD, CNS spectr 16 (5): 579-587

Monastra VJ, Lubar JF, Linden M. (2001) The development of quantitative electroencephalographic scanning process for attention deficit-hyperactivity disorder: reliability and validity studies. Neuropsychology 15(1):136-144 
Muëller A. (2006) Neurobiological diagnostics and therapy in ADHD, in: News in Pediatrics. University of Skopje, Skopje, pp 135-147

Pop-Jordanova N. (1999) Electrodermal response based biofeedback in pediatric patients. Paediatr Croat 43:117-120

Pop-Jordanova N. (2000) Biofeedback mitigation for eating disorders in preadolescents. Int Pediatr 1:76-82

Pop-Jordanova N. (2003) Eating disorders in the preadolescent period: psychological characteristics and biofeedback mitigation, Chapter III. In: Swain P (Ed) Focus on eating disorder research. Nova Biomedical books, New York .

Pop-Jordanova N, Zorcec T. (2004) Child trauma, attachment and biofeedback mitigation. Prilozi 1-2:103-114

Pop-Jordanova N, Markovska-Simoska S, Zorcec T. (2005) Neurofeedback treatment of children with attention deficit hyperactivity disorder. Prilozi 1:71-80

Pop-Jordanova N, Pop-Jordanov J. (2005) Spectrum-weighted EEG frequency ("brain rate”) as a quantitative indicator of mental arousal. Prilozi 2:35-42

Pop-Jordanova N. (2006) Biofeedback modalities for children and adolescents. In: Columbus F. (Ed) New research on biofeedback. Nova Biomedical Book, New York.

Pop-Jordanova N., Muller A., Zorcec T., Markovska-Simoska S. (2007): QEEG subtypes of Macedonian ADHD children, Applied Neuroscience for Healthy Brain Function, Nijmegen, Netherlands, Book of abstracts, p.60

Pop-Jordanova N. (2008) EEG spectra in pediatric research and practice. Prilozi 1: 221-239

Pop-Jordanova N, Cakalaroska I. (2008) Biofeedback modalities for better achievement in high school students school students. MJM 2:25-30, also Revista Espanola de Neuropsicologia, 1:97-98

Pop-Jordanov J, Pop-Jordanova N. (2009) Neurophysical substrates of arousal and attention. Cogn Process. 10(Suppl.1), S71-S79

Pop-Jordanova N. (2009) Biofeedback application for somatoform disorders and attention deficit hyperactivity disorder (ADHD) in children. Int J Med Sci 1(2):17-22

Pop-Jordanov J. Pop-Jordanova N. (2010) Quantum transition probabilities and the level of Consciousness. Journal of Psychophysiology, 24(2):136-140

Schwartz MS (1987) Biofeedback: a practitioner's guide. Guilford Press, New York.

Scott F (1998) EEG biofeedback for children and adolescent: a pediatrician's perspective. Biofeedback 26(3):18-20

Smith and Jonides (1999) Storage and executive processes in the frontal lobe, Science 283 (5408): 1657-1661.

Stroop R. (1935) Studies of interference in serial verbal reactions, Journal of Experimental Psychology, 18: 643-662

Zorcec T, Pop-Jordanova N, Müller A.(2007) Attention Deficit Hyperactivity Disorder in three family generations (case report). Neuroscience today: neuronal functional diversity and collective behaviors, COST B27. EU/ESF Florence, Italy, Book of abstracts p. 25.

Zorcec T., Pop-Jordanova N., Muller A. (2007) QEEG characteristics of children with ADHD, Epilepsy: 111-120

Zorcec T., Pop-Jordanova N., Mueller A., Gjoneska B. (2008) The Role of Q-EEG in Comprehensive Classification of ADHD Children; 2nd Neuromath Workshop, Jena. 28-29 April: $42-43$

Zorcec T., Pop-Jordanova N. (2010) ADHD as executive dysfunction, Prilozi, 31 (2): 171-181 


\title{
EEG Findings in ADHD and the Application of EEG Biofeedback in Treatment of ADHD
}

\author{
Mohammad Ali Nazari \\ University of Tabriz, Tabriz, \\ Iran
}

\section{Introduction}

As defined in the 4th edition of Diagnostic and Statistical Manual of Mental Disorders (American Psychiatric Association, 1994), Attention deficit hyperactivity disorder (ADHD) is characterized by a persistent pattern of inattention, hyperactivity, and impulsiveness, though it can present with or without hyperactivity. ADHD is the most common childhood mental health disorder, with an estimated prevalence of $7 \%$ to $10 \%$ in boys and $3 \%$ in girls aged 4-11 years (Sgrok et al., 2000). This disorder substantially affects the individual's normal cognitive and behavioral functioning. For example, children with ADHD can have a great deal of difficulty focusing on lessons presented by their teachers and remembering how to do their homework. They may often be easily distracted whereby they pay attention to other things than what they should.

The numerous studies support a model that defines ADHD as an inherited disorder whose core symptoms are founded in neuroanatomic, neurochemical, and neurophysiologic abnormalities of the brain (Monastra, 2005). Deficits associated with ADHD support a hypothesis that anatomical and biochemical abnormalities of the prefrontal cortex constitute the physical basis of this disorder (Barkley, 1997). In this line, neurodiagnostic procedures (e.g., positron emission tomography [PET], single photon emission tomography [SPECT] and magnetic resonance imaging [MRI]) studies have provided evidence of the neurological basis of ADHD (Boutros, et al., 2009). Nevertheless, new theories on the pathogenesis of psychopathological phenomena conceptualize as a consequence of the failure to integrate the activity of different brains' areas (Boutros et al., 2009). It needs techniques tapping the dynamics of complex interaction over time among cerebral regions involved in the integration of cognitive processing.

Electrophysiological techniques enable monitoring brain processing in real time, providing the best methods to describe the time course of brain electrical activities. Growth of this field came from the newer and quantifiable techniques such as quantitative electroencephalography (QEEG). QEEG methods provide a set of non-invasive tools that are capable of quantitatively assessing resting and evoked activity of the brain with sensitivity and temporal resolution superior to those of any other imaging methods (Hughes \& John, 1999).

QEEG studies have explored brainwave profile in children with ADHD, compared to normal children. These brainwaves could be trained via operant conditioning (called EEG 
biofeedback or neurofeedback) and it is claimed that self regulation of brain electrical activity result in a therapeutic benefit in ADHD. The main purpose of this chapter is to look at one alternative method of treating children with ADHD. To fulfill this purpose, the present chapter will review:

- description of electroencephalography and QEEG

- QEEG findings in ADHD

- brief history and rationale for neurofeedback development

- description of neurofeedback in practice

- neurofeedback findings in the treatment of ADHD as supported by controlled studies.

Our brain is made up of many cells, including neurons and glial cells. There are about 100 billion neurons in the brain. Neurons are cells that send and receive information to and from the brain and nervous system. The language of these communications throughout the nervous system is electro-chemical signals. An electroencephalography (EEG) is a tool for measuring electrical activity generated in the brain. These electrical activities of neurons are very tiny. Hence, EEG activity always reflects the summation of the synchronous activity of thousands or millions of neurons; when many neurons shift towards being more ready to fire (excitatory) or to not fire (inhibitory) at the same time. The EEG signals are recorded using sensors (electrodes) placed on the scalp. Electrodes are attached to our head and hooked by wires to a computer and then the computer records our brain's electrical activity on the screen. Patterns of neuronal electrical activity recorded are called brainwaves.

An EEG signal is characterized by three major components: phase, frequency and amplitude. Traditional EEG displays waveforms in the time domain, and the interpretation is based on amplitude and dominant frequency. Each brainwave frequency is expressed in Hertz $(\mathrm{Hz})$. One $\mathrm{Hz}$ means 1 cycle per second; it is the rhythm of the wave. Amplitude represents the height (intensity) of the brainwave, and is expressed in microvolt $(\mathrm{mV})$. Brainwaves have traditionally been separated into different frequency bands (Drongelen, 2007):

- $\quad$ Delta rhythm $(\delta): 0.1-4 \mathrm{~Hz}$

- Theta rhythm $(\theta): 4-8 \mathrm{~Hz}$

- $\quad$ Alpha rhythm (a): 8-12 Hz

- Sensory-motor rhythm (SMR): 12 to $15 \mathrm{~Hz}$

- Beta rhythm ( $\beta): 15-30 \mathrm{~Hz}$

- $\quad$ Gamma rhythm (Y): the higher EEG frequencies, usually 30 70 Hz.

Conventional interpretation of the EEG is done visually by a trained specialist. The specialist will examine the EEG, by detecting features of waveshapes (morphology) of the brainwaves to identify certain characteristics that might indicate organic or neurological pathologies. Routine EEG is typically used in the following clinical circumstances:

- to distinguish epileptic seizures,

- to differentiate "organic" encephalopathy or delirium from primary psychiatric syndromes such as catatonia,

- to serve as an adjunct test of brain death,

- to localize the region of brain from which a seizure originates (Niedermeyer and da Silva, 2004). 
A voluminous literature attests to the robustness of conventional EEG studies and their clinical utility in disorders of brain function (Hughes \& John, 1999). However, many functional characteristics of brain activity could not be detected visually. Whereas, quantitative EEG (QEEG) transform the EEG into a format or domain that elucidates relevant information, or associate numerical results with the EEG data for subsequent review or comparison (Nuwer, 1997). Often, neurologically based disorders do not involve a structural abnormality, lesion, or disease process, but abnormalities are expressed in the way the brain evaluates information. These processes can be studied with QEEG techniques, but not with simple visual analysis of the raw EEG (Hoffman et al., 1999). Hence, one can say that QEEG might provide additional measurements and displays of EEG in many different ways that are not possible with visual inspection.

In fact, QEEG reflects the ability of a network to locally synchronize. Such ability to synchronization is related to the integrative capacities of a network and to the characteristics of its inputs. This can be strongly modified by the active state of the brain. Thus, impairment of cognitive processing (i.e. attention) can be monitored by QEEG (Nazari, 2008). Furthermore, QEEG enables precise comparison of the individual patient's record with normative and psychopathologic patient databases (Hughes \& John, 1999). QEEG procedures involve the mathematical processing of digitally recorded EEG. The most commonly used method for EEG quantification is the spectral analysis by means of Fast Fourier Transformation (FFT) algorithm. It provides measures of the power at each frequency of the EEG bands, known as the power spectrum. The test-retest of power spectra has been shown to be high (Hughes \& John, 1999).

The first step for doing a QEEG is digital EEG recording; a cap (usually 19 electrodes at standardized positions) placed on the head and two electrodes are placed on the ears. The electrodes are then made to conduct with the scalp and ears by using a conductive gel. Once this is achieved, a computer interfaces with the EEG machine, and a software program is used to display the traces of the brainwaves generated by the brain, and detected on the scalp. Data is recorded during resting states of eyes open, eyes closed, and in some instances during cognitive tests such as reading or attentional task. Approximately ten minutes of data are recorded in each state. A QEEG typically requires about an hour total in the clinic to complete the data gathering.

After recording the EEG data it is edited to remove artifacts which are distortions in the EEG signal due to muscle movement such as coughs, eye movement, and teeth clenching, muscle tension, pulse and other sources. Artifacts are electric potentials of non-brain origin that are in frequency and voltage range of EEG signals and that are detected by scalp electrodes (Boutros et al., 2009). Clinicians utilizing QEEG must be skillful in recognizing and minimizing artifacts, as well as in careful pre-recording preparation procedures to minimize artifacts in the EEG (Hammond and Gunkelman, 2001). Indeed, it needs to carefully study the raw EEG since abnormalities may be masked by the use of a QEEG alone (Hammond et al., 2004). During the editing process the data is examined visually to identify any patterns that might be of interest for training purposes or would suggest the need to refer to another specialist.

After the editing process is completed the EEG data is subjected to a variety of mathematical and statistical analyses. EEG recordings should be of sufficient quality and of sufficient length so that after artifacting there is a minimum of $40-50$ seconds of artifact-free data 
available for analysis (Hammond et al., 2004). A sample of artifact-free EEG data, usually 1 to 2 minutes, is analyzed, using the FFT to quantify the power at each frequency of the EEG averaged across the entire sample (Hughes \& John, 1999). Results from each electrode can be represented as following measures:

- absolute power: amount of amplitude in each band (total $\left.\mu \mathrm{V}^{2}\right)$,

- relative power: in each band percentage of absolute power/total power,

- power ratio: i.e. absolute power of theta/absolute power of beta (theta/beta ratio),

- coherence: a measure of synchronization between activity in two channels (similarity of frequency between two channels),

- $\quad$ symmetry (the ratio of power in each band between a symmetrical pair of electrodes (no similarity is called asymmetry).

The final analysis is the database comparison. This procedure allows for an individual's EEG to be compared to an 'average' EEG. One can use a reference EEG database to reveal the location and type of EEG feature abnormalities greater than two standard deviations from a normative group (Thatcher, 1998). This comparison data is derived from the analysis of EEG's gathered from hundreds of individuals; same sex, same handedness, approximate same age; who do not exhibit or report historically any significant mental health issues. Often the EEG will be compared to multiple databases. The aspects of an individual's EEG to be analyzed by the QEEG are:

- Does the individual's EEG features differ from the 'average' EEG?

- How does it look different (the level of statistical significance and the degree of difficulty)?

- Where (what areas of the brain) does it look different?

The QEEG data is used to generate a series of analyses presented in tables and graphics in brain map. Brain map is a computerized EEG topography that enables the construction of a bi- or three-dimensional matrix for a topographic representation of Q-EEG parameters, such as instant amplitude or band power (Boutros et al., 2009). Different algorithms have been proposed to localize underlying brain generators. Among the distributed source models, Low Resolution Brain Electromagnetic Tomography-LORETA (Pasqual-Marqui et al., 1994) has been proven to present the smallest localization error (Boutros et al., 2009). The LORETA is one of the QEEG topographic analysis method by which one can provide a 3-D analysis of the EEG identifying localized disruptions in brain activity within the interior of the brain.

An individual who has received specialized training in these fields (see Hoffman et al., 1999; Hammond et al, 2004; Hammond et al, 2011) could examine the QEEG results. Individuals conducting assessment utilizing quantitative EEG or any type of brain mapping should be able to gather reliable data. A much higher standard is required for someone to hold himself or herself out as competent to analyze and interpret QEEG data (Hammond et al., 2004). It is strongly recommended that the QEEG providers should hold diplomate status in QEEG from the Quantitative Electroencephalography Certification Board or be certified by the EEG and Clinical Neuroscience Society (or a comparable neurology board in the case of physicians), or be analyzing data under the supervision of such a certified person, or at a minimum be able to demonstrate thorough education, training, and work product documenting their competence to interpret QEEGs. Otherwise, the QEEG data should be submitted for analysis by an individual with such certification 
(Hammond et al., 2011). For further details about standards and qualifications for doing QEEG and neurofeedback see Hoffman et al., 1999; Hammond et al, 2004 and Hammond et al, 2011.

Due to the non-invasive nature of the procedure, the convenience, not expensive, and specificity of the data the QEEG has been used extensively to examine a variety of aspects of brain function. As mentioned before, with the quantitative EEG and topographic brain maps, it is often possible to observe attributes of brain function that cannot be seen in the raw EEG signal. These processes can be observed and quantified through subtle frequencyrelated and coherence related activities in the QEEG brain maps that index the degree of difficulty of cognitive tasks (Hoffman et al., 1999). Furthermore, it is well known that a great many medications as well as psychoactive drugs can produce some alteration in the EEG (Boutros et al., 2009). The availability of QEEG let to the development of a new research field that named pharmaco-EEG. Pharmaco-EEG methods were included in preclinical studies to identify at early stages of drug development, the therapeutic indications of new drugs, determining onset, peak effect, and duration of drug effect on CNS, and predict therapeutically useful dosage of psychotropic drugs (Boutros et al., 2009).

In the clinical setting, many studies have been reported that QEEG can be useful for the evaluation and understanding of mild traumatic brain injury, learning disabilities, ADHD, alcoholism, depression, and other types of substance abuse (Hoffman et al., 1999). Specifically, QEEG studies have reported different brainwave patterns in children with ADHD than those of the normal population.

Most studies of the electrophysiological correlates of ADHD have compared the QEEG from ADHD sufferers with those of healthy children under resting conditions (for a review, see Barry et al., 2003; for a meta-analysis, see Snyder \& Hall, 2006). However, the allocation of neural resources differs when the subject directs his/her attention to an experimentally controlled situation (Thatcher, 1998). It is therefore important to evaluate a neural network's ability to change from a passive to an active condition. Since inattentiveness and distractibility are the major symptoms of ADHD, assessment of these symptoms would require tasks specifically designed to highlight attentional deficits, such as the continuous performance task (CPT) or the go/no-go task. Hence, in a study, Nazari et al (2011) set out to establish the functional reactivity of frequency-specific EEG activities during eyes-open resting and CPT in children with ADHD. High-resolution EEG was recorded during eyesopen resting and CPT performance in 16 children meeting the DSM-IV criteria (APA, 1994) for ADHD and 16 age-matched controls. Significant CPT vs. eyes-open differences in EEG activities was observed in children with ADHD. In particular, switching to CPT induced an alpha power increase in children with ADHD and an alpha power decrease in controls. Lower alpha power at baseline (eyes-open resting condition) might be interpreted as meaning that children with ADHD are unable to attend to and process visual stimuli as efficiently as healthy children. Klimesch et al (1996) suggested that alpha synchronization during mental inactivity may be important for introducing powerful inhibitory effects, which could prevent a memory search from entering irrelevant parts of neural networks. Based on this explanation, we suggested that impaired inhibition of neural networks in children with ADHD at baseline alters not only energy demands but also control excitatory processes. Opposite alpha changes may also reflect a primary deficit associated with cortical hypoarousal in ADHD. These EEG results agree with behavioral findings leading the 
authors to suggest that dynamic changes in neural network activities are impaired in children with ADHD (Nazari et al., 2011).

Lubar (1995) compared QEEG data for ADHD children with controls. He concluded, "Excessive theta activity and lack of beta activity are the primary neurological landmarks of ADHD" (p. 505). Furthermore, "during academic challenges, there were significant increases in slow (4-8 Hertz) theta activity along the midline and in the frontal regions and decreased beta activity, especially along the midline posteriorly" (p. 502). Lubar's review of the literature revealed the following:

"Abnormalities in EEG were reported in children now classified as ADD and ADHD as early as 1938 (Jasper, Solomon \& Bradley, 1938). There is extensive literature, much of it reviewed in the supplement to the Journal of Child Neurology published in 1991. Basically, EEC studies show excessive slow activity in central and frontal regions of the brain. These studies are supported by recent PET [positron emission tomography] scan and SPECT [single photon emission computerized tomography] scan studies that also indicate abnormalities in cerebral metabolism in these particular brain areas" (p. 50I).

Based on Lubar's finding, studies have repeatedly reported a QEEG pattern that might be present in ADHD but not in controls (normal children, adolescents, and adults). A considerable number of these studies have reported an increase in low-frequency power (predominantly theta) and a decrease in high-frequency power (especially beta) in children with ADHD compared with the age-matched control group (Barry et al., 2003; Snyder \& Hall, 2006). Some researchers have tried to examine the theta/beta ratio as a measure of ADHD-related abnormality with a higher detection power. As reported by Snyder \& Hall (2006) results of 9 DSM-IV studies and the results of 29 pre-DSM-IV studies support that a theta/beta ratio increase is a commonly observed trait in ADHD relative to controls. By meta-analytic statistical extrapolation, the effect size of 3.08 predicts a sensitivity and specificity of $94 \%$, which is similar to values predicted by retrospective studies examining ADHD and normal controls in group comparisons (Snyder \& Hall, 2006).

As emphasized by the committee of the Association for Applied Psychophysiology and Biofeedback (AAPB) and the Society for the Study of Neuronal Regulation (SSNR), QEEG should not be the only tool used for diagnosis of attention-deficit/hyperactivity disorder (Hoffman et al., 1999). There is no single technique that can be solely relied upon for the diagnosis. Manifestations of ADD/ADHD reflect behavior problems, learning style, cognitive processing, social interaction, and many other developmental factors. The current diagnosis of ADD/ADHD depends also on the use of computerized continuous performance tasks, detailed history, school performance, and evaluation for learning disabilities and other comorbidities, as well as other measures. QEEG data complement these other findings by providing for a comparison of brain activity with databases for both normal and ADD/ADHD groups (Hoffman et al., 1999).

Having diagnosed the locations in the brain that are producing high or low activity, it is now possible to intervene with training the brain to normalize the activity of the various locations in the brain. On the other words, the power in being able to define deviations of brain's electrical patterns within a normally distributed measurement set is that one can target deviant measures to "normalize" by a variety of intervention modalities. In fact, the EEG (as a physiological measure) is considered a form of behavior, which is subject to behavior modification through basic "operant conditioning" and "shaping" principles within 
the formwork of learning theory. This brainwave training and learning self regulation of brain activity is called EEG biofeedback or neurofeedback. Neurofeedback postulates that normalizing the target signal will result in a therapeutic benefit. Definition of neurofeedback by the International Society for Neurofeedback and Research (ISNR) is the following:

"neurofeedback is a process in which sensors are placed on the scalp and devices are used to monitor and provide moment-to-moment information that is fed back to the individual about his or her physiological brain activity for purposes of improving brain functioning" (Hammond et al., 2001; p.55). For detailed information about neurofeedback see the website of the ISNR (http:/ / www.isnr.org).

Figure 1 shows the neurofeedback procedure. During neurofeedback training, neuroelectrical activity is detected via surface electrodes (step 1). Note that no electrical current is put into the brain. This activity is then amplified (step 2) and processed by software programs (step 3) that provide contingent auditory or visual feedback to the patient on a computer monitor (step 4); brain activity is monitored and desired changes are rewarded similar to a videogame. The patient watches the dynamic display of the amplitude of the brainwaves in the areas where the electrodes are attached by a gel paste. The computer program gives a reinforcement each time the goal level of the EEG power (an optimal brain state) is reached. This processing continues during the neurofeedback session for a period of 15 to 40 minutes (step 5).

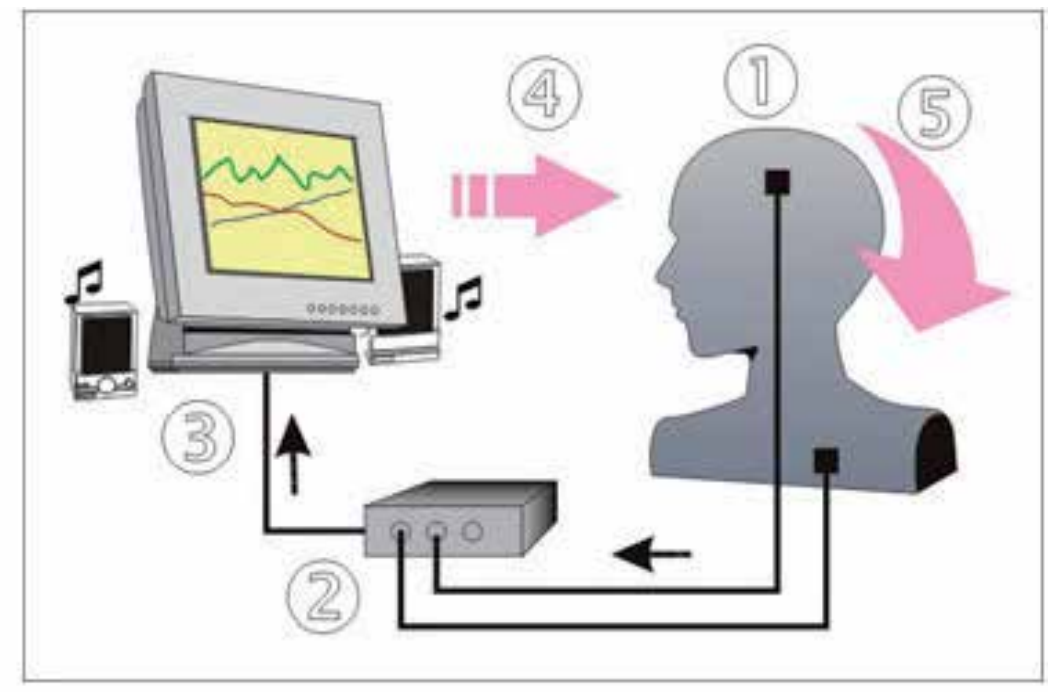

Fig. 1. Neurofeedback procedure

For example, there might be areas of the brain where there is an excess of neurons firing slowly during tasks requiring concentration. This is often the case with ADHD. On the basis of QEEG findings in ADHD, typically the EEG of a person with ADHD will reveal excess theta activity, but diminished beta activity. Hence, during the neurofeedback training a puzzle advances and sounds a tone whenever a child with ADHD maintains waves in the 15-18 Hz range above a certain amplitude threshold (beta increasing) while keeping waves in the $4-8 \mathrm{~Hz}$ range below a certain threshold (theta decreasing). Clients require 20 to 60 
training sessions to achieve their goals. Training takes place 1-3 times for at least one hour of training per week. Once the original goals of treatment have been met, the client continues to train for an additional 5 to 10 sessions to prevent relapse (Demos, 2005).

Prior to beginning neurofeedback training an assessment is conducted to examine presenting problems, client history, contributing factors, current medications the patient may be taking, and other relevant information. Interviews, symptom checklists, computer based tests (i.e. CPT, TOVA, IVA), and review of relevant documentation are common components of the assessment. A pre- and post treatment objective assessment of the client's QEEG should be performed. The QEEG objectively assess the functioning of the brain in comparison with normative database (Hammond et al., 2011). One can use QEEG database and topographical brain maps to evaluate the location and type of EEG feature to target for neurofeedback training.

After reviewing the data gathered during the assessment a training protocol is developed. The neurofeedback protocols cover the following questions:

- $\quad$ Power of which frequency bandwidth targeted to be changed?

- Which areas of the brain are to be trained (electrodes location)?

- Which montage must be used (referential or bipolar)? Which locations are chosen for active electrode, reference and ground?

- How threshold levels are set for each client?

The rationale for neurofeedback protocols is based on solid research and clinical practice. Initially, neurofeedback treatments for ADHD are founded on the groundbreaking research conducted by Sterman (roth et al., 1967; sterman and Wyrwicka, 1967; Wyrwicka and sterman, 1968; sterman et al., 1969 and Lubar and Shouse, 1976; Lubar and Lubar, 1984). A brief history could be interesting. Sterman's research team conducted a systematic examination of EEG patterns and identified the sensory motor rhythm-SMR $(12-15 \mathrm{~Hz})$ over the Rolandic cortex. They were able to train cats to increase production of this rhythm by providing food as an immediate reward. In later research those cats exhibited a significant improvement in stability when exposed to Hydrazine observed to evoke seizure activity in cats that had not received the SMR increasing. Subsequently, they demonstrated that patients with seizure disorders could develop improved control over epileptiform activity by learning self-regulation of the SMR (Sterman, 2000).

Sterman's procedure was replicated by Lubar who used the same training to reduce the symptoms exhibited by 'hyperkinetic' children. Initially, Lubar and Shouse (1976) reported some improvements in a hyperactive child who had learned to reduce theta and increase production of SMR. Subsequently, Lubar and Lubar (1984) reported that children diagnosed with an attention deficit disorder demonstrated improved attention and behavioral control after being trained to increase production of EEG activity in a fast frequency range (beta) while learning to suppress slow wave activity (theta).

These two primary training approaches provide the foundation for each of the protocols that have been examined in the controlled group studies of neurfeedback for ADHD. In a review study, Monastra (2005) has summarized three neurofeedback protocols that have been investigated in controlled group studies. These research-based protocols are the following (Monastra, 2005): 
Protocol 1- SMR enhancement/theta suppression: in this protocol, patients (ADHD who present with primary symptoms of hyperactivity and impulsivity) instructed to increase their SMR (12-15 Hz) over one of two sites (C3 or C4) while simultaneously suppressing the production of theta $(4-7$ or $4-8 \mathrm{~Hz})$ activity. EEG recordings are obtained from one active site, referenced to linked earlobes. Auditory and visual feedback is provided based on patient success in controlling power of theta below and SMR above pretreatment thresholds.

Protocol 2- Theta suppression/beta1 enhancement: In this protocol, patients are reinforced for increasing production of beta1 activity $(16-20 \mathrm{~Hz})$ while suppressing theta activity (4-8 $\mathrm{Hz}$ ). Recordings are obtained at $\mathrm{Cz}$ with linked ear references, at $\mathrm{FCz}-\mathrm{PCz}$ with single ear reference, or at $\mathrm{Cz}-\mathrm{Pz}$ with ear reference. A variation of this protocol also has been reported in the treatment of ADHD, predominately inattentive type (Fuchs et al., 2003). In this training protocol, theta suppression and beta enhancement are reinforced at C3.

Protocol 3- SMR enhancement/beta2 suppression: in this protocol, children with ADHD, predominately hyperactive/impulsive type, are trained to increase SMR (12-15 Hz) while suppressing beta2 activity (22-30 Hz) (Fuchs et al., 2003) Recordings are obtained at C4 with linked ear reference. In ADHD, combined type, this protocol is used during half of each session. During the other portion of each training session, SMR enhancement/theta suppression at C3 is used. Selection of a neurofeedback protocol should be based on level of experience and training, accreditation, the fraction of the therapist's practice devoted to neurofeedback, reports from clients and objective assessments, and the therapist's specific experience in treating AD/HD (for more information see Monastra, 2005; Demos, 2005; Hammond et al., 2011).

Since the work of Lubar and Shous (1976), numerous studies have used neurofeedback approaches for treating ADHD and reported successful diminution of inattentivity and hyperactivity, and improvement in academic performance and concluded that despite some limitations, neurofeedback may be worthy of further consideration as a viable treatment approach for ADHD (Shouse and Lubar , 1979; Lubar and Lubar, 1984; Lubar et al., 1995; Rossiter and La Vaque, 1995; Linden et al., 1996; Thompson and Thompson, 1998; Kaiser and Othmer, 2000; Carmody et al., 2001; Monastra, 2002; Fuchs et al., 2003; Heywood and Beale, 2003; Cho et al., 2004; Heinrich et al., 2004; Rossiter et al., 2004; Xiong et al., 2005; Kropotov et al., 2005; Beauregard and Levesque, 2006; Levesque et al., 2006; Strehl et al., 2006; Gevensleben et al., 2010; Nazari et al., 2011; for review see Rossiter, 2004; ; Vernon et al., 2004; Monastra, 2005; Butnik, 2005; Friel, 2007; Toplak et al., 2008; John and Prichep, 2009; Coben and Evans, 2011). In an excellent meta-analytic study, Arns et al (2009) investigated results of 15 controlled studies. They concluded that neurofeedback treatment for ADHD can be considered "efficacious and specific" with a high effect size for inattention and impulsivity and a medium for hyperactivity (Arns et al., 2009).

Gevensleben et al (2009) conducted a randomized controlled trial encompassing 102 children with ADHD. In this trial behavioral and neurophysiological effects of neurofeedback, were analyzed in comparison to a computerised attention skills training (as a semi-active control group). They have shown neurofeedback to be superior to control group (Gevensleben et al., 2009). They reported follow-up behavioral data assessed 6 
months after completion of the training (either neurofeedback training or attention skills training). Improvements in the neurofeedback group at follow-up were superior to those of the control group and comparable to the effects at the end of the training. They concluded that "though treatment effects appear to be limited, the results confirm the notion that neurofeedback is a clinically efficacious module in the treatment of children with ADHD" (Gevensleben et al., 2010).

In a clinical outcome study, Nazari et al (2011) investigated whether neurofeedback compared to methylphenidate achieves an equally effective outcome. Participants were 39 children: 13 children with ADHD were trained to enhance the amplitude of the beta1 activity and decrease the amplitude of the theta activity, 13 of which were treated with methylphenidate alone, and 13 healthy children did not receive intervention. Several behavioral, neuropsychological and experimental tests were administered before and after intervention. While behavioral measures were improved by both types of method, methylphenidate was significantly more effective than neurofeedback. Response inhibition (assessed by Stroop) was improved only by neurofeedback. Both neurofeedback and methylphenidate were associated with improvements on the variability and accuracy measures of computerized attention tests. Intellectual ability (measured by full version of WISC-III) increased also by both methods. Although averaged effect size for methylphenidate seems to be greater than for neurofeedback, the difference was not significant. In conjunction with other studies they concluded that neurofeedback can significantly improve several behavioral and cognitive functions in children with ADHD and it might be an alternative treatment for ADHD, particularly for those their parents favor a nonpharmacological treatment (Nazari et al., 2011).

Neurofeedback is contraindicated with subjects under age six years, or subjects with mental retardation, developmental delay or other significant medical, neurological, or psychiatric disease. Subjects from families with significant marital discord that could interfere with participation in the treatment process (Friel, 2007).

Side effect can sometimes occur during neurofeedback and practitioners should be aware that occasionally negative effects may occur (Hammond \& Kirk, 2008; Hammond et al., 2001; Lubar \& Shouse, 1976; Todder et al., 2010) if training is not being supervised by a knowledgeable and certified professional. Adverse effects that have been reported by some clinicians include increased anxiety and agitation, headaches, fatigue, sleep disturbance, anger and irritability, crying and emotional lability, enuresis, an increase in depression, increase in somatic symptoms (including tics and twitches), seizures, and temporary disorientation. These reports are uncontrolled case reports from which one cannot know the degree to which other confounding events in the patients' lives may have contributed to these negative symptoms (Hammond \& Kirk, 2008). However, neurofeedback provider as a health-related profession should promote the welfare of their clients. Therefore, they should perform appropriate and objective assessments prior to, during and after providing neurofeedback to assess regularly the effectiveness of the services provided, and they inquire frequently about any side effects or adverse reactions. When it is observed that side effects or negative effects are occurring, providers document the details, discuss them with the client, and take appropriate action to remediate negative effects as quickly as possible. Such action may include modifying neurofeedback protocols, verifying the amount or frequency of treatment, utilizing adjunctive 
treatments, and seeking consultation (Hammond et al., 2011). It should be mentioned that patients with a history of epilepsy should only receive neurofeedback from practitioners who are well versed in neurofeedback for seizure disorders.

Some people interested in alternative health react to the neurofeedback with hesitation. Neurofeedback has been considered as a relatively unstudied treatment, and the studies that have been conducted have reportedly been problematic, due to methodological problems such as confounded treatments, inconsistent use of dependent measures, small sample size, and a lack of clinically meaningful dependent measures (Kline, Brann, \& Loney, 2002; Waschbusch \& Hill, 2003; Loo and Barkley, 2005; Holtmann and Stadler, 2006). In this line, there are some fundamental questions:

- Does neurofeedback result in the intended EEG changes?

- Is there really an effect that leads to significant modifications in cognition and behavior?

- Could these changes be reliably linked to neurofeedback training?

- How does it compare to the current standard of treatment?

- $\quad$ Are these changes retained over time?

- How does neurofeedback work?

For validating purpose, some controlled studies on healthy subjects (i.e. Egner and Gruzelier, 2001; Egner et al., 2002; Vernon et al., 2003; Egner and Gruzelier, 2004) assessed specific cognitive, neuropsychological and electrocortical effects from training of specific frequency bands. They concluded that the modulation of specific frequency bands led to significant and protocol-specific effects. It seems that despite these validation works much remains to be done to provide a scientific basis for neurofeedback.

It has been argued that a potential explanation of the effects of neurofeedback could be cognitive-behavioral training effect as well as client-therapist relationship effect since children are engaging in a training for often 30-50 sessions. Such concerns could be addressed by double-blind controlled studies. Considering the ethical problem of including untreated patient or patient undergoing placebo and the difficulty of conducting a doubleblind placebo controlled study in neurofeedback, some groups (Drechsler et al., 2007; Gevensleben et al., 2009) have still addressed these concerns by comparing neurofeedback group with a semi-active control group (can be considered a credible sham control). In these studies neurofeedback in comparison to this semi-active control group still had medium to large ES for inattention and impulsivity, and small to medium ES for hyperactivity (Arns et al., 2009).

La Vaque and Rossiter (2001) pointed out that, rather than comparing a new treatment (e.g., neurofeedback) to a no-treatment placebo, it should be compared to a protocol of 'known efficacy' to determine whether such an intervention would result in an equivalent effect. This type of design is often referred to equivalent study (Vernon et al., 2004). Regarding the well established efficacy of methylphenidate, several studies have compared the effects of neurofeedback and methylphenidate. Results revealed that although averaged effect size for methylphenidate was greater than for neurofeedback, both were in medium range and the difference was not significant (i.e. Nazari et al., 2011). None of the studies comparing neurofeedback with stimulant medication used random assignment. Although self-selection 
the treatment may bias these findings, self selection potentially maximizes the effects of expectancy in both groups. However, more studies using randomization and larger sample sizes are needed to investigate further how neurofeedback compares to stimulant medication in the treatment of ADHD.

Several follow-up studies (Monastra et al., 2002; Strehl et al., 2006; Gani et al., 2008; Gevensleben et al., 2010) showed that improvements in behavior and attention turned out to be stable. Test results for attention and some of the parents' ratings once more improved significantly. Based on these researches, it can be concluded that the clinical effects of neurofeedback are stable and might even improve further with time. This, in contrast to stimulant medication where it is known that when the medication is stopped often the initial complaints will come back again and recent evidence showing that temporary treatment with stimulant medication is not likely to improve long-term outcomes (Molina et al., 2009).

Yet another domain in need of further investigation involves the theoretical basis and the underlying mechanisms of neurofeedback impact. Today, to understand "how does neurofeedback work?" is one of the most interesting and challenging tasks. It is not surprising that the field of neuroscience attracts a lot of researchers try to answer this question.

Despite some limitations, neurofeedback may be worthy of further consideration as a viable treatment approach for ADHD (See Vernon, 2005; Friel, 2007; Toplak et al., 2008; Yucha and Montgomery, 2008; Arns et al., 2009). On the basis of currently available research results, the success of this therapeutic method is indicated by widespread utilization, and reports of carefully designed studies suggest the utility of this method (John and Prichep, 2009). EEG biofeedback therapy for $\mathrm{AD} / \mathrm{HD}$ results in significant improvement in cognitive functioning for 75-85 percent of patients. It is possible that faster and better outcomes might be achieved by combining other alternative therapies with EEG biofeedback (Friel, 2007). Neurofeedback meets the American Academy of Child and Adolescent Psychiatry criteria for clinical guideline for treatment of ADHD. As mentioned before, meta-analysis results of Arns and his colleagues (2009) demonstrated that neurofeedback treatment for ADHD can be considered "efficacious and specific".

Frank H. Duffy, M.D., Professor and Pediatric Neurologist at Harvard Medical School, stated in an editorial in the January 2000 issue of the Journal Clinical Electroencephalography that the scholarly literature suggests that neurofeedback should play a major therapeutic role in many difficult areas:

"In my opinion, if any medication had demonstrated such a wide spectrum of efficacy it would be universally accepted and widely used" ( $p$. v). "It is a field to be taken seriously by all." ( $p$. vii).

Until 2005, neurofeedback was reportedly used by more than 1500 practitioners (Butnik, 2005) and the last years have seen a rapid growth of the field of neurofeedback in the US and at least 27 countries (Budzynski et al., 2009). There are more than 100 health-related professions in Iran that using neurofeedback in their routine clinical practice. All of them have been trained by the Biofeedback Foundation of Europe-BFE (www.bfe.org) instructors in the Paarand Specialized Center for Human Enhancement-PSCHE (www.paarand.org). 


\section{References}

American Psychiatric Association. Diagnostic and Statistical Manual of Mental Disorders, 4th edn, American Psychiatric Press, Washington DC. 1994

Arns M, de Ridder S, Strehl U, Breteler M, Coenen A. Efficacy of neurofeedback treatment in ADHD: The effects on inattention, impulsivity and hyperactivity: A meta-analysis. Clinical EEG and Neuroscience. 2009;40(3):180-189.

Barkley RA. Behavioral inhibition, sustained attention, and executive functions: constructing a unifying theory of ADHD. Psychol Bull. 1997;121(1):65-94.

Barry RJ, Clarke AR, Johnstone SJ. A review of electrophysiology in attentiondeficit/hyperactivity disorder: I. Qualitative and quantitative electroencephalography. Clin Neurophysiol. 2003;114(2):171-183.

Beauregard M, Levesque J. Functional magnetic resonance imaging investigation of the effects of neurofeedback training on the neural bases of selective attention and response inhibition in children with attention-deficit/hyperactivity disorder. Appl Psychophysiol Biofeedback. 2006;31(1):3-20.

Boutros NN, IaconoWG, Galderisi S. Applied electrophysiology. In Sodock, B. J., Sodock, V. A. and Rouiz, P. (editors). Kaplan and Sodock's comprehensive textbook of psychiatry. Ninth edition, Philadelphia: Lippincott Williams \& Wilkins. 2009.

Budzynski TH, Budzynski HK, Evans JR, Abarbanel A. Introduction to Quantitative EEG and Neurofeedback: advanced theory and applications. Elsevier Inc. 2009.

Butnik SM. Neurofeedback in adolescents and adults with attention deficit hyperactivity disorder. J Clin Psychol. 2005;61(5):621-5.

Carmody DP, Radvanski DC, Wadhwani S, Sabo MJ, Vergara L. EEG biofeedback training and Attention-Deficit/Hyperactivity Disorder in an elementary school setting. Journal of Neurotherapy. 2001;4:5-27.

Cho BH, Kim S, Shin DI, Lee JH, Lee SM, Kim IY, et al. Neurofeedback training with virtual reality for inattention and impulsiveness. Cyberpsychol Behav. 2004;7(5):519-26.

Coben and Evans (eds). Neurofeedback and neuromodulation techniques and applications. Elsevier Inc. 2011

Demos JN. Getting Started with Neurofeedback. (2005). W.W. Norton \& Co.

Drechsler R, Straub M, Doehnert M, Heinrich H, Steinhausen H, Brandeis D. Controlled evaluation of a neurofeedback training of slow cortical potentials in children with ADHD. Behav Brain Funct. 2007; 3:35.

Drongelen W van . Signal Processing for Neuroscientists: Introduction to the Analysis of Physiological Systems. 2007. Elsevier, Amsterdam.

Egner T, Gruzelier JH. EEG biofeedback of low beta band components: frequency-specific effects on variables of attention and event-related brain potentials. Clin. Neurophysiol. 2004;115:131-139.

Egner T, Gruzelier JH. Learned self-regulation of EEG frequency components affects attention and event-related brain potentials in humans. NeuroReport. 2001;12:41554159. 
Egner T, Strawson E, Gruzelier JH. EEG signature and phenomenology of alpha/theta neurofeedback training versus mock feedback. Appl. Psychophysiol. Biofeedback. 2002;27:261-270.

Friel PN. EEG Biofeedback in the Treatment of Attention Deficit/Hyperactivity Disorder. Alternative Medicine Review. 2007;12(2):146-151.

Fuchs T, Birbaumer N, Lutzenberger W, Gruzelier JH, Kaiser J. Neurofeedback treatment for attention-deficit/hyperactivity disorder in children: a comparison with methylphenidate. Appl Psychophysiol Biofeedback. 2003;28(1):1-12.

Gani C, Birbaumer N, Strehl U. Long term effects after feedback of slow cortical potentials and of theta-beta-amplitudes in children with attentiondeficit/hyperactivity disorder (ADHD). Int J Bioelectromagn. 2008;10(4):209-232.

Gevensleben H, Holl B, Albrecht B, Schlamp D, Kratz O, Studer P, Rothenberger A, Moll GH, Heinrich H. Neurofeedback training in children with ADHD: 6-month followup of a randomized controlled trial. Eur Child Adolesc Psychiatry. 2010;19(9):71524.

Gevensleben H, Holl B, Albrecht B, Vogel C, Schlamp D, Kratz O, et al. Is neurofeedback an efficacious treatment for ADHD? A randomised controlled clinical trial. J Child Psychol Psychiatry. 2009;50(7):780-789.

Hammond D. Corydon , Bodenhamer-Davis, Genie , Gluck, Gerald , Stokes, Deborah , Harper, Sara Hunt , Trudeau, David , MacDonald, Margaret , Lunt, Joy and Kirk, Lynda .Standards of Practice for Neurofeedback and Neurotherapy: A Position Paper of the International Society for Neurofeedback \& Research. Journal of Neurotherapy. 2011;15(1):54-64.

Hammond DC, Gunkelman J. The art of artifacting. Corpus Christi, TX: International Society for Neuronal Regulation. 2001.

Hammond DC, Kirk L. First, do no harm: Adverse effects and the need for practice standards in neurofeedback. Journal of Neurotherapy. 2008;12(1):79-88.

Hammond DC, Stockdale S, Hoffman D, Ayers ME, Nash J. Adverse reactions and potential iatrogenic effects in neurofeedback training. Journal of Neurotherapy. 2001;4(4):5769.

Hammond DC, Walker J, Hoffman D, Lubar JF, Trudeau D, Gurnee R, Horvat J. Standards for the use of QEEG in neurofeedback: A position paper of the International Society for Neuronal Regulation. Journal of Neurotherapy. 2004;8(1): 5-26.

Heinrich H, Gevensleben H, Freisleder FJ, Moll GH, Rothenberger A. Training of slow cortical potentials in attention-deficit/hyperactivity disorder: evidence for positive behavioral and neurophysiological effects. Biol Psychiatry. 2004;55(7):772-5.

Heywood C, Beale I. EEG biofeedback vs. placebo treatment for attentiondeficit/hyperactivity disorder: a pilot study. J Atten Disord. 2003;7(1):43-55.

Hoffman DA, Lubar JF, Thatcher RW, Sterman MB, Rosenfeld PJ, Striefel S, et al.. Limitations of the American Academy of Neurology and American Clinical Neurophysiology Society paper on QEEG. Journal of Neuropsychiatry \& Clinical Neuroscience. 1999;11(3):401-405. 
Holtmann M, Stadler C. Electroencephalographic biofeedback for the treatment of attentiondeficit hyperactivity disorder in childhood and adolescence. Expert Rev Neurotherapeutics 2006; 6(4): 533-540.

Hughes JR, John ER. Conventional and quantitative electroencephalography in psychiatry. J Neuropsychiatry Clin Neurosci. 1999;11(2):190-208.

Jasper HH, Solomon P, Bradley C. Electroencephalographic analysis of behavior problems in children. American Journal of Psychiatry, 1938;95:641-658.

John ER, Prichep LS. Principles and applications of quantitative electroencephalography in psychiatry. In Sodock, B. J., Sodock, V. A. and Rouiz, P. (editors). Kaplan and Sodock's comprehensive textbook of psychiatry. Ninth edition, Philadelphia: Lippincott Williams \& Wilkins. 2009.

Kaiser DA, Othmer S. Effects of neurofeedback on variables of attention in a large multicenter trial. J Neurotherapy. 2000;4(1):5-5.

Klimesch W, Schimke H, Doppelmayr M, Ripper B, Schwaiger J, Pfurtscheller G. Eventrelated desynchronization (ERD) and the Dm-effect: does alpha desynchronization during encoding predict later recall performance? Int. J. Psychophysiol. 1996; 24: 47-60.

Kline JP, Brann CN, Loney BR. A cacophony in the brainwaves: A critical appraisal of neurotherapy for Attention Deficit Disorders. The Scientific Review of Mental Health Practice. 2002;1:46-56.

Kropotov JD, Grin-Yatsenko VA, Pomarev VA, Chutko LS, Yakovenko EA, Nikishena IS. ERPs correlates of EEG relative beta training in ADHD children. Int J Psychophysiol. 2005;55:23-34.

La Vaque TJ, Rossiter T. The ethical use of placebo controls in clinical research: the Declaration of Helsinki. Appl Psychophysiol Biofeedback. 2001;26(1):23-37.

Levesque J, Beauregard M, Mensour B. Effect of neurofeedback training on the neural substrates of selective attention in children with attention-deficit/hyperactivity disorder: a functional magnetic resonance imaging study. Neurosci Lett. 2006;394(3):216-21.

Linden M, Habib T, Radojevic V. A controlled study of the effects of EEG biofeedback on cognition and behavior of children with attention deficit disorder and learning disabilities. Biofeedback Self Regul. 1996;21(1):35-49.

Loo SK, Barkley RA. Clinical utility of EEG in attention deficit hyperactivity disorder. Appl Neuropsychol 2005; 12(2): 64-76.

Lubar JF, Shouse MN. EEG and behavioral changes in a hyperkinetic child concurrent with training of the sensorimotor rhythm (SMR): a preliminary report. Biofeedback Self Regul. 1976;1(3):293-306.

Lubar JF, Swartwood MO, Swartwood JN, O'Donnell PH. Evaluation of the effectiveness of EEG neurofeedback training for ADHD in a clinical setting as measured by changes in T.O.V.A. scores, behavioral ratings, and WISC-R performance. Biofeedback Self Regul. 1995;20(1):83-99.

Lubar JO, Lubar JF. Electroencephalographic biofeedback of SMR and beta for treatment of attention deficit disorders in a clinical setting. Biofeedback Self Regul. 1984;9(1):123. 
Lubar JF. Neurofeedback for the management of attention- deficit/hyperactivity disorders. In M. S. Schwartz (Ed.), biofeedback: A practitioner's guide. 2nd edition, New York: Guilford Press. 1995.

Molina BS, Hinshaw SP, Swanson JM, Arnold LE, Vitiello B, Jensen PS, Epstien JN, Hoza B, Hechtman L, Abikoff HB, et al. Prospective follow-up of children treated for combined type ADHD in a multisite study. J Am Child Adolesc Psych. 2009;48(5):461-462.

Monastra VJ, Monastra DM, George S. The effects of stimulant therapy, EEG biofeedback, and parenting style on the primary symptoms of attention-deficit/hyperactivity disorder. Appl Psychophysiol Biofeedback. 2002;27(4):231-49.

Monastra VJ. Electroencephalographic biofeedback (neurotherapy) as a treatment for attention deficit hyperactivity disorder: rationale and empirical foundation. Child Adolesc Psychiatr Clin N Am. 2005;14(1):55-82.

Nazari MA, Querne L, de Broca A, Berquin P. Effectiveness of EEG biofeedback as compared with Methylphenidate in the treatment of attention-deficit/hyperactivity disorder: A clinical outcome study. Neuroscience \& Medicine. 2011;2:78-86.

Nazari MA, Wallois F, Aarabi A, Berquin P. Dynamic changes in quantitative electroencephalogram during continuous performance test in children with attention-deficit/hyperactivity disorder. Int. J. Psychophysiol. 2011 Jul 14. [Epub ahead of print]

Nazari MA. Electroencephalographic characteristic of children with attentiondeficit/hyperactivity disorder and evaluation of the effects of neurofeedback on attentional fonctions.Ph.D. dissertation, University of Picardie Jules Verne, Amiens, France. 2008.

Niedermeyer E, da Silva FL. Electroencephalography: Basic Principles, Clinical Applications, and Related Fields. Lippincot Williams \& Wilkins. 2004.

Nuwer M. Assessment of digital EEG, quantitative EEG and EEG brain mapping: report of the American Academy of Neurology and the American Clinical Neurophysiology Society. Neurology. 1997;49:277-292.

Pasqual-Marqui RD, Michel CM, Lehmann D. Low resolution electromagnetic tomography: a new method for localizing electrical activity in the brain. Int J Psychophysiol. 1994;18:49-65.

Rossiter T. The effectiveness of neurofeedback and stimulant drugs in treating AD/HD: Part I. Review of methodological issues. Appl Psychophysiol Biofeedback. 2004;29(2):95112.

Rossiter T. The effectiveness of neurofeedback and stimulant drugs in treating AD/HD: part II. Replication. Appl Psychophysiol Biofeedback. 2004;29(4):233-43.

Rossiter TR, La Vaque TJ. A comparison of EEG biofeedback and psychostimulants in treating attention deficit/hyperactivity disorders. Journal of Neurotherapy. 1995;1:48-59.

Roth SR, Sterman MB, Clemente CC. Comparison of EEG correlates of reinforcement, internal inhibition, and sleep. Electroencephalogr Clin Neurophysiol .1967;23:50920. 
Sgrok, M., Roberts, W., Grossman, S., \& Barozzine, T. School board survey of attention deficit/hyperactivity disorder: Prevalence of diagnosis and stimulant medication therapy. Pediatric Child Health, 2000; 5, 12_23.

Shouse MN, Lubar JF. Operant conditioning of EEG rhythms and ritalin in the treatment of hyperkinesis. Biofeedback Self Regul. 1979;4(4):299-312.

Snyder SM, Hall JR. A meta-analysis of quantitative EEG power associated with attentiondeficit hyperactivity disorder. J Clin Neurophysiol. 2006;23(5):440-455.

Sterman MB, Wyrwicka W, Roth SR. Electrophysiological correlates and neural substrates of alimentary behavior in the cat. Ann N Y Acad Sci. 1969;157:723-39.

Sterman MB, Wyrwicka W. EEG correlates of sleep: evidence for separate forebrain substrates. Brain Res. 1967;6:143- 63.

Sterman MB. Basic concepts and clinical findings in the treatment of seizure disorders with EEG operant conditioning. Clin Electroencephalogr. 2000;31:45-55.

Strehl U, Leins U, Goth G, Klinger C, Hinterberger T, Birbaumer N. Self-regulation of slow cortical potentials: a new treatment for children with attention-deficit/hyperactivity disorder. Pediatrics. 2006;118(5):1530-40.

Thatcher TW. EEG normative databases and EEG biofeedback. Journal of Neurotherapy. 1998; 2(4):8-39.

Thompson L, Thompson M. Neurofeedback combined with training in metacognitive strategies: effectiveness in students with ADD. Appl Psychophysiol Biofeedback. 1998;23(4):243-63.

Todder D, Levine J, Dwolatzky T, Kaplan Z. Case report: Impaired memory and disorientation induced by delta band down-training over the temporal brain regions by neurofeedback treatment. Journal of Neurotherapy. 2010;14:153-155.

Toplak ME, Connors L, Shuster J, Knezevic B, Parks S. Review of cognitive, cognitivebehavioral, and neural-based interventions for Attention-Deficit/Hyperactivity Disorder (ADHD). Clin Psychol Rev. 2008; 28(5):801-823.

Vernon DJ. Can neurofeedback training enhance performance? An evaluation of the evidence with implications for future research. Appl Psychophysiol Biofeedback, 2005;30(4):347-364.

Vernon D, Frick A, Gruzelier J. Neurofeedback as a Treatment for ADHD: A Methodological Review with Implications for Future Research. Journal of Neu-rotherapy. 2004;8(2):53-82..

Vernon D, Egner T, Cooper N, Compton T, Neilands C, Sheri A, Gruzelier JH. The effect of training distinct neurofeedback protocols on aspects of cognitive performance. Int. J. Psychophysiol. 2003;47:75-85.

Waschbusch DA, Hill, GP. Empirically supported, promising, and unsupported treatments for children with Attention-Deficit/ Hyperactivity Disorder. In S. O. Lilienfield, S. Jay Lynn, \& J. M. Lohr (Eds.), Science and pseudoscience in clinical psychology. New York: Guilford Press, 2003; 333-362.

Wywricka W, Sterman MB. Instrumental conditioning of sensorimotor cortex EEG spindles in the waking cat. Physiol Behav. 1968;3:703-7. 
Xiong Z, Shi S, Xu H. A Controlled study of the effectiveness of EEG biofeedback training on children with attention deficit hyperactivity disorder. J Huazhong Univ Science Tech. 2005;25(3):368-370.

Yucha CB, Montgomery D. "Evidence-based practice in biofeedback and neurofeedback". Faculty Publications (N), 2008. 


\title{
The Effect of Psycho-Educational Therapy on Electroencephalographic Biofeedback Scores in Attention Deficit Hyperactivity Disorder
}

\author{
Irene Nikaina ${ }^{1}$, Aspa Paspali², \\ Georgia Kleidaria ${ }^{2}$ and Antigone Papavasiliou ${ }^{1}$ \\ ${ }^{1}$ Department of Neurology, Pendeli Children's Hospital, Athens \\ ${ }^{2}$ DIKEPSY, Athens \\ Greece
}

\section{Introduction}

Attention deficit/Hyperactivity (ADHD) disorder, a neuropsychiatric disorder characterized by inattention, hyperactivity and impulsivity, has been precisely described by the American Psychiatric Association (APA, DSM-IV, 2000). According to the diagnostic criteria these main symptoms must appear before the age of 7 years, be present for at least 6 months, be evident in at least two settings, and the impairment must contribute to social, academic, or occupational dysfunction. An increasing number of patients, mostly children, are diagnosed with ADHD. Recent studies focusing on the epidemiology of the disorder, reported variable prevalence of ADHD in childhood that reached in some studies the percentage of $14 \%$ (Barbaresi et al, 2004; Froehlich et al, 2007; Pliszka, 2007; Merinkagas et al, 2010; CDC, 2010). It is estimated that $30-70 \%$ of children with ADHD continue to experience ADHD symptoms into adulthood (Vollmer, 1998; Wender, 1997; Mannuzza et al, 1991; Elia et al, 1999).

The therapeutic approach of ADHD includes several treatment modalities (pharmacotherapy, behavioral, psychological and educational interventions). The choice of treatment depends mainly on the patient's characteristics such as age at diagnosis, the presence of co-morbid conditions and the therapeutic goals. Behavioral interventions alone have not been effective in ameliorating the core symptoms of ADHD to a significant extent (The Multimodal Treatment study of Children with ADHD [MTA], 1999; Brown et al, 2005). Their main contribution is the improvement of the behavioral problems that frequently accompany ADHD (Kolko et al, 1999; Pelham et al, 1998). Pharmacotherapy is one of the most efficacious treatment approaches both in children and adolescents. Stimulants have been for many years the first-line treatment for ADHD with a response rate of approximately $70 \%$ or more when patients are strictly complying with the treatment (MTA, 1999; Miller et al, 1998; Schachter et al, 2001). Atomoxetine seems to be about equally effective to stimulants, even though in a recent meta-analysis it was reported that stimulants (especially the long-lasting ones) demonstrated greater efficacy than atomoxetine during the short duration of 
treatment evaluation ( $\geq 2$ weeks) (Wang et al, 2007; Faraone \& Glatt, 2010). In most of the patients it is beneficial to combine medications with psychological and educational interventions especially when there is a suboptimal response to pharmacotherapy, a comorbid disorder and stressors in the family. A systematic review of 11 randomized controlled trials, including 428 children 5-18 years old, demonstrated that combination therapy with methylphenidate and behavioral/psychological treatments significantly improved ADHD behaviors, symptoms, and measures of academic achievement compared to behavior/psychological treatments alone (Lord \& Paisley, 2000). Guidelines on the management of ADHD in children include all aforementioned options, alone or in combination (American Academy of Pediatrics, 2001; Golgman et al, 1998; Dulcan, 1997).

The next important issue on ADHD management is the duration of the positive effects of these interventions. For example, the long term efficacy of medications has not been demonstrated. The Multimodal Treatment Study of Children with the combined type of ADHD (MTA study), which compared pharmacotherapy- short acting methylphenidate versus behavioral therapy or their combination or the standard care provided by community providers, reported in 2004 that treatment with stimulants for 24 months had a reduced effect, as compared to the effectiveness at 14-months published by the same group (MTA, 2004). At 3 years of follow-up of the same patients, the advantage of medications in comparison to all other treatment options disappeared although in all treatment groups an improvement on ADHD symptoms was observed compared to baseline (Jensen et al, 2007). The possible explanations of the loss of the pharmacotherapy advantage over all other treatments were an age-related decline in ADHD symptoms, changes in the intensity of medical management, and alternating periods of treatment/no-treatment after the 14 month study period. In 2002, Monastra et al demonstrated that patients treated with short acting stimulants (Ritalin) lost the benefits derived from the drugs one year after cessation of the drug (Monastra et al, 2002). Similar results had also been presented on the long term effectiveness of atomoxetine, 9 months after drug discontinuation (Michelson et al, 2004). All these observations along with the fact that $25-40 \%$ of children with ADHD do not respond to medications and other conservative treatments makes the research on alternative therapeutic approaches an interesting field (MTA, 1999; Pelham et al, 1998; Miller et al, 1998; Swanson et al, 1993; Barkley, 1998).

Neurofeedback, a form of biofeedback linked to brain activity, was first described as an option for ADHD treatment in the mid-1970's, by Lubar and Shouse (Lubar \& Shouse, 1976). The hypothesis behind the use of this method is that it may enable the user to modify brainwave activity in order to improve attention, reduce impulsivity and control hyperactive behavior. This modification is based on self-regulation of neuronal oscillations recorded by EEG. The goal is to enhance some types of EEG activity or reduce some others in one or more frequency bands by visual or auditory feedback; for example in ADHD, the aim is to enhance beta activity and to inhibit theta activity. There are two major categories of neurofeedback training protocols: one that uses frequency bands of the brain activity and a second which involves training of slow cortical potentials (SCPs), with the first being far more studied than the latter (Vernon et al, 2004). Concerning the first training strategy, three parameters are usually used in children with ADHD: theta frequency (4-8 Hz), beta frequency $(15-20 \mathrm{~Hz})$ and sensorimotor rhythm (SMR) $(12-15 \mathrm{~Hz})$. The target of the patient under training is to decrease the power of theta activity and/or to increase power of beta 
activity and SMR activity, in order to improve attention skills. Research protocols usually combine two or even more parameters. SCPs were first described by Heinrich et al in 2004 (Heinrich et al, 2004). They are event-related shifts of cortical activity lasting from several hundred milliseconds to several seconds (Birbaumer et al, 1990). The training of the SCPs involves an effort to increase positivity or negativity over sensorimotor cortex, in order to enable the subject to control cortical regulation and to allocate resources efficiently, functions that are supposed to be impaired in ADHD (Sergeant, 2000; 2005).

Several studies have demonstrated that neurofeedback has a favorable effect on ADHD symptomatology and cognitive function, as compared to both placebo and psychostimulants, regardless of the protocol used (Monastra et al, 2002; Arns et al, 2009; Gevensleben et al, 2009a, Drechsler et al, 2007; Heinrich et al, 2007; Levesque et al, 2006; Rossiter, 2004; Fuchs et al, 2003). Furthermore, in 2010, Gevensleben $\mathrm{H}$ et al published a 6 months follow-up study providing evidence for long-term benefits of neurofeedback training in children with ADHD (Gevensleben et al, 2010).

In spite of these promising results considerable controversy continues to surround neurofeedback as a therapeutic option for ADHD patients. The skepticism towards this type of training is based mostly on methodological shortcomings of the published research. Small sample size, non-randomized group assignment, and control conditions that do not control for confounding factors since they lack a double-blind setup are the major design problems, that are not easy to overcome (Heinrich et al, 2007). It is questionable whether behavioral improvements after neurofeedback training can be related to the improved cortical regulation or rather to non-specific treatment effects such as invested time and attention, therapist- patient interaction, expectancies, changes in the parental attitude, enhanced awareness of the problem or maturation-related factors. Since systematic reinforcement and a positive relationship to a therapist are integral parts of the neurofeedback training and of any other behavioral intervention especially with children, selective effects of the neurofeedback are difficult to isolate (Drechsler et al, 2007). The best way to resolve this is the correct choice of a control group. However, the implementation of a control condition for neurofeedback training is also difficult. For research purposes, the best control condition would be a placebo neurofeedback condition in which provided feedback is similar to neurofeedback, but not related to the child's own brain activity. The main advantage of this type of control condition would be that it could allow participating children, parents and trainers to be blinded for group allocation; thus confounding factors would be minimized. However, implementing a placebo control condition in a randomized controlled trial may lead to ethical concerns, mostly because this design also involves withholding evidencebased treatment (LaVaque \& Rossiter, 2001; Strehl et al, 2006). Moreover, it has been argued that placebo neurofeedback training may not be feasible since positive or negative therapeutic effects will be perceived by the patient or the therapist (Kotchoubey et al, 2001). Additionally, in a placebo-controlled study it is important to monitor adverse events during the training period in order to test the safety of the placebo condition, that could potentially represent a problem in this type of setting (Birbaumer et al, 1992). For these reasons, the use of a placebo-control study design is rather limited. To our knowledge, only one double-blind placebo-controlled trial has already been published (Lansbergen et al 2011). Other types of control groups that have been utilized are a waiting list control group (Heinrich et al, 2004; Levesque et al, 2006), children undergoing group therapy (Drechsler et al, 2007), and a group 
undergoing computerized attention training (Gevensleben et al, 2009a). Group therapy and computerized attention training may effectively control for unspecific effects such as invested time and treatment expectancies. Waiting list, on the other hand, may introduce bias because of maturational changes, multiple testing or changed parental attitudes (Drechsler et al, 2007). Finally, it has also been argued that neurofeedback training protocols may have a selection bias by design. Given the financial investment and time commitment required for the completion of neurofeedback training program, the majority of children who receive such a treatment are most likely from "stable, upper socioeconomic households, who may have simply improved over time because of their advantaged position" (Baydala \& Wikman, 2001).

Nevertheless, the evidence provided by the most recent and better designed studies is considered to be substantial if not yet conclusive and the use of neurofeedback training for ADHD had gained empirical support. In addition to the accumulating evidence on the effectiveness of neurofeedback in patients with ADHD, it is now suggested that this treatment may be integrated in a multimodal therapeutic approach (Gevensleben et al, 2009a; MTA, 1999). The combination of neurofeedback with standard therapies such as pharmacotherapy, and/or social skills training and parental interventions could result in additional improvements not only to the primary symptoms of the disorder but to other related areas such as learning, behavior and emotional stability. The aim of this study was to explore the interactions between neurofeedback and a program of psychological and educational interventions prescribed for the management of ADHD. Our hypothesis was that the combination of these treatments may induce larger improvements of the examined electrophysiological parameters studied than neurofeedback alone, suggesting a synergistic effect of these two treatment modalities on brain training.

\section{Methods}

\subsection{Patients}

Children consecutively evaluated in the outpatient Child Neurology clinic with an ADHD diagnosis according to DMS IV criteria were considered as candidates for this study. Only children that scored within the normal IQ range (IQ>80), according to WISC III, were included in the study. The presence of co-morbidities other than learning disorders was an exclusion criterion. After an initial evaluation by the clinical psychologist, the teacher of special education, the speech therapist and the occupational therapist, 30 children who fulfilled our inclusion and exclusion criteria were recruited for this study. All of them had learning difficulties in reading and writing.

\subsection{Interventions}

Two kinds of therapeutic interventions were proposed with the intention to be combined: a psycho-educational program (2 hours/week) and a biofeedback training protocol (2 hours/week). The combined program was not accepted by several families due mostly to financial reasons (the biofeedback was covered by most insurance agencies), or due to time restrictions. As a result we ended up with two groups: the first group included 15 children who attended the biofeedback program only (NF group) whereas in the second group $(n=15)$ children were treated with both biofeedback and psycho-educational treatment (NF+PSE group). 


\subsubsection{Neurofeedback}

The biofeedback protocol that was used was the Sensorimotor rhythm (SMR) enhancement/theta suppression. This is based on the concept that improved attention and behavioral control is achieved when the child learns to reduce the production of theta and increase the production of the 12-15 Hz sensorimotor rhythm generated over the electrodes $\mathrm{C} 3$ or $\mathrm{C} 4$ that are positioned over the motor cortex. EEG recordings were obtained from one active site (C3 or $\mathrm{C} 4)$, referenced to linked earlobes. Theta/ Beta ratio was measured before the training initiation, after the $20^{\text {th }}$ session and after the $40^{\text {th }}$ session. In each of these instances, the ratio was obtained in three conditions: Eyes closed, eyes opened and during an attentional task (eyes fixed task). Mean values were calculated over these three conditions.

The neurofeedback training program was designed to last for 40 sessions. Each neurofeedback session lasted for about 45 minutes. Each child worked individually under the supervision of a psychologist (certified neurofeedback trainer). Children were seated facing a computer screen. Once the electrodes were synchronized with the trainer's computer, the children were directed to start playing a computer game. In all the neurofeedback games, the children were asked to move a target, by using their gaze and by remaining concentrated on the target. For as long as the children remained with their gaze perfectly concentrated on the target, they gained points and completed the game by fulfilling the defined goal. Visual (animated figures) feedback was provided based on the patient's success in controlling a) the amplitude in microvolts of theta or sensorimotor rhythm (SMR) or b) the percentage of time that theta rhythm was below pre-treatment threshold or SMR was above pre-treatment threshold. Patients were informed via auditory (tone) and visual feedback when they produced 0.5 seconds of desired EEG activity. By the end of each game, the children got a sum of points, depending on their performance. The goal for each child was to achieve a score that is closer to what is considered appropriate for his/hers age level. Throughout a session the children played as many as 6 games. In between the games, they received immediate feedback on their performance so that they could then proceed to adjustments of the attention level. After a break of 1 to 2 minutes, they proceeded to the next one. This process was repeated throughout the whole session. At the end of each session, children and parents were informed on the total score.

\subsubsection{Psycho-educational}

This was based on the cognitive-behavioral approach for children with ADHD and was scheduled for 2 hours/week right after the neurofeedback session. The intervention program addressed both reading and writing difficulties and at the same time the specialized therapist provided support to the children who due to their learning difficulties struggled at school. Furthermore, both patients and parents received practical counselling with regard to time organisation and scheduled activities management.

\subsection{Statistical analysis}

Mean Theta/Beta ratio scores at the first session and after the $20^{\text {th }}$ and the $40^{\text {th }}$ (last) session, were tested for normality using Shapiro-Wilk distributional test. Normal data were expressed as mean values \pm standard variation (SD), whereas non normal data as median 
values $\left(25^{\text {th }}-75^{\text {th }}\right.$ percentile). The comparison of mean neurofeedback scores between the two treatment options was assessed with t-test when normally distributed or with MannWhitney U Test when non-normal. Categorical variables were compared with Chi square test or Fisher's exact test according to number of observations per cell. P-values for the tests applied in two different occasions (after the $20^{\text {th }}$ and after the $40^{\text {th }}$ session) were corrected with Bonferroni Correction for multiple comparison test, in order to reduce Type I error. Finally, mixed effects model was applied to test the longitudinal evolution of mean Theta/Beta ratio, taking into account the therapy group in which each patient is assigned. In these models, patient and number of sessions were treated as random variables.

\section{Results}

The patients ( $\mathrm{N}=30,15$ boys and 15 girls) had a mean age of 7 years 7 months $( \pm 1$ year 2 months). The two groups (NF and NF+PSE group) consisted of 15 patients each and they were similar in terms of age and sex. All patients followed their treatment consistently and there were no dropouts.

At baseline (first neurofeedback session), mean Theta/Beta ratios were not significantly different between the two therapy branches $(3.13 \pm 0.02$ for the NF+PSE group versus $3.10 \pm$ 0.2 for the NF group, p-value=0.265). Two out of the patients of the NF group managed to obtain the target ratio $(<3)$ already during their first neurofeedback training session.

\begin{tabular}{|c|c|c|c|}
\hline & $\begin{array}{c}\text { Theta/Beta ratio at } \\
\text { First session }\end{array}$ & $\begin{array}{c}\text { Theta/Beta ratio } \\
\text { after 20th session }\end{array}$ & $\begin{array}{c}\text { Theta/Beta ratio after } 40^{\text {th }} \\
\text { session }\end{array}$ \\
\hline NF group & $3.10 \pm 0.08$ & $3.03 \pm 0.10$ & $2.95 \pm 0.13$ \\
\hline $\begin{array}{c}\text { NF+PSE } \\
\text { group }\end{array}$ & $3.13 \pm 0.06$ & $3.05 \pm 0.06$ & $2.94 \pm 0.06$ \\
\hline
\end{tabular}

Table 1. Mean Theta/Beta values for children the two treatment groups at discrete observation times

Table 1 displays mean Theta/Beta ratios for children in both treatment groups. All patients presented a decrease in Theta/Beta ratio values during their treatment. According to the mixed effects model applied, this observed decrease was significant as treatment evolved ( $\mathrm{p}$ value $<0.001$ ). However, the size effect of time elapsed was quite small with the ratio after the $20^{\text {th }}$ session being as much as 0.078 units lower than its initial value, and after the $40^{\text {th }}$ session being as much as 0.172 units lower than its initial value. Similarly, age of patients was a significant predictor of mean Theta/Beta values obtained ( $p$-value $<0.001$ ). More specifically, children 8 or more years old had slightly lower mean values than those younger than 8 years, adjusted for treatment group and time of observation (coefficient $=-0.082,95 \%$ $\mathrm{CI}=$ between -0.127 and -0.038 ).

On the other hand, mean values of Theta/Beta ratios were not significantly different between the two treatment groups, at a precise time point (after the 20th or after the $40^{\text {th }}$ neurofeedback session $-\mathrm{p}$-value $=0.841$ ). These results are graphically demonstrated in Fig 1 . However, when we compared the percentages of patients who succeeded to obtain the 
target ratio between the therapy groups, we were able to display that significantly more patients of the NF+PSE group reached the target, as compared to the NF group only after the $40^{\text {th }}$ session $(14$ versus 6 patients respectively, $\mathrm{p}$-value $=0.006)$. The difference after the $20^{\text {th }}$ session was not significant (4 patients of the NF+PSE group versus 4 patients of the NF group, p-value=0.841) (Fig 2). In this analysis, we omitted the two patients of the NF group who had a successful first session, although their inclusion does not really affect the results.

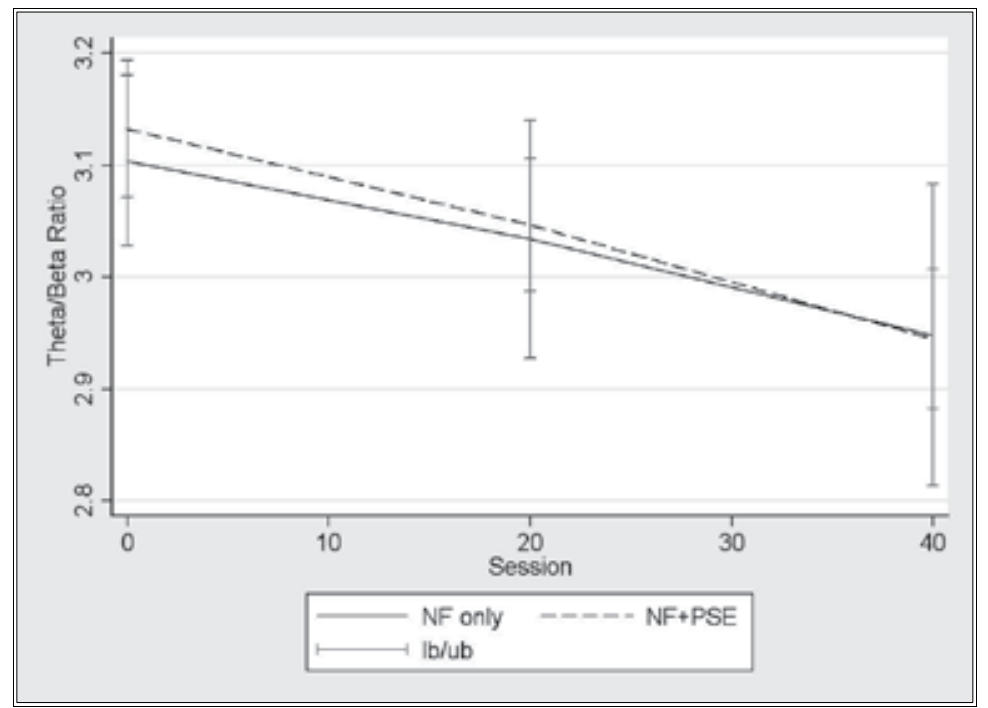

Fig. 1. Longitudinal evolution of mean Theta/Beta ratio for patients of the two treatment groups

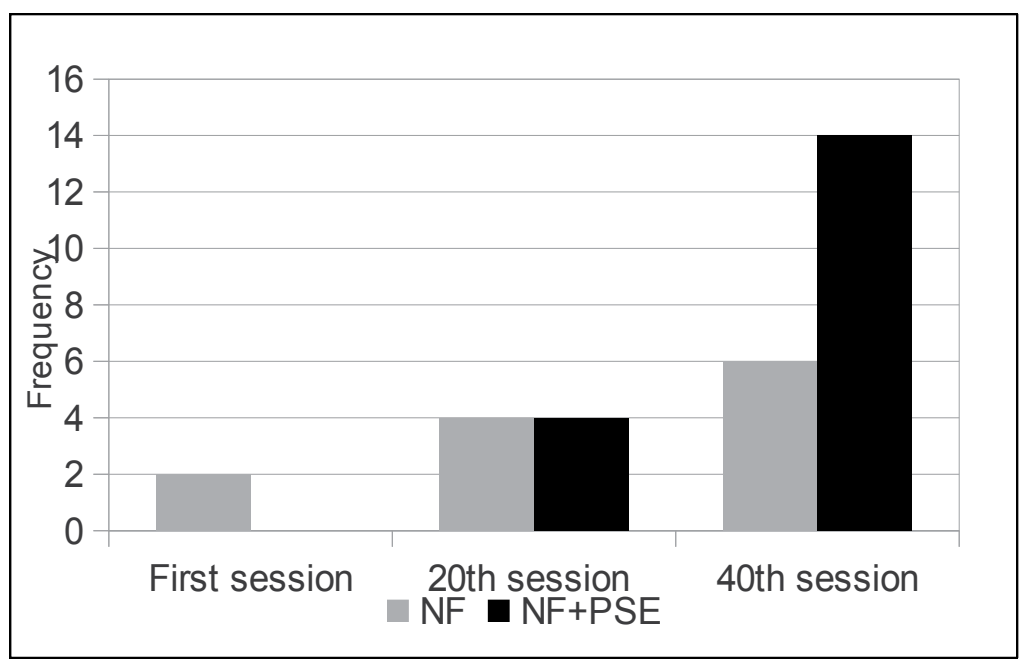

Fig. 2. Frequency of children who had achieved the goal of Theta/Beta $<3$ at the first, after the 20 th and after the $40^{\text {th }}$ session 


\section{Discussion}

The main result in this study was that the addition of psycho-educational treatment to EEG biofeedback resulted in normalization of Theta/Beta ratio and thus in reducing the slowing of the frontal brain activity. To our knowledge, this is the first study to report changes on neurofeedback studied parameters in relation to the psycho-educational intervention.

Up to the present time, only a few studies have assessed the effects of pharmacotherapy, mostly psychostimulants, on EEG patterns in children with ADHD. It has been demonstrated that the use of psychostimulants produce a decrease in Theta/Beta ratio in children with ADHD, to the point of reaching normal values and this was more prominent in the right hemisphere (Clarke et al, 2001a; Clarke et al, 2007; Dixit et al, 2002; Kerdar et al, 2007, Hermens et al, 2005). In their study in 2005, Song DH et al demonstrated that the treatment with methylphenidate induced an increase in Theta/Beta ratio in the right frontal and parieto-occipital region in 20 boys with ADHD, during continuous performance test (CPT) and not in resting states (Song et al, 2005).

When used as part of multimodal treatment in ADHD the psycho-educational intervention aims to maximize the likelihood of the child's academic success by developing areas of strength, by adapting to special needs and by remediating knowledge and skill deficits (William \& Smucher, 2001). There is great variability concerning the form of psychoeducational intervention in bibliography. It may include informative sessions on ADHD, behavioral strategies (parental training, child self-control instruction training, school-based techniques, family counseling) and/or educational packages in combination with behavioral techniques (Montoya et al, 2011).

In the present study, we were able to demonstrate that the psycho-educational program which was added to the treatment of ADHD children could enhance the effect of the neurofeedback protocol used. We have shown that the percentage of patients who reached EEG activity normalization was higher in the NF+PSE compared to the NF group. However, when we compared the longitudinal evolution of Theta/Beta ratio between the treatment groups, the psycho-educational program did not have an effect. Taking into account the fact that all children learned to reduce brain slowing but not all of them achieved normal Theta/Beta ratios during the 40 sessions programmed, it can be postulated that the implementation of a psycho-educational program may accelerate the neurofeedback training process. Whether the effect of the psycho-educational program is modified by other unspecified factors needs to be further studied .

One factor that has been demonstrated to be an important moderating factor of behavior at home, especially in children treated with neurofeedback, was parental support. In the study of Monastra et al, when "systematic" approaches towards parent mediated reinforcement strategies were used with patients treated with both neurofeedback and standard therapies, improved behavioral ratings were demonstrated not only at school environment but also at home, even when medication was discontinued (Monastra et al, 2002). On the contrary, when electrophysiological parameters were examined, as in the study of Drechsler et al, there was no evidence of a direct influence of parent directed approaches on successful cortical control, as $50 \%$ of the children who performed well in neurofeedback training and $66 \%$ of those who performed bad, belonged to the high parental support group (Drechsler et 
al, 2007). In the current study, we did not examine parental support as a factor that could affect the results of the prescribed treatment.

Table 1 reports absolute values of Theta/Beta ratios on three different occasions: at first session, after the $20^{\text {th }}$ session and after the 40th session. To our knowledge, only few neurofeedback studies focus on brain activity modification itself and EEG data are rarely available. Initial values for Theta/Beta ratio at first session were not similar to those reported by Monastra et al in 1999 for a group of ADHD children of the same age (Monastra et al, 1999). In this study, authors used a Theta/Beta value that was at least 1.5 SD higher than the value reported for normal age peers as a cutoff point for ADHD diagnosis. In the current study, mean Theta/Beta values at first session (practically before training) were lower as compared to those reported in the aforementioned study for children with ADHD but above the threshold for normal children 6-11 years old (which was set at the value of 3). On the other hand, the Theta/Beta ratios reported by Song et al in 20 patients with a mean age of 8.6 years (1.4 years) were lower than those presented in our study, in all brain regions that were examined (Song et al, 2005).

One explanation for this discordance in Theta/Beta values may be due to the positioning of electrodes. In some studies focusing on neurofeedback methodology, it was observed that when electrodes were fixed frontally, where more theta and less beta activity in patients with ADHD is found, then artifacts by eye movements may be a significant risk (Mann et al, 1992; Lubar et al, 1995; Chabot \& Serfontein, 1996). For this reason it is suggested that electrodes are placed in a more central position at C3f (= halfway between C3 and F3) and C4f (= halfway between C4 and F4) (Monastra et al, 1999). In the current study electrodes were positioned at $\mathrm{C} 3$ or $\mathrm{C} 4$ positions (a bit posteriorly in relation to the positions described previously), whereas in the study of Song et al Theta/Beta ratios were recorded in all 30 regions defined by the International 10/20 system but not over C3-C4 or C3f-C4f (Song et al, 2005; Monastra et al, 1999). A second explanation may be the difference in the methodology used and more precisely in the calculation of the mean Theta/Beta ratio. In both the aforementioned studies and in the current study, the Theta/Beta ratio was measured in different conditions (performance tasks, resting states, eyes open/closed) and therefore the produced mean values are not expected to be similar.

Furthermore, in the present study we did not make a discrimination of the ADHD type. The composition of the study group in our study could also account for the observed differences in the Theta/Beta values from the study of Monastra et al (Monastra et al, 2002). According to some authors children with ADHD-Combined Type may exhibit more absolute and relative theta activity, and higher Theta/Alpha and Theta/Beta ratios when compared to those with ADHD-Inattentive type (Clarke et al, 1998; Clarke et al, 2001b; Clarke et al, 2003). Although these results were not reproduced by other authors, they may suggest that children with ADHD- Combined Type show the classic pattern of greater underarousal and maturational delay more so than the children with ADHD- Inattentive type. In contrast, the latter group of patients exhibited more relative alpha activity in the posterior regions than those with ADHD-Combined Type, which is consistent with reports of slower cognitive processing and increased rates of daydreaming among these kids (Monastra et al, 2001; Loo \& Barkely, 2005).

As we have shown, Theta/Beta ratio decreased substantially, from the first to the $40^{\text {th }}$ session. Similar results were reported previously (Monastra et al, 2002; Thompson L \& Thompson M, 1998; Lubar et al, 1995; Loo \& Barkley, 2005; Gevensleben et al, 2009b; Leins et 
al, 2007). In the first of these studies, patients who underwent neurofeedback training presented a significant reduction on their Theta/Beta ratio values from above 5.03 (pretreatment) to less than 3 (post-treatment) (Monastra et al, 2002). A decrease in Theta/Beta ratio was also observed in a randomized study published in 2009 by Gevensleben and colleagues (Gevensleben et al, 2009). In this study, the modification of EEG activity demonstrated in 72 children with ADHD was attributed to a marked decrease of theta activity in posterior midline position. The findings of Leins et al are also in accordance, although they used a different approach (Leins et al, 2007). They studied Theta/Beta ratios in activation and in deactivation tasks and in feedback and transfer conditions, in order to produce similar states with the SCP training program. They observed that Theta/Beta ratio changed significantly in deactivation tasks but not in activation tasks and only in feedback conditions. In activation tasks, a decrease in Theta/Beta ratio was observed already within the first sessions of the treatment but, in the end, it did not reach significance. It should be also noted that in the current study just as in the study published in 2002 by Monastra et al, Theta/Beta values by the end of neurofeedback training were similar to values produced by non-ADHD age peers. However, this finding was not reproduced in the study of Carmody et al, in which normalization of spontaneous EEG after frequency neurofeedback training was not found (Carmody et al, 2001).

Another important aspect of the current study is that all members of the study sample proved to be capable of altering their brain wave frequency. The percentage of patients who learn how to increase cortical activity varies across the studies published. In most of them, this percentage was quite high. As in the present study, Lubar et al reported, in a part of their research, that all patients managed to slow cortical activity (Lubar et al, 1995). In support of these data, the majority of patients in the study of Lansbergen et al learned to increase SMR and decrease theta activity (Lansbergen et al, 2011). Nevertheless, it has also been reported that some participants fail to obtain satisfactory training results during EEG feedback or transfer (Drechsler et al, 2007; Lubar et al, 1995; Strehl et al, 2006; Kropotov et al, 2005). This result was mostly observed in training SCPs protocols and it was attributed to the protocol itself since the SCP training, which is rather strategic in nature and prone to artifacts, may be more difficult for children with ADHD than a neurofeedback training based on frequency ratios (Drechsler et al, 2001).

Finally, it was shown that the effect of psycho-educational program on neurofeedback parameters is present only after 40 sessions. In most studies, it seems that a total of 40 sessions is needed in order to achieve improvements in ADHD symptoms and in daily functioning. In the study of Monastra et al, the EEG neurofeedback training was stopped when participants obtained Theta/Beta ratio in the normal range for age (within 1.0 SD of age peers) for at least $40 \mathrm{~min}$ and for 3 consecutive treatment sessions (Monastra et al, 2002). The average number of sessions needed to reach this goal was 43 (range: 34-50). However, fewer sessions may also be enough to produce the desirable effect. In the study of Gevensleben et al, the alteration of brain activity required only 18 sessions (Gevensleben et al, 2009b). Similar were the results of Leins et al who suggested that patients learn to alter EEG activity at a very early stage of the training but they don't show any improvement during the following sessions (Leins et al, 2007). When it comes to long-term benefits from neurofeedback, Fox et al suggested that for a long lasting change on EEG frequencies, many sessions, up to 60 , may be required, even though it was demonstrated that $30 \%$ of patients treated with $\mathrm{ADHD}$ are capable of changing their brain waves frequency for a long time 
after as few as 20 sessions of neurofeedback training (Fox et al, 2005; Rossiter \& LaVaque, 1995). Importantly, the desired improvement on behavioral rating scales and the Test of Variables of Attention (TOVA) was also achieved in 20 sessions (Rossiter \& LaVaque, 1995).

The current study has several limitations. The main ones are the relatively small number of participants, the study design and the absence of follow-up data. Patients included in the research are not randomly assigned to the treatment groups and although they are similar for age and sex, they are not matched. Additionally, the procedure is not blinded, which in combination with the absence of randomization or matching, may allow confounding factors (such as parental expectation and style) to interfere with the results. Consequently, our results may not be easily generalized. Furthermore, although it was shown that the addition of a psycho-educational program does induce greater changes on Theta/Beta ratios towards normalization than neurofeedback alone, this effect is only apparent immediately after the completion of the training. We did not examine if that effect retained in the long term. If that proved to be true, it would greatly support the simultaneous use of neurofeedback with other behavioral interventions, based on a long standing, and hopefully permanent brain activity modification.

Another limitation of the current study is the lack of data concerning changes on patients' clinical characteristics. However, at this point of our investigation, the study was not designed to examine the correlation between changes presented in Theta/Beta ratios and behavioral alterations or if the addition of the psycho-educational program produced greater improvements on ADHD symptoms, in comparison with the presumed improvements due to neurofeedback training alone. In 1995, Lubar and coworkers found that all children who undertook the neurofeedback training showed improvements in behavior and attention, although not statistically significant. These improvements were present in both children who managed or did not manage to alter brain activity (Lubar et al, 1995). However, more recent research demonstrated that the extent of ADHD symptoms improvement along with the extent of neurophysiological parameters' improvement is positively correlated to the degree to which the patient is able to learn to alter his/her brain activity during training sessions. This correlation suggests that functional improvement is based on "the specific action of feedback-guided learning."(Hirshberg, 2007; Gruzelier \& Egner, 2005).

\section{Conclusion}

To conclude, the decision to combine a psycho-educational intervention with neurofeedback in management of children with ADHD may be an advantageous option. The psychoeducational program seems to accelerate the ability of the child to alter brain activity and to reach the normal range of values of electrophysiological parameters. Further research should be focused on the clarification of variables involved in this bi-modal approach e.g. the exact type of the psycho-educational intervention applied, with detailed description of patient and parent derivatives and the best neurofeedback protocol for each ADHD subtype, with the goal to achieve the maximum effect not only on the brain activity but also on the ADHD symptoms. It would also be of importance to examine long term effects of the two treatments, on brain electrophysiolology and on clinical parameters such as ADHD symptoms, academic achievement and even quality of life. 


\section{References}

American Academy of Pediatrics. Subcommittee on Attention-Deficit/Hyperactivity Disorder and Committee on Quality Improvement. (2001) Clinical practice guideline: treatment of the school-aged child with attention-deficit/hyperactivity disorder. Pediatrics, vol. 108, No. 4, pp 1033-44

American Psychiatric Association. (2000).Diagnostic and statistical manual of mental disorders (DSM-IV-TR) (4th edition., text revision). American Psychiatry Association, Washington, DC.

Arns, M.; de Ridder, S.; Strehl, U.; Breteler, M. \& Coenen, A. (2009). Efficacy of neurofeedback treatment in ADHD: the effects on inattention, impulsivity and hyperactivity: a metaanalysis. Clinical EEG and Neuroscience, vol.40, No. 3, pp 180-189

Barbaresi, W.; Katusic, S.K.; Colligan, R.C.; Weaver, A.; Pankratz, V.; Mrazek, D. \& Jacobsen, S. (2004). How common in attention-deficit/hyperactivity disorder? Towards resolution of the controversy: results from a population-based study. Acta Paediatrica Supplement, vol. 93, No. 445 pp 55-9.

Barkley, R.A. (1998). Attention-deficit hyperactivity disorder: A handbook of diagnosis and treatment (2nd ed.) Guilford Press. New York.

Baydala, L. \& Wikman, E. (2001).The efficacy of neurofeedback in the management of children with attention deficit/hyperactivity disorder. Paediatrics $\mathcal{E}$ Child Health, vol 6, No. 7, pp 451-55

Birbaumer, N.; Elbert, T.; Canavan, A.G. \& Rockstroh, B. (1990). Slow potentials of the cerebral cortex and behavior. Physiological Reviews, vol. 70, No. 1, pp 1-41.

Birbaumer, N.; Elbert, T.; Rockstroh, B.; Daum, I.; Wolf, P. \& Canavan, A. (1992). Clinicalpsychological treatment of epileptic seizures: a controlled study. In: Ehlers, A.; Fiegenbaum, W.; Florin, I. \& Margraf J (eds). Perspectives and Promises of Clinical Psychology. Plenum Press, New York, pp 81-96

Brown, R.T.; Amler, R.W.; Freeman, W.S.; Perrin, J.M.; Stein, M.T.; Feldman, H.M.; Pierce, K. \& Wolraich, M.L. (2005) Treatment of attention-deficit/hyperactivity disorder: overview of the evidence. Pediatrics, vol. 115, No. 6, pp 749-57.

Centers for Disease Control and Prevention. (2010). Increasing prevalence of parentreported attention-deficit/hyperactivity disorder among children - United States, 2003 and 2007. MMWR Morbidity and Mortality Weekly Report, vol. 59, pp 1439-1443.

Chabot, R.A. \& Serfontein, G. (1996). Quantitative electroencephalographic profiles of children with attention deficit disorder. Biological Psychiatry, vol. 40, No. 10, pp 951-963.

Clarke, A.R.; Barry, R.J.; McCarthy, R. \& Selikowitz, M. (1998). EEG analysis in AttentionDeficit/Hyperactivity Disorder: A comparative study of two subtypes. Psychiatry Research, vol. 81, No. 1, pp 19-29.

Clarke, A.R.; Barry, R.J.; Bond, D.; McCarthy, R. \& Selikowitz, M. (2001a). Effects of stimulant medications on the EEG of children with attention-deficit/hyperactivity disorder. Psychopharmacology (Berl), vol. 164, No. 3, pp 277-84.

Clarke, A.R.; Barry, R.J.; McCarthy, R. \& Selikowitz, M. (2001b). Age and sex effects in the EEG: differences in two subtypes of attention-deficit/hyperactivity disorder. Clinical Neurophysiology, vol. 112, No. 5, pp 815-826.

Clarke, A.R.; Barry, R.J.; McCarthy, R. \& Selikowitz, M. Clarke DC, Croft RJ. (2003). Effects of stimulant medications on children with attention-deficit/hyperactivity disorder and excessive beta activity in their EEG. Clinical Neurophysiology, vol. 114, No. 9, pp 1729-1737. 
Clarke, A.R.; Barry, R.J.; McCarthy, R.; Selikowitz, M. \& Johnstone, S.J. (2007) Effects of stimulant medications on the EEG of girls with Attention-Deficit/Hyperactivity Disorder. Clinical Neurophysiology, vol. 118, No. 12, pp 2700-8.

Carmody, D.P.; Radvanski, D.C.; Wadhwani, S.; Sabo, M.J. \& Vergara, L. (2001). EEG biofeedback training and attention-deficit/hyperactivity disorder in an elementary school setting. Journal of Neurotherapy, vol. 43, pp 5-27.

Dixit, S.P.; Pandey, M.N. \& Dubey, G.P. (2002). Management of attention deficit/hyperactivity disorder--use of an effective paradigm. Indian Journal of Medical Sciences; vol. 56, No. 8, pp 376-80.

Drechsler, R.; Straub, M.; Doehnert, M.; Heinrich, H.; Steinhausen, H.C. \& Brandeis, D. (July 2007). Controlled evaluation of a neurofeedbacktraining of slow cortical potentials in children with Attention deficit/hyperactivity disorder (ADHD). Behavioral and Brain Functions, 3:35. In: BioMed Central Available from: http:/ / www.behavioralandbrainfunctions.com/Content/3/1/35

Dulcan, M. (1997) Practice parameters for the assessmentand treatment of children, adolescents, and adults with attention-deficit/hyperactivity disorder. Journal of American Academy of Child and Adolescent Psychiatry, vol. 36, No. 10 (Suppl), pp 85S-121S

Elia, J.; Ambrosini, P.J. \& Rapoport, J.L. (1999). Treatment of attention-deficit-hyperactivity disorder. New England Journal of Medicine, vol. 340, No. 10, pp 780-88

Faraone, S.V. \& Glatt, S.J. (2000). A comparison of the efficacy of medications for adult attention-deficit/hyperactivity disorder using meta-analysis of effect sizes. The Journal of Clinical Psychiatry, vol. 71, No. 6, pp 754-63, 2010

Froehlich, T.E.; Lanphear, B.P.; Epstein, J.N.; Barbaresi, W.J.; Katusic, S.K. \& Kahn, R.S. (2007). Prevalence, recognition, and treatment of attention-deficit/hyperactivity disorder in a national sample of US children. Archives of Pediatric $\mathcal{E}$ Adolescent Medicine, vol. 161, No. 9, pp 857-64.

Fox, D.J.; Tharp, D.F. \& Fox, L.C. (2005). Neurofeedback: an alternative and efficacious treatment for Attention Deficit Hyperactivity Disorder. Applied Psychophysiology and Biofeedback, vol. 30, No. 4, pp 365-73.

Fuchs, T.; Birbaumer, N.; Lutzenberger, W.; Gruzelier, J.H. \& Kaiser, J. (2003). Neurofeedback treatment for attention-deficit/ hyperactivity disorder in children: A comparison with methylphenidate. Applied Psychophysiology and Biofeedback, vol. 28, No. 1, pp 1-12.

Gevensleben, H.; Holl, B.; Albrecht, B.; Vogel, C.; Schlamp, D.; Kratz, O.; Studer, P.; Rothenberger, A.; Moll, G.H. \& Heinrich, H. (2009a). Is neurofeedback an efficacious treatment for ADHD? A randomised controlled clinical trial. Journal of Child Psychology and Psychiatry, vol.50, No. 7, pp 780-789.

Gevensleben, H.; Holl, B.; Albrecht, B.; Schlamp, D.; Kratz, O.; Studer, P.; Wangler, S.; Rothenberger, A.; Moll, G.H. \& Heinrich, H. (2009b). Distinct EEG effects related to neurofeedback training in children with ADHD: a randomized controlled trial. International Journal of Psychophysiology, vol. 74, No. 2, pp 149-57.

Gevensleben, H.; Holl, B.; Albrecht, B.; Schlamp, D.; Kratz, O.; Studer, P.; Rothenberger, A.; Moll, G.H. \& Heinrich H. (2010). Neurofeedback training in children with ADHD: 6-month follow-up of a randomised controlled trial. European Child $\mathcal{E}$ Adolescent Psychiatry, vol. 19, No. 9, pp 715-724.

Golgman, L.S.; Genel, M.; Bezman, R.J. \& Slnetz, P.J. (1998) Diagnosis and treatment of attention-deficit/hyperactivity disorder in children and adolescents. Council on Scientific Affairs. American Medical Association. JAMA, vol. 279, No. 14 pp 1100-17. 
Gruzelier, J. \& Egner, T. (2005) Critical validation studies of neurofeedback. Child $\mathcal{E}$ Adolescent Psychiatric Clinics of North America, vol 14, No. 1, pp 83-104

Heinrich, H.; Gevensleben, H.; Freisleder, F.J.; Moll, G.H. \& Rothenberger A. (2004). Training of slow cortical potentials in ADHD: evidence for positive behavioral and neurophysiological effects. Biological Psychiatry, vol. 55, No. 7, pp 772-775.

Heinrich, H.; Gevensleben, H. \& Strehl, U. (2007) Annotation: neurofeedback - train your brain to train behaviour. Journal of Child Psychology and Psychiatry, vol. 48, No. 1, pp 3-16.

Hermens, D.F.; Williams, L.M.; Clarke, S.; Kohn, M.; Cooper, N. \& Gordon, E. (2005) Responses to methylphenidate in adolescent AD/HD: evidence from concurrently recorded autonomic (EDA) and central (EEG and ERP) measures. International Journal of Psychophysiology, vol. 58, No. 1, pp 21-33.

Hirshberg, L.M. (2007). Place of electroencephalographic biofeedback for attention deficit/hyperactivity disorder. Expert Review of Neurotherapeutics, vol. 7, No.4, pp 315-19

Jensen, P.S.; Arnold, L.E.; Swanson, J.M.; Vitiello, B.; Abikoff, H.B.; Greenhill, L.L.; Hechtman, L.; Hinsahaw, S.P.; Pelham, W.E.; Wells, K.C.; Conners, C.K.; Elliott, G.R.; Epstein, J.N.; Hoza, B., March, J.S.; Molina, B.S.; Newcorn, J.H.; Severe, J.B.; Wigal, T.; Gibbons, R.D. \& Hur, K. (2007) 3-year follow-up of the NINH MTA study. Journal of American Academy of Child and Adolescent Psychiatry, vol. 46, No. 8, pp 989-1002.

Kerdar, M.S.; Scheuerpflug, P.; Srdinko, P.; Wewetzer, C.; Warnke, A. \& Romanos, M. (2007) Quantitative effect of treatment with methylphenidate on EEG--a pilot study. Zeitschrift fur Kinder -und Jugendpsychiatrie und Psychotherapie, vol. 35, No. 4, pp 247-55

Kolko, D.J.; Bukstein, O.G. \& Barron, J. (1999) Methylphenidate and behavior modification in children with ADHD and comorbid ODD or CD: main and incremental effects across settings. Journal of American Academy of Child and Adolescent Psychiatry, vol. 38, No. 5, pp 578-86.

Kotchoubey, B.; Strehl, U.; Uhlmann, C.; Holzapfel, S.; Konig, M,; Froscher, W.; Blankenhorn, V. \& Birbaumer, N. (2001) Modification of slow cortical potentials in patients with refractory epilepsy: a controlled outcome study. Epilepsia, vol. 42, No. 3, pp 406-416

Kropotov, J.D.; Grin-Yatsenko, V.A.; Ponomarev, V.A.; Chutko, L.S.; Yakovenko, E.A. \& Nikishena, IS. (2005) ERPs correlates of EEG relative beta training in ADHD children. International Journal of Psychophysiology, vol. 55, pp 23-34.

Lansbergen, M.M.; van Dongen-Boomsma, M.; Buitelaar, J.K. \& Slaats-Willemse, D. (2011) ADHD and EEG-neurofeedback: a double-blind randomized placebo-controlled feasibility study. Journal of Neural Transmission, vol. 118, No. 2, pp 275-84.

La Vaque, T.J. \& Rossiter, T. (2001) The ethical use of placebo controls in clinical research: the Declaration of Helsinki Applied Psychophysiology and Biofeedback, vol 26, No. 1, pp 23-37

Leins, U.; Goth, G.; Hinterberger, T.; Klinger, C.; Rumpf, N. \& Strehl, U. (2007) Neurofeedback for Children with ADHD: A Comparison of SCP and Theta/Beta Protocols. Applied Psychophysiology and Biofeedback, vol. 32, No. 2, pp 73-88.

Levesque, J.; Beauregard, M. \& Mensour, B. (2006) Effect of neurofeedback training on the neural substrates of selective attention in children with attentiondeficit/hyperactivity disorder: a functional magnetic resonance imaging study. Neuroscience Letters, vol. 394, No. 3, pp 216-221

Loo, S.K. \& Barkley, R.A. (2005) Clinical Utility of EEG in Attention Deficit Hyperactivity Disorder. Applied Neuropsychology, vol. 12, No. 2, pp 64-76

Lord, J. \& Paisley, S. The clinical effectiveness and cost-effectiveness of methylphenidate for hyperactivity in childhood. National Institute for Clinical Excellence. Version 2. London. 
Lubar, J.F. \& Shouse, M.N. (1976) EEG and behavioral changes in a hyperactive child concurrent with training of the sensorimotor rhythm (SMR): A preliminary report. Biofeedback and Self-Regulation, vol. 1, No. 3, pp 293-306.

Lubar, J.F.; Swartwood, M.O.; Swartwood, J.N. \& O'Donnell, P.H. (1995) Evaluation of the effectiveness of EEG neurofeedback training for ADHD in a clinical setting as measured by changes in T.O.V.A. scores, behavioral ratings and WISC-R performance. Biofeedback and Self-Regulation, vol. 20, No. 1, pp 83-99.

Mann, C.A.; Lubar, J.F.; Zimmerman, A.W.; Miller, C.A. \& Muenchen, R.A. (1992) Quantitative analysis of EEG in boys with attention deficit hyperactivity disorder: Controlled study with clinical implications. Pediatric Neurology, vol. 8, No. 1, pp 30-36

Mannuzza, S.; Klein, R.G.; Bonagura, N.; Malloy, P.; Giampino, T.L. \& Addalli, K.A. (1999). Hyperactive boys almost grown up. V, Replication of psychiatric status. Archives of General Psychiatry, vol. 48, No. 1, pp 77-83

Merinkagas, K.R.; He, J.P.; Brody, D.; Fisher, P.W.; Bourdon, K. \& Koretz, D.S. (2010) Prevalence and treatment of mental disorders among US children in 2001-2004 NHANES. Pediatrics, vol. 125, No. 1, pp 75-81.

Michelson, D.; Buitelaar, J.K.; Danckaerts, M.; Gillberg, C.; Spencer, T.J.; Zuddas, A.; Faries, D.E.; Zhang, S. \& Biederman, J. (2004) Relapse prevention in pediatric patients with ADHD treated with atomoxetine: a randomized, double-blind, placebo-controlled study Journal of American Academy of Child and Adolescent Psychiatry., vol. 43, No. 7, pp 896-904

Miller, A.; Lee, S.K.; Raina, P.; Klassen, A.; Zupancic, J. \& Olsen, L. (1998). A review of therapies for attention-deficit/hyperactivity disorder. Ottawa: Canadian Coordinating Office for Health Technology Assessment (CCOHTA). Available from: http://www.ccohta.ca/main-e.html.

Monastra, V.J.; Lubar, J.F.; Linden, M.K.; VanDeusen, P.; Green, G.; Wing, W. ; Phillips, A. \& Fenger, T.N. (1999) Assessing attention deficit hyperactivity disorder via quantitative electroencephalography: An initial validation study. Neuropsychology, vol. 13, No. 3, pp 424-433.

Monastra, V.J.; Lubar, J.F. \& Linden, M. (2001) The development of a quantitative electroencephalographic scanning process for attention deficit-hyperactivity disorder: Reliability and validity studies. Neuropsychology, vol. 15, No. 1, pp 136-144.

Monastra, V.J.; Monastra, D.M. \& George, S. (2002) The effects of stimulant therapy, EEG biofeedback, and parenting style on the primary symptoms of attentiondeficit/hyperactivity disorder. Applied Psychophysiology and Biofeedback, vol. 27, No. 4, pp 231-249.

Montoya, A.; Colom, F. \&, Ferrin, M. (2011) Is psychoeducation for parents and teachers of children and adolescents with ADHD efficacious? A systematic literature review. European Psychiatry, vol. 26, No. 3, pp 166-75.

MTA Cooperative Group. Multimodal treatment Study of Children with ADHD. (1999) A 14month randomized clinical trial of treatment strategies for attention-deficit/ hyperactivity disorded. Archives of General Psychiatry, vol. 56, No. 12, pp 1073-86.

MTA Cooperative Group. National Institute of Mental Health (2004) Multimodal Treatment Study of ADHD follow-up: Changes in effectiveness and growth after the end of treatment. Pediatrics, vol. 113, No. 4, pp 762-9.

Pelham, W.E.Jr.; Wheeler, T. \& Chronis, A. (1998) Empirically supported psychosocial treatments for attention deficit hyperactivity disorder. Journal of Clinical Child Psychology, vol. 27, No. 2 pp 190-205. 
Pliszka, S.; AACAP Work Group on Quality Issues. (2007). Practice parameter for the assessment and treatment of children and adolescents with attention-deficit/hyperactivity disorder. Journal of American Academy of Child and Adolescent Psychiatry, vol. 46, No. 7, pp 894-921.

Rossiter, T.R. \& La Vaque, T.J. (1995). A comparison of EEG biofeedback and psychostimulants in treating attention deficit/hyperactivity disorders. Journal of Neurotherapy, vol. 1, No. 1, pp 48-59

Rossiter, T. (2004) The effectiveness of neurofeedback and stimulant drugs in treating AD/HD: part II. Replication. Applied Psychophysiology and Biofeedback, vol.29, No. 4, pp 233-243.

Schachter, H.M.; Pham, B.; King, J.; Langford, S. \& Moher, D. (2001) How efficacious and safe is short-acting methylphenidate for the treatment of attention-deficit disorder in children and adolescents? A meta-analysis. Canadian Medical Association journal, vol. 165, No. 11, pp 1474-88.

Sergeant, J.A. (2000) The cognitive-energetic model: an empirical approach to attention-deficit hyperactivity disorder. Neuroscience and Biobehavioral Reviews, vol. 24, No. 1, pp 7-12.

Sergeant, J.A. (2005) Modeling attention-deficit/hyperactivity disorder: a critical appraisal of the cognitive-energetic model. Biological Psychiatry, vol. 57, No. 11, pp 1248-55.

Song, D.H.; Shin, D.W.; Jon, D.I. \& Ha, E.H. (2005) Effects of methylphenidate on quantitative EEG of boys with attention-deficit hyperactivity disorder in continuous performance test. Yonsei Medical Journal, vol. 46, No. 1, pp 34-41.

Strehl, U.; Leins, U.; Goth, G.; Klinger, C.; Hinterberger, T. \& Birbaumer, N. (2006) Selfregulation of slow cortical potentials: a new treatment for children with attentiondeficit/hyperactivity disorder. Pediatrics, vol. 118, No. 5, pp 1530-40.

Swanson, J.; McBurnett, T.; Wigal, T.; Pfiffner, L.; Lerner, M.; Williams, L.; Christian, D.L.; Tamm, L.; Willcutt, E.; Crowley, K.; Clevenger, W.; Khouzam, N.; Woo, C.; Crinella, F.M. \& Fisher, T.D. (1993) Effect of stimulant medication on children with attention deficit disorder: A "review of reviews. Exceptional Children, vol.60, pp 154-162.

Thompson, L. \& Thompson, M. (1998) Neurofeedback combined with training in metacognitive strategies: effectiveness in students with ADD. Applied Psychophysiology and Biofeedback, vol. 23, No. 4, pp 243-63

Vernon, D.; Frick, A. \& Gruzelier, J. (2004) Neurofeedback as a treatment for ADHD: A methodological review with implications for future research. Journal of Neurotherapy, vol. 8, No. 2, pp 53-82.

Vollmer, S. (1998) AD/HD: it's not just in children. Family Practice Recertification, vol. 20, pp 45-68,

Wang, Y.; Zheng, Y.; Du, Y.; Song, D.H.; Shin, Y.J.; Cho, S.C.; Kim, B.N.; Ahn, D.H.; MarquezCaraveo, M.E.; Gao, H.; Williams, D.W. \& Levinem L,R. (2007) Atomoxetine versus methylphenidate in paediatric outpatients with attentiondeficit hyperactivity disorder: a randomized, double-blind comparison trial. The Australian \& New Zealand Journal of Psychiatry, vol. 41, No. 3 pp 222-30.

Wender, P.H. (1997). Attention-Deficit Hyperactivity Disorder in Adults. Oxford University Press, New York.

William, D. \& Smucher MH. (2001) Evaluation and Treatment of ADHD. Americal Family Physician, vol. 64, No. 5, pp 817-30 

